\title{
Hispanic Environmental and Waste Management Outreach Project
}

Final Report to the United States Department of Energy Office of Environmental Management

October 1,1997 -July 25,1998 
Seli Rruance Foundanon

Hispanic Environmental and Waste Management Outreach

Project Identification No. DE-FC26-97FT34346

\section{Table of Contents}

$\begin{array}{lc} & \text { Page } \\ \text { Project Overview } & 1 \\ & \\ \text { Environmental Literacy Plan } & 1 \\ \text { DOE - EM Communication Plan } & 2 \\ \text { Project Metrics } & 4 \\ \text { Third Quarter Activity Summary } & 5 \\ \text { Bucando la Belleza Radio Affliates } & 9 \\ \text { Planeta Azul Radio Affliates } & 10\end{array}$

Analysis - $1^{\text {s and }} 2^{\text {a }}$ Caller Demographics:

$11-14$

- Figure 1: Number of Episodes Broadcast

- Figure 2: DOE-EM Callers by Gender

- Figure 3: DOE-EM Callers by Age

- Figure 4: DOE-EM Callers by English Proficiency

- Figure 5: DOE-EM Callers by Educarion Level

Appendices:

Program Guide for Buscando la Belleza

Program Guide for Planeta Azul

II.

Radio Afrliate List for Buscando la Belleza

III.

Radio Affliate List for Planeta Azul

IV.

DOF - EM Caler Reports

V. 


\section{Marketron/ Arbitron Ratings Analysis}

\section{Disclaimer}

This repont was prepared an account of work sponsored by an agency of the Urited States Goverment Nether the Unied States Gowernmant nor any agency thereof nor any of their employees, make any waranty, express or impled, or assumes amy legal liability or responsibility for the accurncy, completeness, or useluhess of any information, apparatus, produce, or process disclosed, or represents that its use would not infmge protely owned rights. Reference heren to any specific commercial product, process, or service by trade name, tradenak, manufacturer, or otherwise does not necestarly constitute or imply its endorsemenl, recommendation, or favomg by the United Srates Govemmen or any agenoy thereof. The hews and opinions of auhors expressed heren do not necesarily state or reheci those of the United States Govermeni or agercy thereof 
Sel Reliance Foundation Hispanic Evvironmental and Waste Managenent Outreach

\section{Project Overview}

The Department of Energy Office of Environmental Maragenent (DOE.EM) in cooperation with the Self Reliance Foundation (SRF) is conducting the Hisparic Environmental and Waste Managenent Outreach Project (HEWMO) to increase science and environmental literacy, specificaly that related to nuclear engineering and waste management in the nuclear industry, among the US. Hisparic population. The project will encourage Hispanic youth and young adults to pursue careers through the regular presentation of Spantishpeaking scientists and engineers and other role models, as well as creer information on nationally broadcast radio programs reaching youth and parents. This project will encourage making seience, mathenaties, and technology a contscious part of the everyday life experiences of Hispanic youth and Gamilies.

The SRF in collaboration with the Hispanc Radio Network (MRN) will produce and broadcast radio progrms to address the topies and meet the objectives as outtred in the Environmental Literacy Plan and DOE-EM Communtcations Plan in this document. The SRI hus in place a toll-free " $800^{*}$ " number Information and Resource Referral (IRRR) service that national ndio program listeners ean call to obtain information and resource referzals as well as give their reactions to the radio programs that will air. HRN will use this feature to put listeners in touch with loal organizations and resources that can provide them with further information and assiskance on the related program topics.

\section{Task 1 - Environmental Literacy Plan}

The HEWMO Proper Wil:

- produce and broadeast interviews with Hispanic nen and women role models who have science cateers at all levels, from technical positions not requing a college degree to

Ph.D.'s engaged in cutting edge research and in waste management in the nudear industry:

- provide information, via ndio prognatrs and I \& RR serwices, on:

1) Jacadenic and career resources 2) stuccess stories by highlighting the steps needed for academic success in the pursuit of a science career 3) examples of Hisparic students who have employed these successfully 4) career opportunities for women 5.) financial aid resources and trends; 
Self Relance Foundation Hispanic Environmental and

Waste Management Ouireach

- produce and broadcast interviews with Hispanic physicsts, chemists, biologists, and engineers working at Stanford, Los Aliznos, Sandia, Liwermore, Berkeley, and other DOE funded labonatories and facilites. Selentists can serve as role radels and also explain the achievenents of the National Labontories;

- provide information, via radio programs and I \& RR services, about internship and job opportunties in sciertific and engineering fields avalable to Hispaaic students;

- produce and broadcast radio programs that discuss how chiddren leam science, etmphasizing that children are ratumally curious, learn well in groups, and are fascinated by many basic areas of science: water, light and color, plants, the body, and many ober topics:

- produce and broadcast radio programs that educate the Hispanic communty on how chemistry is a part of everyday life in the kitchen and other parts of the household; simple kitchen chemistry activities, such as themical reactions from mixing ingredients in cooking, changes in remperature while baking, the chemical effects of houschold cleaning products; and inform Hispanics, via mdio programs and I \& RR services, where to get more information in Spanish or English on chemistry activities at home, anong others;

- produce and broadcast ndio programs that provide examples of and opportunines for the involvement of chlldren and youth in organized science activities, as volunteers in: 1) science museums 2) is summer science programs 3.) ini environmestal projects, and 4.) in programs provided by Hispanic organizations;

- produce and broadcast mdio prognams that prowide informution about new technological advances that are helping enwironmental scientists preserve our natumal resources and recycle usable materials like aluminum and plastics, and control the waste stream.

\section{Task 2 - DOE - EM Communication Plan}

The HEWMO Project Will.

- produce and broadcast radio programs that conmunicate the mission, goals and activities of the Department of Energy Office of Environmental Management to the Hisparic cotamunity;

- produce and broadcast radio programs dua provide information regarding the spes of waste managed by DOEEM, clenup and restontion plans for nuclear sttes, and ways of treating, recyding, and disposing of nuder waste;

- produce and broadcast radio programs that inform the Hispanic community about the nuclear age history and current federal plans, nuclear disamament stmtegies, the test ban treaty, the mission of the National Labs, and the role of Hisparic scientists in the Labs;

- produce and brodeast radio programs that provide biographies of key scientists 
Self Reliance Foundation Hispanic Environmental and

Waste Managenent Outreach

that developed nuclear energy technology and projects:

- produce and broadcast radio programs that educate the Hispanic community about types of radiation and its sources, radioactivity and fission, biological effects of mdiation, types and amounts of waste, wasteful policies on tritium, uranium and plutonim, transportation of nuclear waste, and the importance of nuclear reactors.

The SRF will achieve the HEWMO Project objectives by conducting national outreach through the Hispanic Radio Network's ndio program series "Plantata Azu" and "Buscando la Belleza" which air dally via HRN radio station affinates in all major U.S. Spanish-speaking markets.

With guidance from DOEFM Contrating Officer's Representative (COR), SERF/HRN will identify, research and develop subject information to be formulated into radio program scripts and content representative of the topics outlined in tasks one and cwo of this document.

HRN produced and distribute for broadcast the following radio programs:

\section{Planera Azul:}

Volume 50, Prograns 451 - 457

- Educates listeners about nuclear encrgy, its war applications and how it affects us in our everyday lives.

Volume 51, Programs $456-469$

- Educates listeners about the good and the bad impacts of mudear energy on humars kind. This includes nuclear medicine and the effect this energy can cause to the human body.

Volume 52 , Programs $470-483$

- This volume combines the history of nuclear energy-induding the worst nudear accident in history (Chernoby), and the unvelling of this country's nuclear dirty secrets by the DOE during Clinton's first administration.

Volume 53, Programs $484-497$

- Educates listeners about the DOE and its efforts to diminish the negative impacts of nuclear energy on the environment. Describes programs DOE-EM is conductirg to clear up the environment and how the community can get involved in these efforts.

Volume 54, Programs $498-511$ 
Saf Relance Foundation

Fispanic Environmental and

Waste Management Outreach

- Biographical profies of some of the most outstanding nuclear scientists in listory axe presented at role model for listeners.

Volume 61, Programs $603-609$

Volume 62, Programs $617-623$

Volume 66, Programs 673-679

Volume 67, Programs $680-686$

- Profiles Hispanic role models working on dirrinishing the negative inpacts of nudear energy and how they can influence young students in order for them to purtue a career in sciences.

- Identifies summer intcrnship and job opportunities in scientific and engineering field avalible to Hispanics anterested in scientific and environanental carcers through public and private industries.

\section{Bracando La Belleza:}

Volume 295, Programs 4067-4068

- Interviews with Hispanic men and women who have science careers at all levels engaged in cutting edge research and waste managenent in the nudear industry.

Volume 296, Progran 4072

Volume 298, Prograns 4105, 4106, 4108, 4113

- Acudemic and career resources/ success stories. Highlights of steps needed for acadernic success in the pursuit of a science career, career oppontunities and frranchl aid resources and trends.

Volume 296, Programs 4076, 4097, 4080, 4081, 4087

Volutie 297, Programs 4089, 4086, 4097

Volume 298, Ptograms 4099,4109

Volume 299, Program 4121

Volume 300, Programs 4128, 4133, 4135

Volune 301, Programs 4142, 4153, 4146, 4150

- Science literacy and family involvement topics: Examples of and oppontunties for 
Self Reliance Foundation Hispanic Enwironmental and

Waste Management Outreach the involvement of children and youth in organized science activities in science musenms, in summer science programs, in environmental projects, and ix programs provided by Hispanic organizations. Information that will help parents teach science to their children, emphasizing that children are naturally curious, leam well in groups, and are fascitated by many basic areas of science: water, light and color, plants, the body, and many other topics.

Volurne 302, Programs 4161,4164, and 4165

Volume 303, Programs 4168,4171, and 4177

Volume 304, Progratris 4184 and 4192

Volume 310 , Programs $4269,4271,4274,4276$, and 4279

- Profiles Hispanic physicists, chemists, biologiss, and engineers.

\section{Project Metrics}

Focas groups will be conducted in September 1998 in order to test bow the radio programs infuence environmental attitudes within the Hispanic commutuity. HRN has held several focus group sessions regarding the attitudes, interests, and lanowledge of its listenets relative to the previous and current radio episode topics. HRN has included diverse Hispanis participants, so that it can address differences in educational levels and interests. Response to the proposed programming is generally very positive wikh all participants feeling comfortable with the accets of the program hosts.

HRN will continue focus group sessions to get continual feedback on its programming. As a group of episodes is developed for the environmental series, HRN will select indwidual episodes representative of the scope of programming featured in each series, have focts groups of tweive Hispanic listen to them, and immcliately afterward, respond to questions concening their ovenill appeal and content/delivery comprehension istes.

HRN will develop a systematic means for documenting the use of the tollfree Information and Resource Referml (I \& RR) services. HRN will also conduct followup interviews with users of the toll-free number to leam more about the effec of using this service.

HRN also conducts its own monitoring of the project, including tracking the calls to the toll-free number and recording denographic profiles of callers. Solicting both open ended and directed feedback from program directors of the stations carrying the 
Self Reliance Foundation

Hispanic Environmental and

Waste Management Outreach

programs. Montoring Arbitron tatings, which give precise dernographic breakdowns for the actual listening audience in ench community. Obtaining contunal feedback from cooperating educational outreach and community based organizations and prograns. HRN is fully committed to using the results of the evaluations and our own monitoring to continually revise and improve programming.

\section{Third Quarter Activity Summary}

Self Reliance Foundation in collaboration with the Hispanic Radio Network produced and broadcast 40 DOE-EM sponsored radio episodes during the $3^{\text {nd }}$ quarter of the HEWMO

Project. These episodes covered a warety of enwironmental topics ensuring a wide listening audience.

Self Relance Foundation's analysis of Outreach listeners/callers dernographics for the Third Quarter show:

- $60 \%$ of listeners/callers are men

- $39 \%$ of listeners/ alles are of Mexican Heritage

- 32\% of listeters/callers are able to speak English

- $26 \%$ of listener/culter last level of cducation completed was ligh school 
Self Reliance Foundation Hispanic Envirommental and Waste Management Outreach

During the Third Quarter of the HEWMO Project, the following episodes wete produced and broadcast in MRN's Buseando L Belleza and Planetu Azul series:

\section{Buscando la Belleza}

\begin{tabular}{|c|c|c|}
\hline Air Date & No. of Erogram & Tille \\
\hline June 11, 1998 & 4269 & $\begin{array}{l}\text { Planta de desechos nueleares } \\
\text { Entrevisa a Howard Vasquez I } \\
\text { Waste Isolation Power Plant } \\
\text { Interwew wilh Howard Vasquez I }\end{array}$ \\
\hline June 13, 1998 & 4271 & $\begin{array}{l}\text { Residuos nucleares e hispanos } \\
\text { Entrevista a Howard Vasquez II } \\
\text { Nuclear Waste } \\
\text { Interview with Howard Vasquez II }\end{array}$ \\
\hline June 16,1998 & 4274 & $\begin{array}{l}\text { Carlsbad y los residuos nucleares } \\
\text { Entrevista a Howard Vasquez III } \\
\text { Carlsbad and Nuclear Waste } \\
\text { Interview wih Howard Vasquez II }\end{array}$ \\
\hline June 18, 1998 & 4276 & $\begin{array}{l}\text { Transporte de plutonio } \\
\text { Entrevista a Howard Vasquez IV } \\
\text { Transporting Pltornim } \\
\text { Interview will Howard Vasque IV }\end{array}$ \\
\hline June 21,1998 & 4279 & $\begin{array}{l}\text { Seguridad nuclear } \\
\text { Entrevista a Howard Vasquez V } \\
\text { Nuclear Safety } \\
\text { Interview with Hownd Vasquez V }\end{array}$ \\
\hline
\end{tabular}



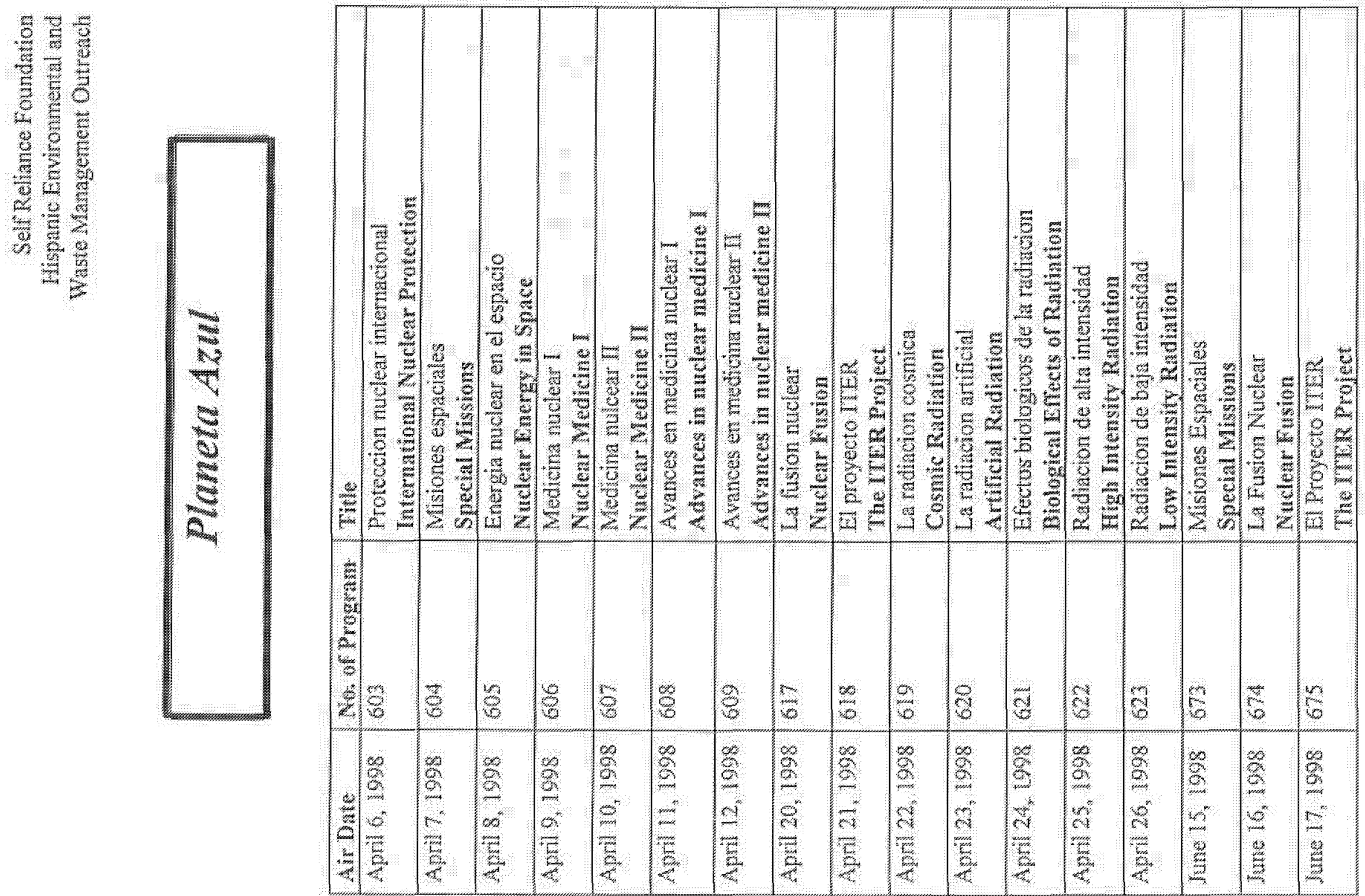
Sol Relance Foundation

Hevanc Enviromental and

Waste Managenent Outreach

\begin{tabular}{|c|c|c|}
\hline Jure 18,1998 & 676 & $\begin{array}{l}\text { La Radiacion Cosmita } \\
\text { Cosmic Rudiation }\end{array}$ \\
\hline June 19,1998 & 677 & $\begin{array}{l}\text { La Radacion Artificial } \\
\text { Artifcia Radiation }\end{array}$ \\
\hline June 20,1998 & 678 & $\begin{array}{l}\text { Efectos Biologicos de la Radiacion } \\
\text { Biological Cffects of Radiation }\end{array}$ \\
\hline June 21,1998 & 679 & $\begin{array}{l}\text { Las Intensidades y Efectos de la Radia } \\
\text { Intensity and he effect of Radiation }\end{array}$ \\
\hline June 22,1998 & 680 & $\begin{array}{l}\text { El rol de ha opimon publica } \\
\text { The Role of Yublic Opinion }\end{array}$ \\
\hline June 23, 1008 & 681 & $\begin{array}{l}\text { Tnstancias democraticas } \\
\text { Democratic Instances }\end{array}$ \\
\hline June 24, 1908 & 682 & $\begin{array}{l}\text { Vertederos y encrgia } \\
\text { Dumping and Lnergy }\end{array}$ \\
\hline June 25,1998 & 683 & $\begin{array}{l}\text { Rotativas Grupales } \\
\text { Team Rotary } \\
\end{array}$ \\
\hline June $26, \$ 998$ & 684 & $\begin{array}{l}\text { Nwevas Ponticas de decision } \\
\text { New Decision Making Policies }\end{array}$ \\
\hline June 27, 1998 & 685 & $\begin{array}{l}\text { Quien sabe donde poner el desedho nuclear? } \\
\text { Who knows where to dispose nuclear waste? }\end{array}$ \\
\hline Jume 28,1998 & 686 & $\begin{array}{l}\text { Desafios para la cuidadania } \\
\text { Chnllenges for Cinizens }\end{array}$ \\
\hline June 20,1998 & 687 & $\begin{array}{l}\text { Trilium, al camaleon de los bosques } \\
\text { Trillum, the Chameleon of the Forest }\end{array}$ \\
\hline Jure 30,1998 & 688 & $\begin{array}{l}\text { Wl proyecto "Ro Condo" } \\
\text { "Ro Condor" Project }\end{array}$ \\
\hline July 1. 1998 & 689 & 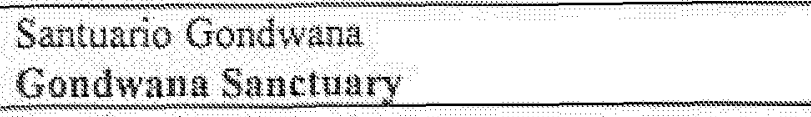 \\
\hline July 2, 1998 & 690 & $\begin{array}{l}\text { Cerwificacion forestal } \\
\text { Forest Cortificution }\end{array}$ \\
\hline JuY 3.9998 & 691 & $\begin{array}{l}\text { El rmpacto de las tranksacionales } \\
\text { The Transmational lmowet }\end{array}$ \\
\hline Suly 4, 1908 & 692 & $\begin{array}{l}\text { Mundo sustentable } \\
\text { A Sustainable Wordd }\end{array}$ \\
\hline July 5,3998 & 693 & $\begin{array}{l}\text { Cronica de un mucre anumciada } \\
\text { Chronology of an advertised Deali }\end{array}$ \\
\hline
\end{tabular}


Self Reliance Foundaton

Hispanic Enwionmental ard Waste Managennent Outreach

\section{Buscando la Belleza Radio Affiliates}

During the 3 " Quarter of the HEWMO Project, Buscando La Belleze (In Search of Beaury and Health) wa comptised of 68 affliated Spanish laxguage radio stations broadcating HRN's radio programming covering $73.06 \%$ of the HOM $12+$ (Hispanic Consumer Market) population* or 15.7 million Hispanics.

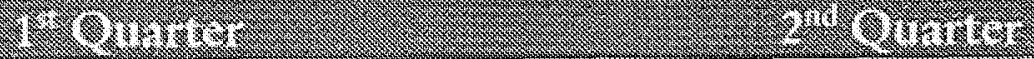

$71.20 \%$ or 15.3 million Hispanics $\quad 73 \%$ or 15.7 million Hispanics

\section{Third Quarter: \\ Buscando la Belleza Program Coverage of the Hispanic Consumer Market (HCM) Age 12+}

$73.06 \%$

15.7 million 
Self Reliance Foundation Hispanic Envirommentsl and Waste Management Outreach

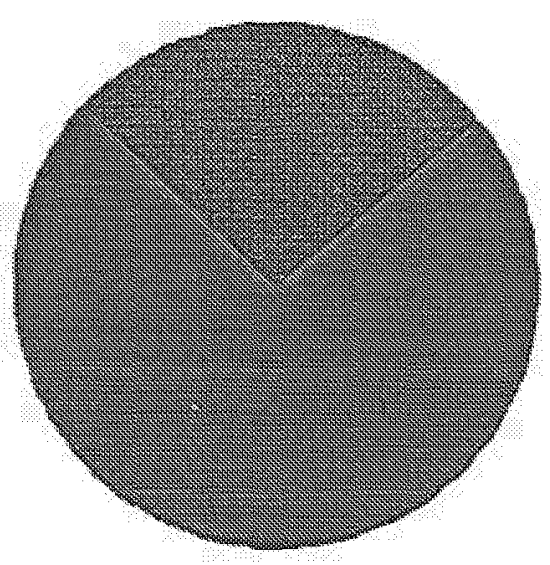

\section{Planeta Azul Radio Affiliates}

During the 3rd Quarter of the HEWMO Project, Planets Awn (The Blue Planet) was comprised of 71 radio affliated Spanish language radio stations broadcasting HRN's radio programming covering $71.35 \%$ of the HCM $12+$ (Hispanic Consunner Makket) population. * or 15.4 million Hispanics.

\begin{tabular}{|c|c|}
\hline 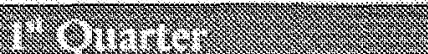 & zumpuricse \\
\hline $77.20 \%$ or 16.6 million & $71.25 \%$ or 15.3 million \\
\hline
\end{tabular}


Sef Reliance Foundation

Hispanic Environmental and Waste Managentent Outreach
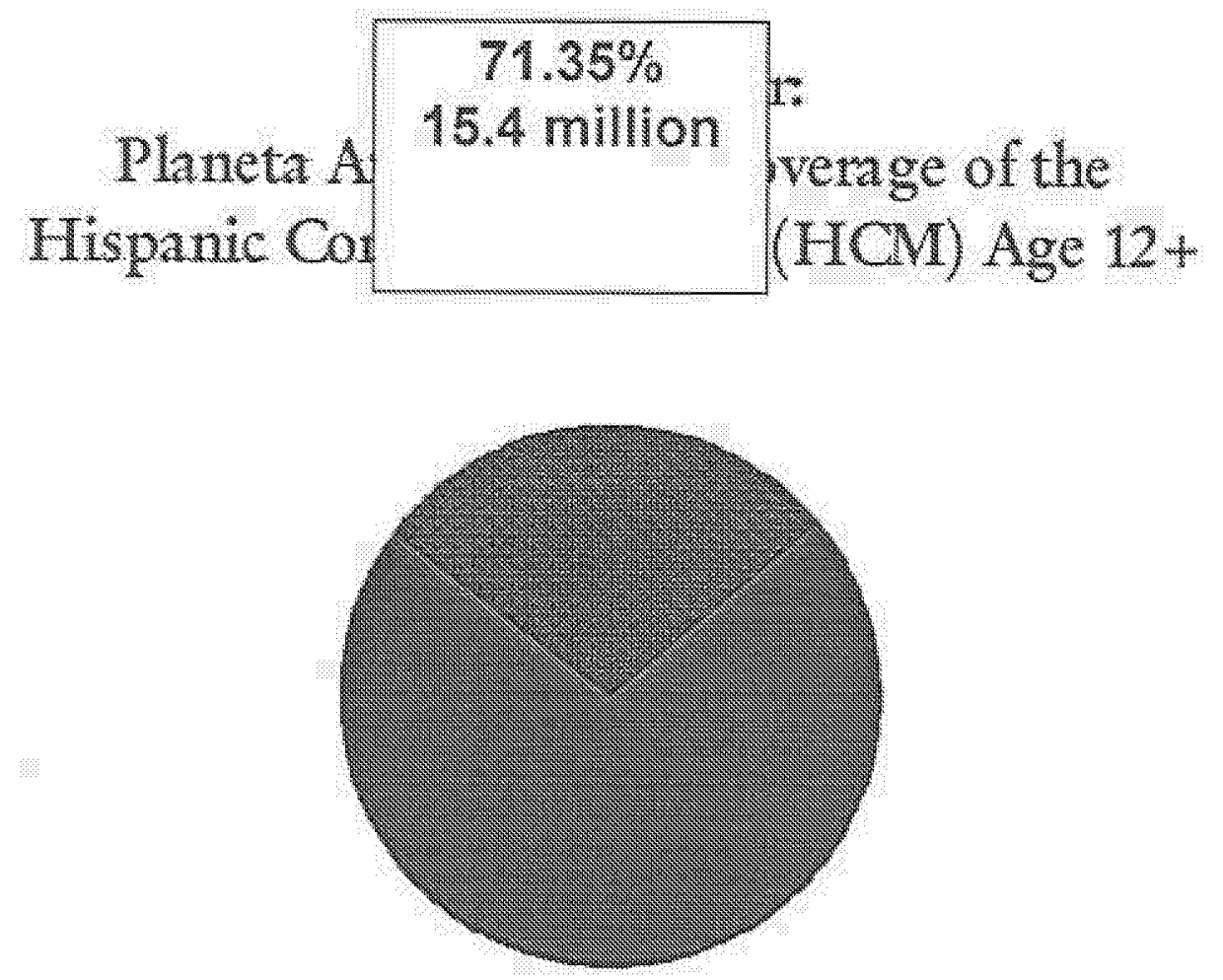


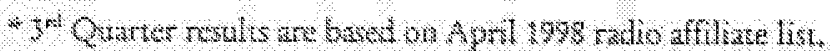

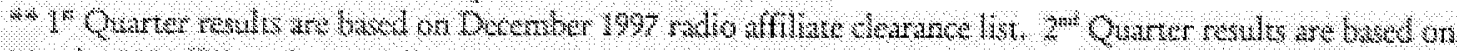

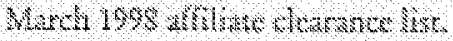

\section{Analysis of $1^{\text {th }} \& 2^{\text {td }}$ Quarter Listener / Caller Demographics}

\section{Figure 1:}

Number Of Episodes Broadcast

\begin{tabular}{|c|c|c|c|}
\hline 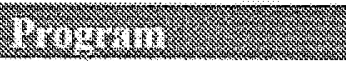 & 20 женит & $203002 \pi$ & 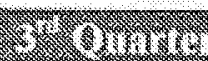 \\
\hline Buscando la Belleza & 35 & 15 & 5 \\
\hline Planeta Azul & 70 & 4 & 35 \\
\hline Total & 105 & 9 & 40 \\
\hline
\end{tabular}

Figure 2:

DOE EM Environmental Callers by Gender

\begin{tabular}{|c|c|c|c|}
\hline 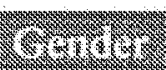 & st & y & 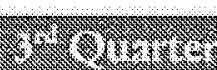 \\
\hline Male & $49 \%$ & $44 \%$ & $60 \%$ \\
\hline Fermate & $510 \%$ & $56 \%$ & $40 \%$ \\
\hline
\end{tabular}


Self Relance Foundation

Hispanic Enwromental and Waste Munagement Outreach

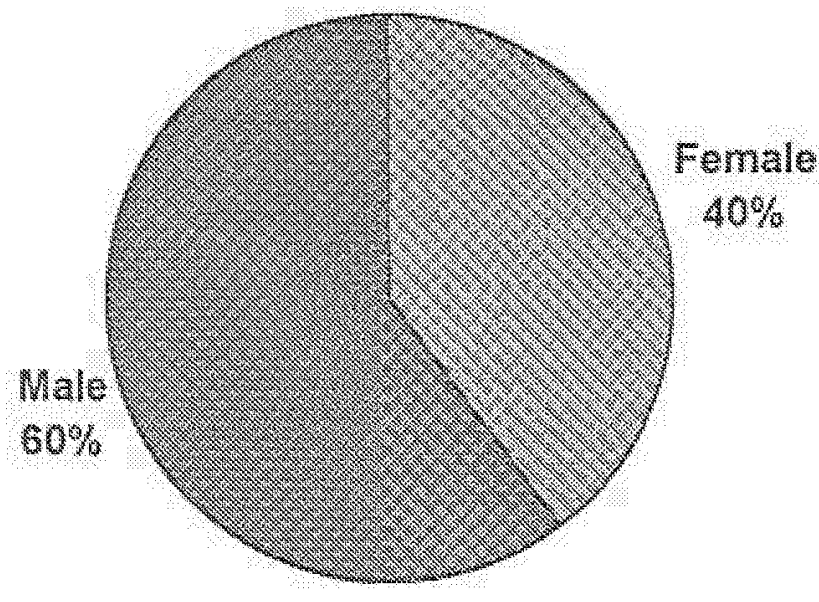


Self Relance Foundation

Mispanic Ervironnental and

Waste Management Outreach

Figure $3:$

DOE-EM Environmental Callers by Age

\begin{tabular}{|c|c|c|c|}
\hline 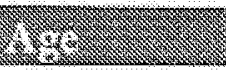 & g. & 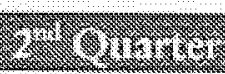 & 3. \\
\hline 178 Unde: & $0 \%$ & $3 \%$ & $0 \%$ \\
\hline $8-35$ & $40 \%$ & $42 \%$ & $41 \%$ \\
\hline $36-45$ & $24 \%$ & $30 \%$ & $36 \%$ \\
\hline $46+$ & $22 \%$ & $20 \%$ & $23 \%$ \\
\hline
\end{tabular}




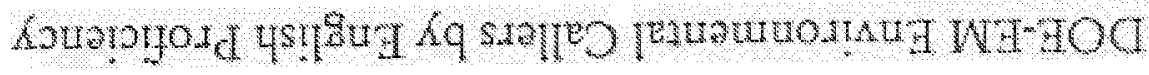
: $2 \operatorname{man} \pi$

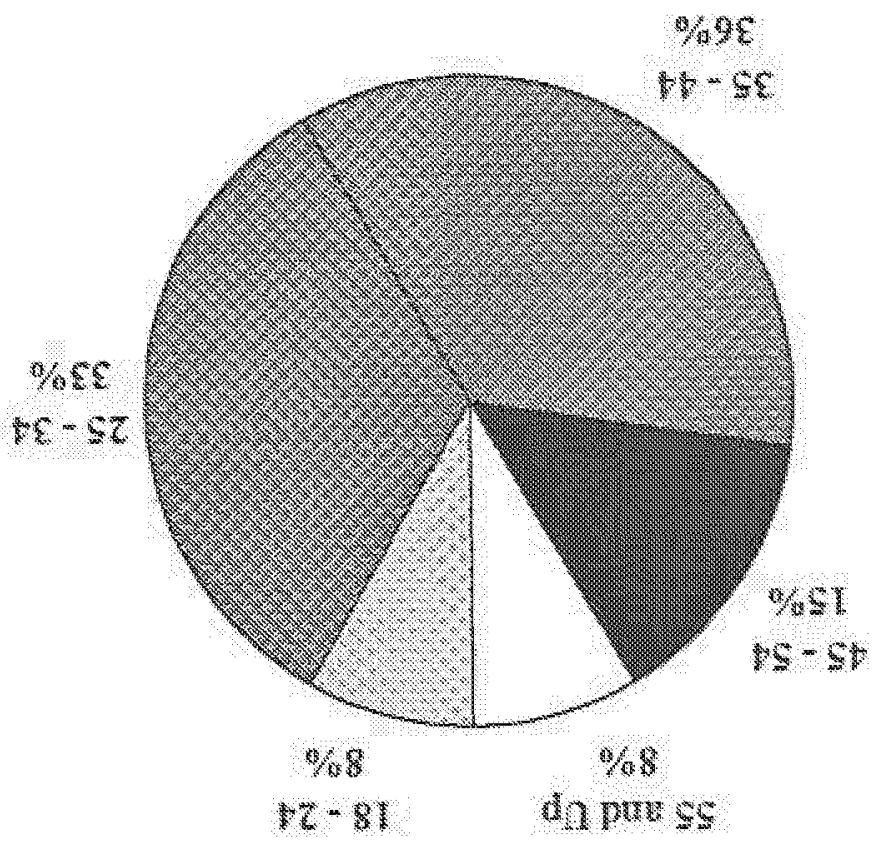

\section{अ:6}

tpranno 7ustreftue

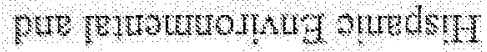

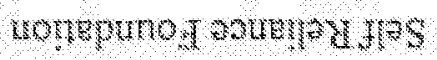


Self Reliance Foundation

Hispanic Envirommental and Waste Managenent Outreach

\begin{tabular}{|l|l|l|l|}
\hline Does Not Speak Erglish & $17 \%$ & $16 \%$ & $26 \%$ \\
\hline Speaks Some Errglixh & $30 \%$ & $33 \%$ & $34 \%$ \\
\hline Speaks Engish & $53 \%$ & $51 \%$ & $32 \%$ \\
\hline
\end{tabular}

\section{DOE- EM Callers By English Proficiency: Sp:eak}

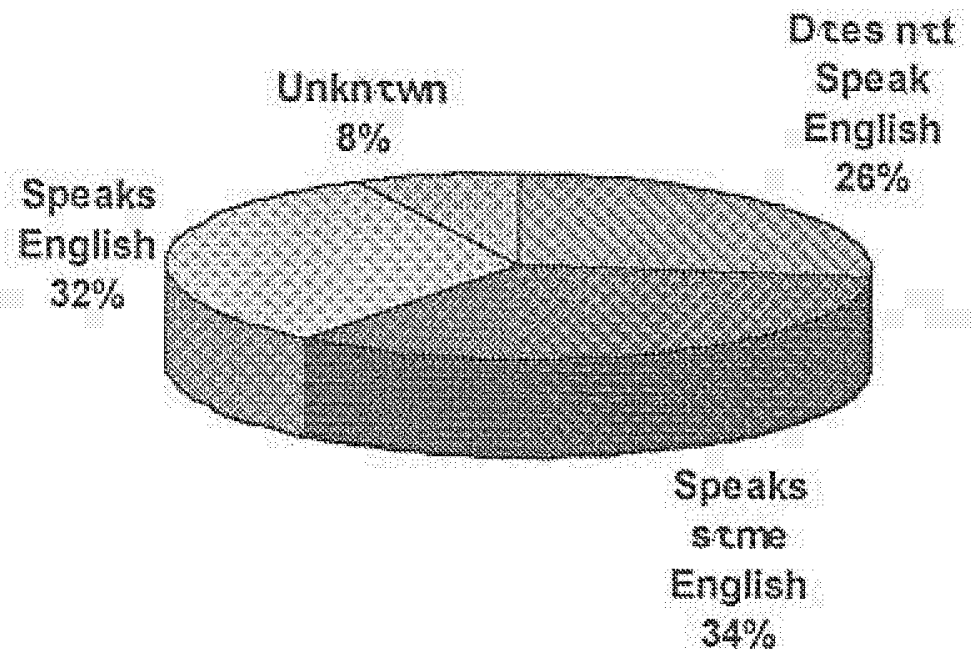

Figure 5 
Self Reliance Foundation Hispanic Envirommental and Waste Management Outreach

DOE-EM Environmental Callers by Education Level

\begin{tabular}{|c|c|c|c|}
\hline 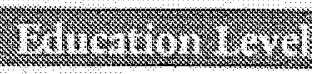 & 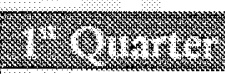 & 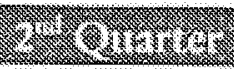 & $x^{2} \cos x=$ \\
\hline None & $0 \%$ & 18 & $2 \%$ \\
\hline Elenrentaty & $37 \%$ & $17 \%$ & $15 \%$ \\
\hline Jr. High School & $7 \%$ & $7 \%$ & $6 \%$ \\
\hline High School & $28 \%$ & $41 \%$ & $26 \%$ \\
\hline College & $25 \%$ & $31 \%$ & $34 \%$ \\
\hline Post Gradonte & $2 \%$ & 39 & $2 \%$ \\
\hline
\end{tabular}

\section{DOE-EM Callers By Education}

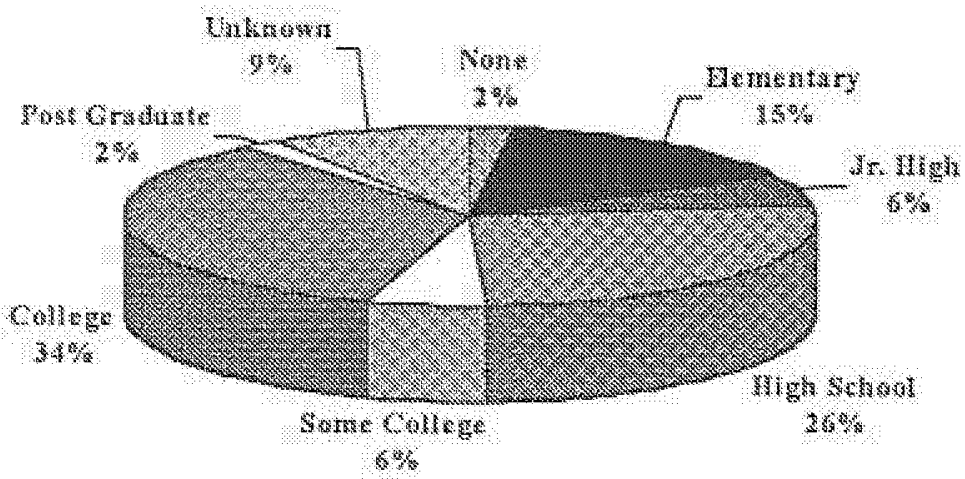




\section{DOE- EM Callers By Ethnic Heritag:e}

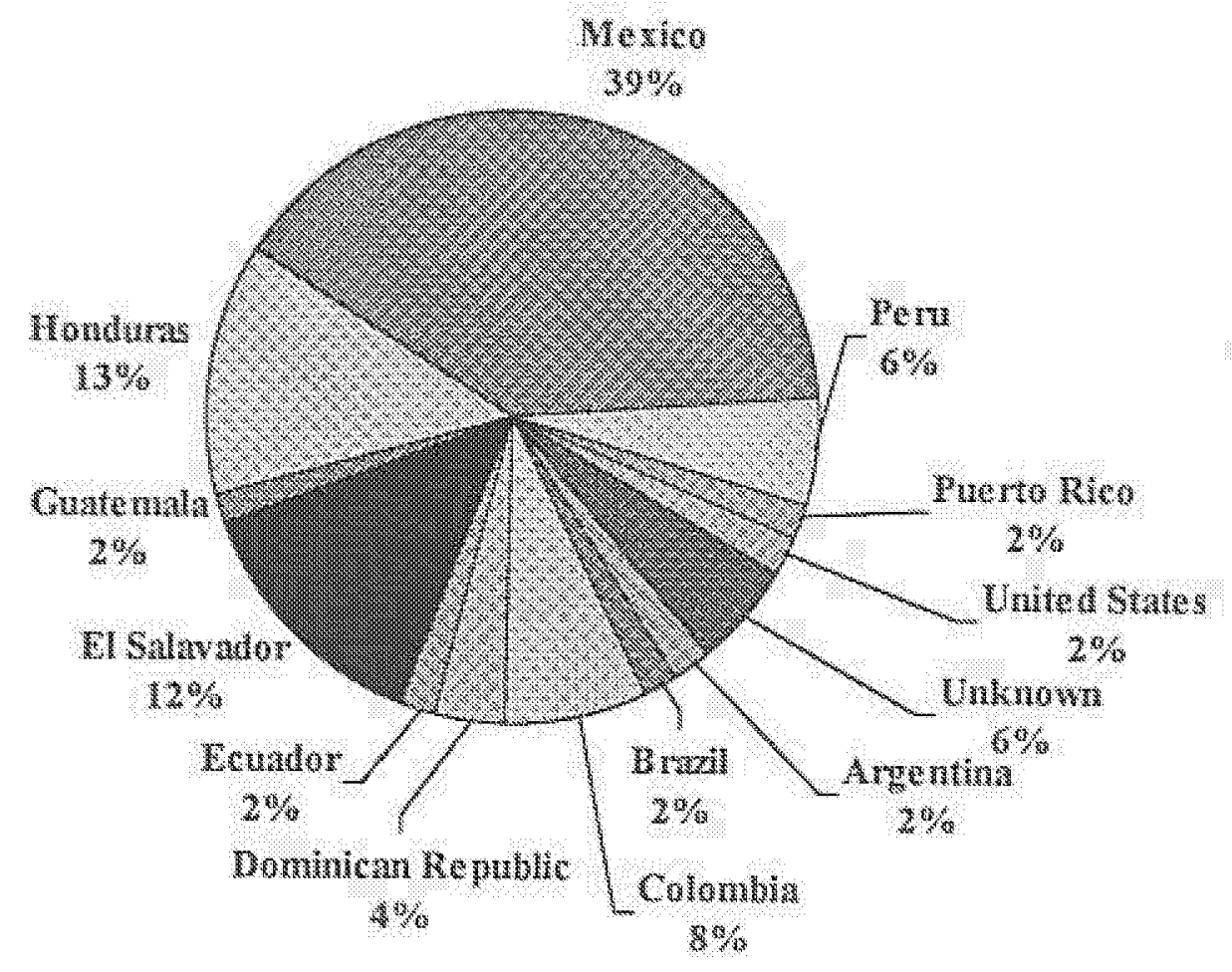


Self Reliance Foundation Hispanic Enwrontnental and Waste Management Outreach

\section{APPENDICES}


Self Relance Foundation

Hispasic Environnental and Waste Management Outreacis

\section{I. \\ Program Guide For Buscando la Belleza}




\section{Inz $\forall$ eloueld NO oping uedbodd \\ "II}

4ptam

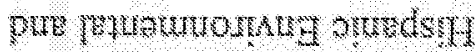

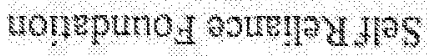


Self Reliance Fowndation

Hispanic Enyitonmental and

Waste Mandagenent Outreach

\section{III. \\ Radio Affiliate List \\ For \\ Buscando la Belleza}




\section{Inzb elaueld IOI

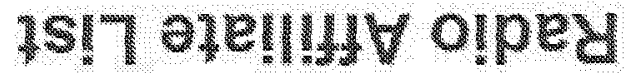 \\ "N}

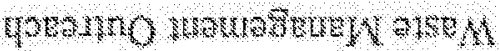

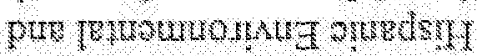
uoyepuno 1 sumyey Jos 
Self Relance Foundation

Mispanic Frwirommental and Waste Mrragentert Outreach

\section{V. DOE-EM Caller Reports}




\section{s!sर́peu

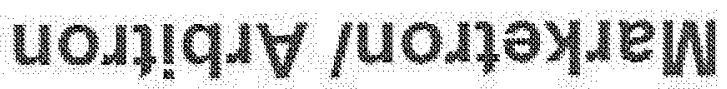 \\ "IA}

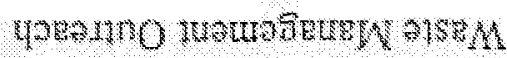

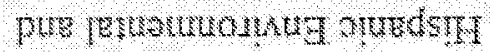

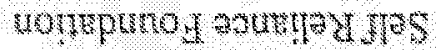




\section{Buscando la Belleza Radio Affiliates}

During the $3^{\text {ri }}$ Quarter of the HEWMO Project, Buscando LA Belleza (In Search of Beaty and Health) was comprised of 68 affihted Spanish language radio stations broatcasting HRN's thdio programming covering $73.06 \%$ of the HCM $12+$ (Hispanic Constutzer Market) population* or 15.7 million Mispanics.

\begin{tabular}{|c|c|}
\hline 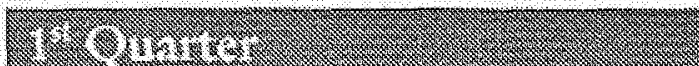 & 24 orwher \\
\hline $71.20 \%$ or 15.3 million Hisp & Hispar \\
\hline
\end{tabular}

\section{Third Quarter: \\ Buscando la Belleza Program Coverage of the Hispanic Consumer Market (HCM) Age 12+}

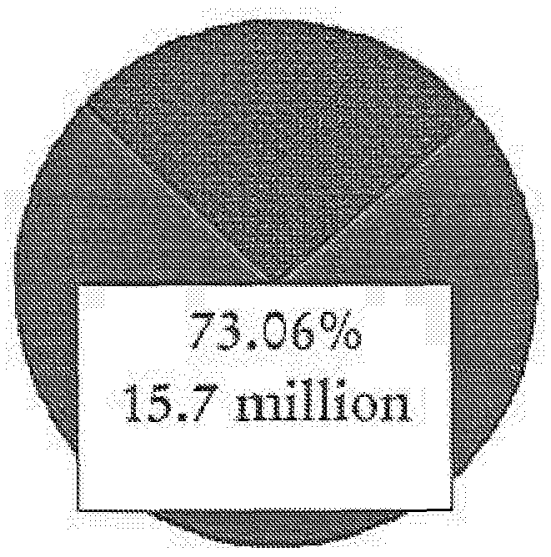




\section{Planeta Azul Radio Affiliates}

During the 3rd Quarter of the HEWMO Project, Planed Azal (The Blue Planet) was comprised of 71 madio affitated Spanish language ndio stations brodcasting HRN's ndio programming covering $71,35 \%$ of the HCM 12+ (Hispanic Consuner Market) popitation, *or 15.4 million Hispantics.

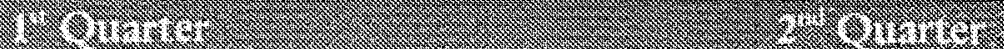

\begin{tabular}{l|l}
$77.20 \%$ or 16.6 million & $71.25 \%$ or 15.3 million \\
\hline
\end{tabular}

\section{Third Quarter: \\ Planeta Azul Program Coverage of the Hispanic Consumer Market (HCM) Age 12+}




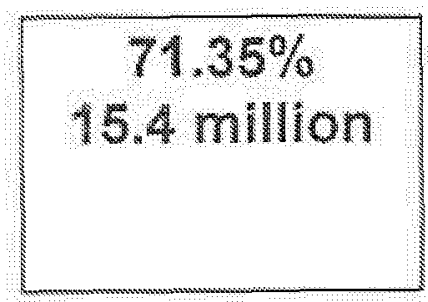

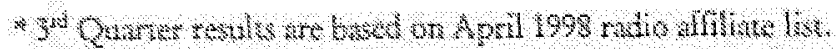

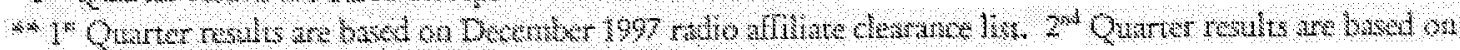
Marh 1908 anthase dearane kist.

\section{Analysis of $1^{\text {st }} \& 2^{\text {nd }}$ Quarter Listener / Caller Demographics}

Figure 1:

Number Of Episodes Broadcast

\begin{tabular}{|c|c|c|c|}
\hline $3 \times 2 \times 4 \times 1 \%$ & 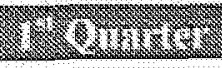 & $(2)$ (x) & 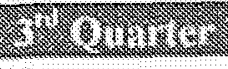 \\
\hline Buscrndo la Belleza & 35 & 15 & 5 \\
\hline Planet A ARI & 70 & 4 & 35 \\
\hline Total & 105 & 9 & 40 \\
\hline
\end{tabular}


Figure 2:

DOE-EM Environmental Callers by Gender

\begin{tabular}{|c|c|c|c|}
\hline estuty & 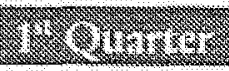 & 2 plunis? & 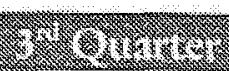 \\
\hline Male & $49 \%$ & $44 \%$ & $60 \%$ \\
\hline Female & $51 \%$ & $56 \%$ & $40 \%$ \\
\hline
\end{tabular}

DOE- EM Callers By Gender

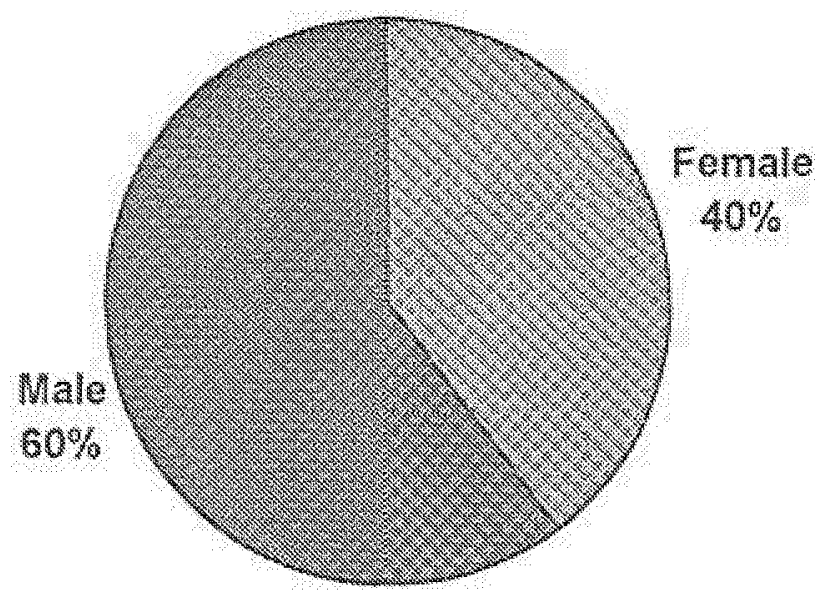


Figure 3:

DOE-EM Environmental Callers by Age

\begin{tabular}{|c|c|c|c|}
\hline 14 & rovimes & 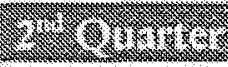 & 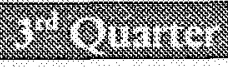 \\
\hline 178 Under & $0 \%$ & $3 \%$ & $0 \%$ \\
\hline $18-35$ & $40 \%$ & $42 \%$ & $41 \%$ \\
\hline $36-45$ & $24 \%$ & $30 \%$ & $36 \%$ \\
\hline $46+$ & $22 \%$ & $20 \%$ & $23 \%$ \\
\hline
\end{tabular}




\section{DOE-EM Callers By Age}

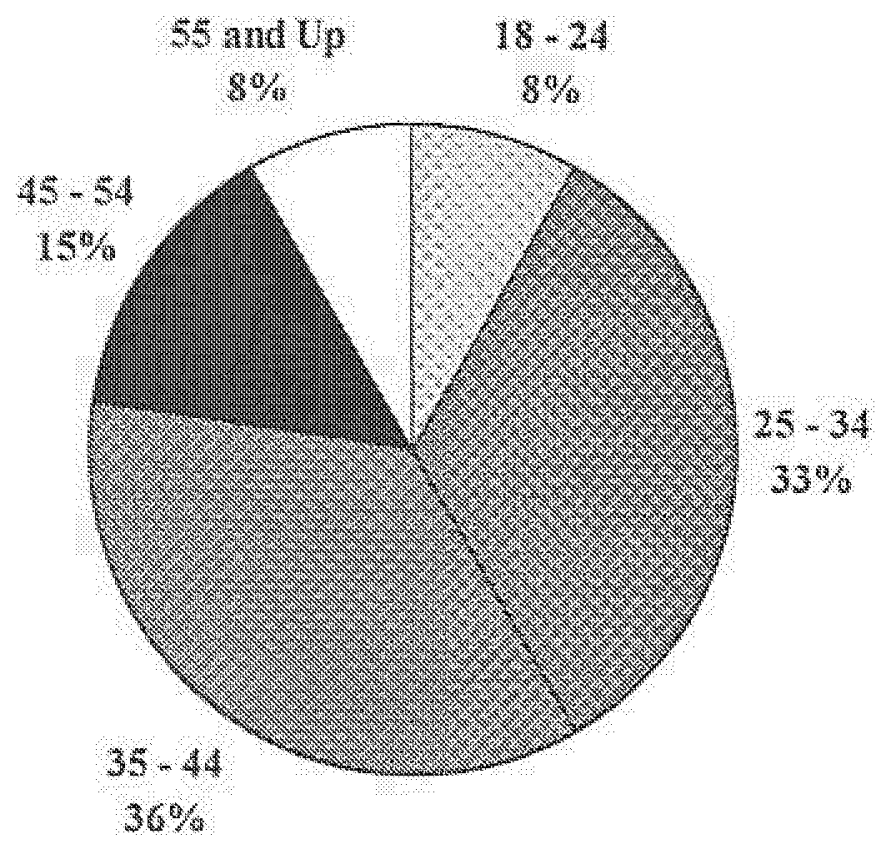

Figure 4:

DOE-EM Environmental Callers by English Proficiency

zнам.




\begin{tabular}{|l|l|l|l|}
\hline Does Not Speak Engith & $17 \%$ & $16 \%$ & $26 \%$ \\
\hline Speaks Sone English & $30 \%$ & $33 \%$ & $34 \%$ \\
\hline Speaks English & $53 \%$ & $51 \%$ & $32 \%$ \\
\hline
\end{tabular}

\section{DOE- EM Callers By English Proficiency: Speak}

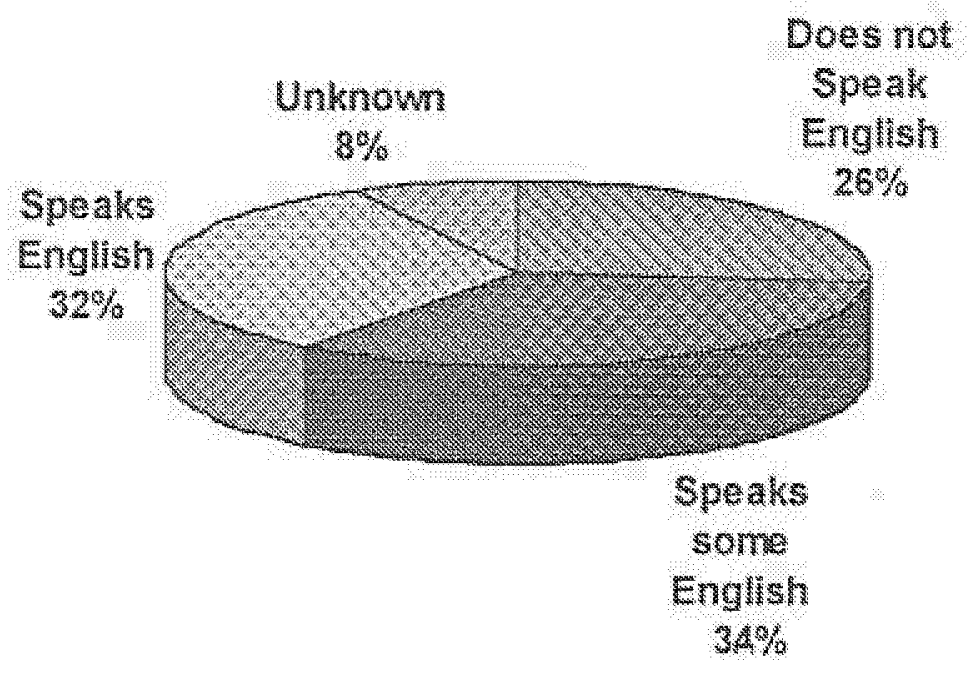

Figure 5

DOE-EM Envionmental Callers by Education Level 


\begin{tabular}{|c|c|c|c|}
\hline 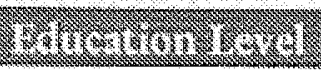 & 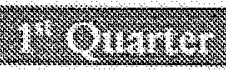 & 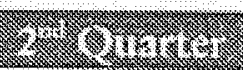 & 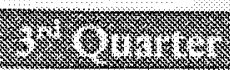 \\
\hline None & $0 \%$ & $1 \%$ & 28 \\
\hline Elementary & $37 \%$ & $17 \%$ & $15 \%$ \\
\hline Jit Higi Schoot & $7 \%$ & $7 \%$ & $6 \%$ \\
\hline High School & $29 \%$ & $41 \%$ & $26 \%$ \\
\hline College & $25 \%$ & $31 \%$ & $34 \%$ \\
\hline Post Graduate & $2 \%$ & $3 \%$ & 28 \\
\hline
\end{tabular}

\section{DOE-EM Callers By Education}

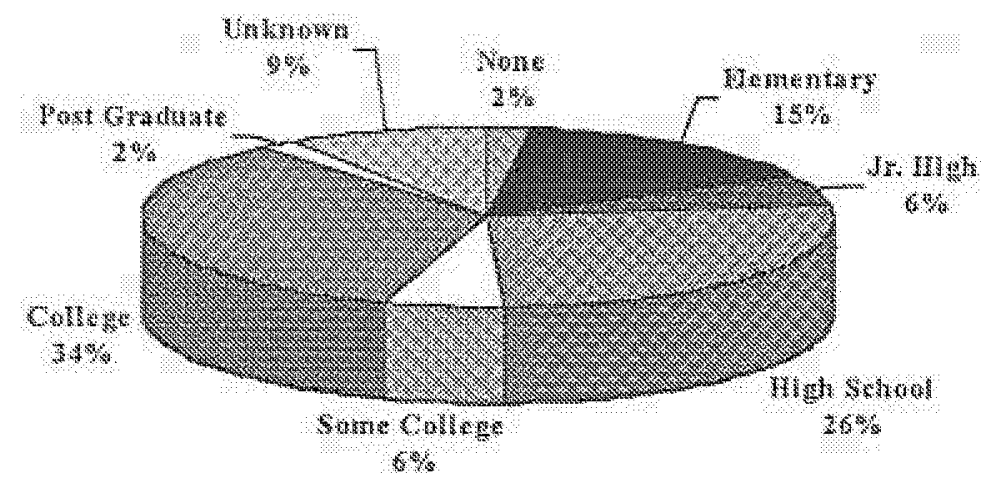




\section{DOE- EM Callers By Ethnic Heritage}

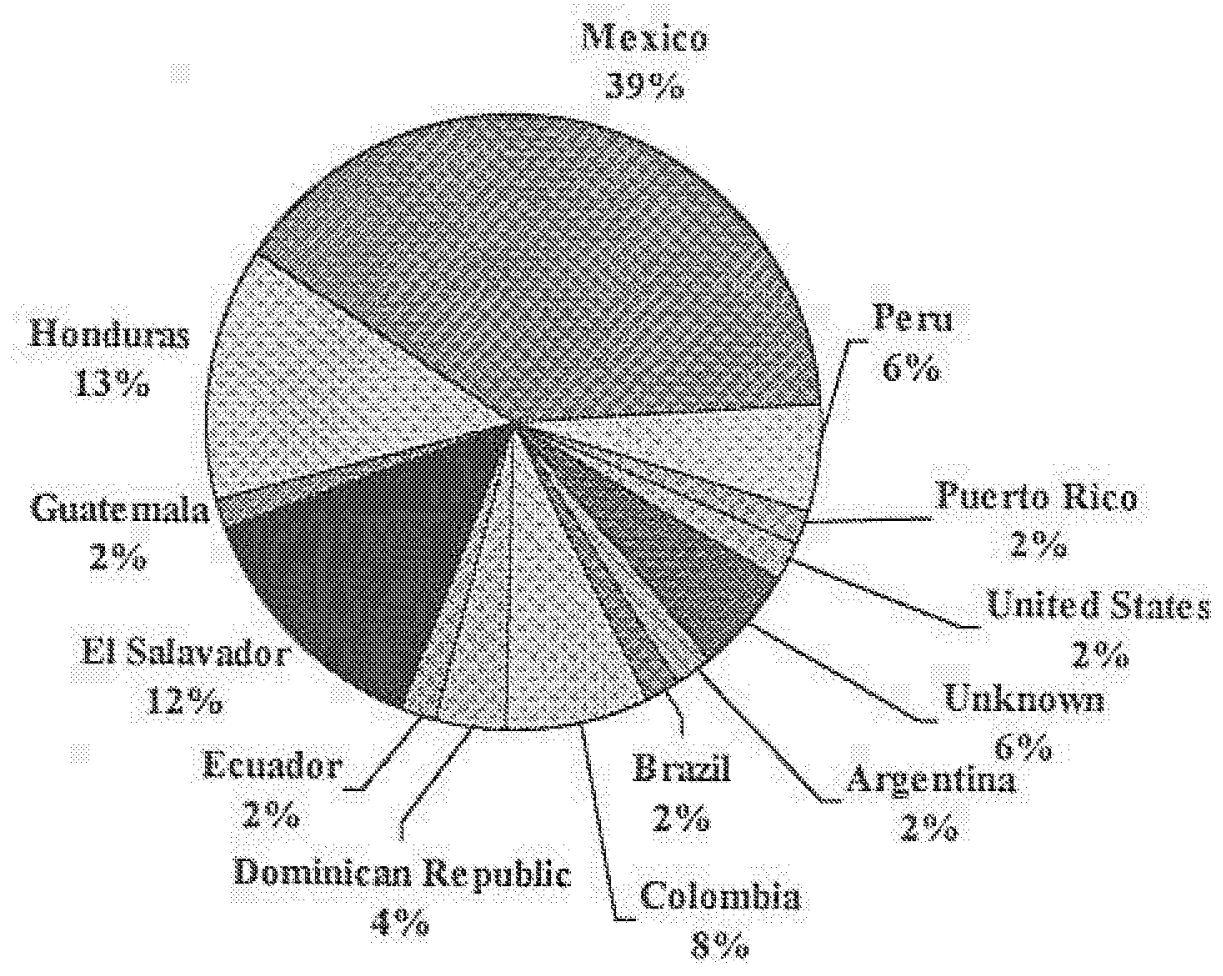


APPENDICES 


\section{I. Program Guide For \\ Buscando la Belleza}




\section{II. \\ Program Guide For Planeta Azul}




\section{III. Radio Affiliate List For Buscando la Belleza}




\section{IV. \\ Radio Affiliate List \\ For \\ Planeta Azul}




\section{V. DOE-EM Caller Reports}




\section{VI. \\ Marketron/ Arbitron}




\section{Self Reliance Foundation}

\section{Hispanic \\ Environmental and Waste Management Outreach Project}

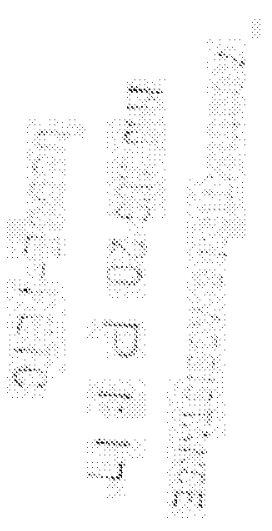

Fimal Report to the

Uated States Department of Linergy Orfice of Environmental Mamagement

October 1, 1997-.. July 25,1998 
Self Reuance Foundation

Hispanic Environmental and Waste Management Outreach

Project Identification No. DE-FC26-97FT34346

\section{Table of Contents}

Project Overvicw

Pag:

Envtronmental Literacy Plan

DOL - GM Communication Plan 2

Propect Metrics 4

Third Quarter Activity Sunmary

Burando La Bolly Radio Afrinates ?

Plonela Asw/Radio Afniates 10

Analyss -1 and 2 " Caller Demographics: $11-14$

Figure 1. Number of Episodes Broadcast

- Figure 2: DOE-FM Callers by Gender

Figure 3. DOL.EM Callers by Age

- Figure 4: DOL EM Callers by English Proficiency

- Figure 5: DOL-EM Callers by Education Level

Appendices:

Progran Gude for Buscando ia Belleza

1.

Progran Gude for Planera Azul

TI.

Radio AThiate List for Buscando la Belleza

III.

Radio Affliate l ist for Planeta Anul

DOT: - GM Caller Report:

V.

Marketron/ Arbitron Raing* Anulysis

VI. 


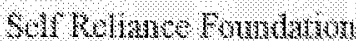

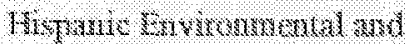

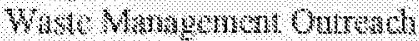

\section{Project Overview}

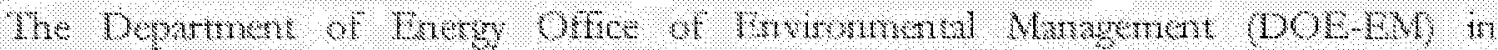

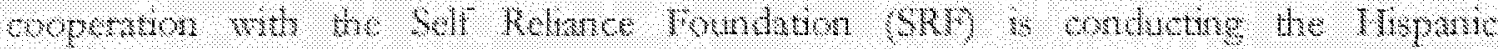

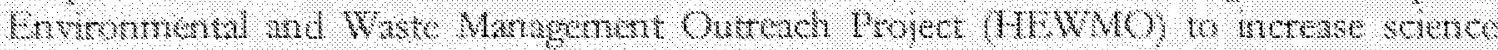

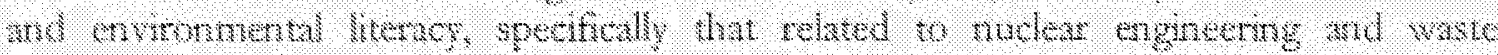

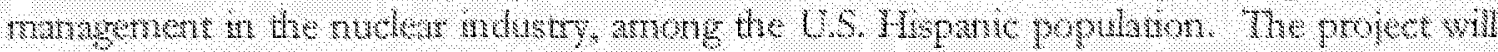

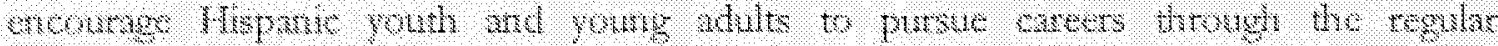

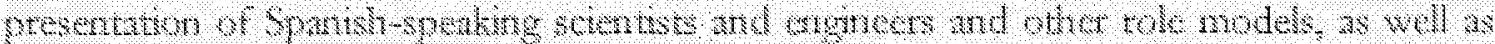

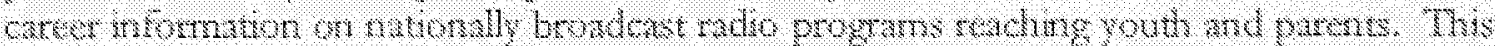

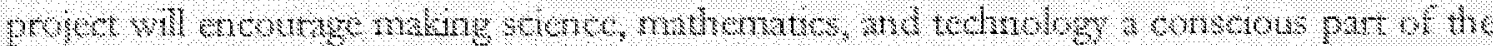

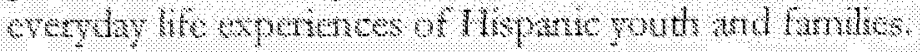

The SRT in collabotation whit the Gepane ladio Netwent. (HRN wil prodisee and

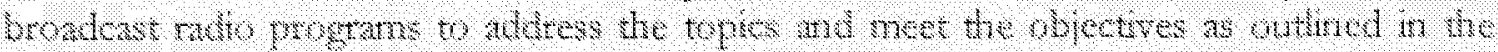

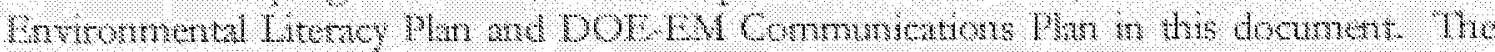

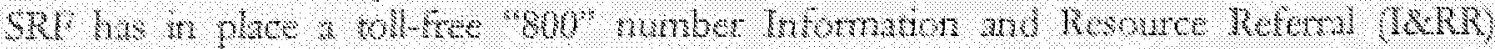

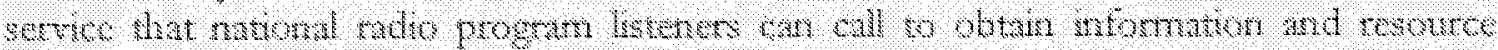

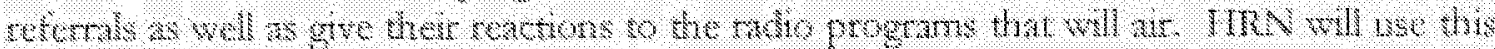

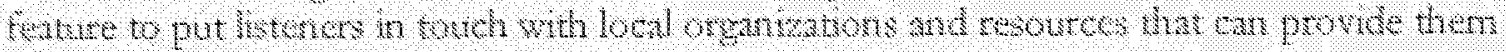
wh hrther informaton and asestance on the related progran topics.

\section{Task 1 - Environmental Literacy Plan}

\section{The MTWWO Proger Wal:}

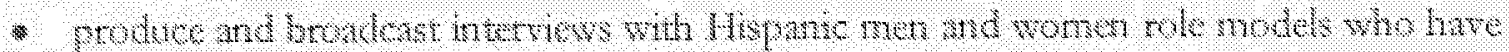
scence cerers at all levels, fron technch postwons not requivig a college degree to

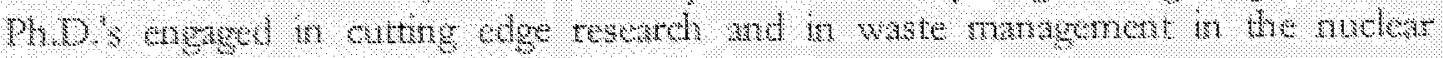
mosetry:

- provte mformatim, wa malio programs and T R TR servece on:

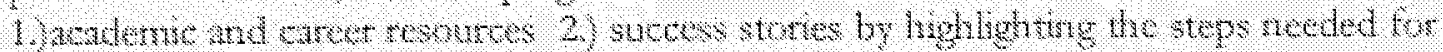

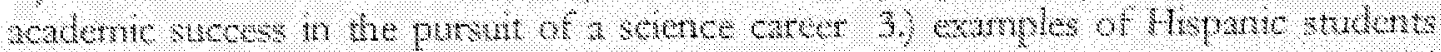
whe have enployed these successtuly. 4. caree opportumes for wonen 5 ) fmanchal ard rerames and trands:

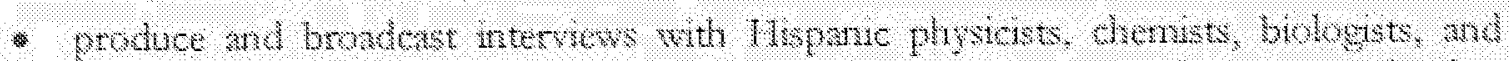

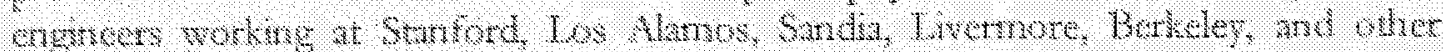

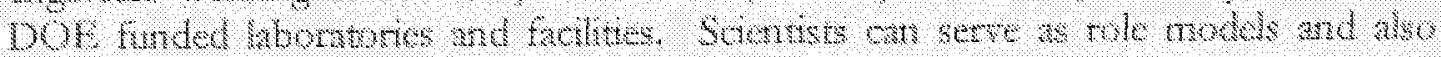
explats the acherentent of the National L womarones?

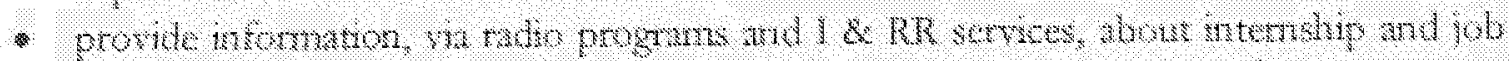

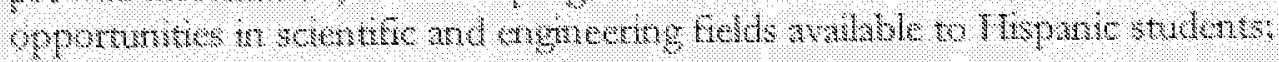

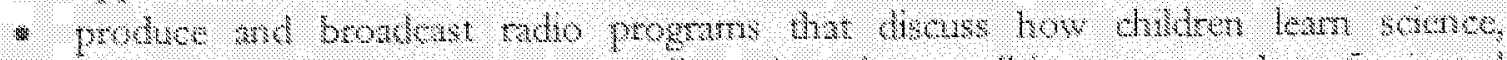
empheitimy that thldten are naturlly currous, feam well th grups, and are fascinated 


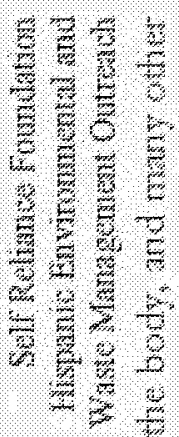

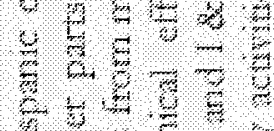

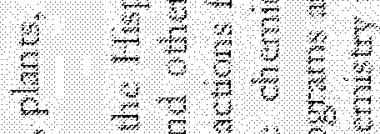

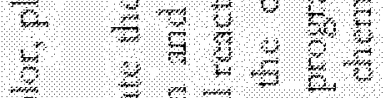

$39 \frac{5}{3} 32$ ?

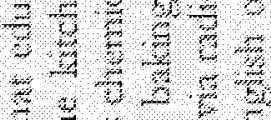

I 920

단 $\frac{2}{3} \frac{2}{3} \frac{4}{5}$

$\varepsilon^{-4}+\frac{5}{3}=\frac{5}{5}$ - $5.5 \mathrm{E}$

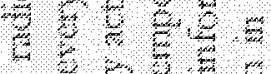

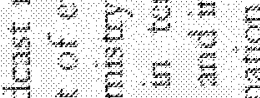

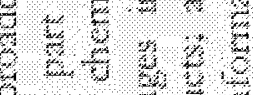

$3+5 \mathrm{~g}$

s

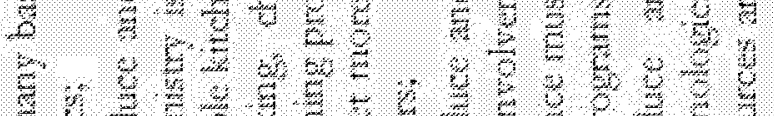

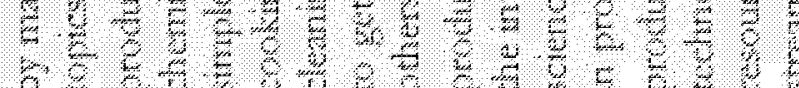

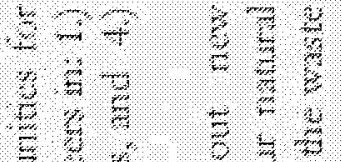

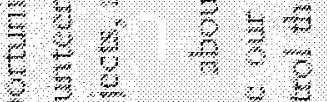

है है ह ह

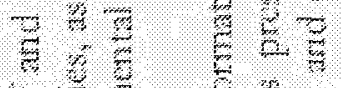

है

ह

है 8

ช.

4.

है

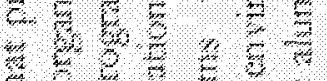

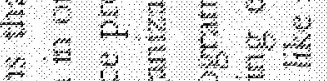

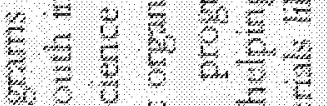

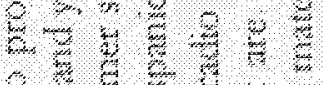

$8 \frac{9}{2}=5$

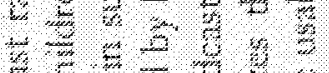

$4+a \frac{3}{4} \mathrm{~g}$

E t

प 5 ह

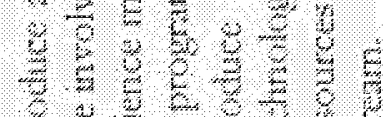

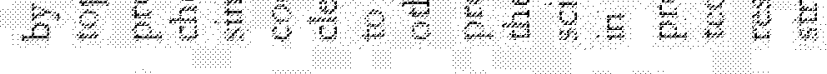

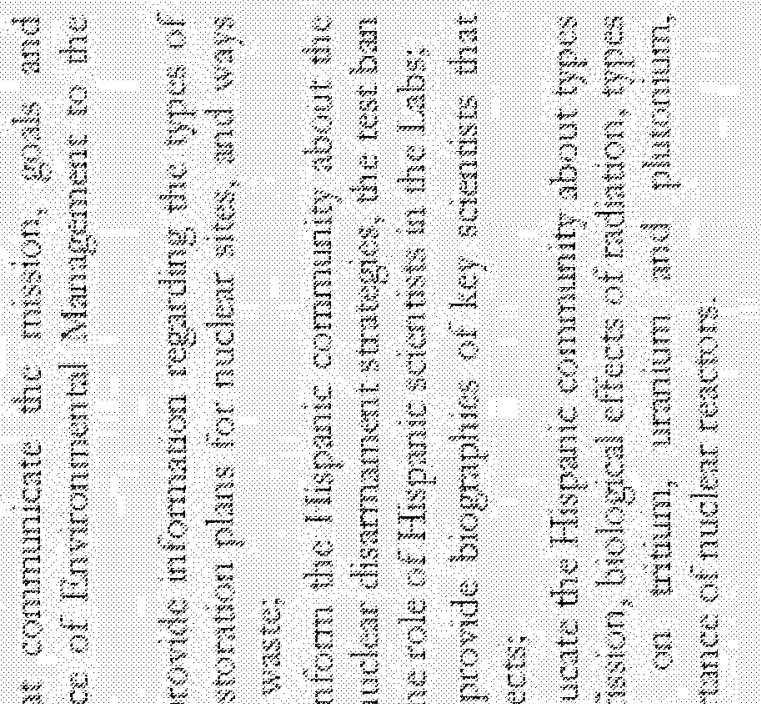

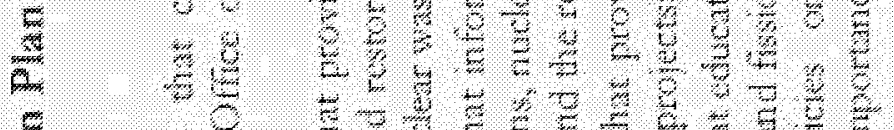
g. E E E

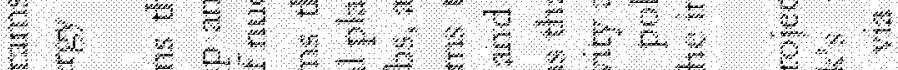

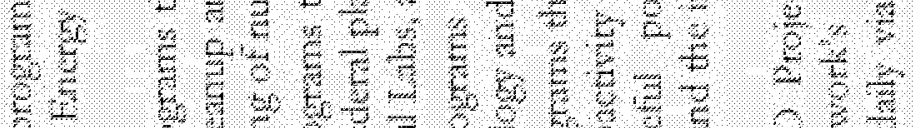

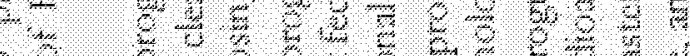

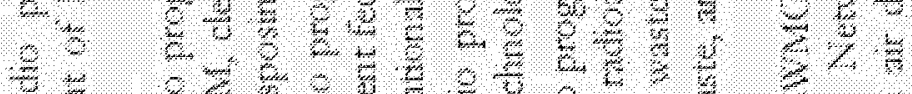

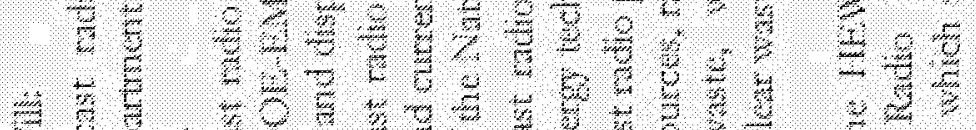

$\sum_{i=1}$

1.45 a

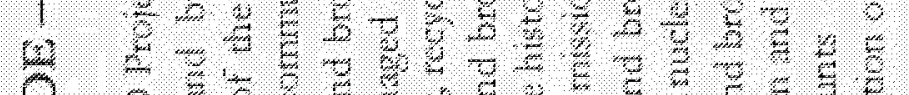

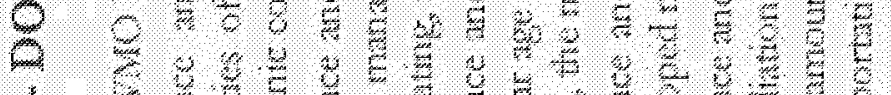

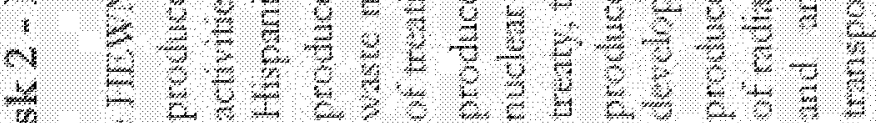

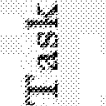

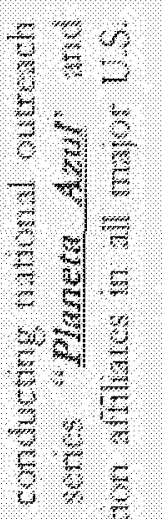

$5 \mathrm{~g}$

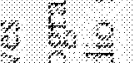

를

है 8

t. $\frac{1}{3}$ है

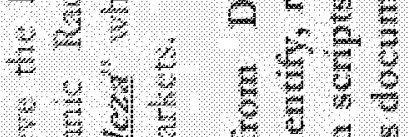

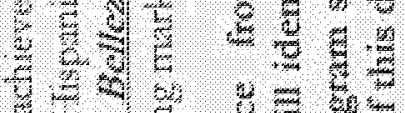
- 7 क्ष 3. 39 $\frac{2}{5}=5$ w 3 ㄴ $\frac{3}{2}=5$ z 64

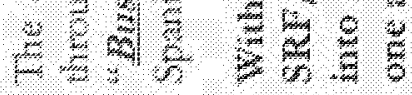



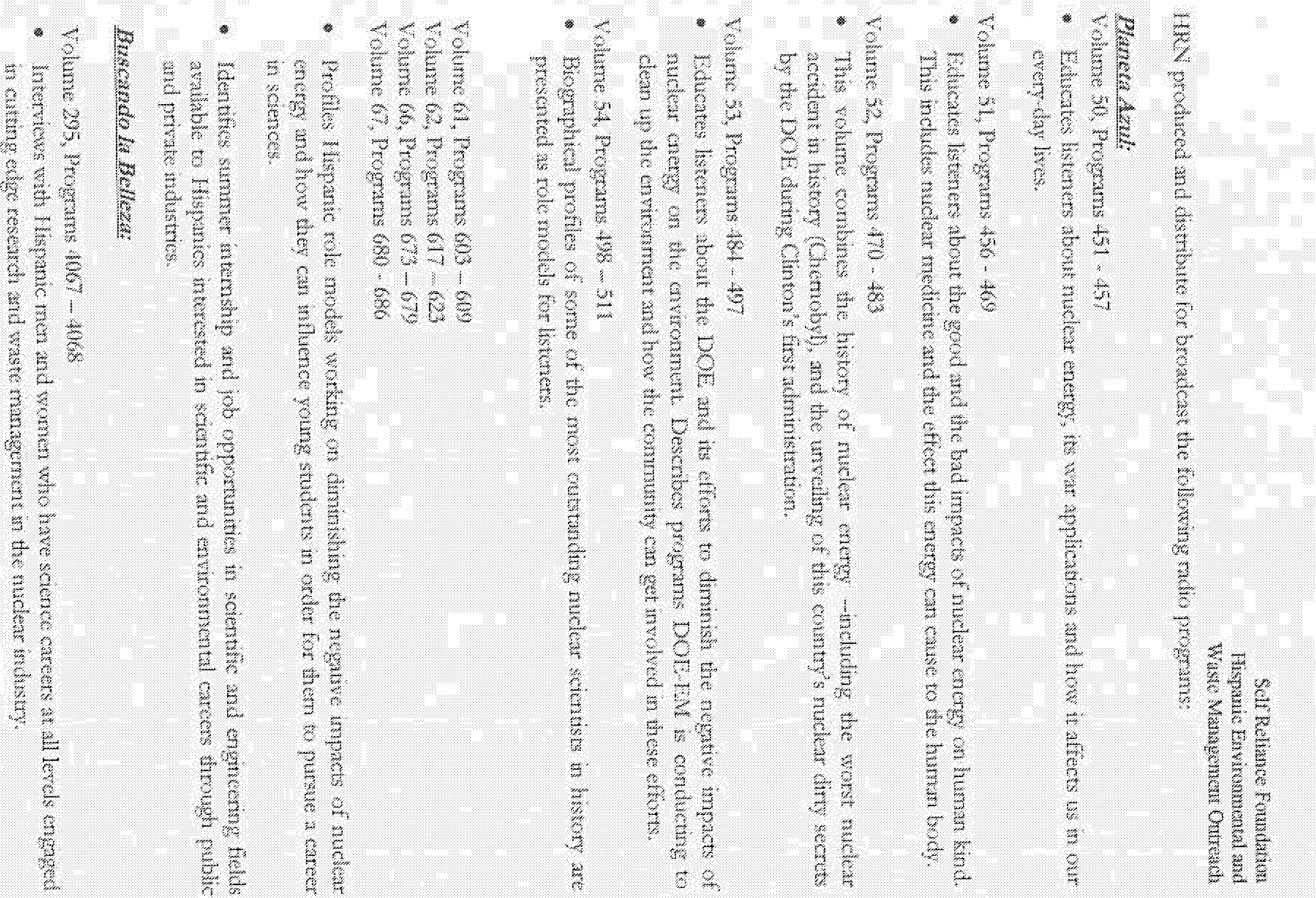
Seif Relinnce Fomdatian

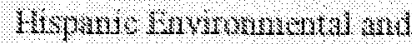

Waste Mandecnen Cutesds

Yolume 206 , Progran 4072

Volurne 298, proberns $405,4106,4108,4113$

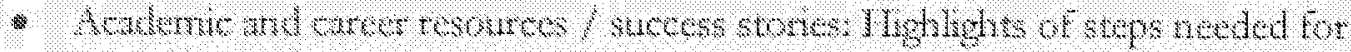

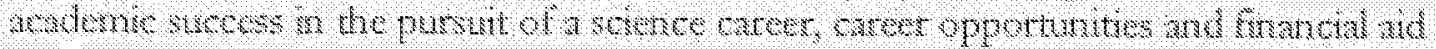
resonces and trends.

Folume 296, Proparms 4076, 4007, 4080, 4081,4037

Yolume 207 , Programs $4089,4086,4097$

Yolame 206, Programs $400 \%, 410 \%$

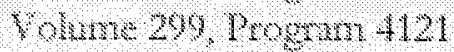

Toinne 300, Programs $4128,4133,4135$

Volsme $301, P_{\mathrm{rog}} \mathrm{mm} 4.42,453,4146,4150$

* Setence literacy and famly mwhement topre: Fxamples of and pportumaes for the

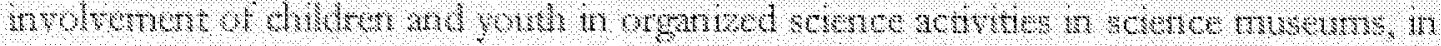
summer setenee proptams, in enwironmental projects, and in prograne prowded by

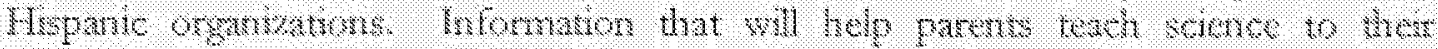

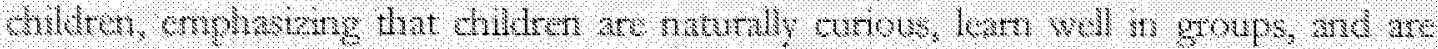
Gavenated by many buste aree of science water, light and coles, plarts. the body, arrd manus ottert terses.

Folune 012 Programs 4161,4164 , and 4165

Yolme 303 , Program 4168,4171 , and 4977

Yolumi 304, Fograme 4184 and 4192

Yohme 310 , Proprans $4260,4271,4274,4276$, and 1279

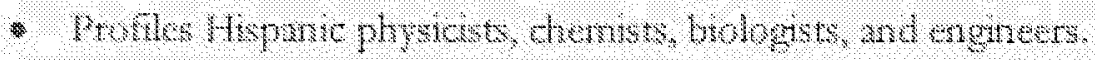

\section{Project Metrics}

Foeds groups wil be conducted in September 1948 in ordet to lest bou the radro progtrms

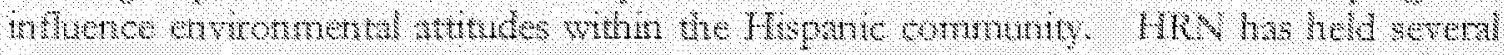

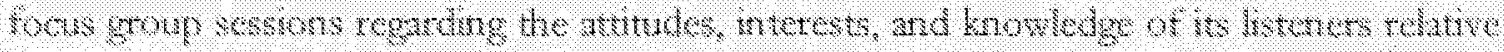

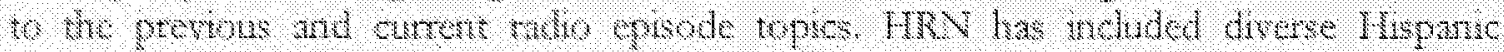

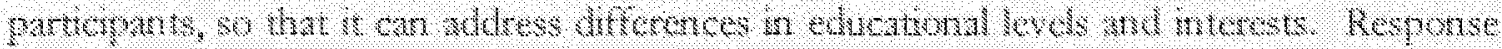

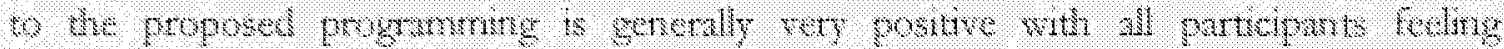
corrortable whth the accents of the program hous.

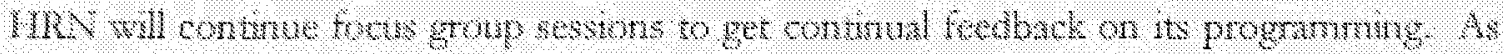
a gromp of eptsodes is serdoped for the envenmmenol seris. MRY wil select ndrutual

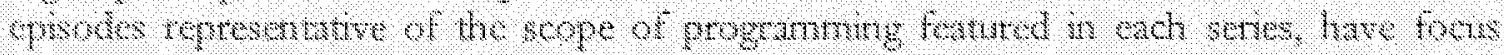
zroups of weive Grepmics likten to them, and immediately aftermard, respond wo guesurns

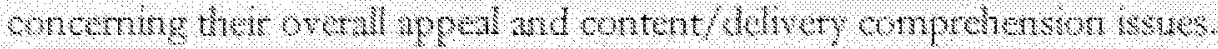


Sell Rulwee Fowndarten

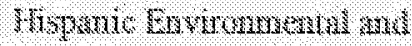

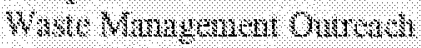

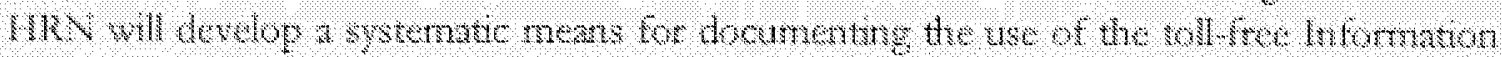

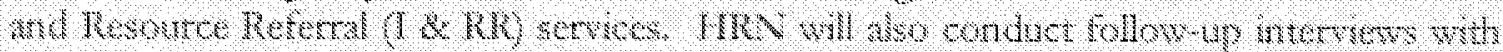

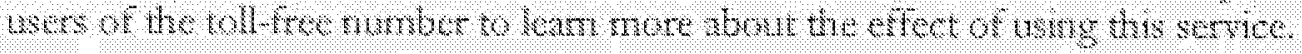

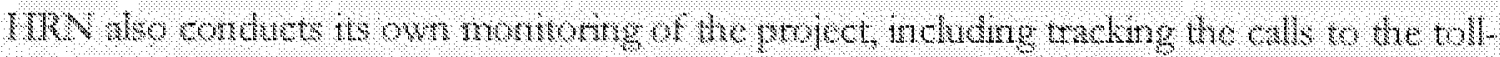

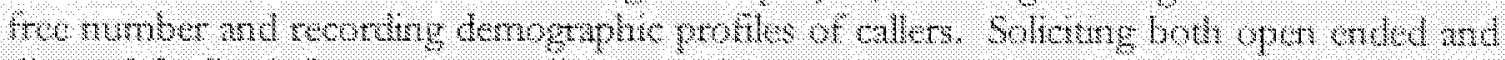

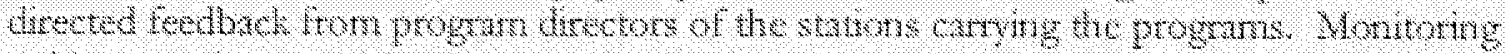

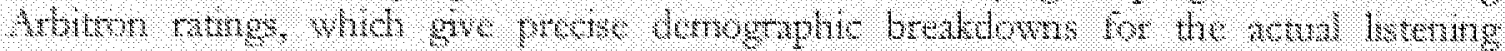

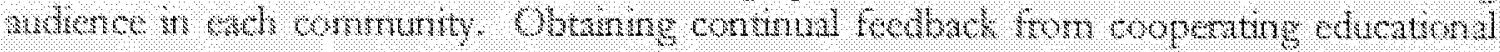

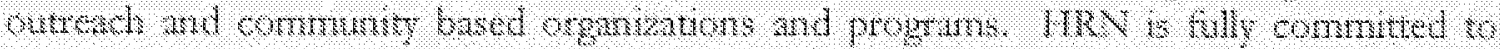

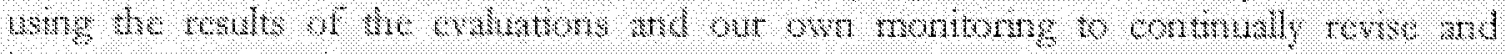
improxe progammane

\section{Third Quarter Activity Summary}

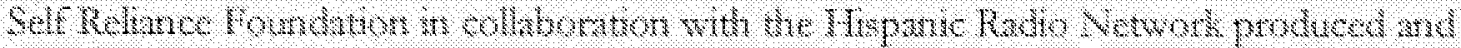

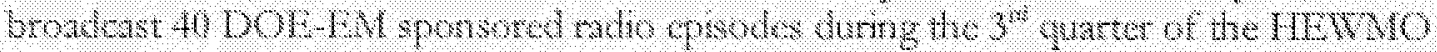

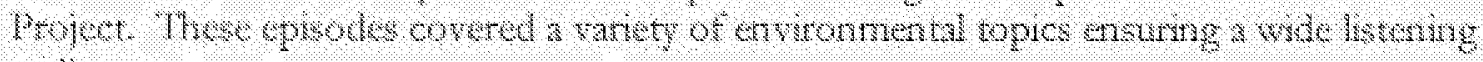
andresece.

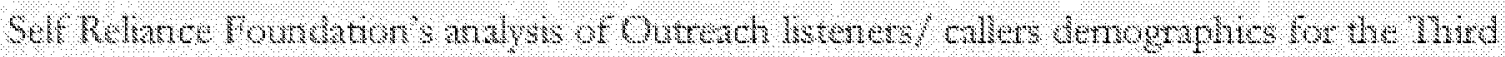
Qunter show:

- 60 o of hiteres/cillets are men

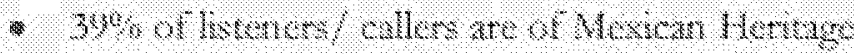

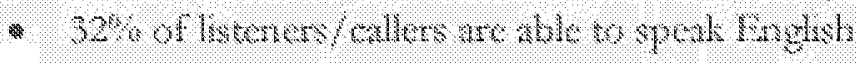

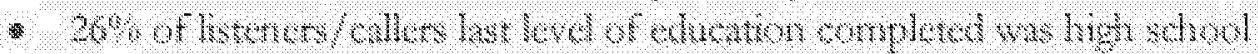



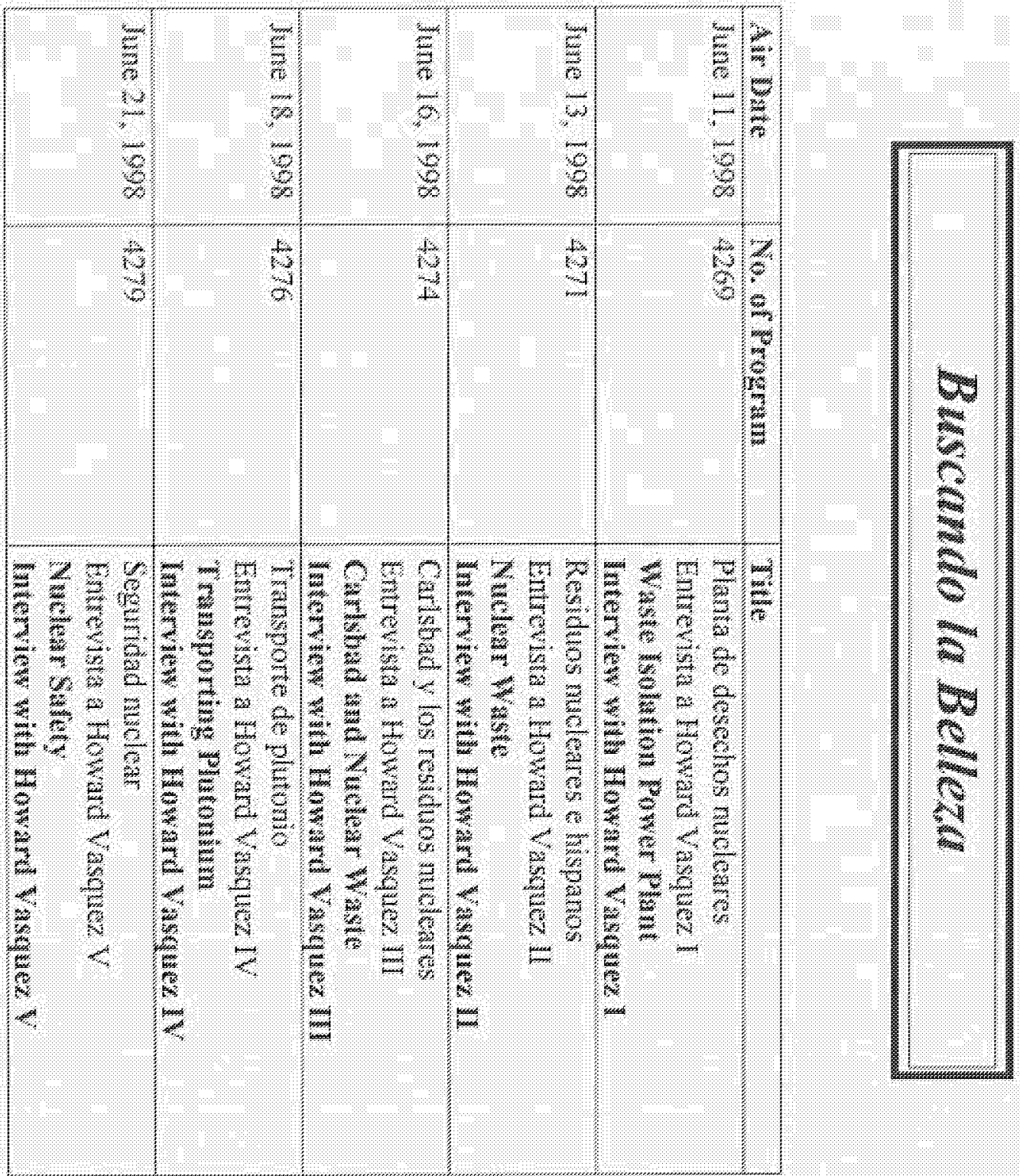


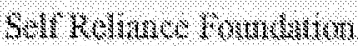

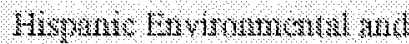

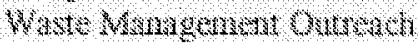

\section{Planeta Azul}

\begin{tabular}{|c|c|c|}
\hline Arr 0746 & No. of Program & Title \\
\hline Aprit 6, 1998 & 603 & $\begin{array}{l}\text { Proteccion nuclear internacional } \\
\text { Interuational Nuclear Protection }\end{array}$ \\
\hline April 7., 1908 & 804 & $\begin{array}{l}\text { Misiones espaciales } \\
\text { Special Missions }\end{array}$ \\
\hline April 8. 1908 & 605 & $\begin{array}{l}\text { Energia nuclear en el espacio } \\
\text { Nuclear Energy in Space }\end{array}$ \\
\hline April9, 1098 & 606 & $\begin{array}{l}\text { Medicina nudear I } \\
\text { Nuclear Medicinel }\end{array}$ \\
\hline April 10.1008 & 607 & $\begin{array}{l}\text { Medicina nulcear II } \\
\text { Nuclear Mcdicine II }\end{array}$ \\
\hline April11, 1968 & 608 & $\begin{array}{l}\text { A wances en medicina nuclear I } \\
\text { Advances in nuclear medicine I }\end{array}$ \\
\hline Apri 12, 1098 & 609 & $\begin{array}{l}\text { Avances en medicina nudear } 11 \\
\text { Advances in mudear mediciae } 11\end{array}$ \\
\hline Apri1 20, 1998 & 617 & $\begin{array}{l}\text { La fusion nuclear } \\
\text { Nuclear Fusions }\end{array}$ \\
\hline April 21. 1908 & 618 & $\begin{array}{l}\text { El proyeeto ITER } \\
\text { The IIER Project }\end{array}$ \\
\hline April 22, 1008 & 619 & $\begin{array}{l}\text { La radiacion cosmica } \\
\text { Cosmic Radiation }\end{array}$ \\
\hline April 23, 1908 & 620 & $\begin{array}{l}\text { La radiacion artificial } \\
\text { Artificiat Radiation }\end{array}$ \\
\hline April 24, 1998 & 621 & $\begin{array}{l}\text { Efectos biologieos de la radiacion } \\
\text { Biological Effects of Radiation }\end{array}$ \\
\hline Apri 25,1998 & 622 & $\begin{array}{l}\text { Radiacion de alta intensidad } \\
\text { High Intensity Radiation }\end{array}$ \\
\hline Apri 26, 1908 & 623 & $\begin{array}{l}\text { Radiacion de baja intensidad } \\
\text { Low Intensity Radiation }\end{array}$ \\
\hline June 15,1908 & 673 & $\begin{array}{l}\text { Misiones Espaciales } \\
\text { Special Missions }\end{array}$ \\
\hline June 16, 1998 & 674 & $\begin{array}{l}\text { La Fusion Nuclear } \\
\text { Nuclear Fusion }\end{array}$ \\
\hline Jume 17.1008 & 675 & $\begin{array}{l}\text { El Proyecto TTER } \\
\text { The ITER Projed }\end{array}$ \\
\hline June 18,1908 & 676 & $\begin{array}{l}\text { La Radiacion Cosmica } \\
\text { Cosmic Radiation }\end{array}$ \\
\hline June 19,1908 & 677 & $\begin{array}{l}\text { La Radiacion Artificial } \\
\text { Artificial Radiation }\end{array}$ \\
\hline
\end{tabular}




\begin{tabular}{|c|c|c|}
\hline June 20.1908 & 678 & $\begin{array}{l}\text { Efectos Biologicos de la Radiacion } \\
\text { Biological Erects of Radinion }\end{array}$ \\
\hline Jure 21.1988 & 679 & $\begin{array}{l}\text { Ras Intensidades y Efectos de la Radia } \\
\text { latensity and the effects of Radiation }\end{array}$ \\
\hline Jwne 22,1908 & 680 & $\begin{array}{l}\text { El rol de la opinion publica } \\
\text { The Role of Public Opinion }\end{array}$ \\
\hline June 23,1998 & 681 & $\begin{array}{l}\text { Instancias democraticas } \\
\text { Democratic lnstances }\end{array}$ \\
\hline June 2., 1908 & 682 & $\begin{array}{l}\text { Vertederos y energia } \\
\text { Dumping and Lnergy }\end{array}$ \\
\hline June 25,1908 & 683 & $\begin{array}{l}\text { Rotativas Grupales } \\
\text { Team Rotary }\end{array}$ \\
\hline June 26,1908 & 684 & $\begin{array}{l}\text { Nuevas Polinicas de decision } \\
\text { New Decision Making Policies }\end{array}$ \\
\hline Wure 27,1098 & 685 & $\begin{array}{l}\text { Quien sabe donde poner el desecho nucleat? } \\
\text { Who knows where to dispose nurclear waste? }\end{array}$ \\
\hline June 28,1998 & 686 & $\begin{array}{l}\text { Desafios para la cuidadania } \\
\text { Crullenges for Citizens }\end{array}$ \\
\hline Jne 20,1098 & 687 & $\begin{array}{l}\text { Trillum, el camaleon de los bosques } \\
\text { Trillium, the Clameleon of the Forest }\end{array}$ \\
\hline June 30,1998 & 688 & $\begin{array}{l}\text { Pl proyecto "Rio Condor" } \\
\text { "Rio Condor" Project }\end{array}$ \\
\hline July 1. 1908 & 689 & $\begin{array}{l}\text { Santuario Gondwana } \\
\text { Gondwana Sanctuary }\end{array}$ \\
\hline July 2, 1998 & 690 & $\begin{array}{l}\text { Certificacion forestal } \\
\text { Forest Certification }\end{array}$ \\
\hline July 3,1998 & 691 & $\begin{array}{l}\text { Ql impacto de las transnacionales } \\
\text { The Transuational Impact }\end{array}$ \\
\hline July 4, 1998 & 692 & $\begin{array}{l}\text { Mundo sustentabie } \\
\text { A Sustainable Wordd }\end{array}$ \\
\hline July 5,1998 & 693 & $\begin{array}{l}\text { Croniea de una muerte anunciada } \\
\text { Chronology of an advertised Death }\end{array}$ \\
\hline
\end{tabular}




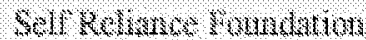

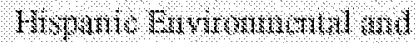

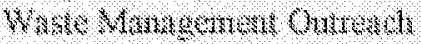

\section{Buscando la Belleza Radio Affiliates}

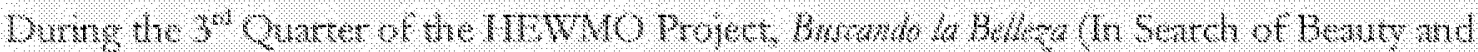

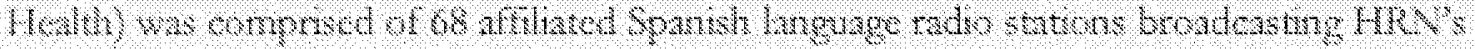

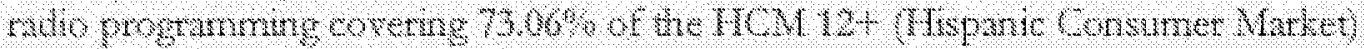
populator or 15.7 million Mispanics.

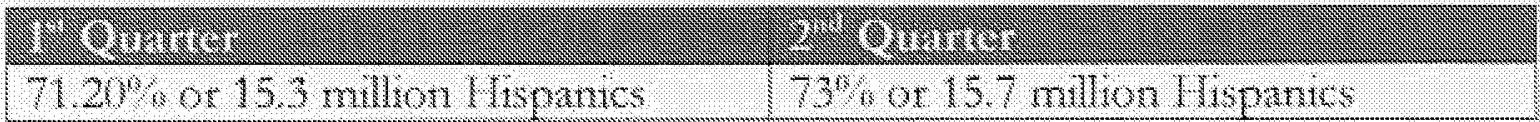

\section{Third Quarter: \\ Buscando la Belleza Program Coverage of the Hispanic Consumer Market (HCM) Age 12+}

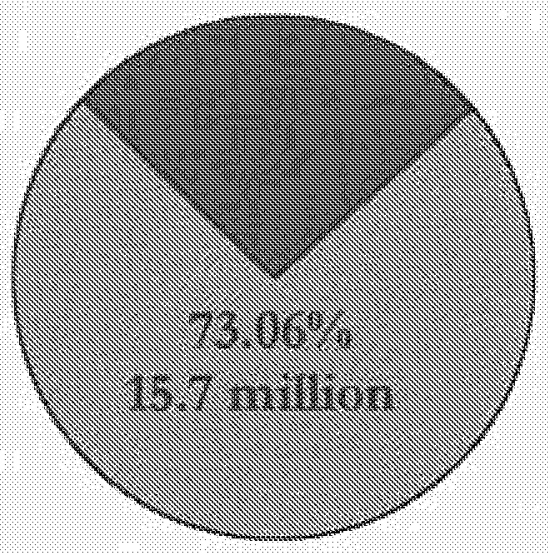


Self Rerhace foumdation

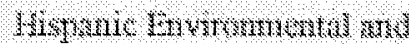

Warte Mangugancar Outread:

\section{Phanera Acu/ Radio Ambliates}

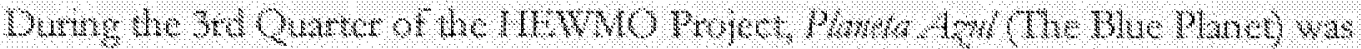

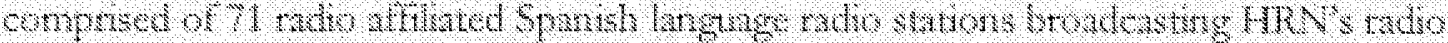

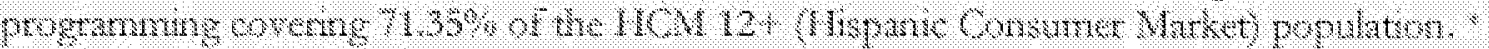
of 15.4 milion lispratics.

2010

\section{Third Quarter:}

\section{Planeta Azul Program Coverage of the Hispanic Consumer Market (HCM) Age 12+}

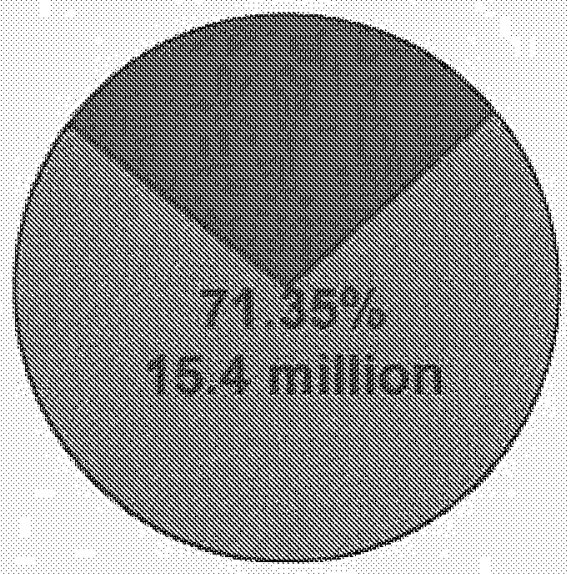

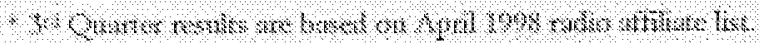

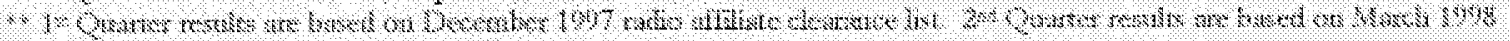

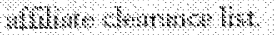


Analysis of II: $82^{\text {nu }}$ Quarter Listencr / Caller Demographics

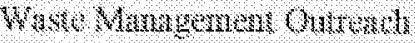

\section{Figure 1:}

Number Of Episodes Broadcast

\begin{tabular}{|c|c|c|c|}
\hline$x_{2}^{2}$ & 1300 & 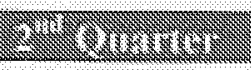 & 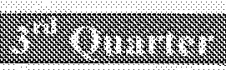 \\
\hline Buscando la Beleza & 35 & 15 & 5 \\
\hline Plancta Azul & 70 & 4 & 35 \\
\hline Total & 105 & 9 & 40 \\
\hline
\end{tabular}

Figure 2:

DOF EM Envimonmental Callers by Gender

\begin{tabular}{|c|c|c|c|}
\hline 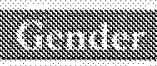 & 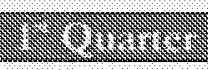 & 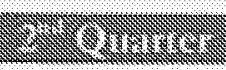 & 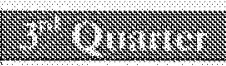 \\
\hline Mals & $40 \%$ & 448 & $60 \%$ \\
\hline fermale & $51 \%$ & $56 \%$ & $40 \%$ \\
\hline
\end{tabular}

\section{DOE- EM Callers By Gender}

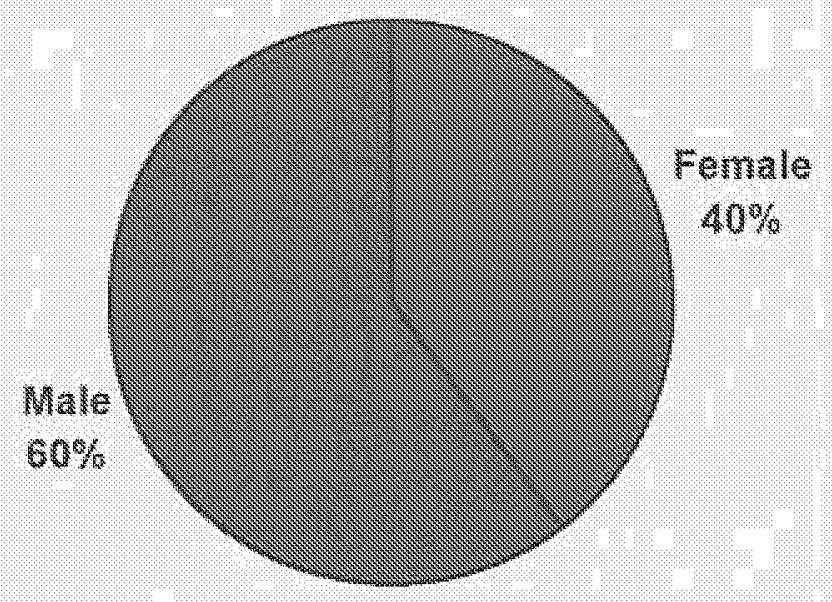


Figure 3:

DOE FM Fnvironmental Callets by Age

\begin{tabular}{|c|c|c|c|}
\hline 10 & 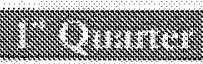 & 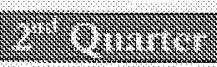 & 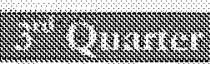 \\
\hline $17 \&$ Irdet & $0 \%$ & 38 & $1 y_{8}$ \\
\hline $18-38$ & $10 \%$ & 428 & $419 \%$ \\
\hline $36-48$ & $24 \%$ & $30 \%$ & $36 \% 9$ \\
\hline $16 \%$ & $22 \%$ & $20 \%$ & 234 \\
\hline
\end{tabular}

\section{DOE-EM Callers By Age}

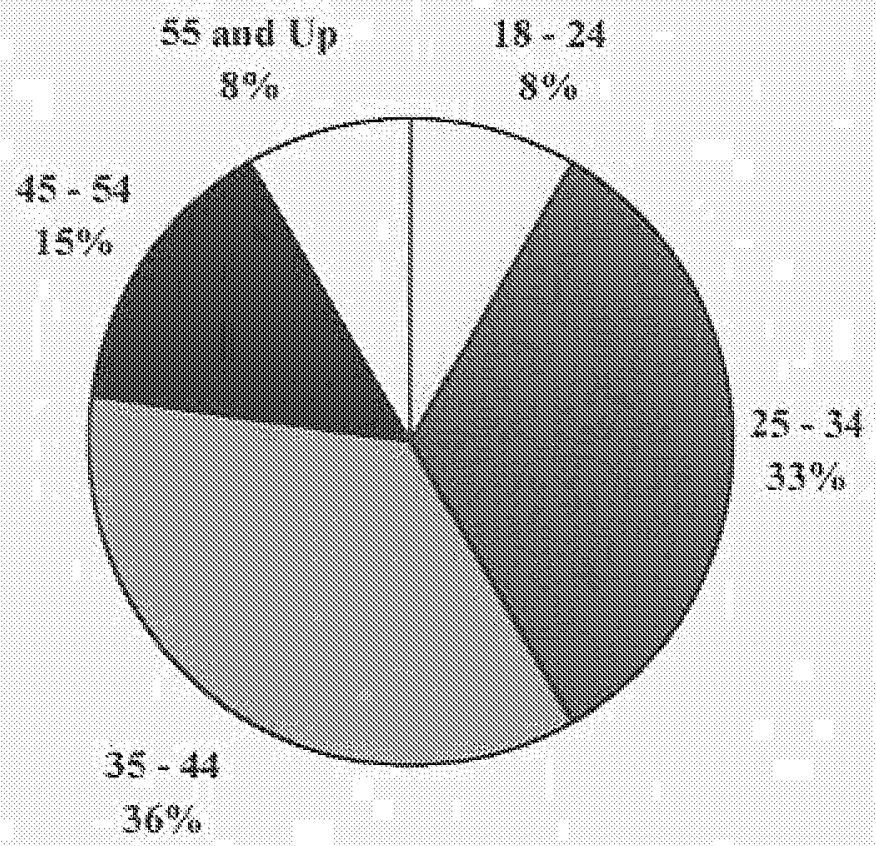




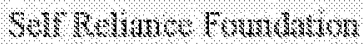

\section{Figure 4:}

DOL - EM Rnvitonmental Callets by English Proficiency

\begin{tabular}{|c|c|c|c|}
\hline 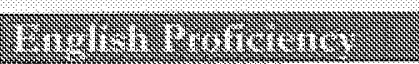 & 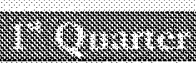 & 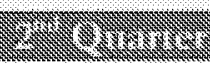 & 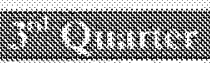 \\
\hline 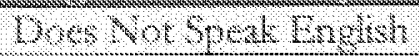 & $17 \%$ & 168 & $26^{\circ}$ \\
\hline 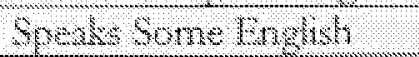 & $30^{\circ}$ & $33^{8} / 2$ & $3 \%$ \\
\hline 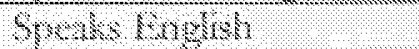 & $33^{*}$ & $31 \%$ & $32 ?^{3}$ \\
\hline
\end{tabular}

\section{DOE- EM Callers By English Proficiency: Speak}

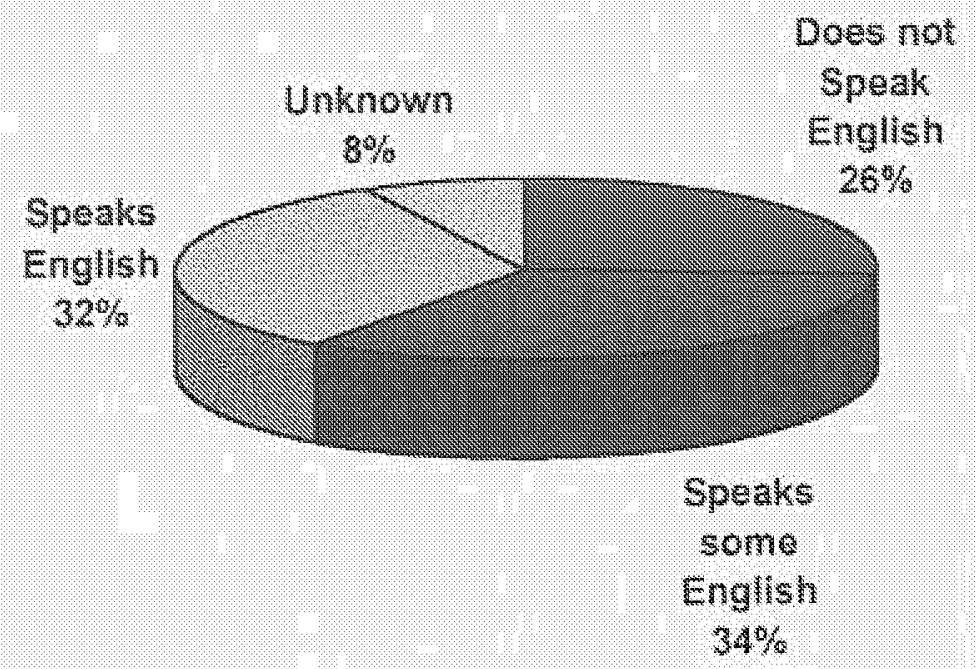




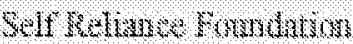

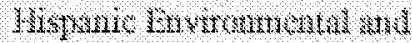

Waste Nenagemess Outratad

\section{Figure 5}

DOF BM Environmental Callers by Education Lexd

\begin{tabular}{|c|c|c|c|}
\hline 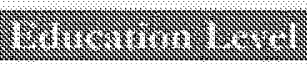 & 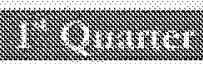 & ॠथख्य & 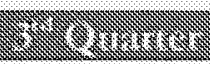 \\
\hline Dotic & $0 \%$ & $1 \%$ & 28 \\
\hline Menertary & $37 \%$ & $11 \%$ & $15 \%$ \\
\hline Is. Truph School & 78 & $7 \%$ & $6 \%$ \\
\hline $\mathrm{Mr}$ h Solroul & $20 \%$ & $41 \%$ & $26 \%$ \\
\hline Sollers & $28 \%$ & $31 \%$ & $34 \%$ \\
\hline Post Gradunte & $2 \%$ & 30 & 28 \\
\hline
\end{tabular}

\section{DOE-EM Callers By Education}

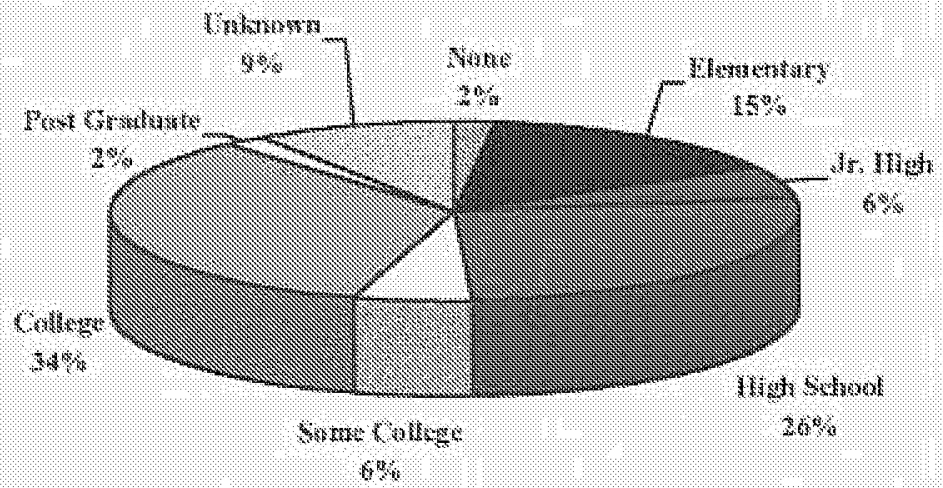




\section{DOE- EM Callers By Ethnic Heritage}

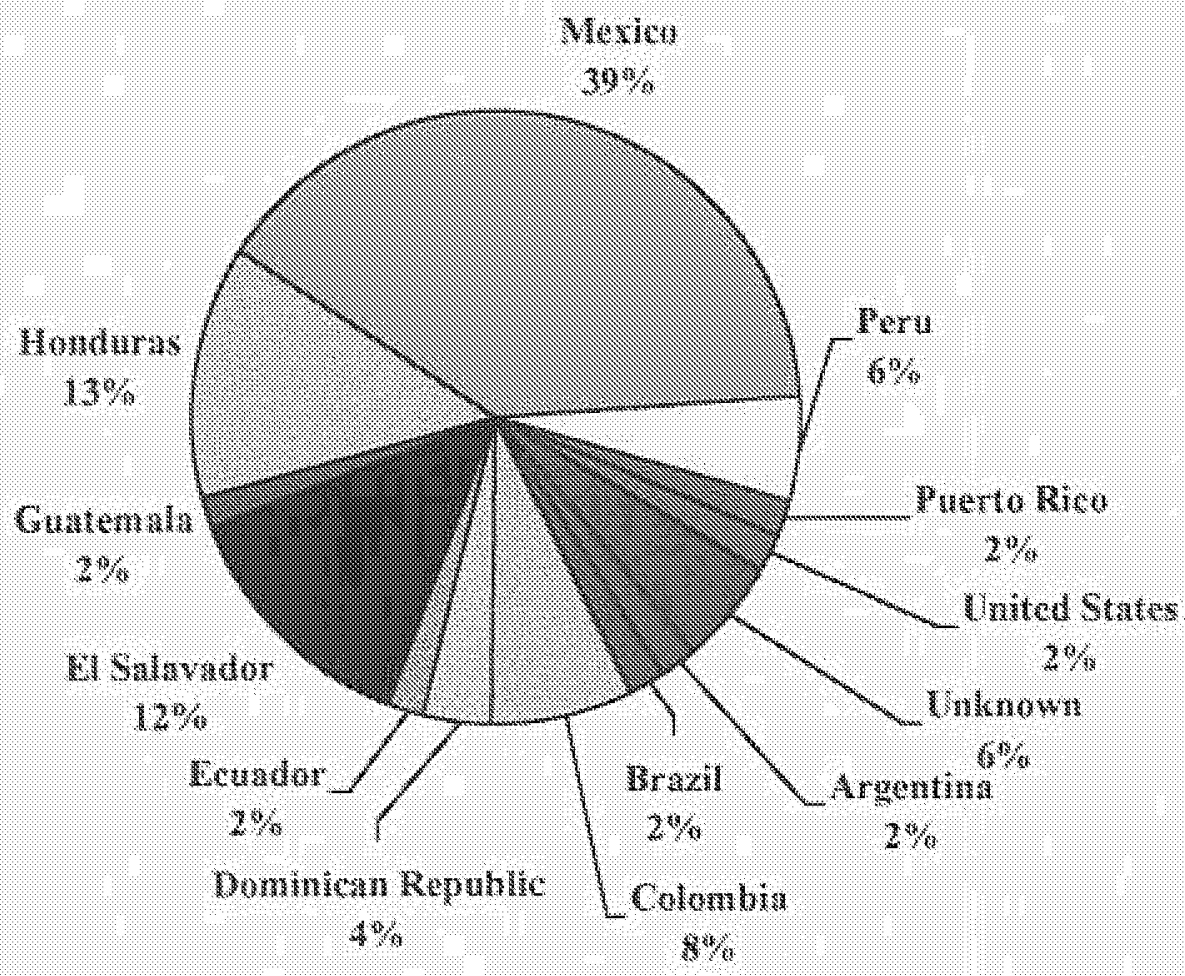




\section{APPENDICES}




\section{1. Program Guide For Buscando la Belleza}




\section{HISPANIC RADIO NETWORK}

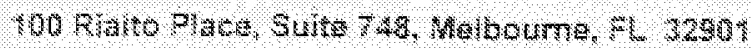

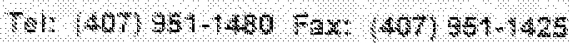

\section{PROGRAM NSERTION ORDER \& AFFIDAVIT OF PERFORMANCE}

\section{Buscando La Belleza / hosted by: Consuelo Luz}

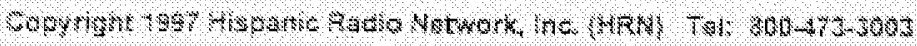

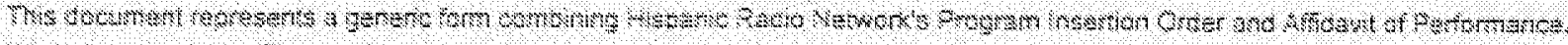

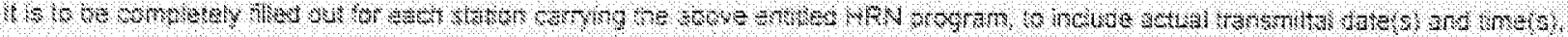

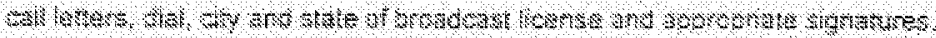

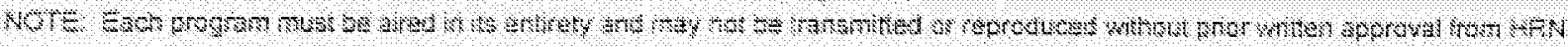

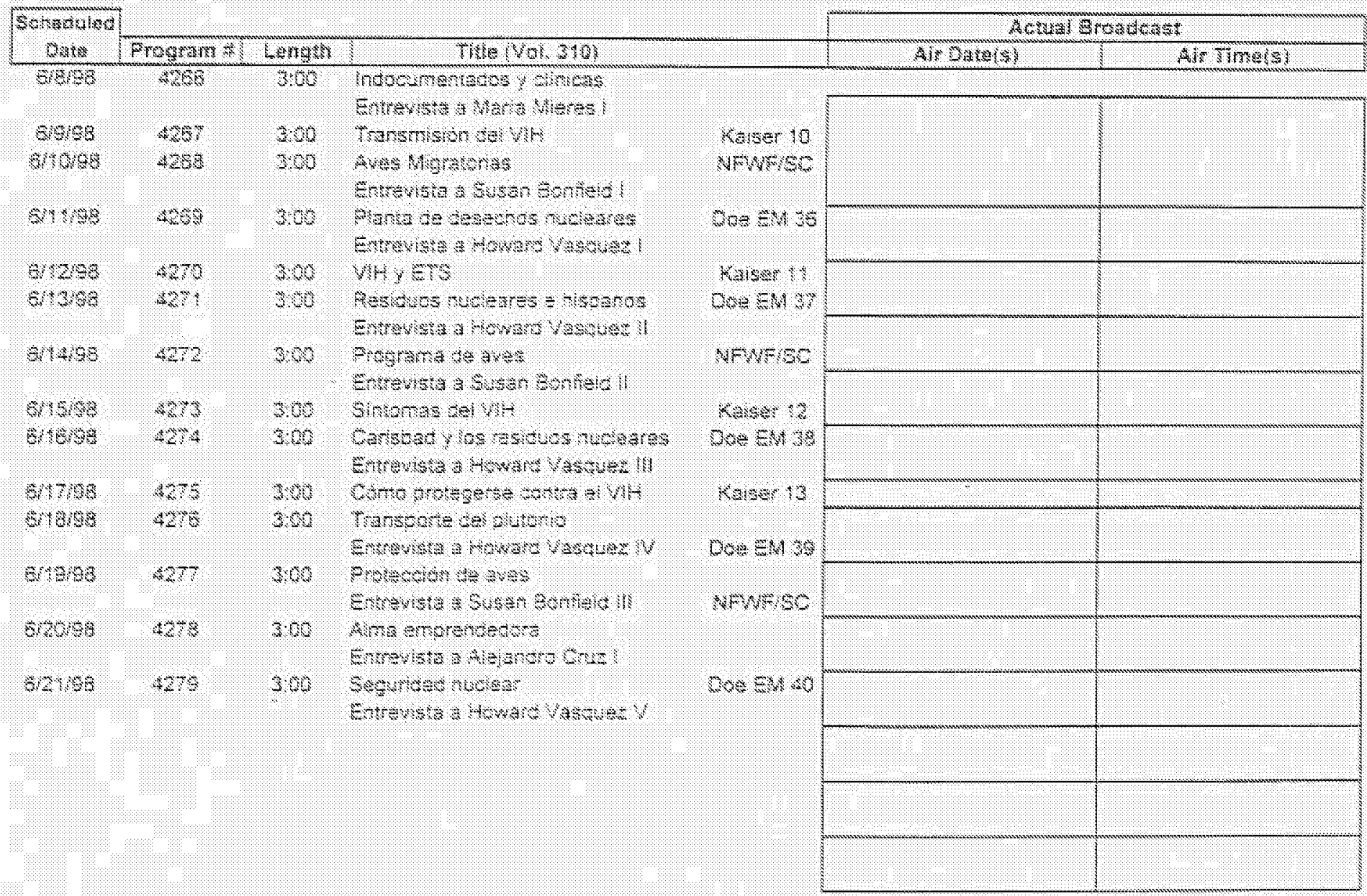

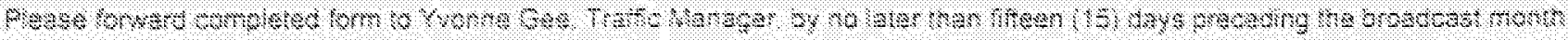

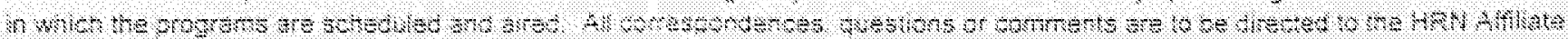

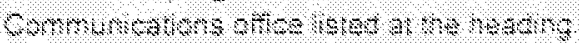

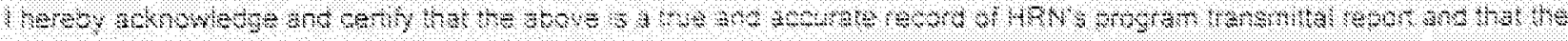

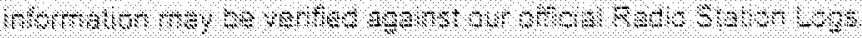

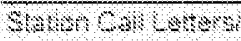

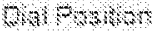

3.

The

\section{$\odot+1 \times 14 \times \%$}

ㄴ:?

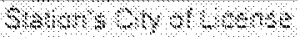


HISPANIC RADIO NETWORK

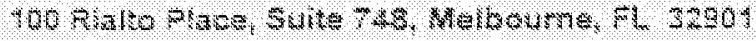

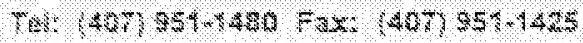

\section{PROCRAM INSERTCV OROER \& AFFDAVT OF PERFORMANCE}

\section{Buscando la Belleza hosted by: Consuelo Luz}

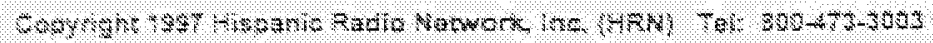

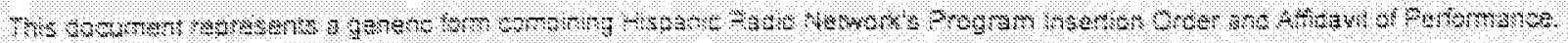

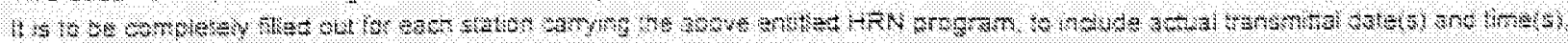

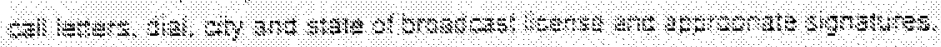

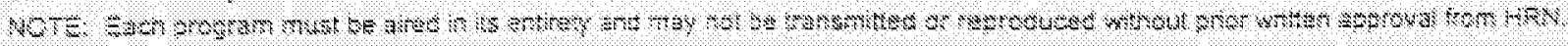

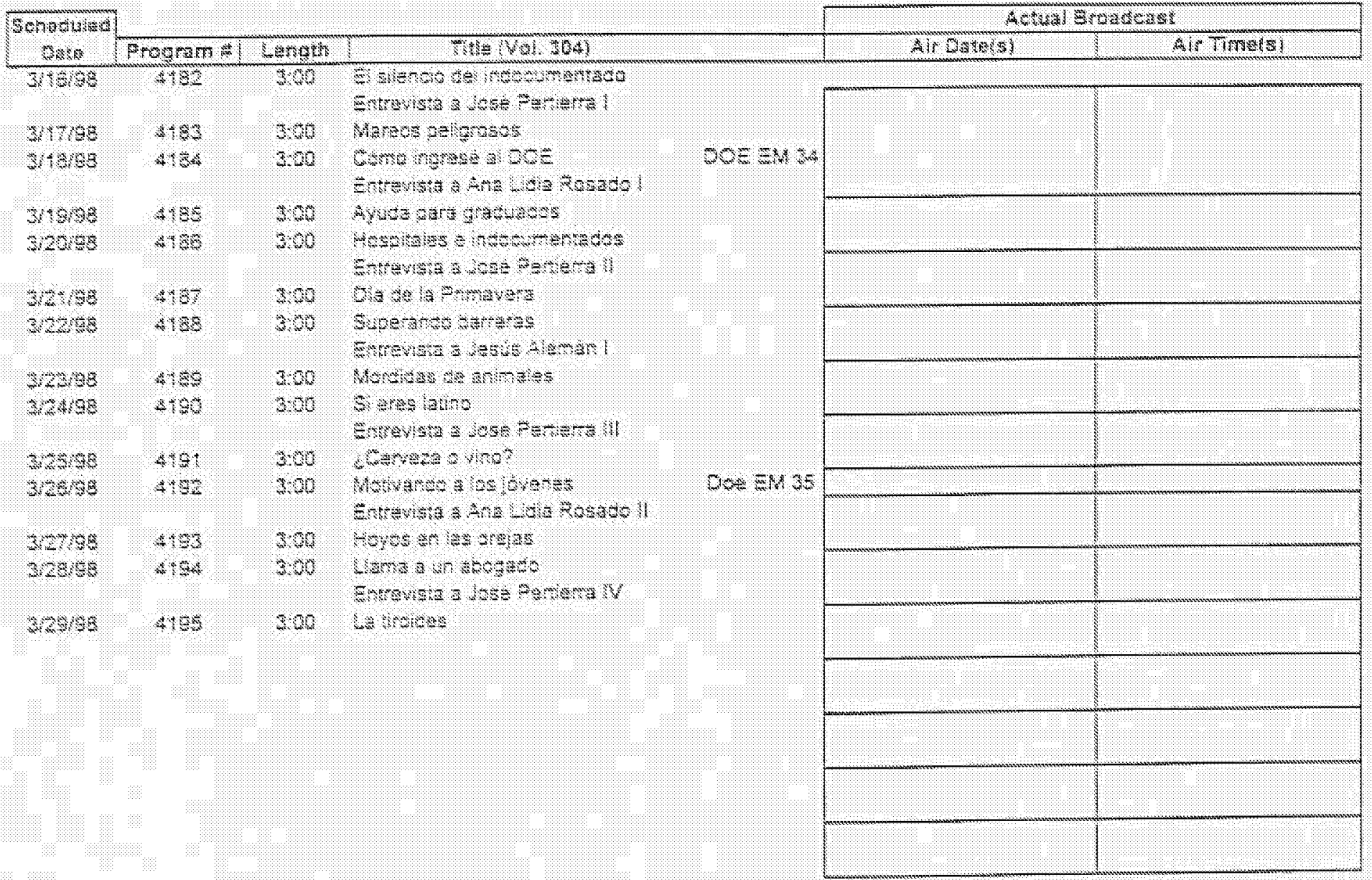

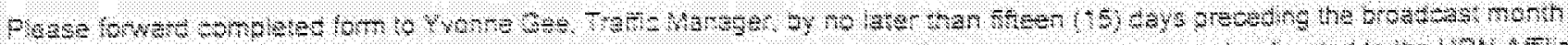

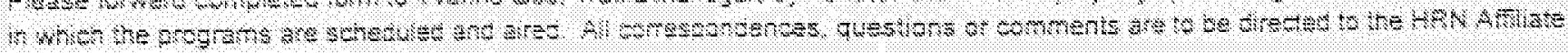

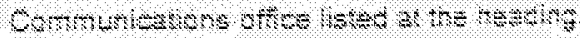

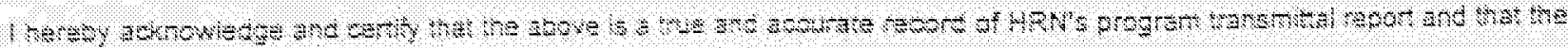

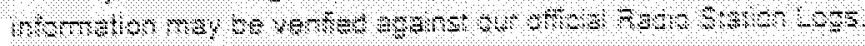

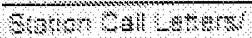

$0+80,+4$

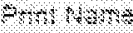

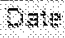

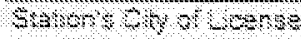

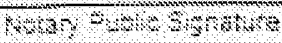




\section{HISPANIC RADIO NETWORK}

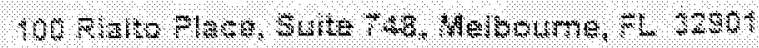

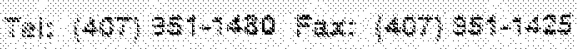

\section{PROCRAW INSERTION OFOER A AFFICAVT OF PERFORWANEE}

\section{Buscrendo La Balleza / hosted by: Consuelo Luz}

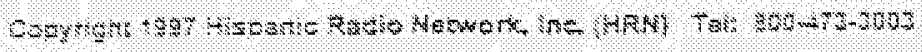

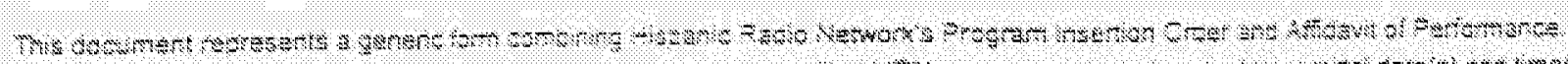

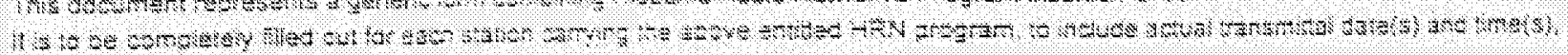

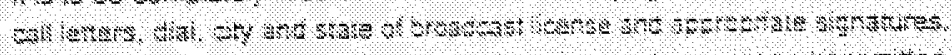
NOP $=$ _

\begin{tabular}{|c|c|c|c|c|c|c|}
\hline \multirow{2}{*}{ 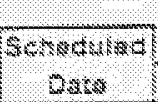 } & \multirow{2}{*}{\multicolumn{4}{|c|}{$760(194303)$}} & \multicolumn{2}{|c|}{ Actual sroancerse } \\
\hline & seormat & & & & 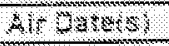 & $4+11780$ \\
\hline 328 & 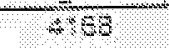 & 300 & A,utas hareists: & Soe $=M 31$ & & \\
\hline & & & 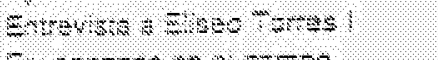 & & & \\
\hline 3348 & $4+58$ & $4, \infty$ & 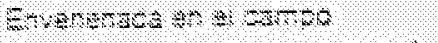 & & & \\
\hline & & & 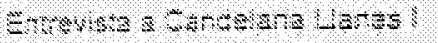 & & & \\
\hline 24,408 & $4 \rightarrow 7$ & 300 & 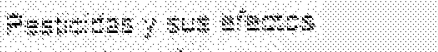 & & & \\
\hline $3, \pi=8$ & 4,4 & 340 & 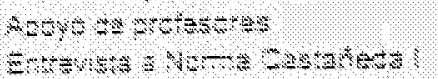 & $00=-1 \times 22$ & & \\
\hline 3609 & 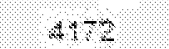 & $3 \%$ & 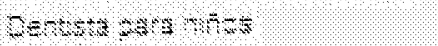 & & & \\
\hline $3+98$ & 40 & 300 & 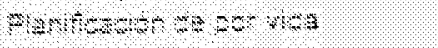 & & & \\
\hline 30,50 & s०4 & $3+4$ & 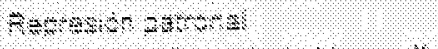 & & & \\
\hline & & & 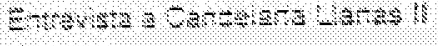 & & & \\
\hline 384:3 & 483 & 340 & 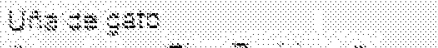 & & & \\
\hline & & & 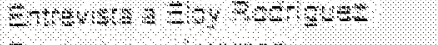 & & & \\
\hline 2408 & 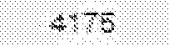 & ssto & 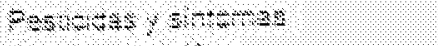 & & & \\
\hline 241,90 & : $\bullet$ & ४०० & 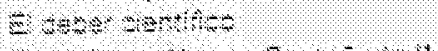 & $000=42$ & & \\
\hline & & & 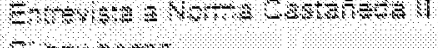 & & & \\
\hline 31203 & 480 & 300 & 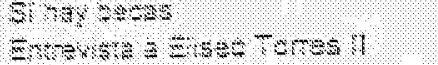 & & & \\
\hline 34380 & 478 & $3 . \infty$ & 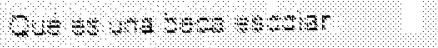 & & & \\
\hline $3 / 1888$ & 480 & $3 \circ 0$ & 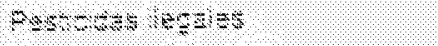 & & & \\
\hline $3+806$ & 48 & 3000 & 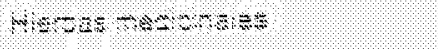 & & & \\
\hline & & & $:$ & & & \\
\hline & & & 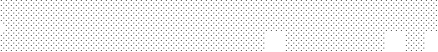 & & & \\
\hline & & & 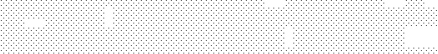 & & & \\
\hline & & . & 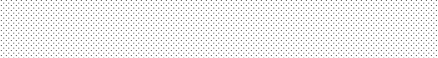 & is & & \\
\hline & & & & & & \\
\hline 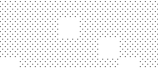 & 9 & & sing & & & \\
\hline
\end{tabular}

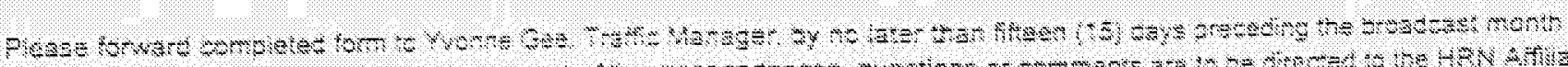

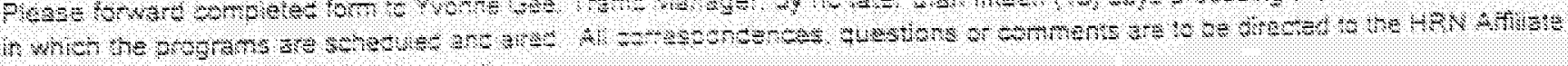

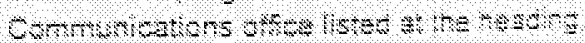

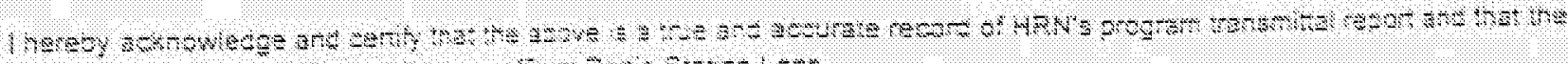

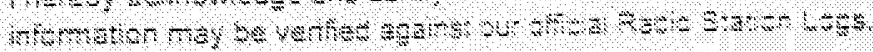

\section{3atsor Col 6 rar}

$\mathrm{ras}+4+6$

Sutors -ry of Lermet

\section{S4.45}

irs

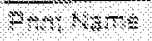

Stre 


\section{HISPANIC RADIO NETWORK}

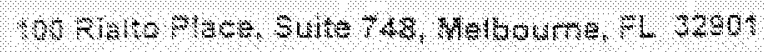

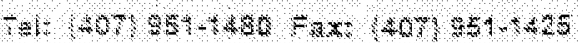

\section{PROCRAM NSERTON OROEF \& AFFIOAUT OF PERFOPUANCE}

\section{Buscando La Bellera / hosted by Consuelo Luz

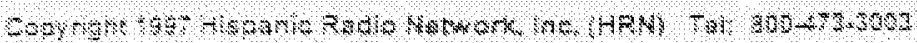

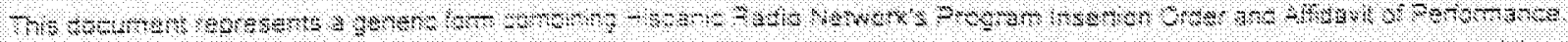

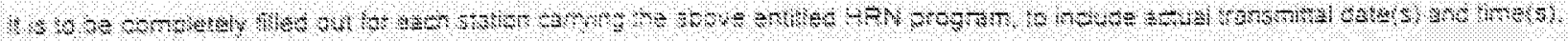

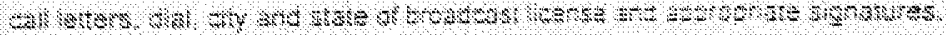

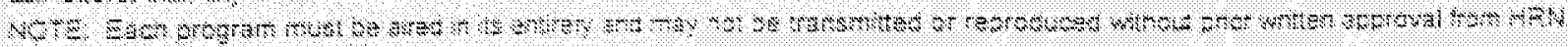

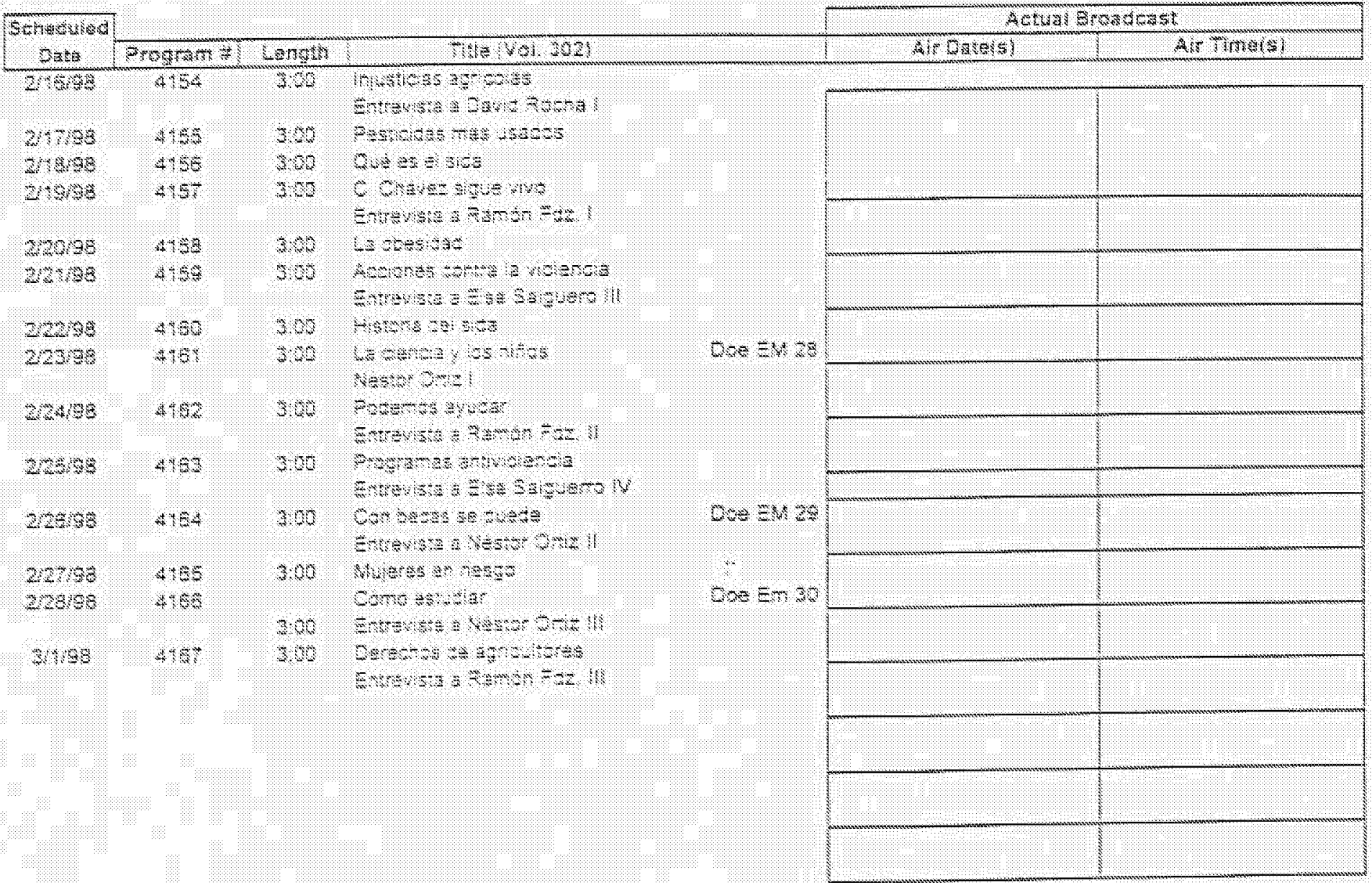

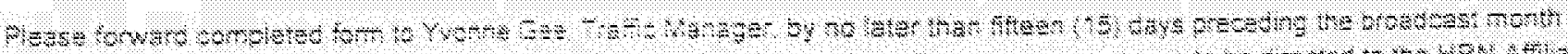

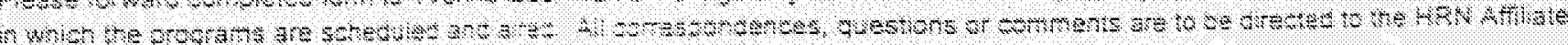

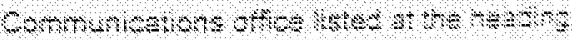

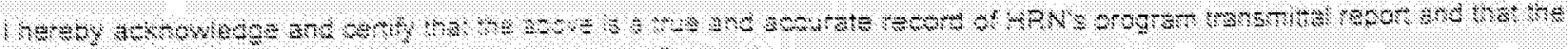

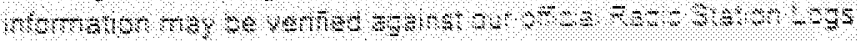

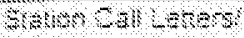

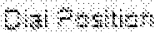

$3.40 \%$

7t: 3monthe

2atse

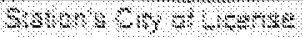




\section{HISPANIC RADIO NETWORK}

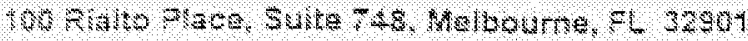

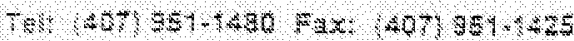

\section{PROGRAW NSERTON ORDER \& AFFIDAVT OF FERFOFMANCE}

\section{Buscando la Belleza / hosted by Consuelo Luz}

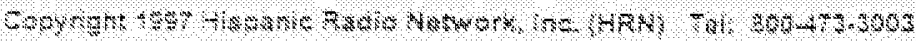

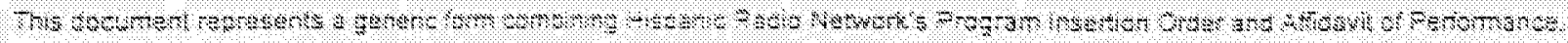

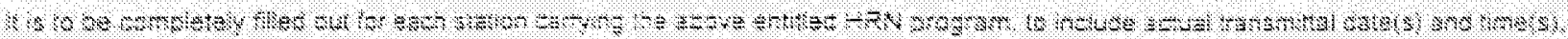

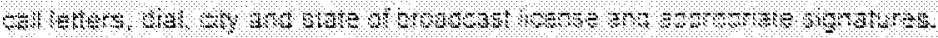

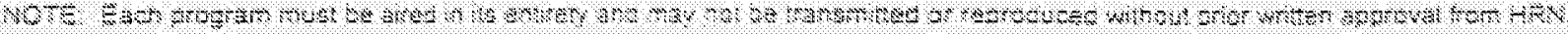

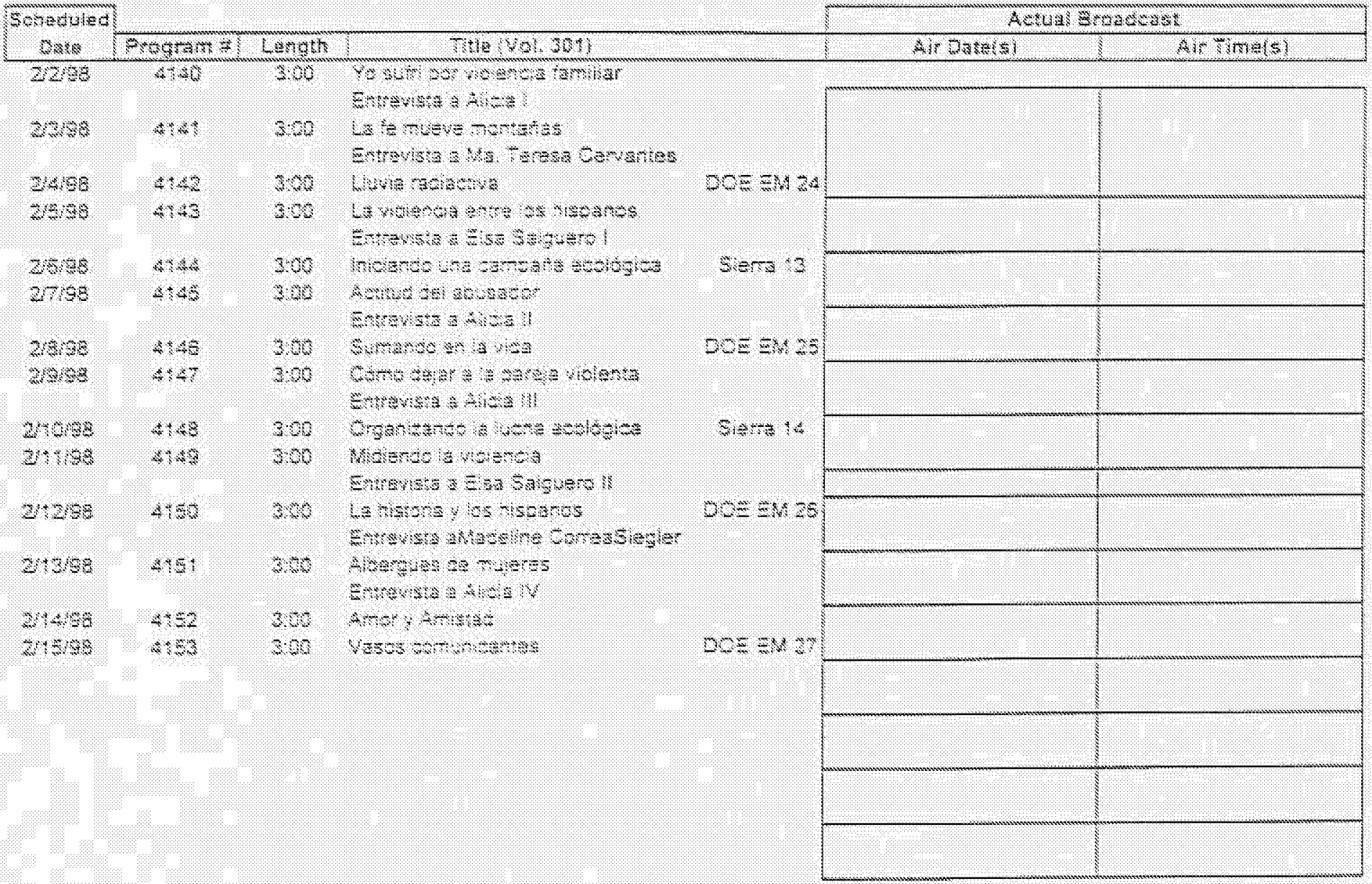

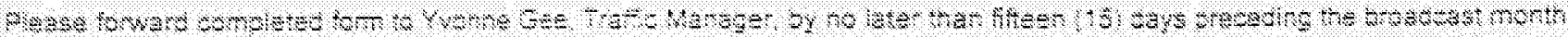

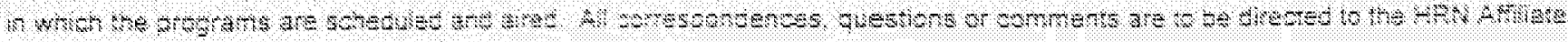

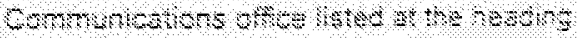

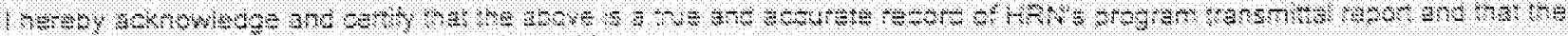

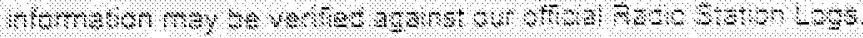

$3006+8+4$

$34+86$
$-1,+4$

30

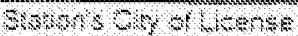




\title{
HISPANIC RADIO NETWORK
}

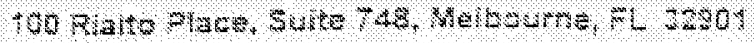

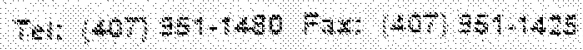

\section{PROCRAM INSERTION ORDER \& AFFDAVT OF RERFORMANCE}

\author{
Euscando la Bellezal hosted by: Consweio Luz

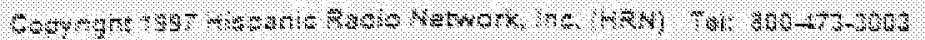

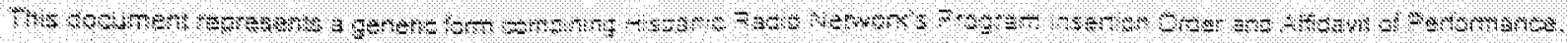

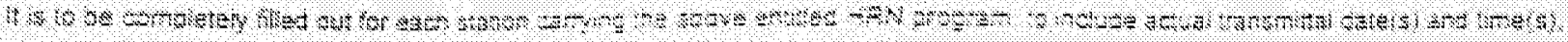

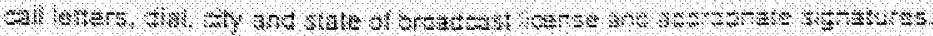

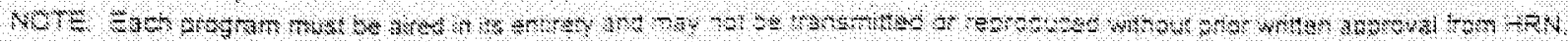

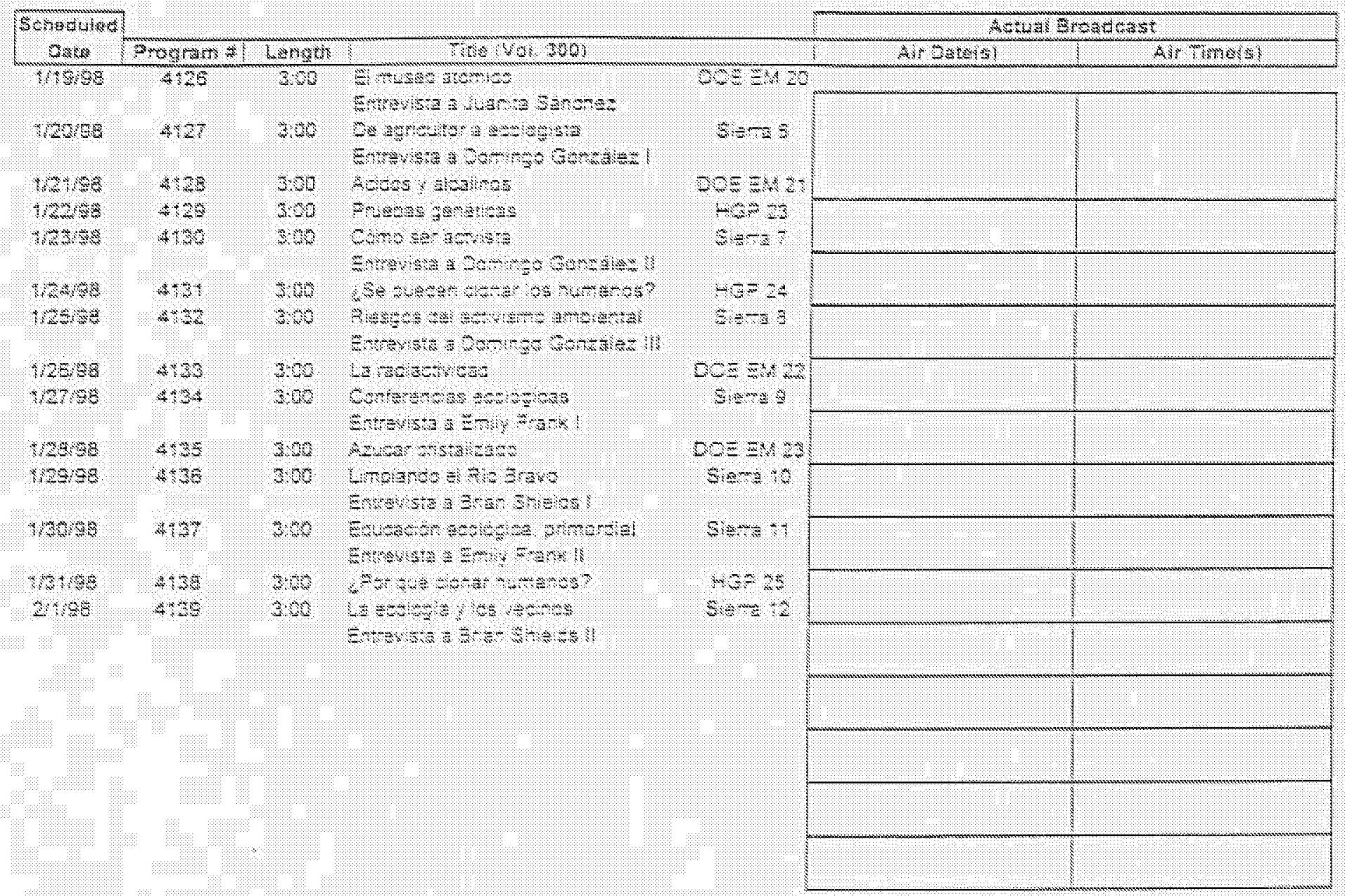

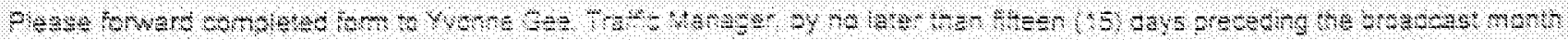

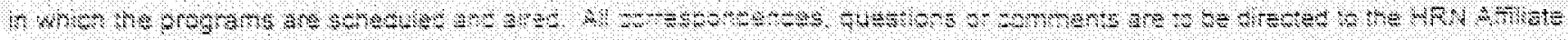

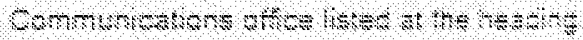

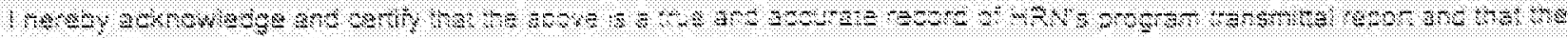

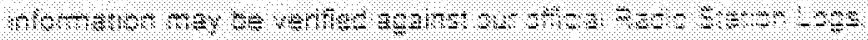

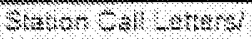

$0.4=5.400$
$370 \times 6 r 6$

\section{Fes}




\section{HISPANIC RADIO NETWORK}

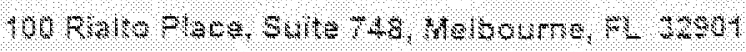

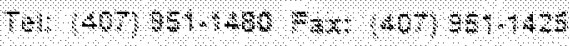

\section{PROGRAM NSERTON ORDER \& AMFOAVT OF PERFORMANCE}

\section{Guscando la Belleza / hosted by: Consuelo Luz

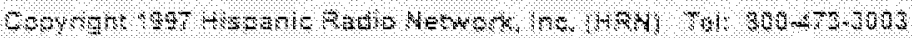

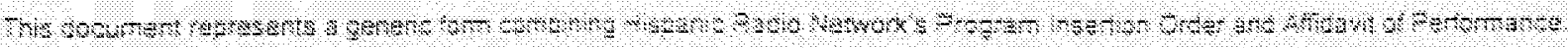

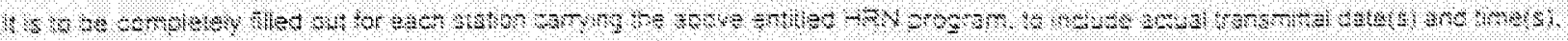

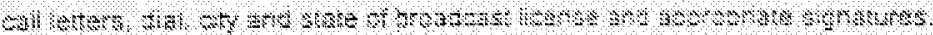

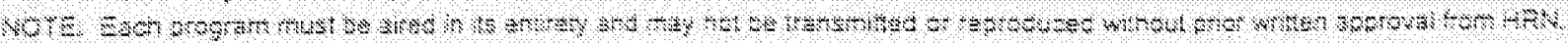

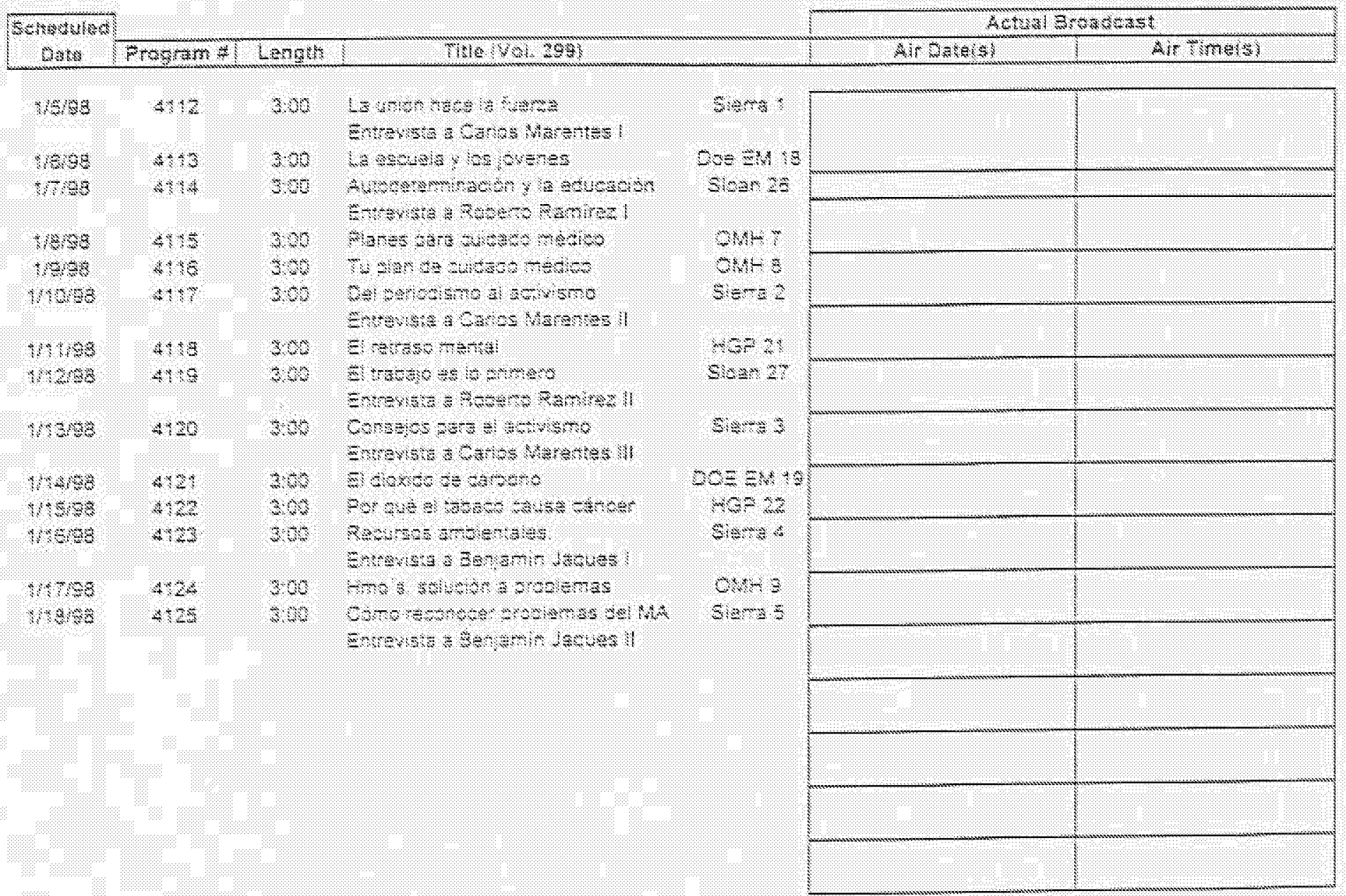

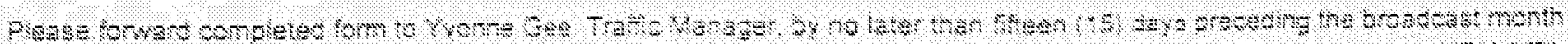

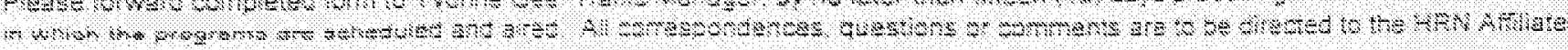

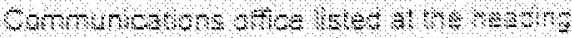

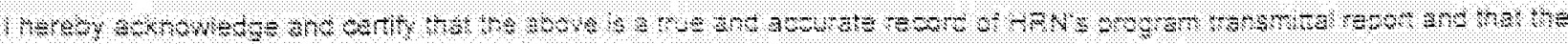

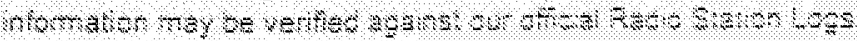

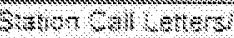

$8+20.600$

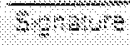$$
\text { क. }
$$

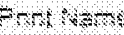

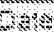




\section{HISPANIC RADIO NETWORK}

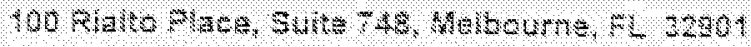

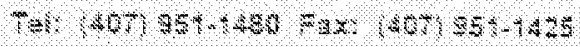

\section{PROORAM NEERTION ORDER A AFFOAVTT OF PERFORMANCE}

\section{Buscando la Belleza / hosted by. Consuelo Luz}

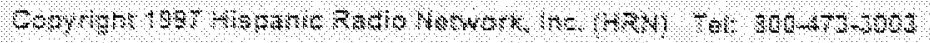

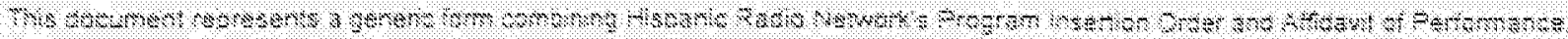

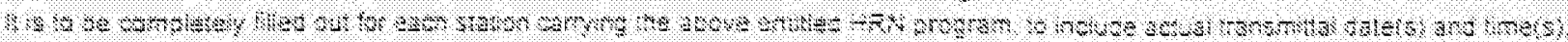

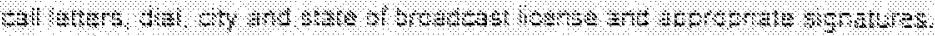

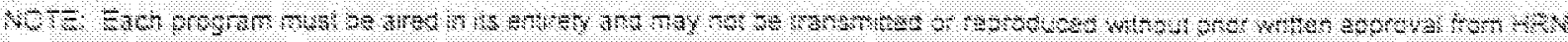

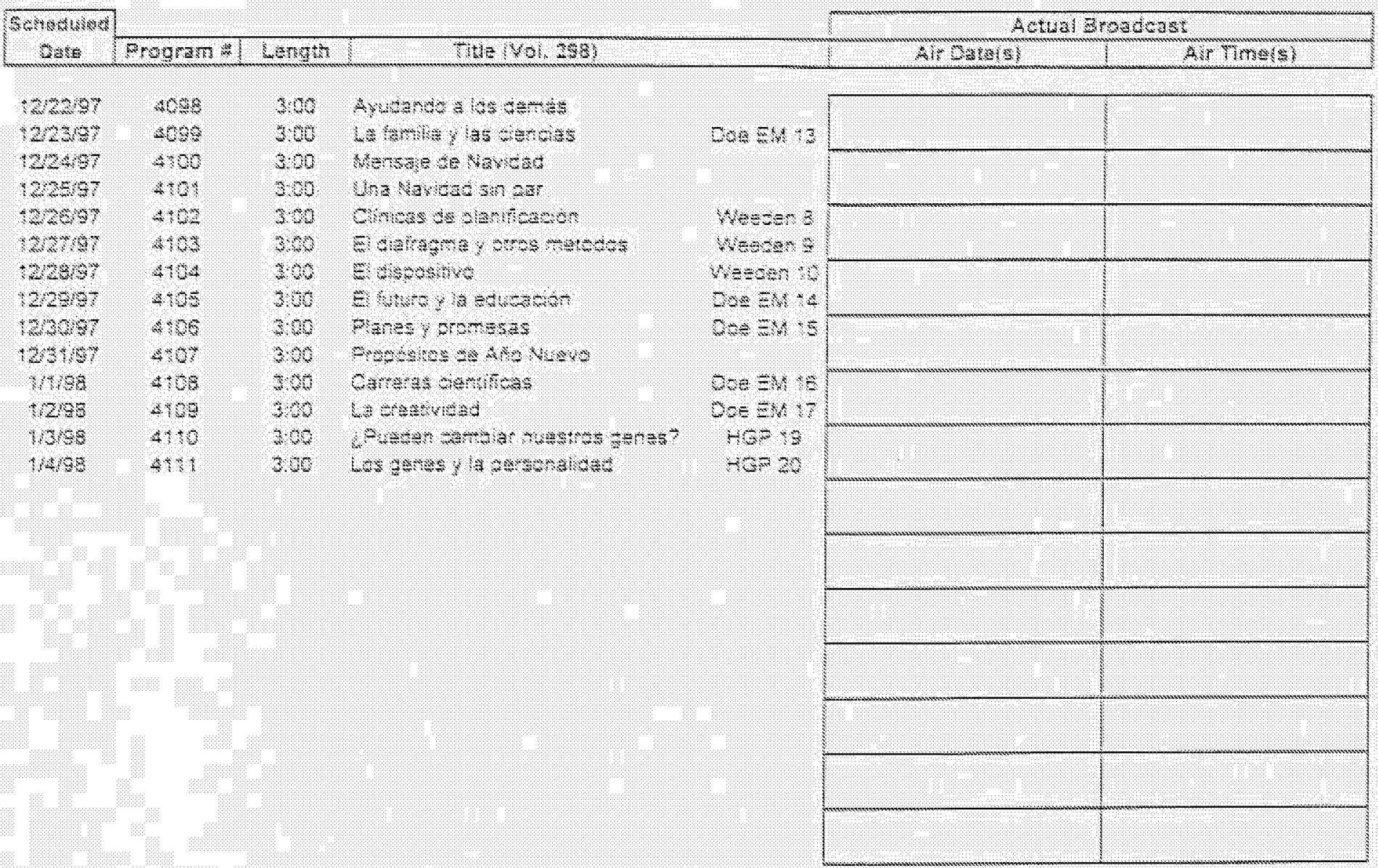

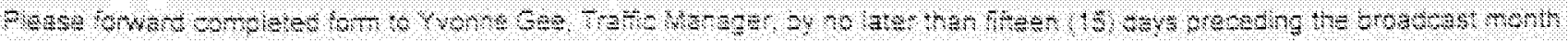

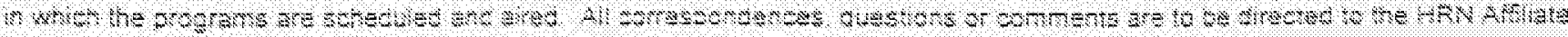

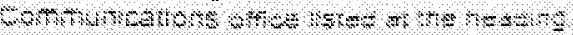

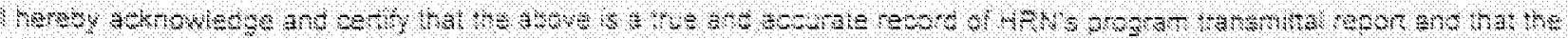

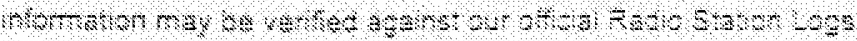

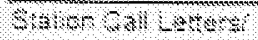

$48-0,106$ 3rotorne

Sarsis

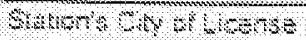

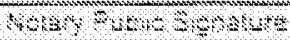




\section{HISPANIC RADIO NETWORK}

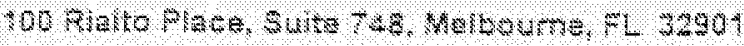

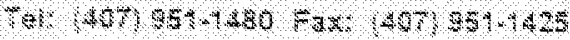

\section{PROCRAM INSERTION ORDER \& AFFIDAVT OF PERFORMANCE}

\section{Buscanco La Pelleza / hosted by: Consuelo Luz}

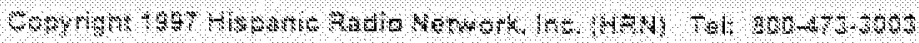

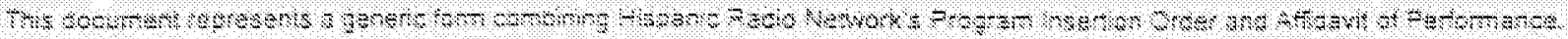

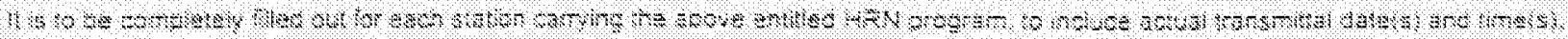

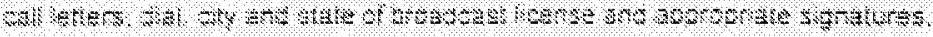

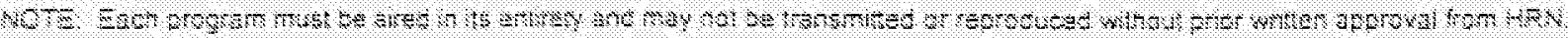

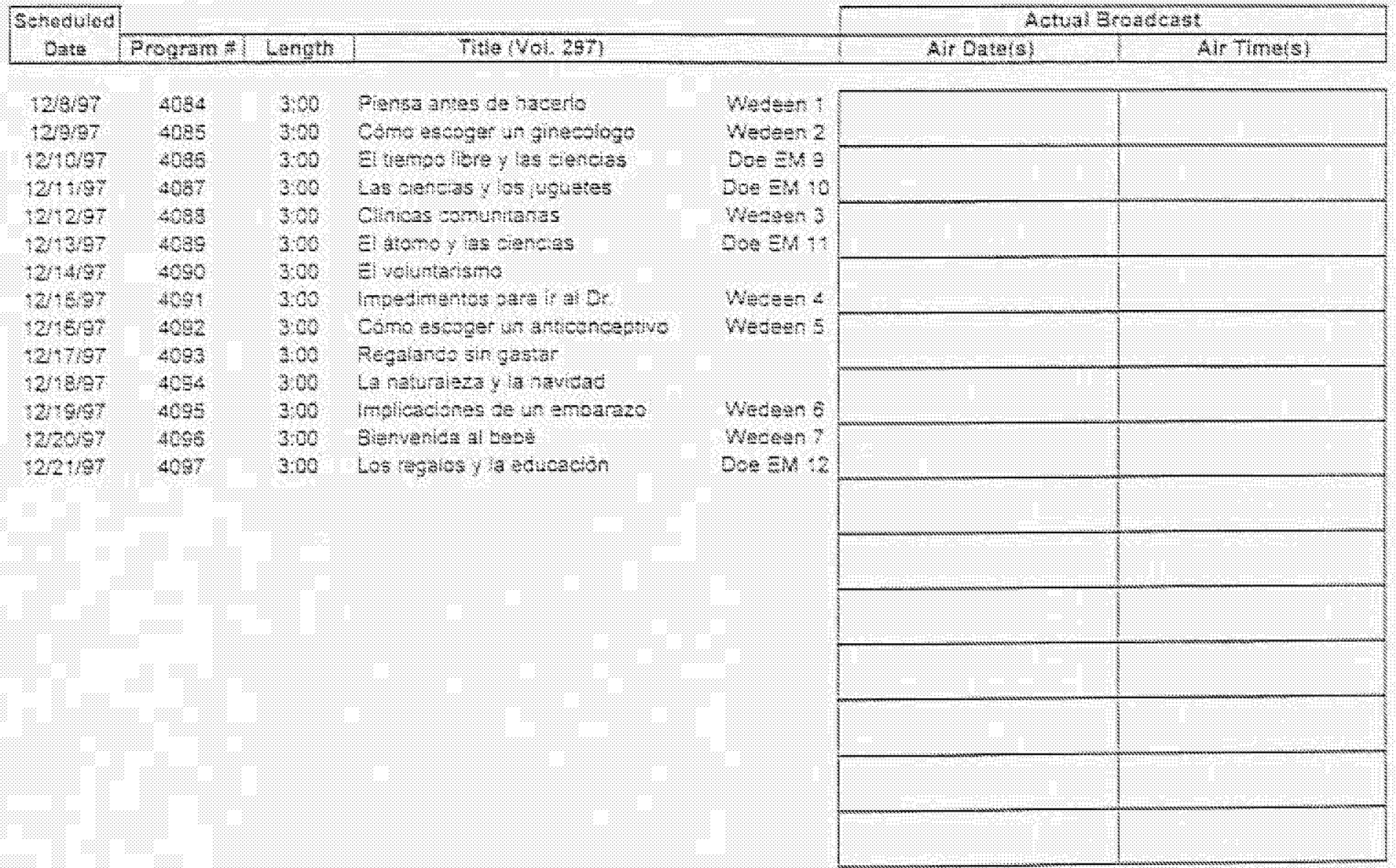

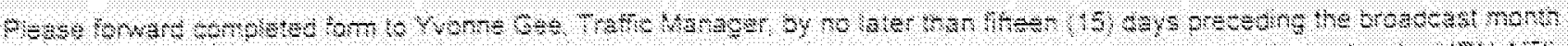

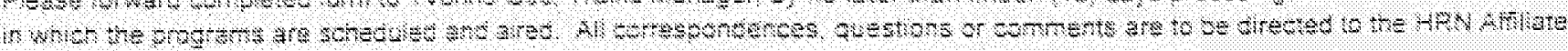

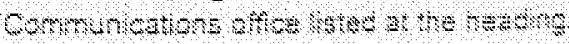

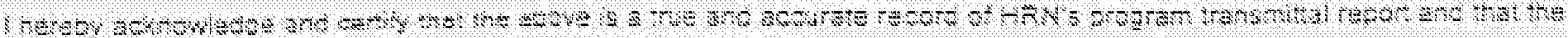

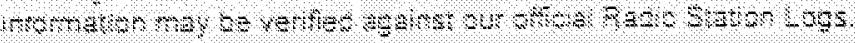

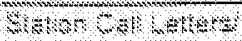

$0+8,60$
Forkents

Sare 


\section{HISPANIC RADIO NETWORK}

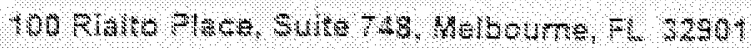

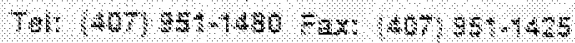

\section{Buscando La Belleza / hosted by: Consuelo Luz}

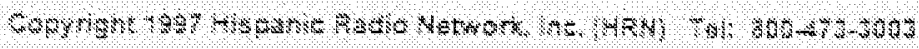

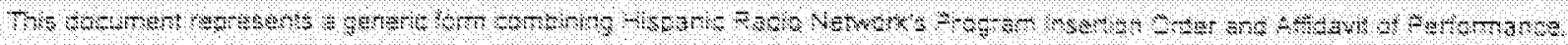

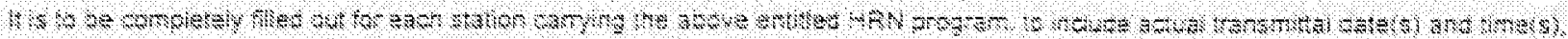

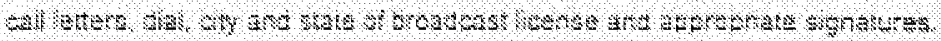

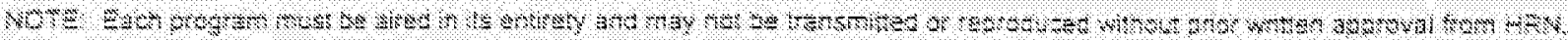

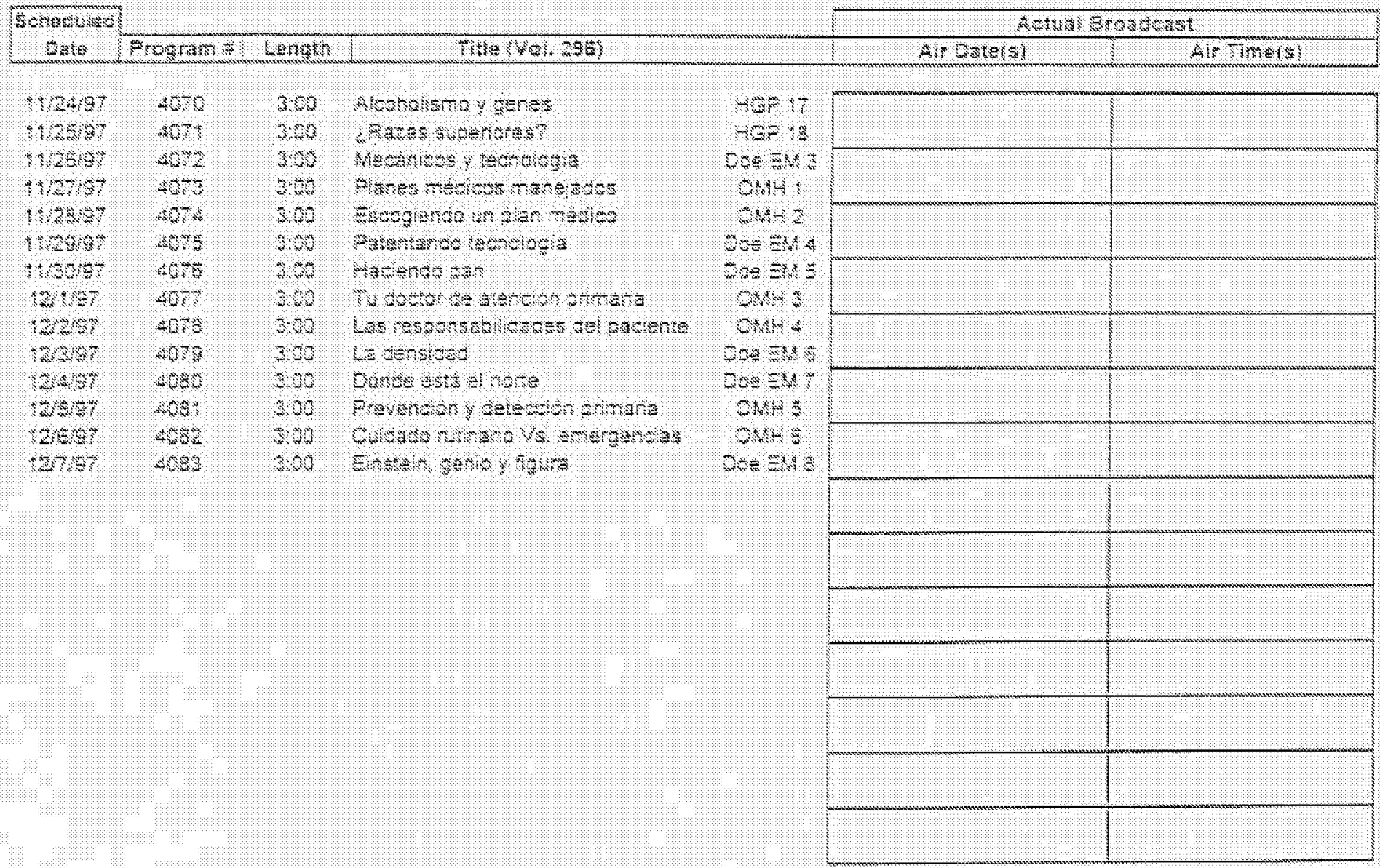

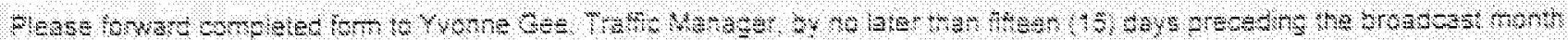

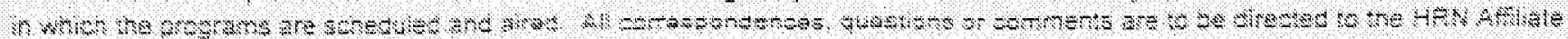

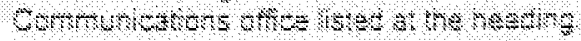

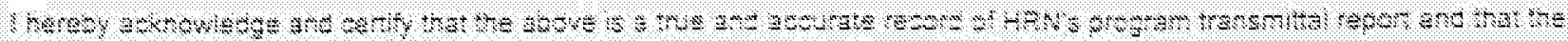

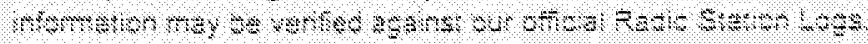

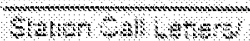

$0,30.0 \% 0 \%$

$3+40 \%$

Thes
Thentis?

Pats

seron 8 rotsense 


\title{
HISPANIC RADIO NETWORK
}

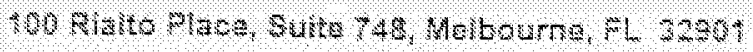

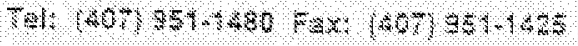

\section{PROQRAM INSERTION ORDER \& AFWDAVT OF PERFORMANCE}

\section{Buscando La Belleza / hosted by: Consuelo Luz}

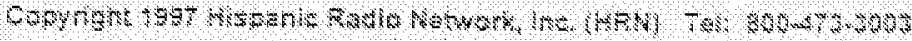

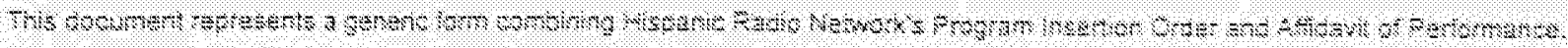

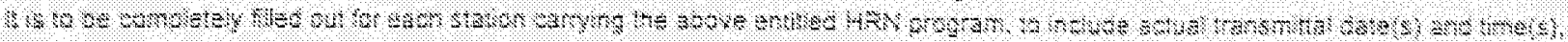

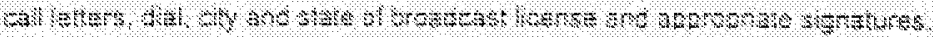

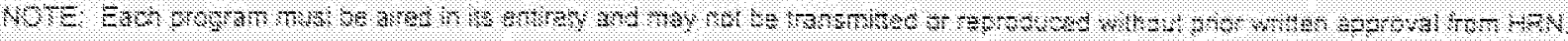

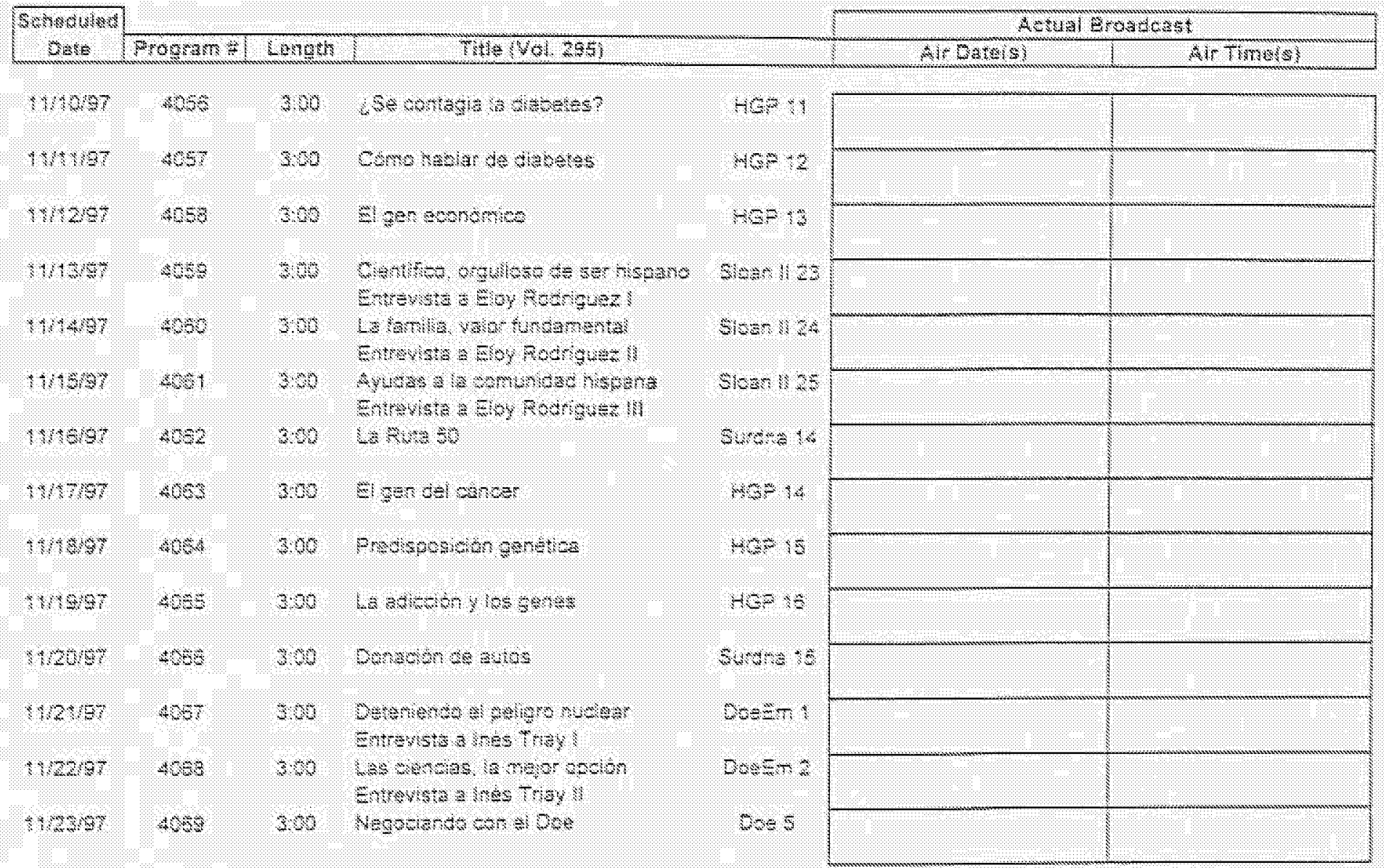

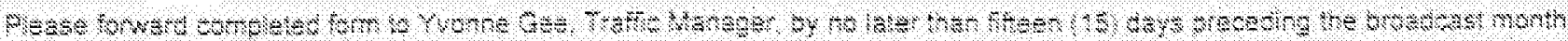

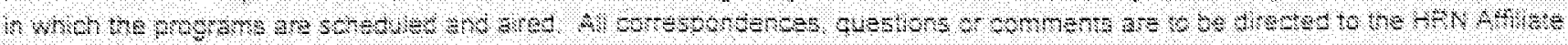

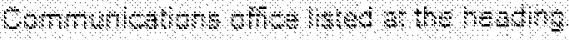

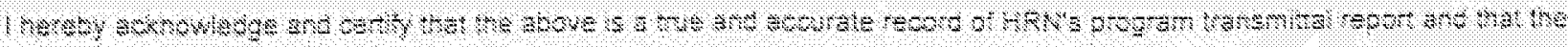

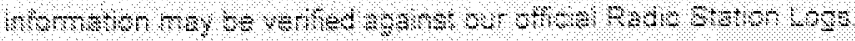

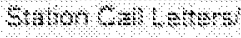

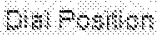

30 ?०?

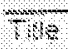

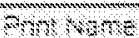

to:s

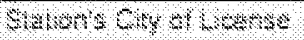




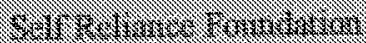

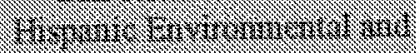

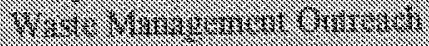

\section{1. Program Guide For Planeta Azul}




\title{
HISPANIC RADIO NETWORK
}

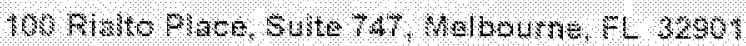

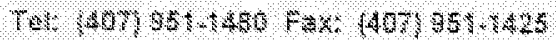

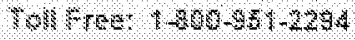

\section{PROORAM IUSERTION OROER I AFFIOAVT OF PERFORHANCE}

\section{Planeta Azul/hosted by: Mario Sole engrid Baena}

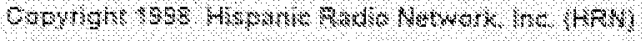

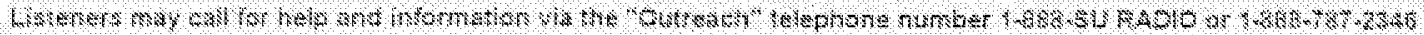

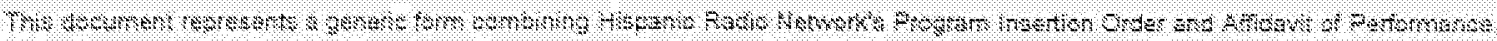

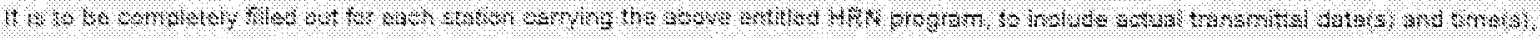

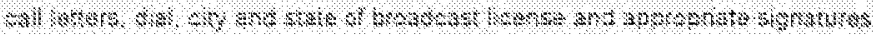

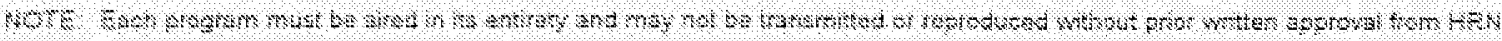

\begin{tabular}{|c|c|c|c|c|c|c|c|}
\hline \multirow{2}{*}{$\begin{array}{r}60 \\
8 k\end{array}$} & \multirow{2}{*}{$\begin{array}{c}\text { Sereoduler } \\
\text { artate }\end{array}$} & \multirow[b]{2}{*}{ Protram by } & \multirow[b]{2}{*}{ Cenoth } & \multicolumn{2}{|l|}{164} & \multicolumn{2}{|c|}{ 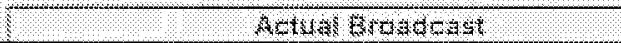 } \\
\hline & & & & Thes volune + -9 & 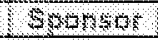 & Mr Dars & Ar $5 \mathrm{~mm}: 5$ \\
\hline 8 & $2+289$ & 80 & 206 & 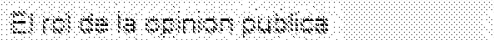 & memexto os 5 & Sherois & \\
\hline 2 & 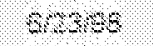 & 88* & 20 & 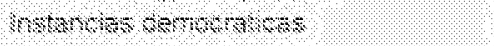 & 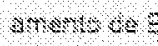 & $8+8+49$ & \\
\hline 3 & $2 \times 1 \times 1$ & $8+4$ & 200 & Werederos, eneros & $8+t a n$ ors 8 & Estorola & \\
\hline 4 & 80800 & 883 & 20 & 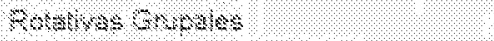 & antert $4=8$ & 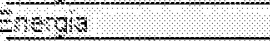 & \\
\hline 8 & 22080 & 80 & 200 & 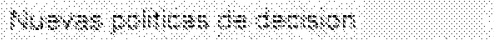 & 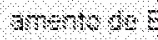 & nesera & \\
\hline 8 & $42+4$ & 88 & 200 & 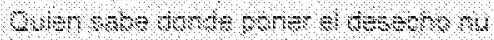 & 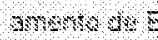 & $e^{2}+3,30$ & \\
\hline 18 & 62046 & 80 & 200 & 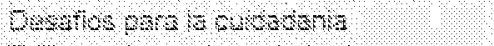 & 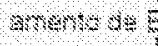 & Enos? & \\
\hline 8 & 648,8 & 8 & 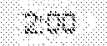 & 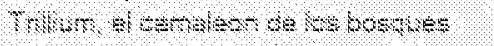 & & & \\
\hline 8 & 20088 & 08 & 200 & e & & & \\
\hline 10 & 10,60 & $8+8$ & 200 & 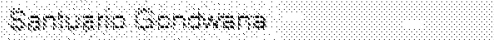 & & & \\
\hline 11 & $\%<>3$ & 400 & 200 & 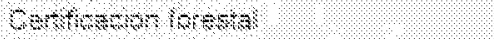 & & & \\
\hline 12 & \%) & 88) & 200 & 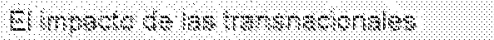 & & & \\
\hline 13 & 4,8 & 84 & 400 & 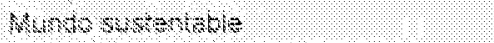 & & & \\
\hline 14 & \%४s & 8 & 300 & 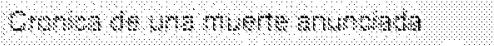 & & $=$ & \\
\hline
\end{tabular}

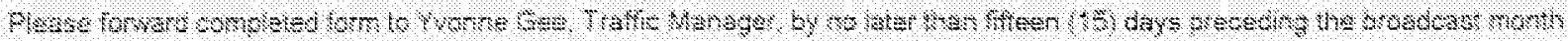

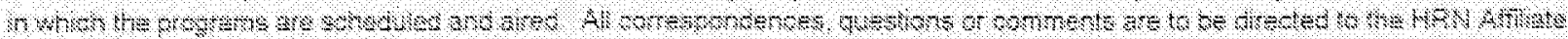

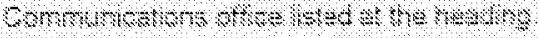

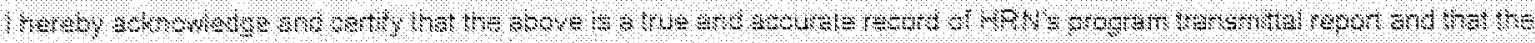

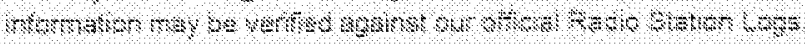

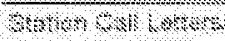

botertos:
Q\$m:

Trmolnos roters

Base

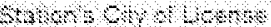

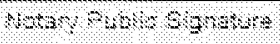




\title{
HISPANIC RADIO NETWORK
}

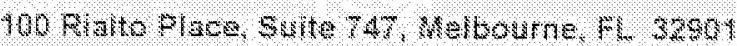

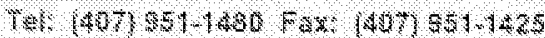

toll k.tee $1,200.351 .2294$

\section{PROGRAM INSERTION OROER \& AFFDAVT OF PERFORMANCE}

\section{Planeta Azul / hosted by: Mario Sol e Ingrid Baena}

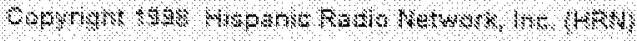

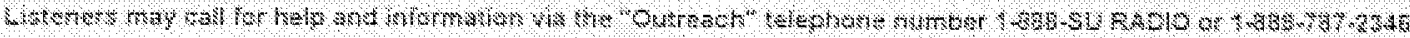

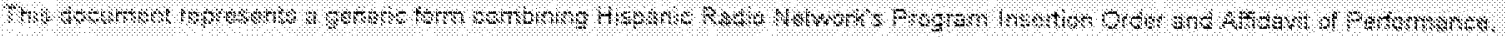

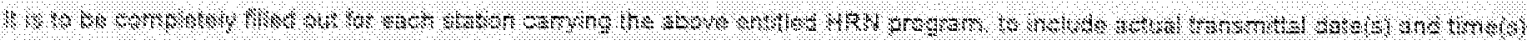

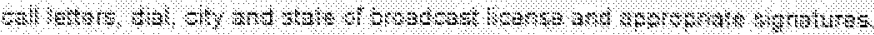

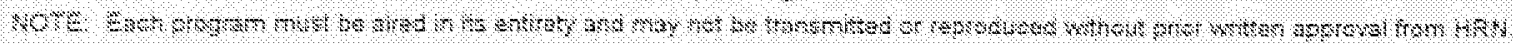

\begin{tabular}{|c|c|c|c|c|c|c|c|}
\hline \multirow{2}{*}{$\begin{array}{c}c \\
\mathrm{~T}\end{array}$} & \multirow{2}{*}{$\begin{array}{c}\text { sencoluter } \\
\text { Bste }\end{array}$} & \multirow[b]{2}{*}{ Propran 4} & \multirow[b]{2}{*}{ Length } & \multirow[b]{2}{*}{ Trelos Nerime * 681} & \multirow[b]{2}{*}{ Sponsor } & \multicolumn{2}{|c|}{ Aroual $\mathrm{Br}$ radcast } \\
\hline & & & & & & A, Dates & Ar $\operatorname{sinals}$ \\
\hline 1 & 4048 & 30 & 206 & 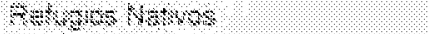 & 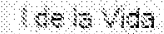 & ho stios s swertse & \\
\hline s. & $20 \% 18$ & $8+$ & 200 & 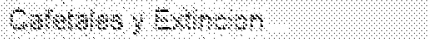 & $10 \times 18$ & & \\
\hline 3 & 8018 & 8 & 2,0 & 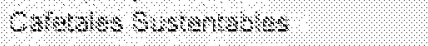 & 18188 & ঊ & \\
\hline 4 & $018 \%$ & 888 & 400 & 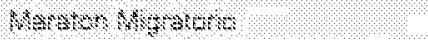 & 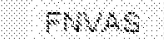 & & \\
\hline 8 & 428 & 8 & 28 & 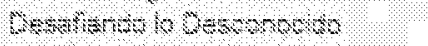 & 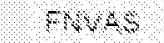 & & \\
\hline 6 & 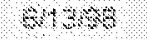 & 6) & $2 \%$ & 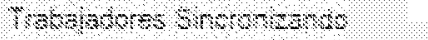 & 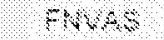 & & \\
\hline ? & 8486 & 642 & 206 & Orow ots manto & 18,8 & & \\
\hline 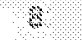 & 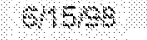 & 87 & 20 & 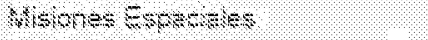 & shes $60 \mathrm{da}$ & Crorars & \\
\hline 4 & $4+19$ & 84 & $2 \%$ & $43+4 \times 4+4,6+48$ & 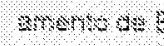 & netris: & \\
\hline 80 & $8+18$ & 63 & 28 & $4+\infty, 4-1+4$ & artiento des: & tretrac & \\
\hline 3 & $3+8,18$ & ४४ & 4 & 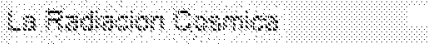 & smancese & Whaturis & \\
\hline 82 & 81898 & 61 & 20 & 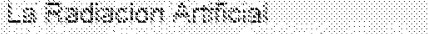 & inmento des & entorat & \\
\hline 3 & 12408 & 80 & 200 & 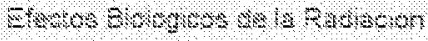 & aments de & neryan & \\
\hline 84 & $2+488$ & 88 & 208 & $40 \mathrm{~s}$ hend & senentches & $=6$ & \\
\hline
\end{tabular}

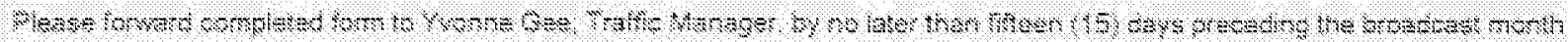

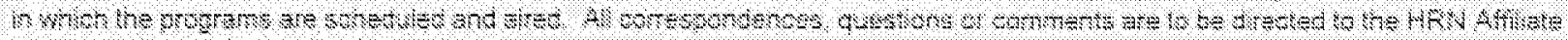

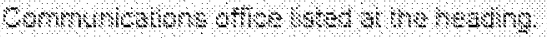

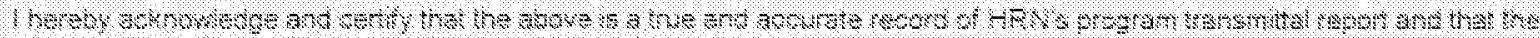

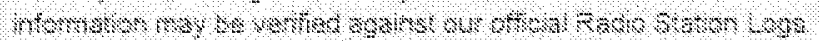

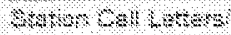

$0,10.6 \%: \%$

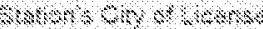

Spmare

Pant kare ras.

7 Ts: 


\section{HISPANIC' ADIO NETWORI presenta:}

\section{PLANETA AZUL con Mario Sol e Ingrid Baena}

ATENCION! SI SUS OYENTES NECESTTAN MAS TNFORMACION GRATUITA O AYUDA PARA HALLAR RECURSOS DISPONIBLES EN SU LOCALIOAD, LLA AMENOS AL 1-800-473-3003.

ESIE VOLUMEN COMIENZA RL LUNES 3 DE ABRIL DE 1998

PIANFTA ATUT. GUIA DE VOLUMEN: 62 PROGRAMAS: 610 -623

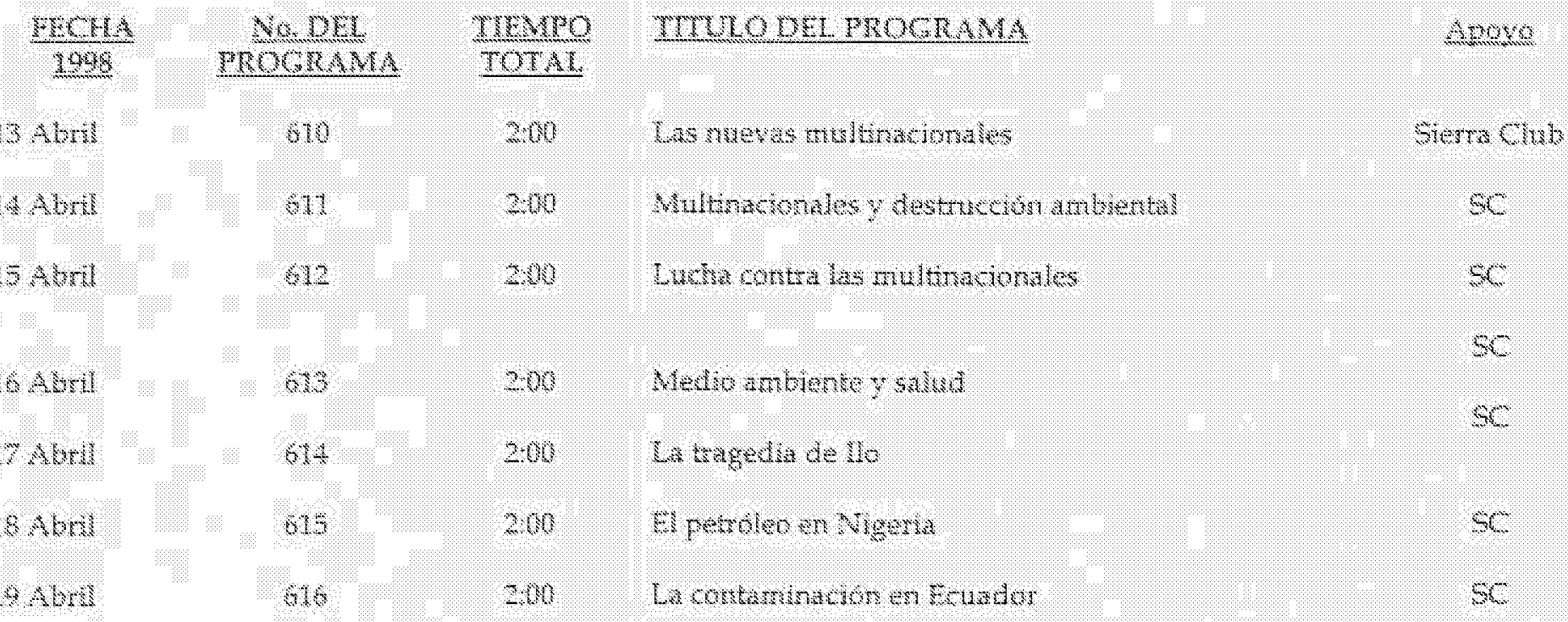

$1 \times 00 ?$

\begin{tabular}{|c|c|c|c|c|}
\hline SoA $4 \times 4$ & का? & 200 & La fusion nueleser & 10: \\
\hline s: & : & & & MOE \\
\hline 21 Abril & 88 & 200 & 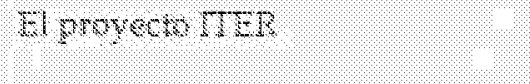 & DOE \\
\hline $24+6-i l$ & 69 & 200 & Las radiatron obsusian & $60 \%$ \\
\hline 28 abril & $\quad 670$ & 200 & 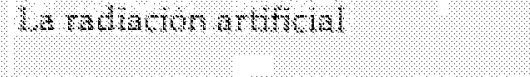 & 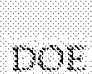 \\
\hline 24 aril & 624 & 200 & Etechos bublogrers de la radiacion & $C$ \\
\hline 23 Arit & & 200 & Ramacion ate ala intersidiad & \\
\hline 36 Abrs & 82 & 2.00 & Radiacoun ae baja stensidad & \\
\hline
\end{tabular}




\section{HISPANIC RADIO NETWOKK presenta:}

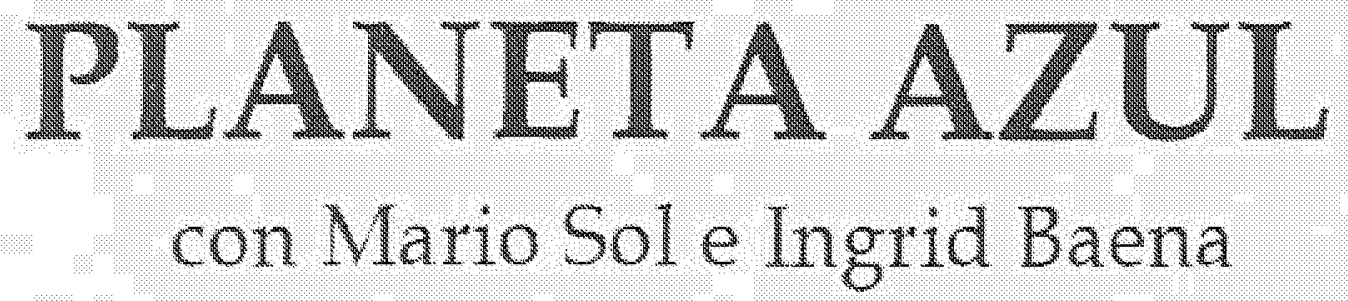

ATENCION! SI SUS ONENTES TECESTTAN MAS INFORMACION GRATUTTA O AYUDA TAEA HALLAR RECURSOS DISTONIBLES EN SU LOCALIDAD, LLAMENOS AL 1-500-473-3003.

ESTE VOLUMEN COMIENZA EL LUNE \$O DE MARZO DE 1998

PLANETAAZUL GULA DE VOLUMEN: 61 PROGRAMAS: $596 \cdot 699$

\begin{tabular}{|c|c|c|c|c|}
\hline$\frac{\text { EECHA }}{1998}$ & $\begin{array}{l}\text { No. OEL } \\
\text { PROCRARES }\end{array}$ & $\begin{array}{l}\text { THEMro } \\
\text { TOTAL }\end{array}$ & MULO DEL PROCRAMA & Arsove \\
\hline 10 Marre & $\$ 96$ & 200 & Sobrepohiacion! madio ambiente & Stera Clwi \\
\hline W Marzo & 904 & 200 & Comuridades amerazadiss & 50 \\
\hline Atril & $5 \% 8$ & 2.00 & Montras y contruninacon & 50 \\
\hline Axril & 399 & 2,10 & La guetra del agrua & $\begin{array}{l}5 \% \\
50\end{array}$ \\
\hline Abril & 600 & $2: 00$ & Ecologistas y justicua ambiental & \\
\hline A trell & sin & 200 & Aire contaminads & 50 \\
\hline iabril & $\omega 2$ & 2.00 & $\begin{array}{l}\text { La gaema de la therrat } \\
\qquad \angle A D O \text { ? }\end{array}$ & 50 \\
\hline
\end{tabular}

\begin{tabular}{|c|c|c|c|c|}
\hline Abril & ats & 200 & Proteccon nudesy intersacional & DOE \\
\hline & & & & DOE \\
\hline Abril & 604 & 2009 & Misiones espacilies & DOE \\
\hline Abril & 60 & 300 & Energh nudeat en el espacio & DOE \\
\hline A bril & 806 & 200 & Medtcina nucleas ! & DOE \\
\hline $04 \mid m i l$ & 607 & 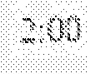 & Medicuna mucient II & D. \\
\hline $34 \mathrm{mal}$ & 608 & 280 & Avances en tredicma surtona ! & \\
\hline Mbrit & sne & 200 & Avances en medicana rudea: II & \\
\hline
\end{tabular}




\section{HISPANIC RADIO NETWORK presenta:}

\section{EL PLANETA AZUL con Mario Sol e Ingrid Baena}

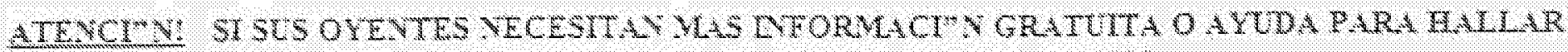

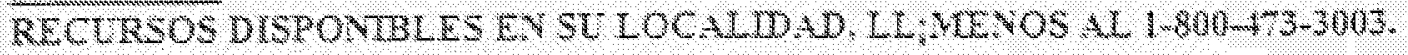

ESTE VOLUMEX COMIEXZ EL LUNES 2 DICREYBRE DE $199 \%$

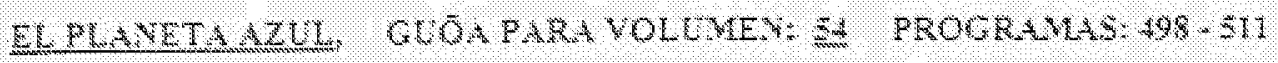

\begin{tabular}{|c|c|c|c|c|}
\hline$\frac{\text { FECSAS }}{1909}$ & 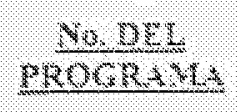 & $\frac{\operatorname{TrEMTO}}{\frac{\mathrm{TOTH}}{\mathrm{TOT}}}$ & TITYLO DEL PROGR MA & $450 \times 0$ \\
\hline Ditertibre & 498 & 2,10 & 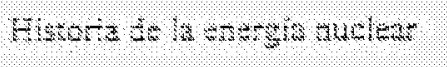 & DOE \\
\hline Dicierthe & 990 & 7.90 & 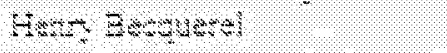 & DI \\
\hline Diclembre & 300 & 3.00 & Emen Rumerord & DOE \\
\hline : & & & & DOH \\
\hline & $58 \%$ & 200 & Meris Brati & DOE \\
\hline Distambre & 302 & $2: 00$ & Jame Cradwiest & \\
\hline belentre & 90 & 300 & 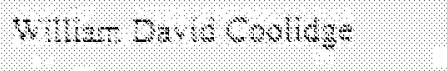 & DOE \\
\hline more & 504 & 2.00 & 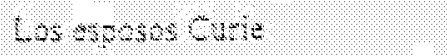 & Q08 \\
\hline
\end{tabular}

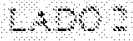

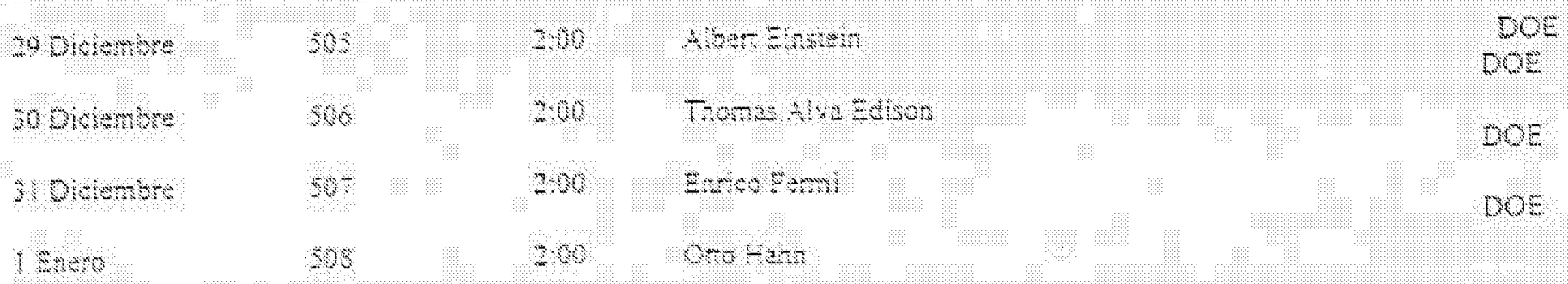




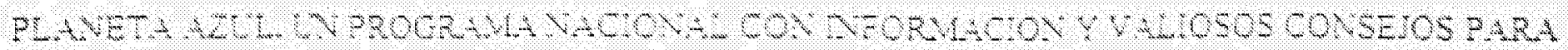

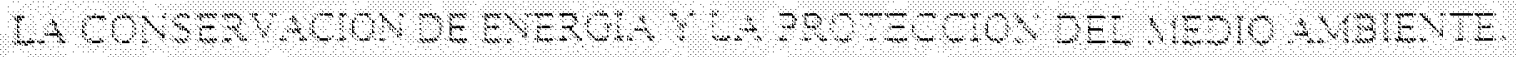

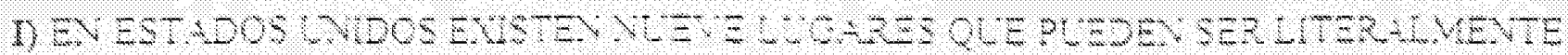

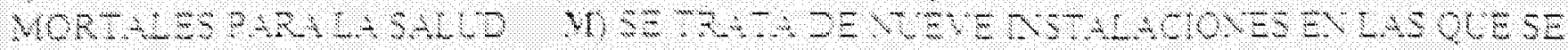

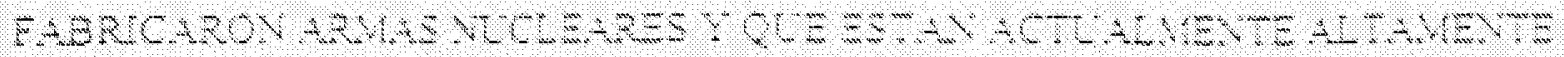

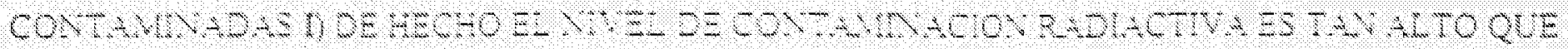

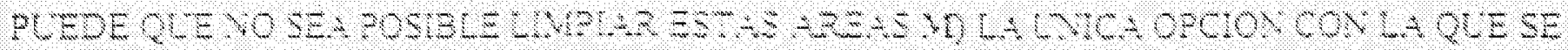

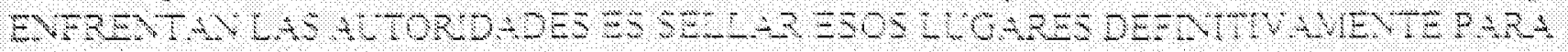

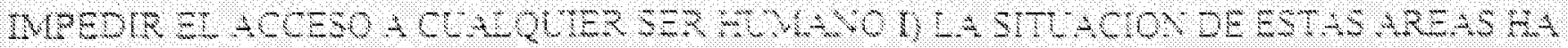

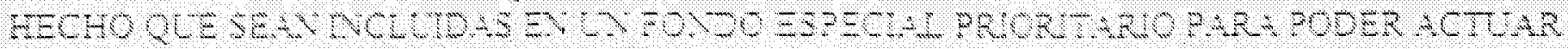

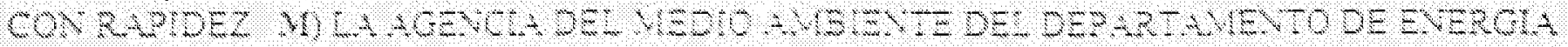
ESTA AL FREVTE DEL PROVECTO DE LMPEZZA Y CONTROL DELOS RESUCLOS RADLACTVVOS EN ESTADOS CXDOS D GR ACLAS \& LOS FCWDOS DESRMUDOS A ESTE CAPITLO DECEVAS

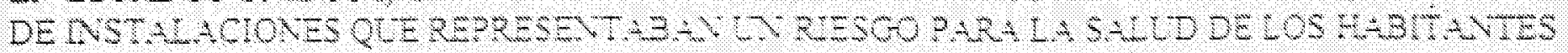
DE LOS AIREDEDORES ESTANATOR. 3-CO CONTROL MO ORR-S HAV PODDO SER

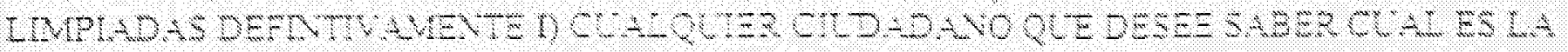

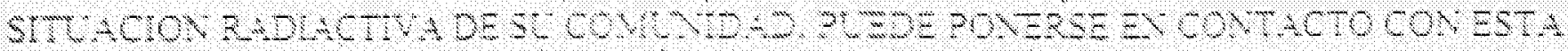

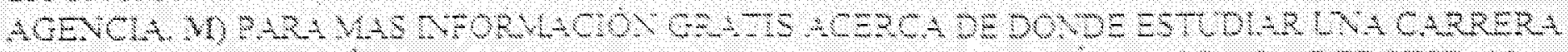

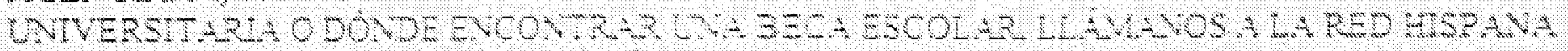

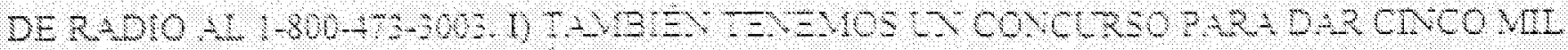

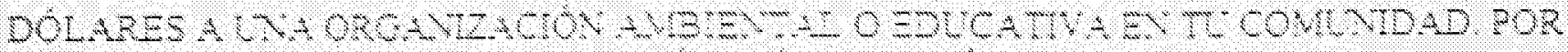

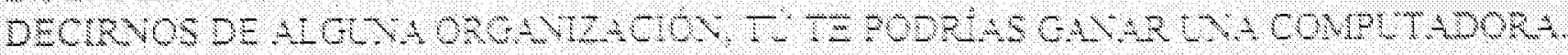

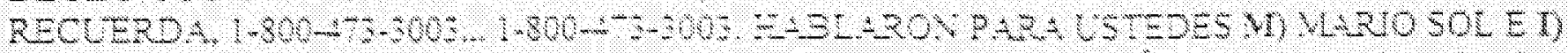

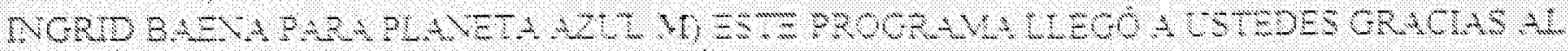
APOYO DEL DEPARTAVENTO DE EVERCLL

\section{HISPANIC RADIO NETWORK presenta:}

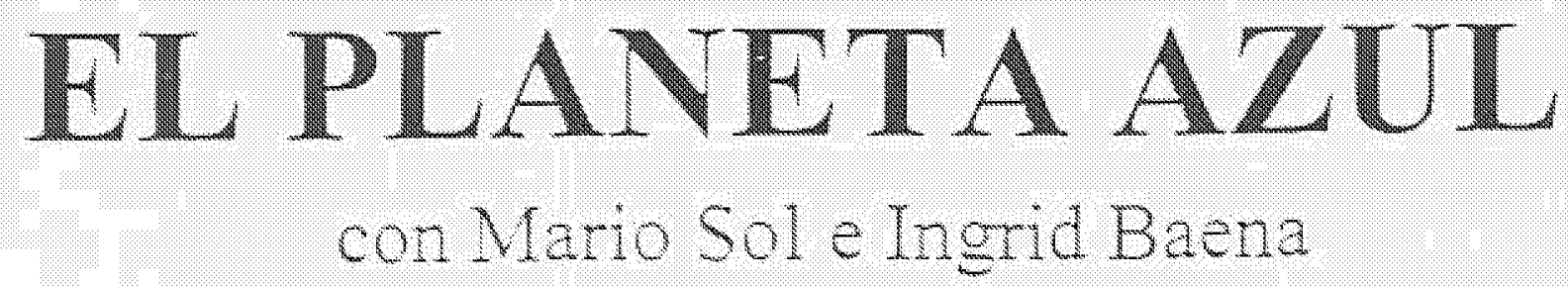

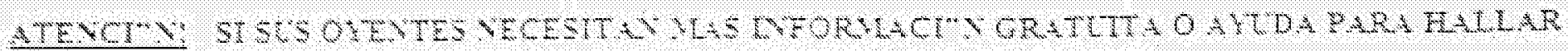

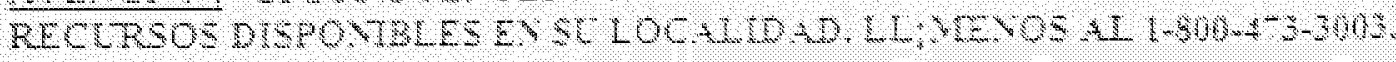

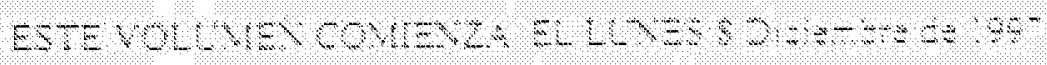

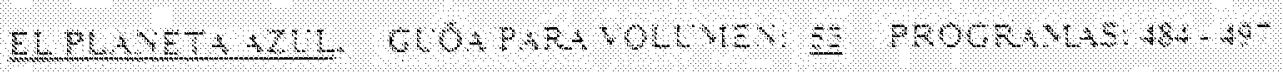

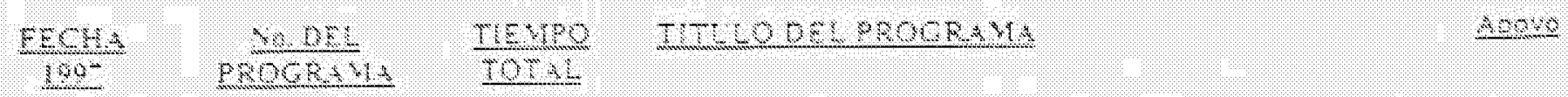




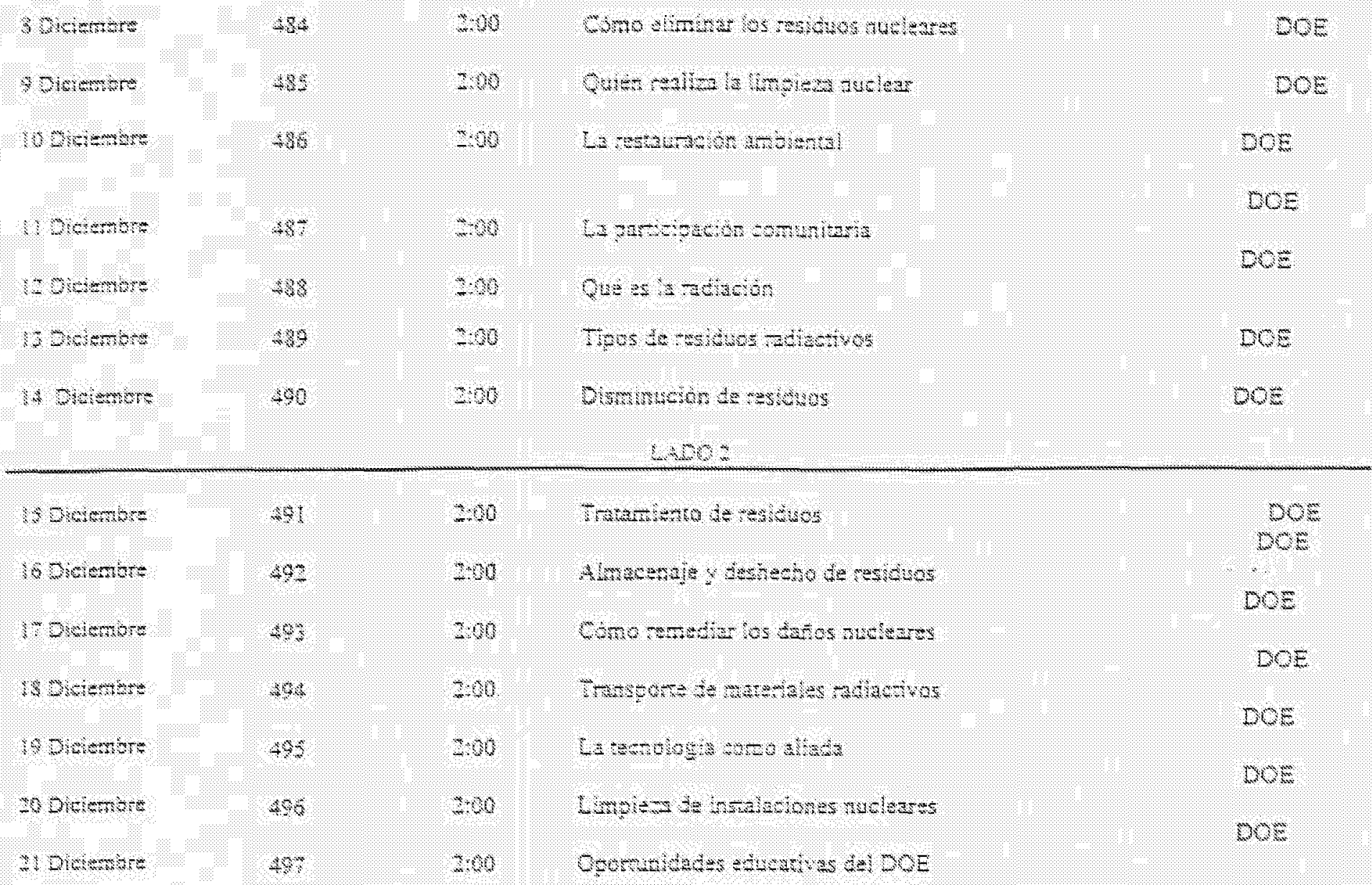

\section{COMO RLMTYAR LOS RESWDLOS NCCLEARES}

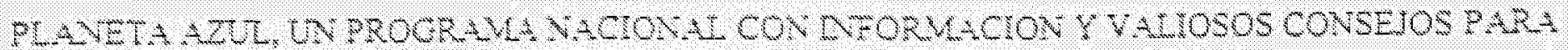
LA COFSERVACION DE EERCL Y Y A PRO ECCIOR DEI MEDIO AMBENTE.

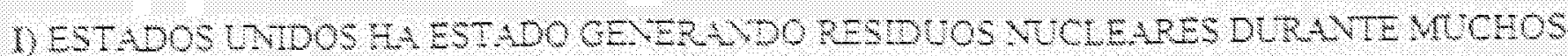

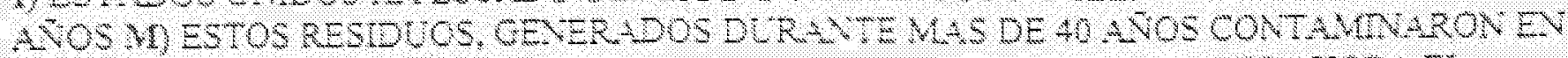

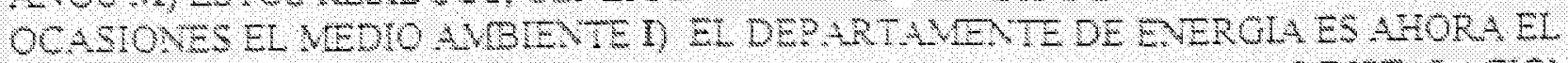

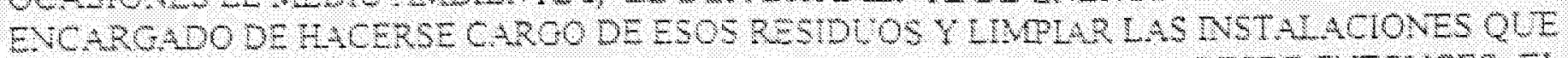

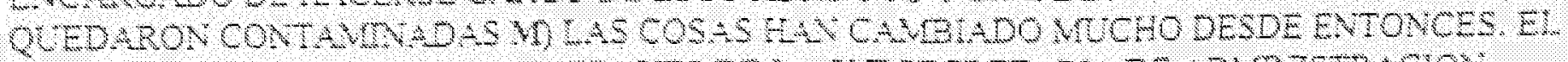
DEPAPTAUENTO DE EREROLA, AR- TES DELA S BSECRETARIA DE ADMMTSTRACIOK

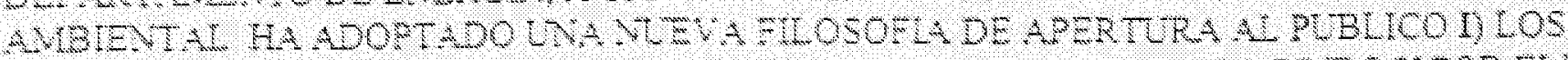
RESDUOS YUCLEARS SOA TR PROBLELA QUE A-ECIA A TOOO EL MUNDO Y POR ELLO ESTA AGENCL QUTER BERER NCLUR A LOS CUDADAVOS EU LA TOMLAE DECISIONES A

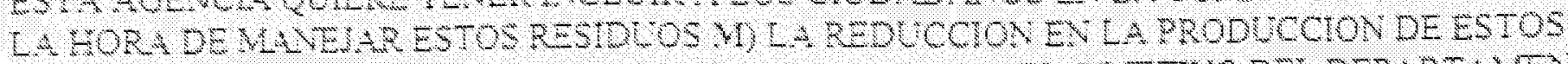

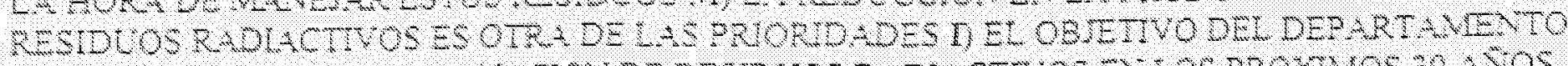

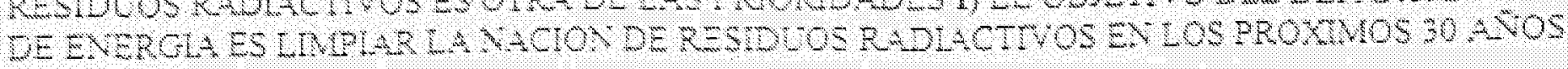




\section{HISPANIC RADIO NETWORK presenta:}

\section{EL PLANETA AZUL con Mario Sol e Ingrid Baena}

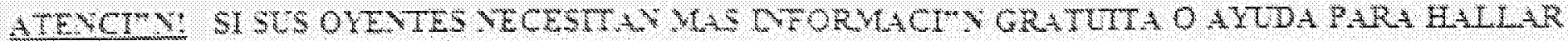

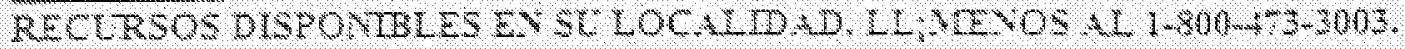

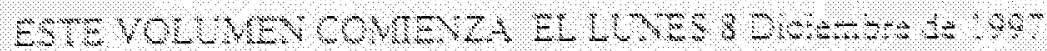

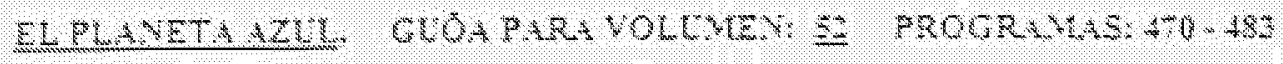

\section{$\operatorname{EeCA}$} $190 \%$

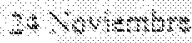

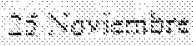

$38,4+6,+8-8$

$-80,+4+4: 8$

$\$ \$, 40,0 \mathrm{or}:$

$3+80+4+8+6$

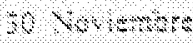

\section{$+4,+,+4$}

$3+4 .+4: 5$

$+8+4+8+$

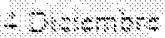

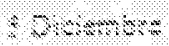

$8+4-8 \mathrm{~s}$

\section{No. DEL PROCRAM}

478

475

42

4

$4-4$

$4-5$

46

$4 *$

$4+8$

48

481

$+18$

43

\section{$\frac{\text { TEYTPO }}{\text { TOT }+2}$}

$+18$

38

$-\infty 06$

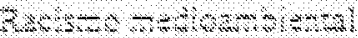

2.08

$+8$

48

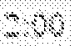

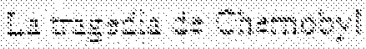

$4 \times-4 \times 3$

38

$4+4+4+4+0 t+46+, 00$

$+4$

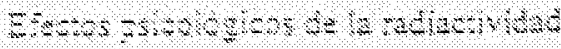

$\$$

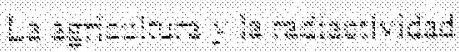

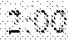

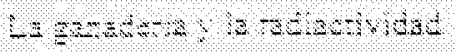

$2+$

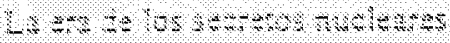

$-\$$ 3nove

DOE

DOE

30

Do:

2us

208

DSE

90 E

1048

50

90

04

003 


\section{EFECTOS DE LA RADIACION MUCLEAR}

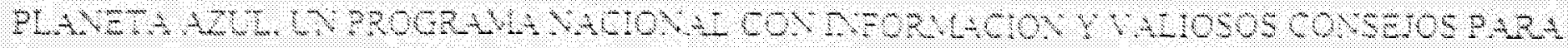

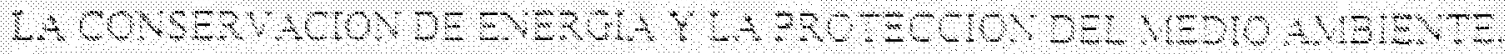

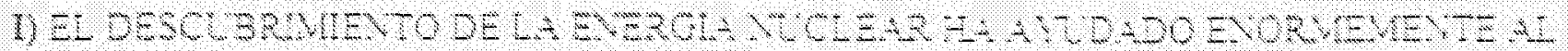

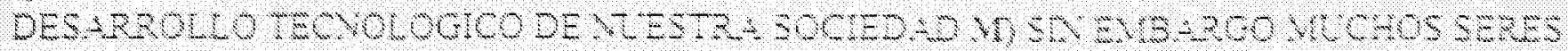

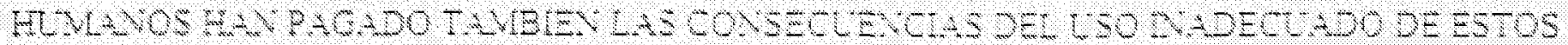

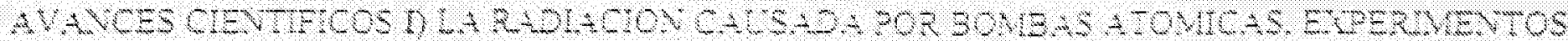

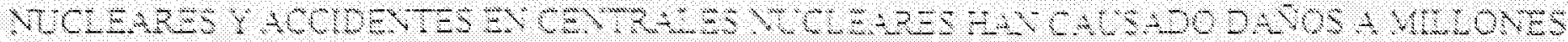

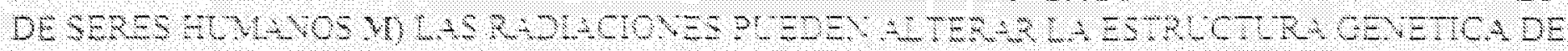

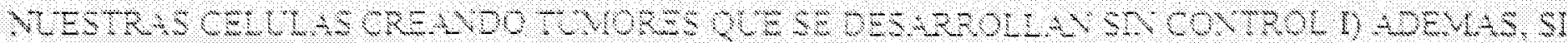

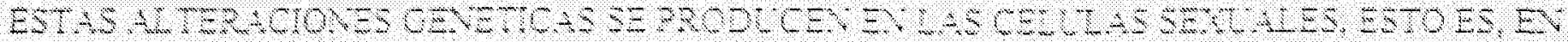

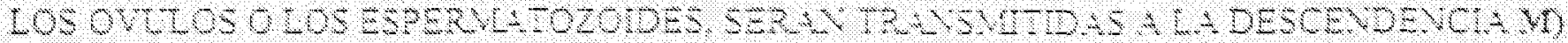

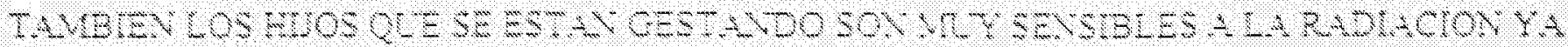

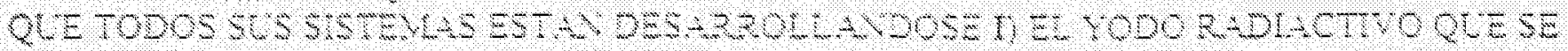

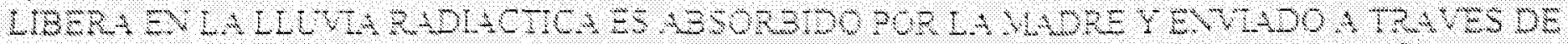

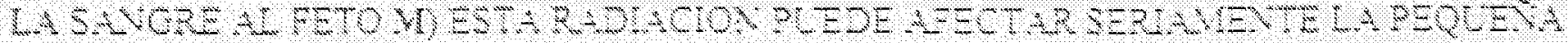

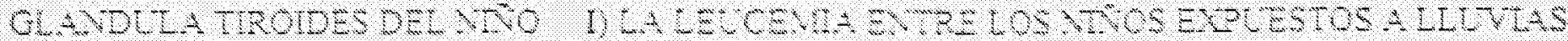

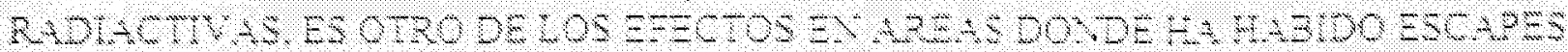

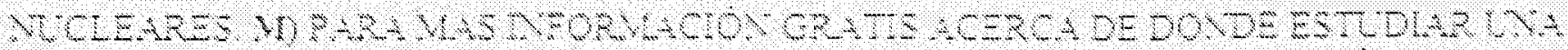

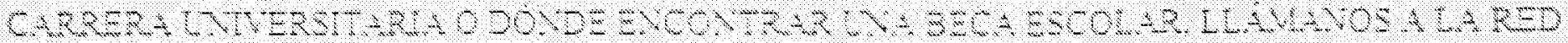

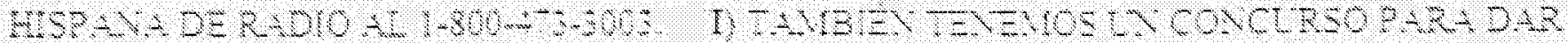

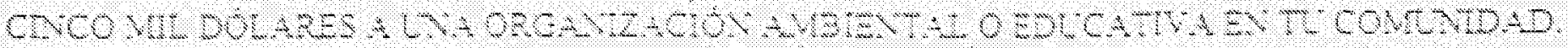

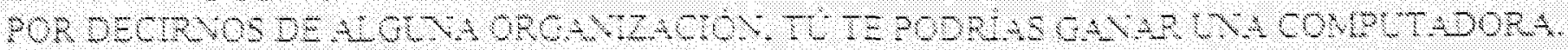

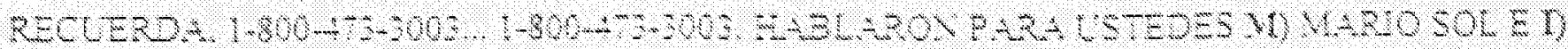

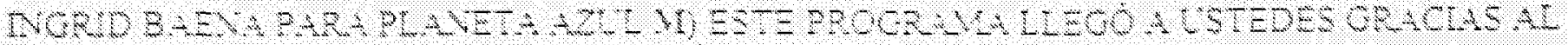

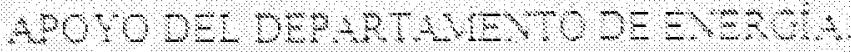

\section{2. $\operatorname{LOSR} \mathrm{R}+\mathrm{OSS}$}

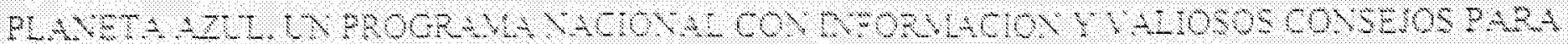

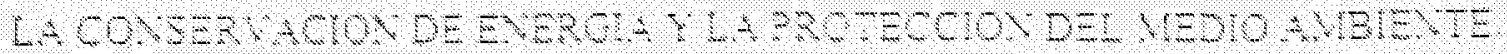

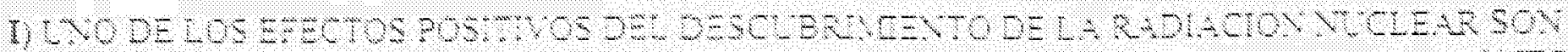

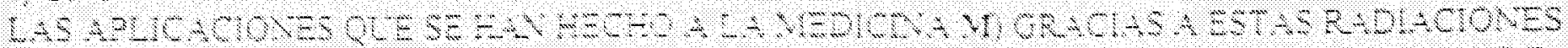

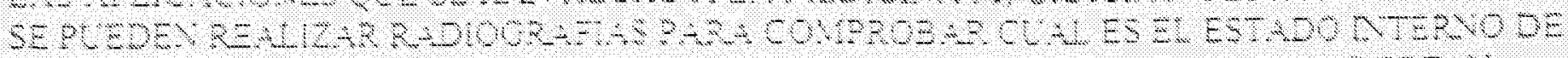

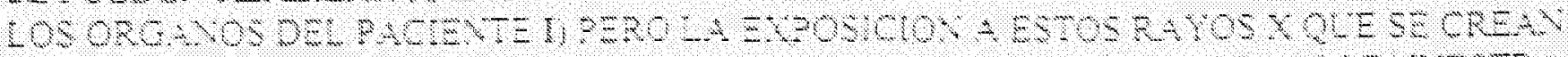

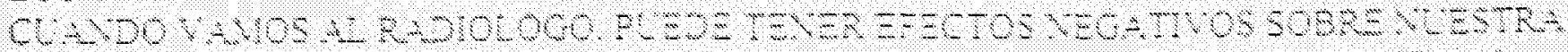

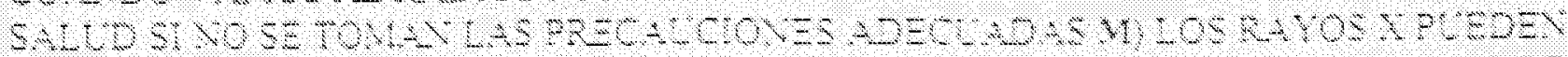

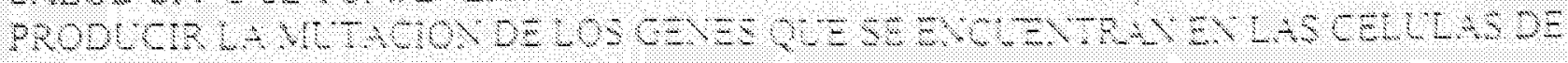




\title{
HISPANIC RADIO NETWORK presenta:
}

\section{EL PLANETA AZUL con Mario Sol e Ingrid Baena}

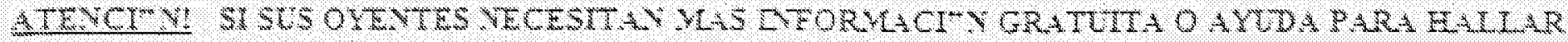

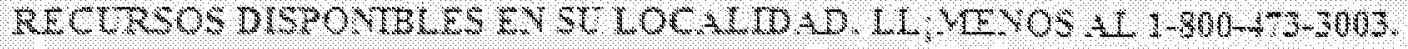

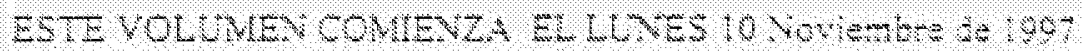

EL PLANETA AZLL GUOA PARA VOLUMEY: 3 PROGRAMA5: $456 \cdot 469$

\begin{tabular}{|c|c|c|c|}
\hline$\frac{\mathrm{Fecs}}{190^{2}}$ & $\begin{array}{c}\text { No. DEL } \\
\text { progR } 44\end{array}$ & $\frac{\text { TE YMO }}{\text { TOTIL }}$ & OEL PROCR \\
\hline
\end{tabular}

$40 \cdots+6$

$3016010+6$

48

$-48$

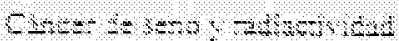

100

$8,804+4 \%: 0$

18

2.1

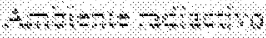

20

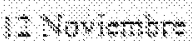

48

404

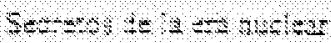

$\mathrm{NOS}$

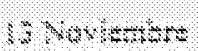

48

2,00

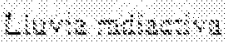

$00=$

14 tersentse

486

$+, 00$

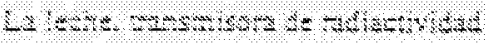

$00=$

15 souerbor

$+4$

s.60

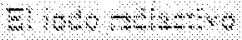

10)

$16960,8+2,00=$

$46^{4}$

300

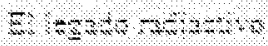

SOE

$1+008$

\begin{tabular}{|c|c|c|c|}
\hline 17 veverabet & 48 & $=60$ & 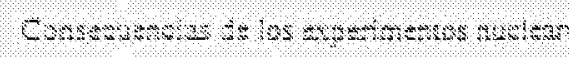 \\
\hline 18 Yonseritese & 864 & $=90$ & 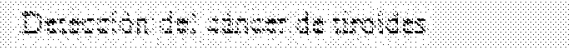 \\
\hline 19 revlemiore & 868 & 340 & 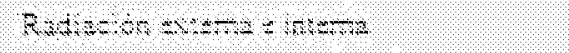 \\
\hline loversibe & 488 & .30 & 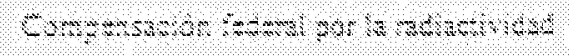 \\
\hline Pistertaterts & 48 & 300 & 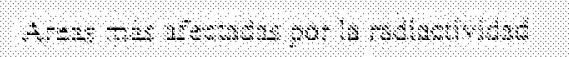 \\
\hline 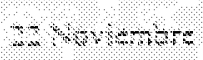 & 368 & \pm 20 & 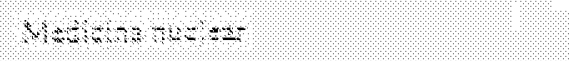 \\
\hline To: & 1\%: & 906 & 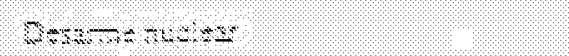 \\
\hline
\end{tabular}

$D O=$

DOE

Dos

DoE

Dos 


\section{HISPANIC RADIO NETWORK presenta:}

\section{EL PLANETA AZUL con Mario Sol e Ingrid Baena}

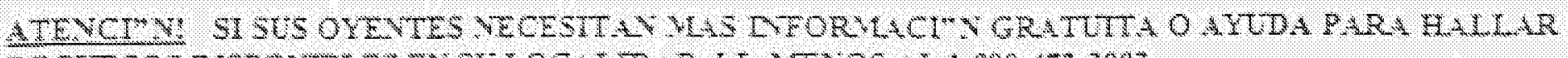

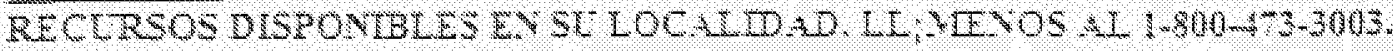

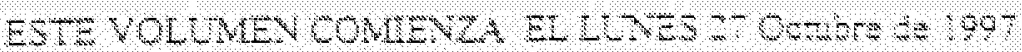

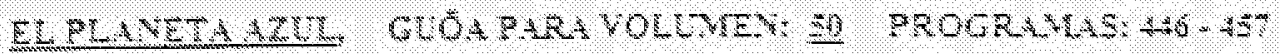

\begin{tabular}{|c|c|c|c|c|}
\hline$\frac{\operatorname{rrcsa}}{100^{-}}$ & 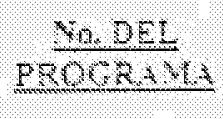 & $\frac{3 C \vee P O}{10+4}$ & THCLO DE, PBOGR, 4 & $A=0 \times x$ \\
\hline$-80 \mathrm{moses}$ & 4.6 & 2.00 & 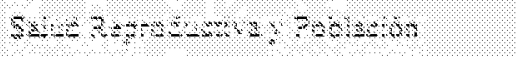 & $84==2$ \\
\hline-8 cowione & 4. & 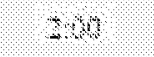 & 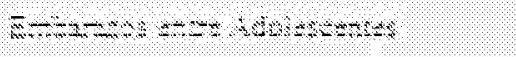 & $8+2 \times 2$ \\
\hline mosise & 48 & $3 . \infty$ & 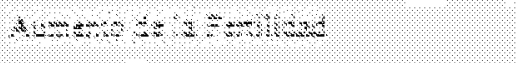 & 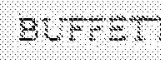 \\
\hline $9000.46=$ & 49 & -20 & 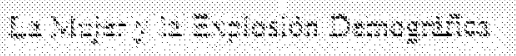 & QUPFET \\
\hline ;: & 40 & 9.80 & 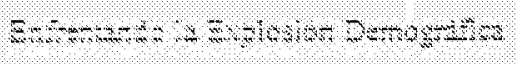 & SHF: \\
\hline $830,4=-6+5$ & $44 \%$ & $=00$ & 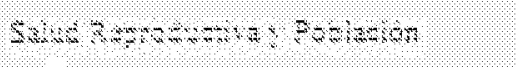 & BUFFE \\
\hline 2 Ronembs: & 478 & 300 & 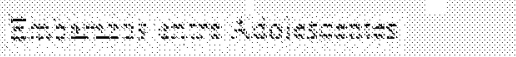 & BC:= = $=3$ \\
\hline \multicolumn{5}{|c|}{$5.2+x=2$} \\
\hline 3 werestats & $4: 1$ & $3 \%$ & 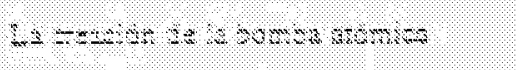 & DoE \\
\hline & & & 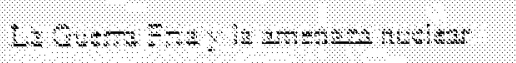 & D०E \\
\hline 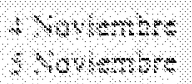 & $+2=3$ & $\begin{array}{l}200 \\
200\end{array}$ & 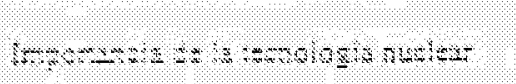 & $50 E$ \\
\hline s.somentive & 48 & $9 \%$ & 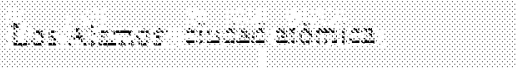 & $P O=$ \\
\hline$-16 \times 1+-6 \cdot=$ & 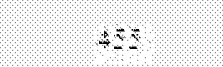 & 200 & 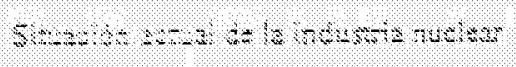 & 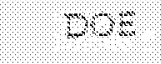 \\
\hline 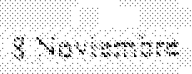 & -80 & $-\infty$ & 1 & $00 \%$ \\
\hline & 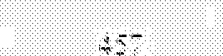 & $=.+0$ & $=$ & 9 \\
\hline
\end{tabular}

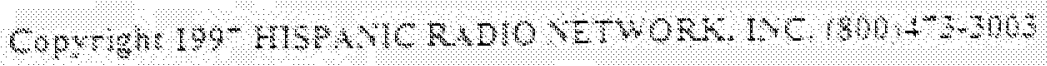




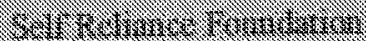

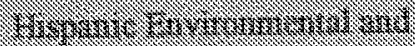

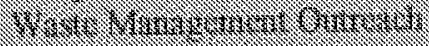

\section{1. \\ Radio Affiliate List \\ For \\ Buscando la Belleza}




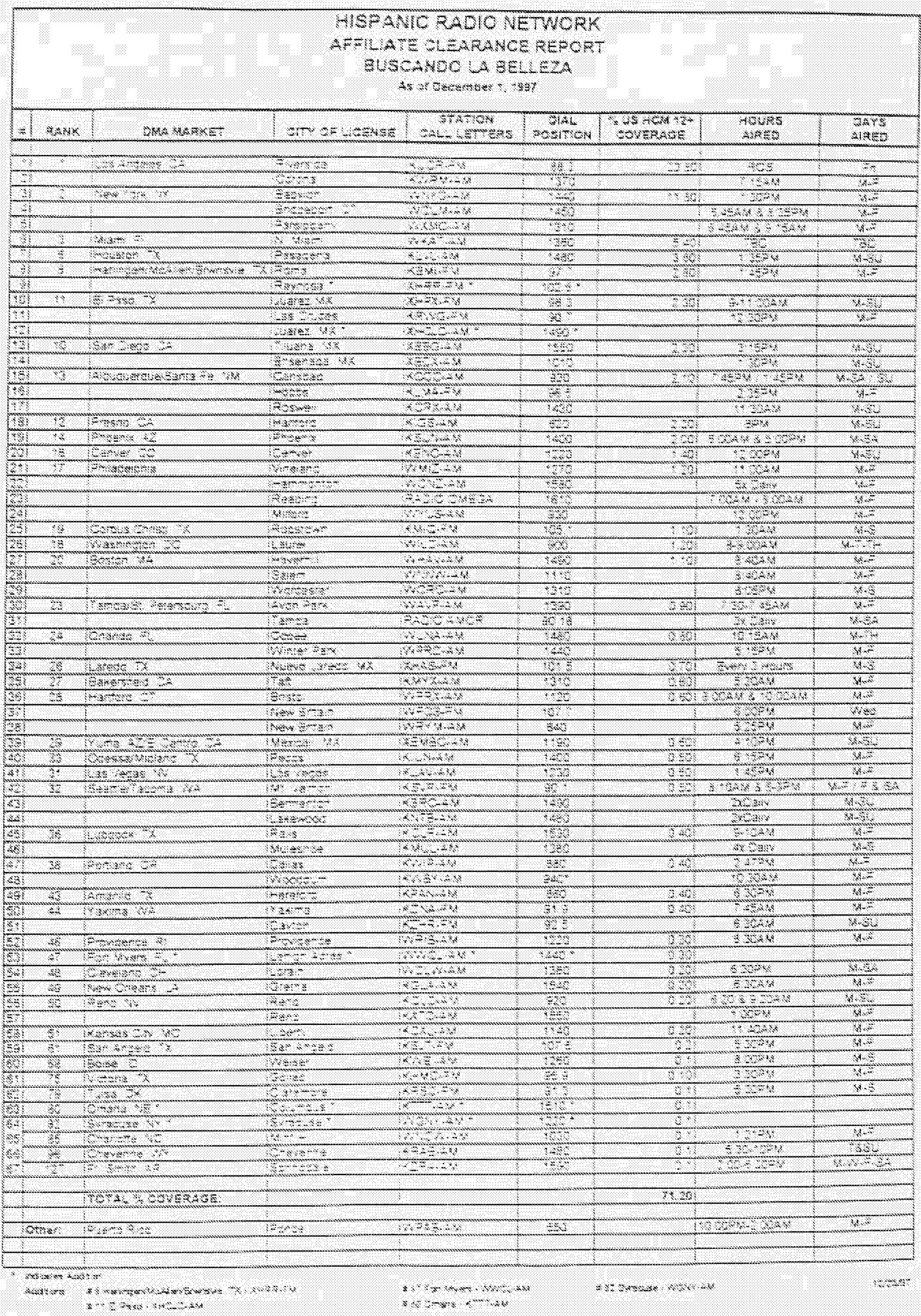




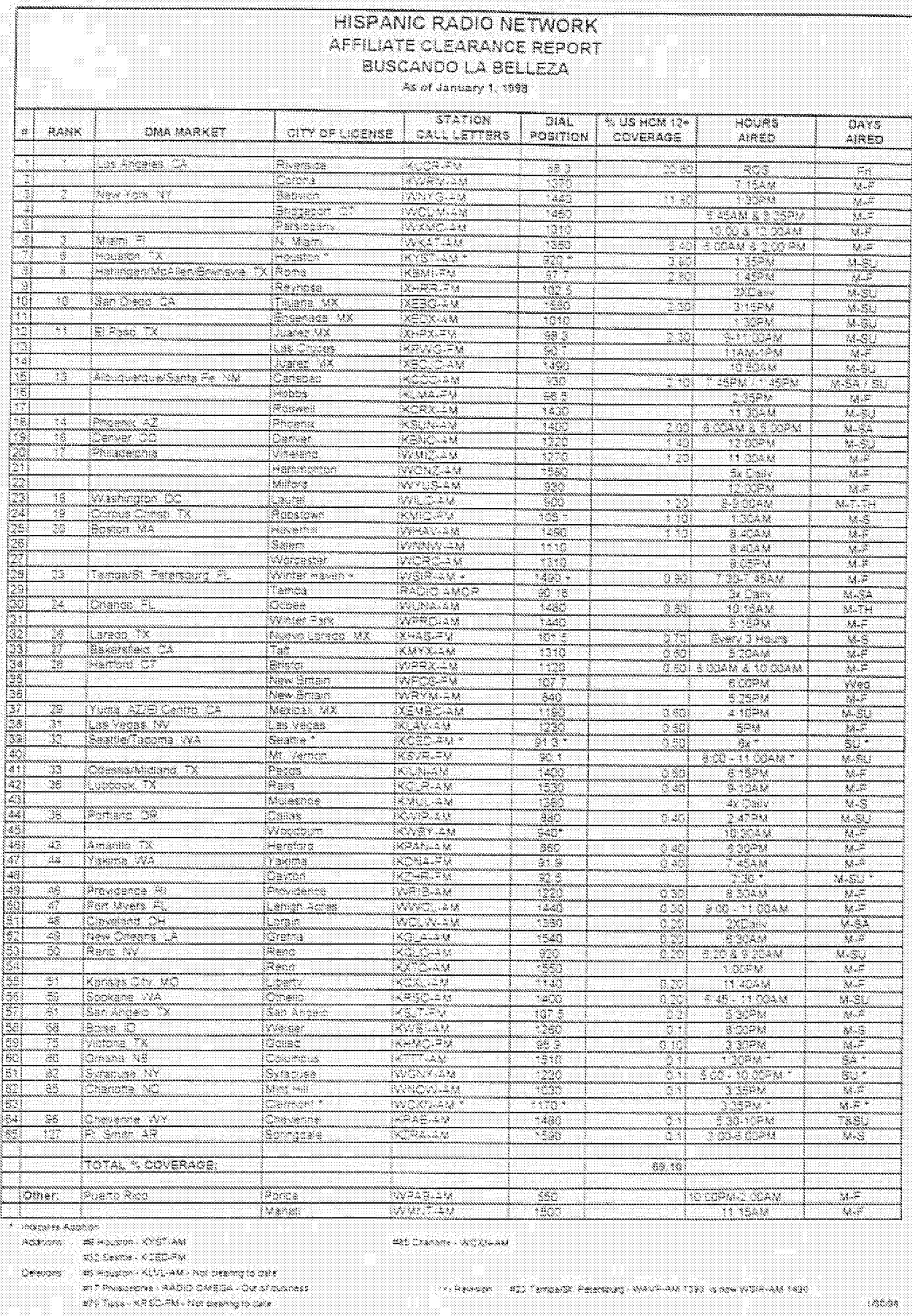




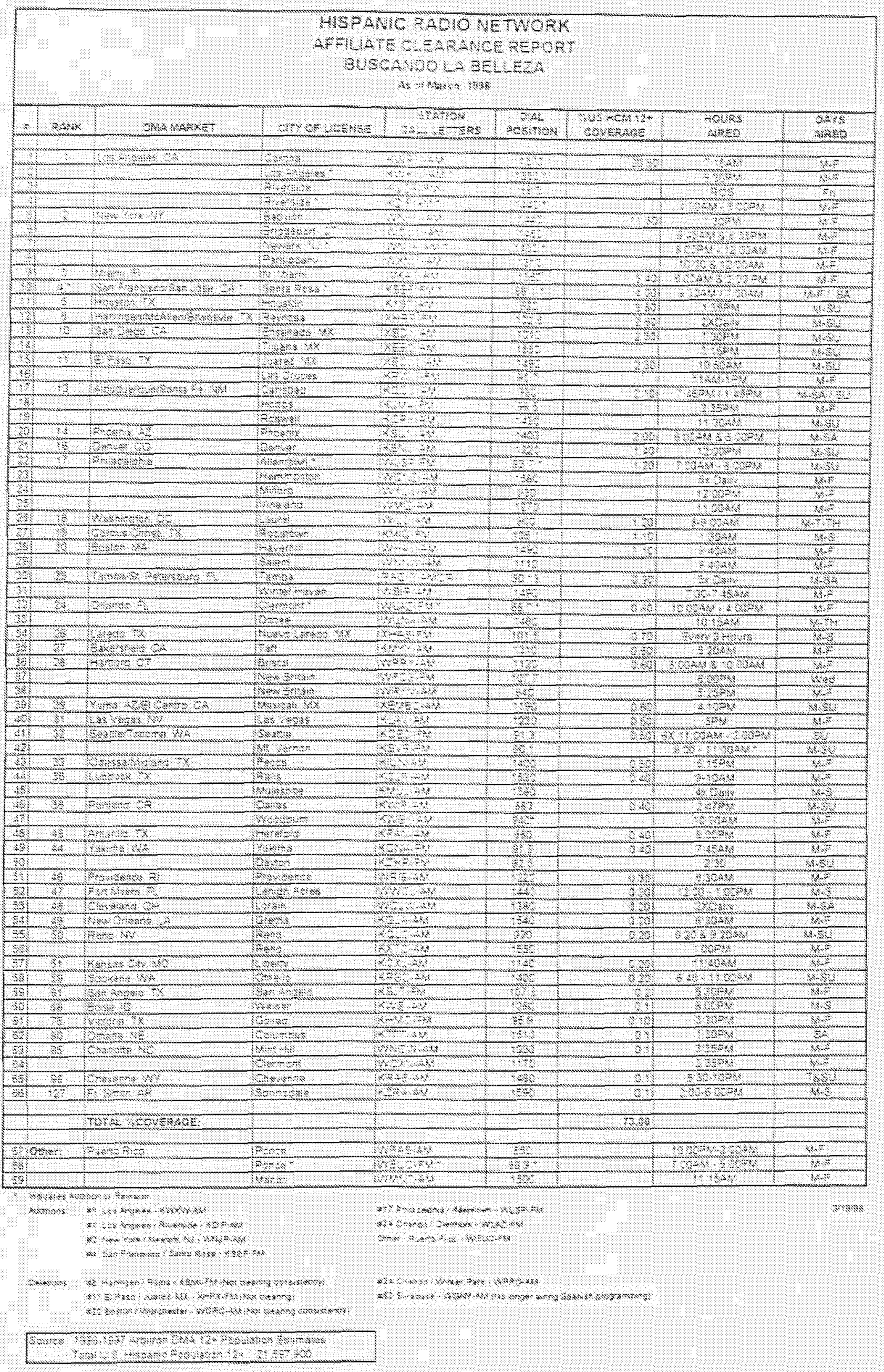




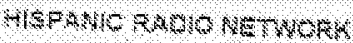

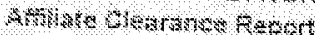

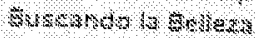

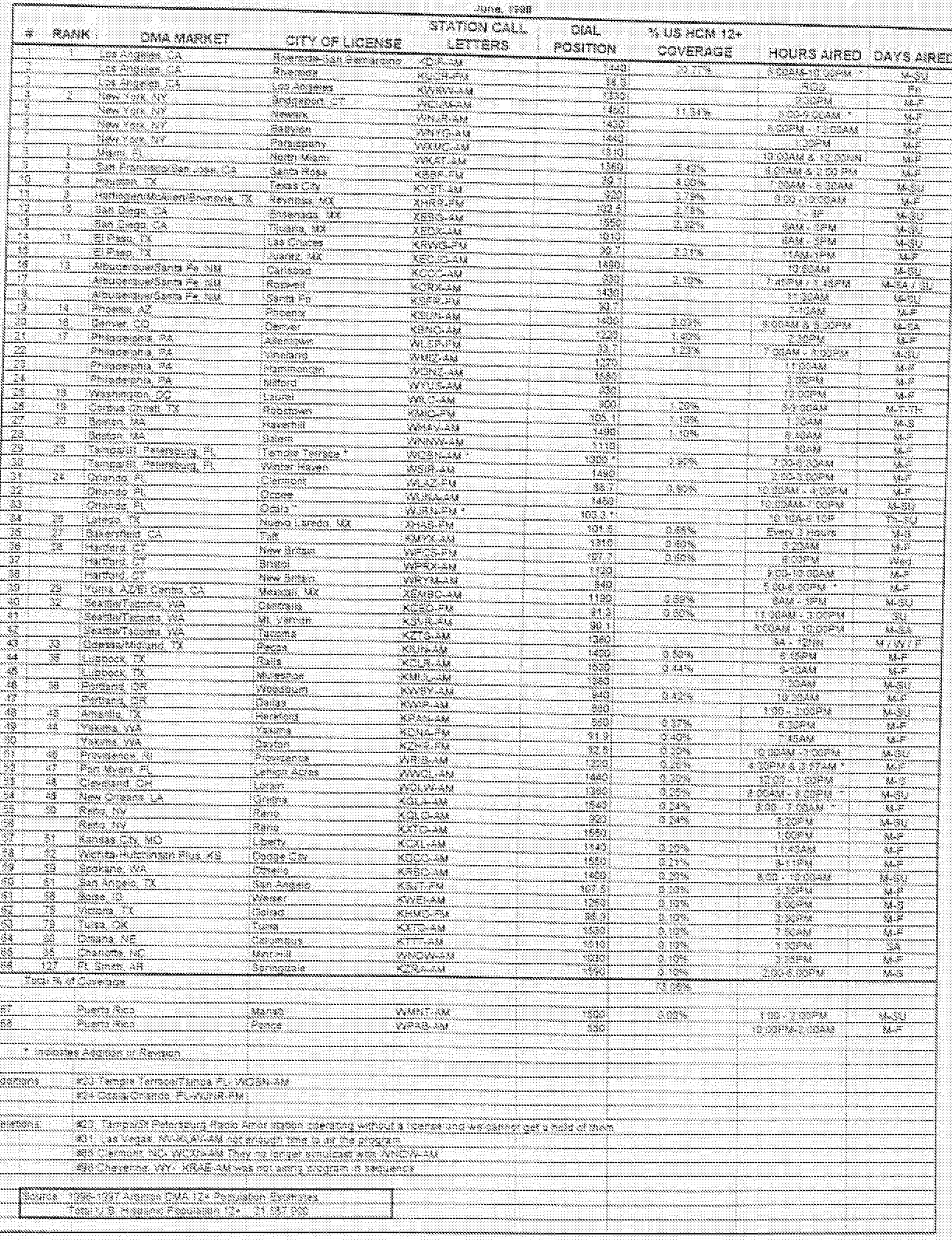




\section{IV. Radio Affiliate List For Planeta Azul}




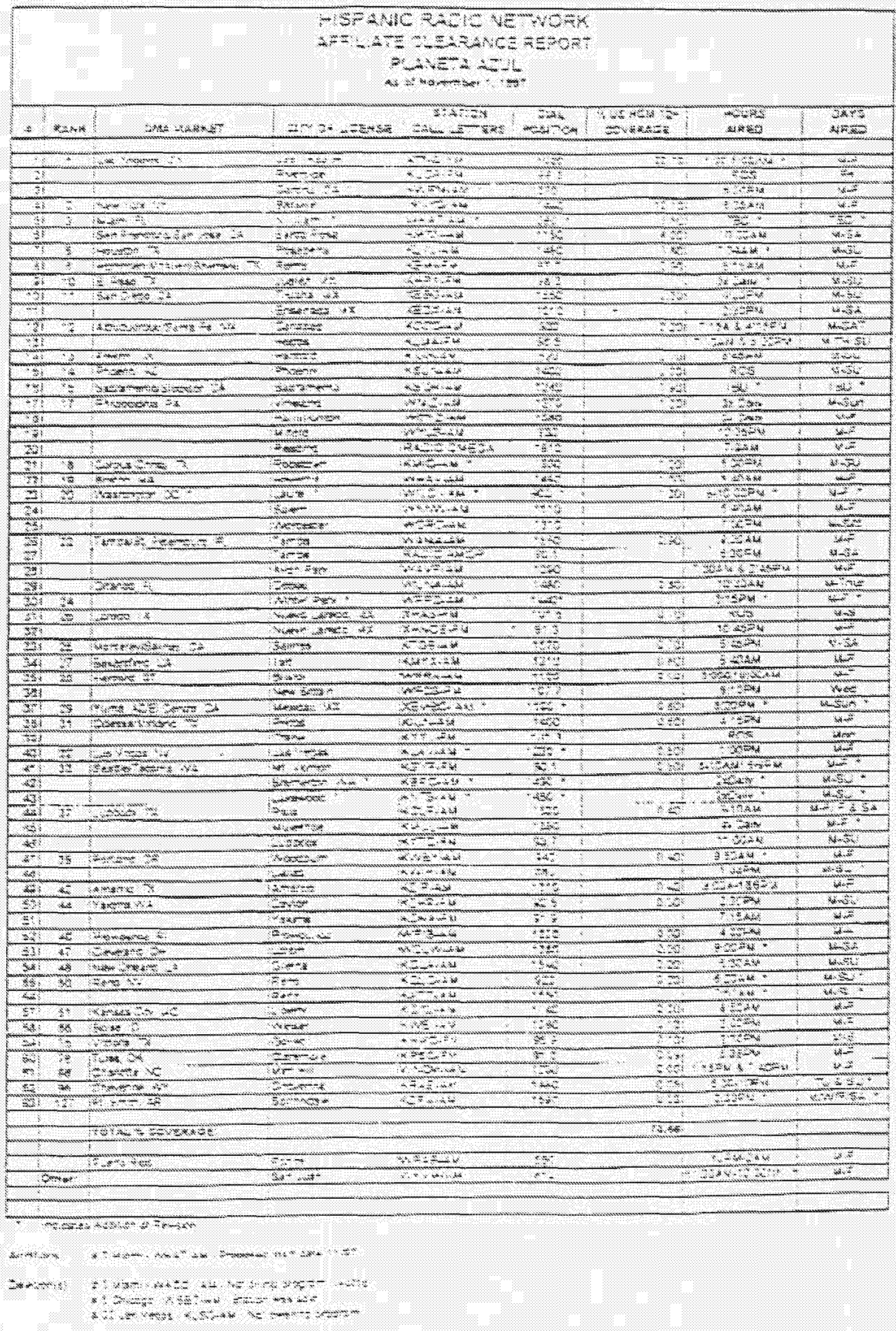




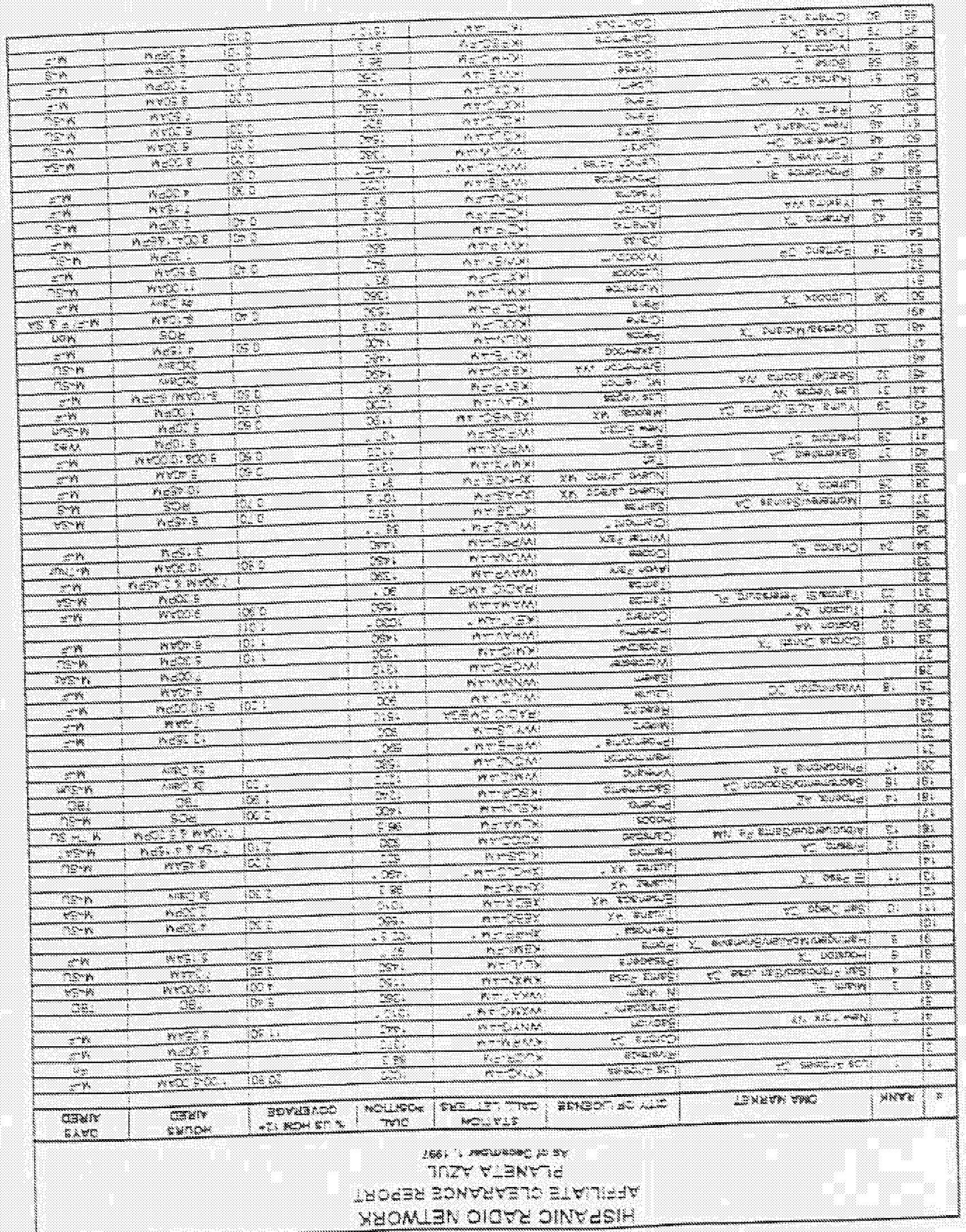




\begin{tabular}{|c|c|c|c|c|c|c|c|c|}
\hline & & aso ararer & 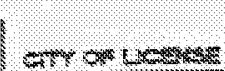 & 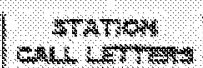 & 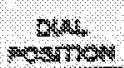 & 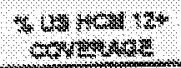 & 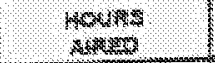 & oxtrot \\
\hline 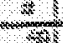 & 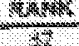 & 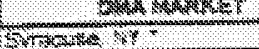 & & 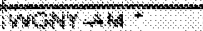 & $\mathrm{m} \times \mathrm{T}$ & mor $17 \mathrm{C}$ & & \\
\hline 4 & -9 & $\frac{4 x}{6 x}$ & $8 y+x$ & 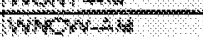 & के & 4 & 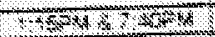 & $8=$ \\
\hline (1) & 3 & constem, & (6) & 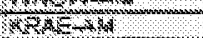 & 400 & $3 t$ & $r+40+8<x$ & एक \\
\hline 31 & 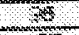 & 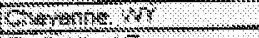 & 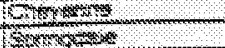 & ros & 380 & 0 & $20+3$ & XWrs \\
\hline 21 & $\frac{21}{21}$ & $8+\operatorname{sen} 43$ & $10 \times 3=$ & & & & & \\
\hline & & & & is & & 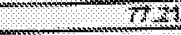 & & \\
\hline & & 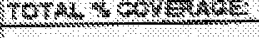 & & & & & & \\
\hline & & & $x_{\infty}$ & 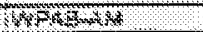 & 283 & & $10 \times 108$ & $x=$ \\
\hline & & 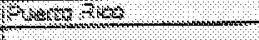 & sentax & 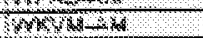 & sito & & 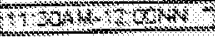 & $x=$ \\
\hline & estrer: & & & & & & & \\
\hline+8 & $x+\infty$ & 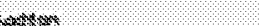 & & \multirow{2}{*}{\multicolumn{5}{|c|}{ 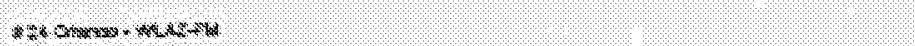 }} \\
\hline \multirow{5}{*}{ Amers } & & 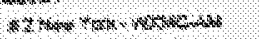 & & \multirow{2}{*}{\multicolumn{5}{|c|}{ 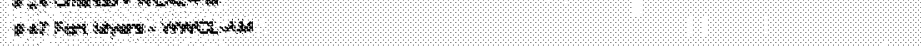 }} \\
\hline & & 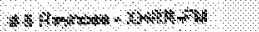 & & & & & & \\
\hline & & 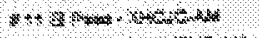 & & \multicolumn{5}{|c|}{$00000 \times 0$} \\
\hline & & 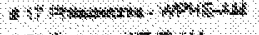 & & & & & & wat \\
\hline & & 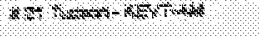 & & & & & & \\
\hline
\end{tabular}




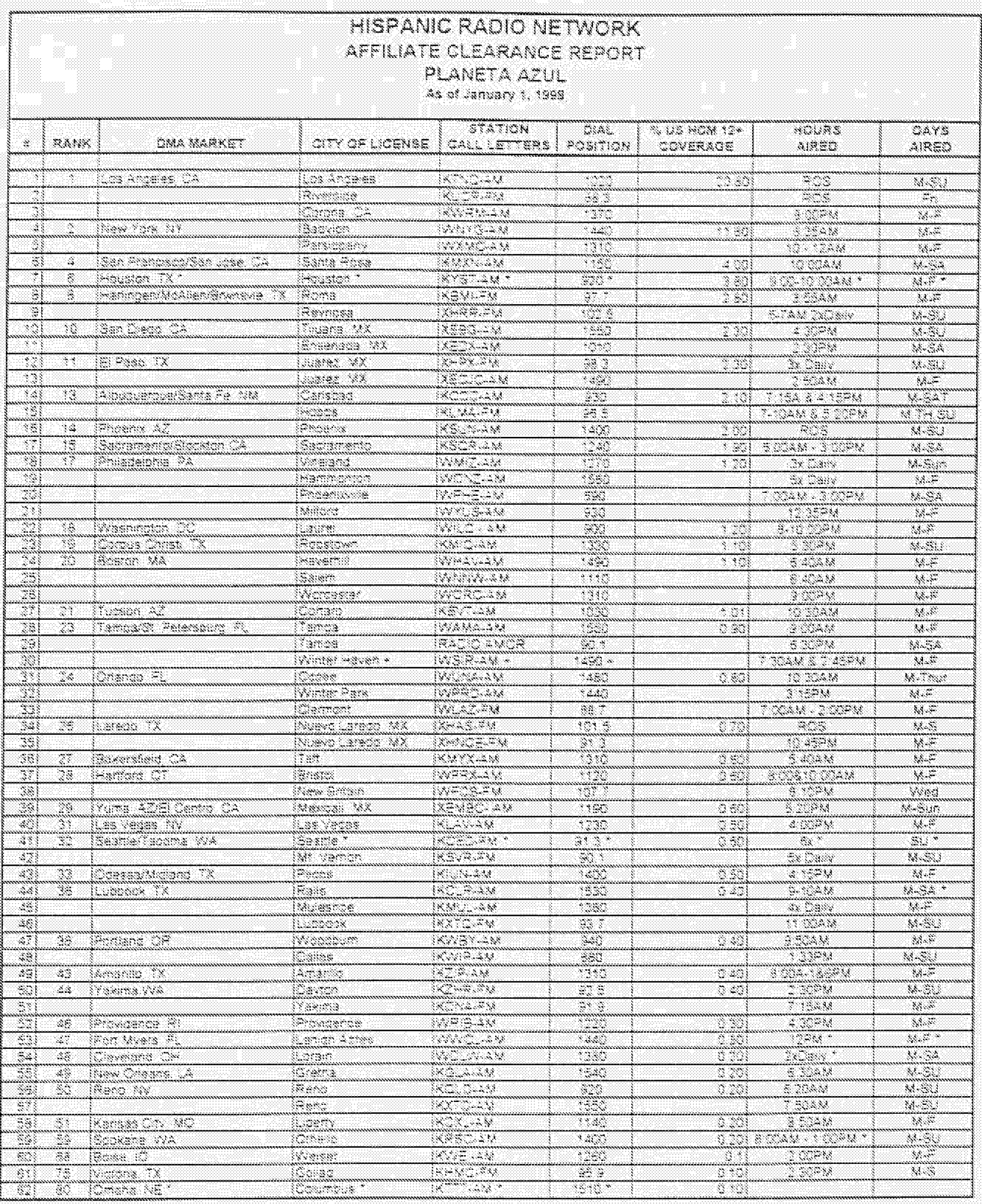




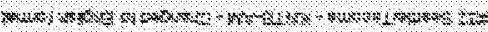

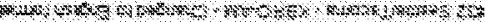

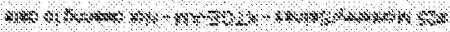

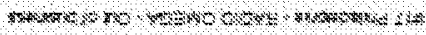

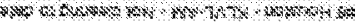

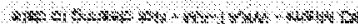

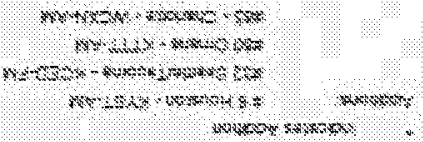

\begin{tabular}{|c|c|c|c|c|c|c|c|c|}
\hline 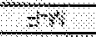 & 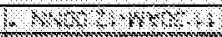 & & 36 & 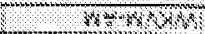 & WOIY uts & & nखw & \\
\hline $4+6$ & 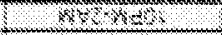 & 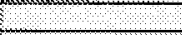 & 03 & H. - Sus & Noto 4 & 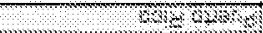 & & \\
\hline & سينتيست & & & is & & & & \\
\hline & & Hon & & 3 & & 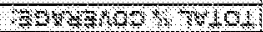 & & \\
\hline & & & & & & montom & & \\
\hline 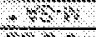 & nons & 6 & 0 & $6 \times-1+3$ & Retheness & 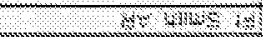 & 3 & 10 \\
\hline 39 & 8.017\% & 1 & 8 & 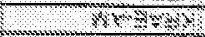 & andestants & 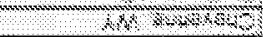 & 3. & 16 \\
\hline$(4,4$ & (X) & & $\$ 2$ & , $10, \mathrm{x}^{2}$ & 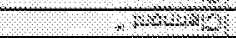 & & & 4 \\
\hline$=8$ & nht 1 & 1 & \$4) & $14 \times 2040$ & Who 131 & 3101404 & 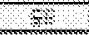 & 4 \\
\hline 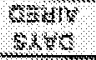 & dortson & 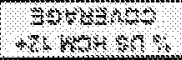 & 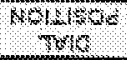 & 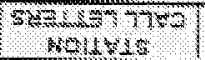 & 35 H. & 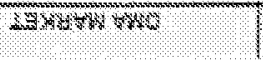 & 2018\% & 3 \\
\hline
\end{tabular}


HSPANIC RADIO NETWORK

AFFULATE CLGARANCE REPORT

PUANETA AZUL

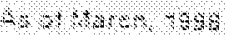

\begin{tabular}{|c|c|c|c|c|c|c|c|c|}
\hline 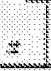 & 1406 & $344 \times 24=-$ & 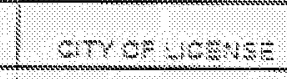 & 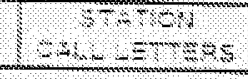 & 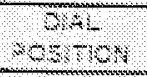 & 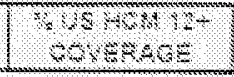 & 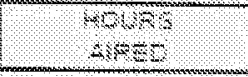 & $\begin{array}{l}088 \\
+880\end{array}$ \\
\hline 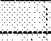 & & & & & & & & \\
\hline 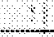 & 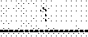 & 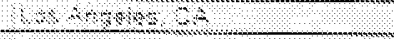 & $3+4+8$ & $346+1$ & 24 & 1989 & 908 & tes \\
\hline 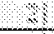 & & & 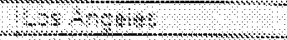 & 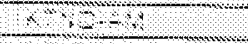 & 10 & & 48 & 18 \\
\hline 3 & & & 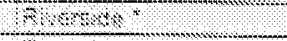 & \$? & 902 & & (4.5०4\% & ४ \\
\hline 4 & & & 10,408 & ४४४ & 8 & & $8+8$ & 3 \\
\hline 3 & 3 & 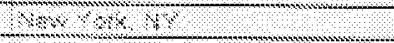 & $8+6$ & mer & 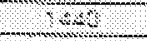 & 78 & $8258 \%$ & $6 \%$ \\
\hline 8 & & & 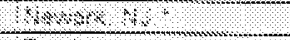 & 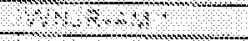 & 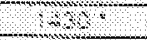 & & $302+4 \%$ & 1.2 \\
\hline 3 & & & 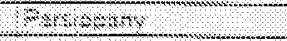 & 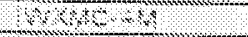 & 3 & & $8 ., 24$ & 19: \\
\hline 9 & 4 & 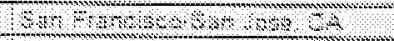 & 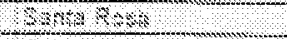 & $189-49^{\circ}$ & 31 & ४Qs & 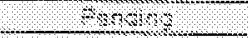 & ?-80ity \\
\hline 3 & 3 & 30304,0 & $488 \%$ & $40,-6$ & 28 & 20 & (4) & $x=$ \\
\hline 01 & 8 & $18+4+\cdots$ & tensers & $18+4$ & 32 & 380 & $290+30044$. & $3 \cdot=$ \\
\hline+8 & 8 & 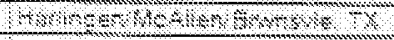 & $8+9$ & 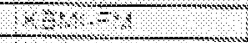 & 84 & 280 & 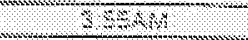 & 26 \\
\hline 41 & & & 84000 & $848+1$ & 48 & & $2-3,6,3, y$ & 48 \\
\hline 01 & 8 & 6,643028 & T.4\% & 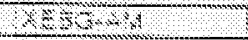 & 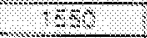 & 20 & $200 \% 10$ & (x) \\
\hline 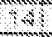 & & & sesenes 9 & $8=2 \times 4$ & 80 & & $3+380$ & $16 \%$ \\
\hline 3 & 1 & $89 \times 2,4$ & $013 r=48$ & 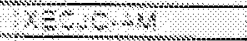 & 480 & 248 & 2.04 & (4) \\
\hline 48 & 3 & 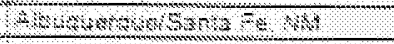 & 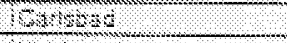 & $18+2,1$ & 93 & 28 & 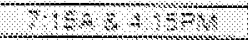 & 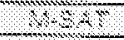 \\
\hline 4 & & & $50+48$ & $8,6,6$ & 95 & & $3 \times 4030304$ & $8+8$ \\
\hline 83 & 18 & $190 \times 4 \times 2$ & $3+4$, & $8801+4$ & 40 & 2.0 & 180,1 & (68 \\
\hline (1) & 17 & 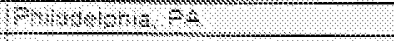 & $30-8 \times m$ & 64081 & 54 & 080 & 35001 & $8+30$ \\
\hline 20 & 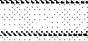 & 4,5 & (x.mmm & $200 \times-4$ & 18 & & 2.08 .1 & $x_{4}=$ \\
\hline 4 & 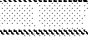 & 18 & $8906+24$ & $x=-8 \%$ & 8, & & $78 \% 30,3<-3$ & ४ $\%$ म \\
\hline 2 & 4 & $1, \$$ & $10 \% 2 \%$ & $20+3-1$ & 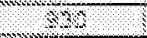 & & $12 \times 14$ & 18 \\
\hline 21 & 1 & (\$) & $3+8,8$ & $00+12 \times 14$ & 82 & & $13, \ldots$ & 140.3 \\
\hline 24 & 8 & 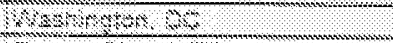 & $\sqrt{8,49}$ & 168028 & 2 & 19 & 8 & 80 \\
\hline 25 & 89 & $8<-<11,42$ & $18+30,0$ & 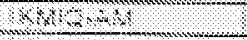 & 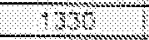 & 10 & 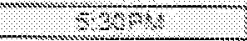 & (x-4) \\
\hline 26 & 20 & $84+0 \times 4$ & $14,4 \times r+\%$ & 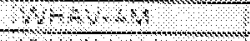 & 40 & 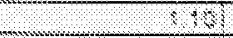 & 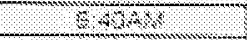 & $4 *$ \\
\hline 8 & & & 8840 & 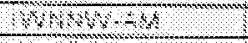 & 184 & & $048 \times$ & 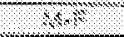 \\
\hline 8 & 8 & & 00,0 & Mors $>1$ & 230 & & $98 \%$ & 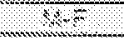 \\
\hline 29 & 81 & $34+4$ & $\sqrt{8+30}$ & $\mathrm{~K}^{2} \mathrm{~N}^{2}+\mathrm{K}$ & 100 & 80 & $1040+1$ & 42 \\
\hline 3 & 33 & 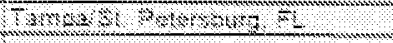 & Thirms & $8-90 \times 40$ & 901 & $(690)$ & $20+44+4$ & $30 \times$ \\
\hline 80 & & & Perseg 4986 & XPS $4+41$ & 393 & & Mstrits & $8,4,2$ \\
\hline 3 & & & Yomet 43,45 & $100-2-8$ & ssis & & 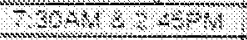 & 34 \\
\hline 31 & 4 & $96-2=$ & $06 \mathrm{rob}$ & $x+-2=1$ & 39 & 0281 & $1+02+1-2+0 \%$ & 24 \\
\hline 24 & & & $8+4 \times$ & 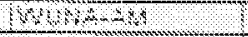 & 148 & & 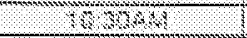 & $0+4 \times 1 \%$ \\
\hline 39 & & & binke $2 \times 6$ & 64084 & $9+4$ & & $3+301$ & 84 \\
\hline 81 & 38 & 46,86 & $869640800,6 \%$ & $2458 \%$ & 194 & 07 & 84 & 14 \\
\hline 31 & & & 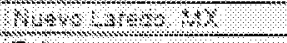 & $2+1+84$ & 48 & & 12480 & $x=$ \\
\hline 38 & 2 & $8 \mathrm{sect}+40$ & 134,1, & $x+x+4$ & 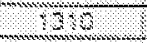 & 8510 & $3+4 \%$ & 34 \\
\hline 98 & 3 & $148680 \%$ & Estro & 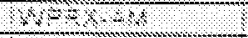 & 130 & 84 & 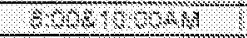 & $4+4$ \\
\hline 80 & & & 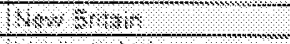 & $00+3=4$ & $3 \times 4$ & & $8 \mathrm{~s} 9 \%$ & sise \\
\hline 4 & 2 & 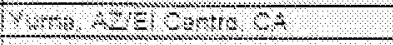 & 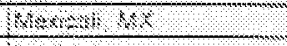 & 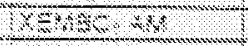 & 14\% & Q & $2+40 \%$ & 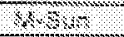 \\
\hline 4 & 31 & 1640435 uy & 1640848 & (4) & 28 & 258 & 124 & 20.4 \\
\hline 4 & 3 & 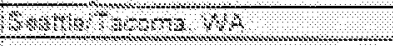 & (1)nary & $x<=-2$ & 963 & 088 & $801,004018>14$ & 3 \\
\hline+8 & & & $14+\quad 4+4 s$ & $18 \times 18=1$ & 80. & & $1,4, \beta_{2}$ & 884 \\
\hline 4 & 3 & 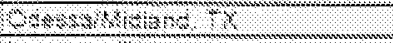 & $18-2$ & $804+-4$ & 180 & Q80 & $-4+201$ & $14=$ \\
\hline 48 & 38 & 46680 & 1844 & $12+249$ & 173 & 040 & 1. 4846 & $3+34$ \\
\hline yt & & & $0 \times 8 \times n$ & $8+8 \div-11$ & 28 & & $2 \times 08$ & 14 \\
\hline 48 & & 4 & 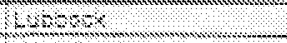 & $8 \% 0+8$ & 42 & & 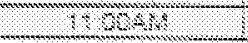 & 860 \\
\hline 4 & 3 & $8+494$ of & $40,3 \mathrm{Br}$ & $008 \times 4$ & प्रिक & 648 & 30214 & 48 \\
\hline 81 & & & 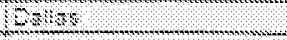 & 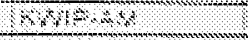 & 98 & & 3394 & 148 \\
\hline 41 & $4^{\circ}$ & $864084^{\circ}$ & $1308+4^{\circ}$ & $14040 \%$ & 18301 & ४०य & $3.4 \% 8 \%$ & 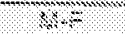 \\
\hline 84 & 48 & $4 \div-48<$ & 4 & $4 \% 46$ & 134 & 840 & $64 \% 44+\% 3$ & 164 \\
\hline 81 & 48 & 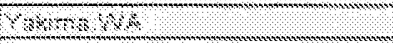 & $18+2$ & 10480 & 28 & 201 & ४४४\% & \%० \\
\hline 84 & & & $10 \times 43$ & $808+, 3$ & 1े? & (2) & 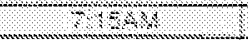 & 40 \\
\hline 8 & 48 & $84 \times 4$ & 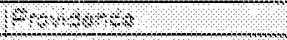 & $1468+4$ & 102 & (1) & 2020 & n०त \\
\hline 80 & + & 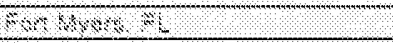 & $384,4 \%$ & 40484 & $4+4$ & 02 & 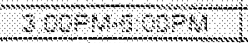 & 4. \\
\hline 8 & 8 & $140.6 \times$ & $\sqrt{4 \times 1}$ & 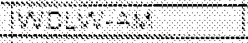 & 108 & 28 & $3 \times 4$ & 40 \\
\hline 88 & 4 & 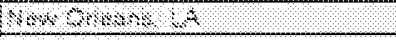 & 840 & $108-4$ & (2.) & 12\% & 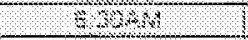 & 68 \\
\hline 8 & s? & 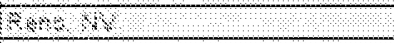 & ४ै: & $30+2$ & 88 & 38\% & 8208 & 40 \\
\hline 84 & & 8 & $88+4$ & $1040-4$ & ९९ & & $790-4$ & 808 \\
\hline 81 & 41 & 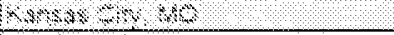 & 1068 & 14 \& \& & 840 & 048 & $2 \div<18$ & 64 \\
\hline 8 & 3 & 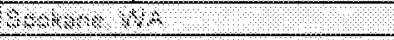 & 1648 & $305+1$ & 140 & 00 & $840<1 \%>>$ & no \\
\hline 3 & 88 & $18 \%$ & $146 \% \% 8$ & $3 \times 4-6$ & $\% ब$ & 9 & $3 \times 3 \%$ & $4 \%$ \\
\hline 3 & 8 & $0+00048$ & 8 & $2+r<-1$ & 89 & 50 & 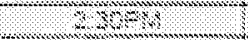 & 80 \\
\hline 88 & 8 & (m, No & 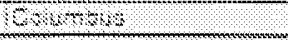 & $80 \div$ & 80 & 20 & 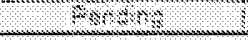 & $13+4$ \\
\hline
\end{tabular}




\begin{tabular}{|c|c|c|c|c|c|c|c|c|}
\hline 8 & $\mathrm{C}, 4$ & $0 \% 4 \quad 4 \times k 8$ & 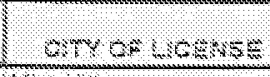 & 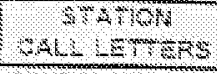 & 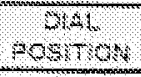 & 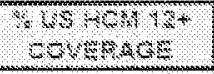 & $\begin{array}{l}\text {-0ros } \\
\text { sines }\end{array}$ & $\begin{array}{l}1748 \\
989\end{array}$ \\
\hline 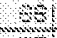 & 26 & 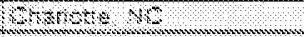 & $16 n+411$ & $2040 \times 44$ & 150 & 18 & 1389 & $8 \%$ \\
\hline 2 & & & Ponmsas & $80 \times 4-36$ & 3170 & & $235+4$ & 48 \\
\hline 8 & 8 & 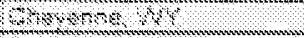 & Ctrovore & 848,4 & 48 & 4 & $8340<$ & $4<48$ \\
\hline 8 & 21 & 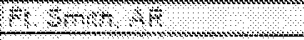 & Ponoras & $2,-4$ & 38 & 11 & ...8p & 30 \\
\hline s. & & & & & & & & \\
\hline 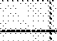 & & 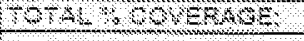 & & & & $(1,8)$ & & \\
\hline 3 & & & & & & & & \\
\hline 8 & Buer & $2+3.68,4$ & Ports & $3 P 3 e-42$ & 88 & & $600 \mathrm{den} \times$ & $4+\%$ \\
\hline 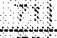 & & & 8 , & Wesc=k & 301 & & 3oration & $8 \times 4,42$ \\
\hline 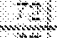 & & & $3 \times 8 \times 10$ & $20815-41 \%$ & 840 & & 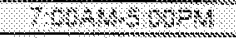 & 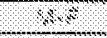 \\
\hline 3 & & & SAn $-4 m$ & $30 \times 4 \times 8$ & 20 & & $188+4.8 \times 14$ & कि \\
\hline
\end{tabular}

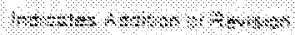

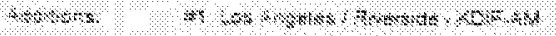

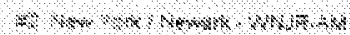

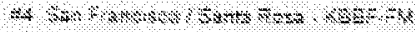

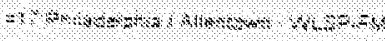

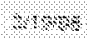

$+4+4,+, 4,4$

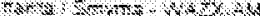

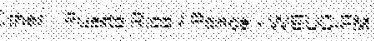

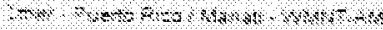

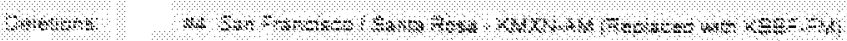

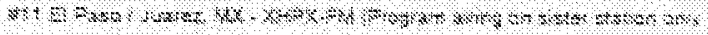

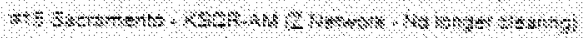

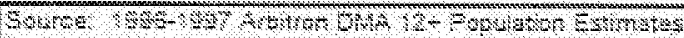

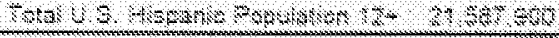




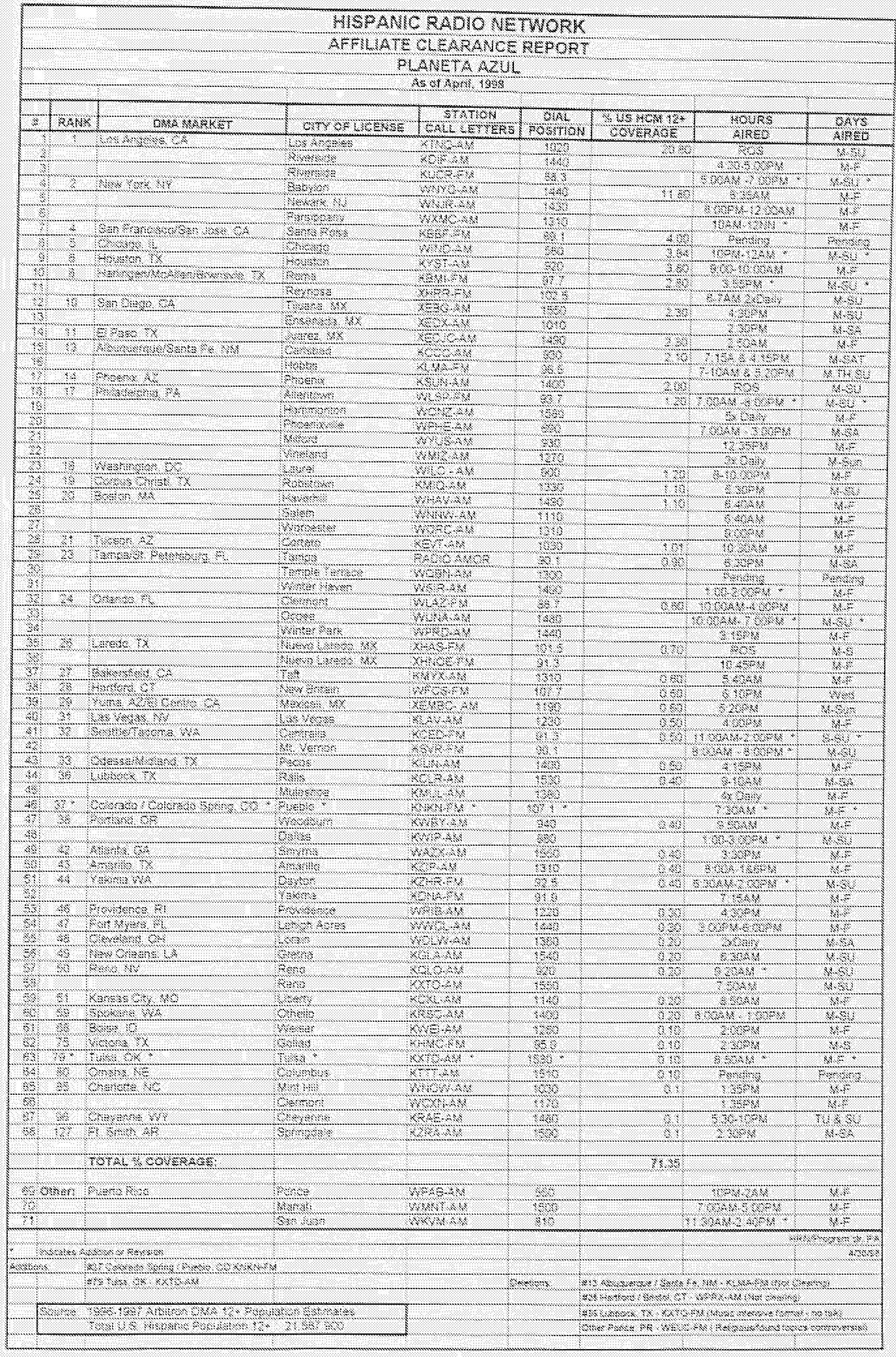




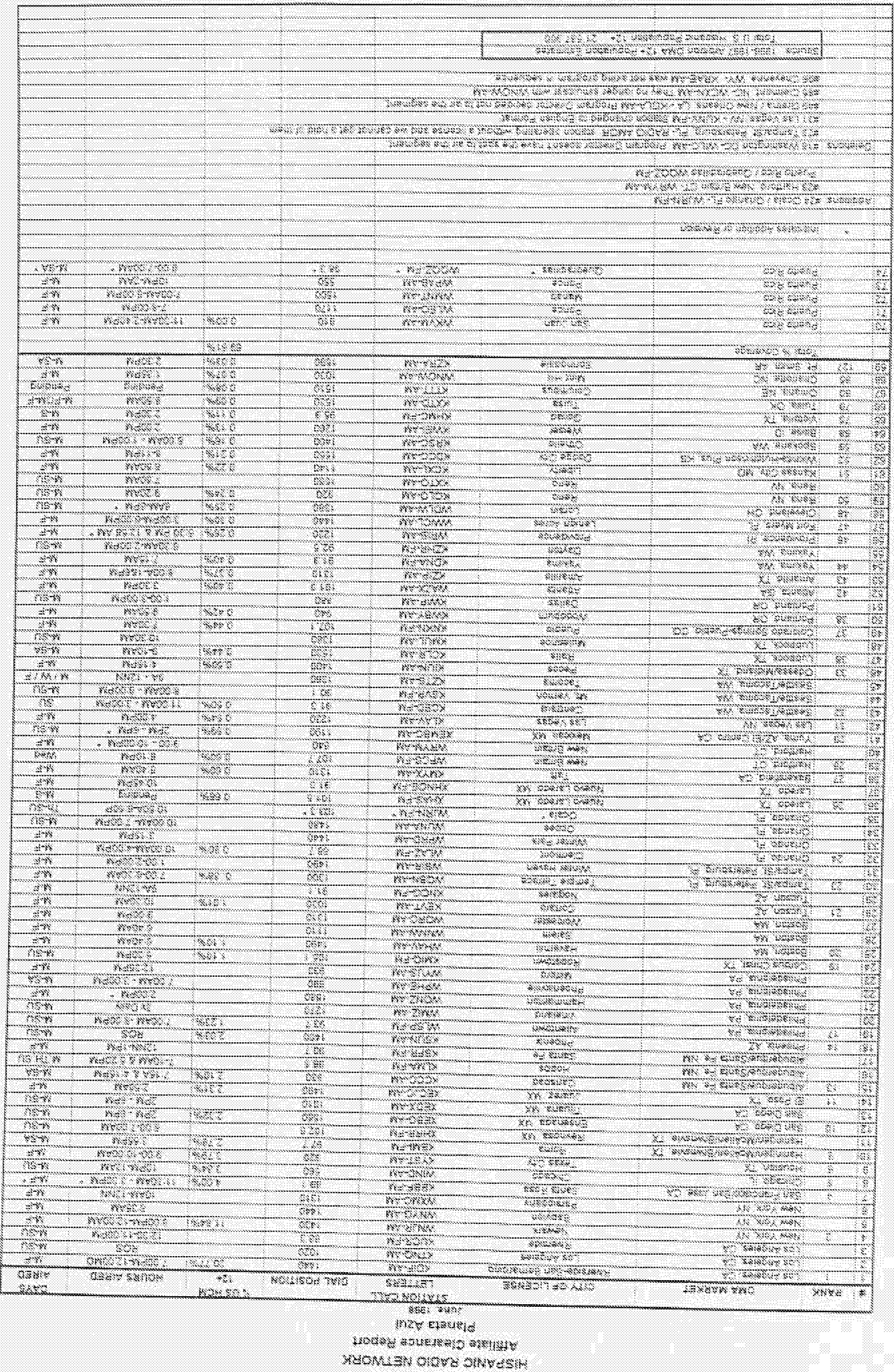




\section{HSPANIC RADO VE TWRK

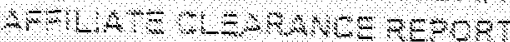

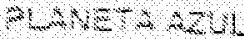

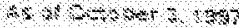

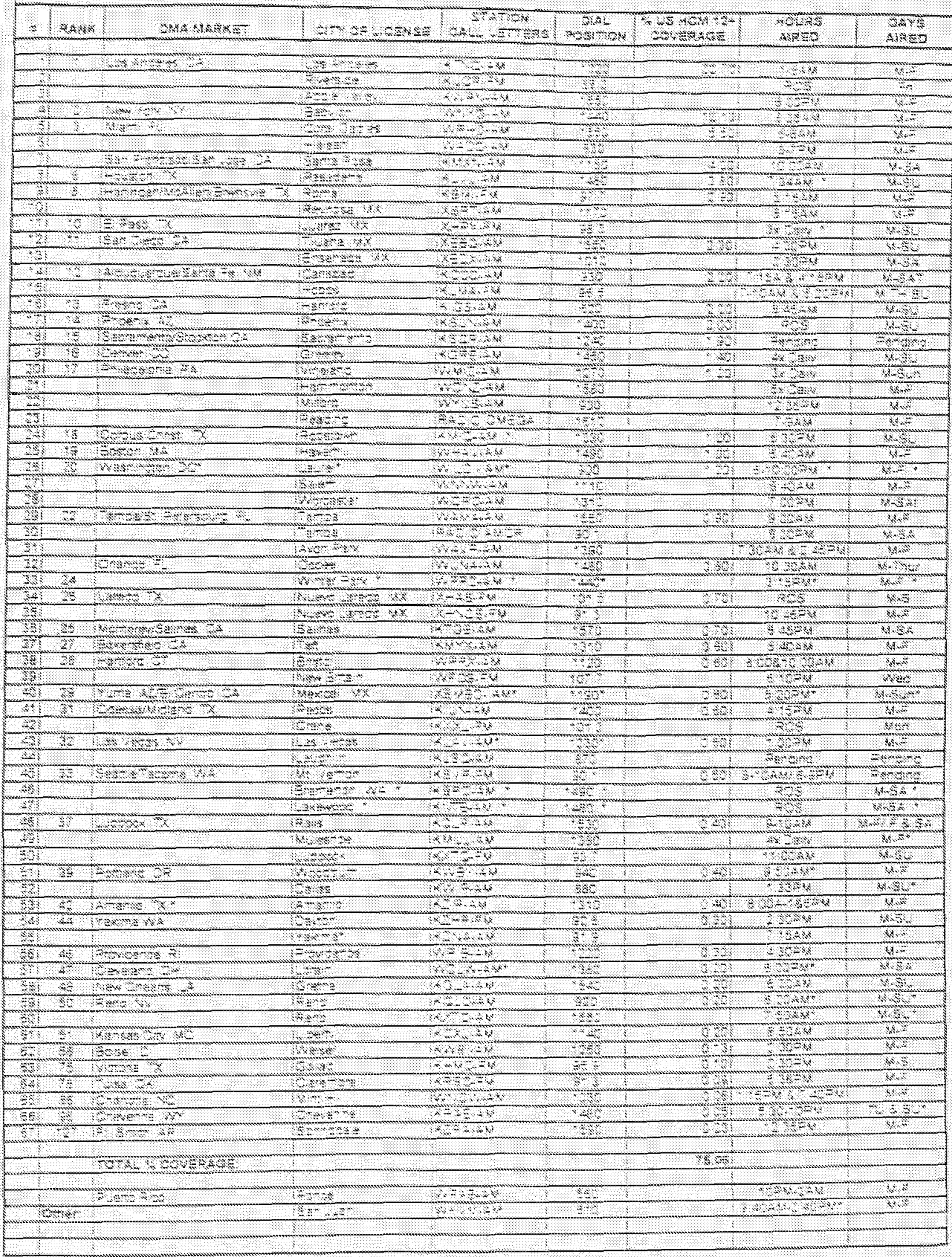

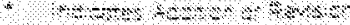

40

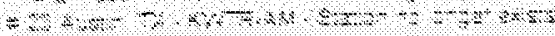




\section{V. DOE-EM Caller Reports}




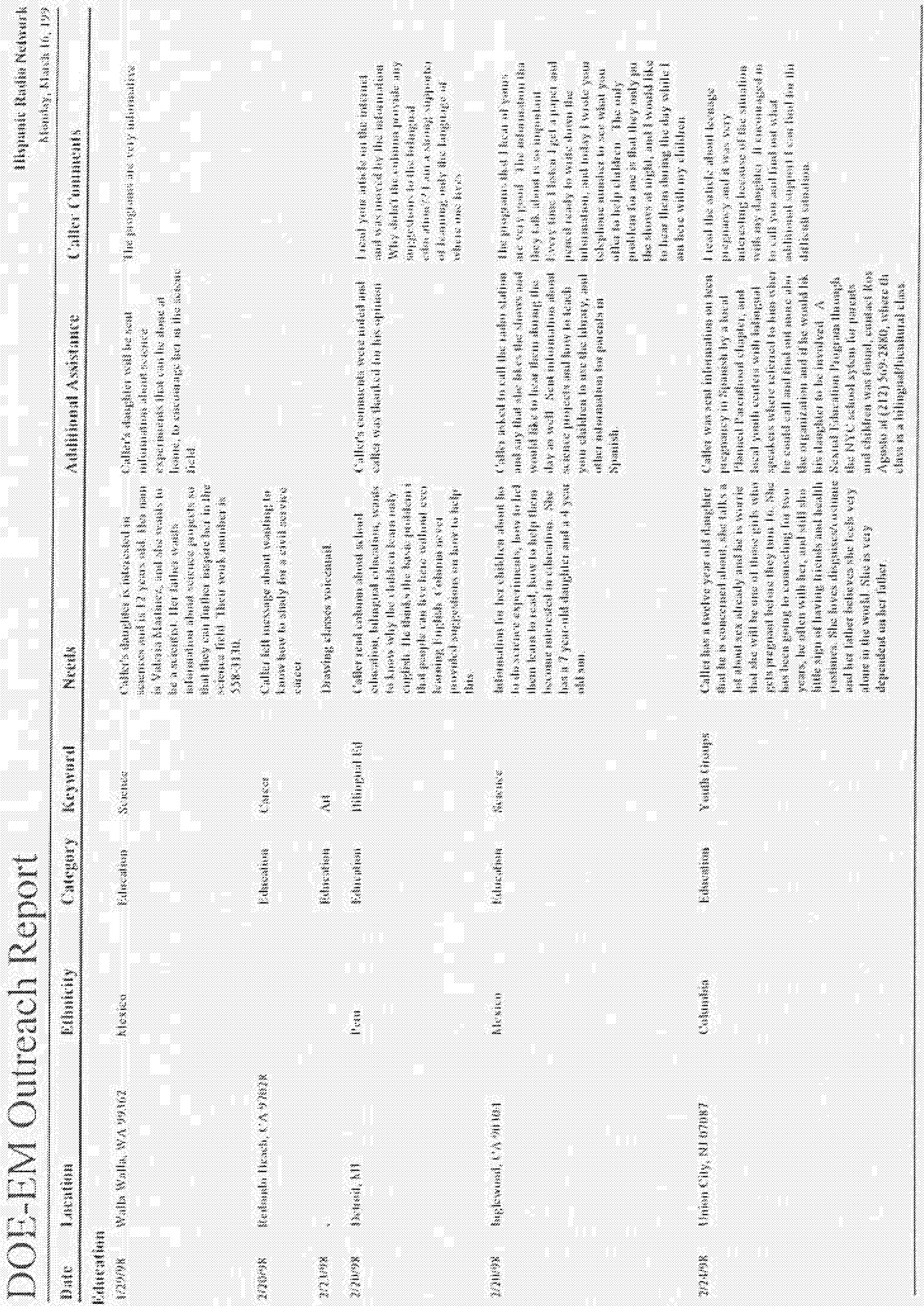




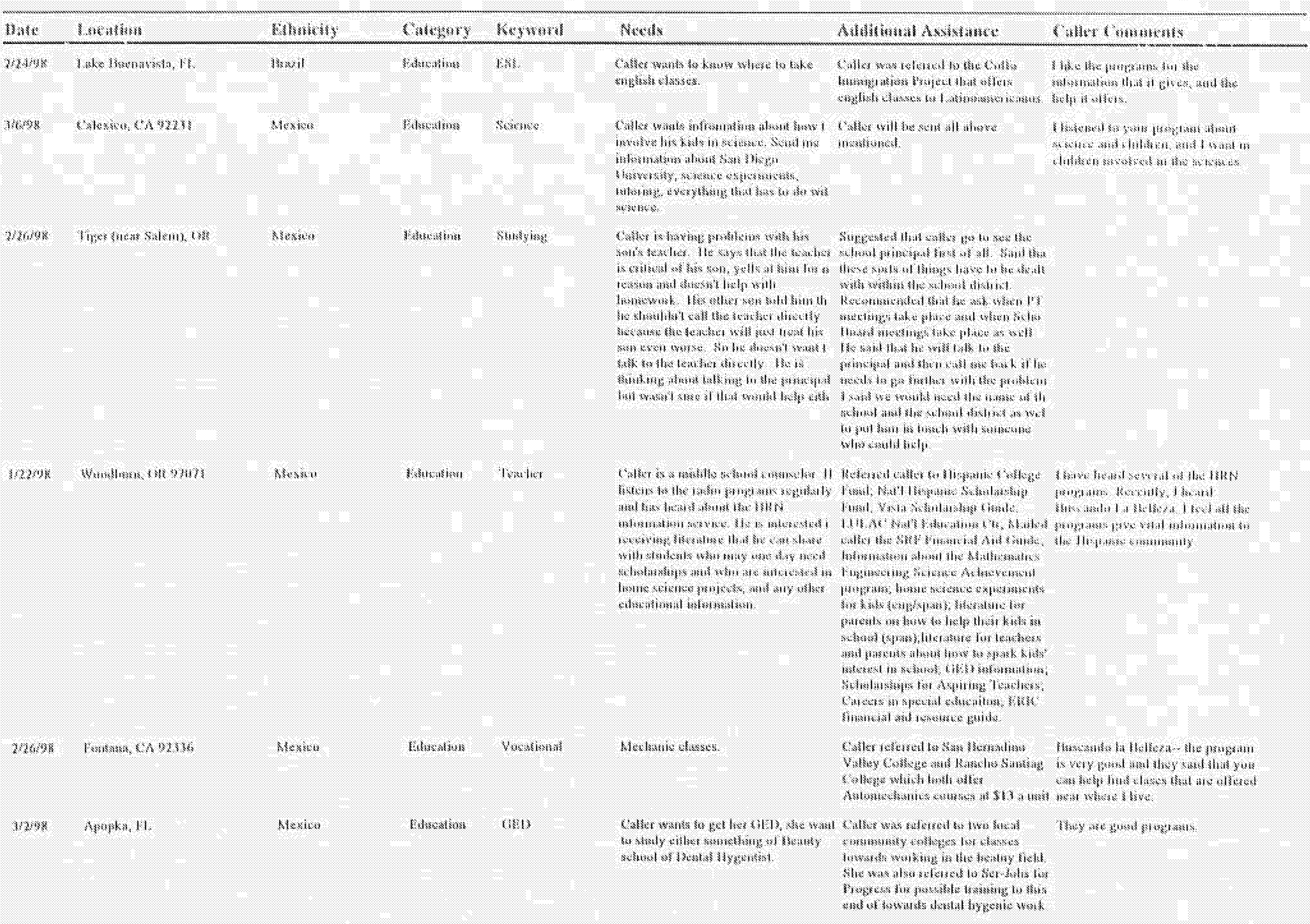




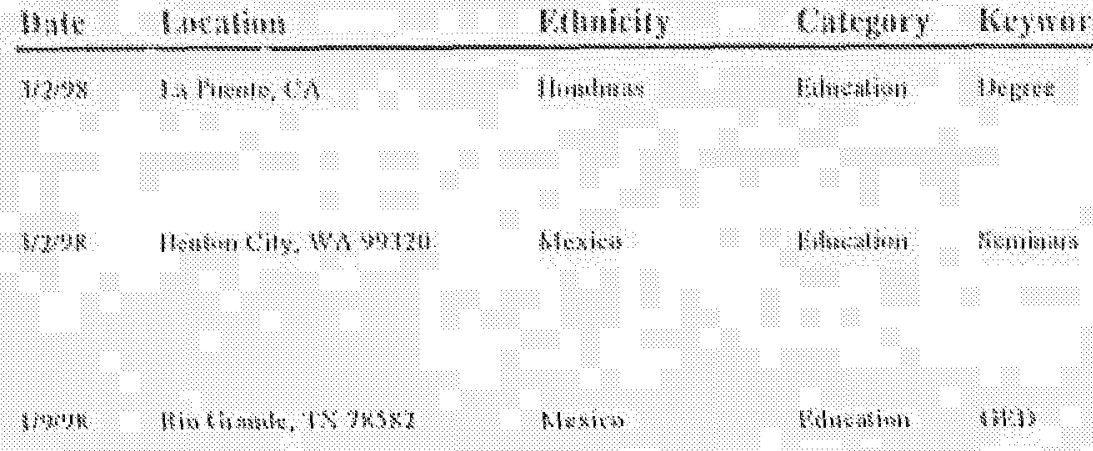

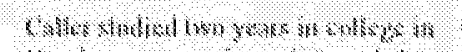

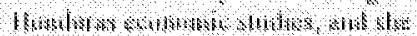

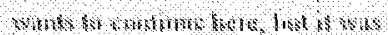

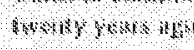

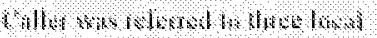

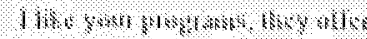

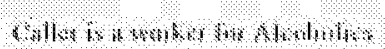

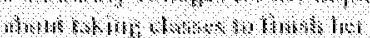

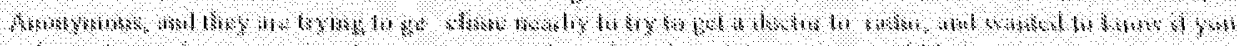

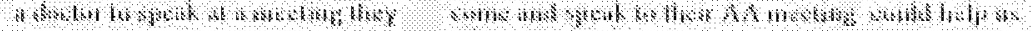

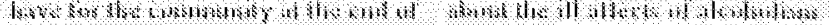
Qh:me:

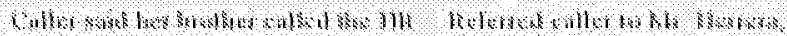

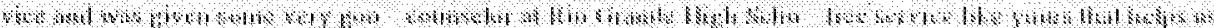

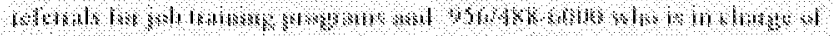

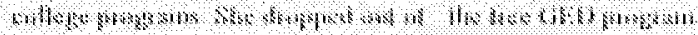

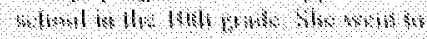

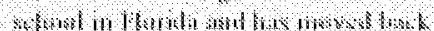

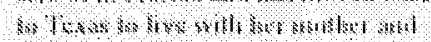

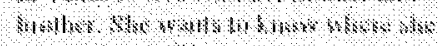

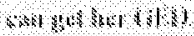

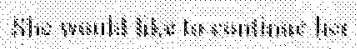

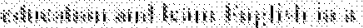
Thos (m) ling

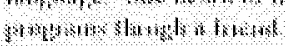

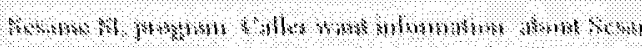

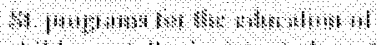

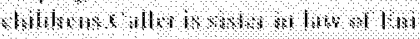

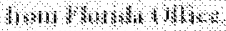

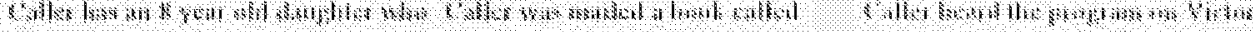

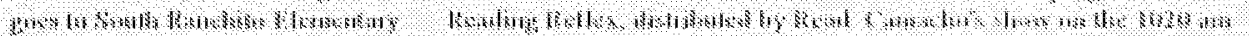
(1) 190.

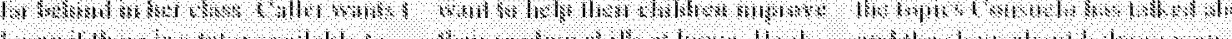

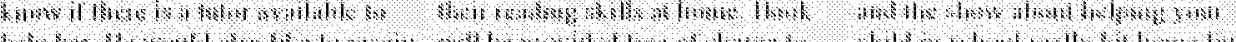

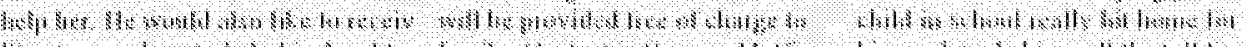

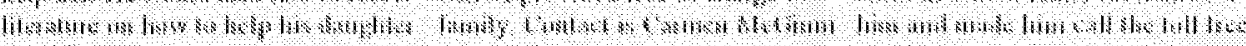

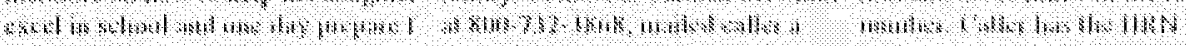

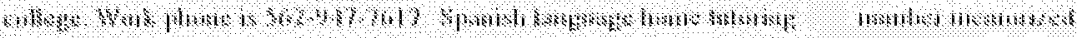

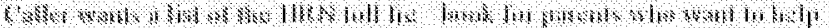

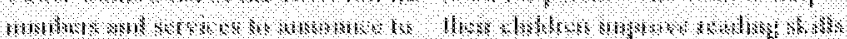

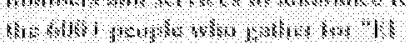
(1)

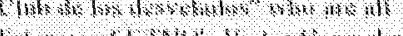

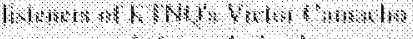

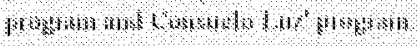

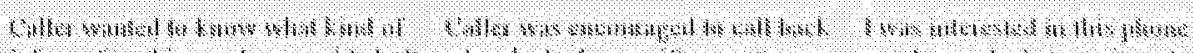

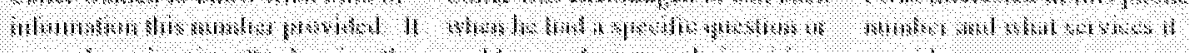

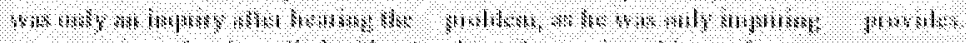

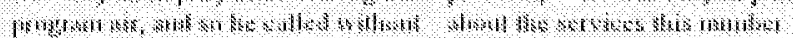

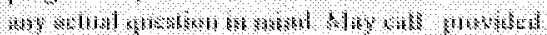

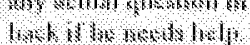




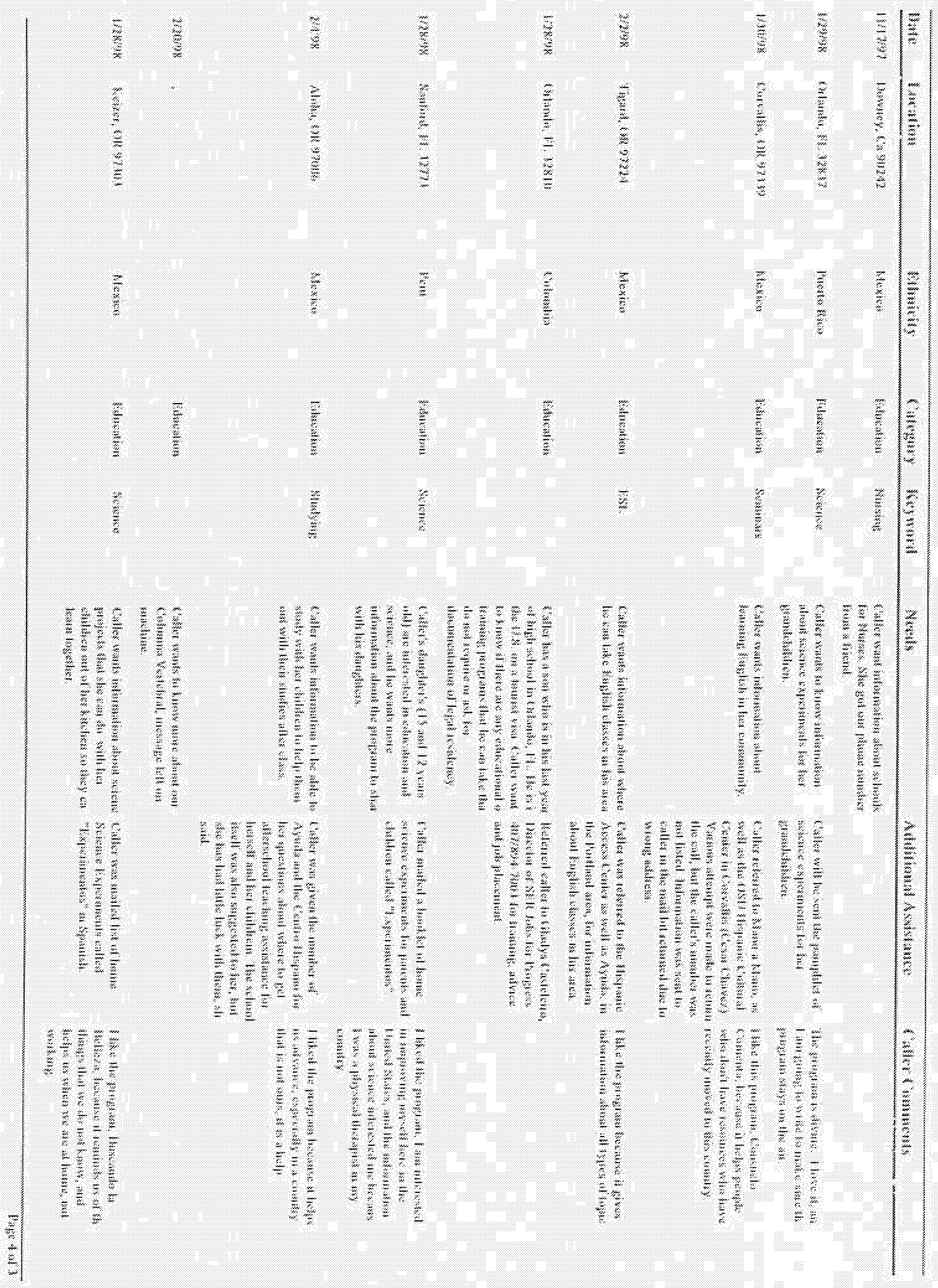




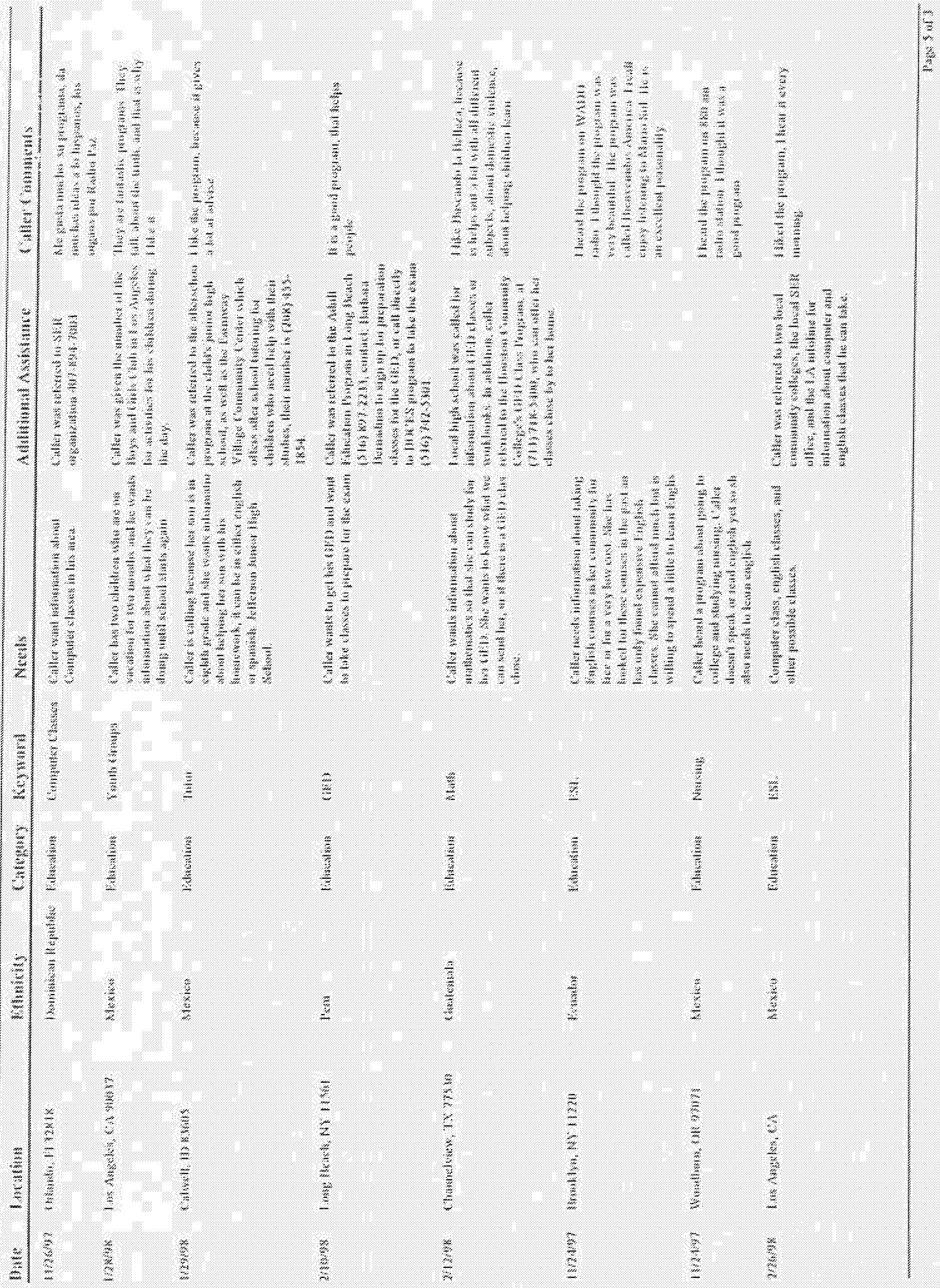




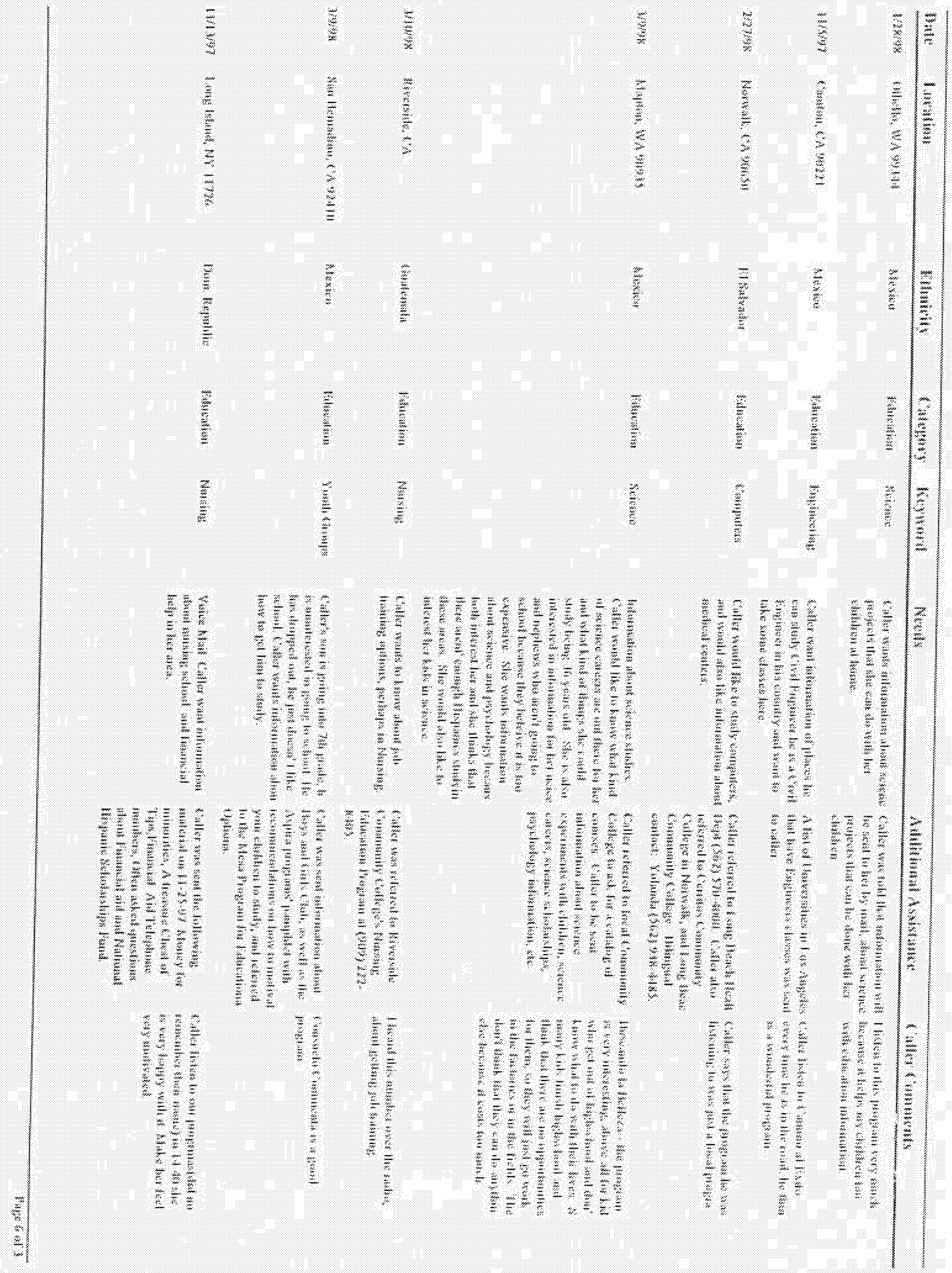




\begin{tabular}{|c|c|c|c|c|c|c|c|}
\hline 6860 & Orencimen & lumately & 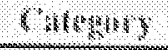 & 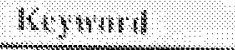 & Nereeds: & 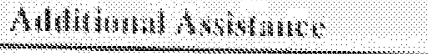 & 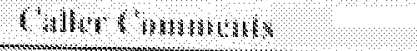 \\
\hline$\left(\frac{10}{2}\right.$ & 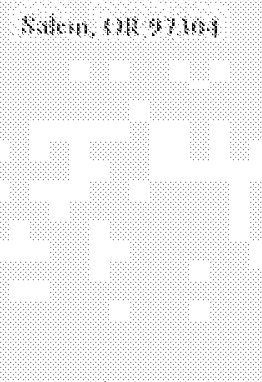 & 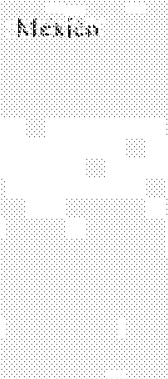 & 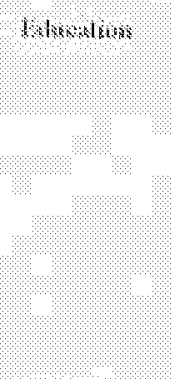 & 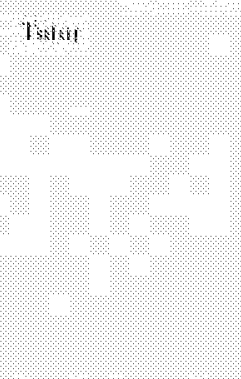 & 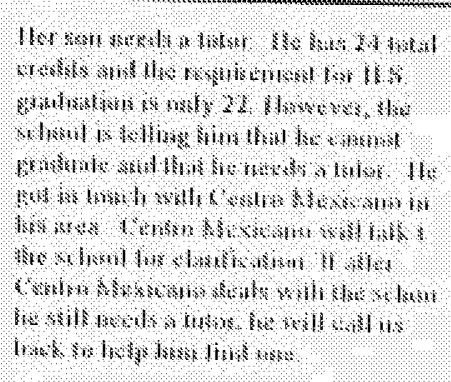 & tas & 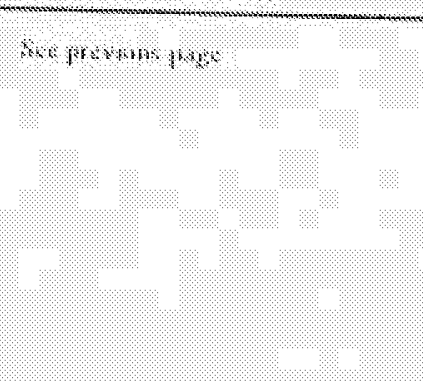 \\
\hline $8100 \%$ & 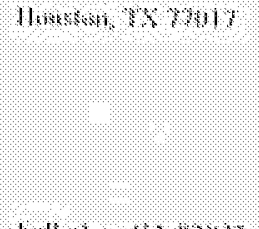 & 111 & 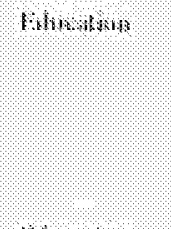 & Shesereres stronimaliess & 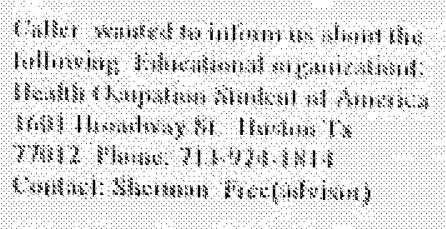 & 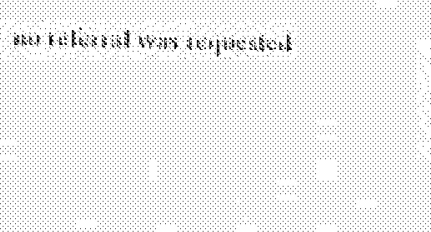 & 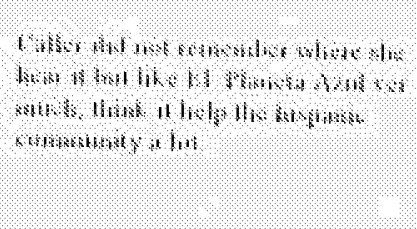 \\
\hline $1,179 \%$ & 1 a & $x(6060)$ & 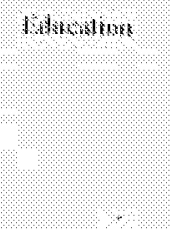 & Mlange & 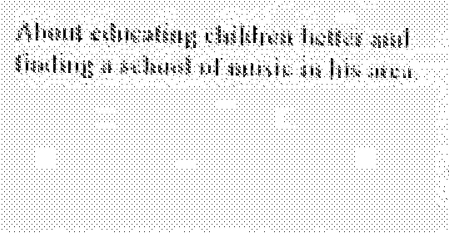 & 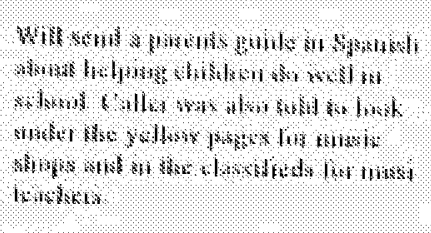 & 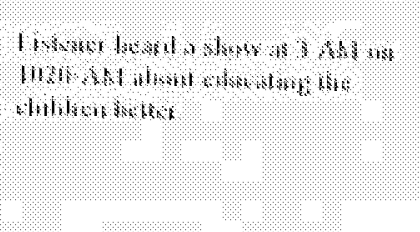 \\
\hline Shomo & Mllowerke. Wh & seresing & 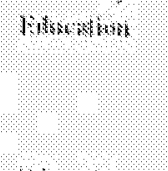 & $|m|$ & 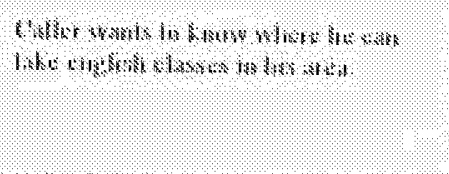 & 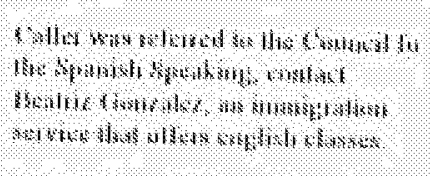 & $\begin{array}{ll}4 \\
4 \\
4\end{array}$ \\
\hline $1010 \times 2$ & 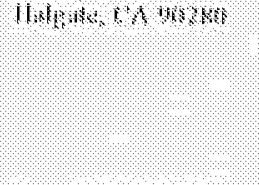 & $016 \% 100$ & 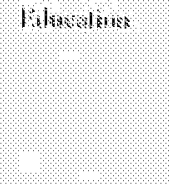 & 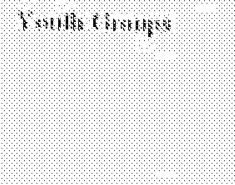 & 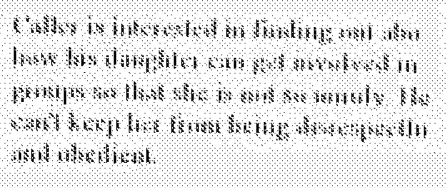 & 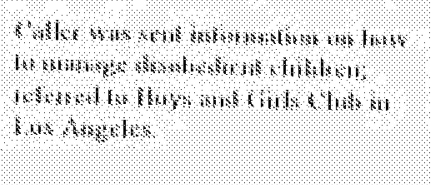 & 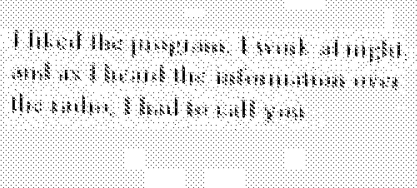 \\
\hline $18181 \%$ & 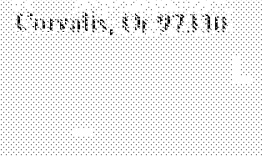 & 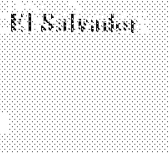 & 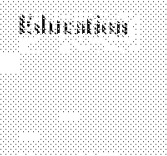 & 488 & 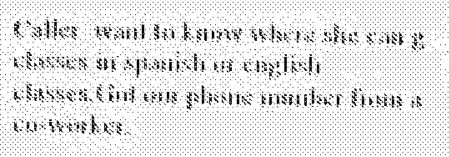 & 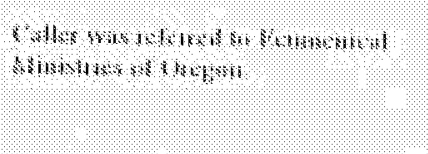 & 8 \\
\hline $10 \% 13$ & 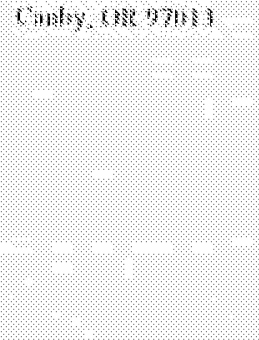 & 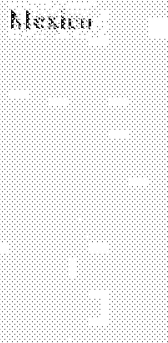 & Mtinolings: & lerenember & 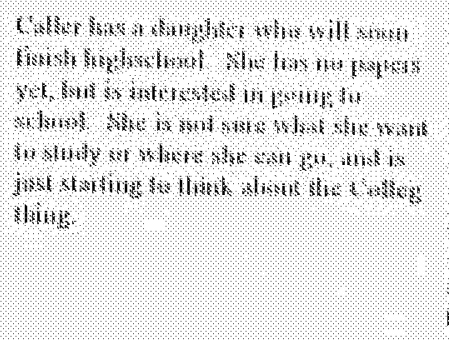 & 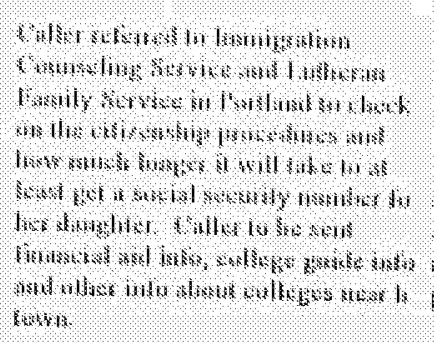 & 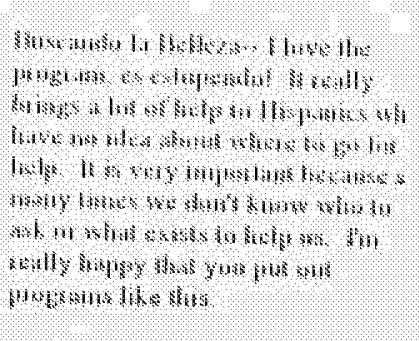 \\
\hline
\end{tabular}




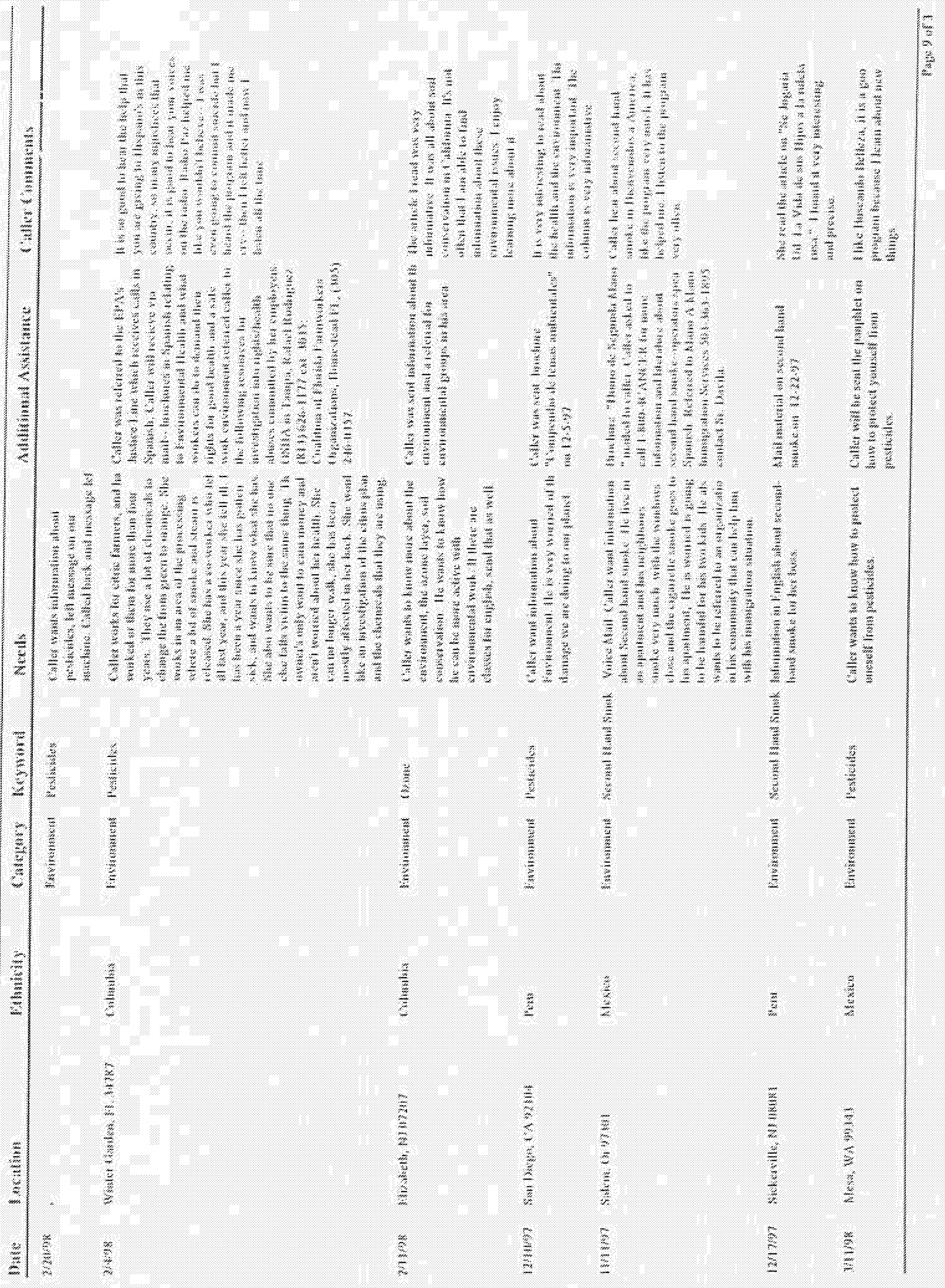




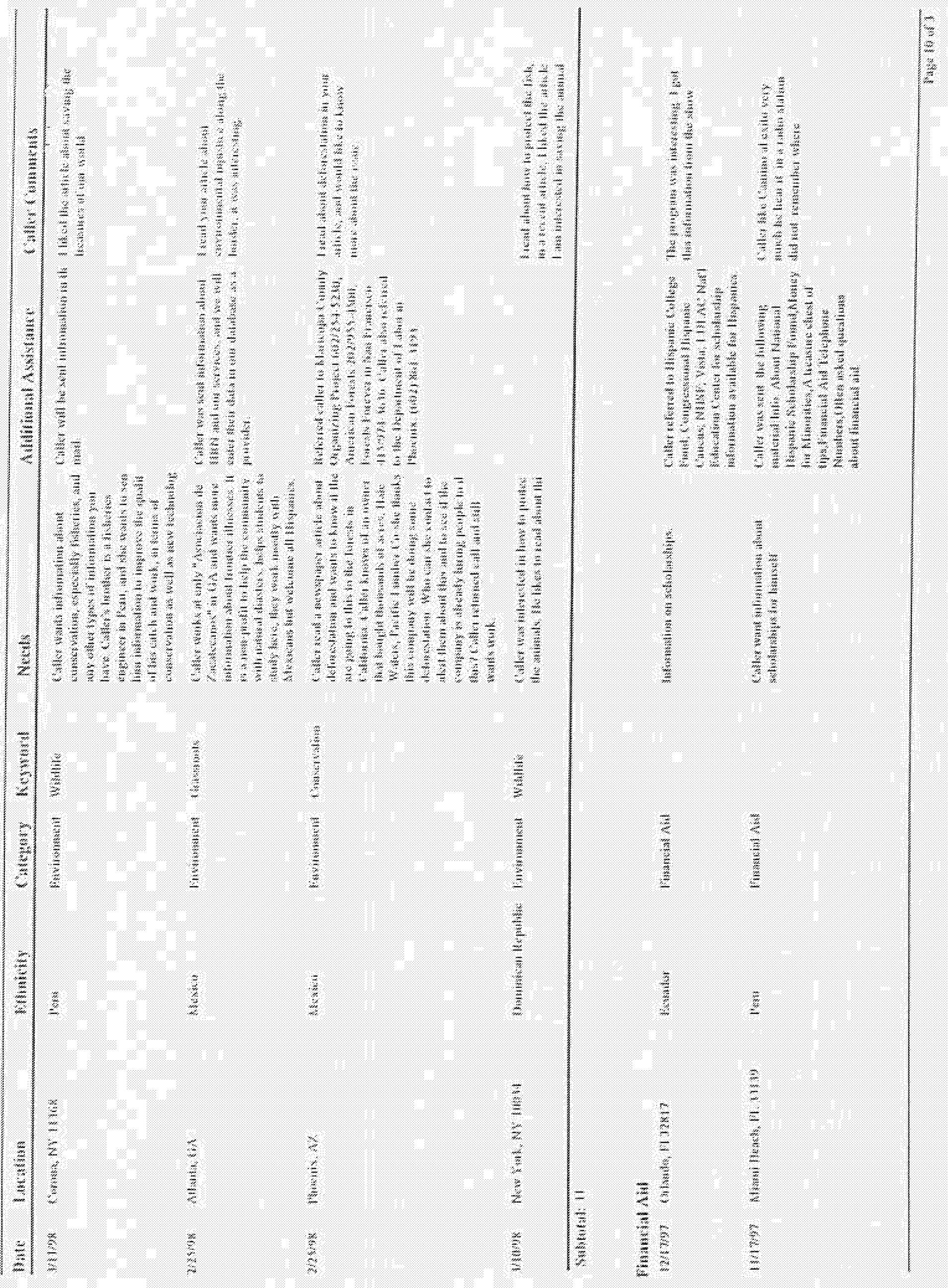




\begin{tabular}{|c|c|c|c|c|c|c|c|}
\hline 0,10 & 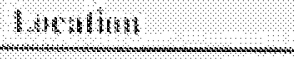 & $1 / 4$ aricis & $(60140+15)$ & 691,180119 & Norils & 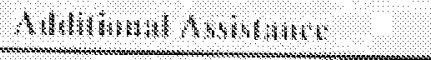 & 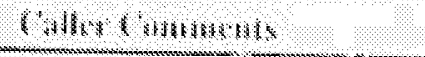 \\
\hline 102004 & 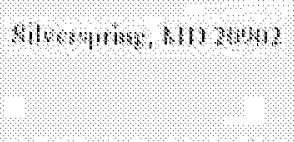 & lontron & In: & & 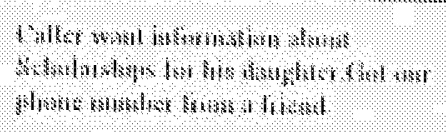 & & \\
\hline 19404 & 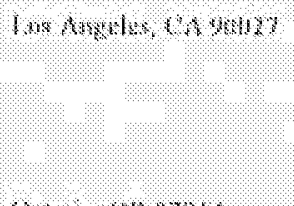 & Atsinat & 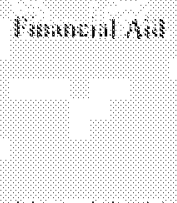 & & 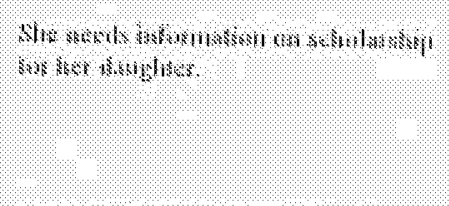 & 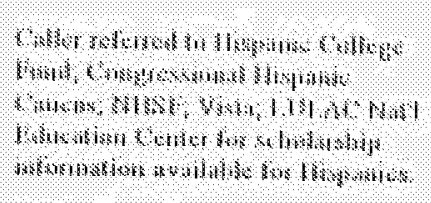 & 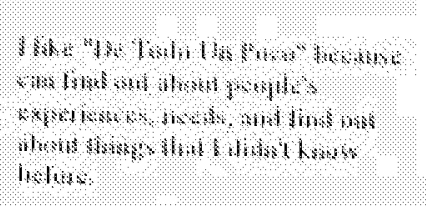 \\
\hline 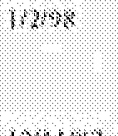 & 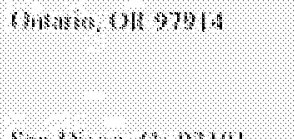 & 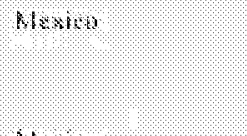 & 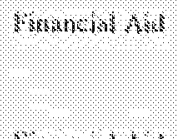 & & 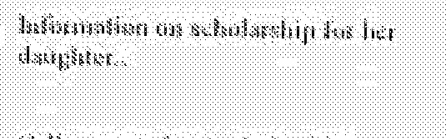 & 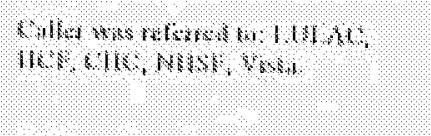 & 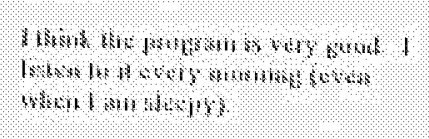 \\
\hline $1218 \%$ & 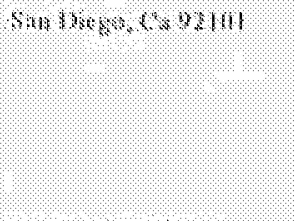 & Mtexisen & 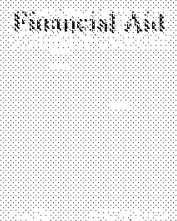 & & 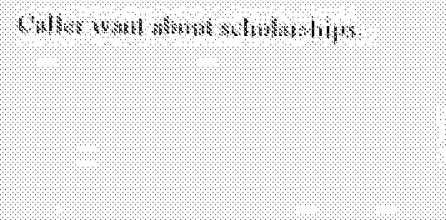 & 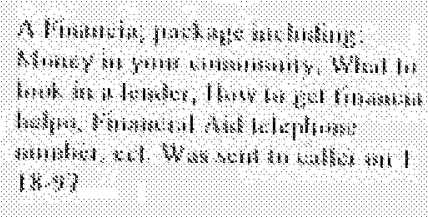 & \\
\hline $81870 \%$ & 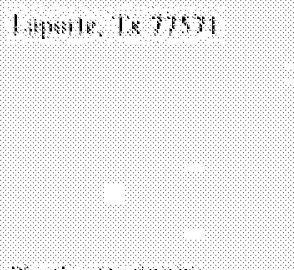 & menser: & 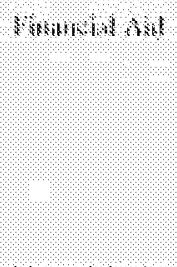 & & 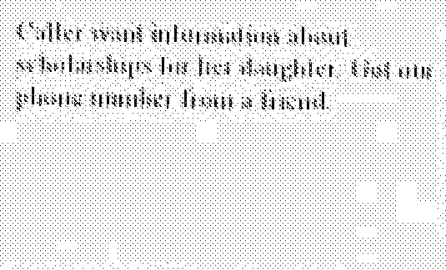 & 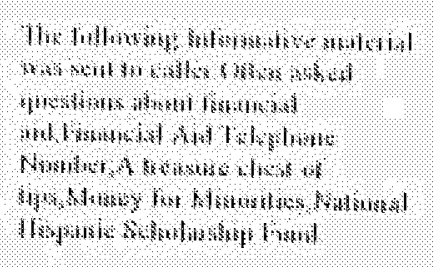 & \\
\hline $144 \%$ & $10,69,680 \% 46$ & $18 \mathrm{~m}$ & 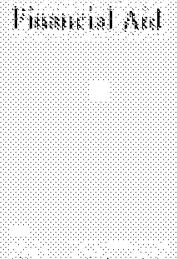 & & 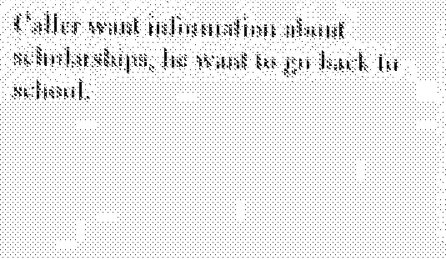 & 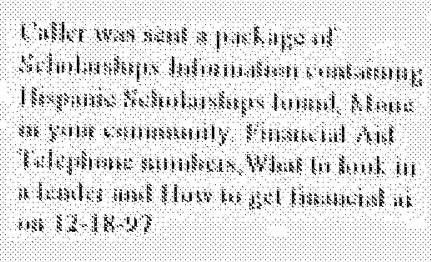 & \\
\hline $10 \% 8 \%$ & 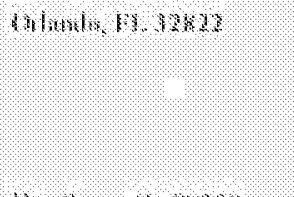 & 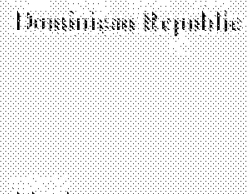 & 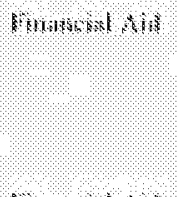 & & 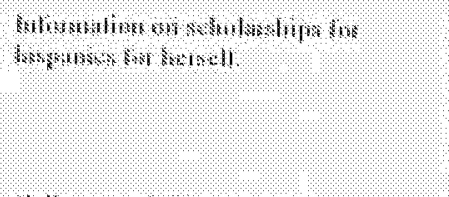 & 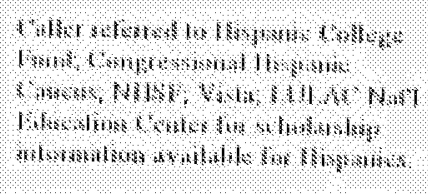 & 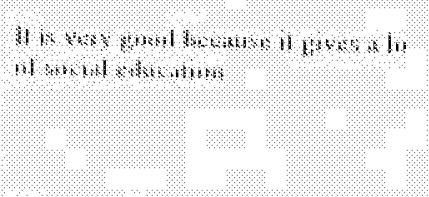 \\
\hline 10491 & 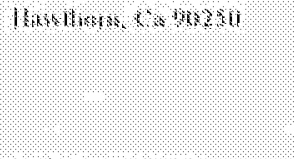 & sisenises & 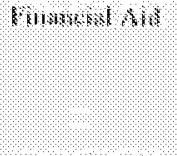 & & 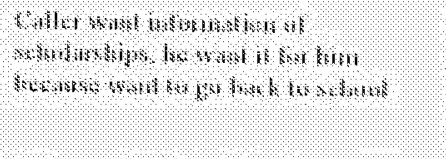 & 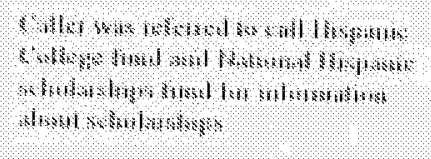 & 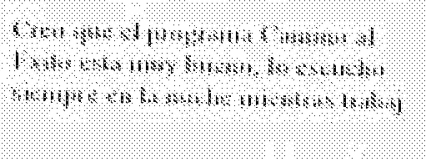 \\
\hline $12 \% 4 \%$ & $\begin{array}{l}\text { My } \\
4\end{array}$ & 111 & reminesul ans & & 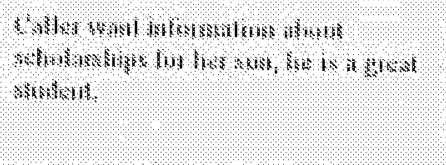 & 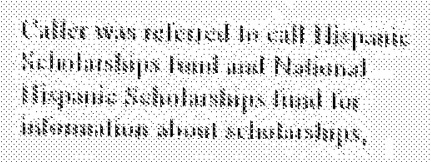 & 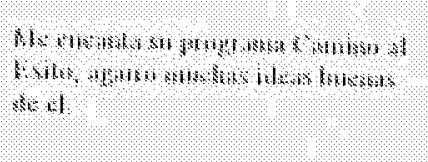 \\
\hline $12.4 m$ & 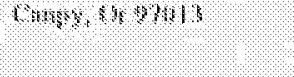 & N1003:00: & $100 \times 66 m i l s$ & & 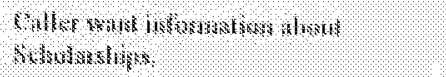 & & \\
\hline
\end{tabular}



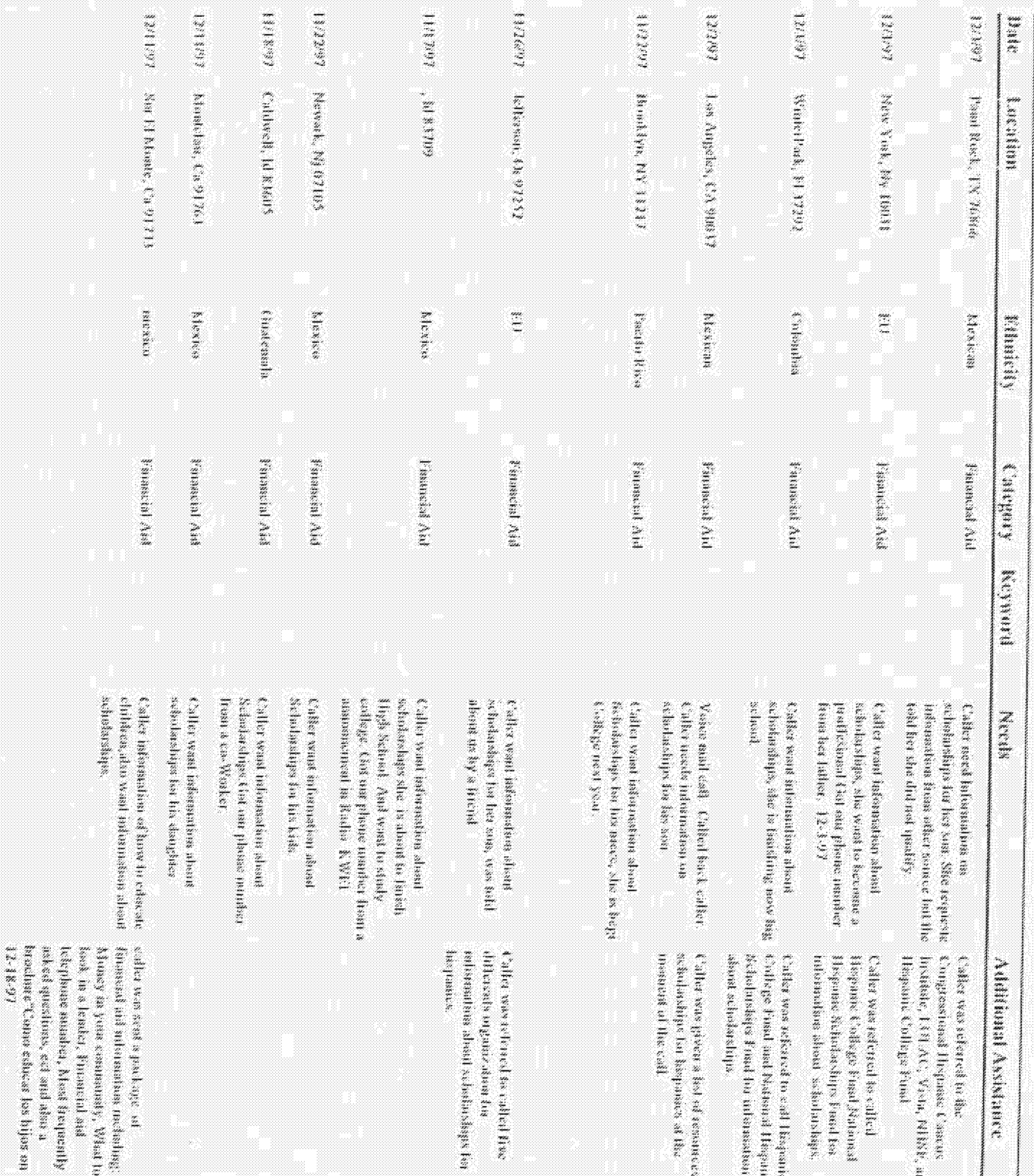

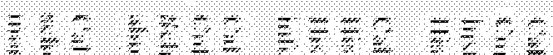

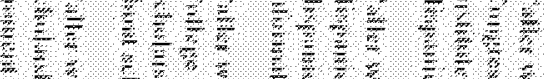

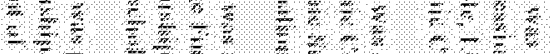

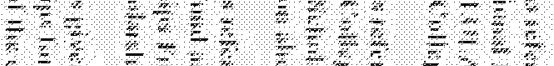

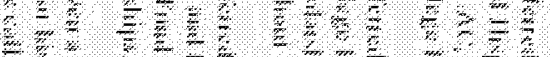

글

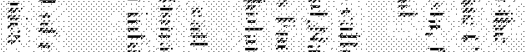

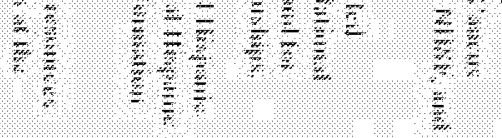

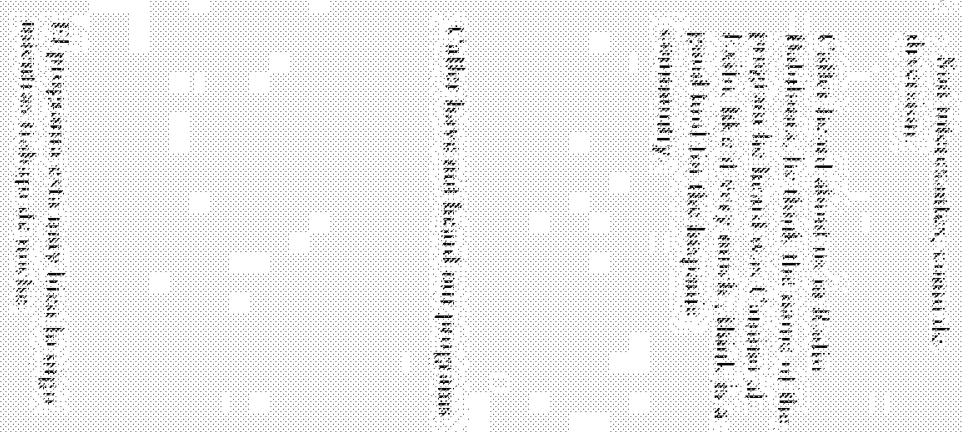




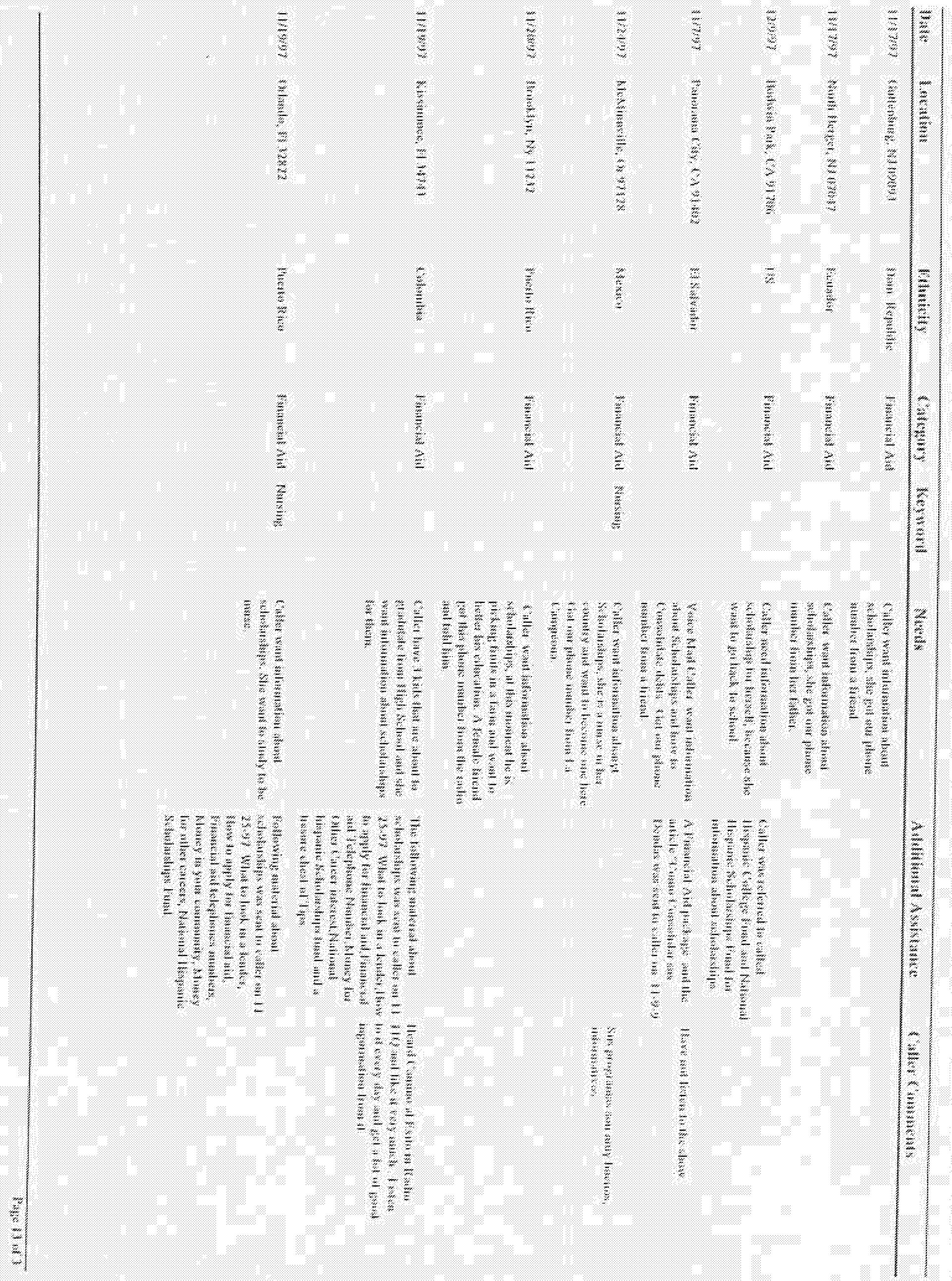




\begin{tabular}{|c|c|c|c|c|c|c|c|}
\hline 10,110 & Hormorass & 186 mesis & 6410.1918 & horaneng & Wrouls & 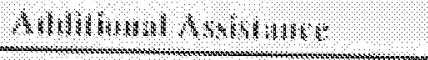 & (C) \\
\hline 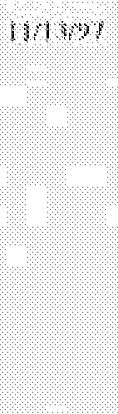 & 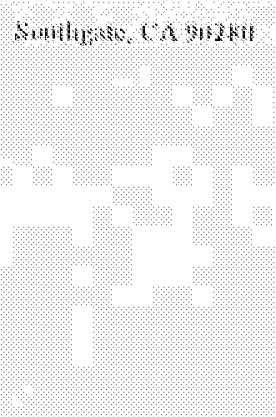 & 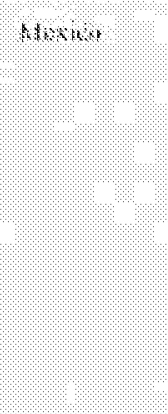 & 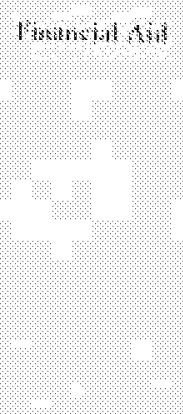 & & 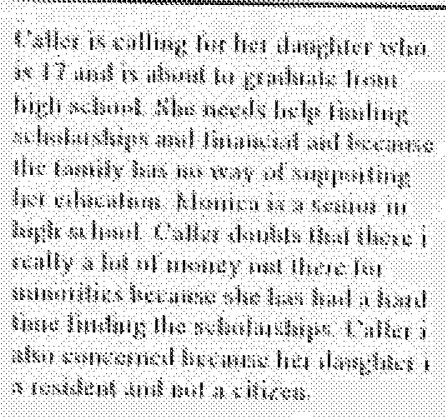 & 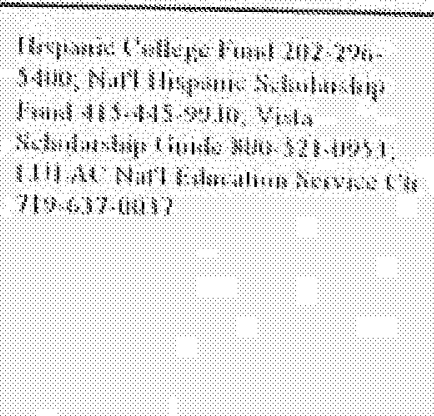 & 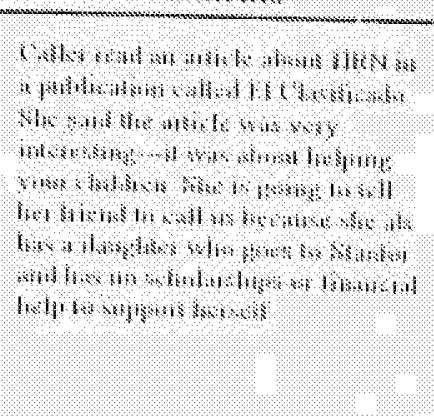 \\
\hline $131818 \%$ & 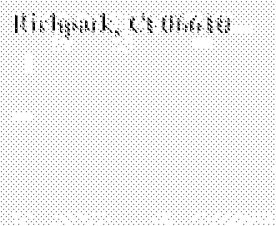 & 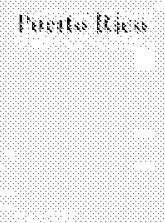 & 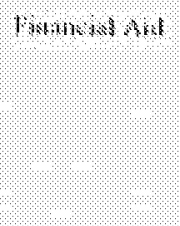 & & 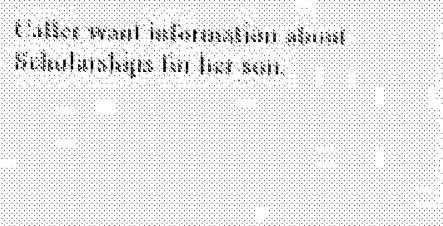 & 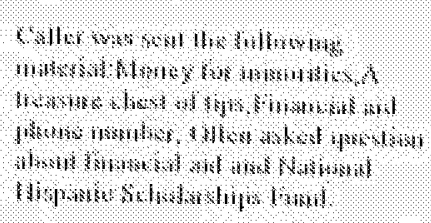 & 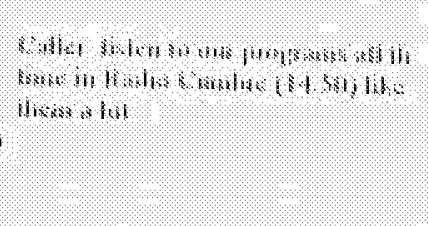 \\
\hline $11120 \%$ & 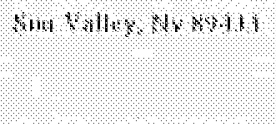 & 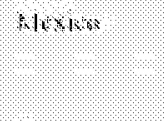 & inneserest hid & & 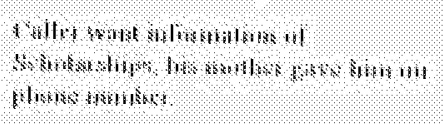 & & \\
\hline $1011, \%$ & 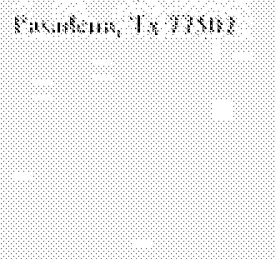 & $1 \%: 30$ & 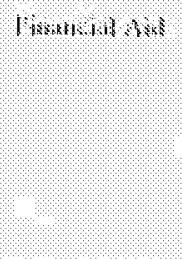 & & 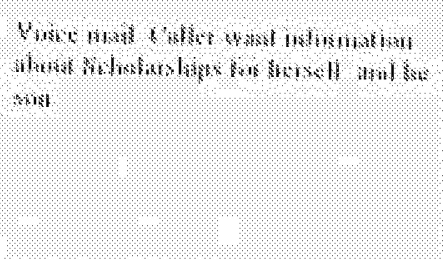 & 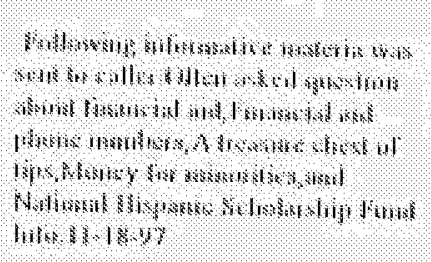 & 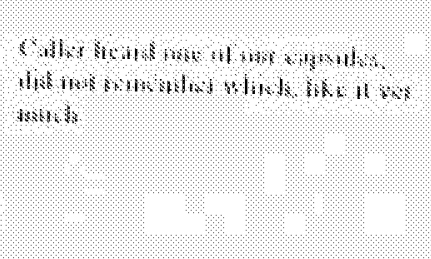 \\
\hline 148184 & 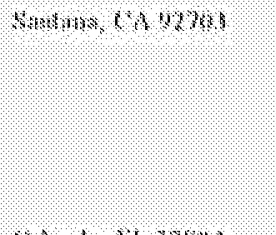 & 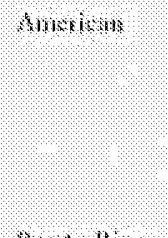 & lennimes $\mathrm{kml}$ & & 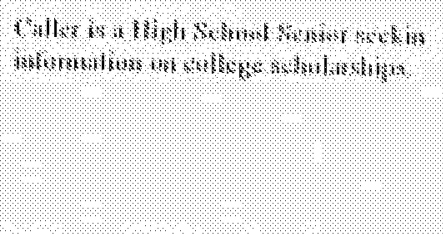 & & 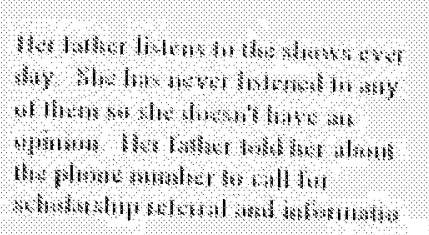 \\
\hline 81812 & 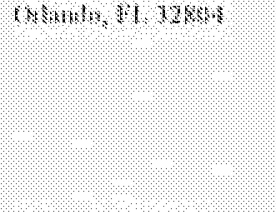 & Vormlle Ris & 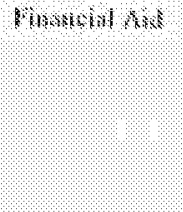 & & 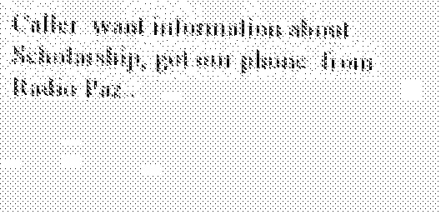 & 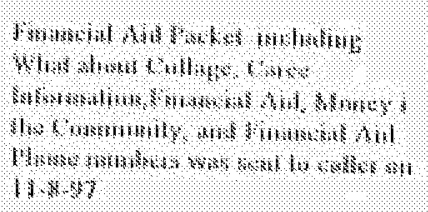 & 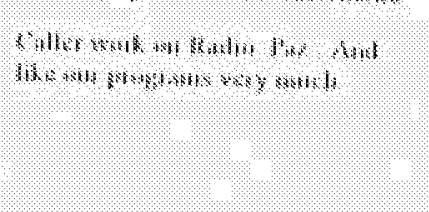 \\
\hline $102810 \%$ & $180210,18,91710$ & & 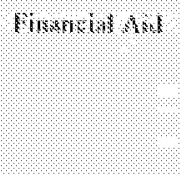 & & 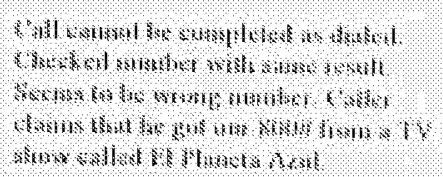 & & \\
\hline
\end{tabular}



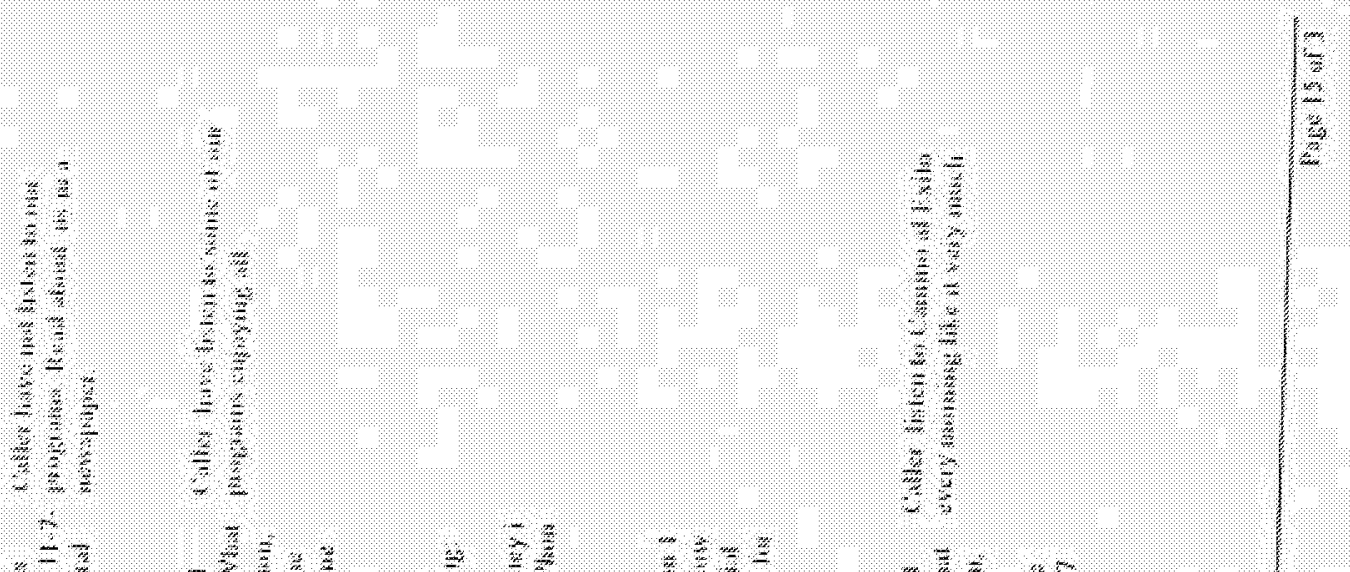

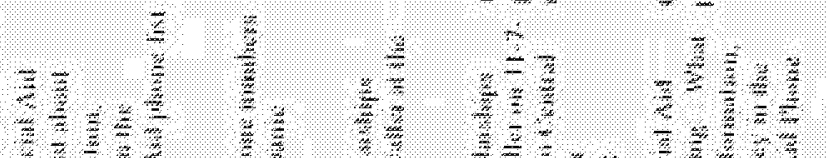

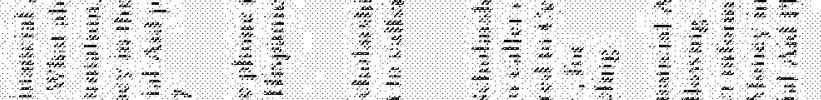

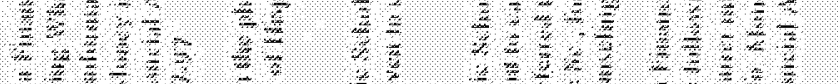

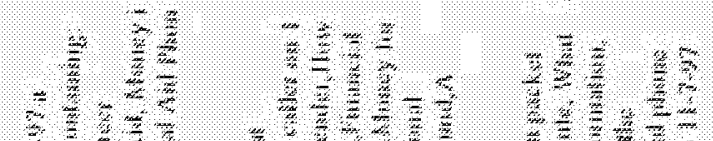

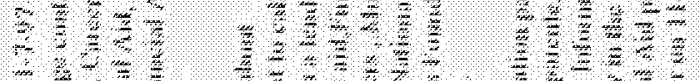

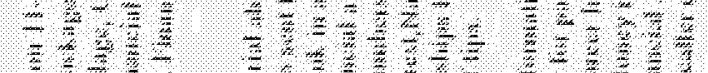

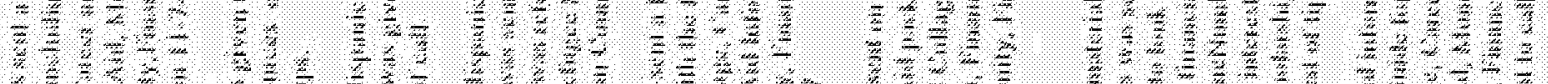

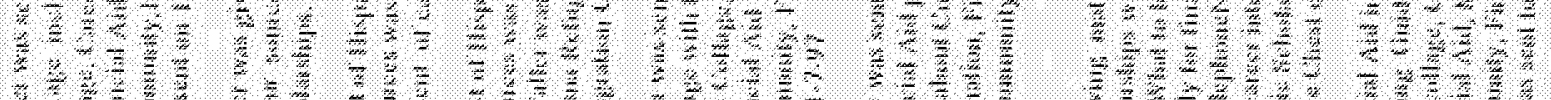

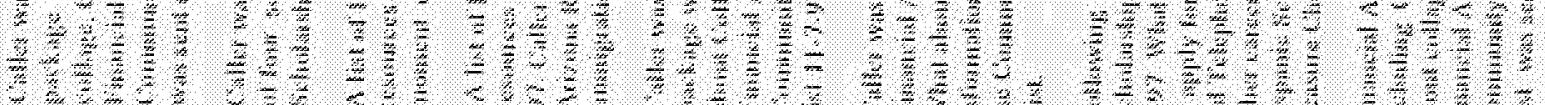

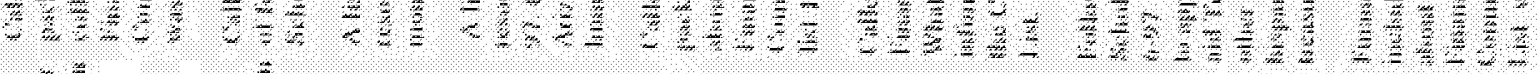

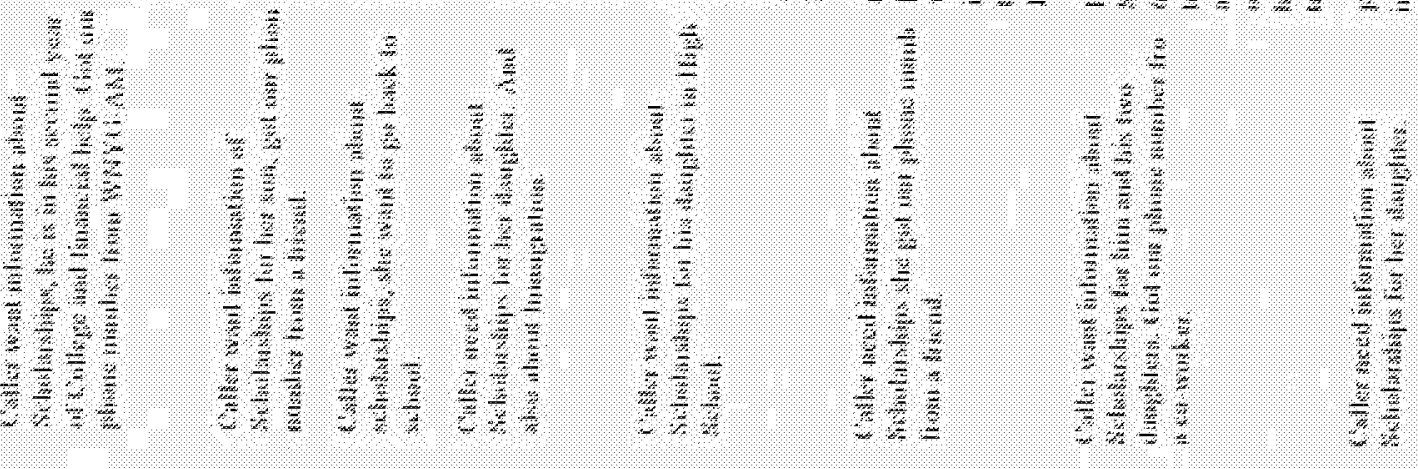

$\stackrel{=}{2}$

$\frac{2}{3}$

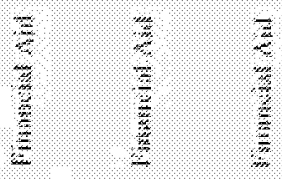

$\frac{3}{3}$

$\begin{array}{lll}\frac{3}{3} & \frac{2}{3} \\ \frac{3}{3} & \frac{3}{3}\end{array}$

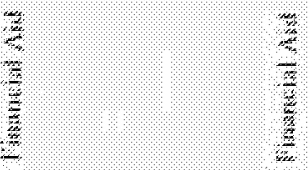

$\frac{\sqrt{2}}{\frac{\pi}{3}}$

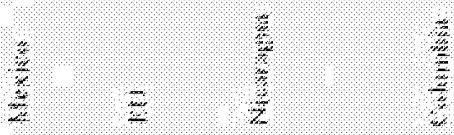

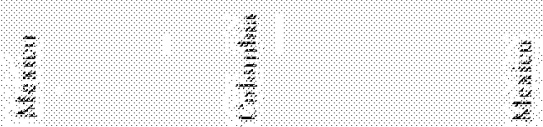

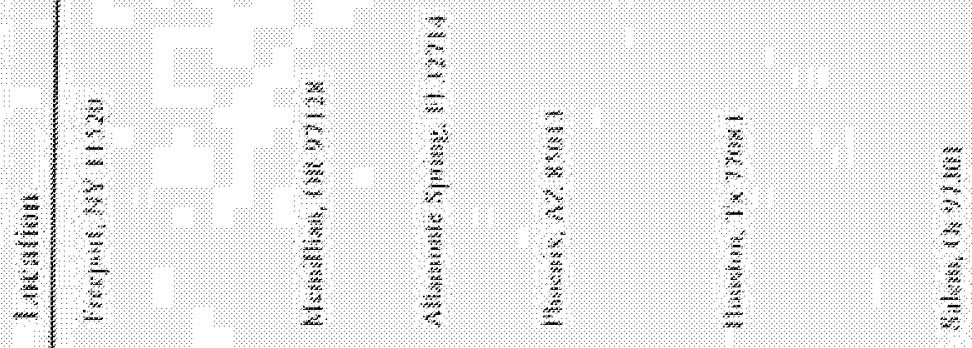

$\frac{9}{2}$
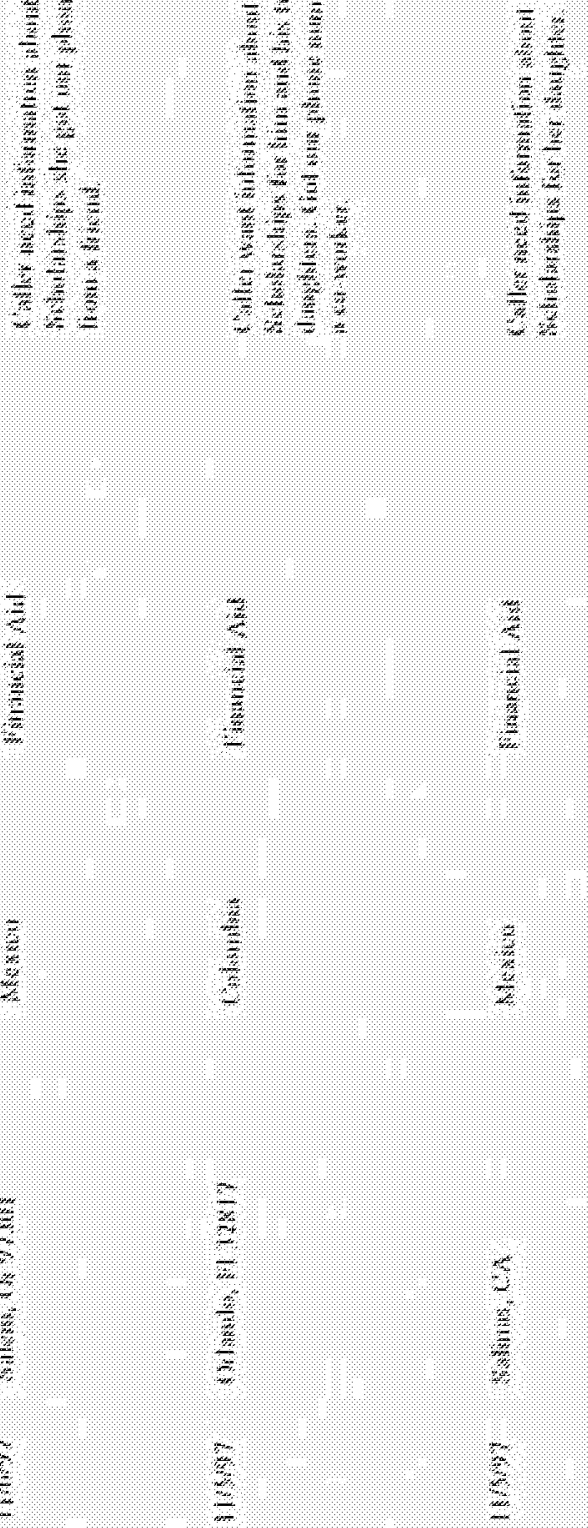

$=\sqrt{2}$

$\frac{3}{2}=\underline{3}$

$\underline{3}$

$\underline{3}$

3 


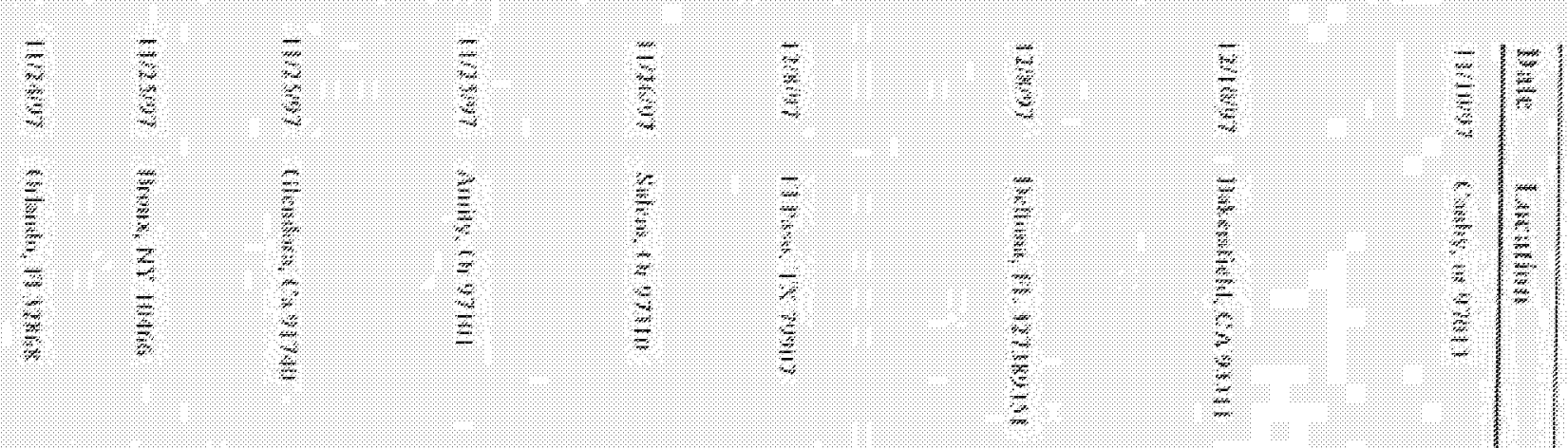

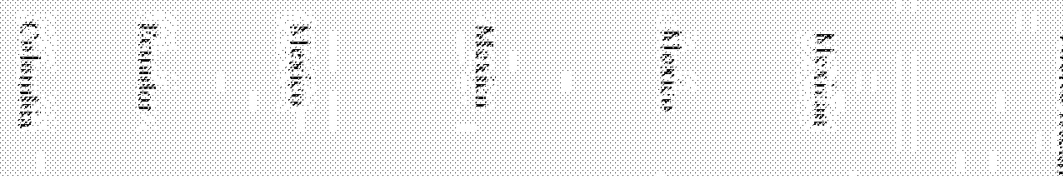

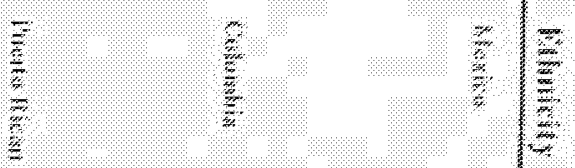

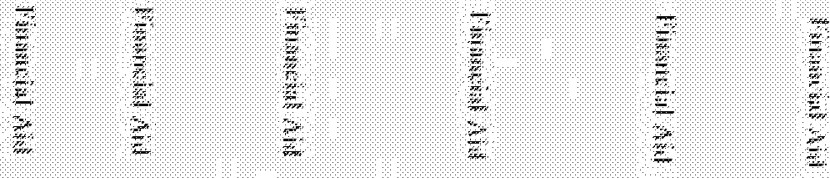

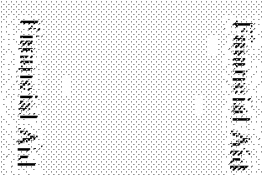

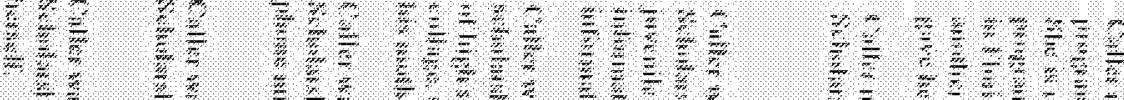

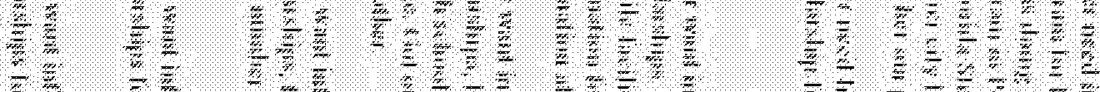

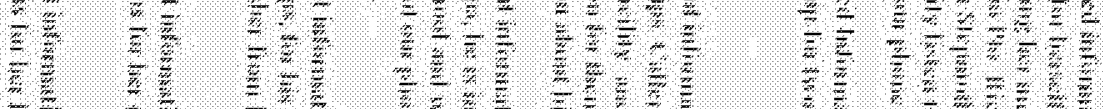

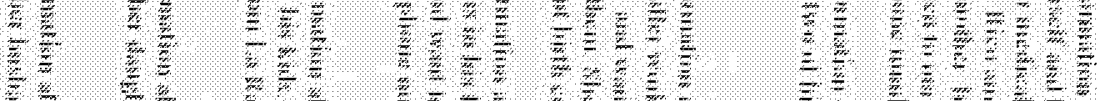

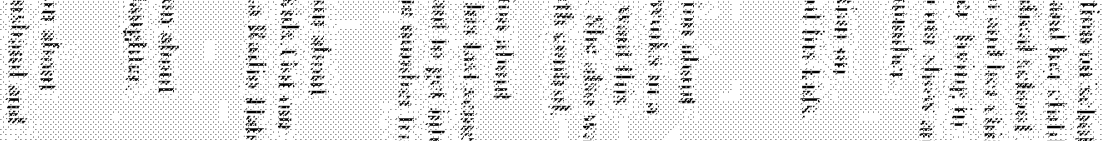

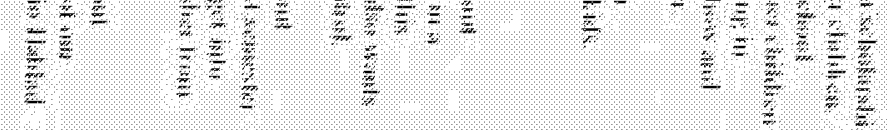

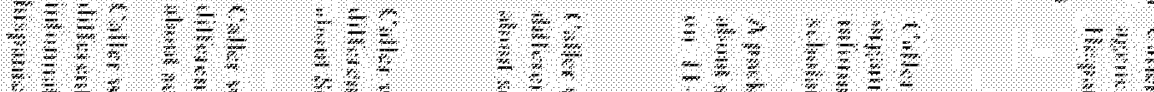

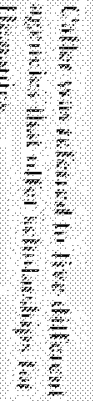

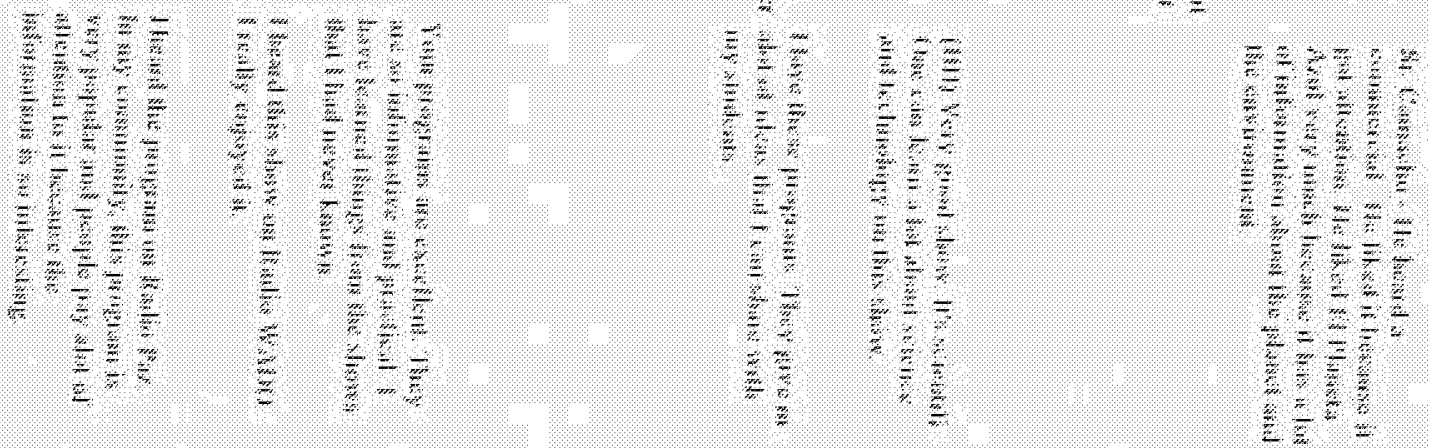

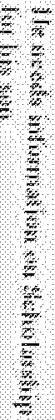

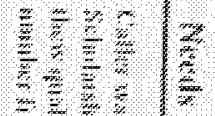

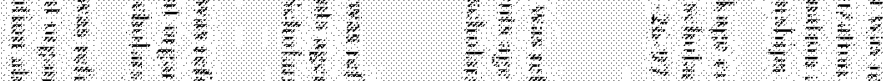

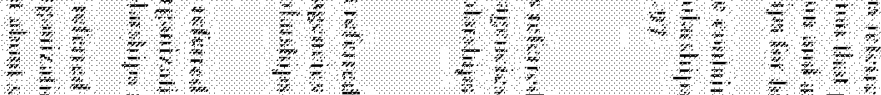

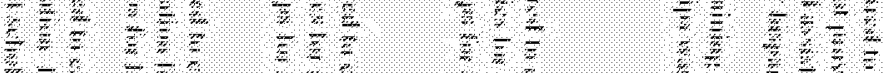

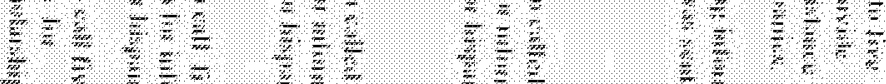

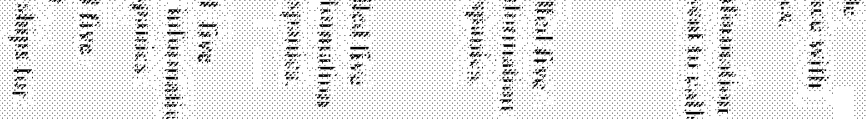

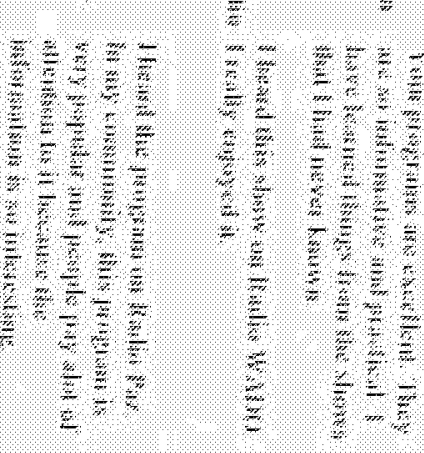

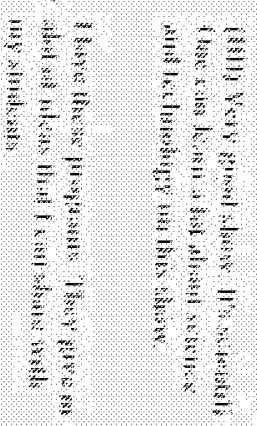

=

5
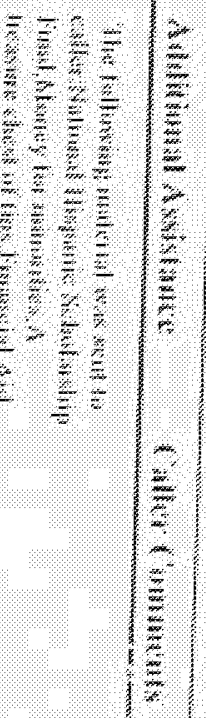


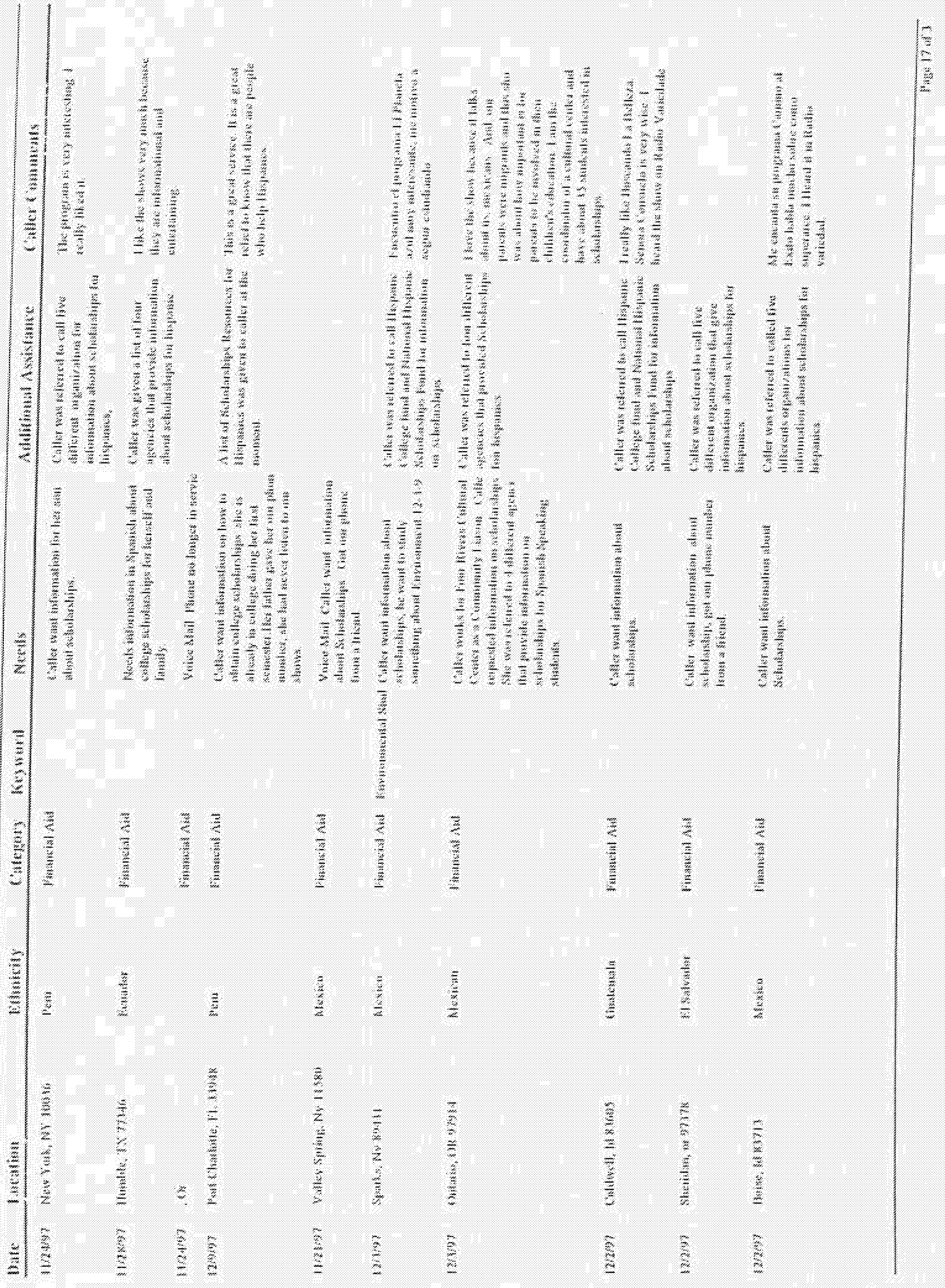




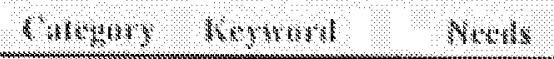

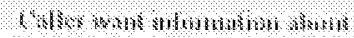

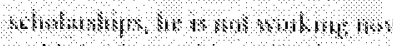

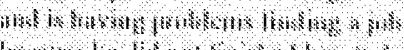

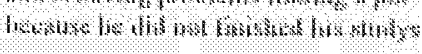

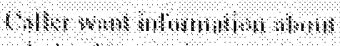

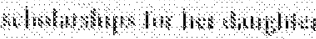

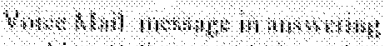

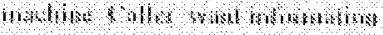

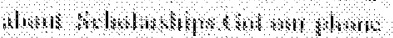

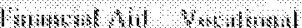

$0.100 \%, 8.18$

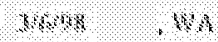

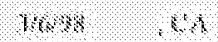

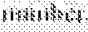

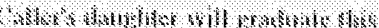

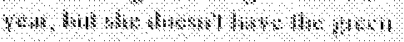

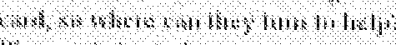

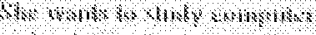

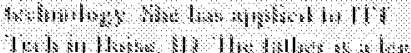

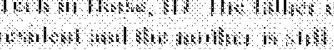

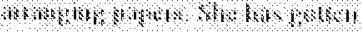

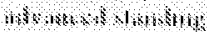

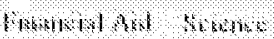

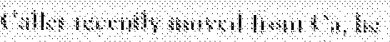

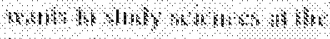

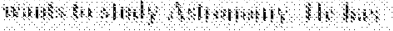

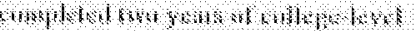
sasuly alkanks:

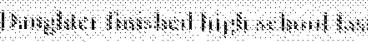

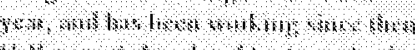

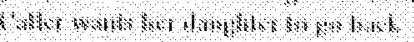

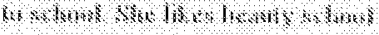

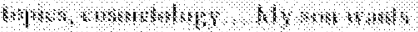

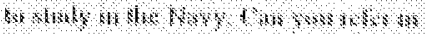

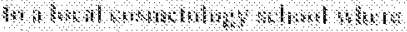

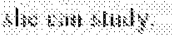

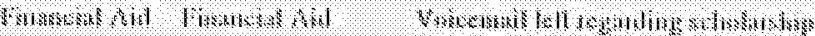
knth

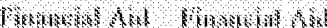

Whenels:at:

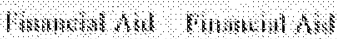

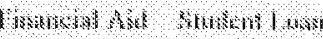

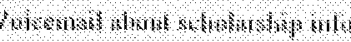

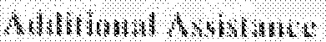

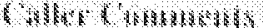

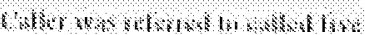

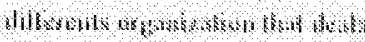

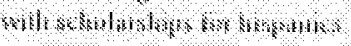

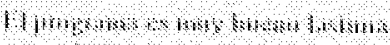

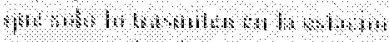

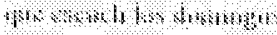

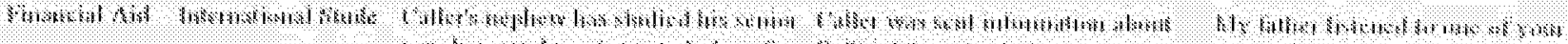

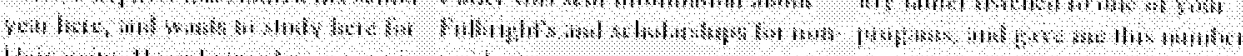

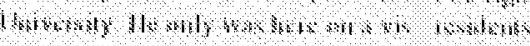

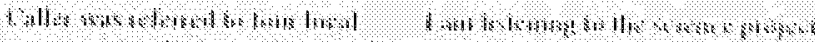

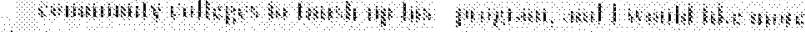

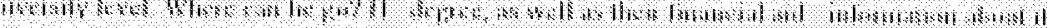
4hom

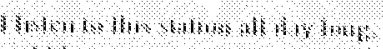

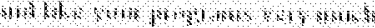

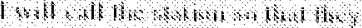

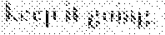

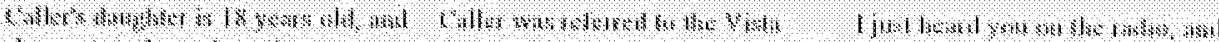

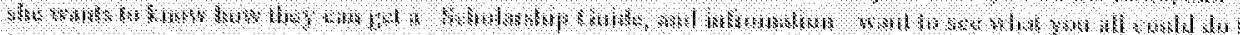

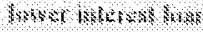

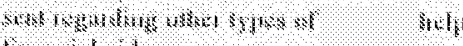

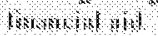




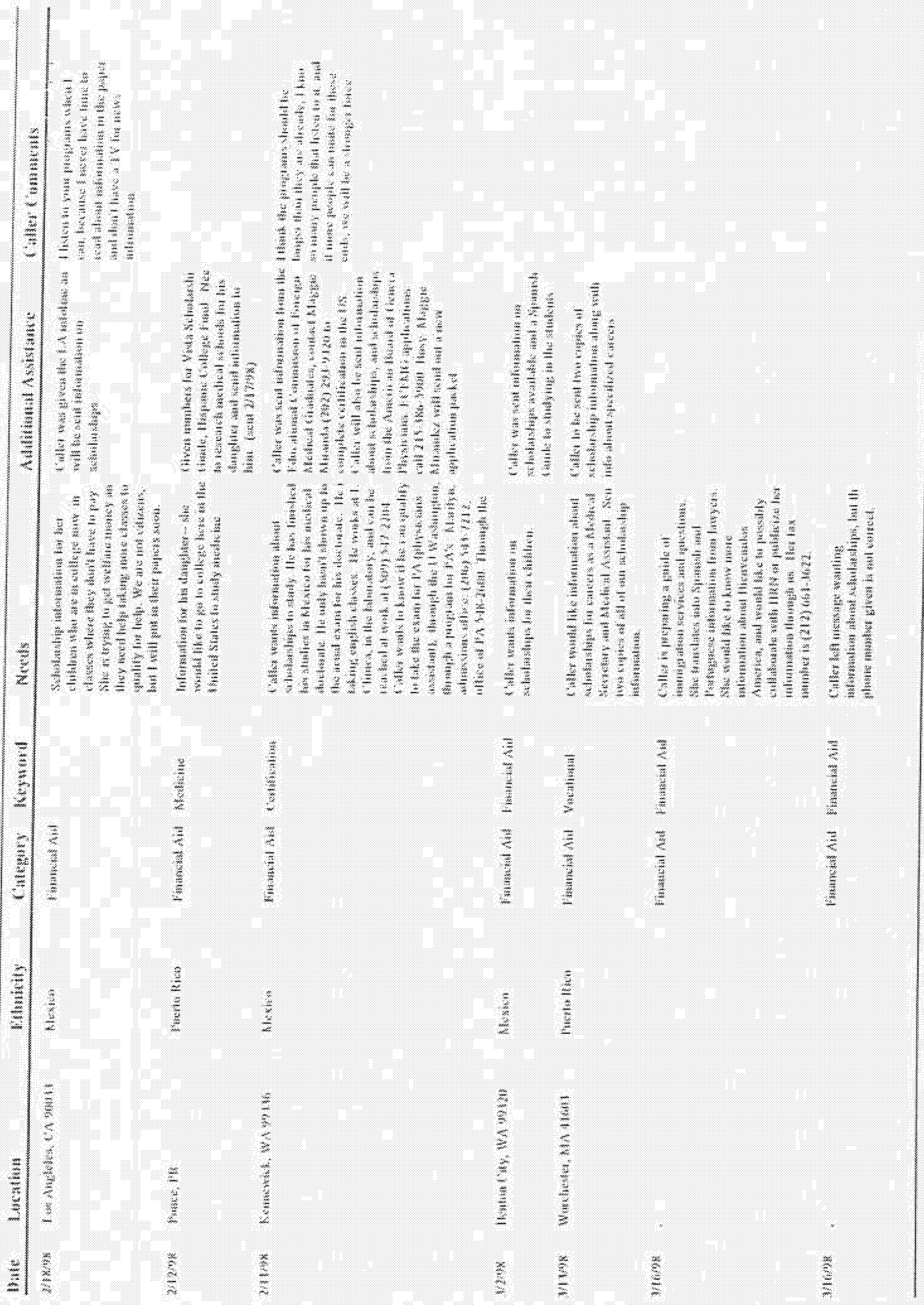




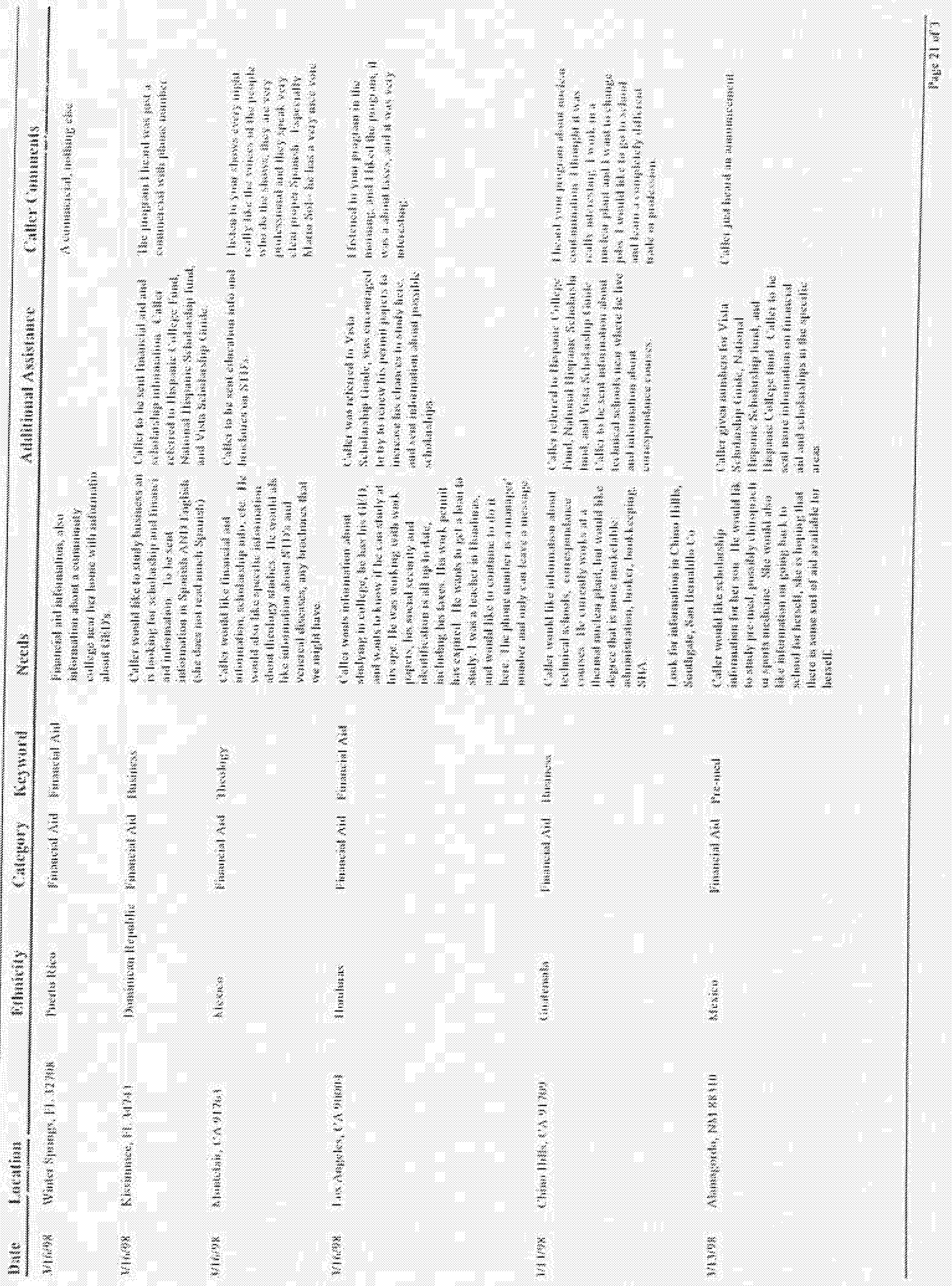




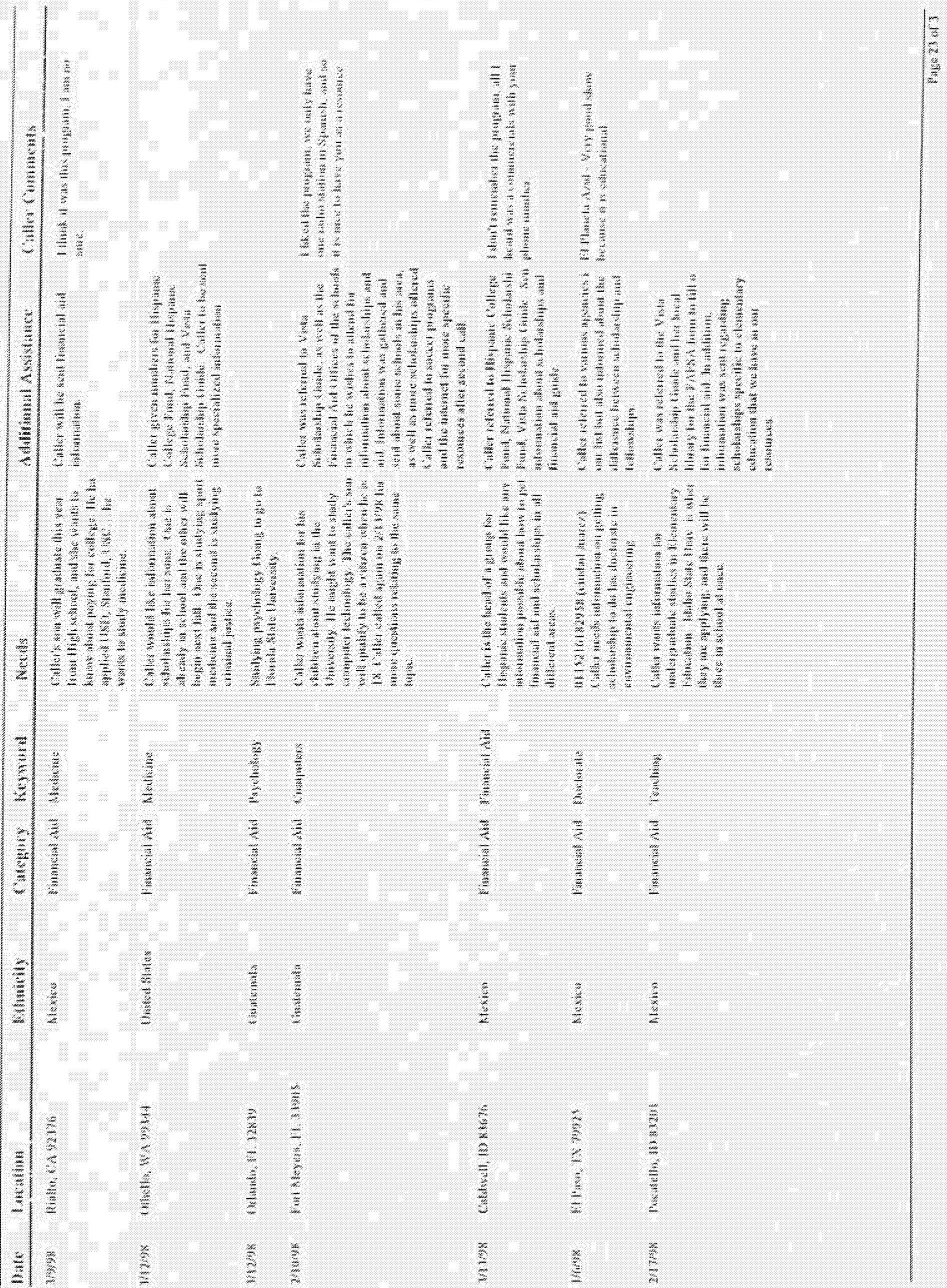




\begin{tabular}{|c|c|c|c|c|c|c|c|}
\hline 1010 & $140601616 \mathrm{~m}$ & $100 \mathrm{~min}$ & Cratuens & key uneatil & Nerils & 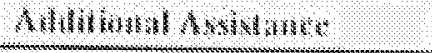 & 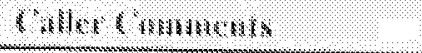 \\
\hline $14 m, m$ & 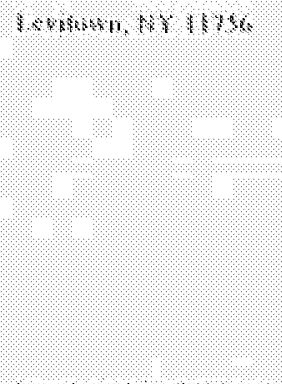 & 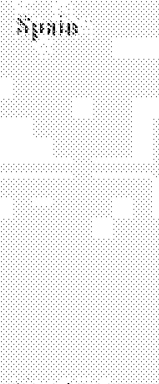 & 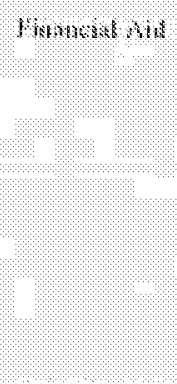 & 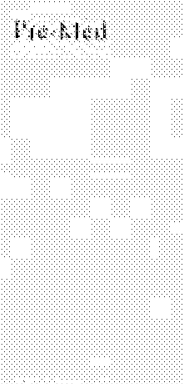 & 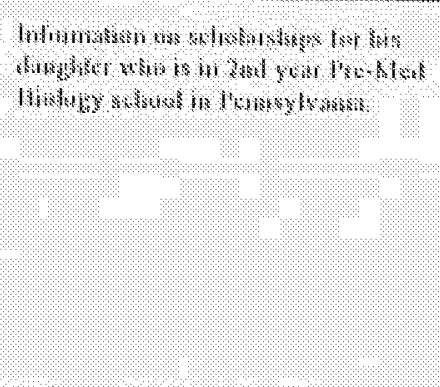 & 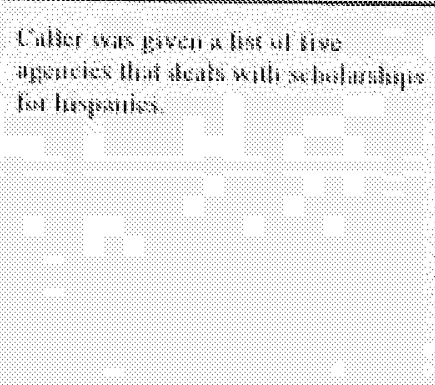 & 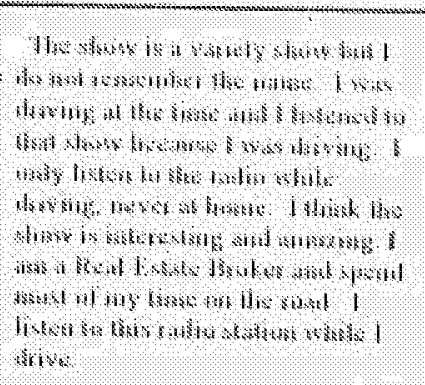 \\
\hline 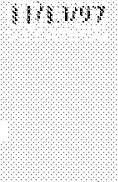 & 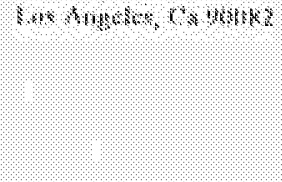 & hin: $1 \mathrm{k}: 8$ & $10 \mathrm{~s}, \mathrm{~m}+\mathrm{s} \mathrm{s}$ & 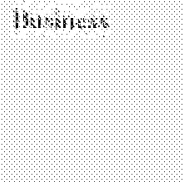 & 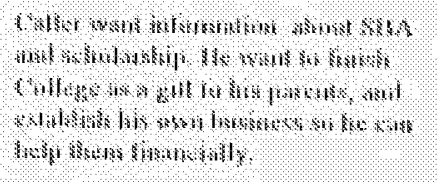 & & 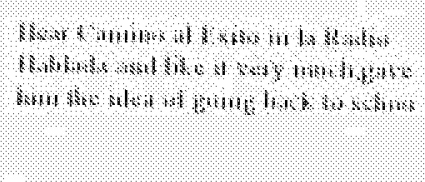 \\
\hline $811000 \mathrm{r}$ & 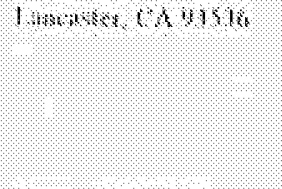 & $48,6 r m u m$ & 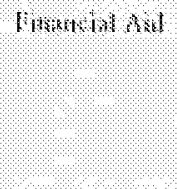 & & 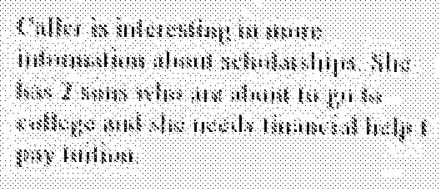 & & 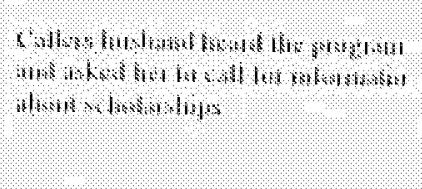 \\
\hline $300 \%$ & 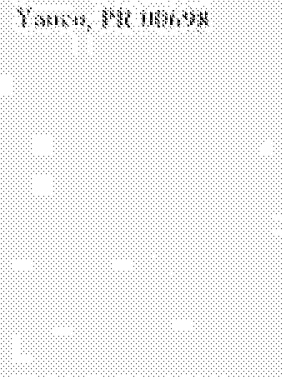 & 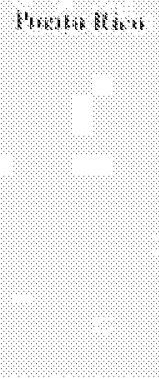 & 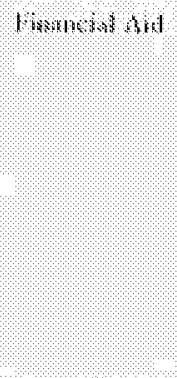 & 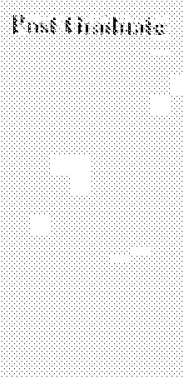 & 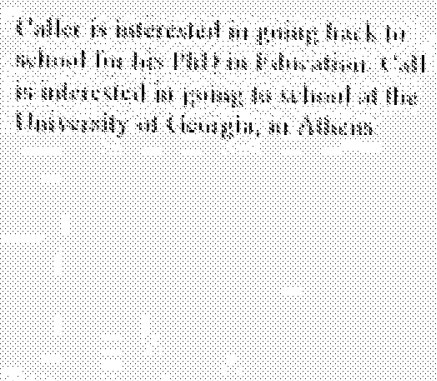 & 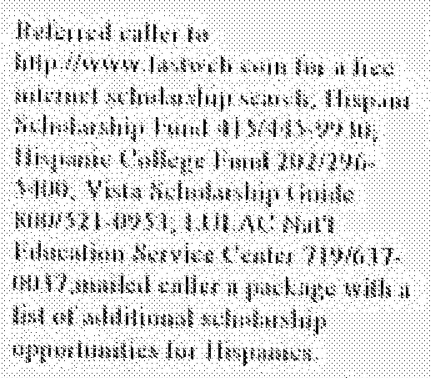 & 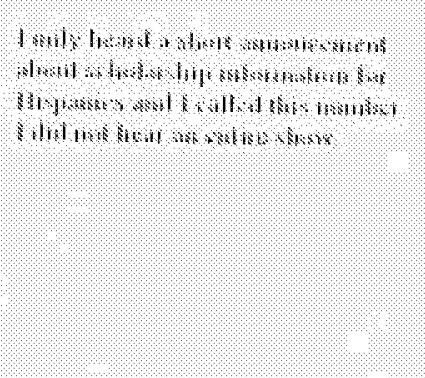 \\
\hline 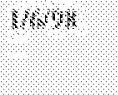 & $416010,31,7,22$ & 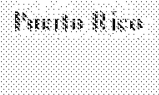 & 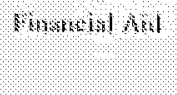 & & 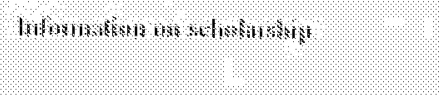 & 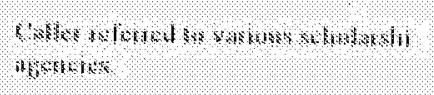 & \\
\hline 2010100 & 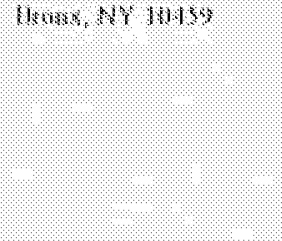 & $1 \%: \operatorname{lik} 360$ & 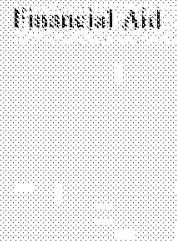 & 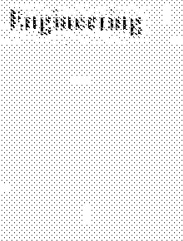 & 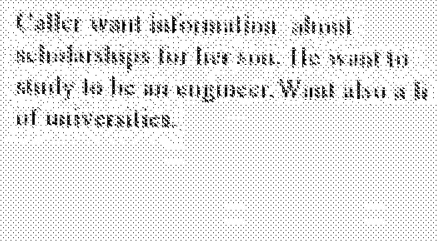 & 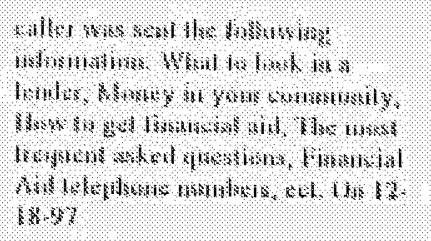 & 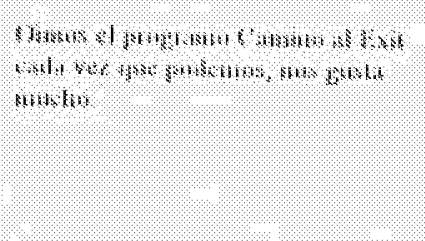 \\
\hline
\end{tabular}




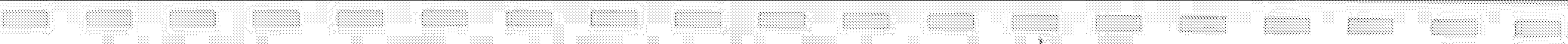

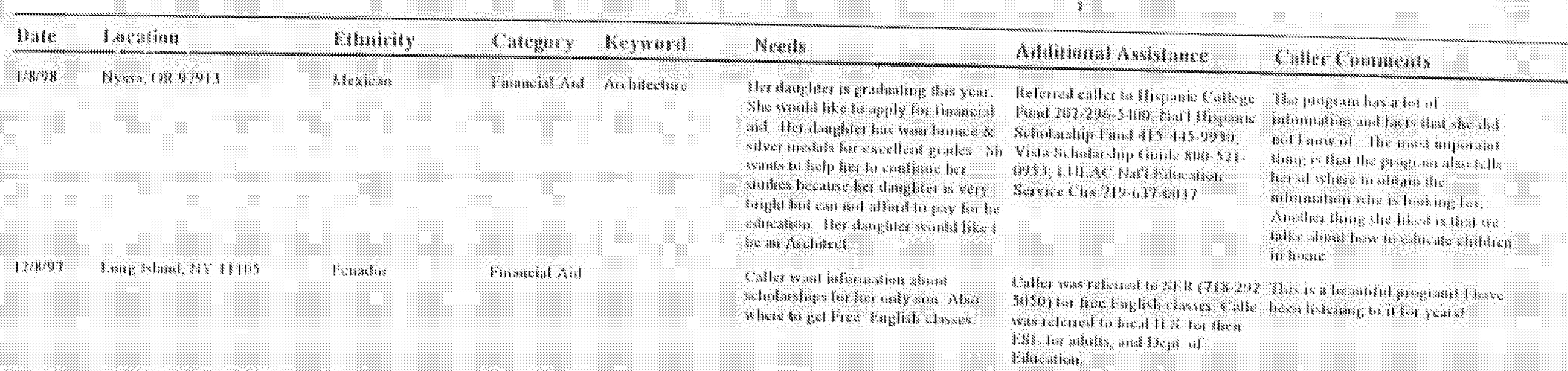
1\%०\%

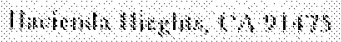

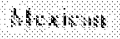
Thes:me

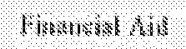

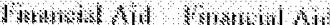

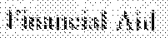

Bratromol 404

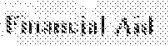

Morersibl hat

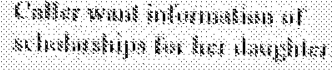

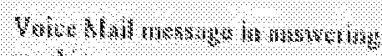
mentembor

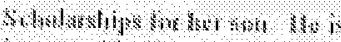

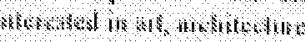

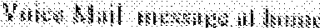

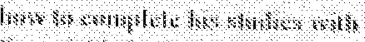

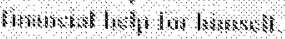

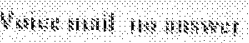

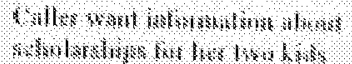

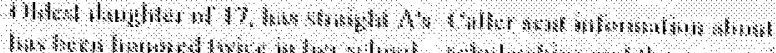

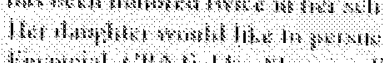

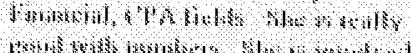

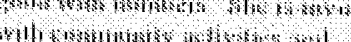

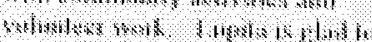

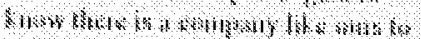

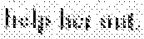

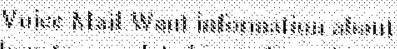

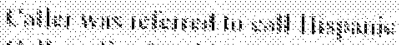

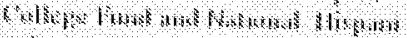

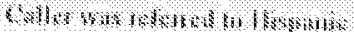

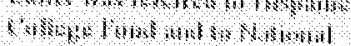

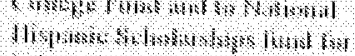
ollowitimisen

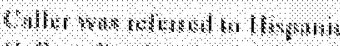

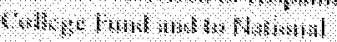

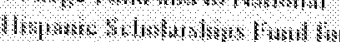

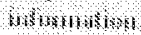

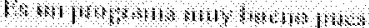

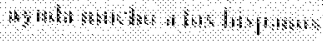

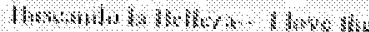

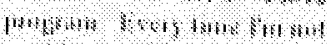

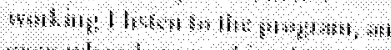

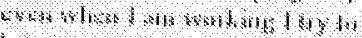

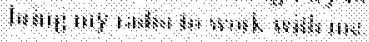

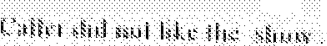

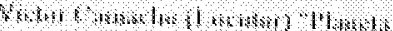 10.5

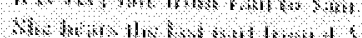

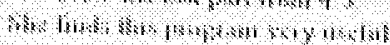

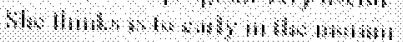 \\ mantiatis

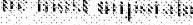

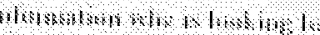

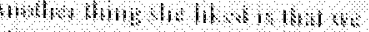

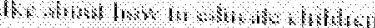

4




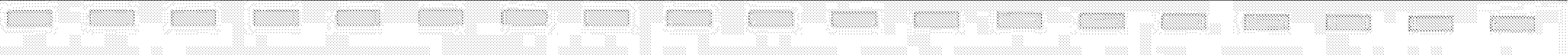

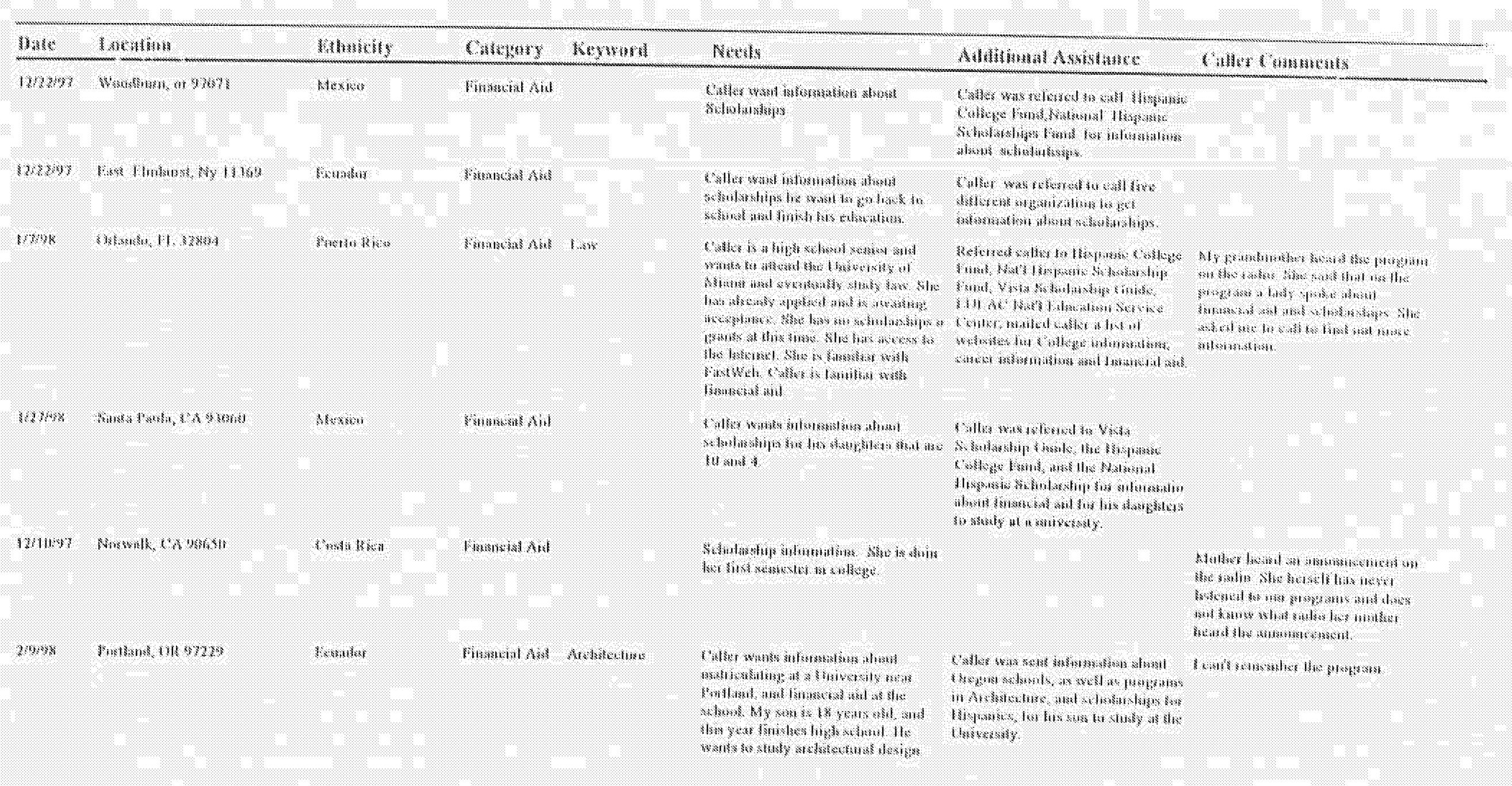




\begin{tabular}{|c|c|c|c|c|c|c|c|}
\hline Yllo & $0180610 \%$ & 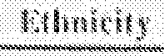 & 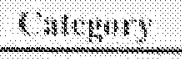 & 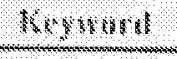 & Brer*ilk & 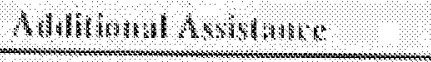 & 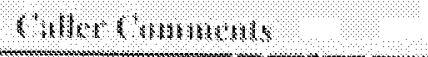 \\
\hline 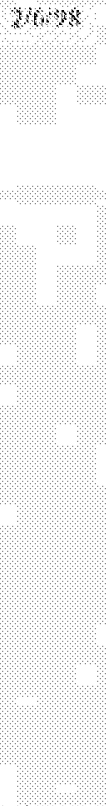 & 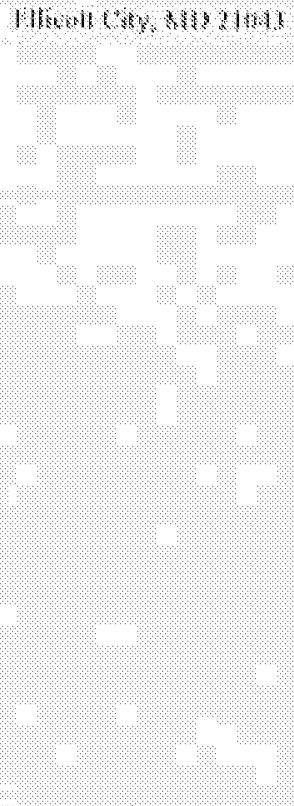 & 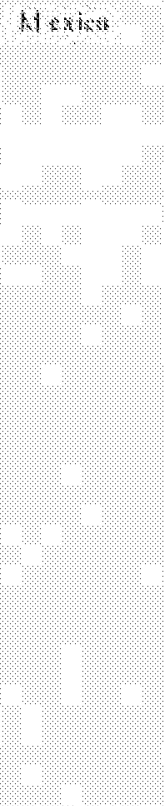 & 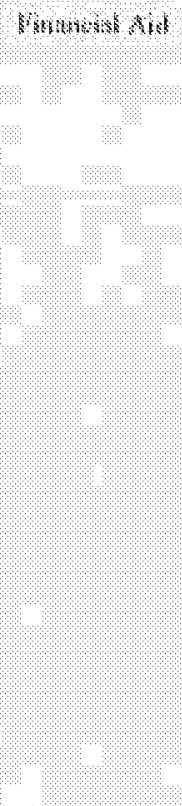 & 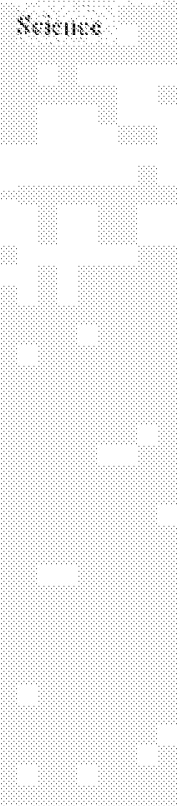 & 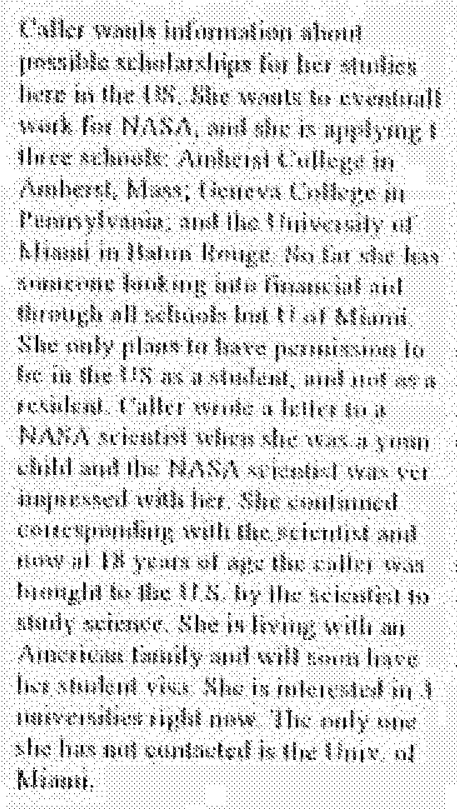 & 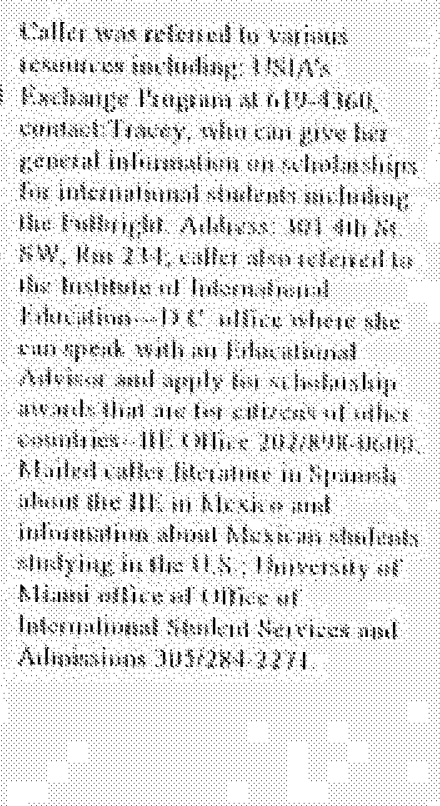 & 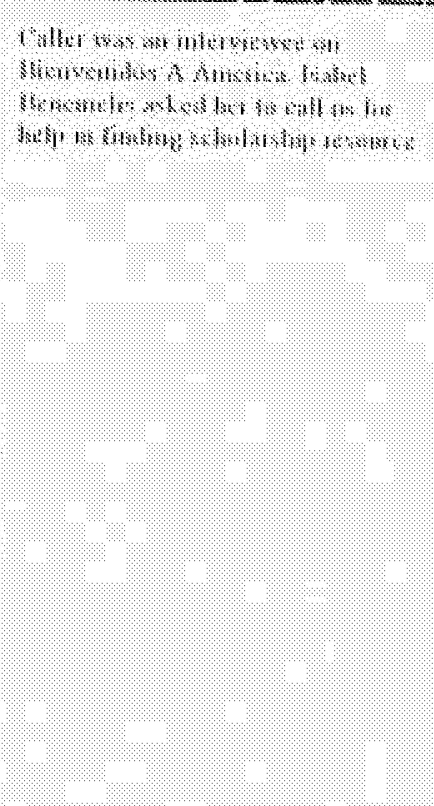 \\
\hline Psok & 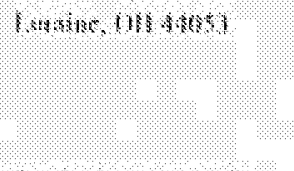 & Irmosetheris & Stmosent hail & $914 \times 51418980$ & 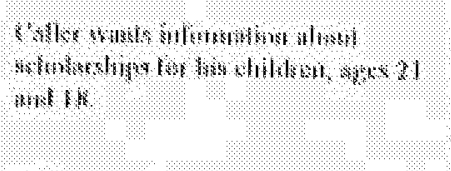 & 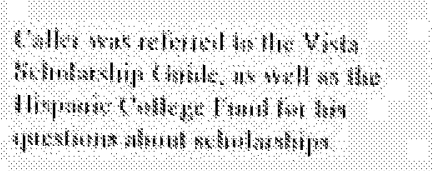 & 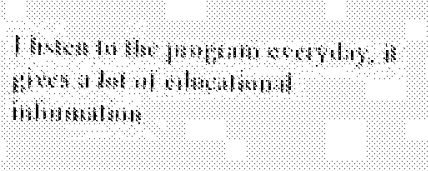 \\
\hline $210 \%$ & 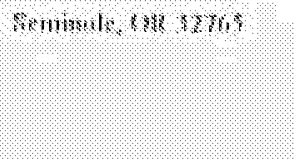 & 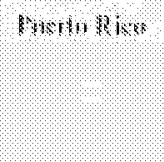 & 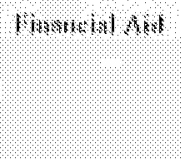 & & 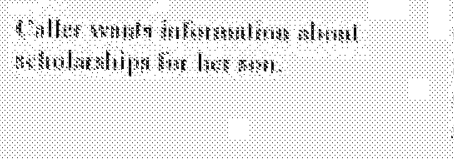 & 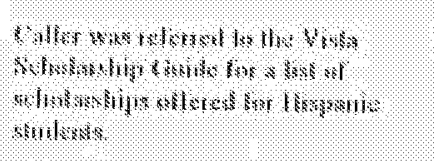 & 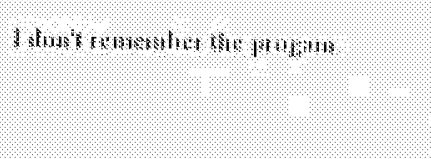 \\
\hline ४०ष & $1+14 \times 84014048$ & 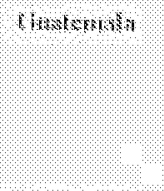 & 1804618480 & Onsmill Kasl & 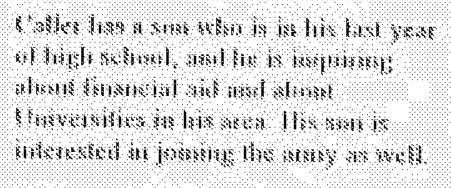 & 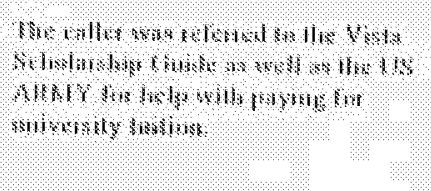 & 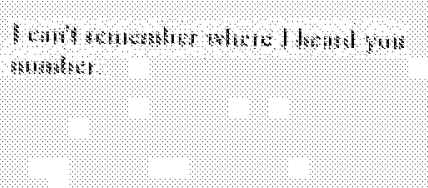 \\
\hline 3288 & 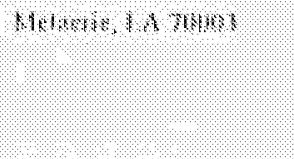 & $86 m 80 \mathrm{~mm} / \mathrm{n}$ & Ploneroll Moll & Mnisinem & 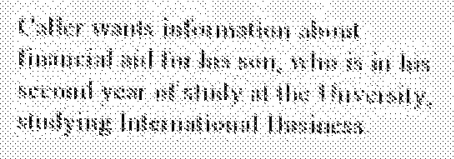 & 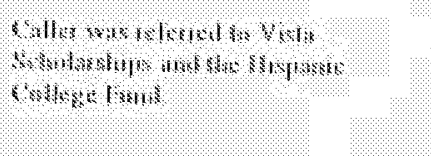 & 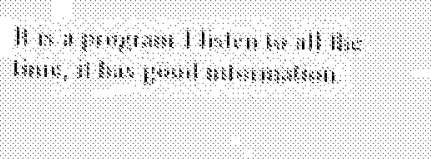 \\
\hline 2010\%8 & 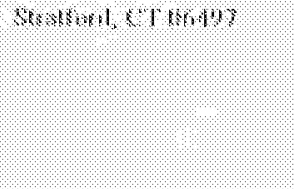 & 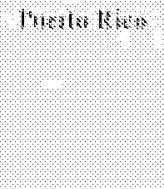 & 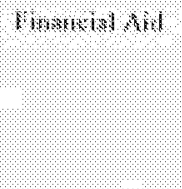 & 18 & 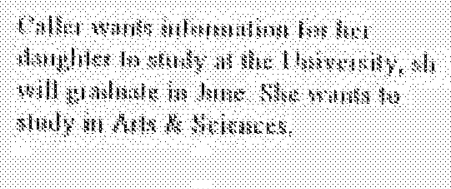 & 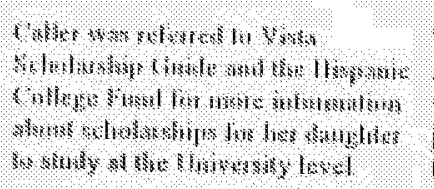 & 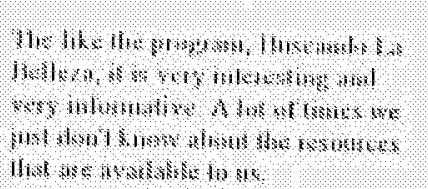 \\
\hline
\end{tabular}




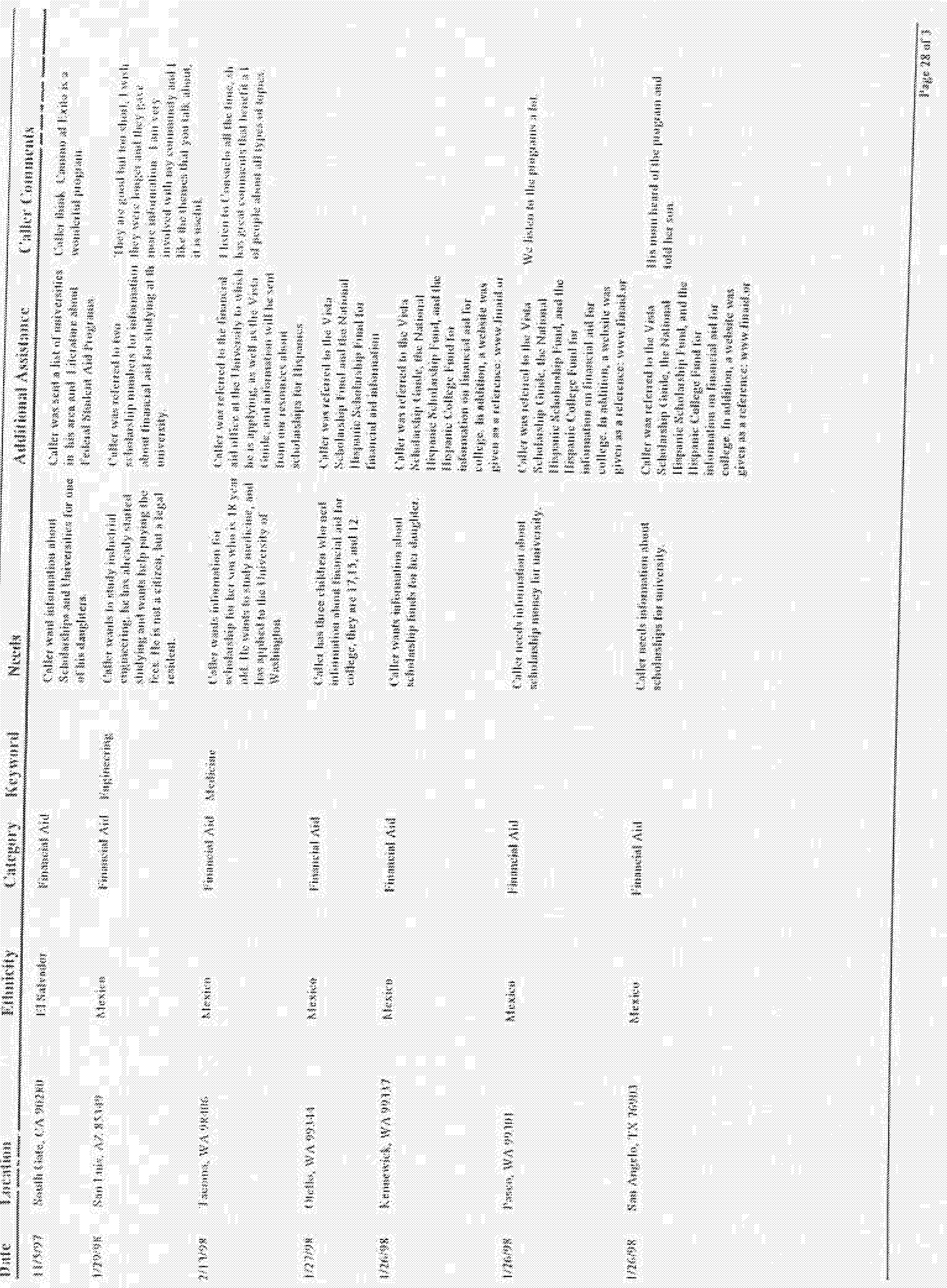




\begin{tabular}{|c|c|c|c|c|c|c|c|}
\hline $1984:$ & Uncosikes & 10641019 & (4saness) & $689400 \mathrm{~m}$ & Nee: $1:$ & 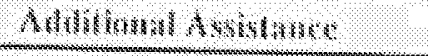 & 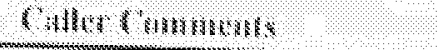 \\
\hline 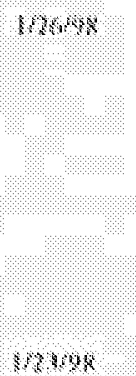 & 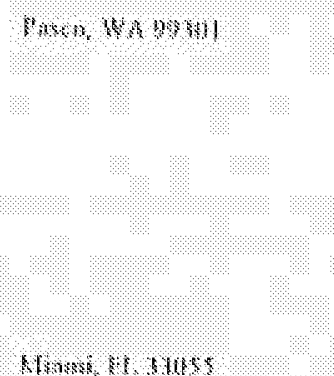 & 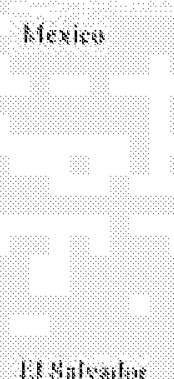 & 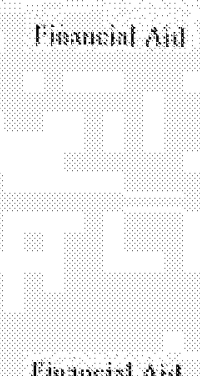 & $x^{2}$ & 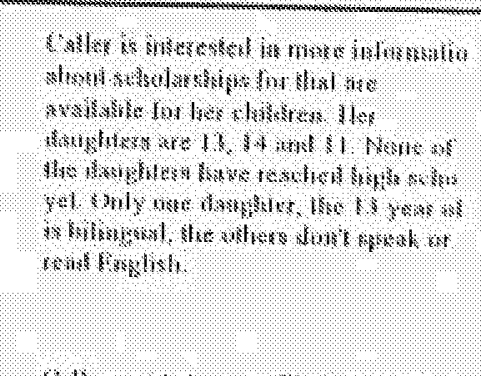 & $\mathrm{P}$ & 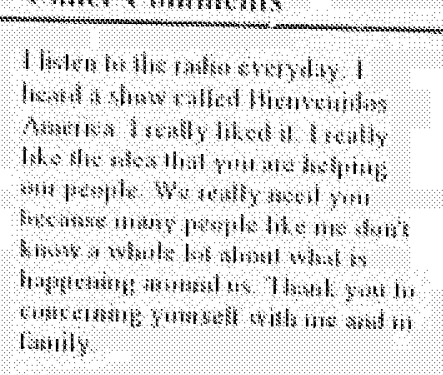 \\
\hline tis & 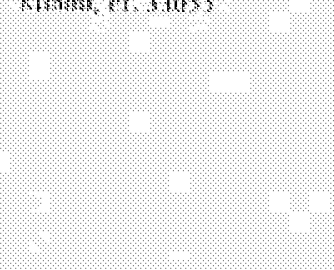 & Wenthifos: & Thromerist Mes! & Broglentary & 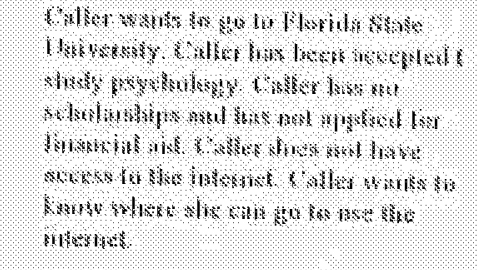 & 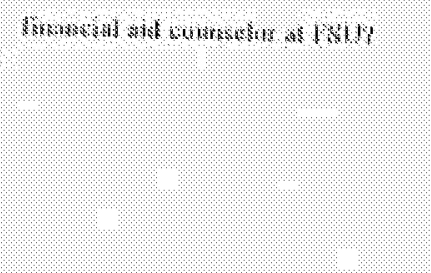 & 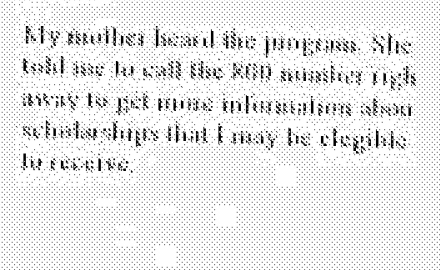 \\
\hline 196\%or & 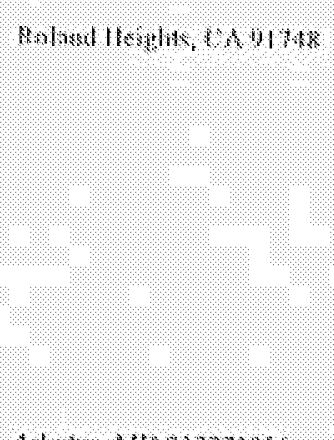 & $18 \mathrm{com}$ & 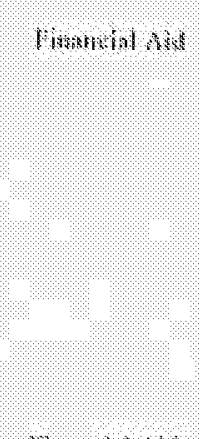 & Gersencerinks & 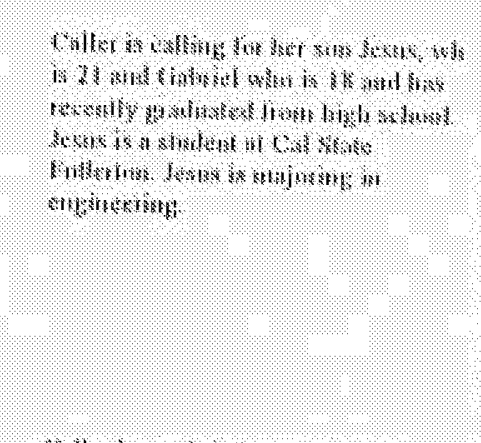 & 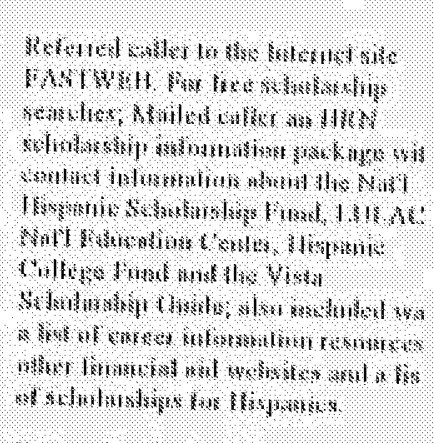 & 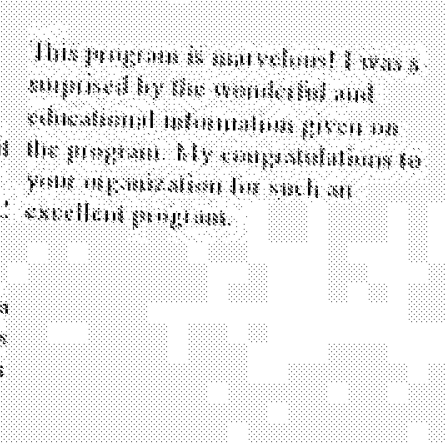 \\
\hline 101848 & 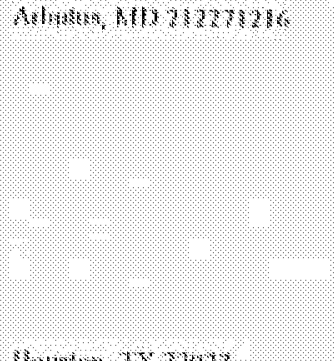 & $18 \%$ & 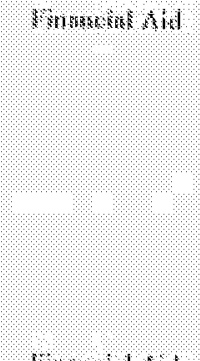 & lav & 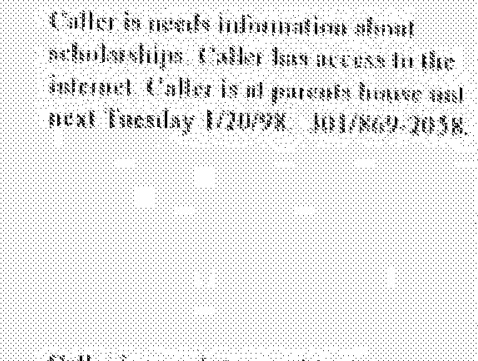 & 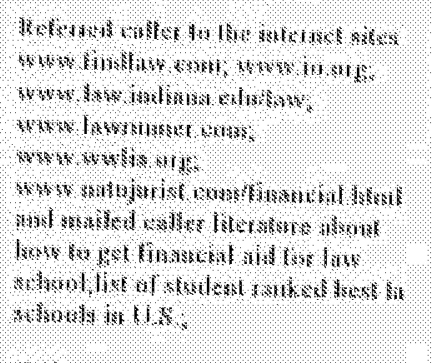 & 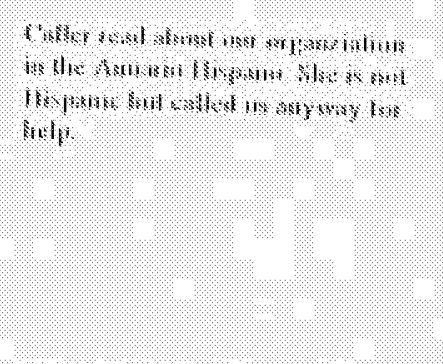 \\
\hline 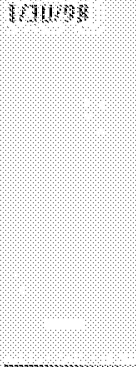 & 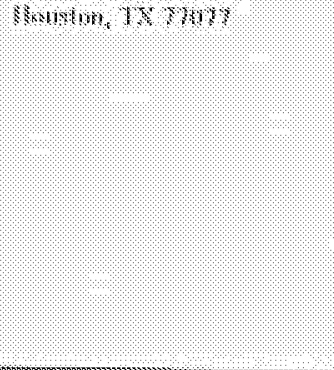 & roversenira & 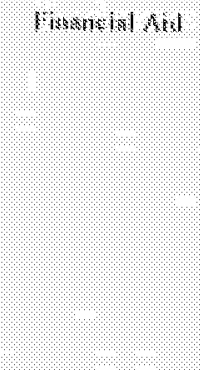 & $10 \mathrm{cll} / \mathrm{s}$ & 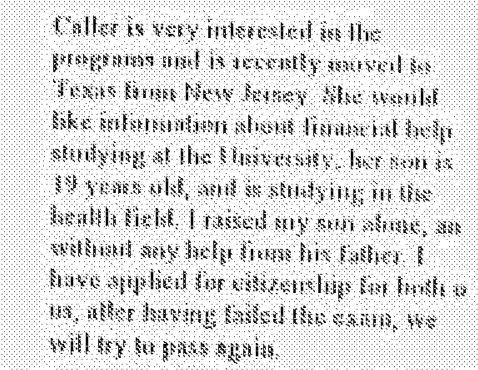 & 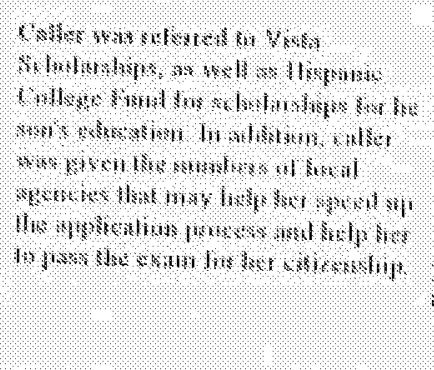 & 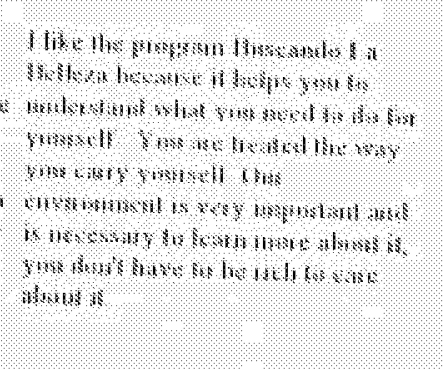 \\
\hline
\end{tabular}




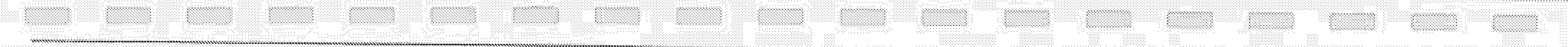

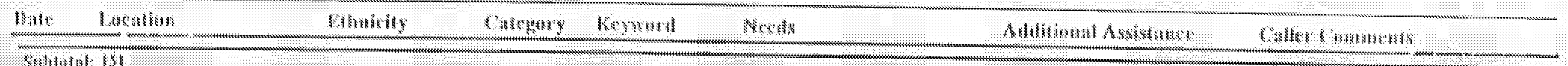

Turmoring 210 


\section{DOE-EM Outreach Report}

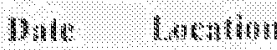

\section{WHeromon}

14tement

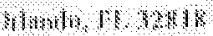

Mansosita:

Now

Forentins:

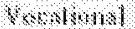

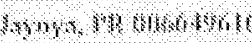

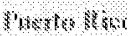

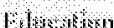

Finmil

\section{JBWE: \\ Mivesterte Ch}

$1010 \%$

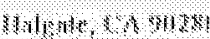

Morile:

Easseriten?

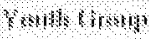

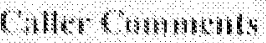

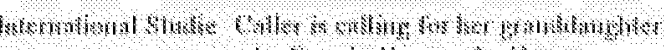

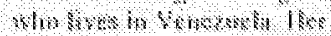

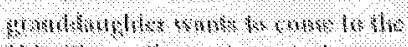

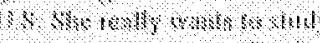

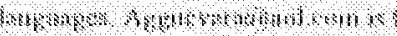

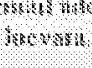

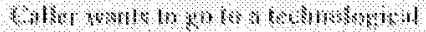

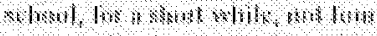

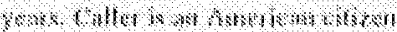

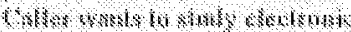

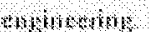

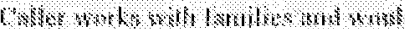
is

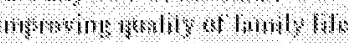

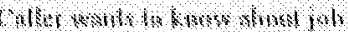

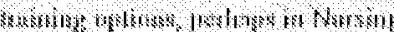

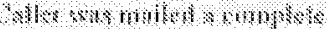

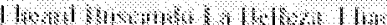
A

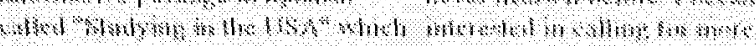

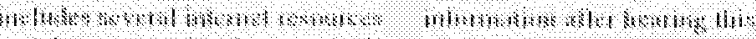

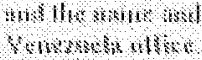

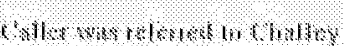

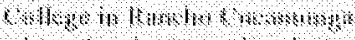

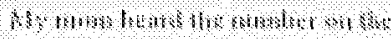

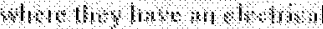

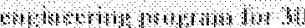
wask,, 27

Coiler

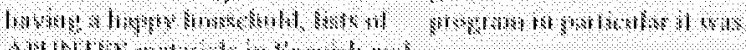

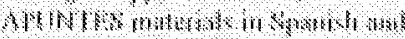

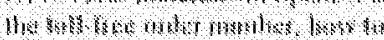

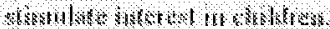

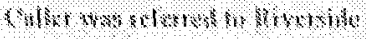

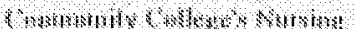

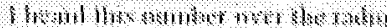

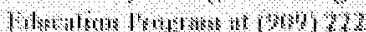
3,405

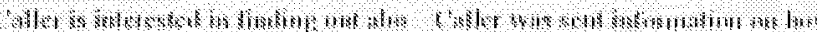

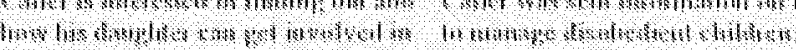

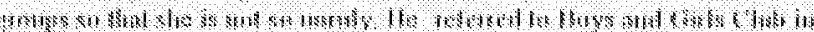

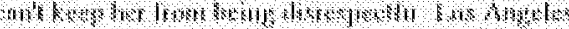

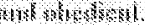

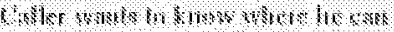

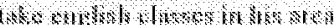

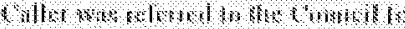

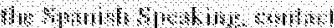

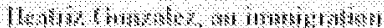

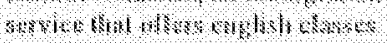

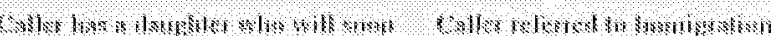

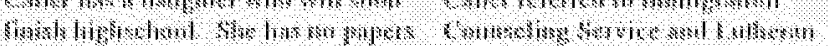

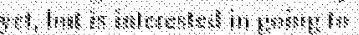

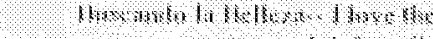

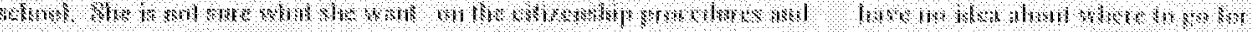

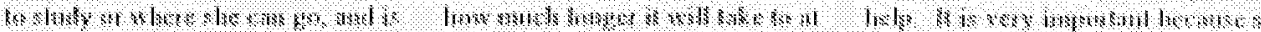

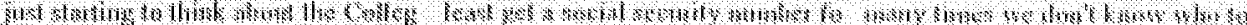
16.0.

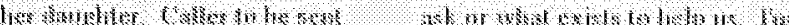

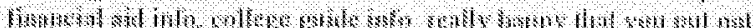

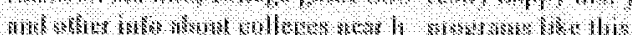



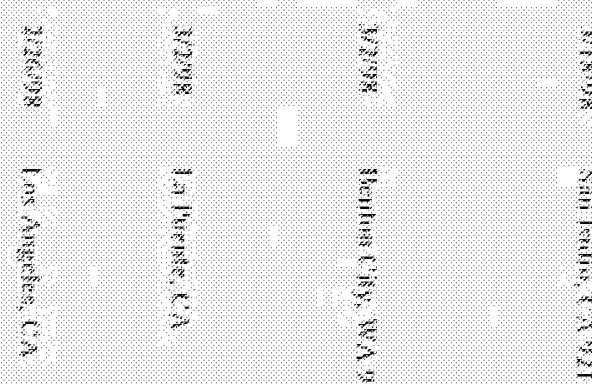

喜

范

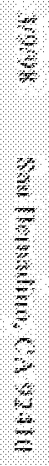

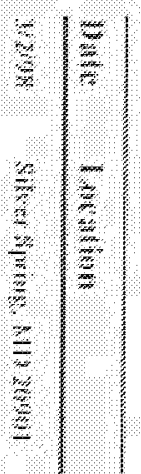

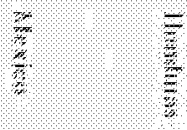

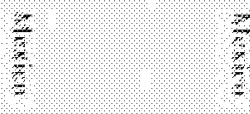

$\frac{5}{8}$

$\mathrm{B}$
$\mathrm{b}$
$\mathrm{b}$

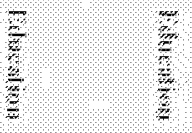

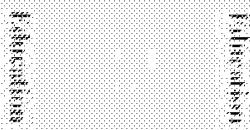

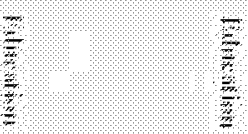

$\frac{3}{3}$

翌

$\frac{10}{3}=$

z

$\frac{1}{3}$

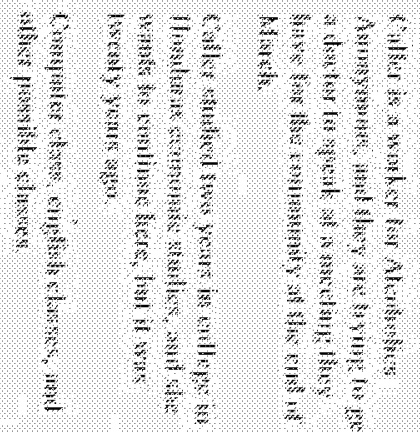

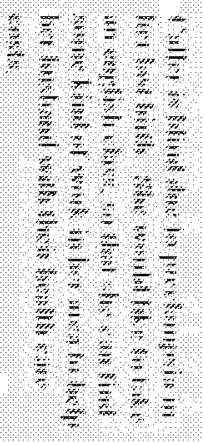

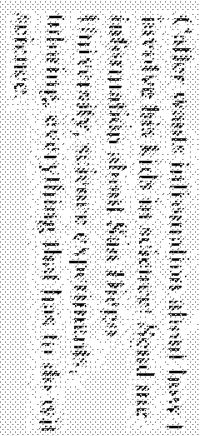
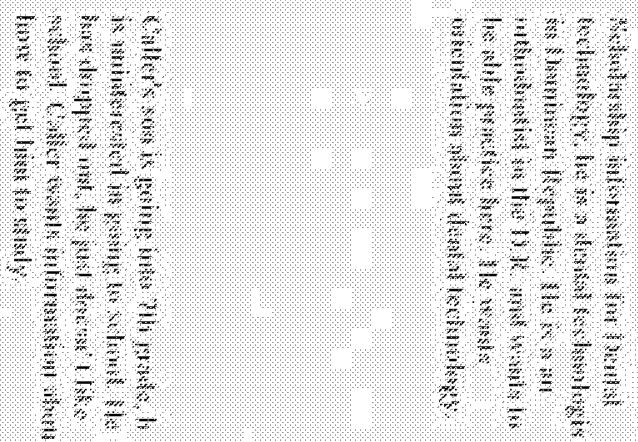

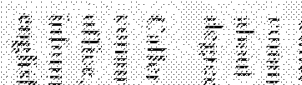

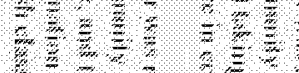

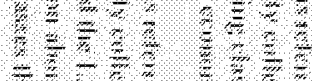

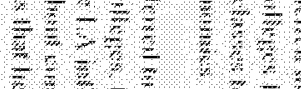

52 , 5 ?

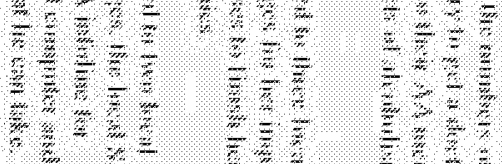

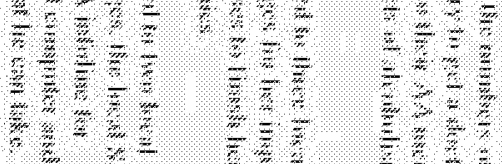

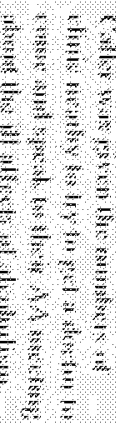

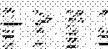

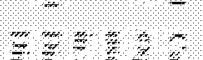

cz- $7=0$

g
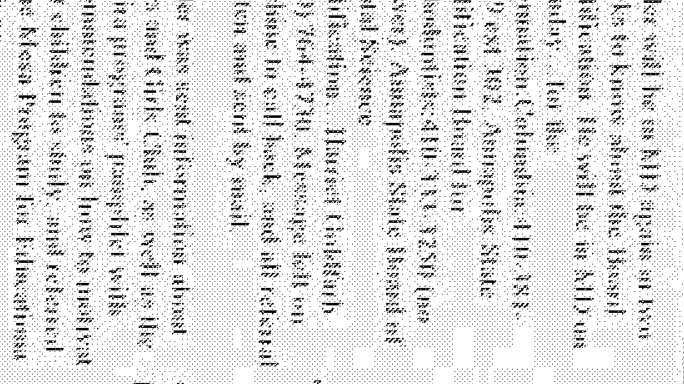

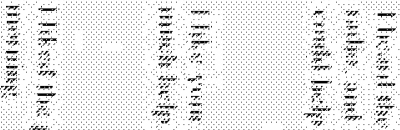

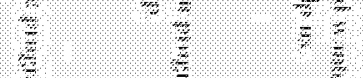

$\frac{2}{2}$

$=\stackrel{5}{2}=$

$7,2 \quad \frac{2}{2}=3$

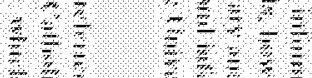

$\frac{-3}{2} \quad \frac{3}{2}=2$

$\frac{3}{3}=\frac{2}{2}=\frac{2}{2}$ $\frac{3}{2}=\frac{3}{2}$ $\frac{2}{2}+3$ $3 \overline{3}$
$\times 3$

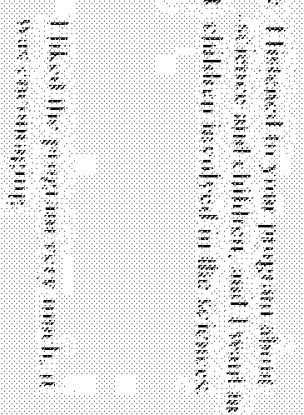

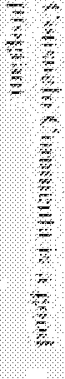




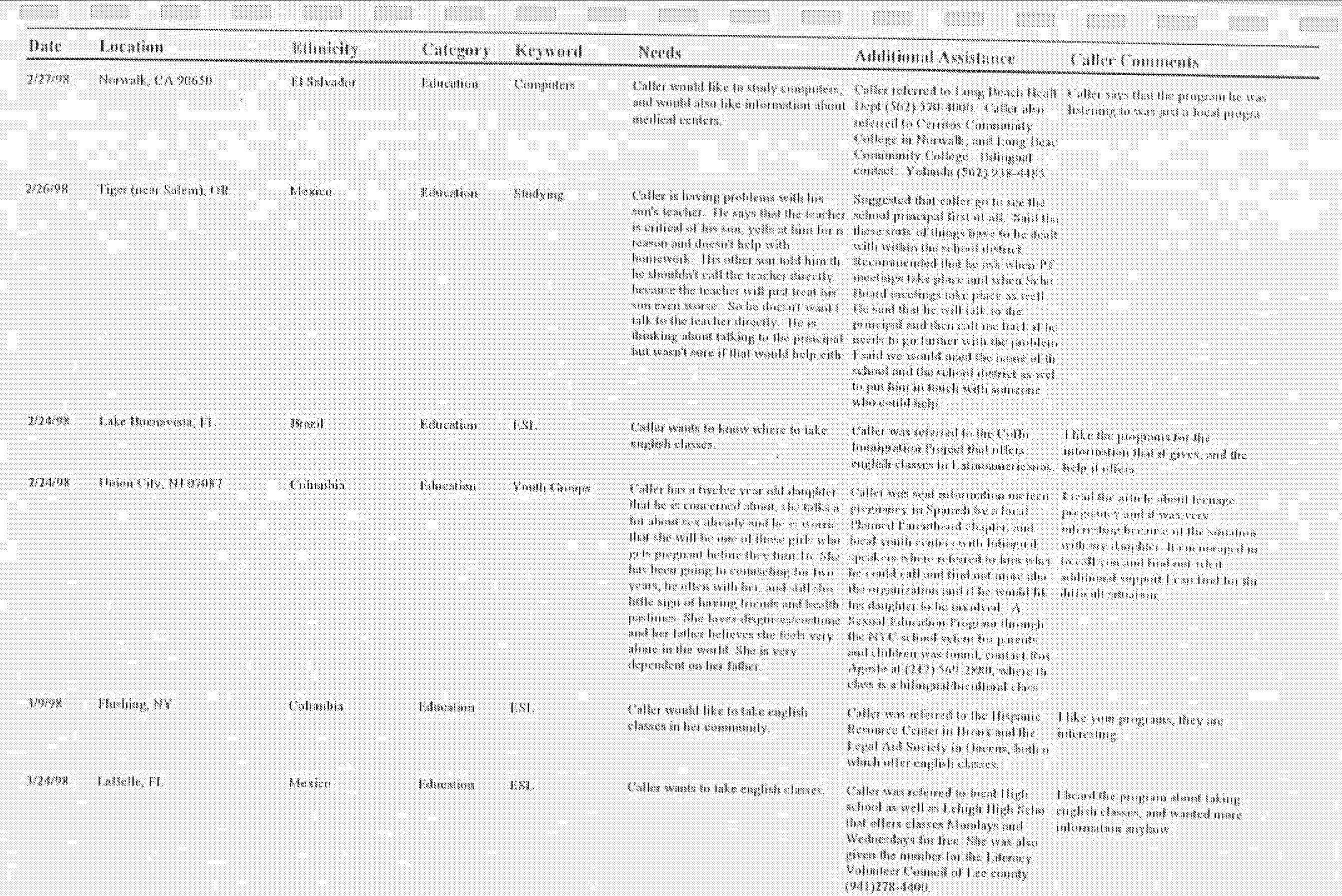




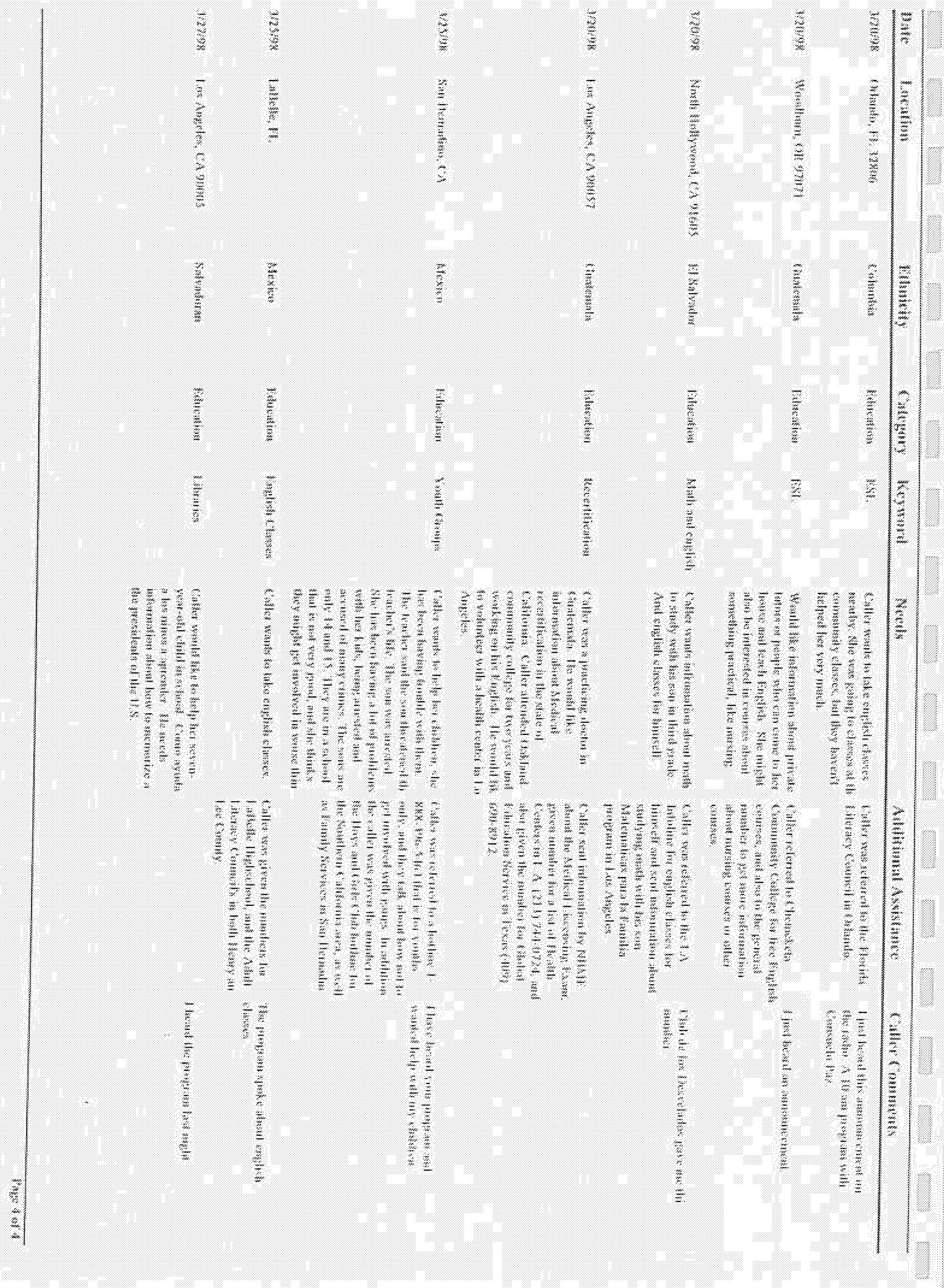





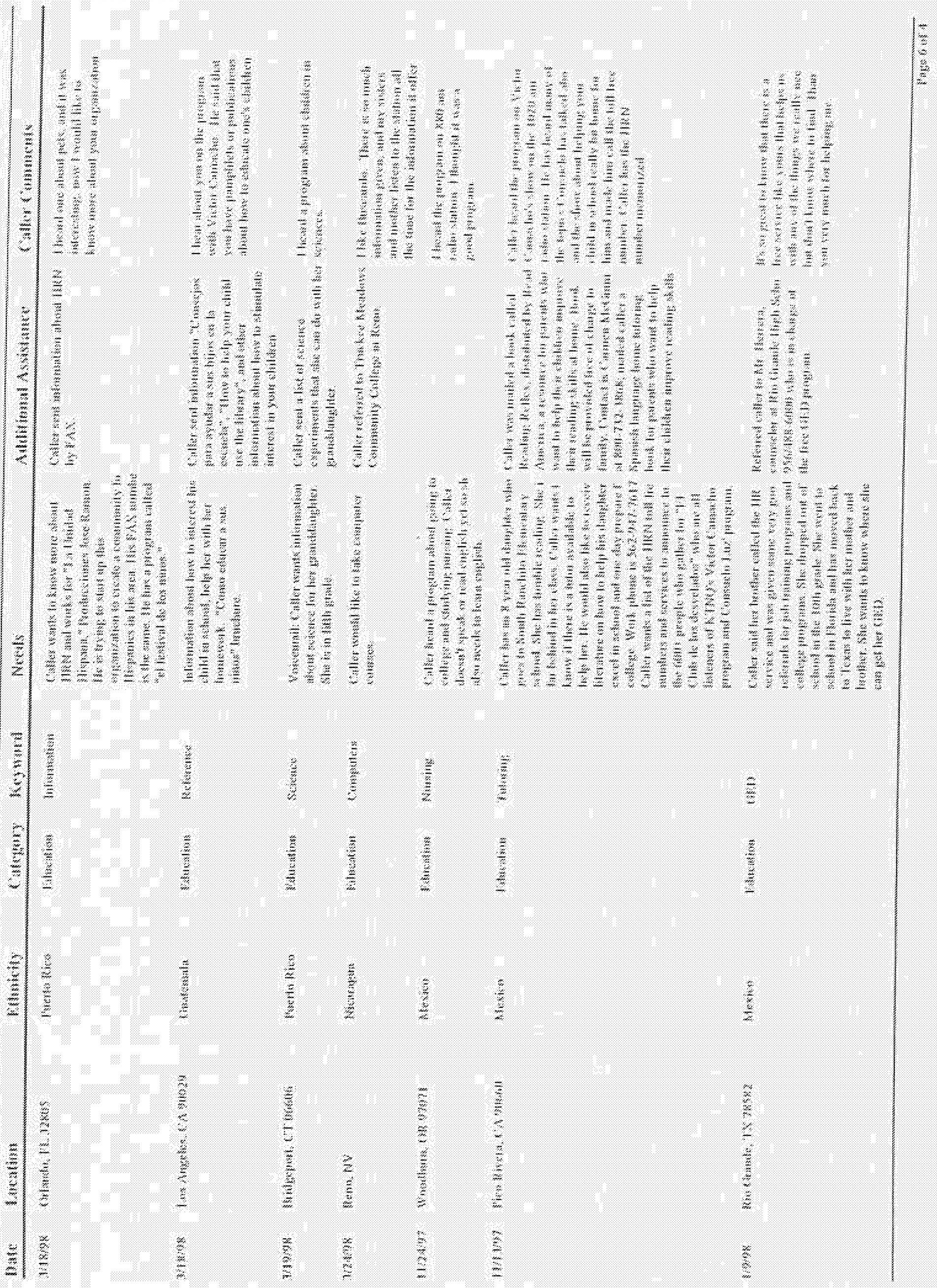




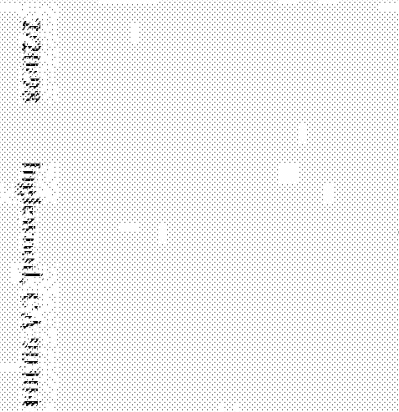

ए)

落

$\frac{2}{3}$

$\frac{2}{3}$

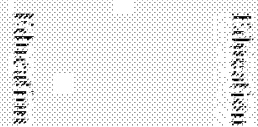

$\frac{\frac{3}{6}}{\frac{2}{3}} \quad 1 \quad \frac{5}{5}$

$\frac{3}{2}$

$\frac{7}{2}$

z

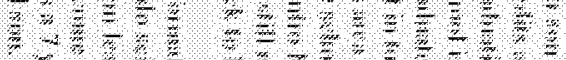

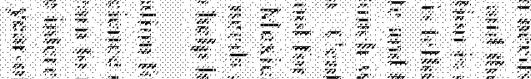

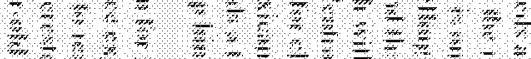

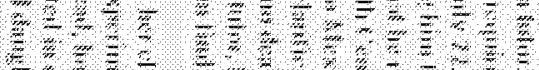

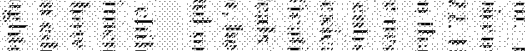

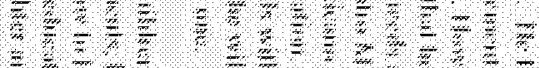

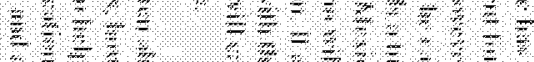

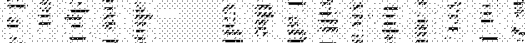

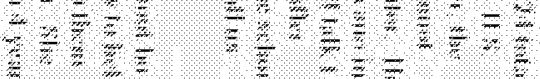

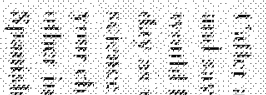

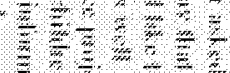

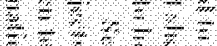

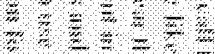
$\underline{z}=\frac{z}{z}=3$

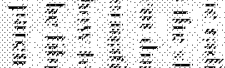

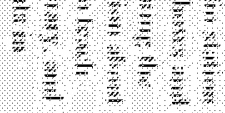

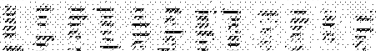

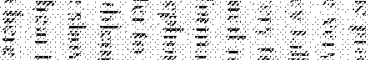

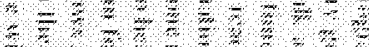
굴 를 等

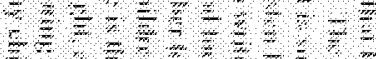

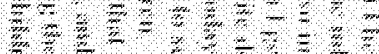

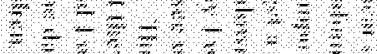

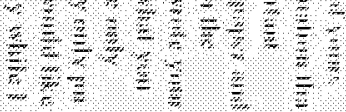
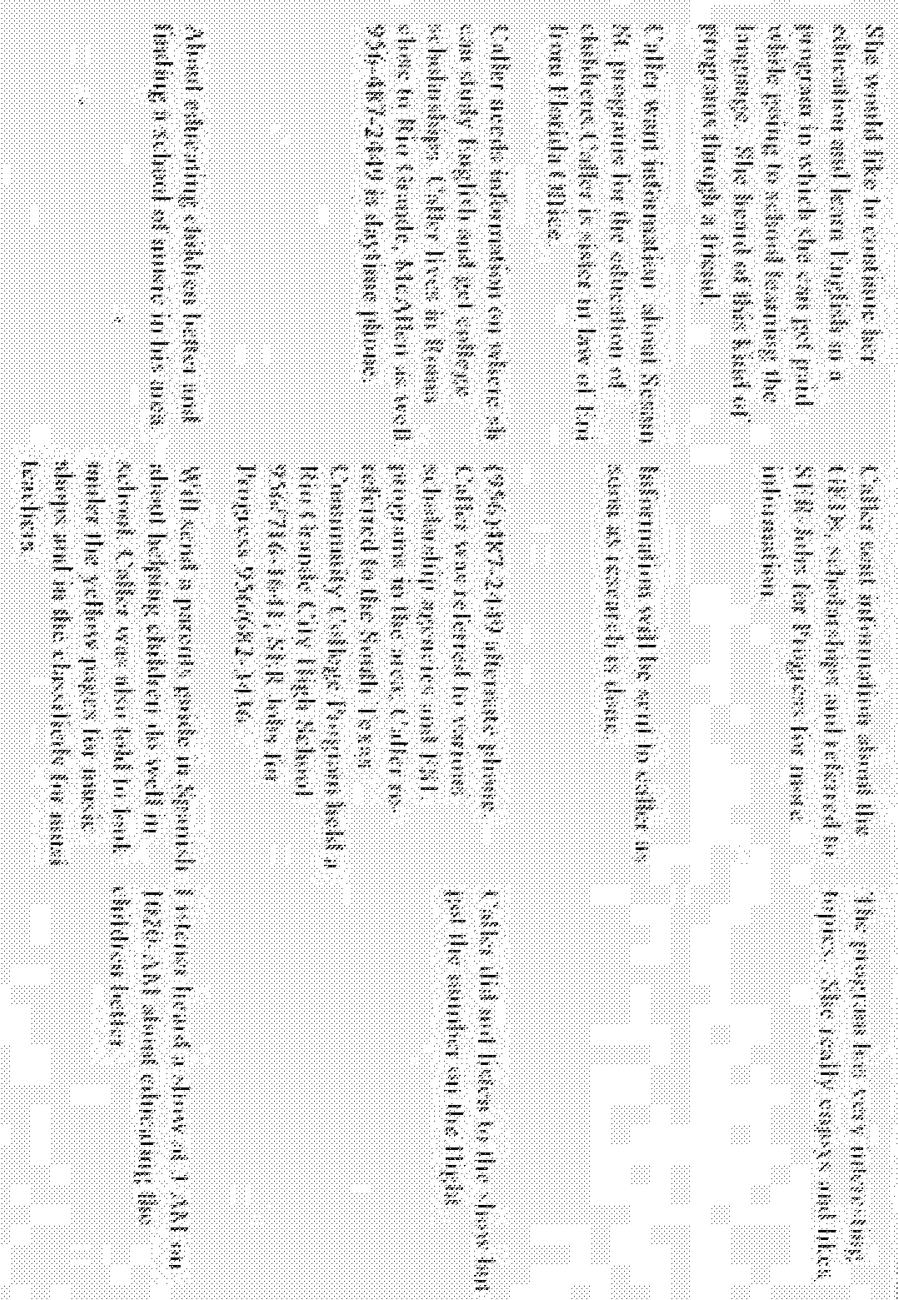


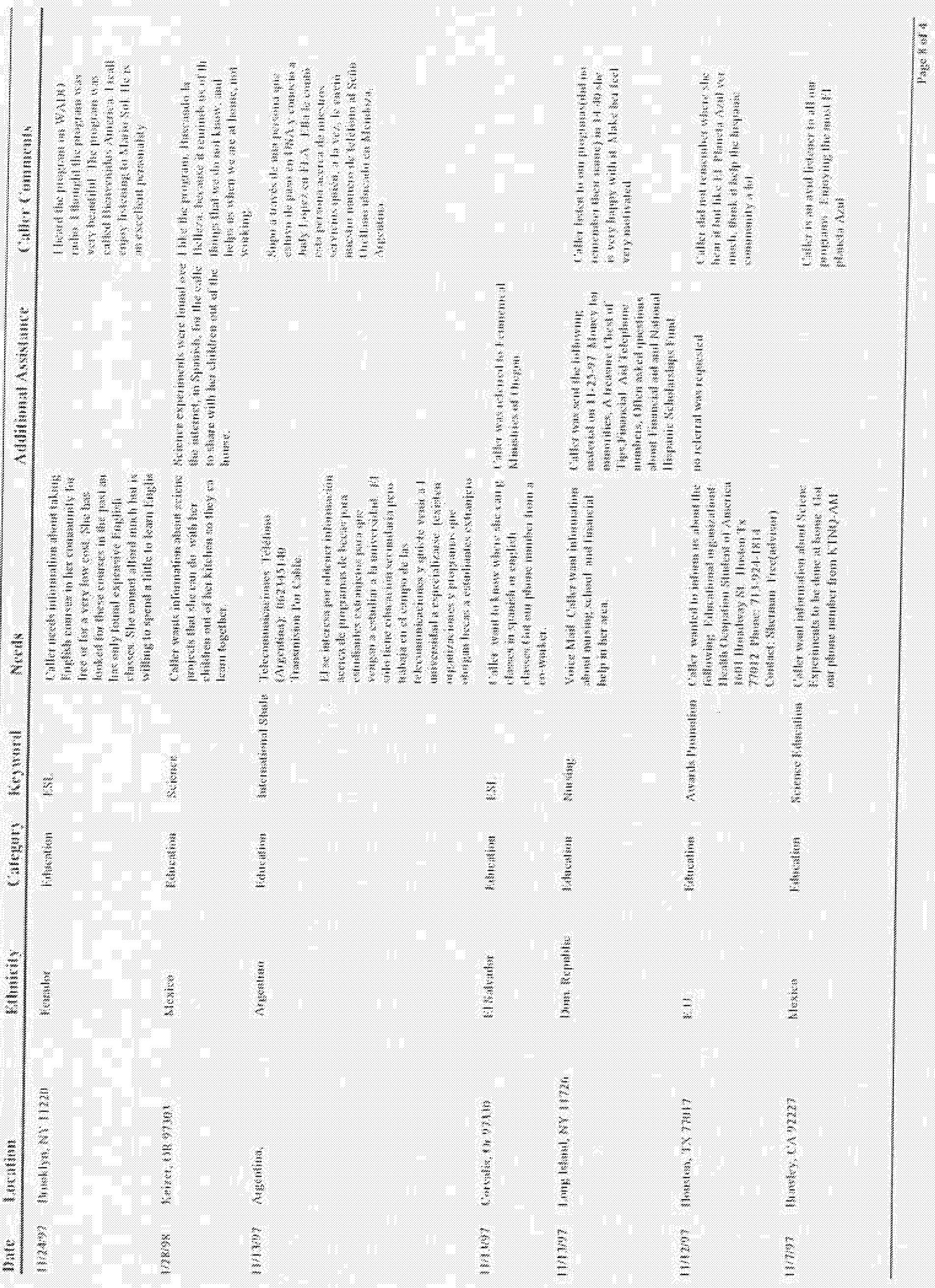




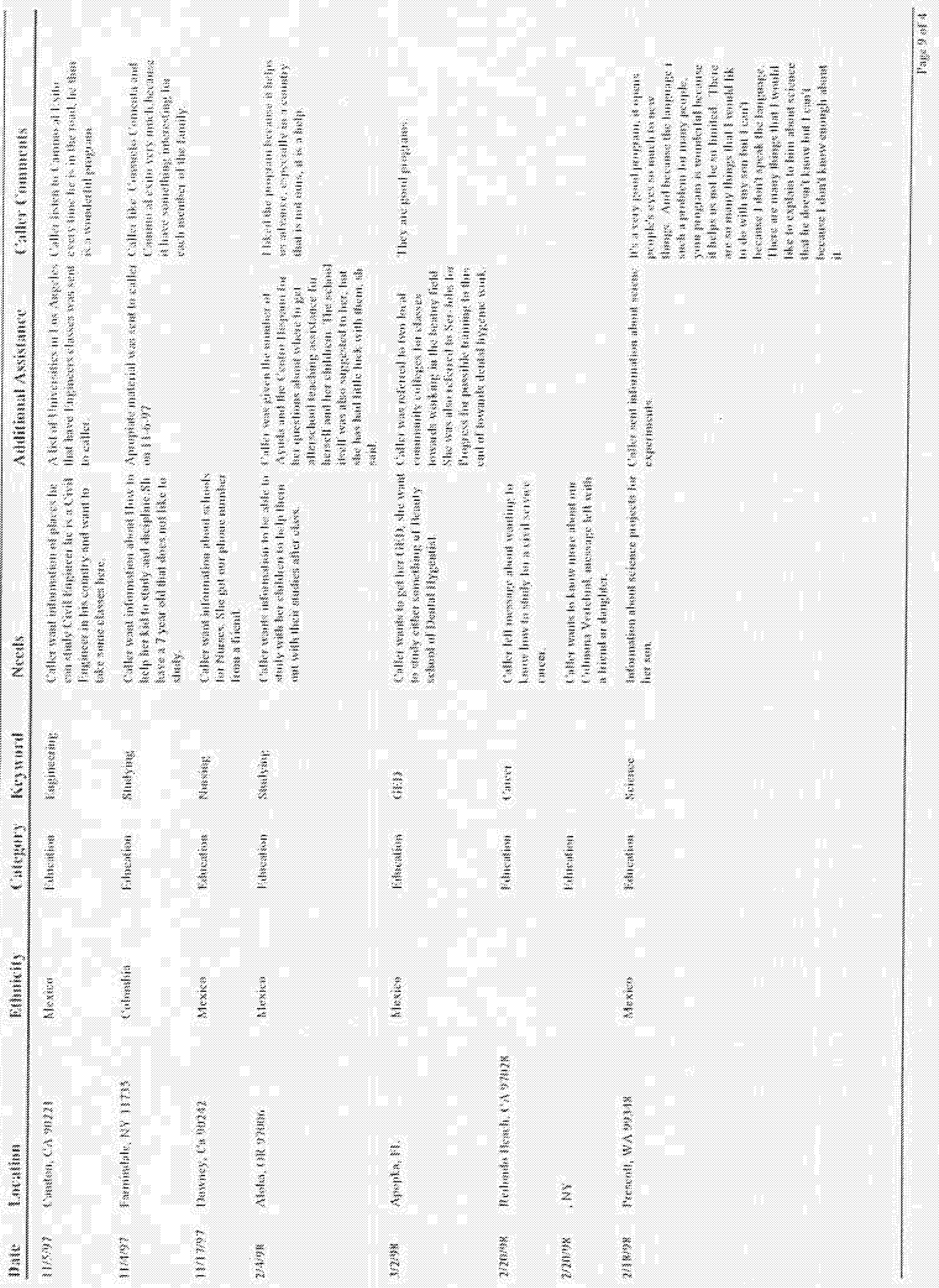




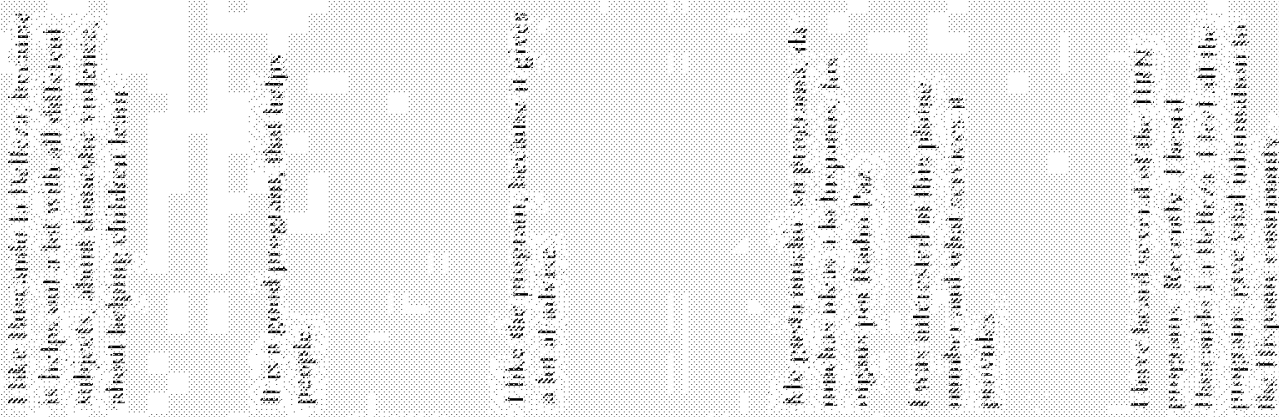

Z

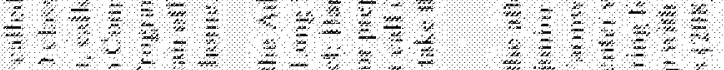

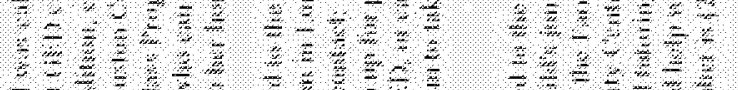

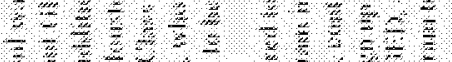

$2 y=5$. 2

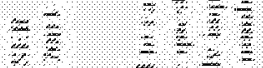

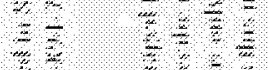

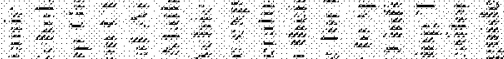

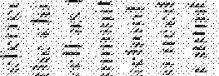

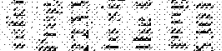

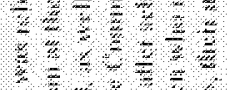

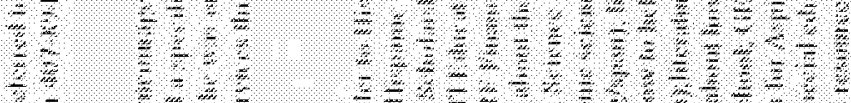

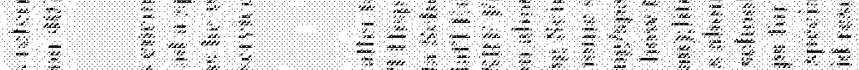

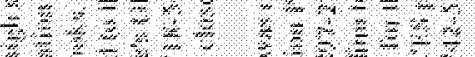

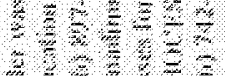

$7 \frac{1}{2}=\frac{1}{2}=$

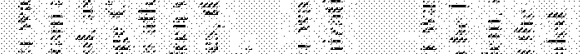

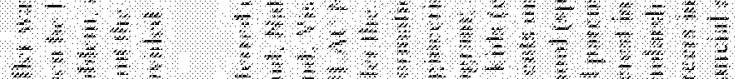

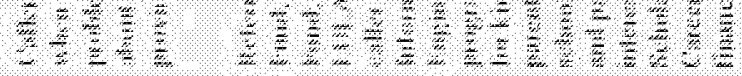

828 is

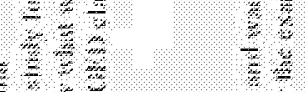

$29 \frac{2}{2}=\frac{2}{2}$

$2 \frac{2}{2}$

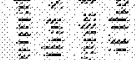

$=2$

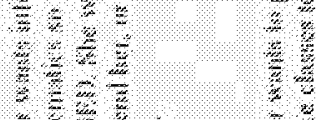

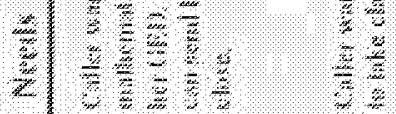

E)

$\frac{1}{2} \quad 2$

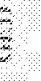

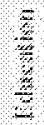

$\frac{5}{2}$

$\frac{3}{3} \div$

$\frac{2}{2}=\frac{3}{2}=$

$\frac{-2}{4} \frac{2}{2}=\frac{3}{2}=$

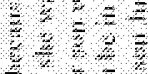

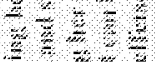

$\frac{2}{4} \div 2=$

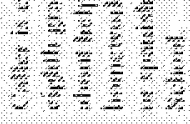

$\frac{2}{4}$

롤

g

$\stackrel{\bar{z}}{3}$

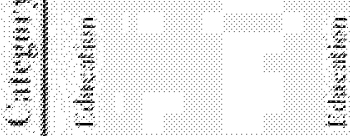

$z^{2}= \pm=$

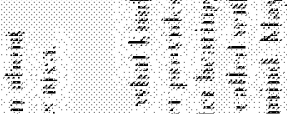

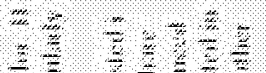

$5=$

\%

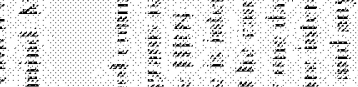

$7 y=2$
$z y$

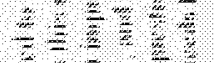

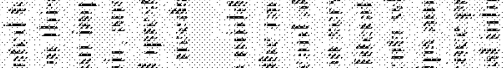

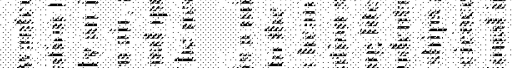

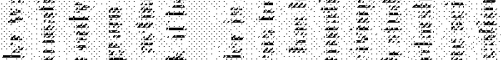

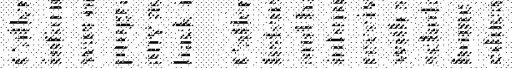

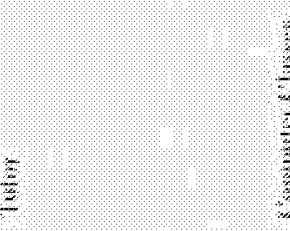

$\frac{8}{\frac{3}{8}}$

$\frac{\frac{3}{2}}{\frac{3}{2}}$

$\frac{\pi}{3}$

$\frac{\overline{4}}{3}$

$\frac{3}{3}$

$\frac{3}{4}$

$\frac{8}{3}$

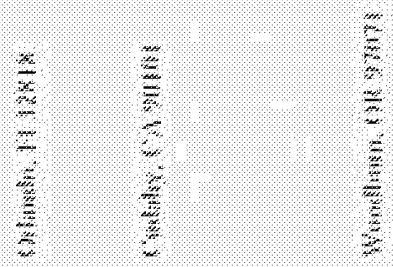

$\pm$

$\stackrel{2}{2}$

$\frac{2}{3} \quad \frac{3}{5}$

8

ह 


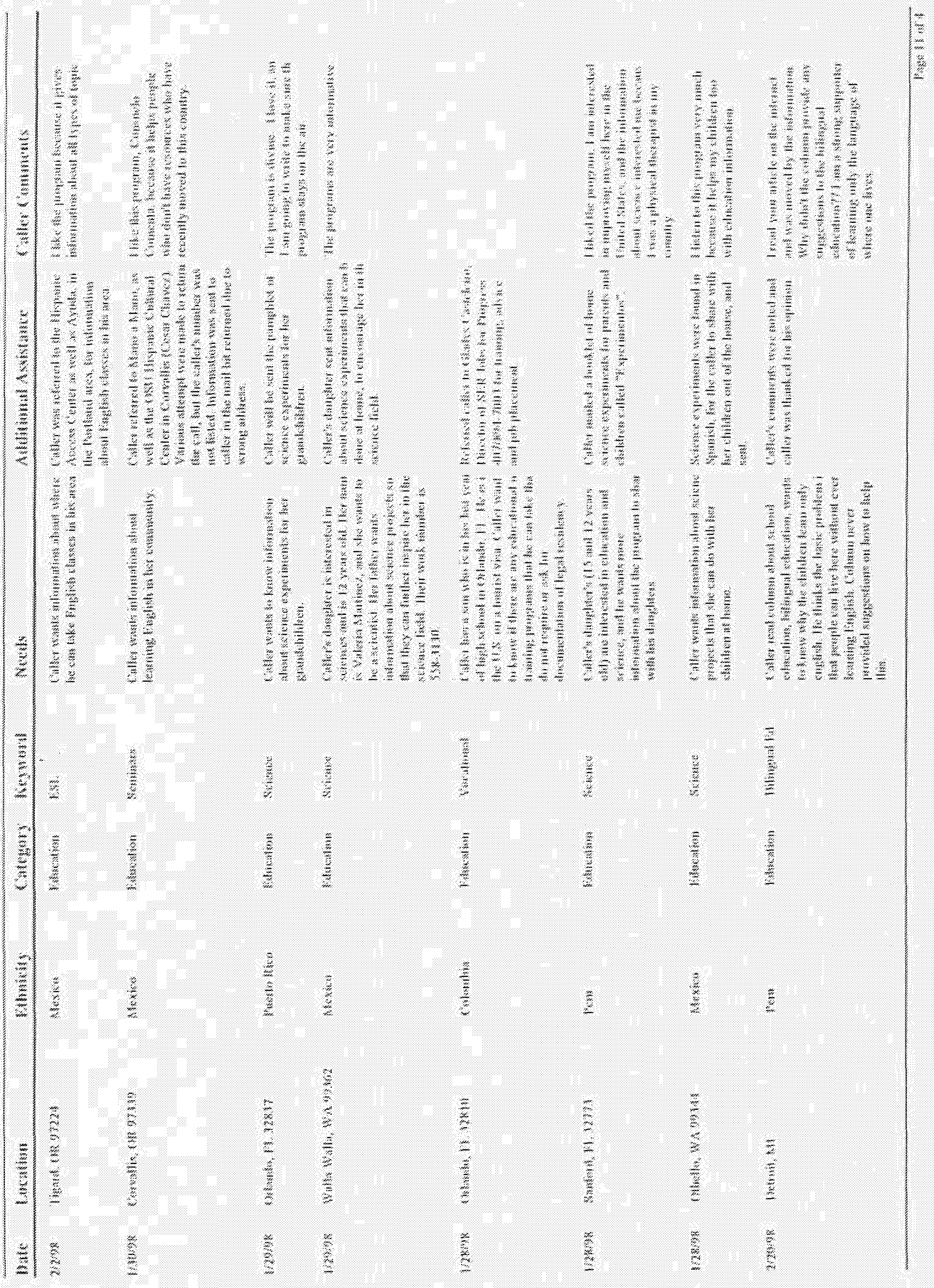


$\begin{array}{ll}\frac{3}{2} & \frac{2}{2} \\ \frac{3}{2} & =\end{array}$

高然

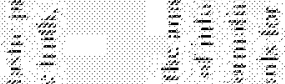

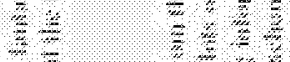

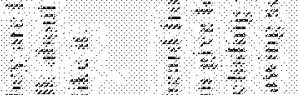

$\underline{2}=\frac{2}{2}=\frac{2}{2}$

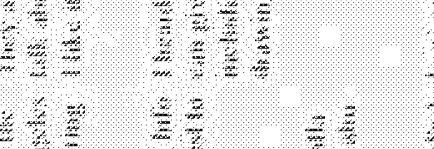

$\frac{2}{2}=\frac{1}{2}$

$\frac{2}{5}=\frac{2}{4}$

$\frac{25}{2}=\frac{2}{2}$

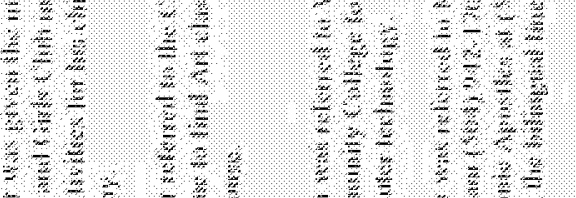

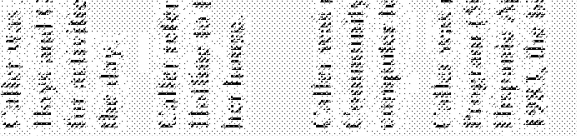

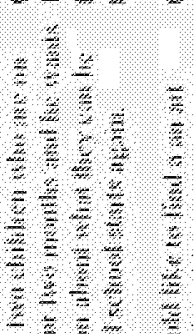

$\stackrel{2}{2}$

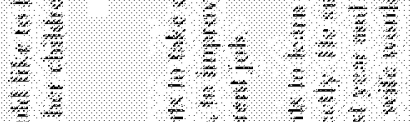

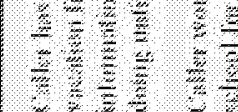

$\frac{7}{3}$

$z$
$z$
$z$
$=$
$z$
$z$

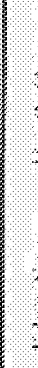

$\frac{\sqrt{2}}{\frac{3}{2}}$

$\frac{3}{3} \quad \frac{3}{3}$

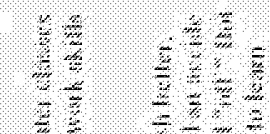

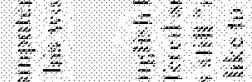

$\frac{2}{2}=2$

$\frac{25}{2}=\sqrt[3]{2}=$

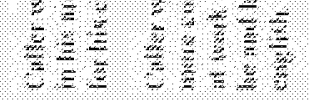

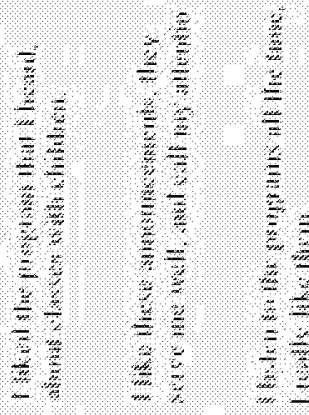

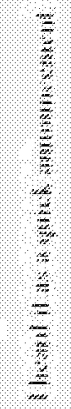

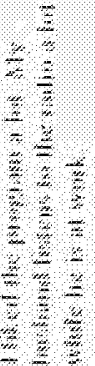

$- \pm-2=$

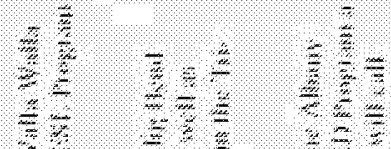

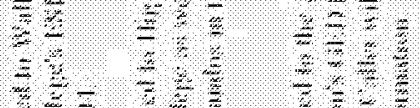

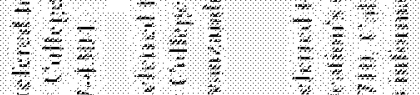

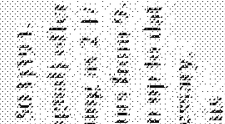

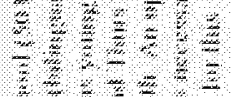

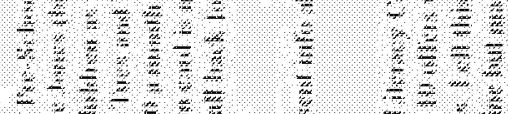

$\frac{2}{4}+2=2$

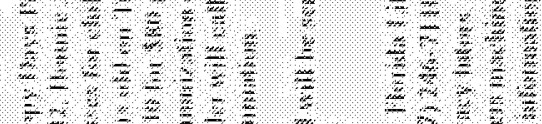

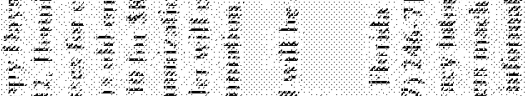

275

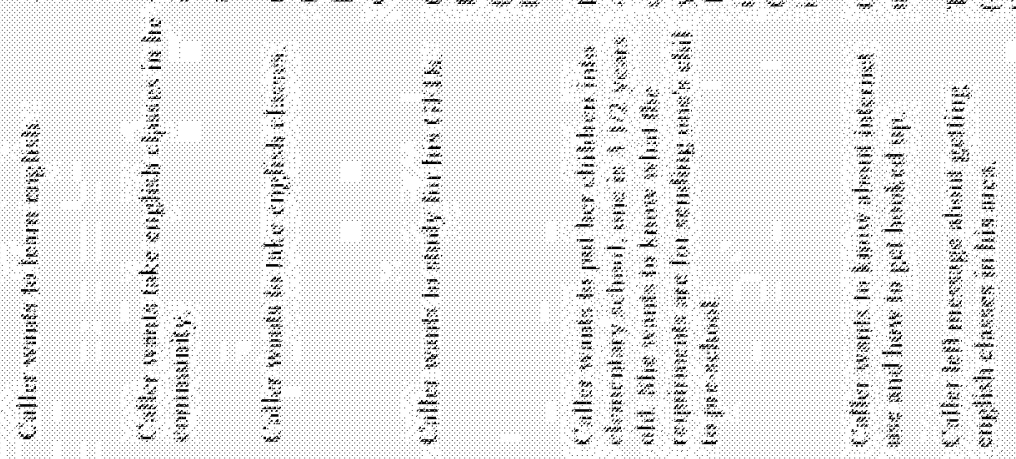

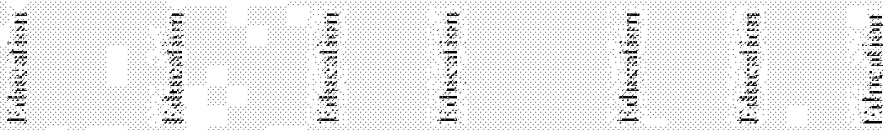

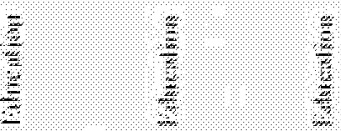

$\frac{3}{4}+\frac{\pi}{3}$

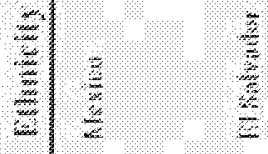

$\frac{2}{\frac{3}{3}}+192$

3

$\frac{3}{2}$
$\frac{3}{2}$

$\frac{5}{2} \quad \frac{3}{5}$

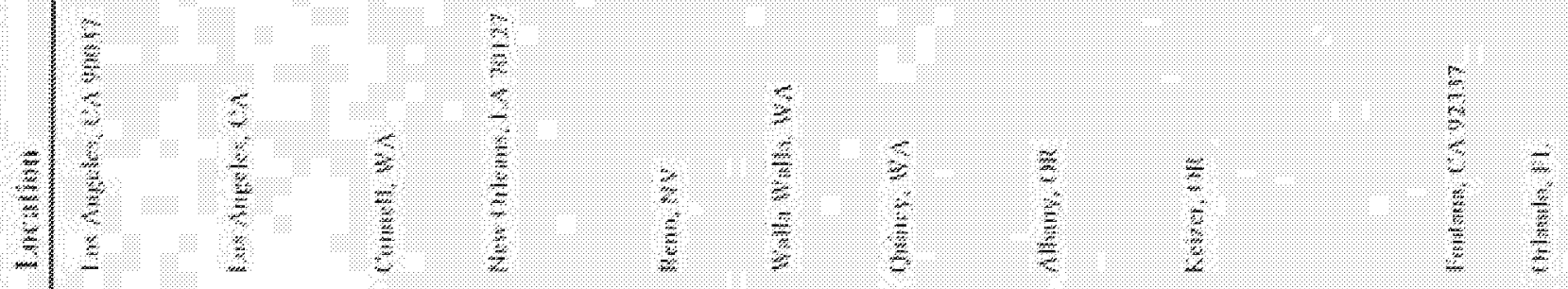

$\frac{3}{3}+\frac{3}{4}=$

$\frac{2}{5}$

$\frac{2}{2} \quad \frac{2}{2}$ 


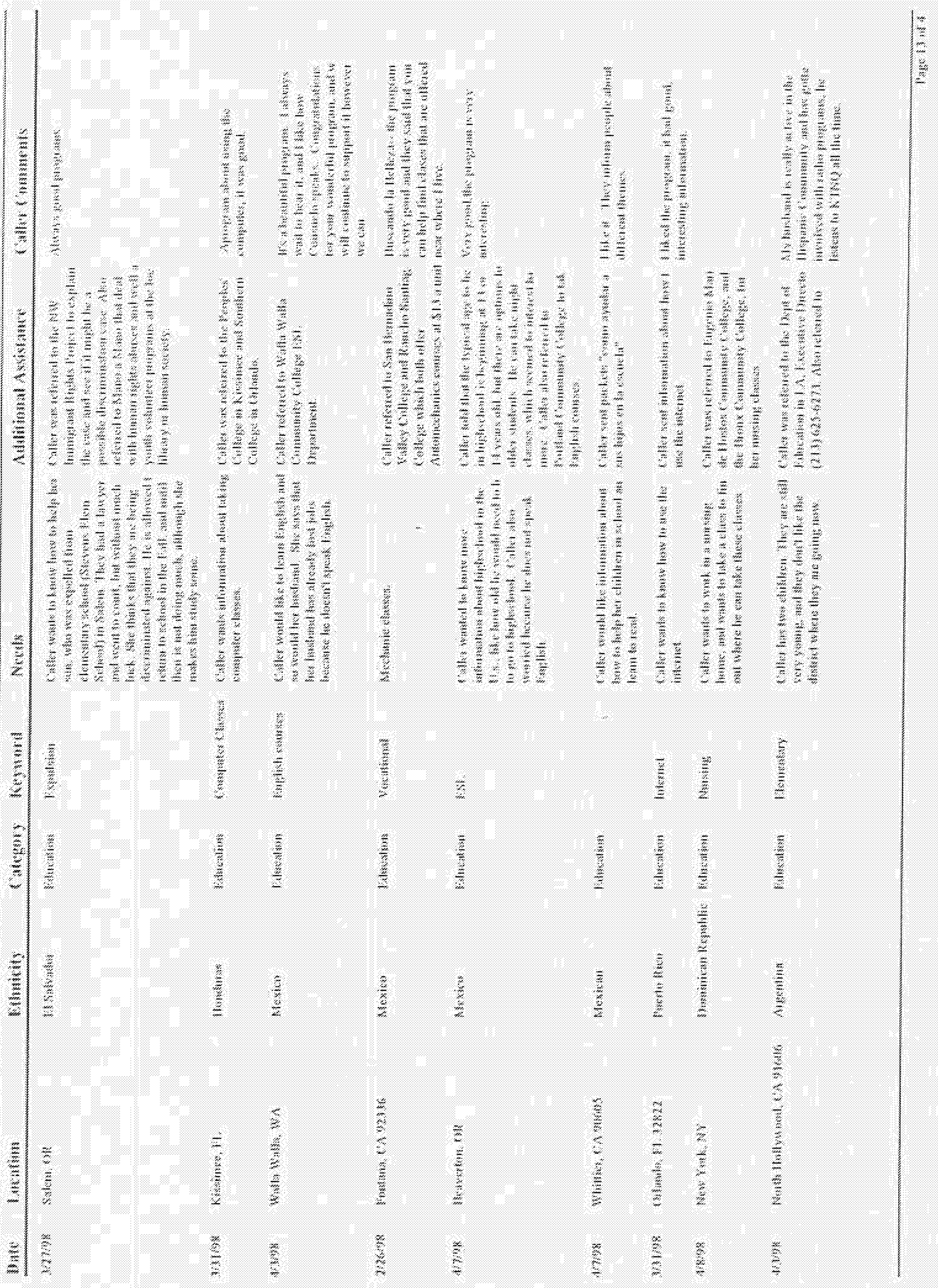




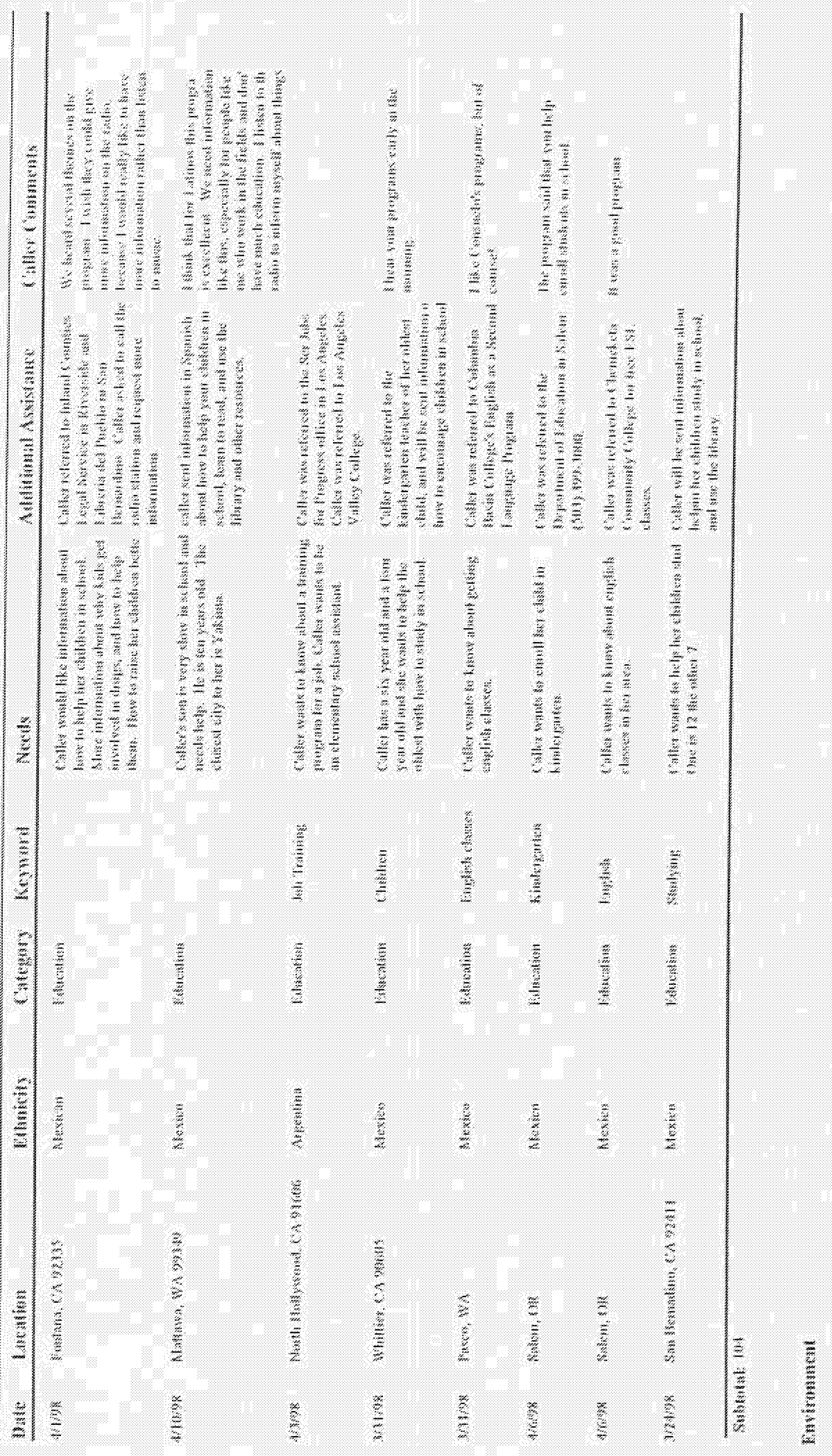




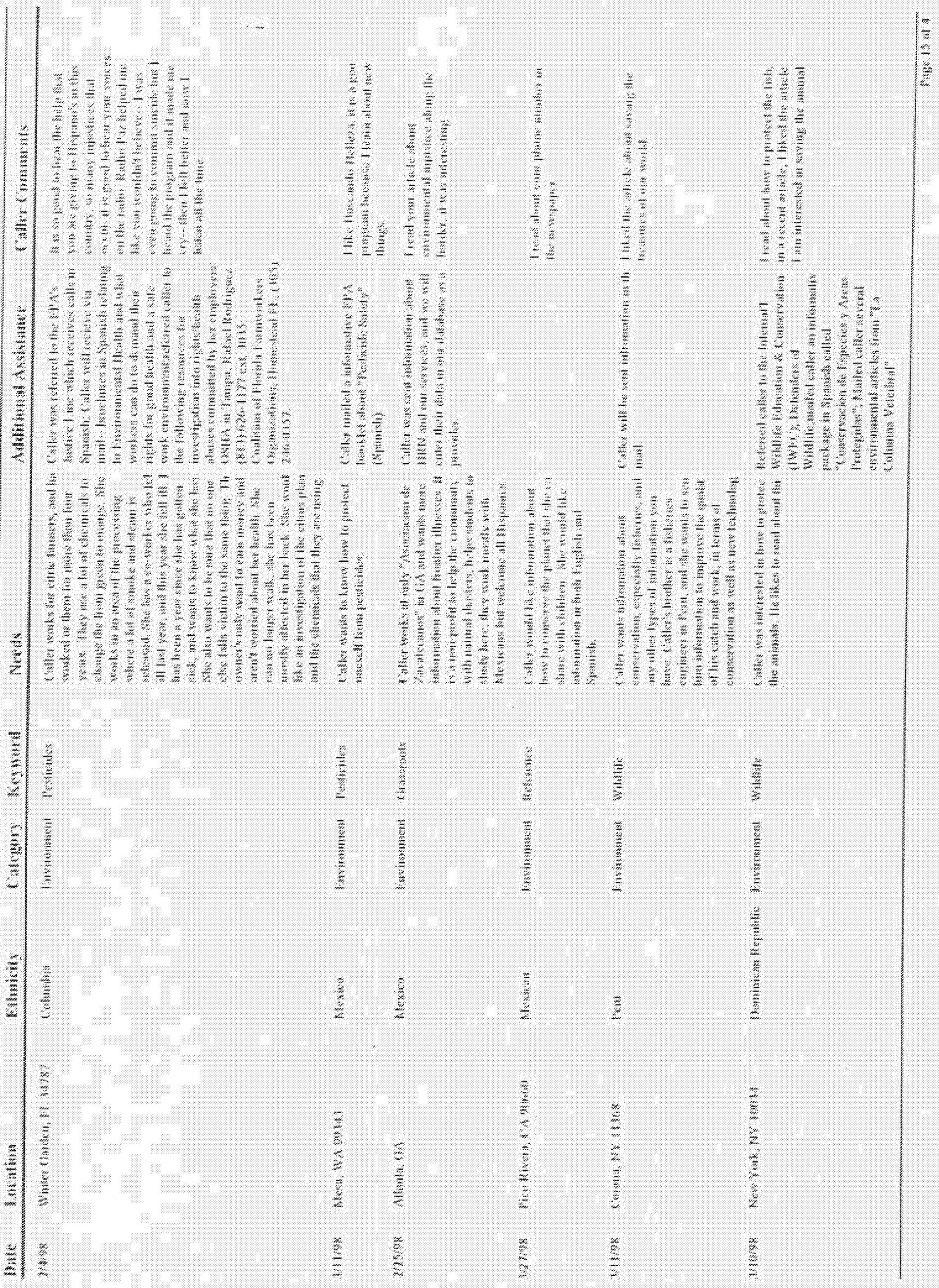




\begin{tabular}{|c|c|c|c|c|c|c|c|}
\hline 18,48 & 10,1016048 & Glankils & 1310,9019 & Bromone & Yis+ot? & 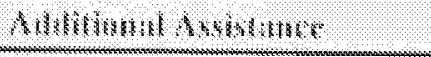 & 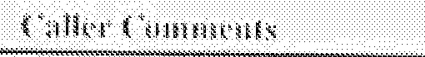 \\
\hline $14 \% \%$ & 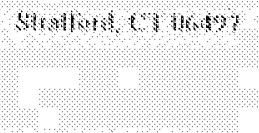 & 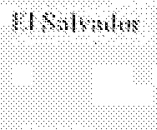 & 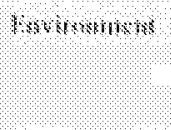 & 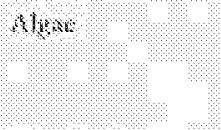 & 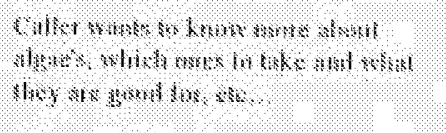 & 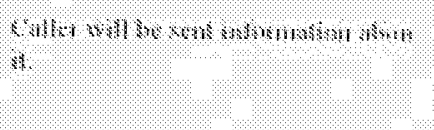 & 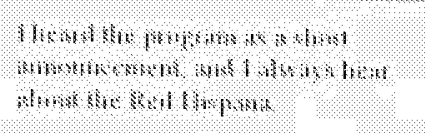 \\
\hline $140 \%$ & 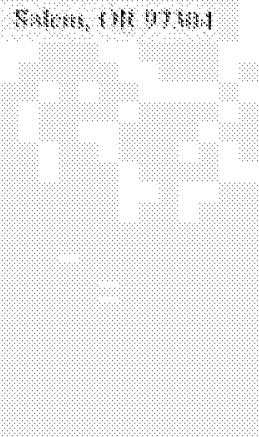 & 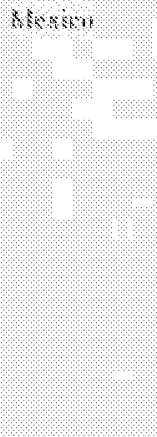 & Syrotronsmor & 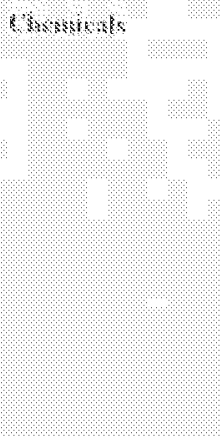 & 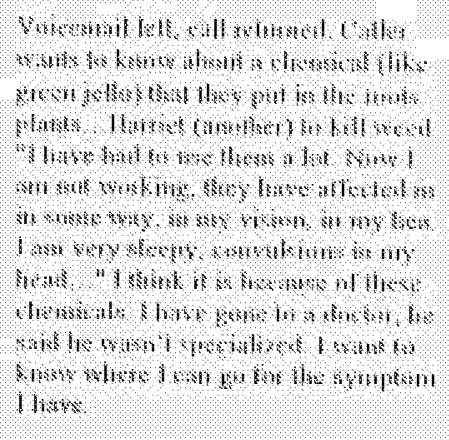 & 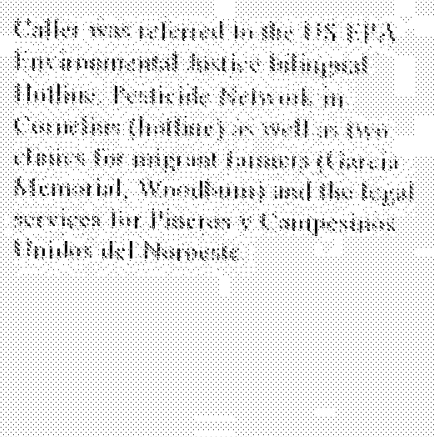 & 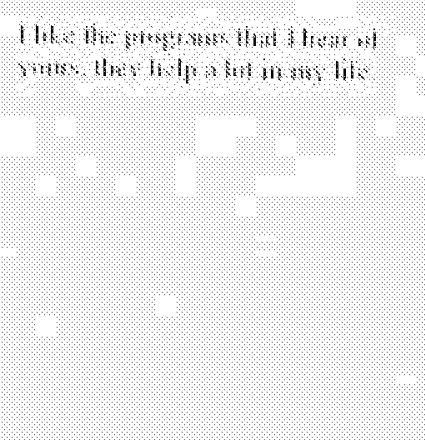 \\
\hline 22481 & $1806 \mathrm{mox} \times 14$ & 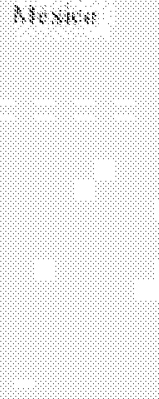 & Ineinesturest & 8 reremslon & 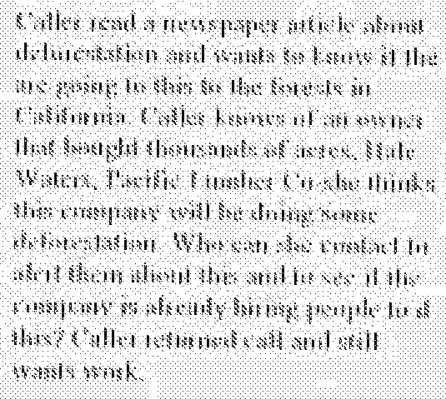 & 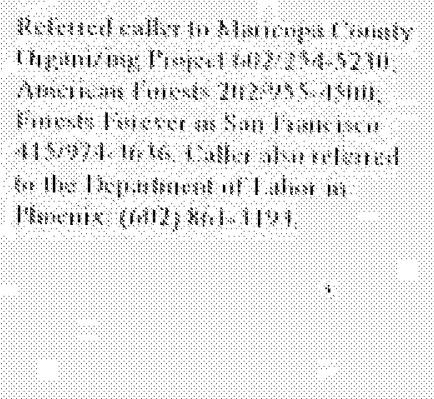 & 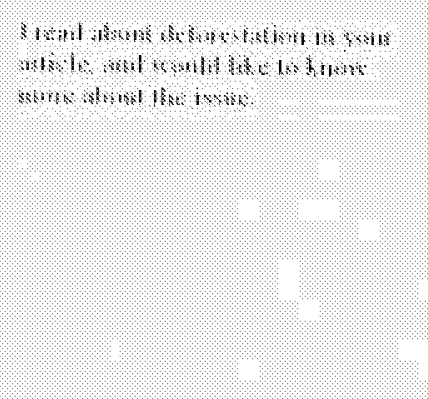 \\
\hline $100 \%$ & vinkm $41 \% 0180$ & $18 \times 180,61$ & 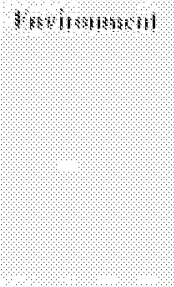 & $81,1,4,8,4$ & 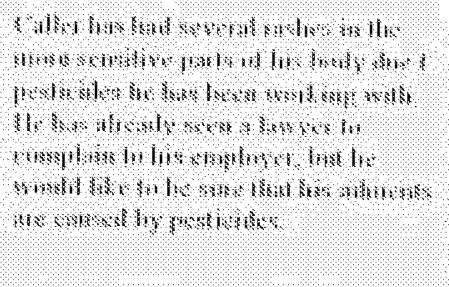 & 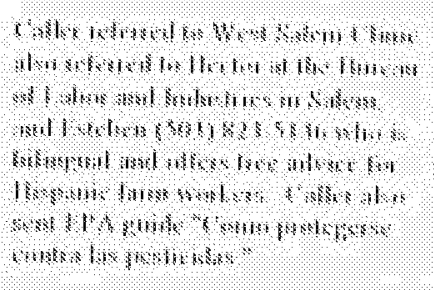 & 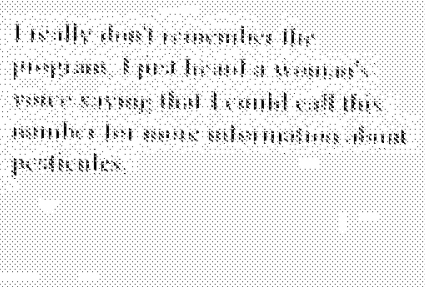 \\
\hline $281 \% 2$ & \% & & rins:nemenks:8 & $1 \%$ in $11 \%$ & 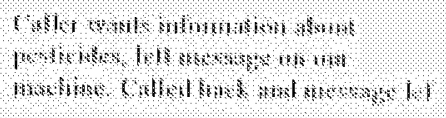 & & \\
\hline 201102 & 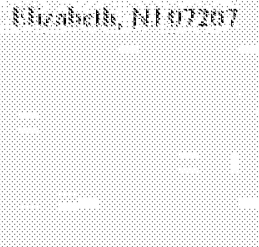 & rellomirs & 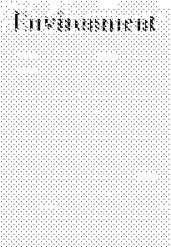 & $810118 \%$ & 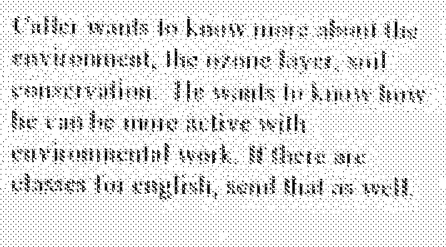 & 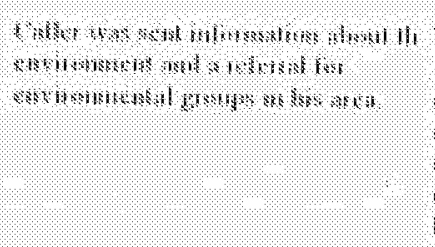 & 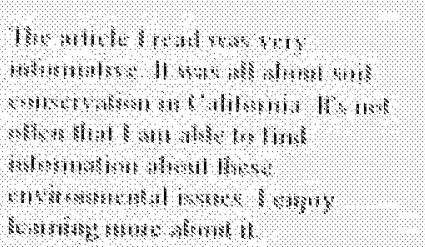 \\
\hline
\end{tabular}




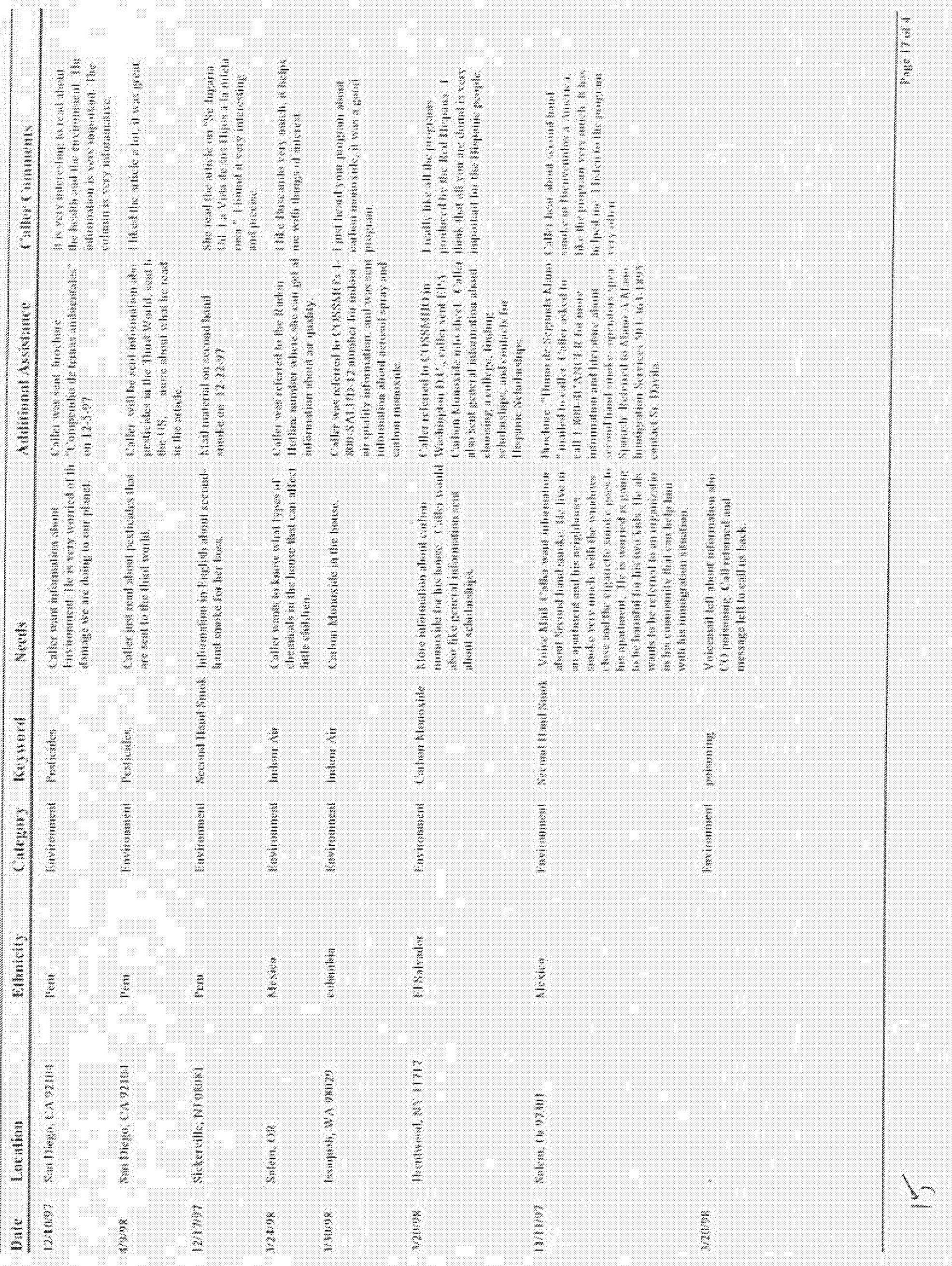




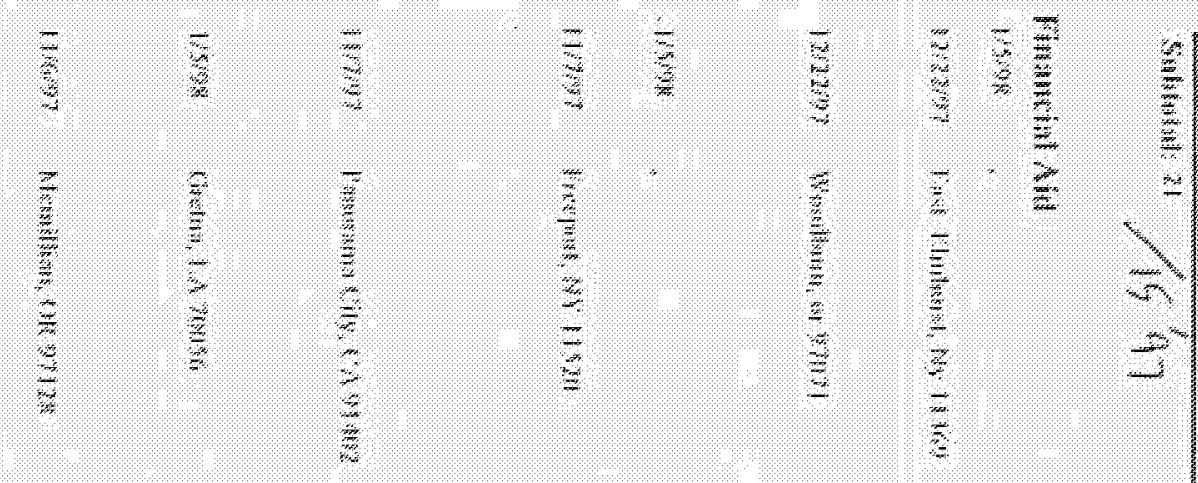

11111

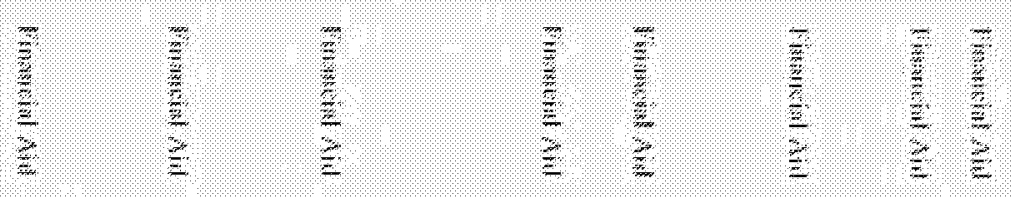

mIn Im!

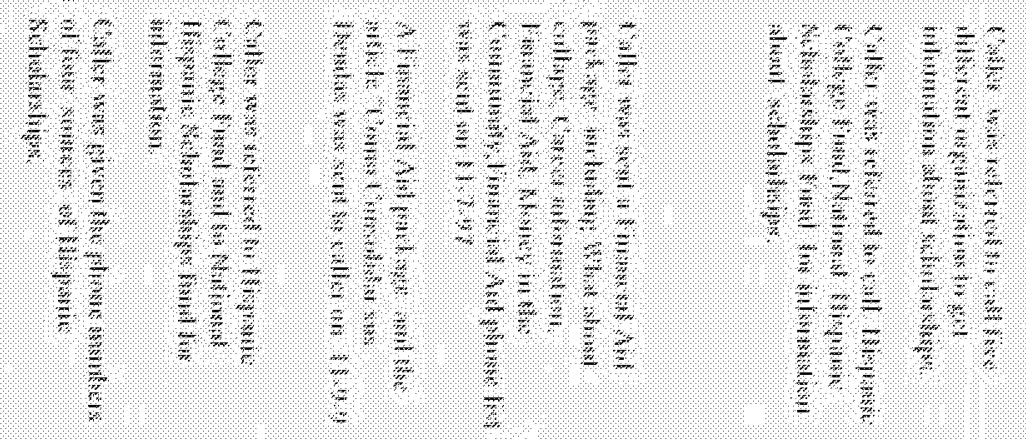

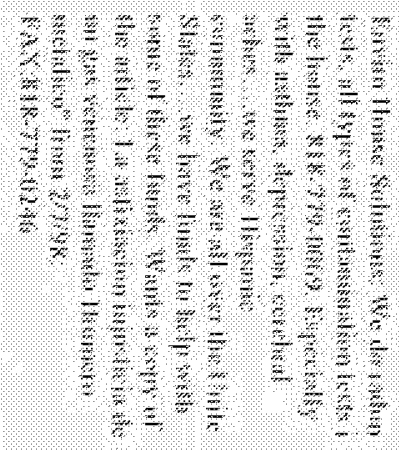




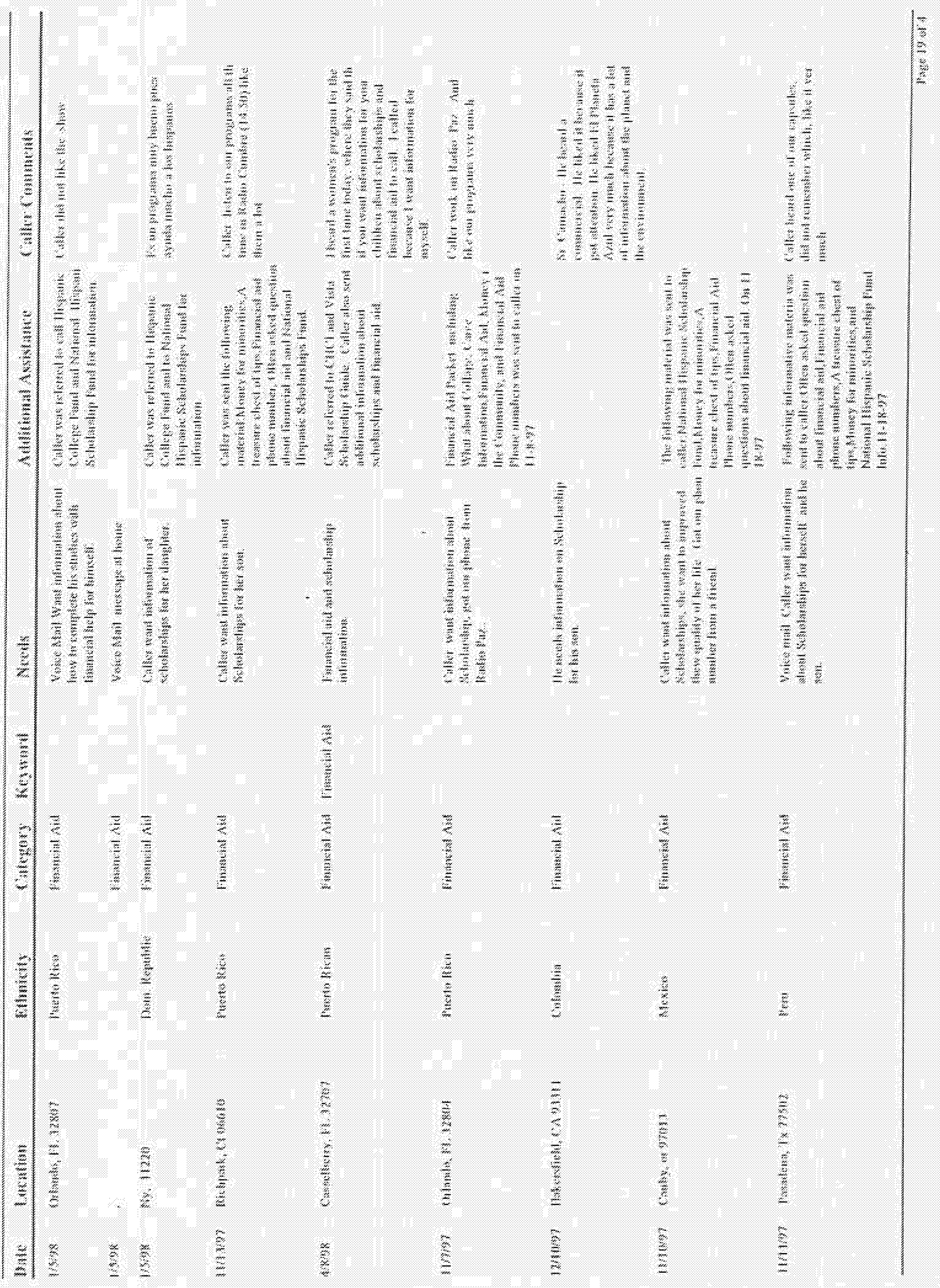




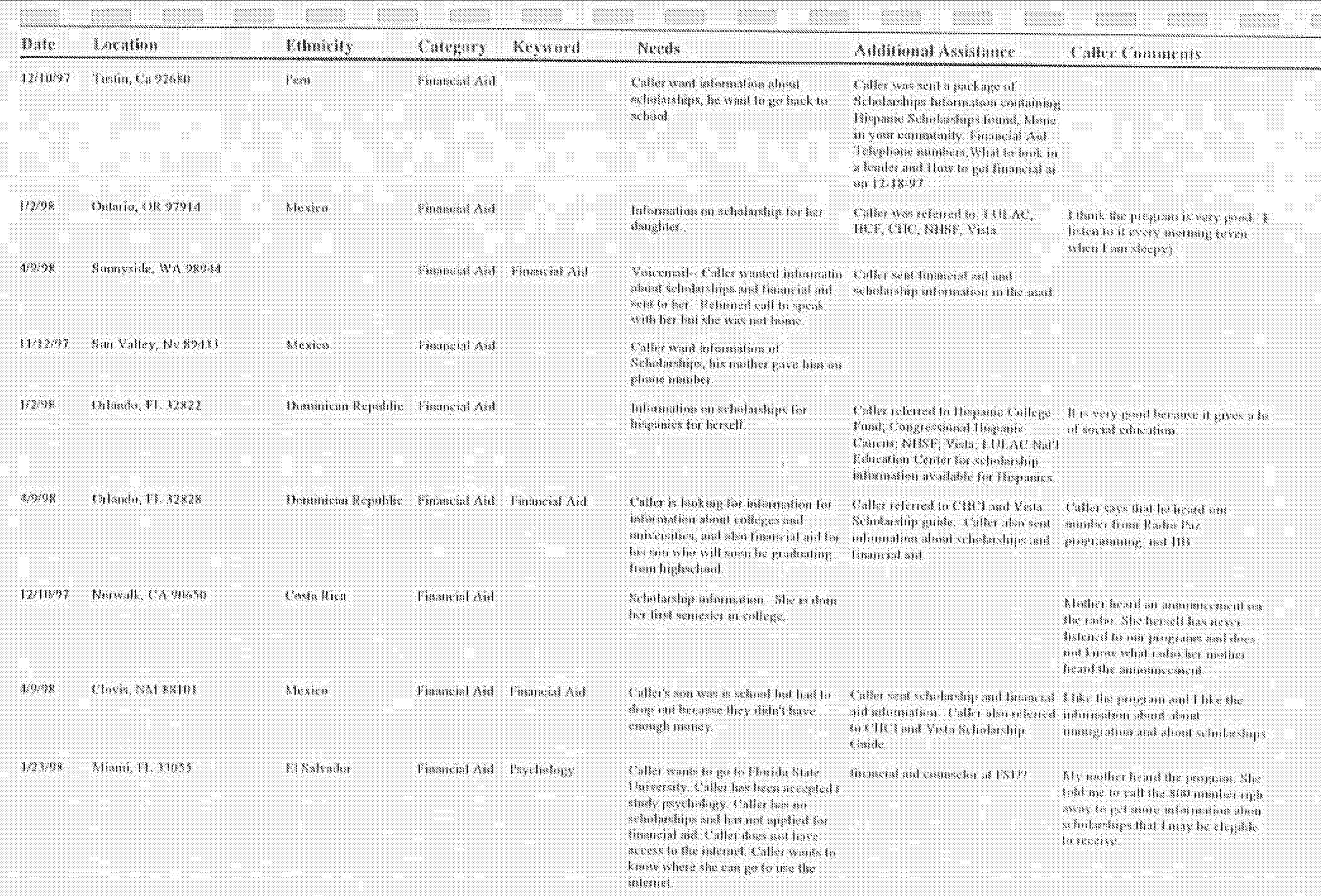




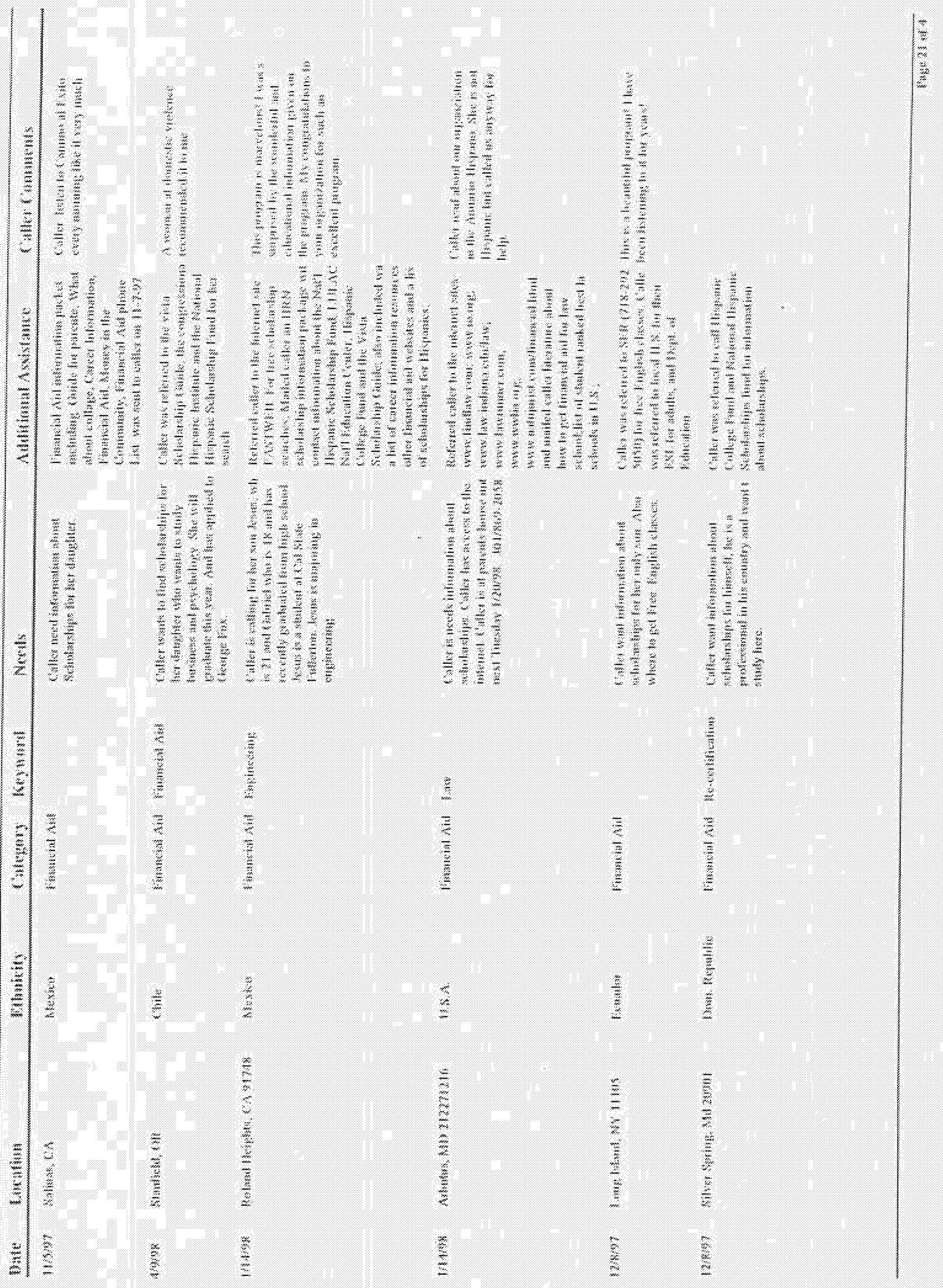




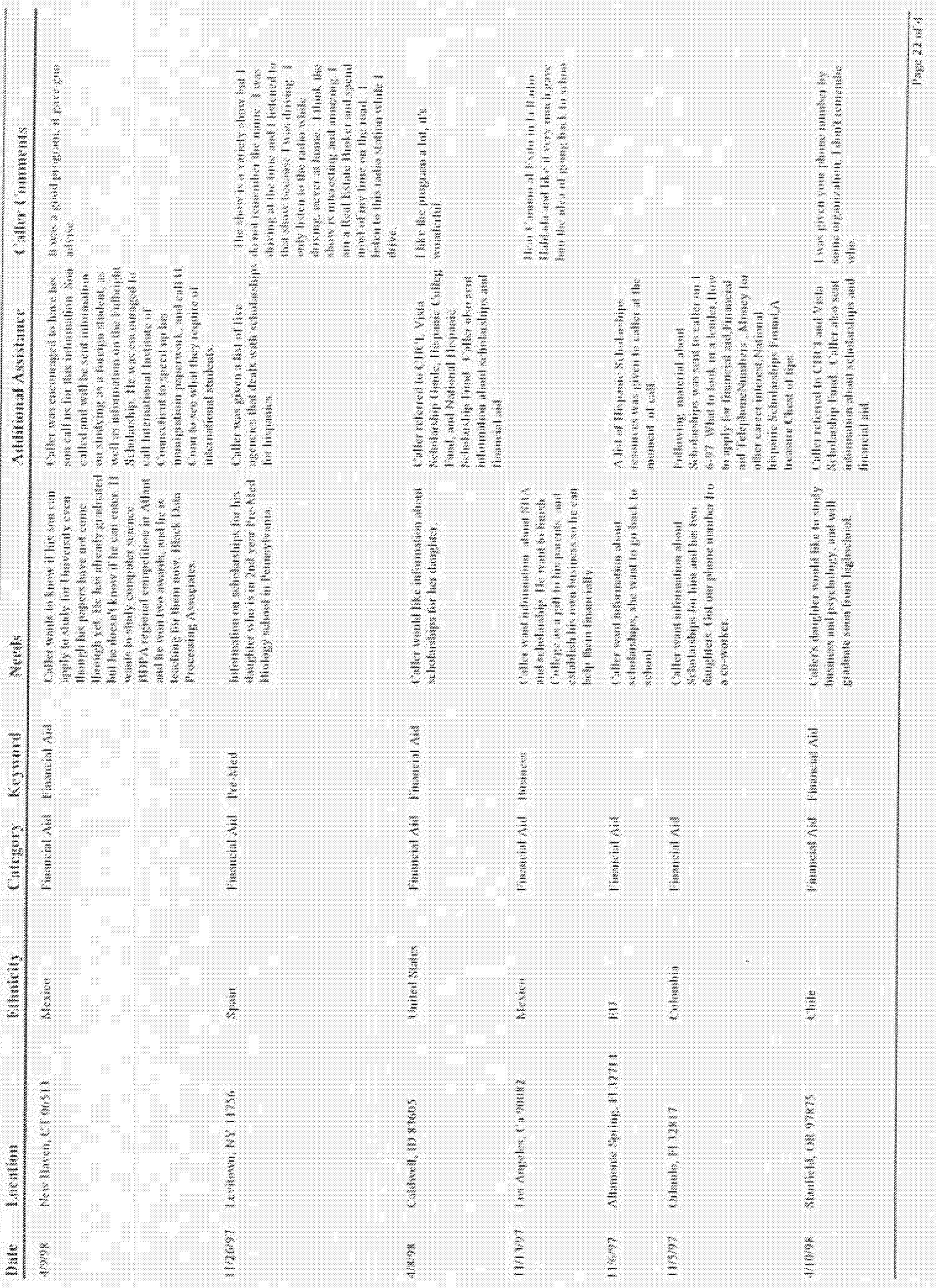




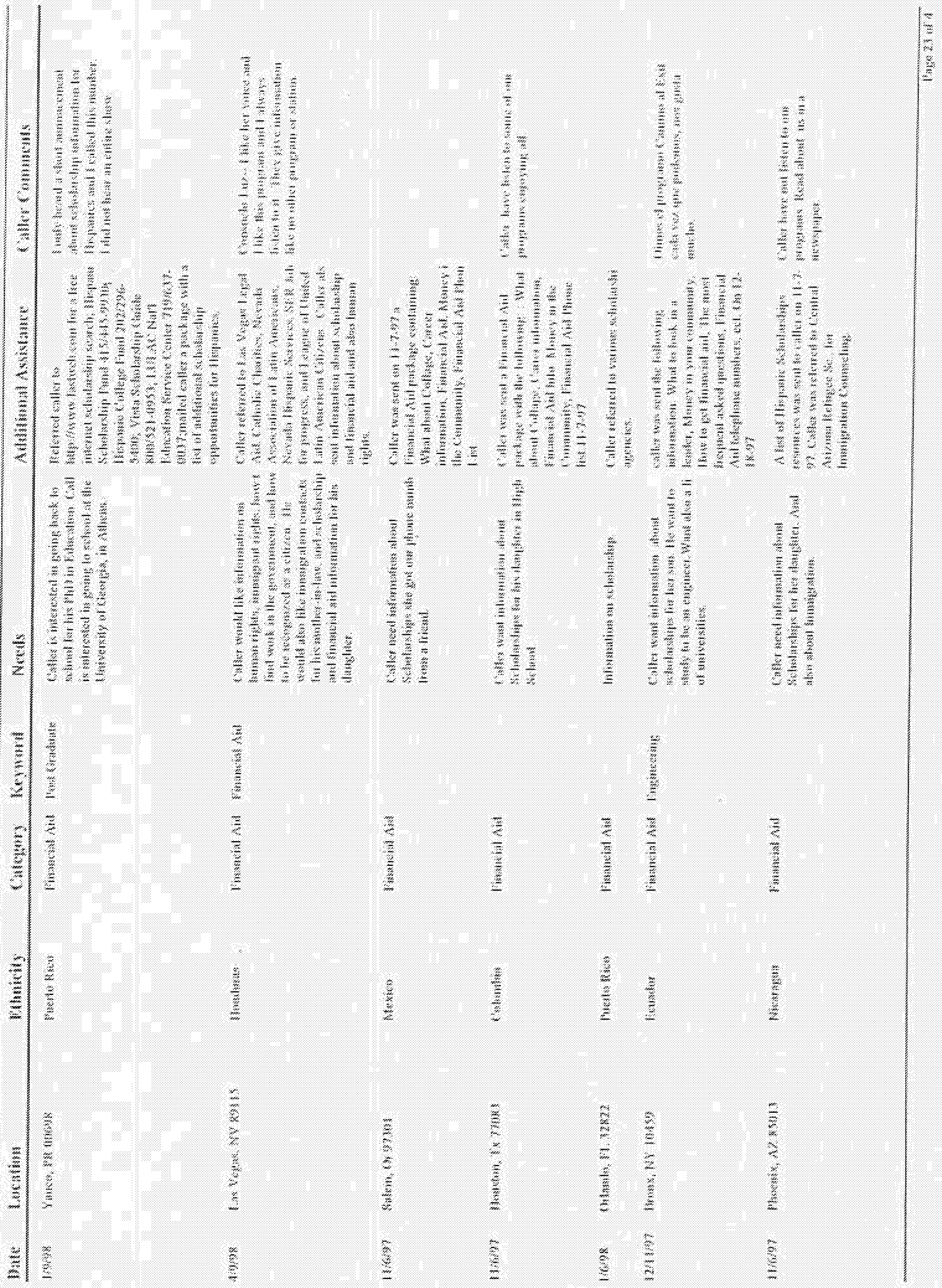




\begin{tabular}{|c|c|c|c|c|}
\hline Ois:10 & 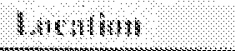 & Bonerists & 6 renesess & 16818 \\
\hline 8448 & 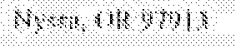 & $81,1,817 \%$ & 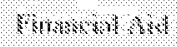 & 8 \\
\hline & 4.8 & & & \\
\hline & & v' & & \\
\hline & . & sis & 48 & \\
\hline & 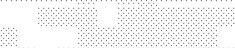 & & 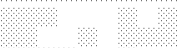 & \\
\hline $17 \% 08$ & 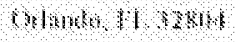 & lescher linse & 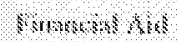 & 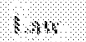 \\
\hline
\end{tabular}

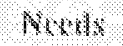

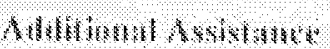

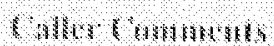

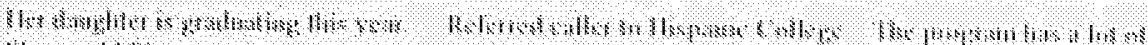

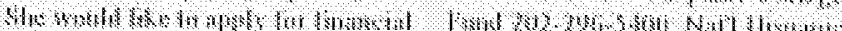

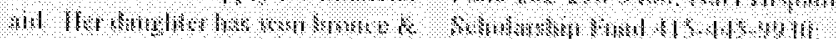

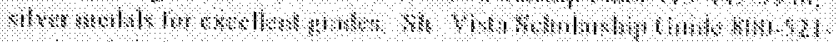

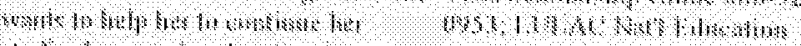

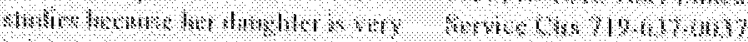

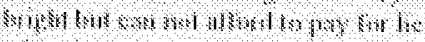

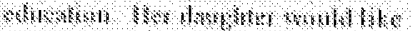

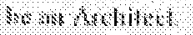

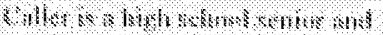
(4)

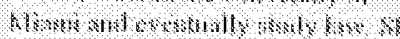

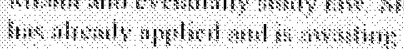

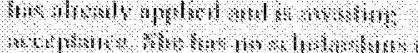

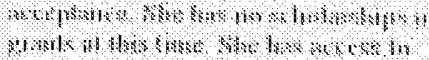

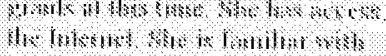

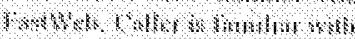
thimblal ais

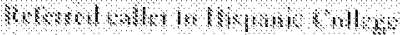

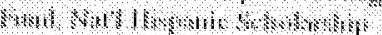

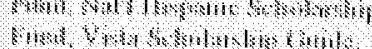

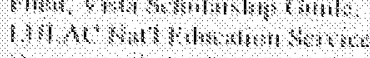

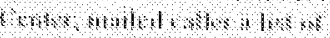

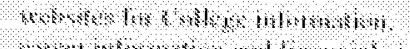

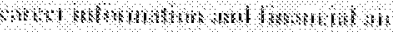

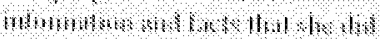

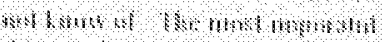

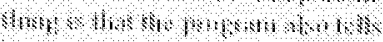

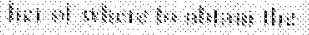

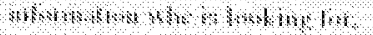

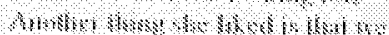

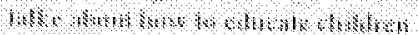

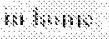

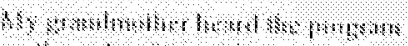

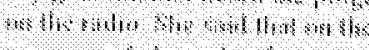
gener:

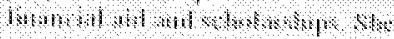

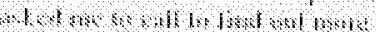

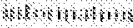

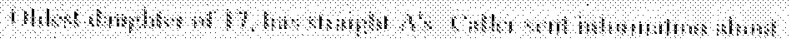

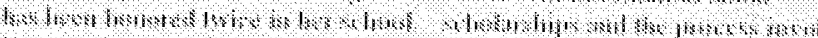

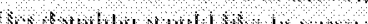
(10.0

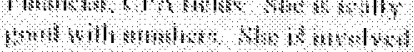

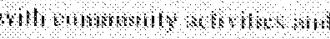

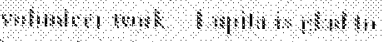

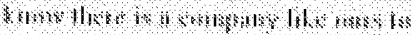

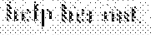

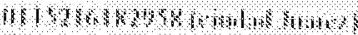

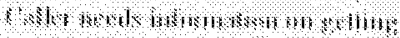

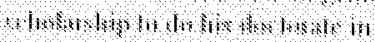
(4)

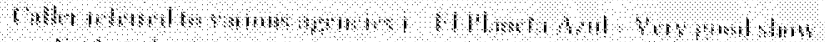

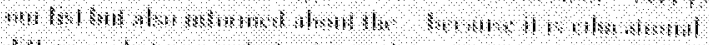

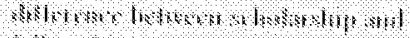

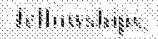

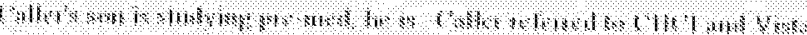

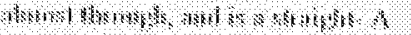

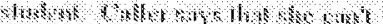

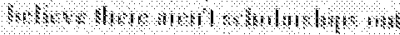

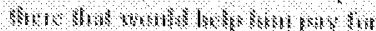

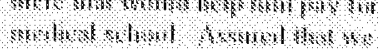

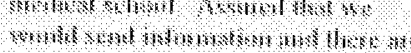

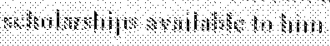

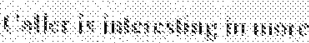

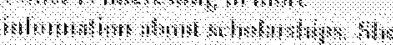

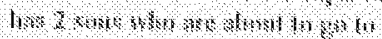

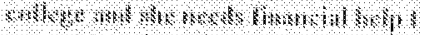

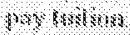

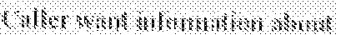

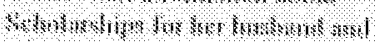
furmall:

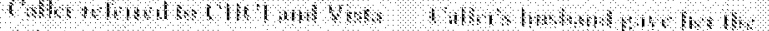

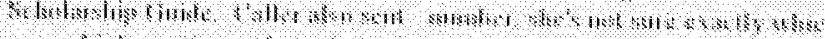

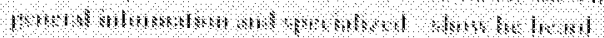

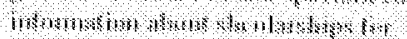

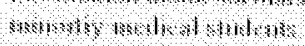

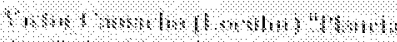
14.

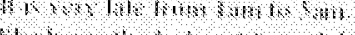

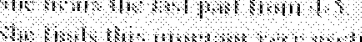

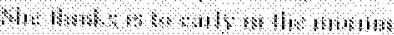

?

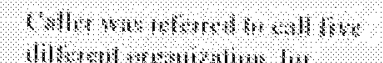

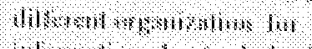

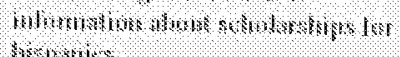
Het:mo

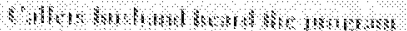

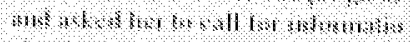

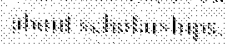

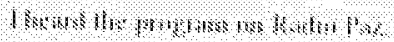

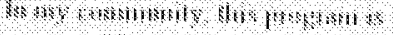

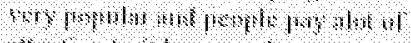

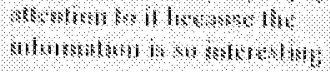




\section{(3)}

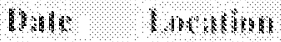

lomiths

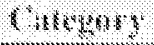

$86,18 \mathrm{rrol}$

virwh

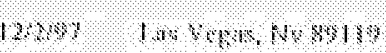

$41 \times 301 \%$ lin:

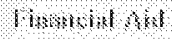

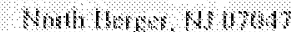

\%orsur:

tisenatian

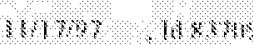

$1110 \%$

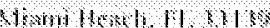

$1 \%$

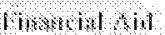

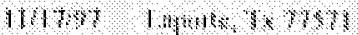

$86 \mathrm{~mm}$

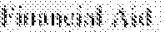

$4.1, \times 1:$

Thmolat het

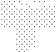

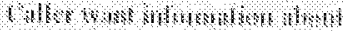

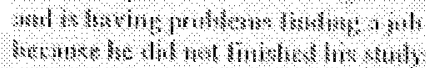

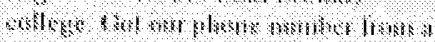

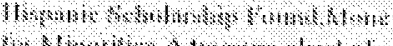
mesters

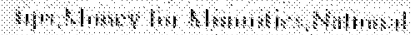

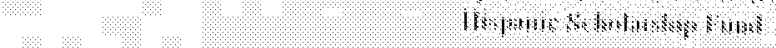

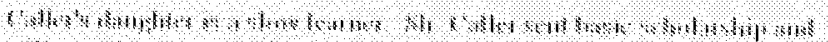

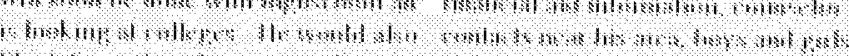

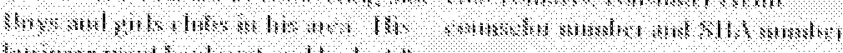

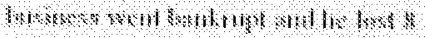

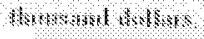

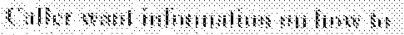

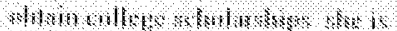

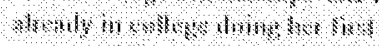

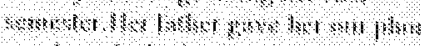

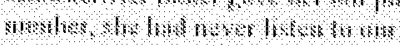
sintr.

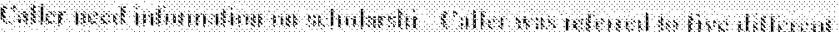

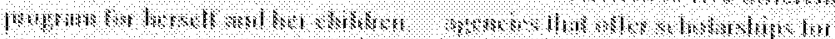

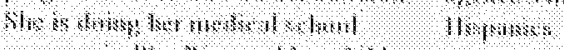

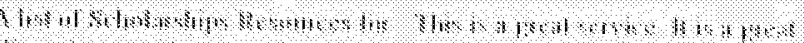

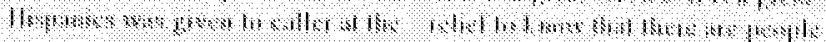

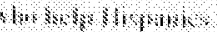

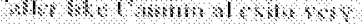

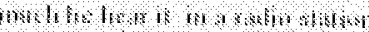

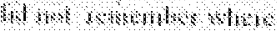

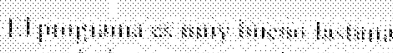

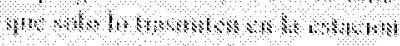

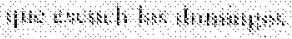

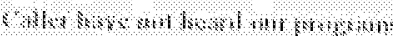
rorimaks

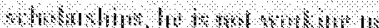

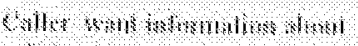

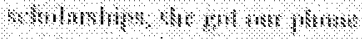

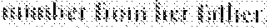

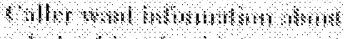

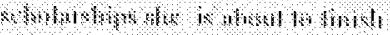

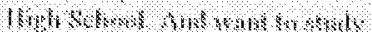

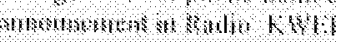

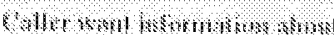

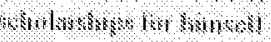

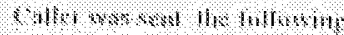

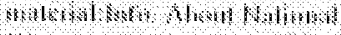

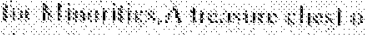

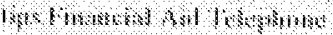

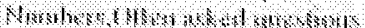

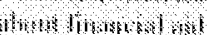

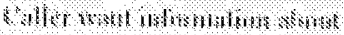

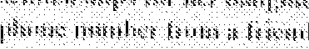

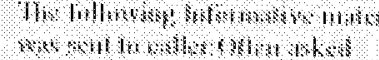

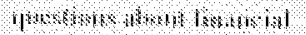
abit. I \&

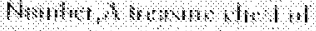

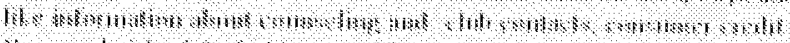
ता:men

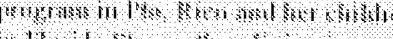

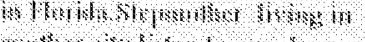

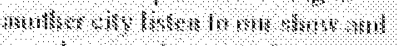

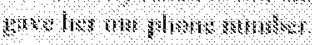

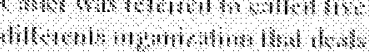
:

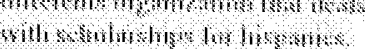




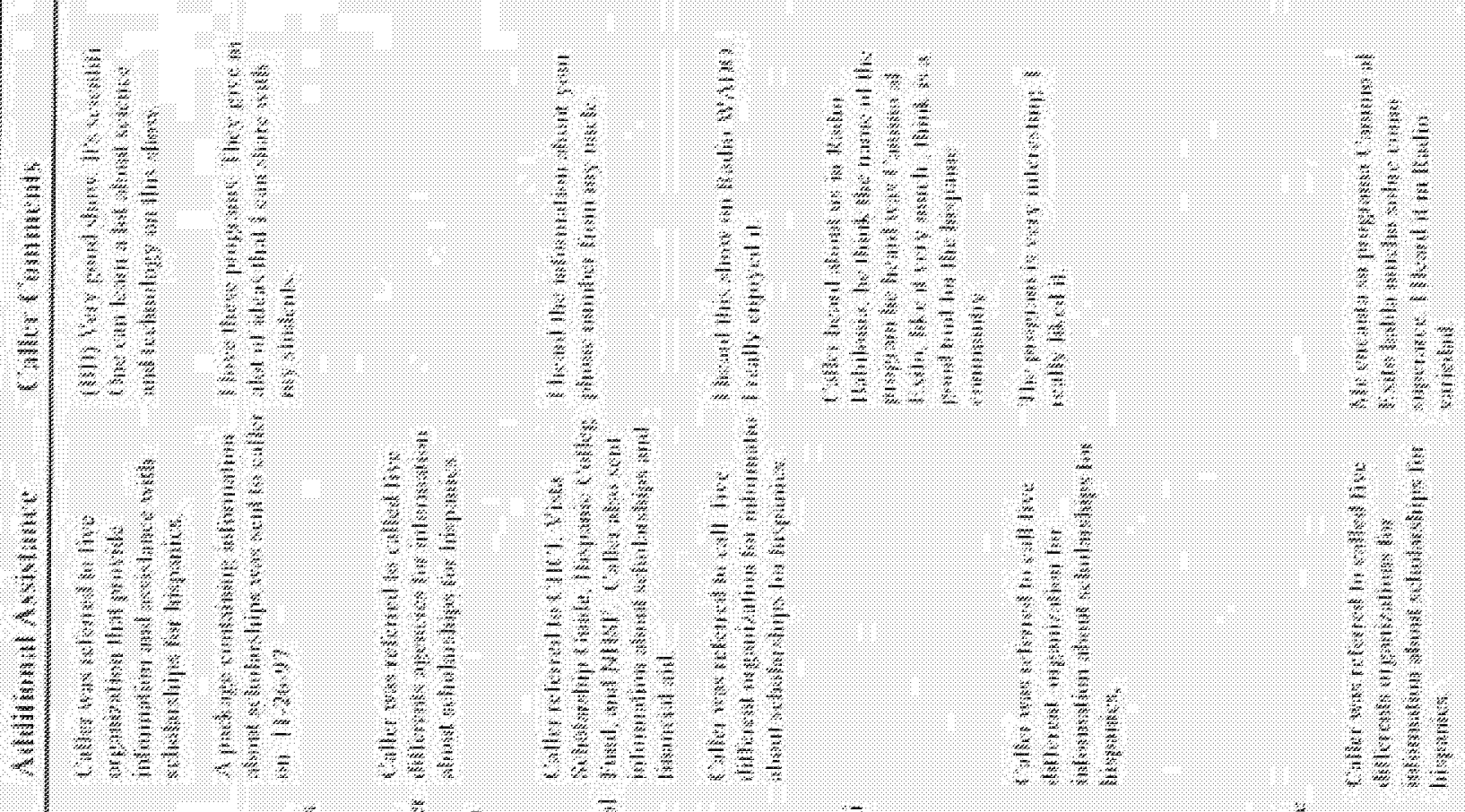
MIIIUM

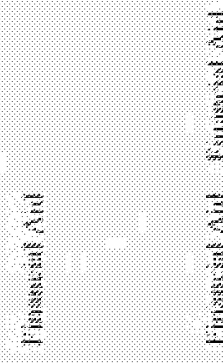

$\frac{3}{3}$
$\frac{3}{2}$
$\frac{3}{3}$
$\frac{7}{4}$
$\frac{7}{2}$

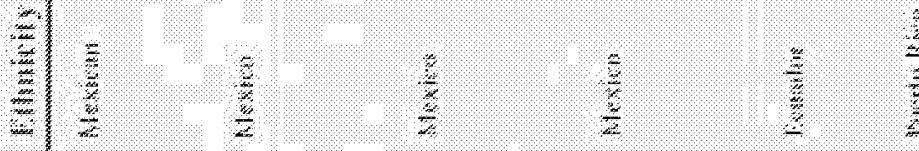
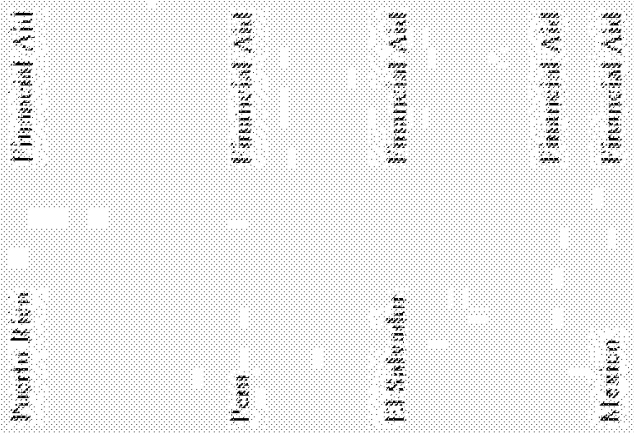

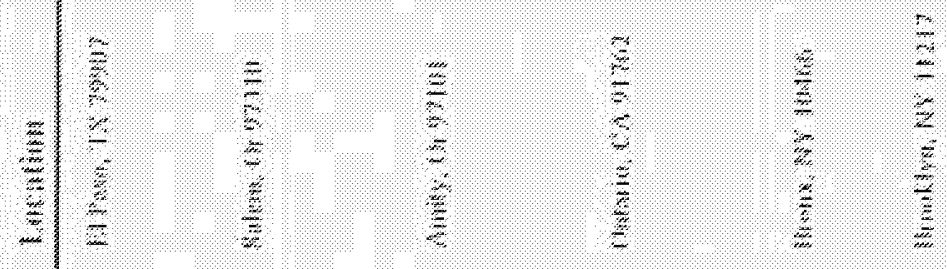

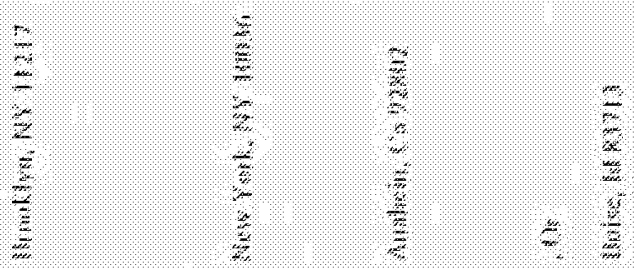

$\frac{2}{2} \quad 3$

$\stackrel{5}{2}$

$\frac{8}{2} \quad \frac{3}{2}$

$\frac{5}{3} \quad 2$

$\frac{25}{2}$ 


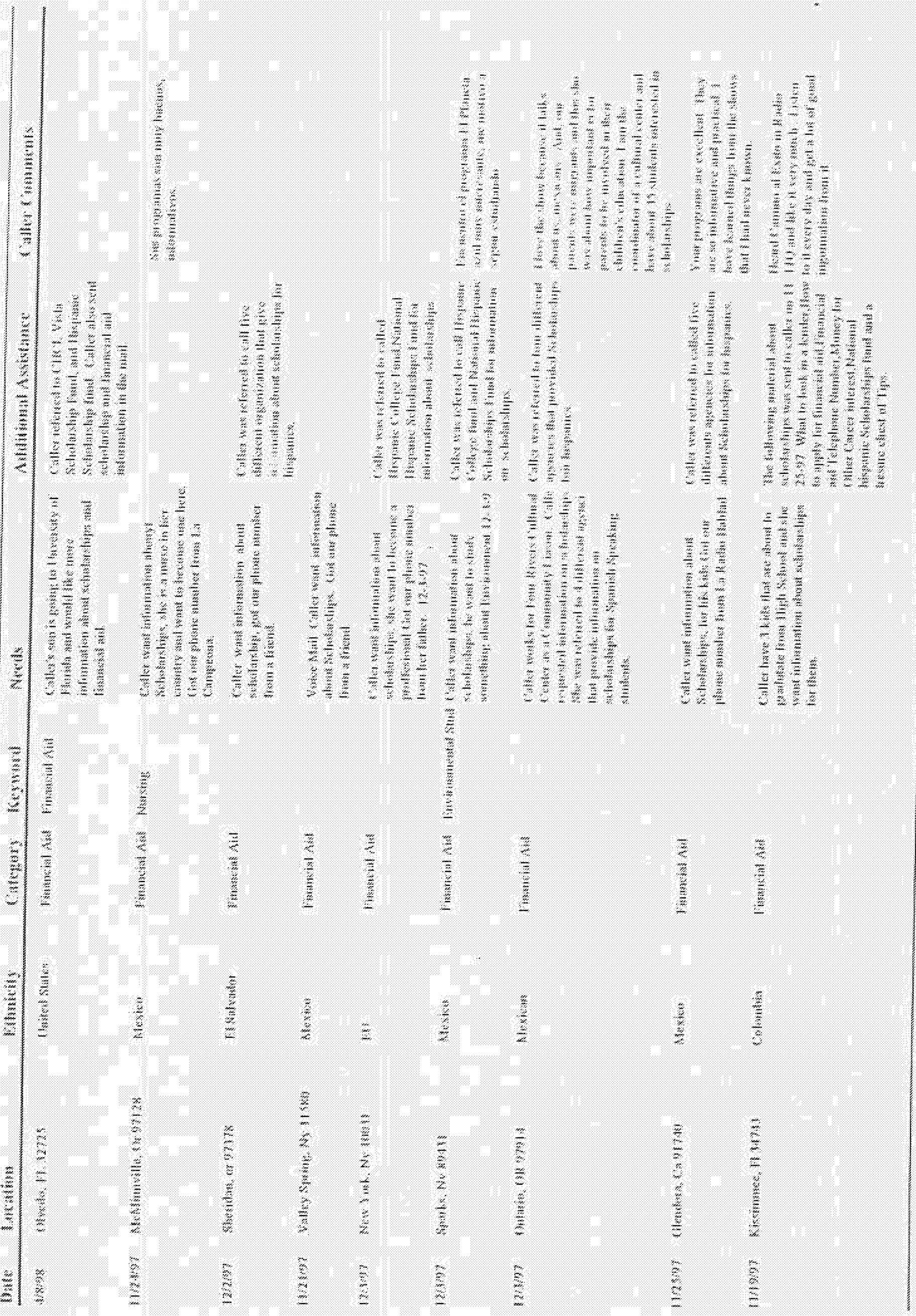




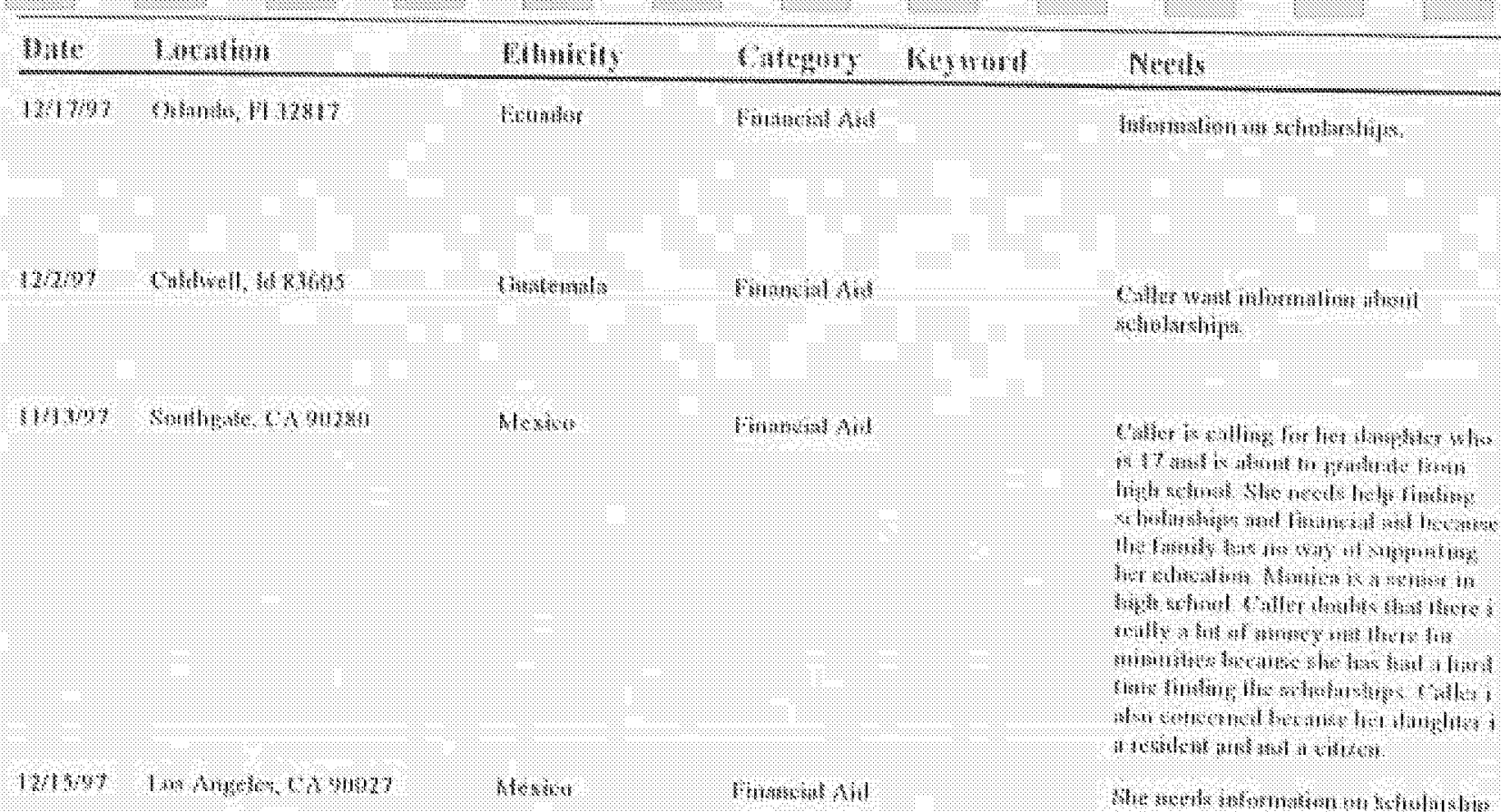

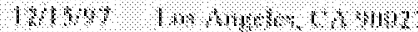

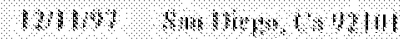

Meskm:

Asiratiki

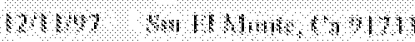

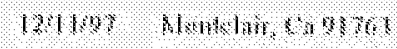

$14 \times 6 \times$

818

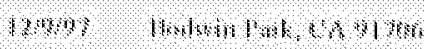

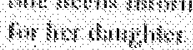

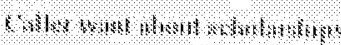

tiramen ail ate?

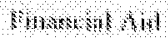

Fammene k kill

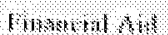
blinkerstive

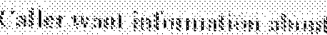

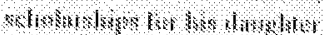

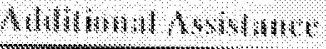

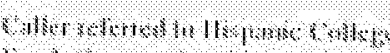

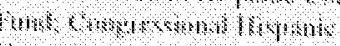

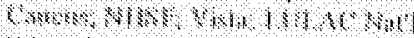

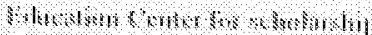

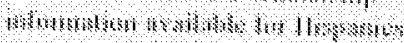

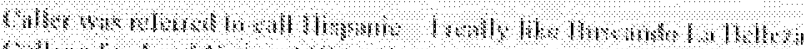

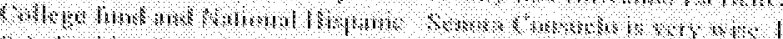

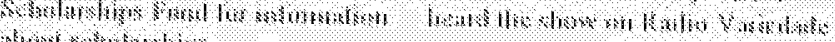
shosit keswhinghijes

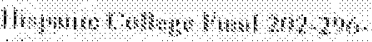

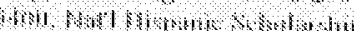

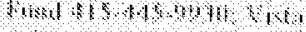

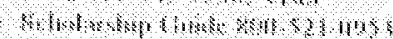
10,101017

2 ! 1

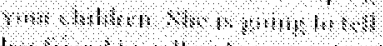

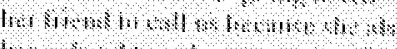

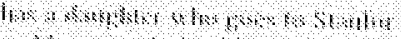

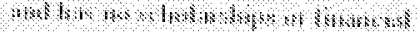

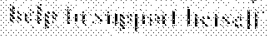

ํ.

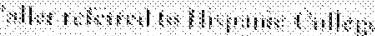

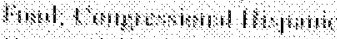

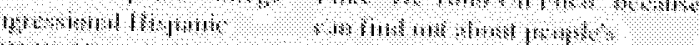
1.6

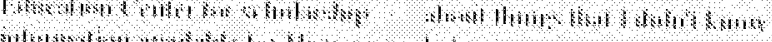

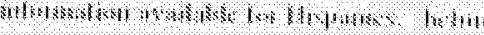

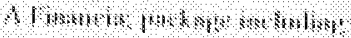

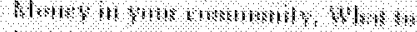

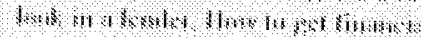

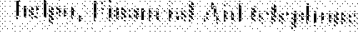

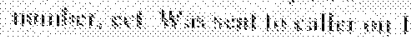
(10.89

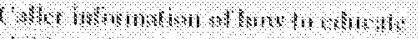

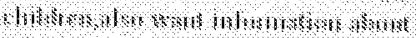

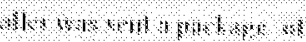

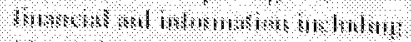

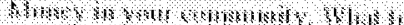

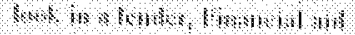

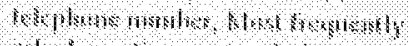

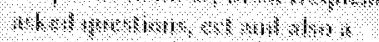

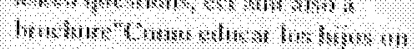
12189

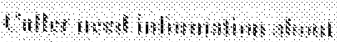

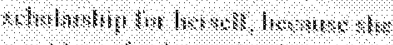

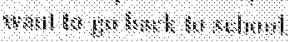

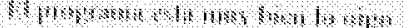

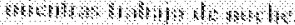

(4)

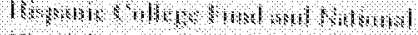

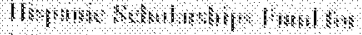
Cle \&

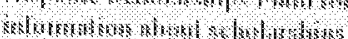




\begin{tabular}{|c|c|c|c|c|c|c|}
\hline Yhe: & 18101110 & 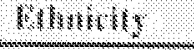 & Cargurs & 8xegratal & Menols & 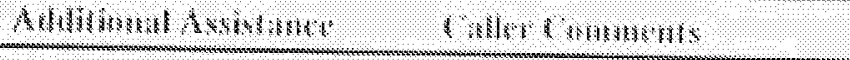 \\
\hline 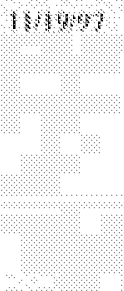 & 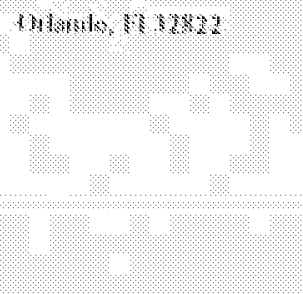 & 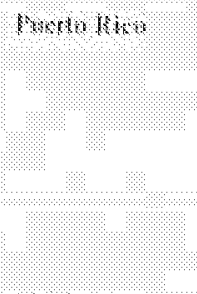 & 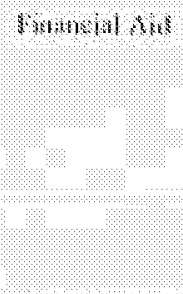 & 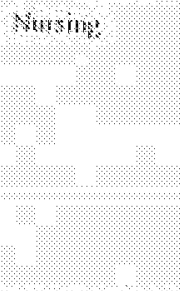 & 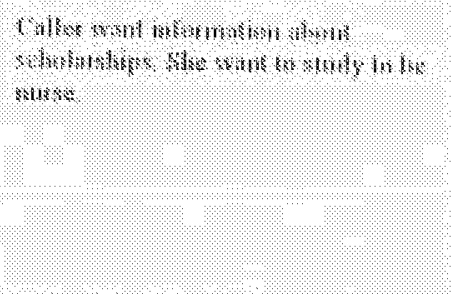 & 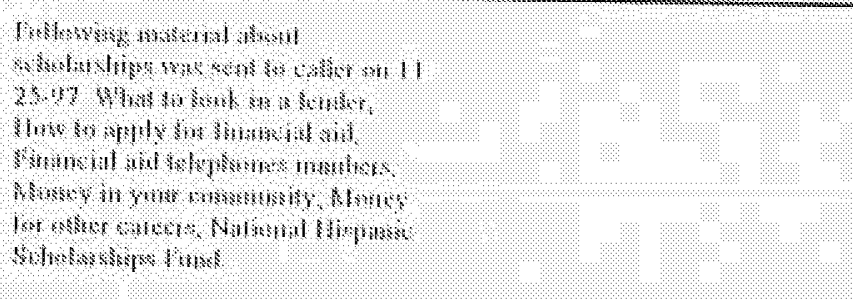 \\
\hline 46,45 & 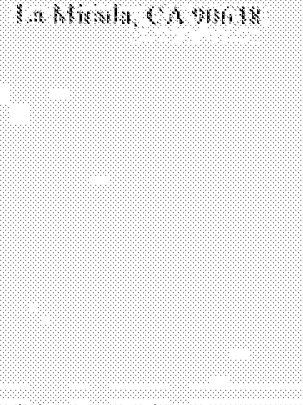 & $41 \mathrm{~s} / \mathrm{ml}$ & linamerest onet & lomenter hos & 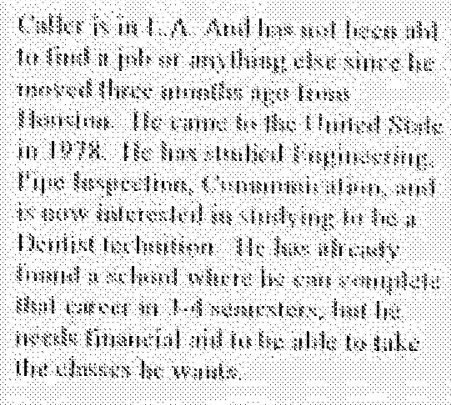 & 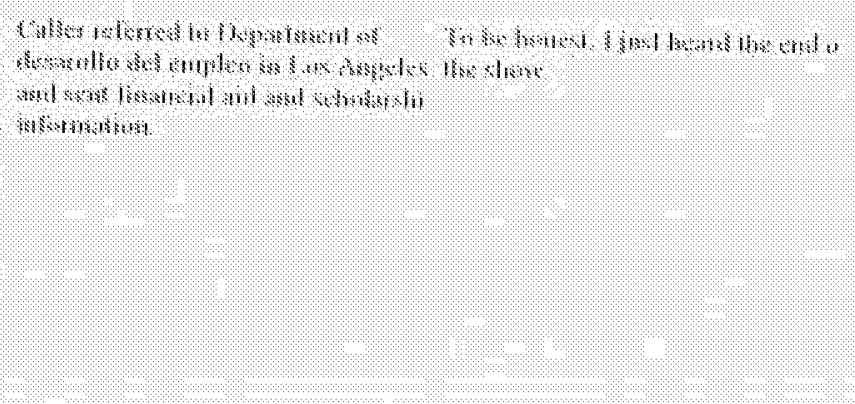 \\
\hline $10 \% 40$ & 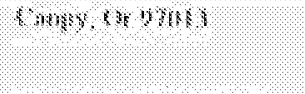 & menkr & Bnmenke aks & & 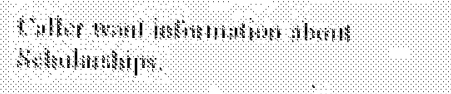 & \\
\hline $141 \%$ & 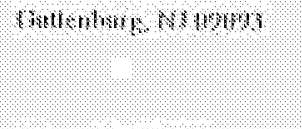 & 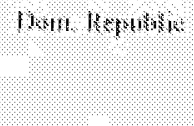 & lisesnctil hitil & & 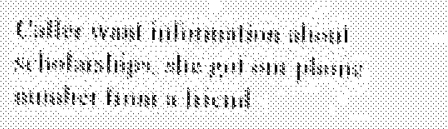 & \\
\hline $12 \% 6$ & 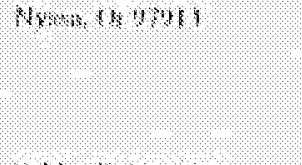 & 101 & $84018 \mathrm{~min} / \mathrm{sin}$ & & 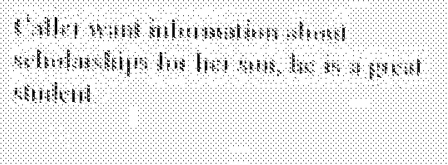 & 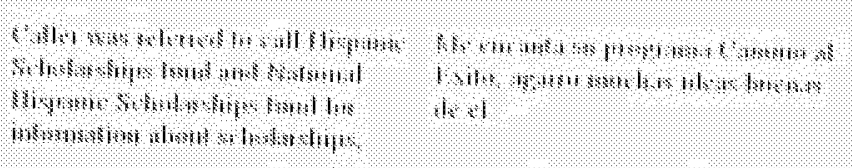 \\
\hline $1411 \% 17$ & m: & 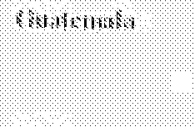 & 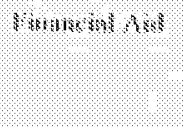 & & 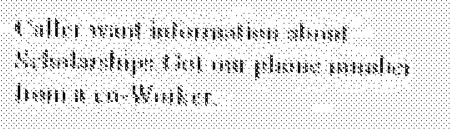 & \\
\hline $10 \% 9$ & 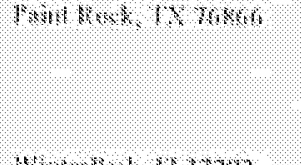 & 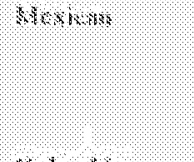 & 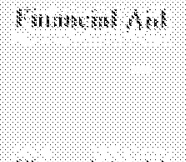 & & 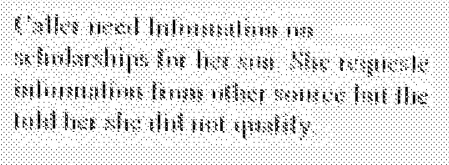 & 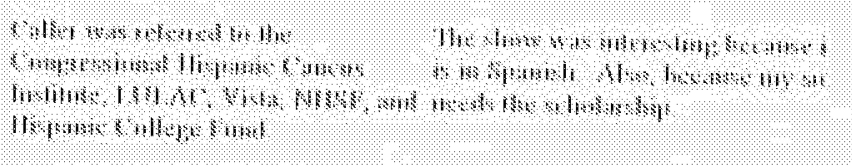 \\
\hline $1200 \%$ & 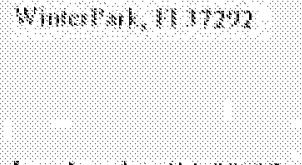 & $1640118 \mathrm{~m}$ & $8 \mathrm{H} \times 8 \mathrm{C} \times \mathrm{Mil}$ & & 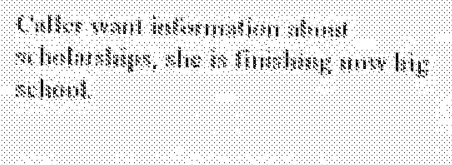 & 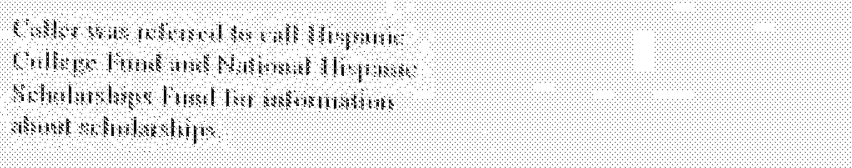 \\
\hline Shorer & 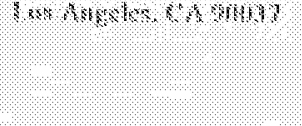 & $408 \mathrm{kn}$ & 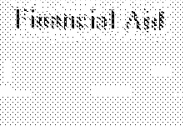 & & 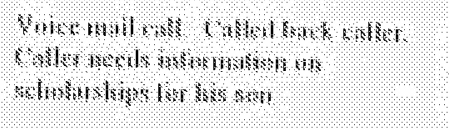 & 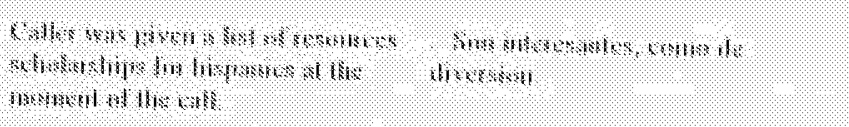 \\
\hline
\end{tabular}




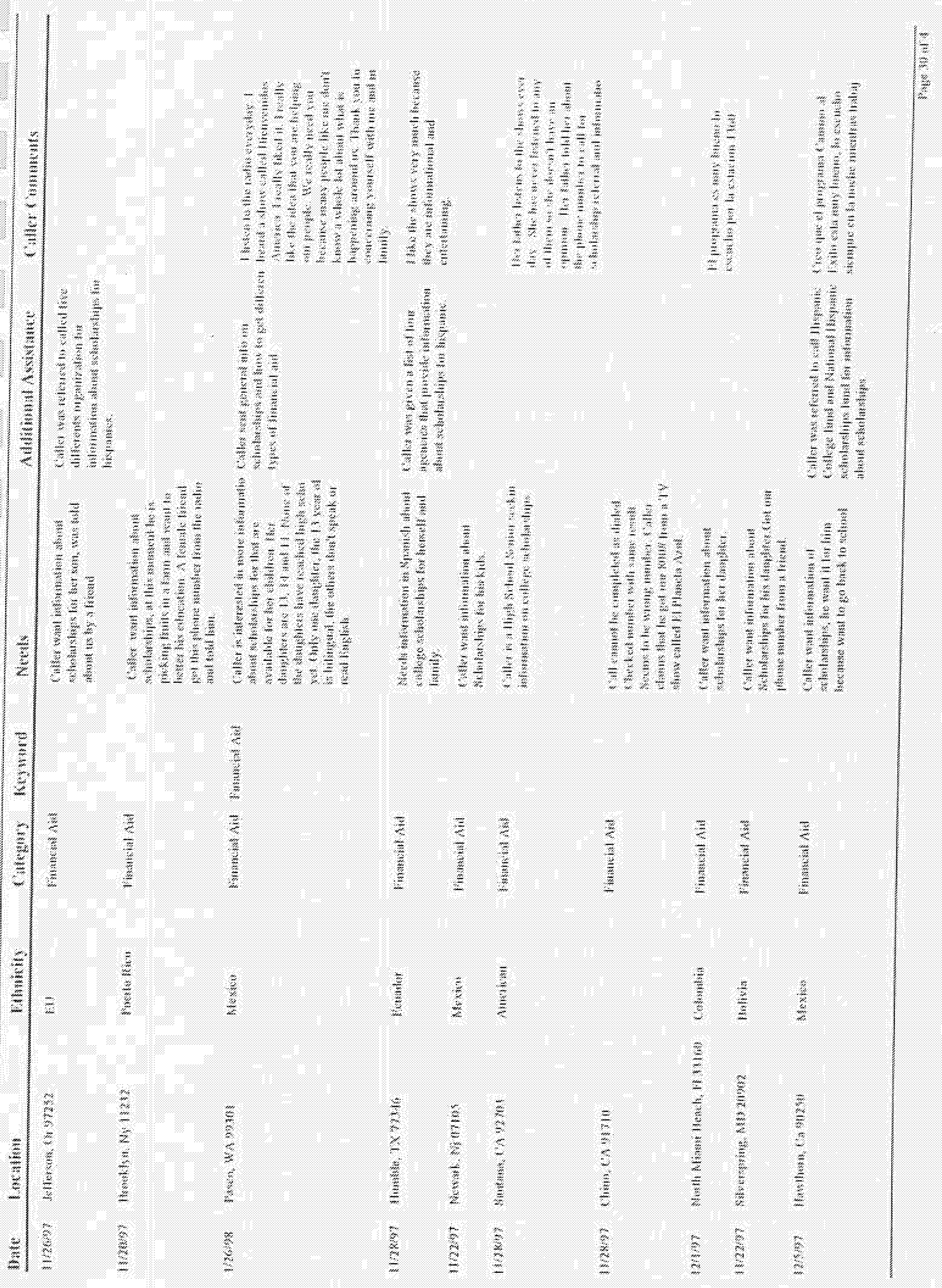




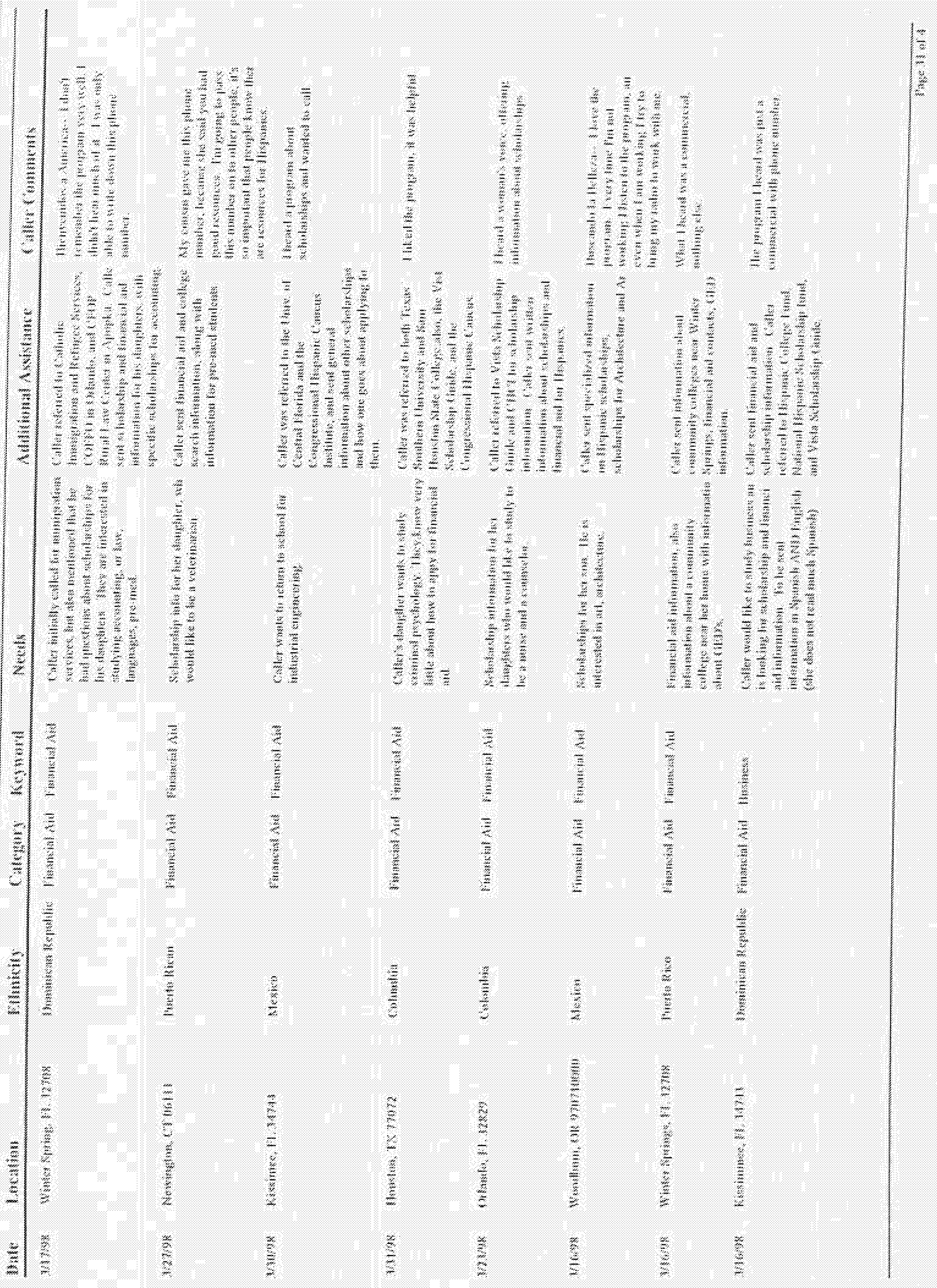



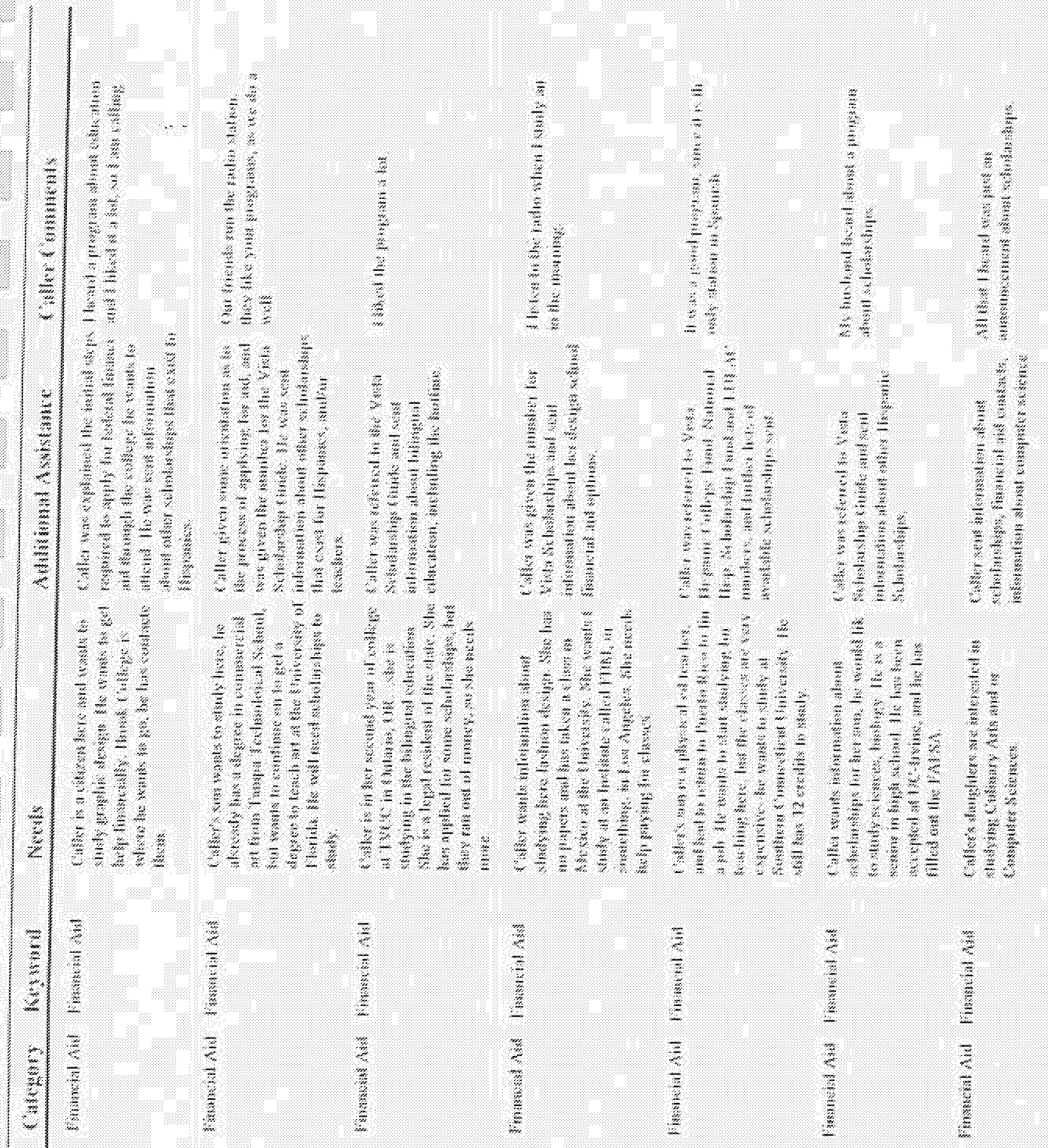

妾
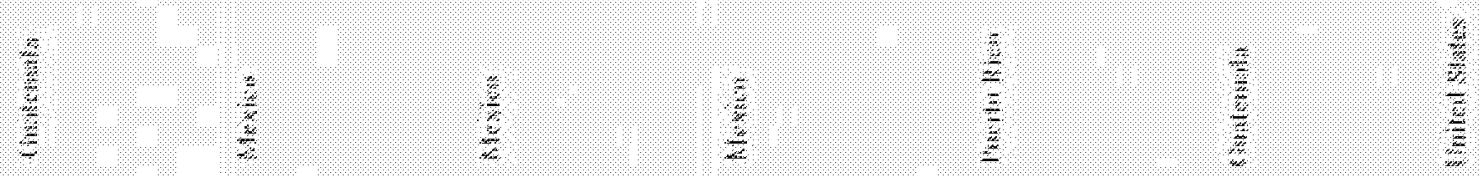

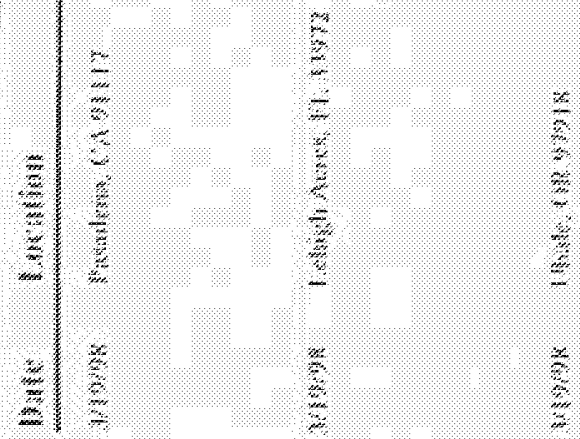

3

3
3
3
3

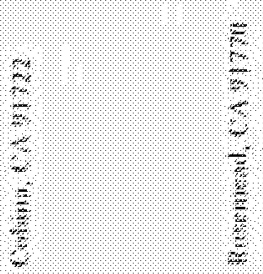

2

8

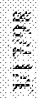



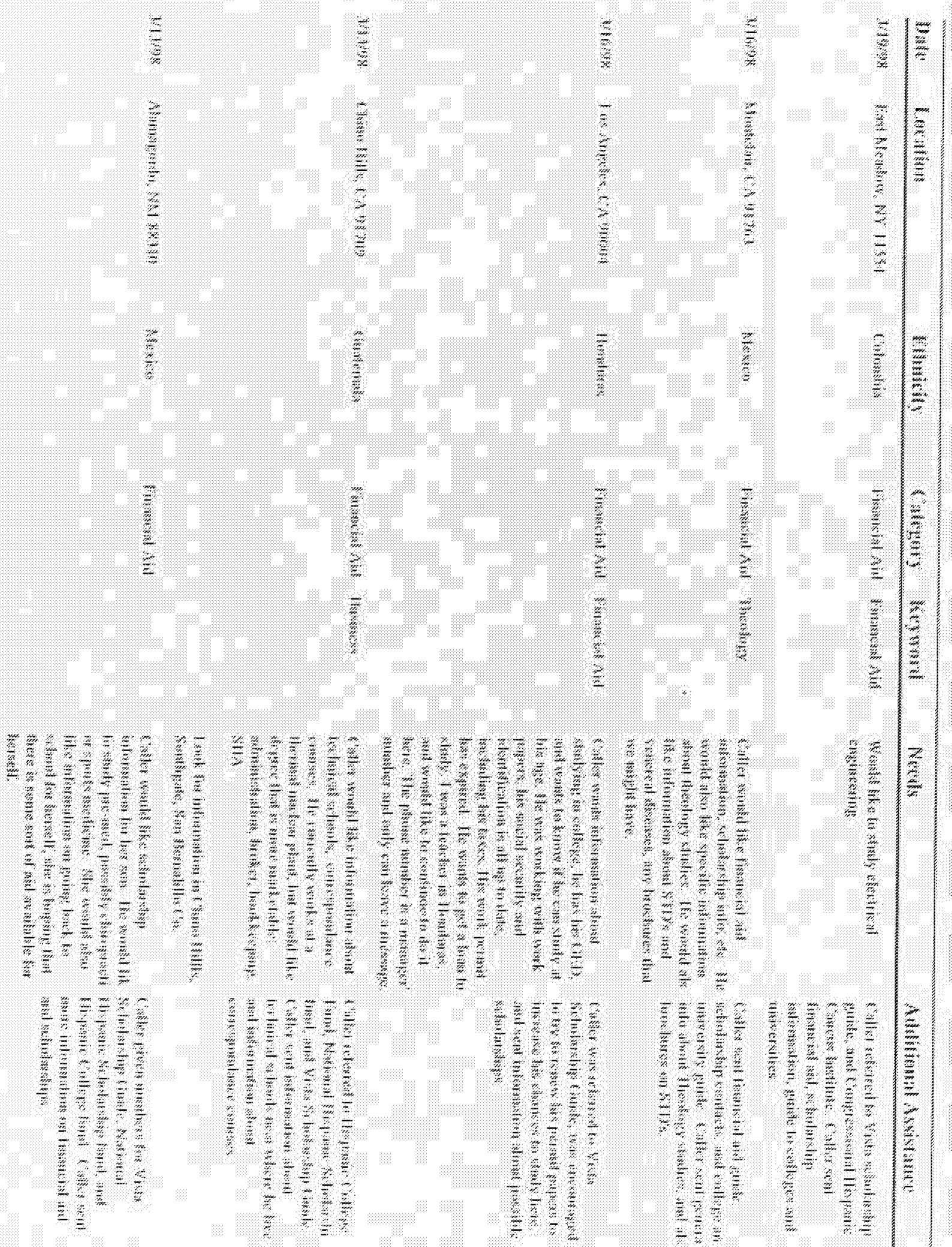

$\Rightarrow \Rightarrow z=$

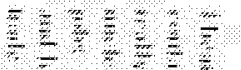

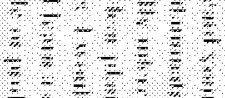

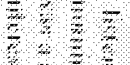

z $=38$ ?

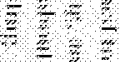

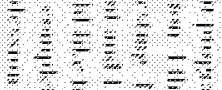

$\frac{7}{2} \div=3$

$\frac{7}{2}=\frac{1}{2}=\frac{2}{4}$

$=\frac{7}{2}=\frac{7}{2}$

$=$

$3=3$

2

E

5

$3 \%$

$\frac{2}{3}=2$

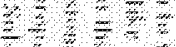

$z=2$.

$\frac{3}{3}=\overline{2} ?$

$4-\frac{1}{2}=0$

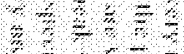

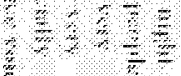




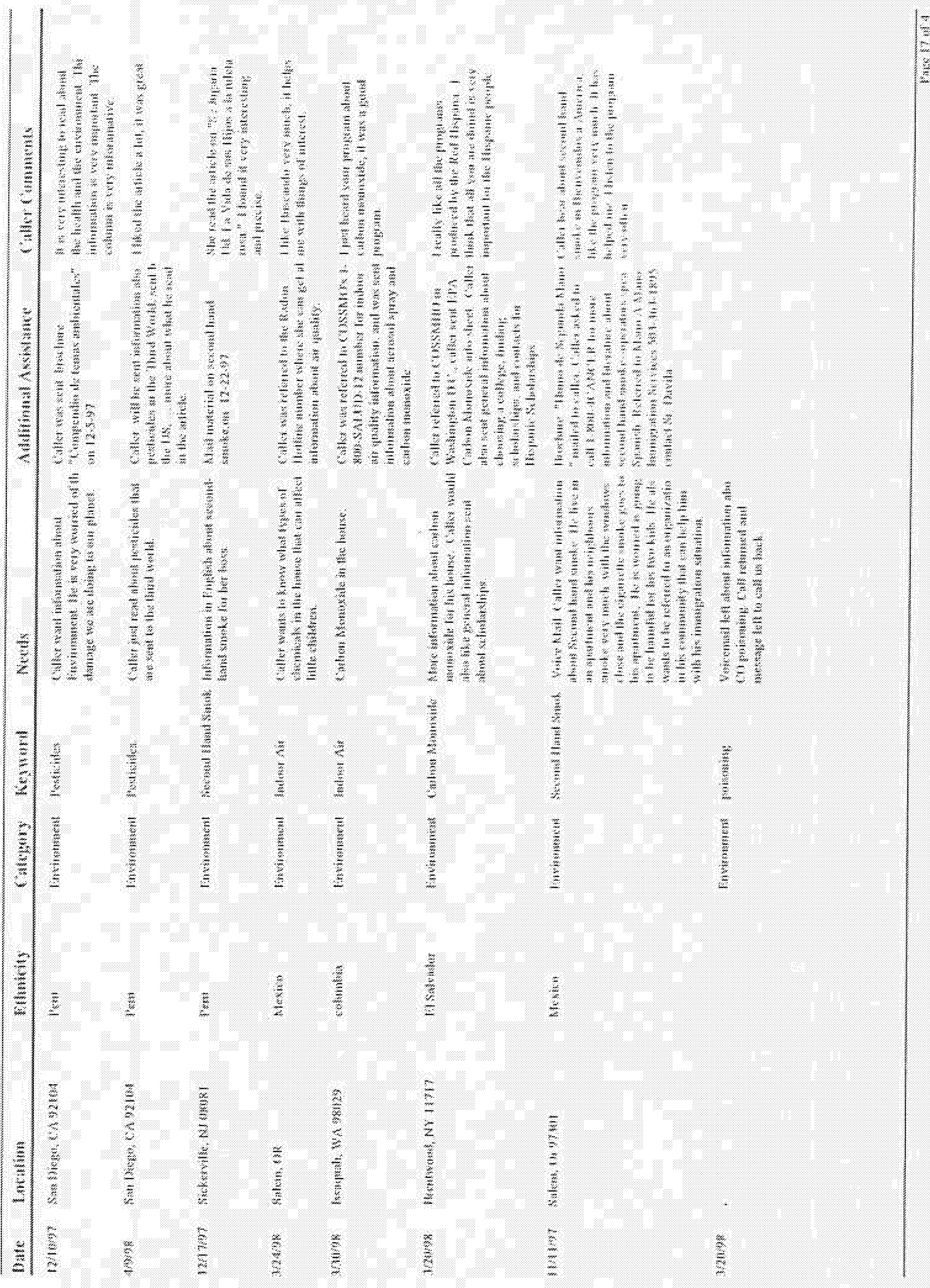




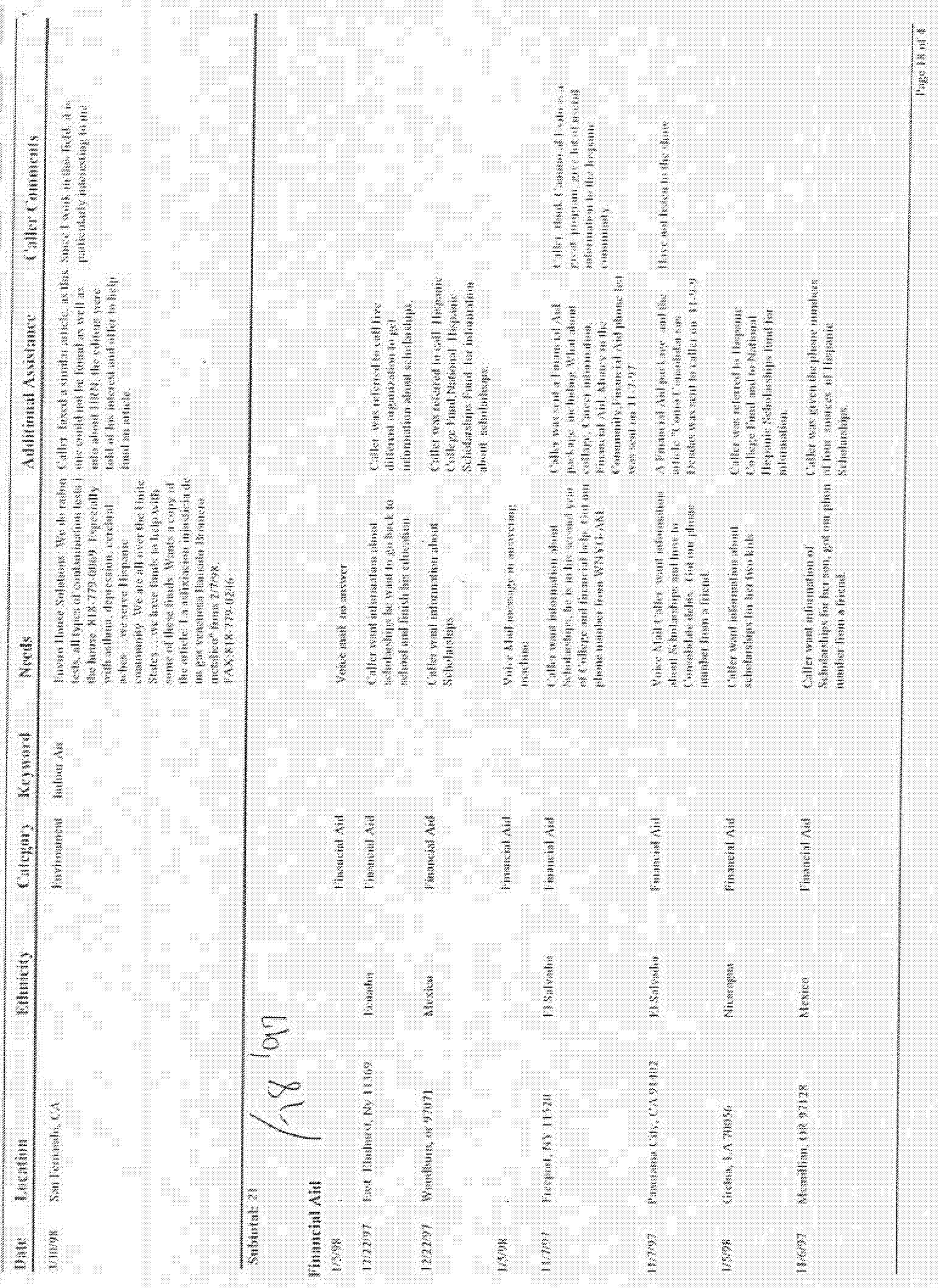




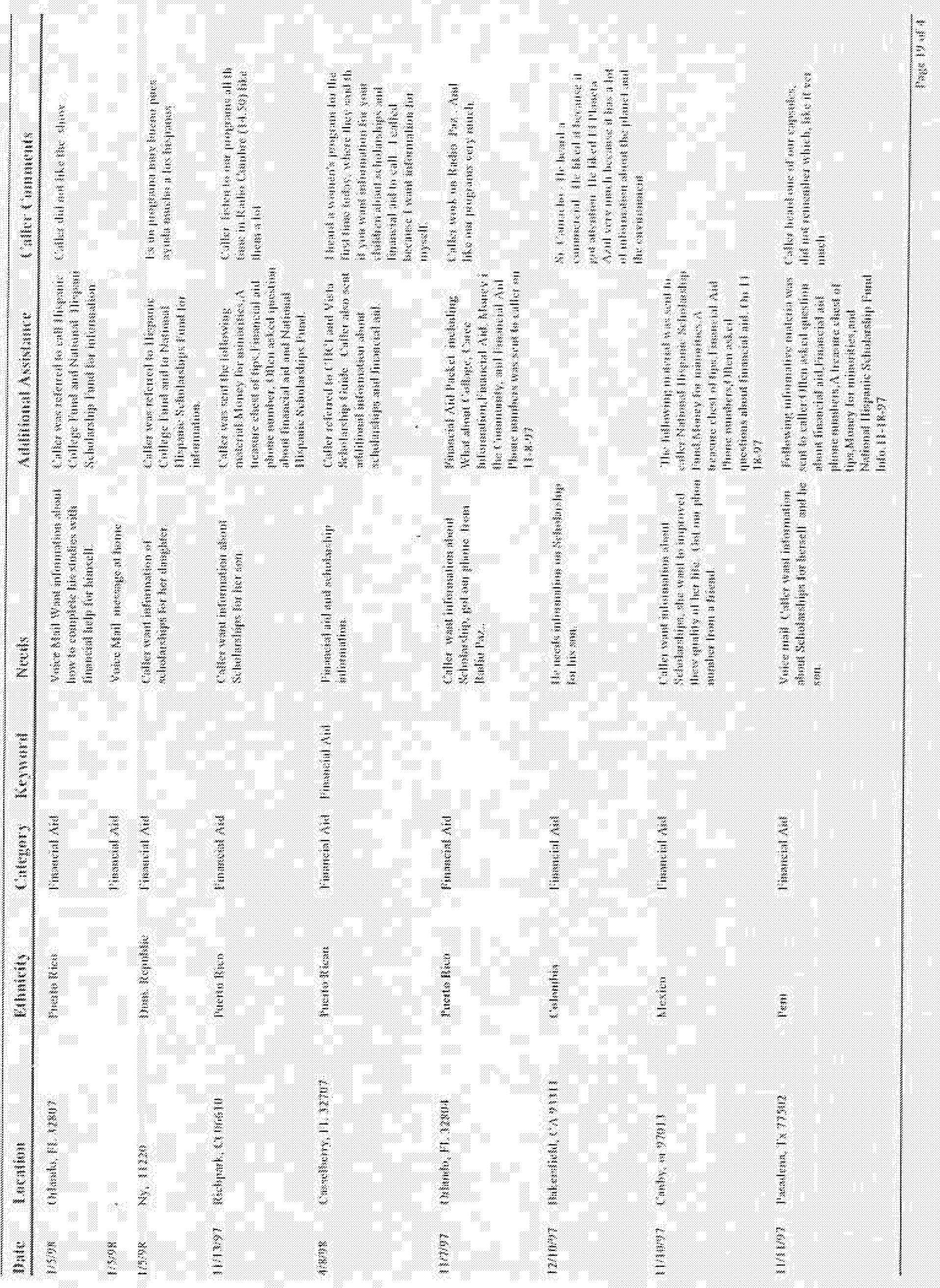





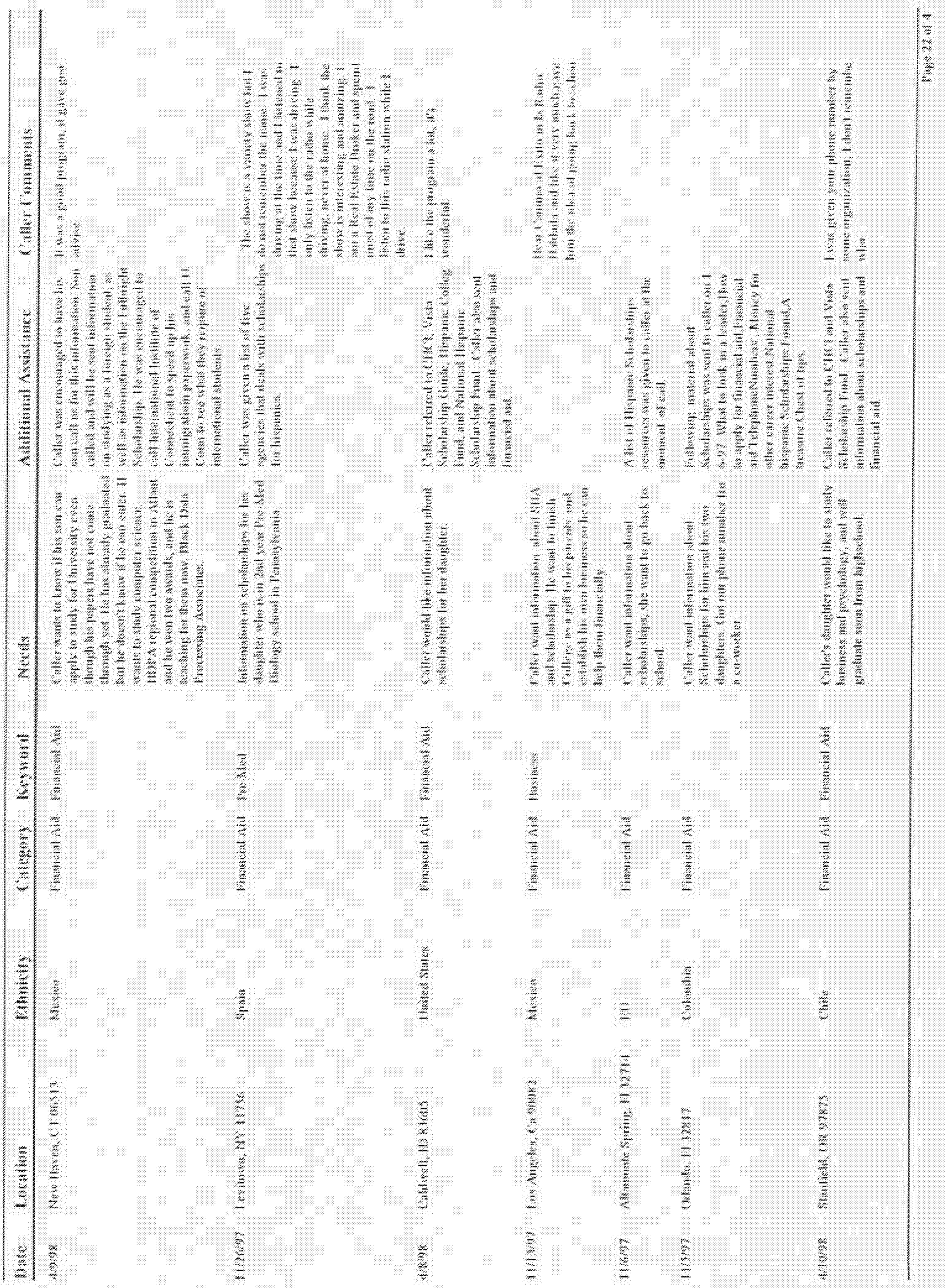




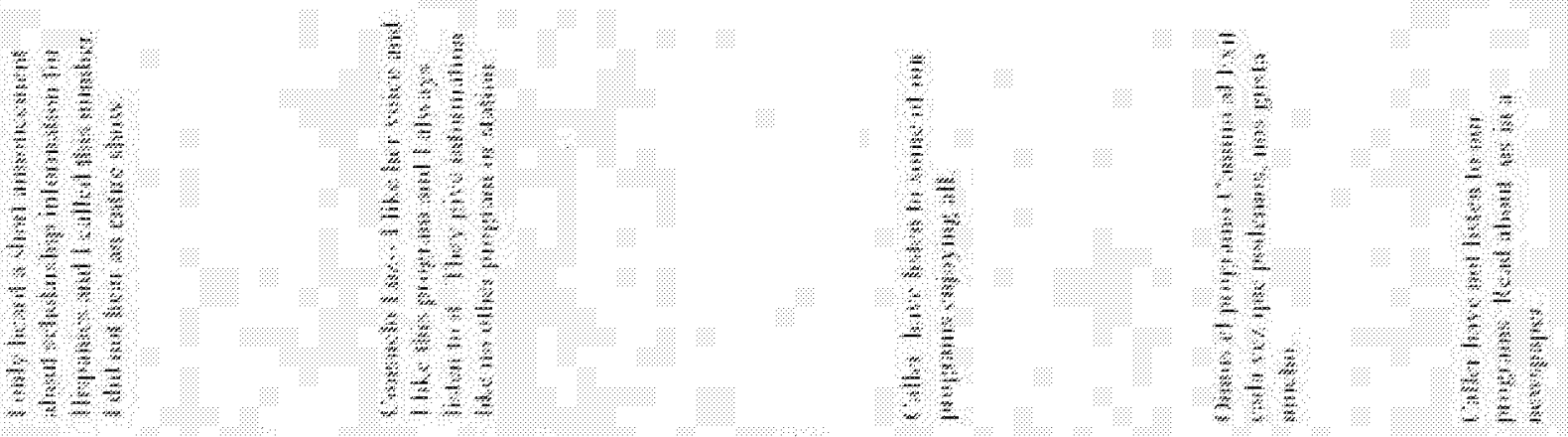

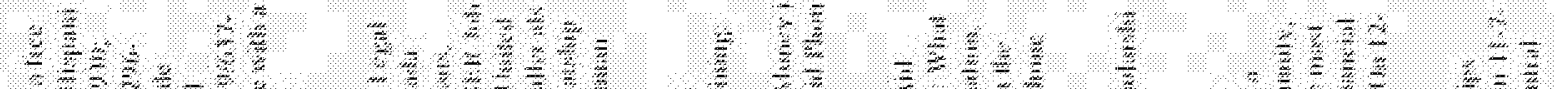

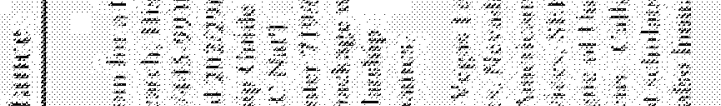

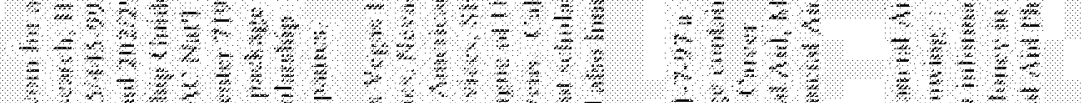

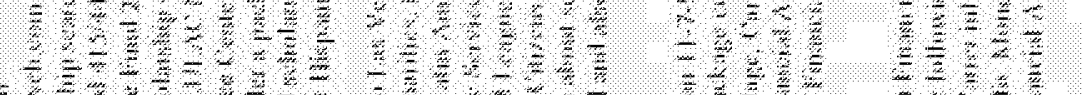

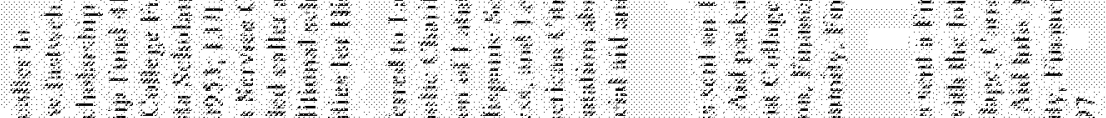

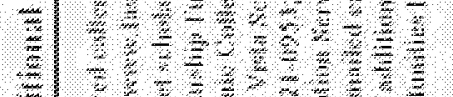

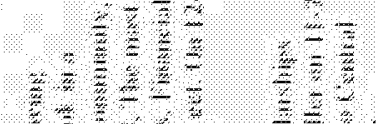

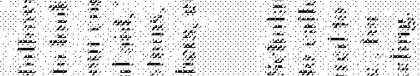

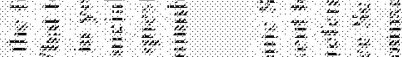

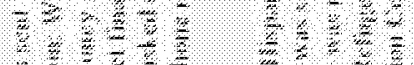

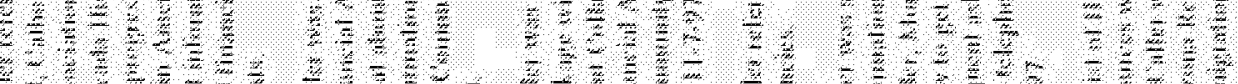

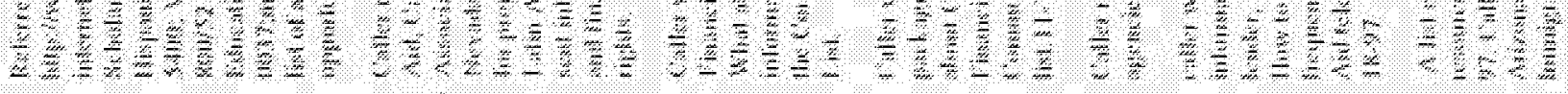

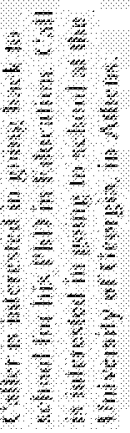

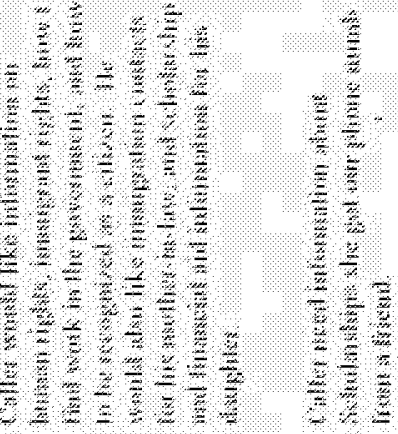

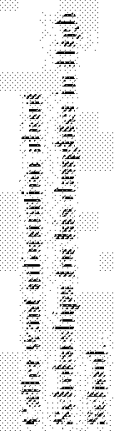

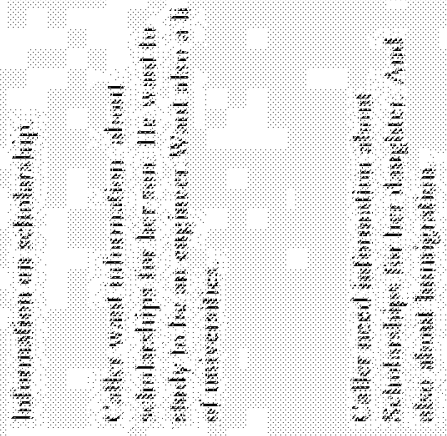

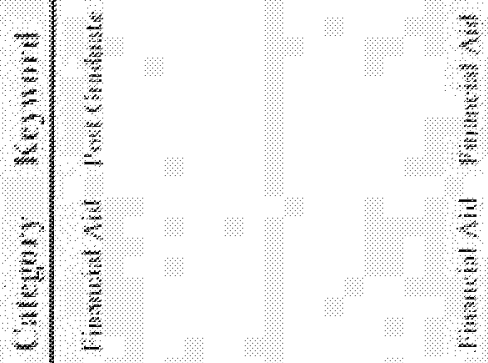
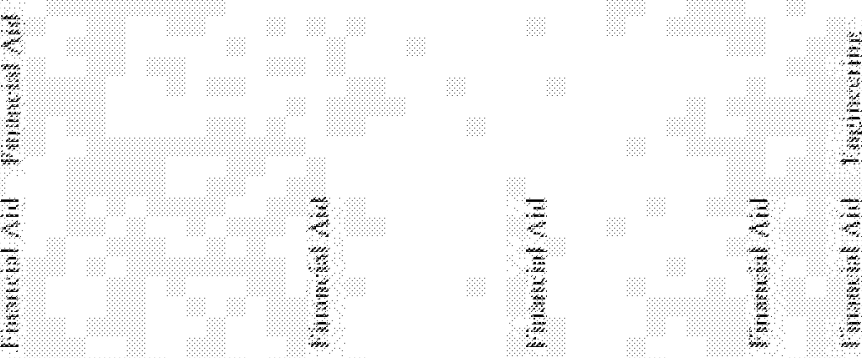

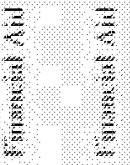

$\frac{3}{3}$

$=$
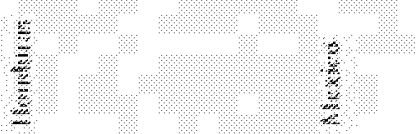

$\begin{array}{llll}\frac{3}{3} & \frac{3}{3} \\ \frac{3}{2} & & \frac{3}{3}\end{array}$

$\frac{3}{3}$

$=$

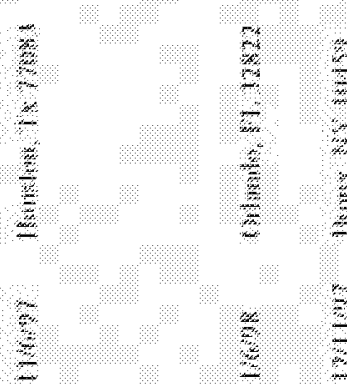

$\frac{3}{3}$
$\frac{3}{4}$
$\frac{2}{2}$
2
$=$ 


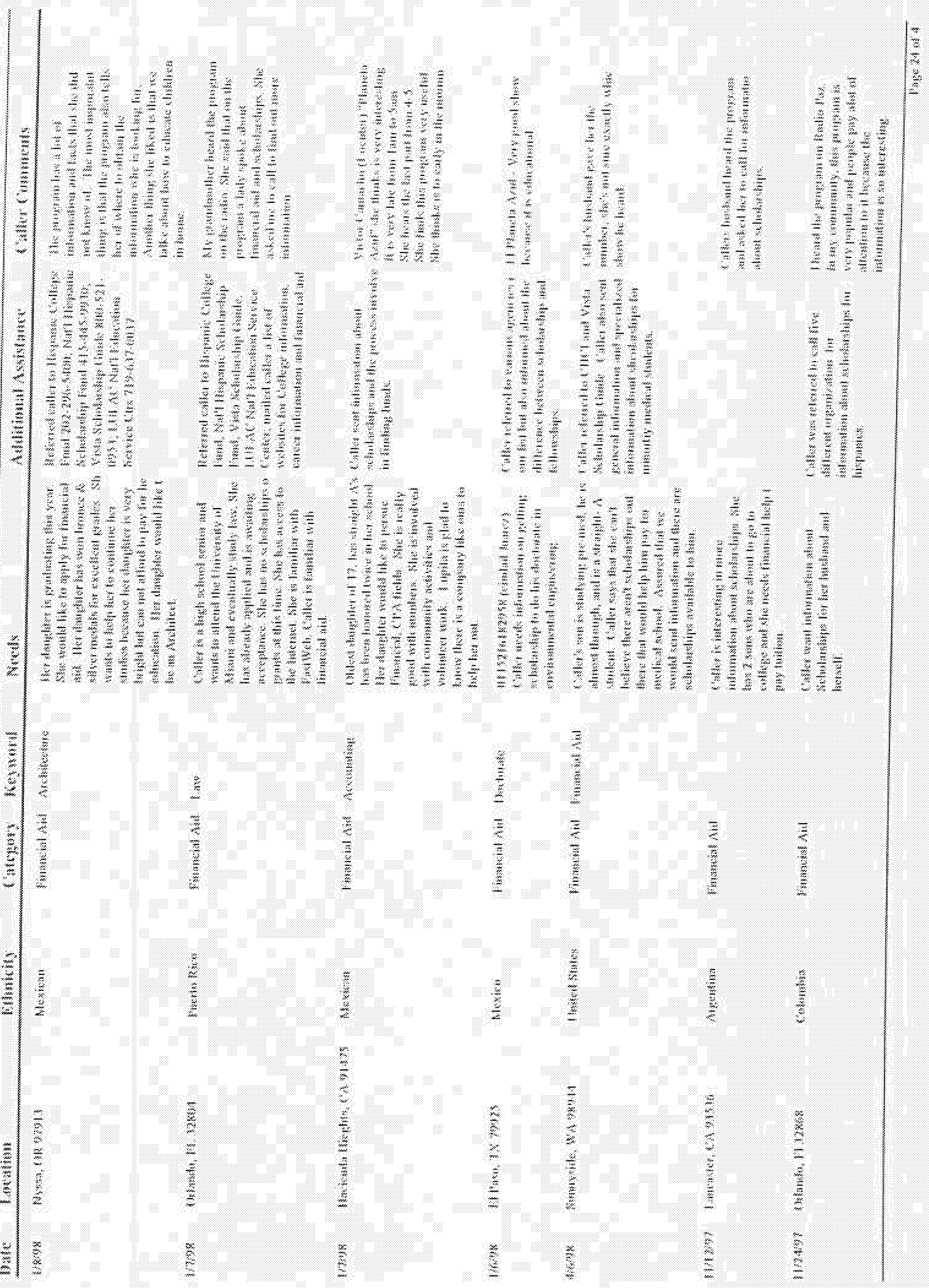




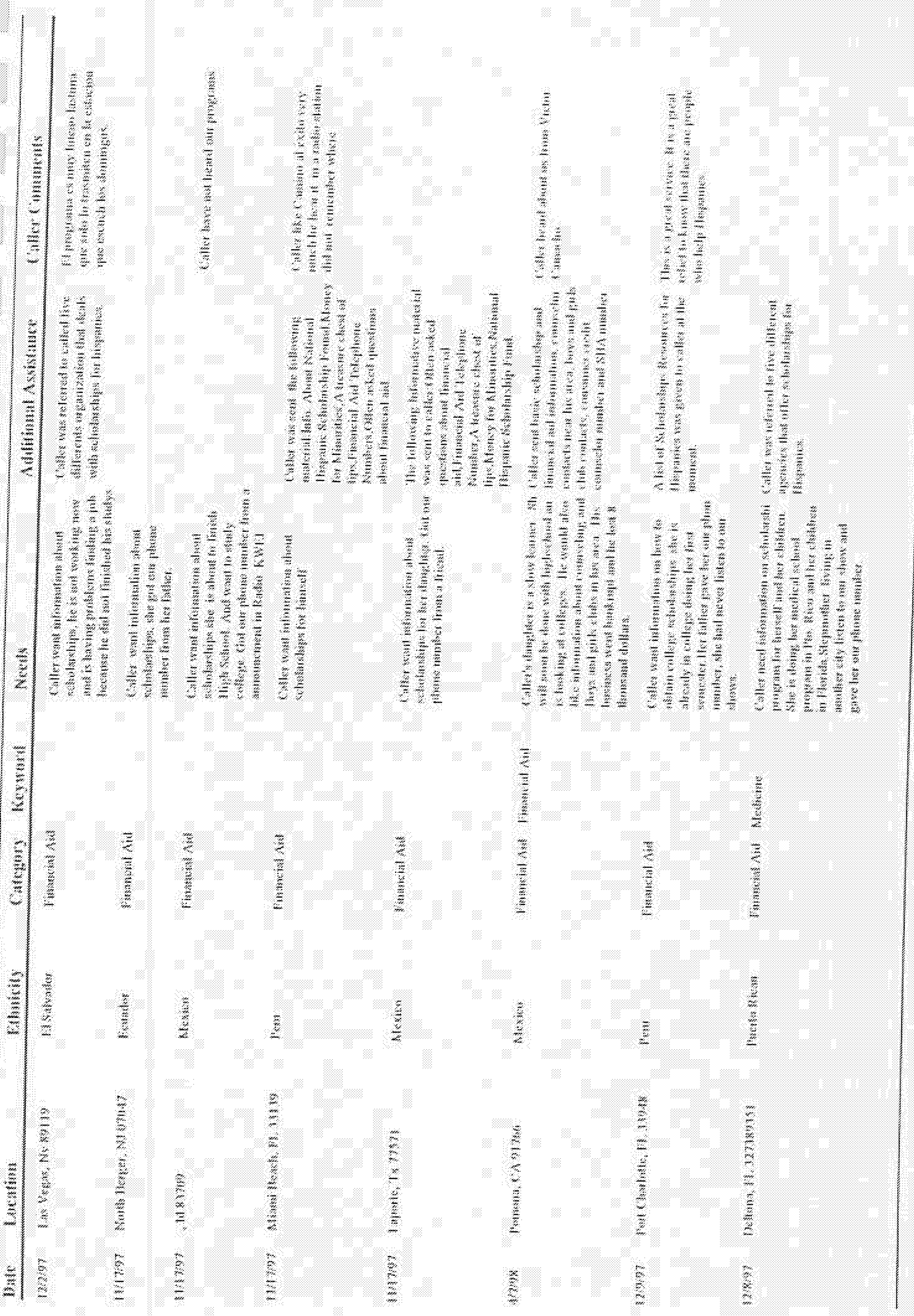






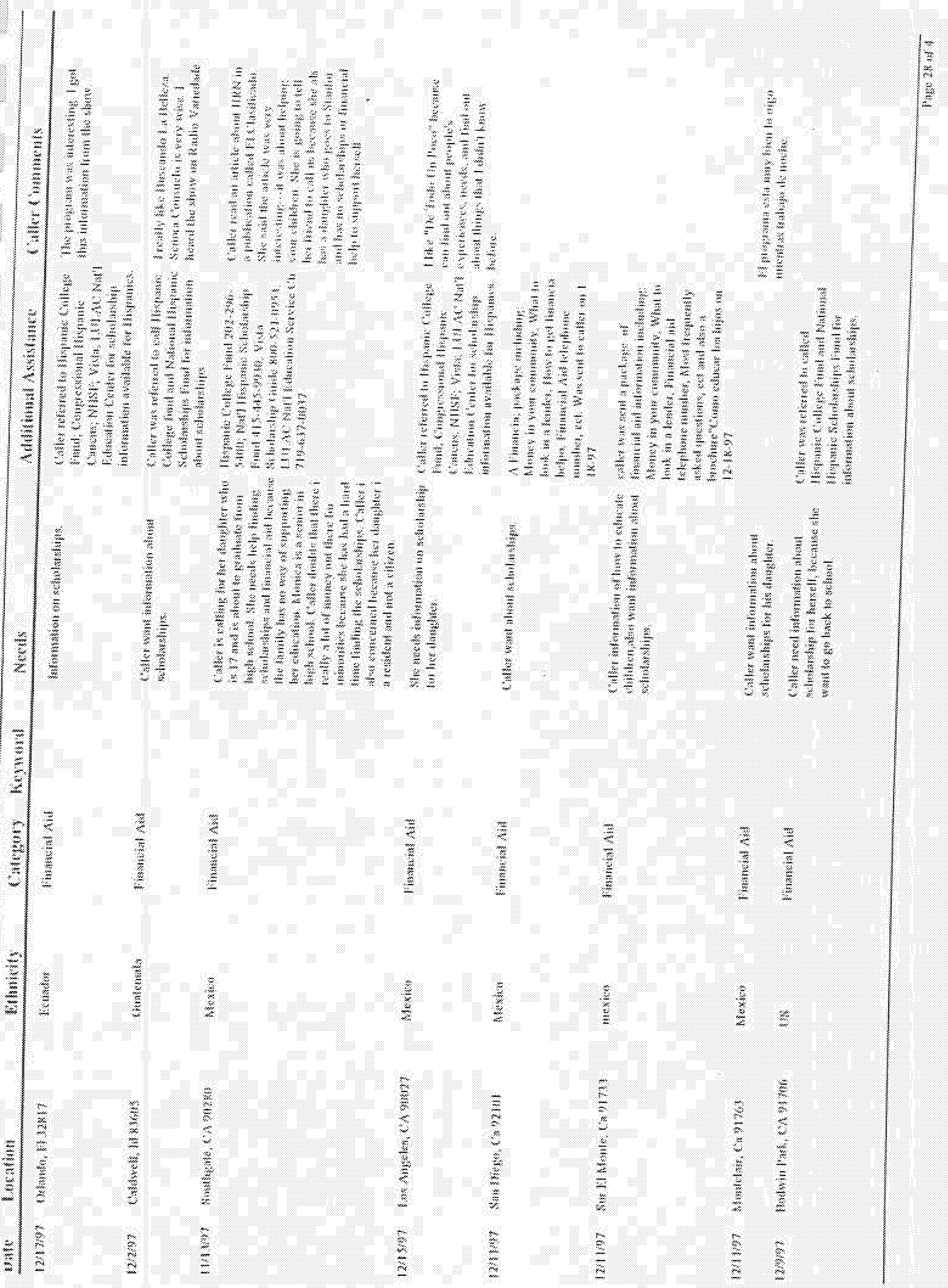




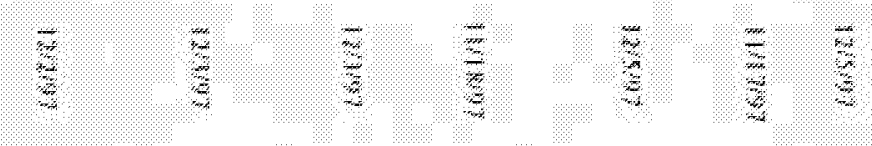

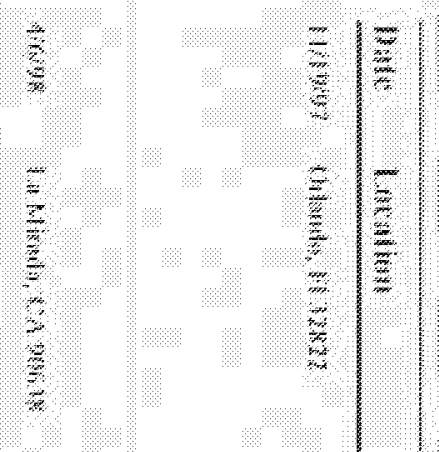

$1+\left.1\right|^{2}=\mid$

$\prod_{1}^{1}$

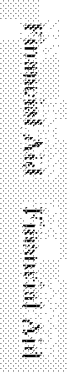

II Im m

m! II

II III

If I"

$+y^{2}$

IIIII

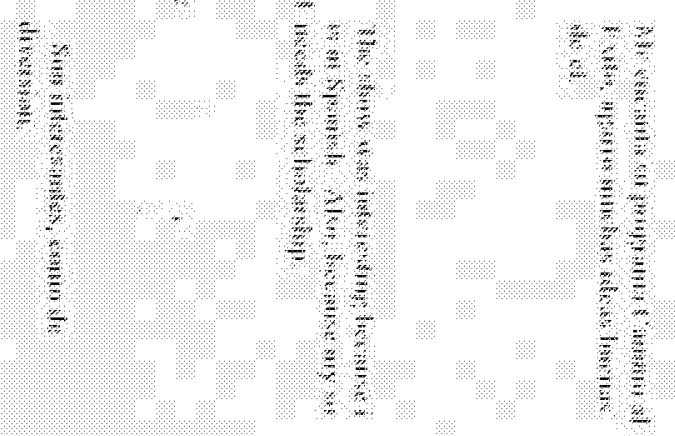

III)

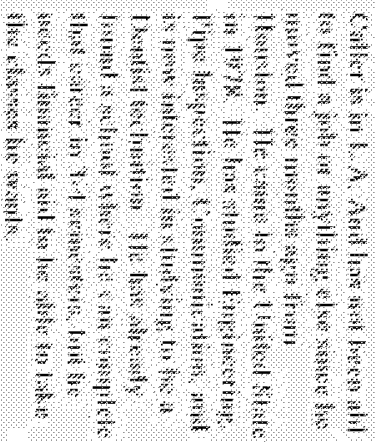
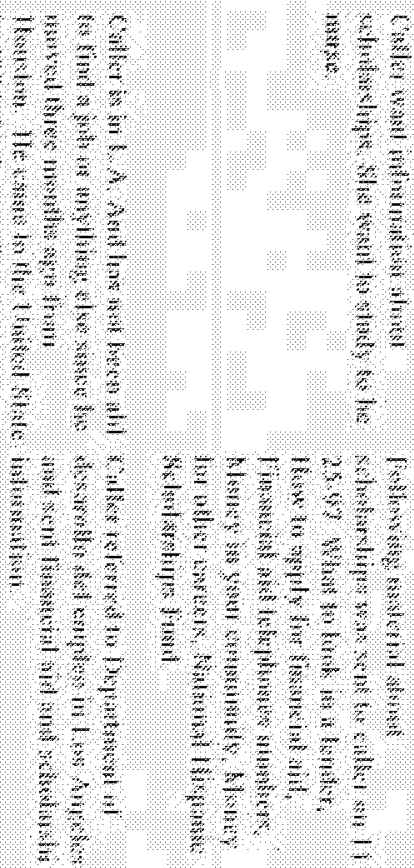

$-=$

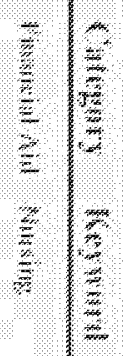

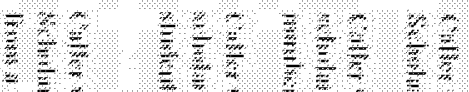

(1)

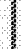

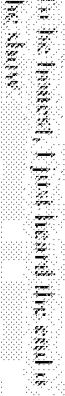




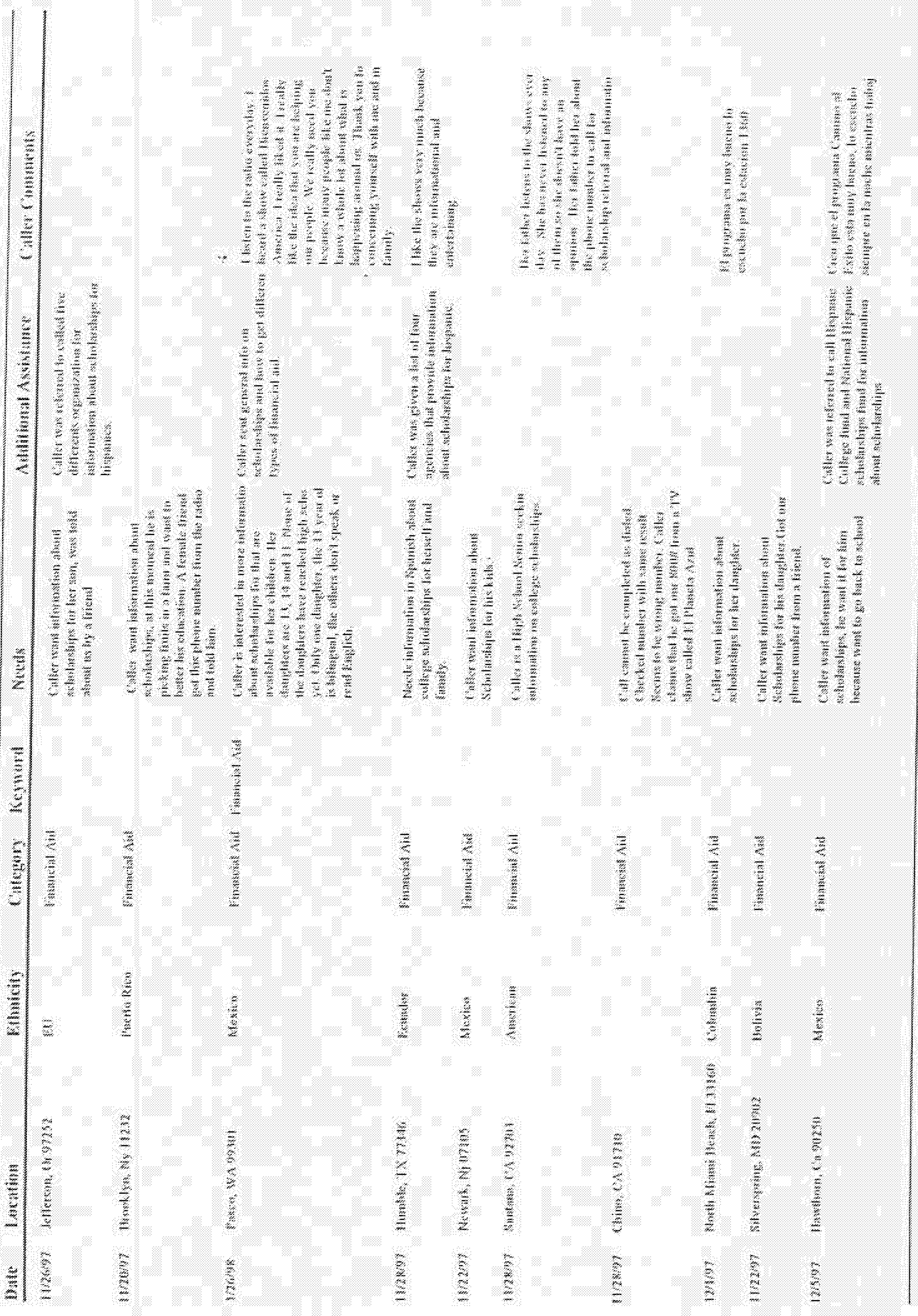




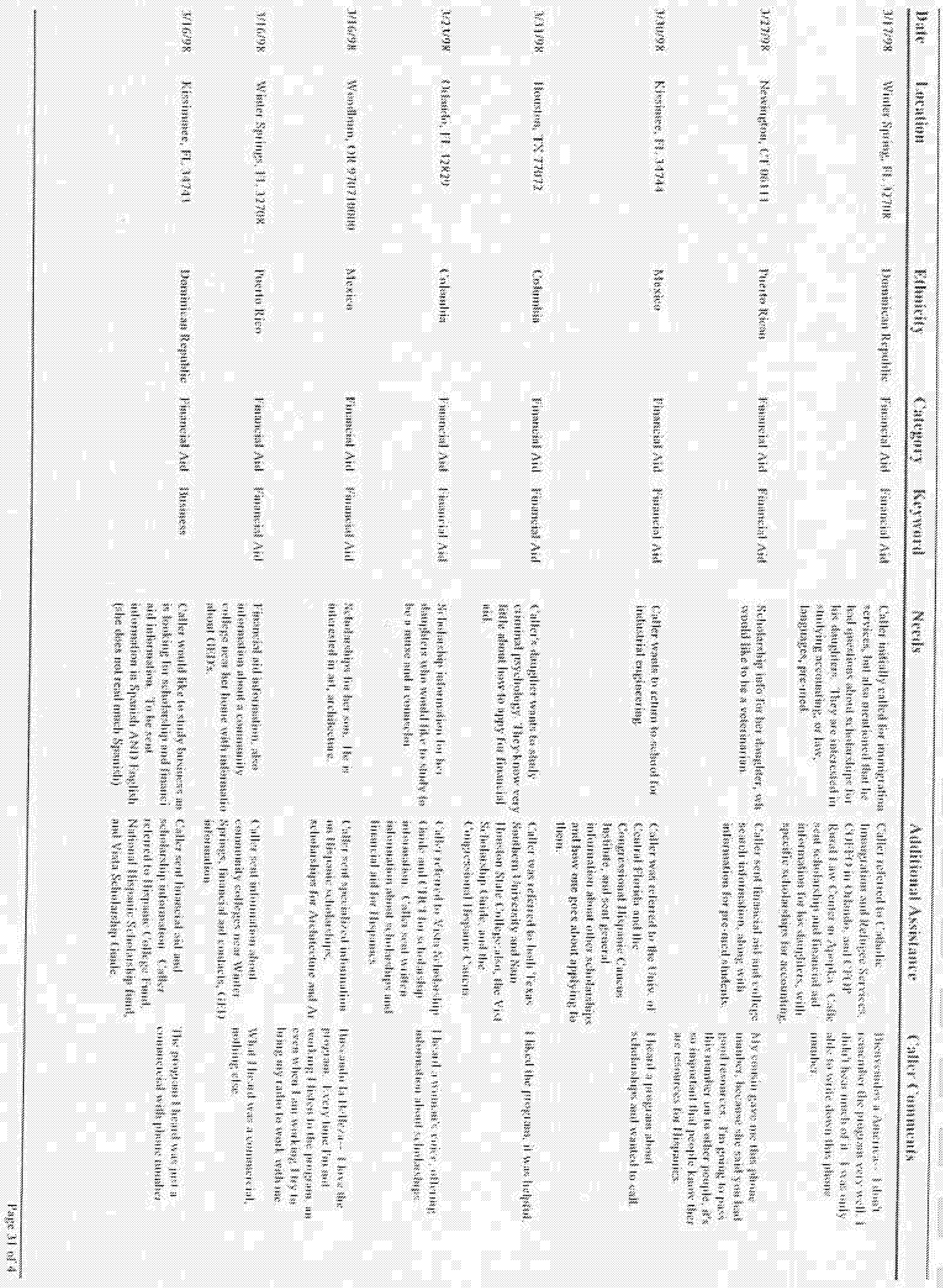




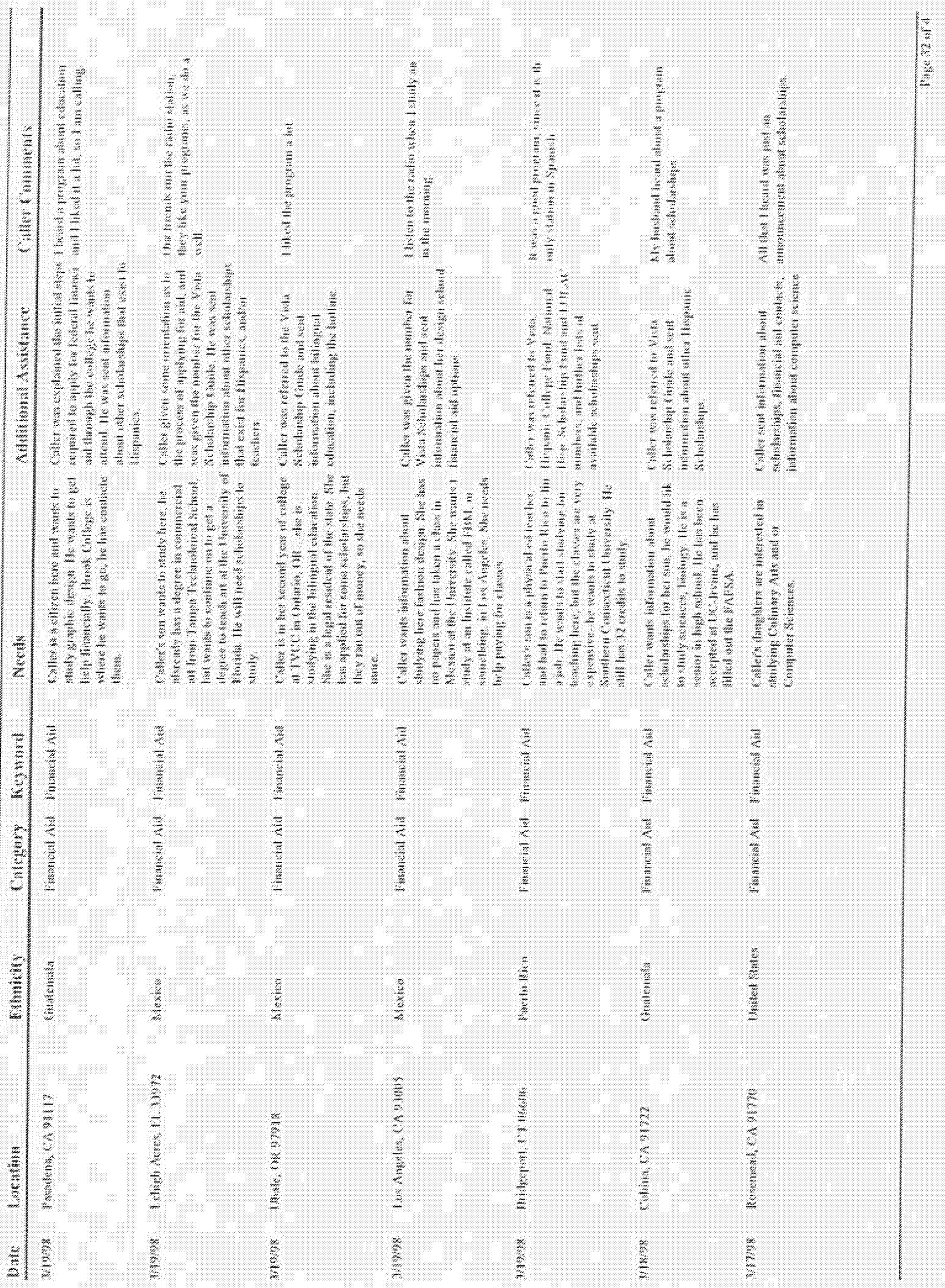




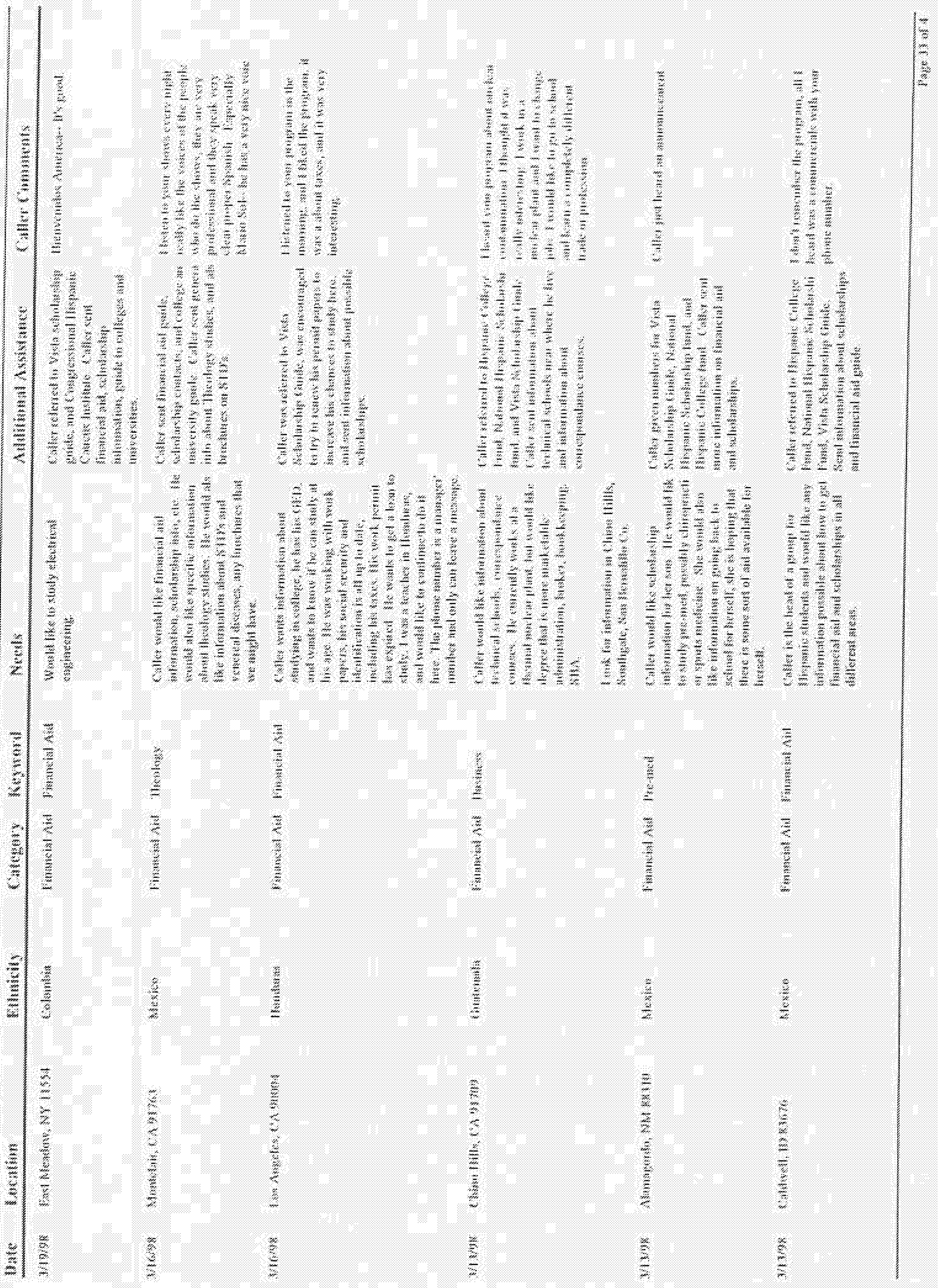




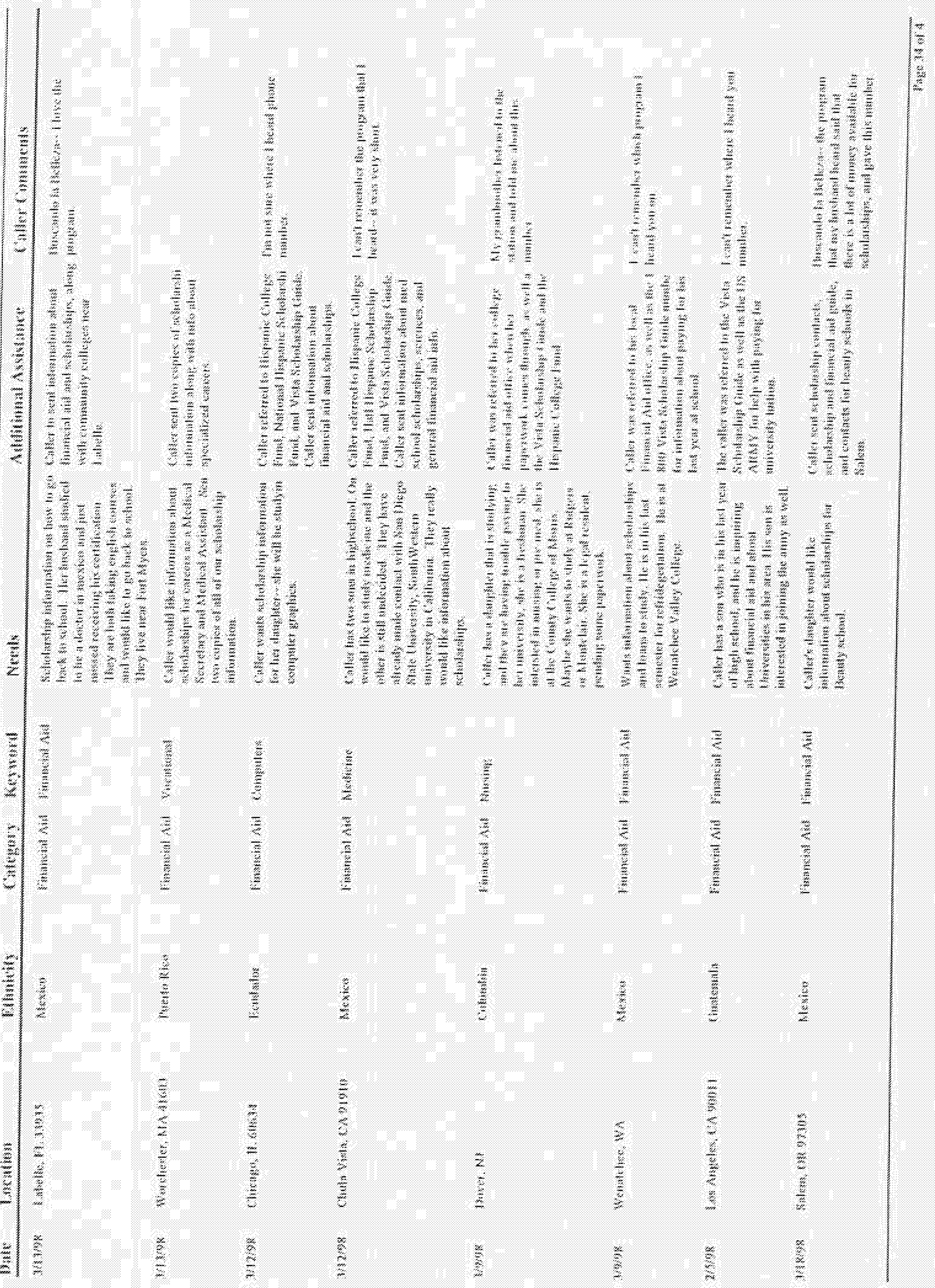




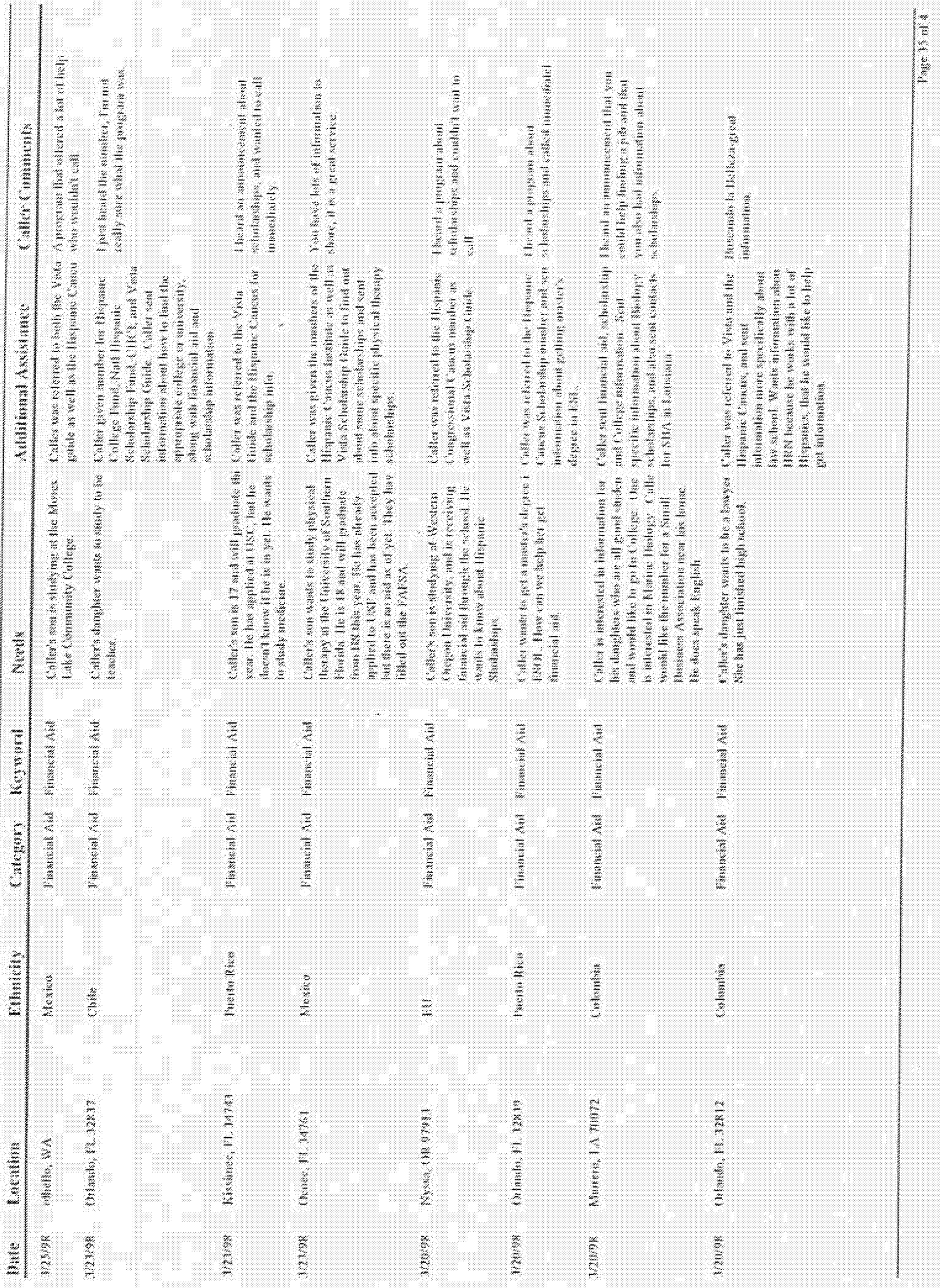




\begin{tabular}{|c|c|c|c|c|c|c|c|}
\hline 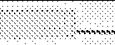 & $\sqrt{4}+4$ & & 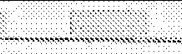 & , & 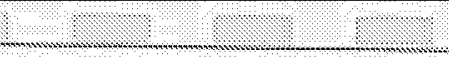 & s. & (3) \\
\hline U.ro & 401411109 & 10101010 & (nomens & Iteyetured & $\mathrm{N}+\mathrm{s}+\mathrm{s}$ & 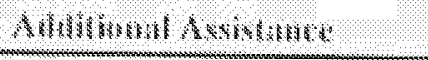 & 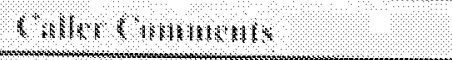 \\
\hline $124 \%$. & 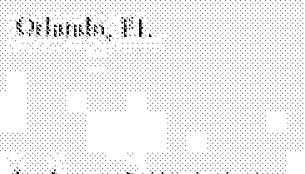 & 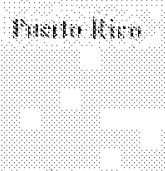 & 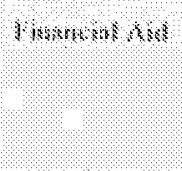 & 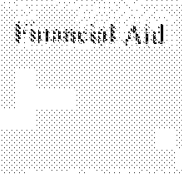 & 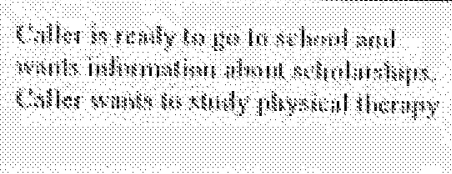 & 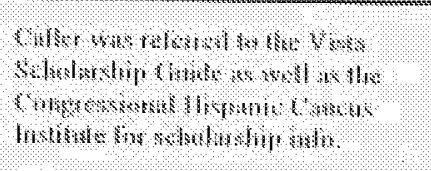 & 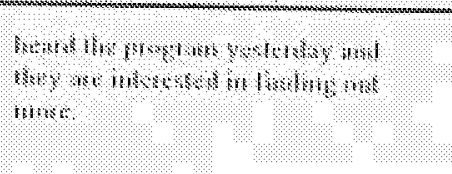 \\
\hline 14\%8: & 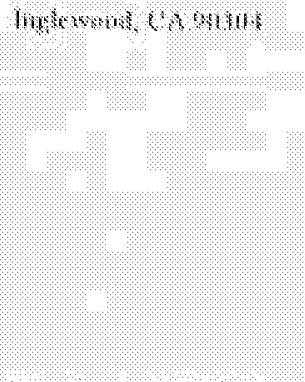 & 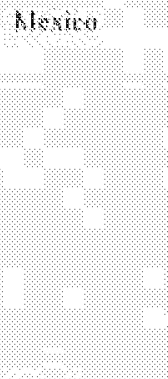 & 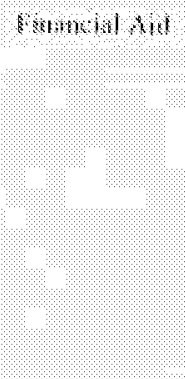 & 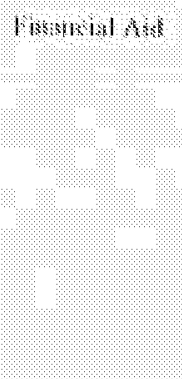 & 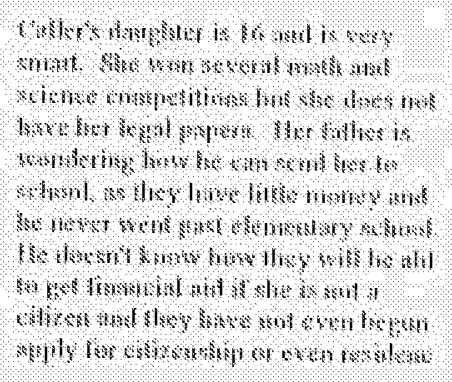 & 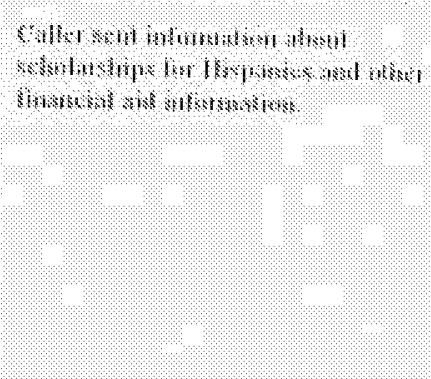 & 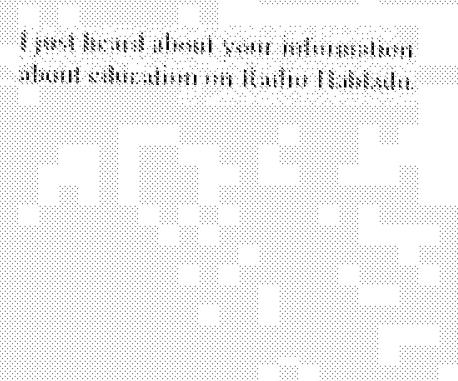 \\
\hline $10000 \%$ & 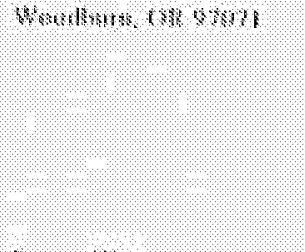 & Menmerer & Finmerial ant & 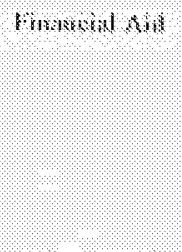 & 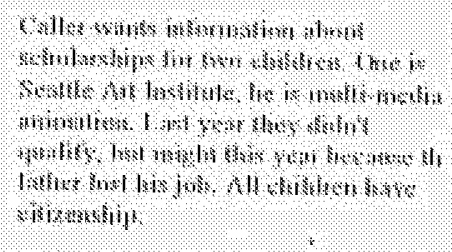 & 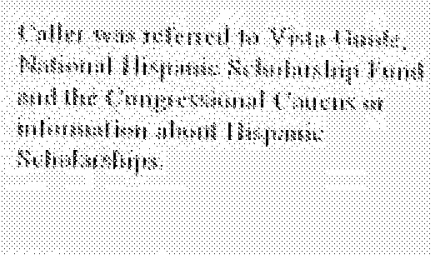 & \\
\hline $10,6 \% 0 \%$ & 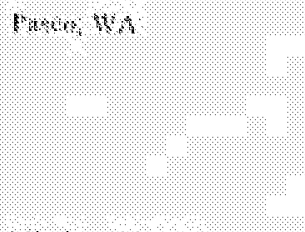 & Iens: & Brsmaths & smmknt bil & 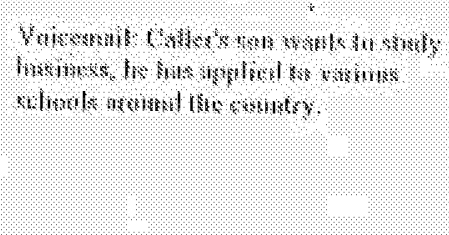 & 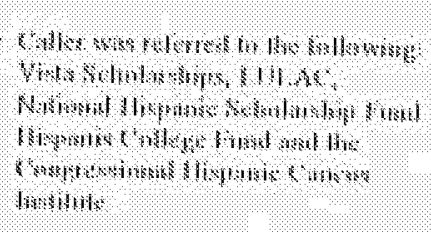 & 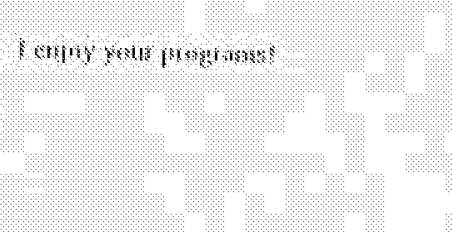 \\
\hline 140.4 & 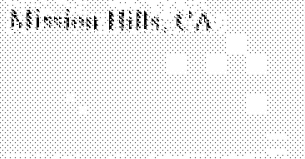 & M1.8198: & 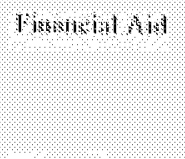 & 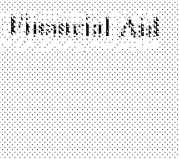 & 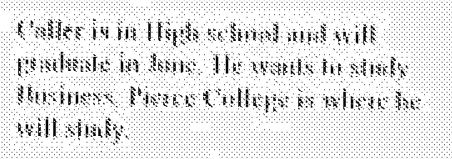 & & 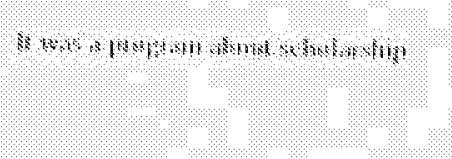 \\
\hline 780863 & 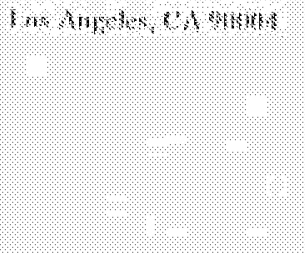 & 1928, s: & 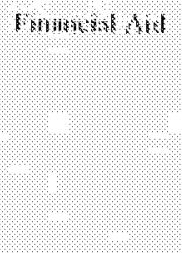 & 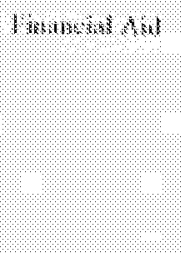 & 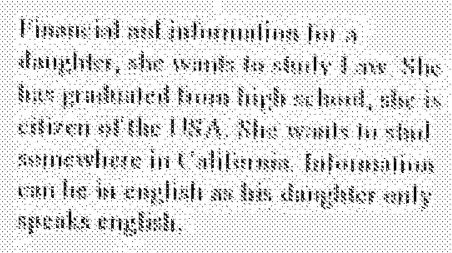 & 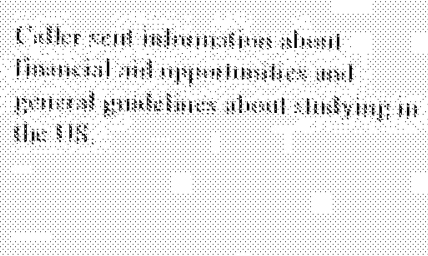 & 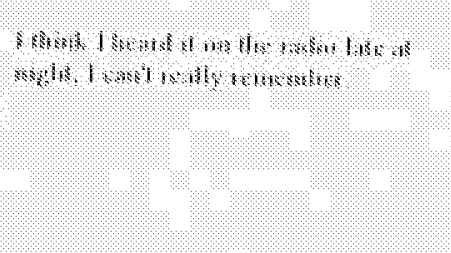 \\
\hline Whores & 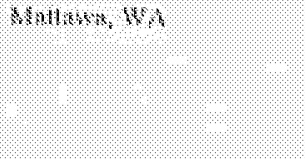 & 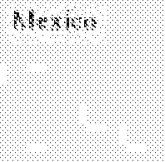 & 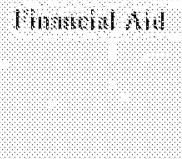 & Ymozerial nia & 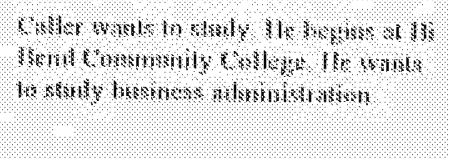 & 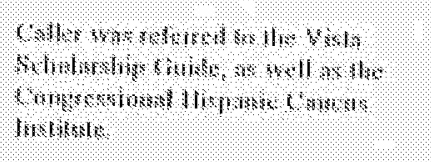 & 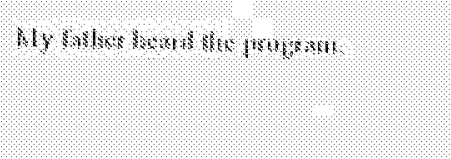 \\
\hline
\end{tabular}




\begin{tabular}{|c|c|c|c|c|c|c|c|}
\hline 1016 & $960 \mathrm{mrlwoss}$ & Aronoricis & 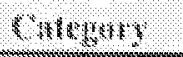 & keyinuens & Noests: & 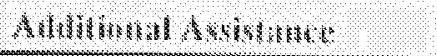 & 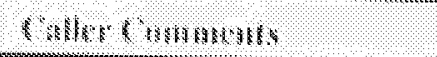 \\
\hline 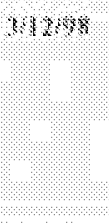 & 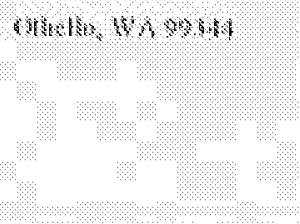 & 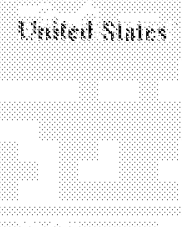 & 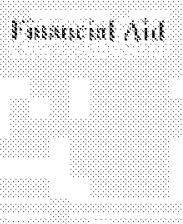 & 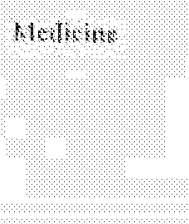 & 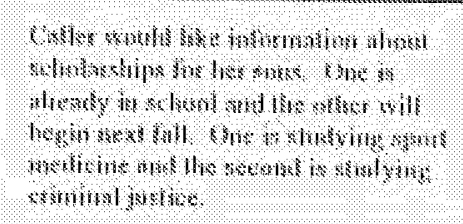 & 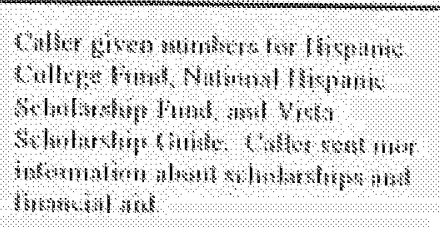 & \\
\hline $120 \% 8$ & 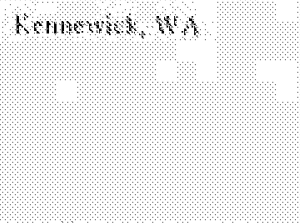 & $40, n$ & 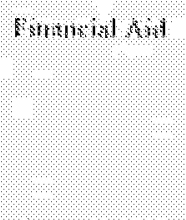 & 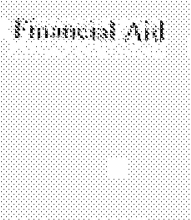 & 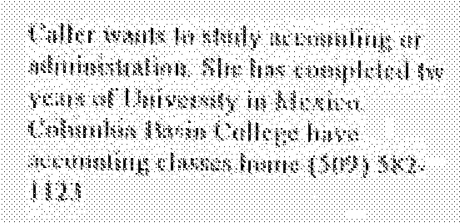 & & \\
\hline $4 \% 5 \%$ & 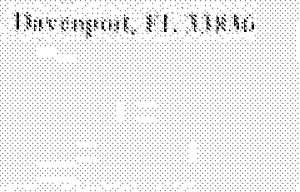 & $8+810041,16$ & 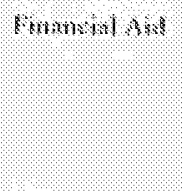 & 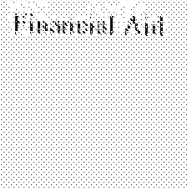 & 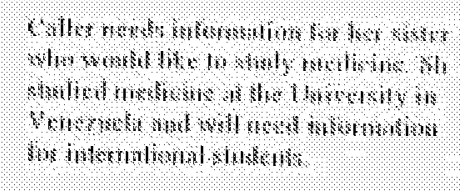 & 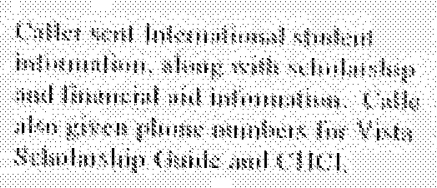 & 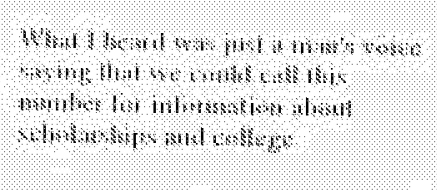 \\
\hline $1028 \%$ & 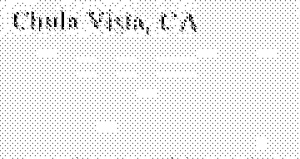 & 11:66:8. & 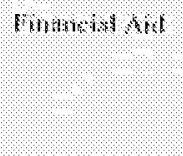 & 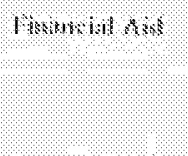 & 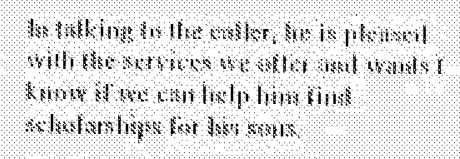 & 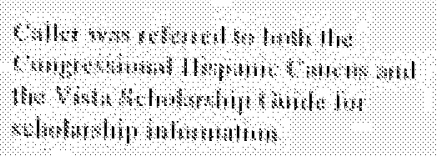 & \\
\hline $120 \% 8$ & 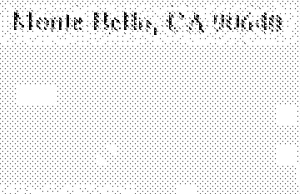 & $4 \operatorname{los} 6$ & TSm & 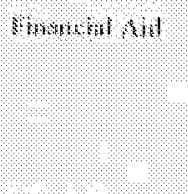 & 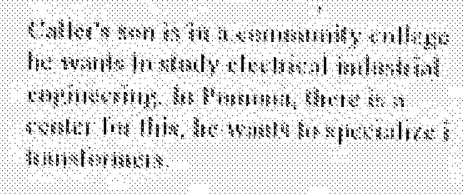 & 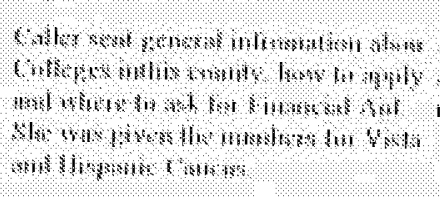 & 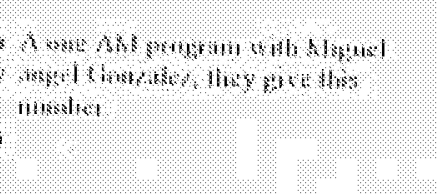 \\
\hline अ४rmk & rmoro: 115 & $4, \times, 1,0$ & 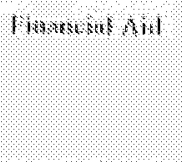 & 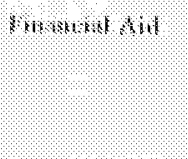 & 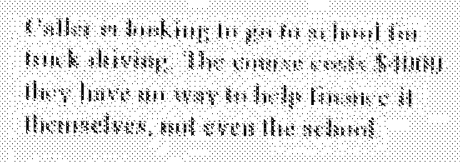 & & \\
\hline 3.1\%1: & 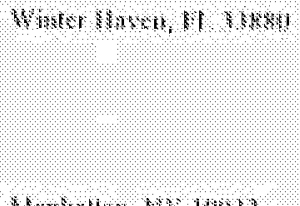 & $4 \times+1 \%$ & $1 / \mathrm{km}, \mathrm{m} / \mathrm{k}, \mathrm{m}$ & 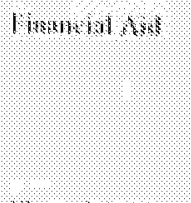 & 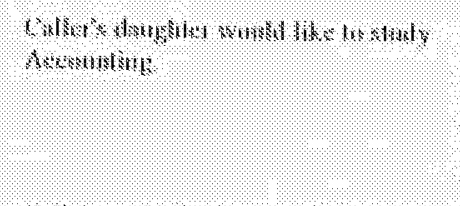 & 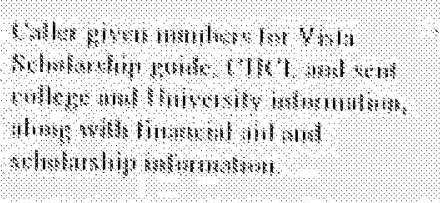 & 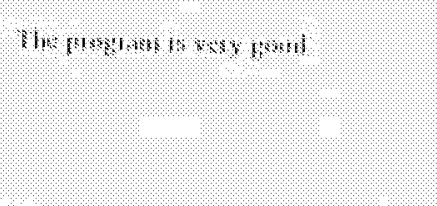 \\
\hline tertent & 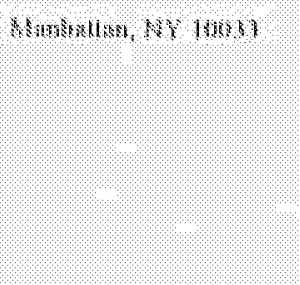 & $1,80817 \%$ & limmist $186 \mathrm{~s}$ & 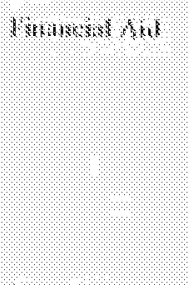 & 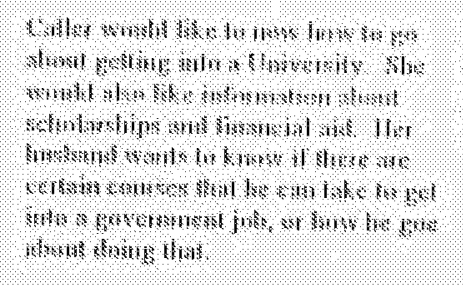 & 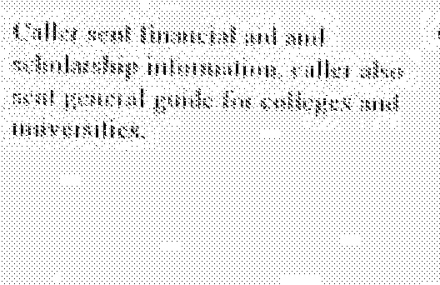 & 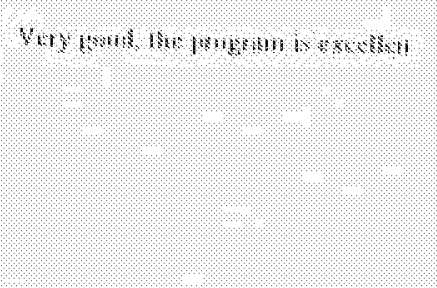 \\
\hline 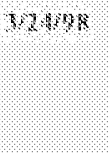 & 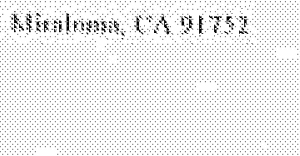 & xis:er: & 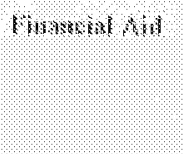 & 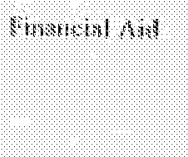 & 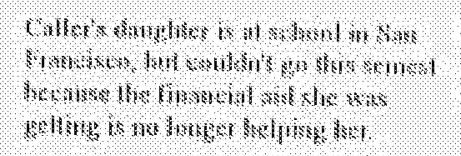 & 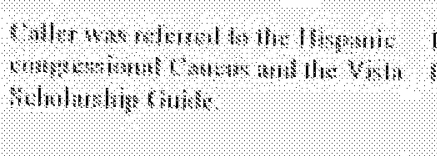 & 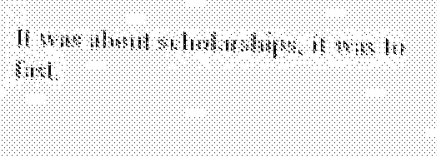 \\
\hline
\end{tabular}




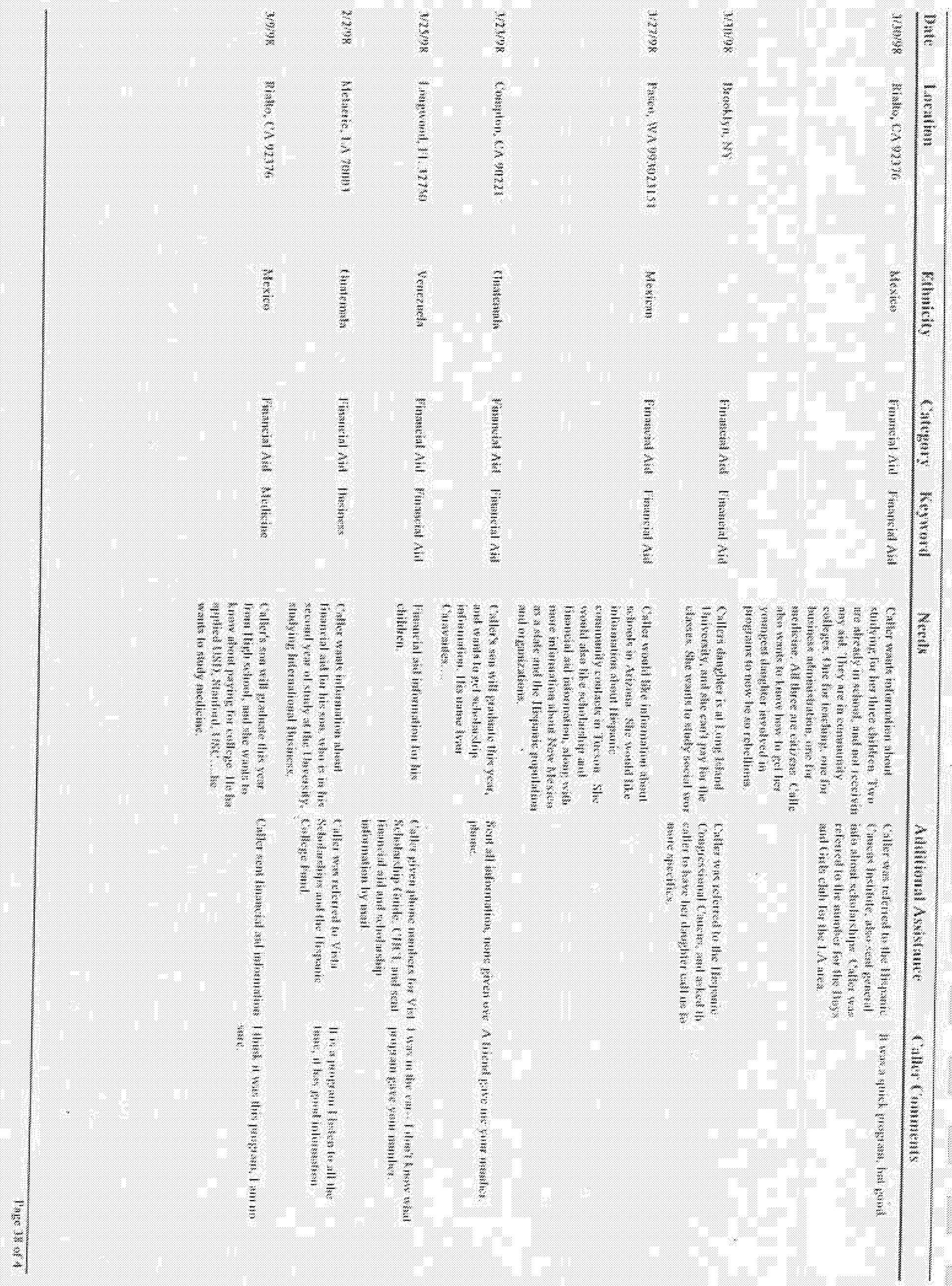




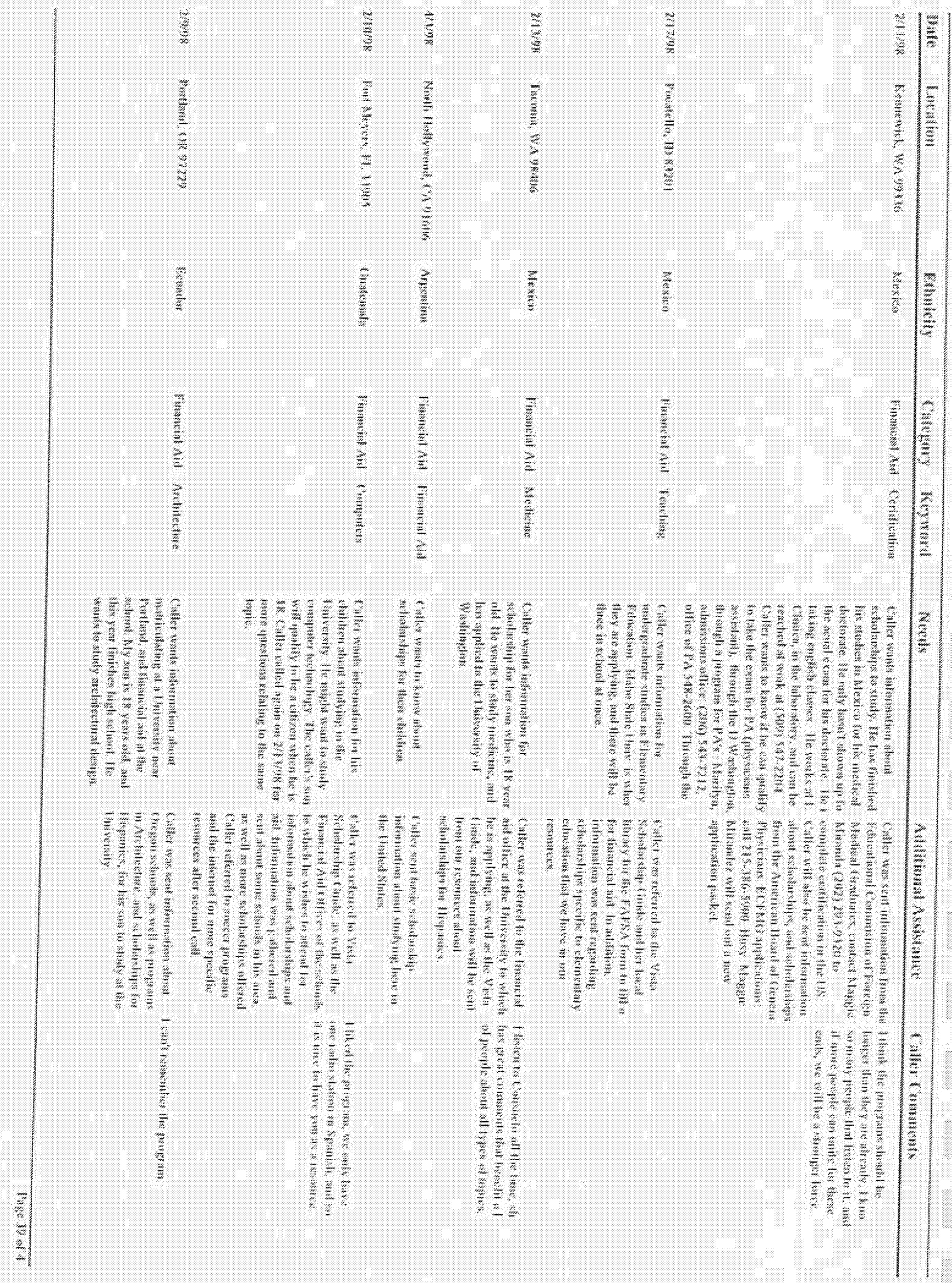





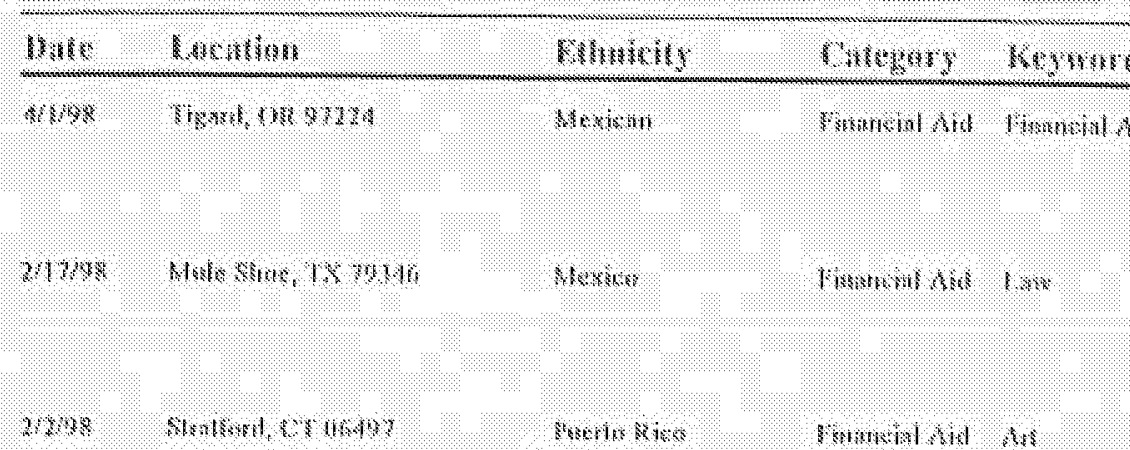

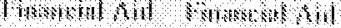

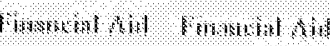

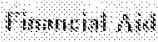

\section{Nowils}

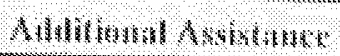

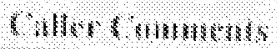

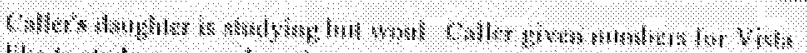

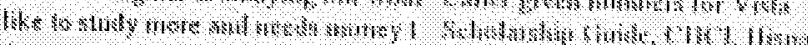

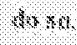

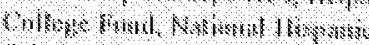

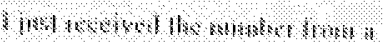

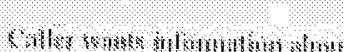

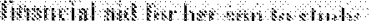

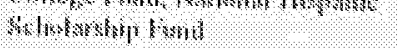

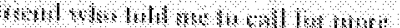

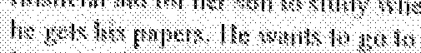

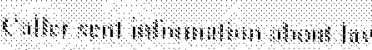
beseschess

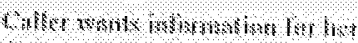

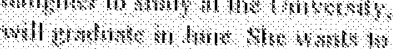

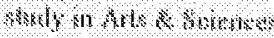

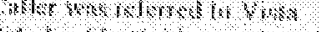

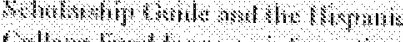

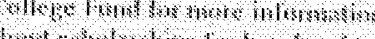

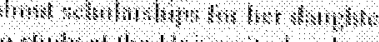

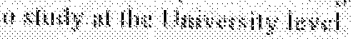

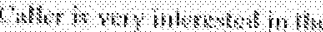

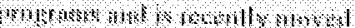
tes

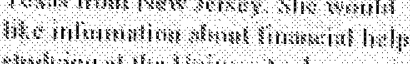

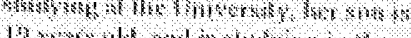

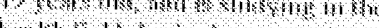

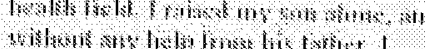

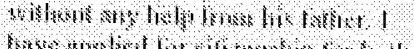

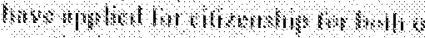

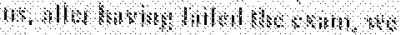

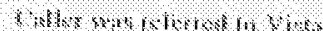

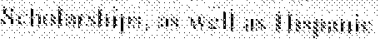

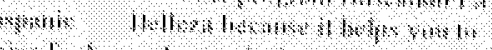

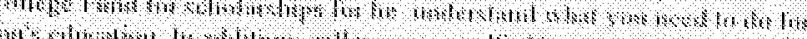

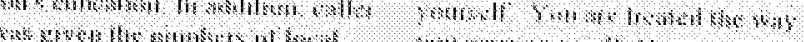

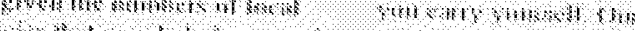

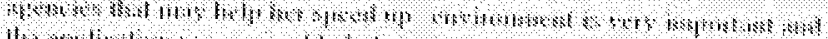

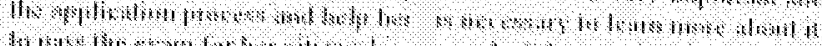
will to lo pars mersibs:

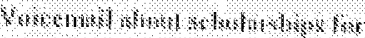

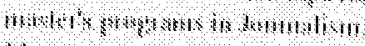

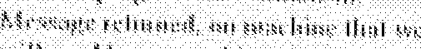

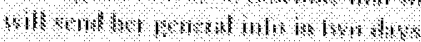

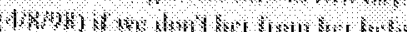

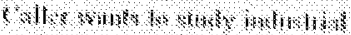

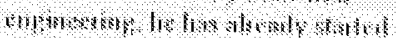

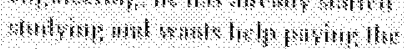

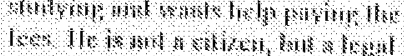
1ost 110

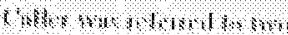

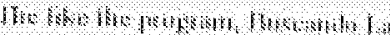

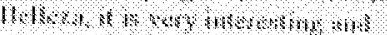

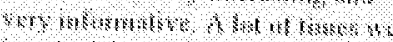

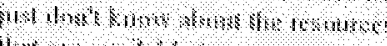

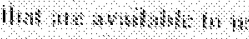

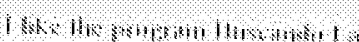

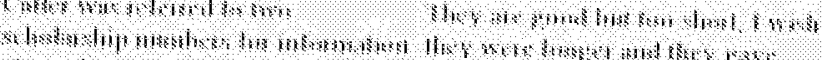

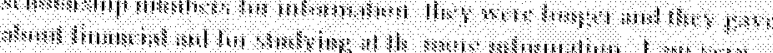
silineross?

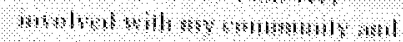

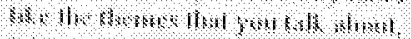
$10,406 \times 64$

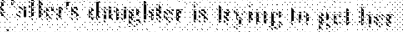

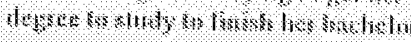

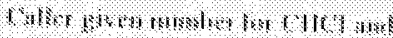

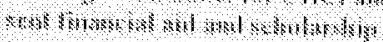
int

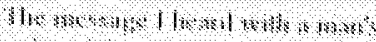

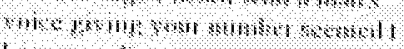
lom mormblas

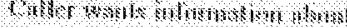

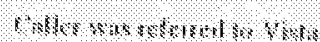

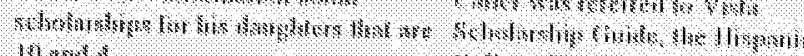
10 ment s:

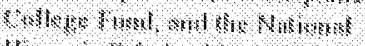

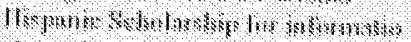

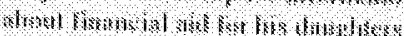

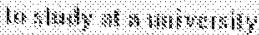





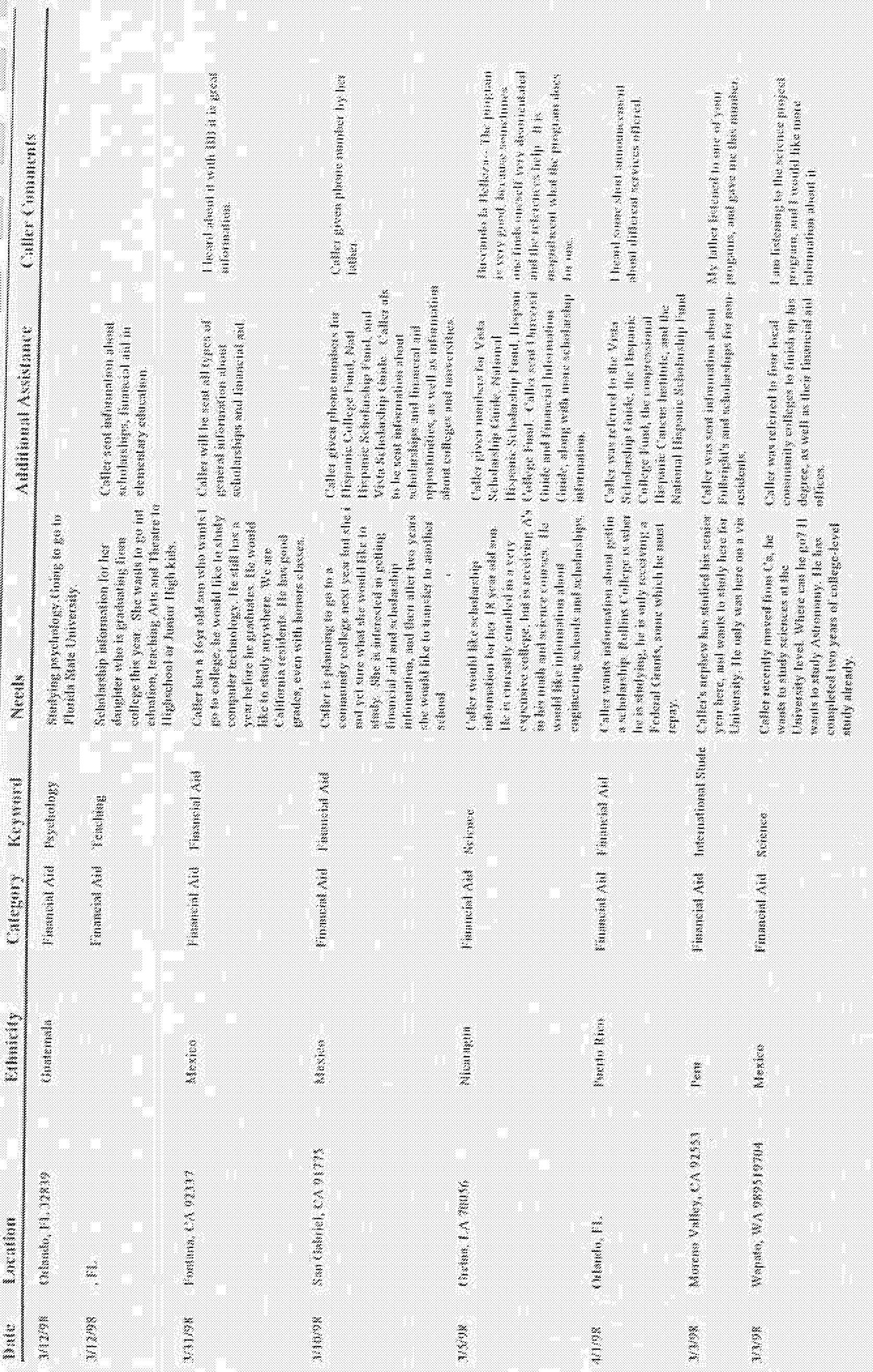




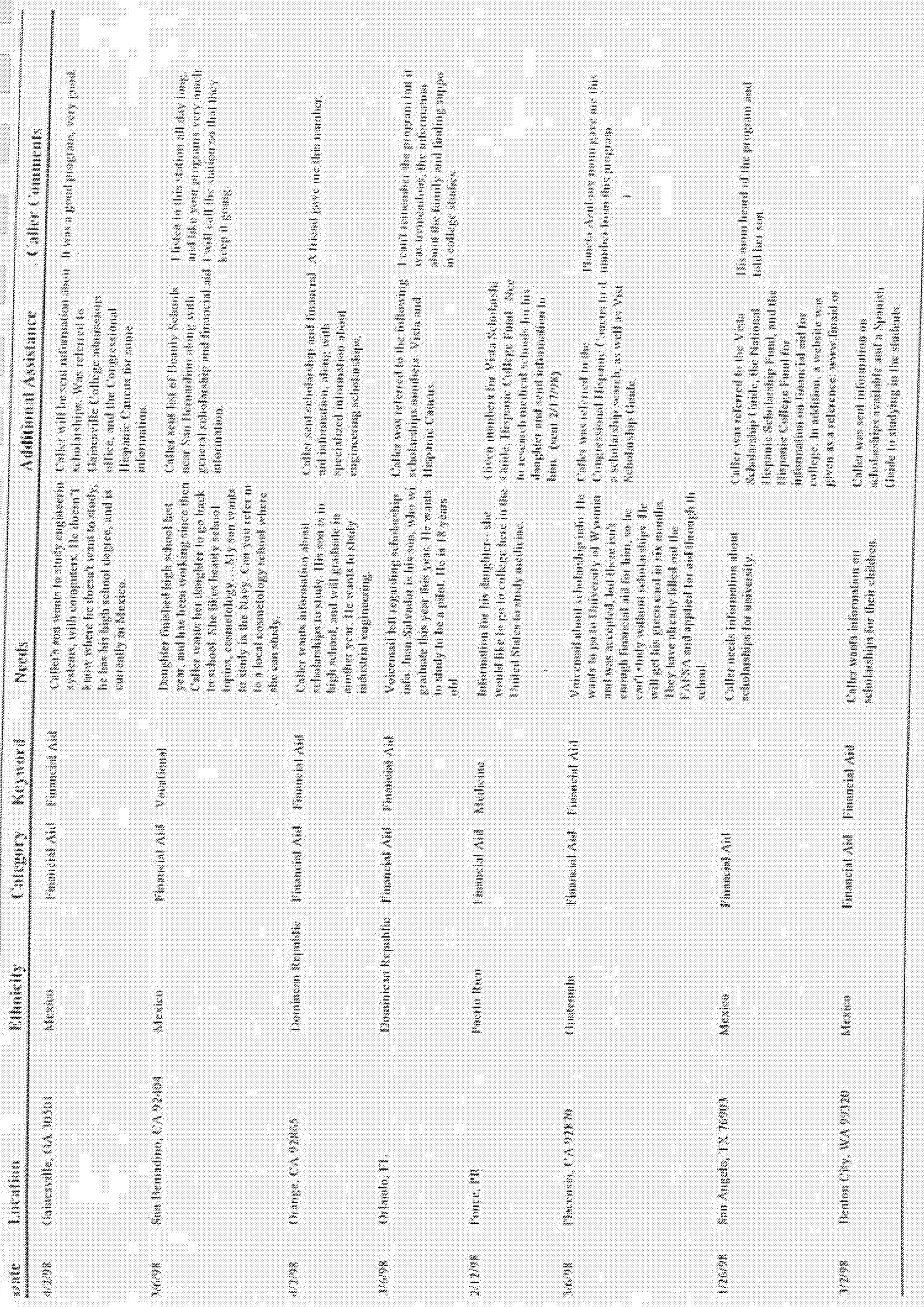




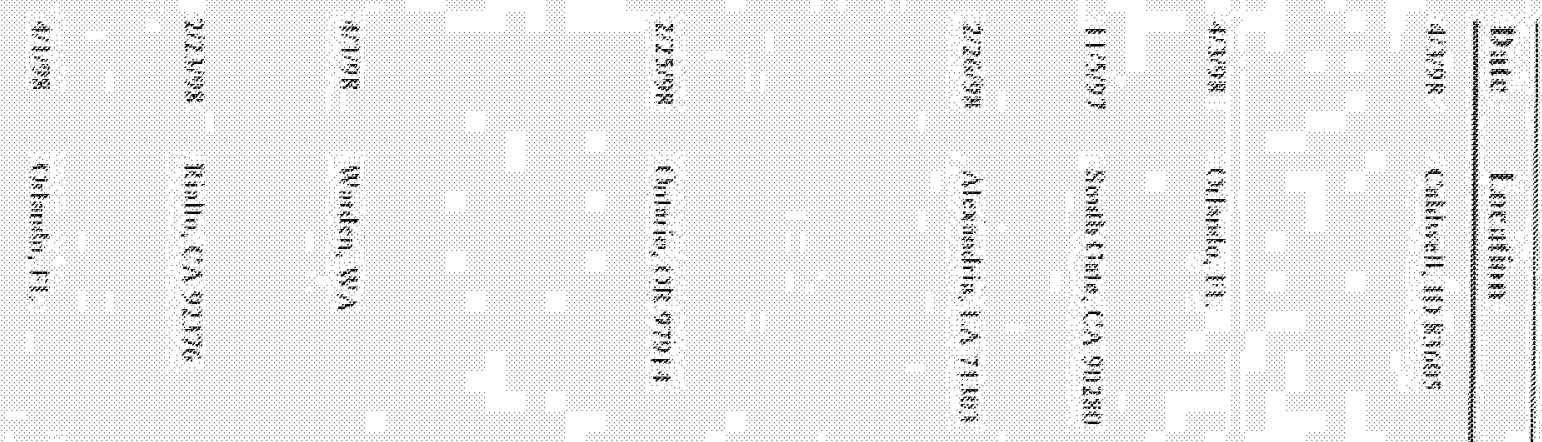

$\frac{1}{3}$

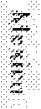

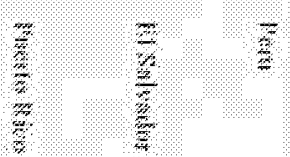
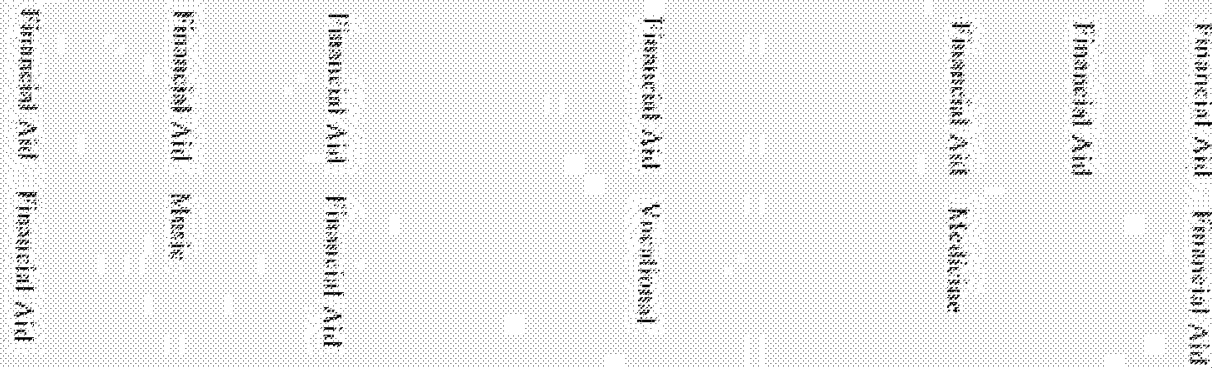

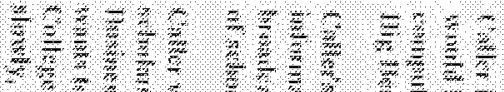

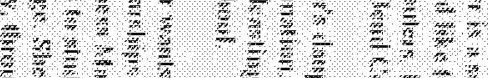

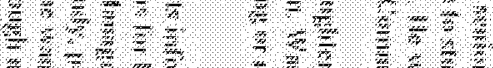

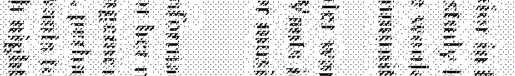

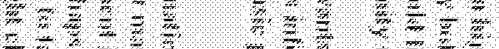

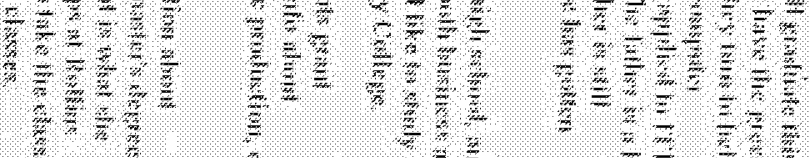

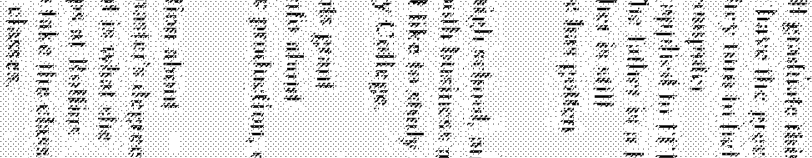

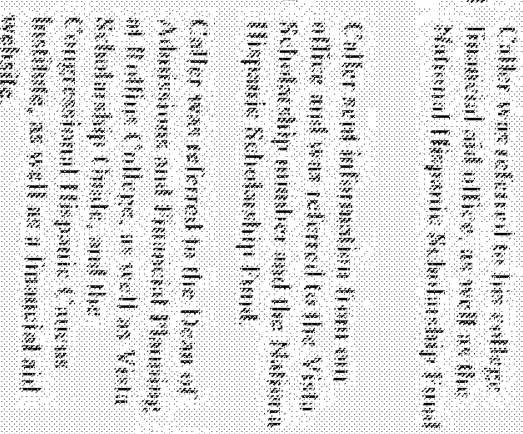

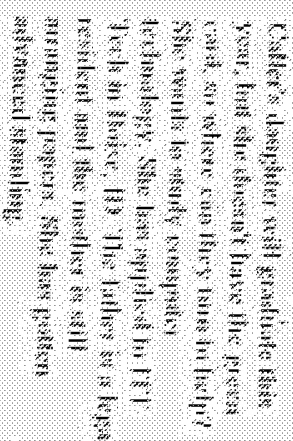

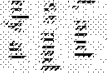
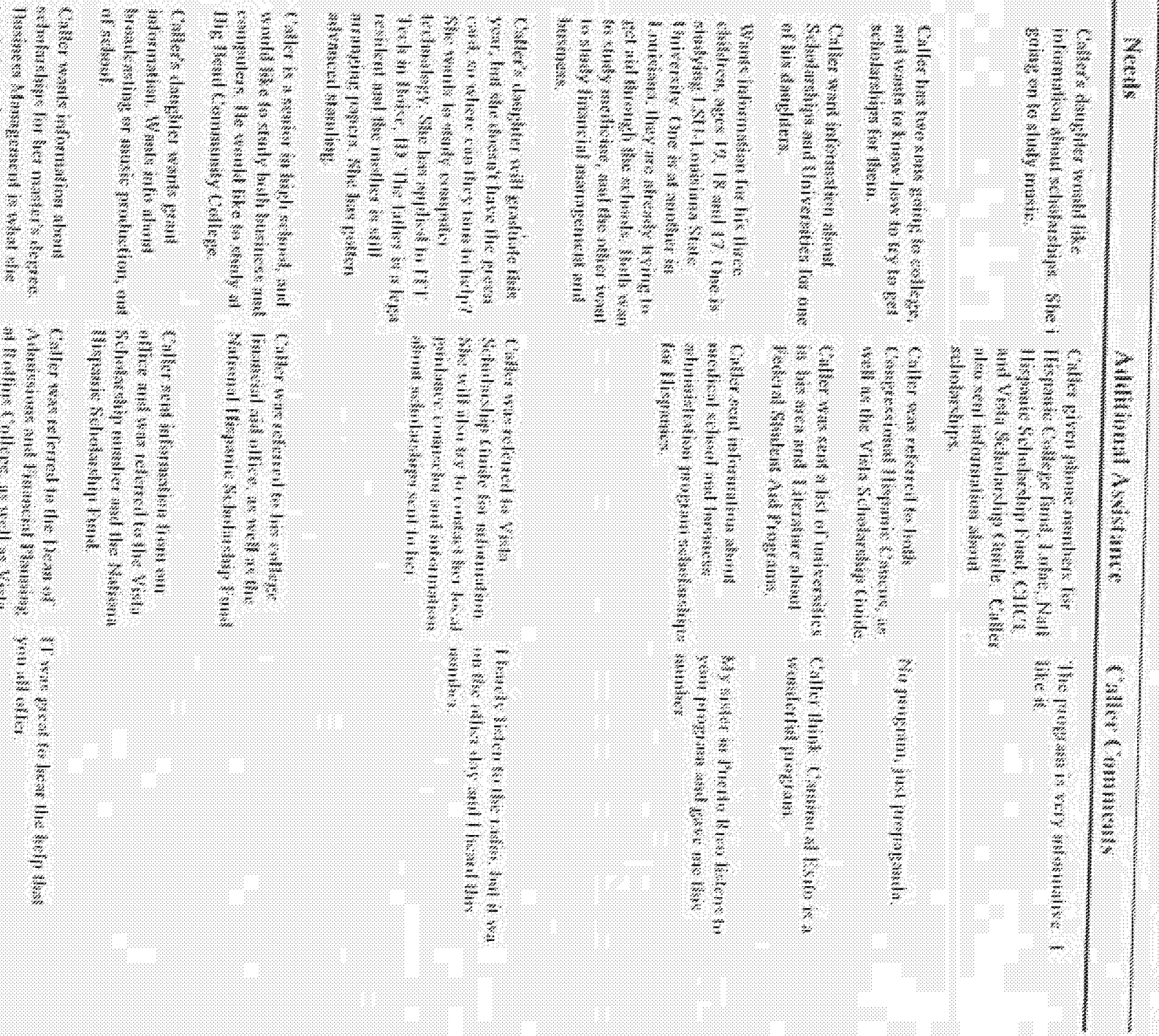


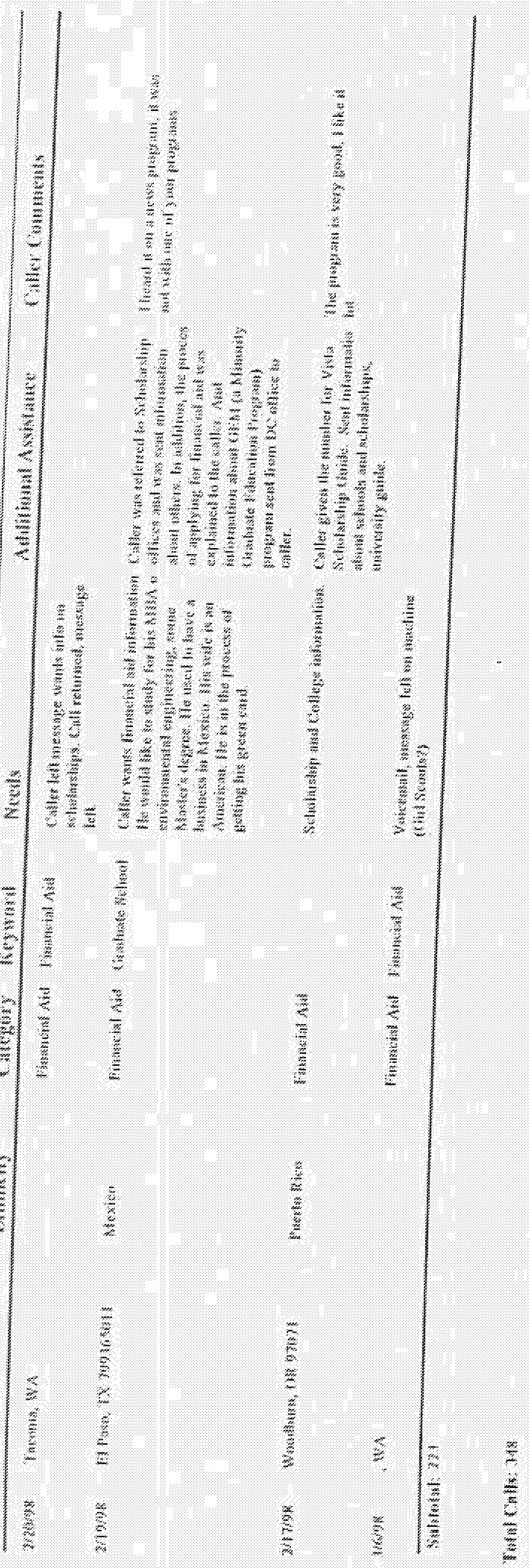




\section{V. \\ Marketron/ Arbitron \\ Ratings Analysis}

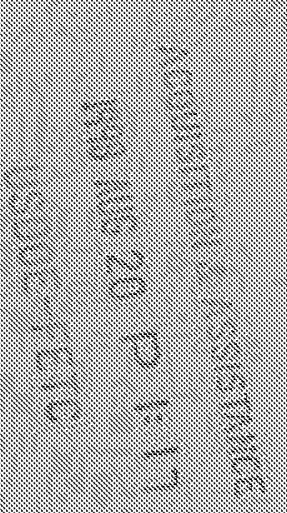




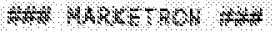

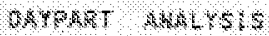
$4+4,4 \%$

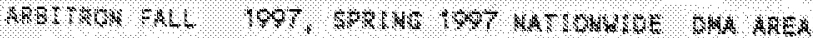

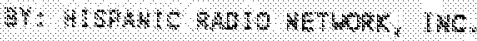

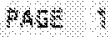

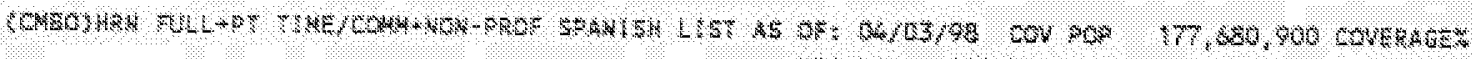

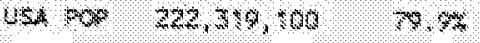

\begin{tabular}{|c|c|c|c|c|c|c|c|c|c|}
\hline A & & Eroson & & & & $4 \cdot 7 \quad 346-704$ & $4-F>+4-60$ & $8 \mathrm{Ar} \times \mathrm{H} \times 7 \mathrm{~m}$ & $48 \times 28$ \\
\hline 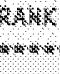 & & terst & 8006 ? & stitis & & HY 134 \% & Wit $14 \operatorname{lin}_{n \rightarrow \infty}$ & $\mathrm{NC} 1 / 4,4 \mathrm{k}$ & 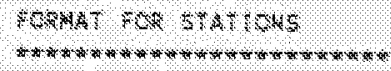 \\
\hline 1 & $4 \%$ & $88 \mathrm{R}$ & 8407 & rastras & 4 & 8 & 0 & $a$ & 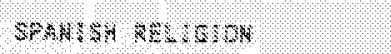 \\
\hline 1 & $88 \%$ & $10 \times$ & 44 & $40-4-4$ & $\approx$ & 8 & 0 & 8 & 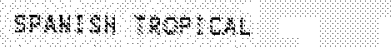 \\
\hline 1 & $\notin=4$ & soks: & 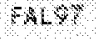 & $8 \times 64$ & 8 & 8 & 8 & 0 & 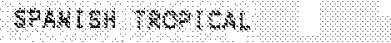 \\
\hline 1 & 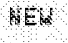 & rox & ar & $4 \times 4+48$ & 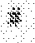 & 8 & 0 & 0 & 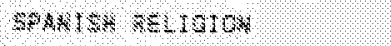 \\
\hline i. & MB & sox & A4\% & $4 \times 1 \times 4$ & 4 & 8 & 0 & 0 & 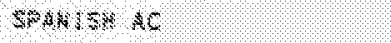 \\
\hline 3 & $8 \times$ & 808x & exs? & $401-4$ & 2 & 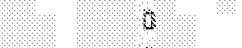 & 0 & 8 & $4 \times 6=8$ \\
\hline$\%$ & xus & $10 \times$ & 84\% & $6 \times-4$ & 8 & 8 & 0 & 6 & 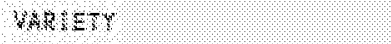 \\
\hline 1 & 464 & rok & 1480 & ४山r, & $\%$ & 0 & 0 & 8 & $4 \times 1,1$ \\
\hline 1. & \$4: & sork & 1407 & $44-A$ & 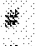 & 0 & 4 & 4 & $18 \times 1 / 7 \mathrm{R} \times$ \\
\hline 18 & $10 \%$ & rak & A4\$? & 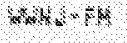 & \% & 8 & 6 & 8 & $0 \times 18 \mathrm{Cl}$ \\
\hline s. & $8 E$ & 10\% & $546 \%$ & $66 \times+4$ & \& & 8 & 0 & 0 & $16 \times 5,84 C$ \\
\hline 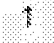 & & $30 \times$ & ABP & $\% 60 \%$ & * & 4 & $\theta$ & 0 & vanst \\
\hline 8 & 8E.4 & sox & (448) & $4+4-4$ & 4 & 6 & 8 & b. & 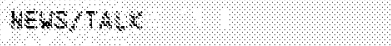 \\
\hline 8 & k & $80 \%$ & $4 \mathrm{~A}$ & $0.46+4$ & 4 & 0 & 8 & 1 & 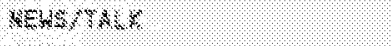 \\
\hline 8 & r & rox & 4007 & $1004-4$ & $\%$ & 8 & 8 & 1 & $1 \times 22$ \\
\hline & 18 & $80 \times$ & $4 \times, 7 \%$ & 6040 & s. & 40 & 0 & 8 & $148<+4$ \\
\hline 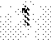 & 464 & $18 \times 8$ & $8+4$ & strato & थ. & 8 & $\mathrm{~d}$ & 0 & 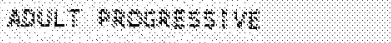 \\
\hline 19 & 104 & $10 \times$ & 4.87 & 6400.14 & & 85,300 & 8,806 & 26,060 & 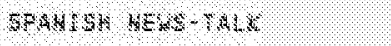 \\
\hline 1 & 106 & Yoxs & $\mathrm{ASO}^{\circ}$ & $13 \mathrm{SP}-\mathrm{AS}$ & & 86,300 & 6,80 & 4,060 & saMus \\
\hline 11 & 1 64 & som & $8 \times 4$ & $480-1 \%$ & & $30,00 \%$ & $7 \cdot, 400$ & 15.400 & 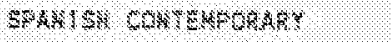 \\
\hline 2 & 185 & MeFles & 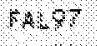 & $16 \times 4+4$ & . & 8 & 8 & 0 & sex \\
\hline z & 198 & MLELES & $=48 \%$ & 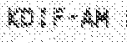 & H. & 0 & 0 & ๑ & 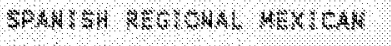 \\
\hline 2 & $10 \%$ & 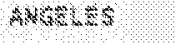 & PASP & $182-4$ & * & 0 & 0 & 4 & 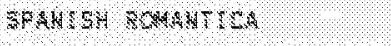 \\
\hline 2 & 808 & nroelss & -1419 & $4 \times 4+4$ & * & 8 & t) & 1 & 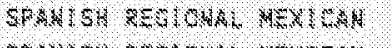 \\
\hline 2 & \$6 & $4 x+4=8$ & FALY & $1000-8 \%$ & $\%$ & 9 & 9 & 0 & 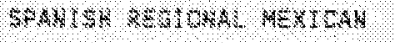 \\
\hline 2 & 189 & skes-s & 84४९ & $8085-78$ & 4 & 8 & 8 & 1 & 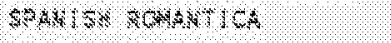 \\
\hline 2 & 404 & $484 \times . \%$ & $\mathrm{AACP}$ & $1 \times 88+4$ & 4 & 8 & 0 & $\mathrm{c}$ & 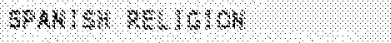 \\
\hline 2 & 808 & 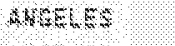 & 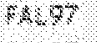 & $x, 38+4$ & . & 8 & 6 & 1 & 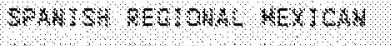 \\
\hline 2 & 80 & 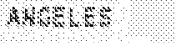 & 84,7 & 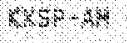 & + & 808 & a & 100 & 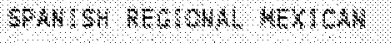 \\
\hline 2 & $\operatorname{ses}$ & $4 \times 6805$ & ALP? & $80 \times 3+M$ & 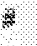 & 8 & 0 & 1 & 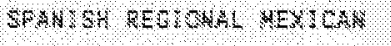 \\
\hline 2 & 88 & a $68<8$ & $84 \%$ & $\mathrm{~m} 3 \mathrm{~s}, \mathrm{k}$ & 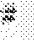 & 8 & 8 & 8 & 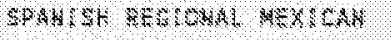 \\
\hline 2 & 106 & 846,4 & 449 & $4 m, k$ & 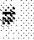 & 4 & ४ & 8 & SRAN SA ROMABT ICA \\
\hline 2 & Los & $4 \times 6<8$ & 840\% & Sur $\times 4$ r & 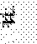 & 8 & $\Delta$ & 8 & 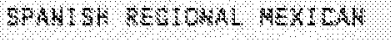 \\
\hline 2 & 408 & atess & $8 \times 40$ & $4612+4$ & \& & 8 & 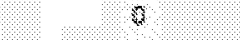 & 4 & 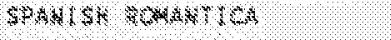 \\
\hline 8 & 48 & she $4=8$ & 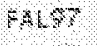 & $80-40$ & * & 9 & 0 & 8 & 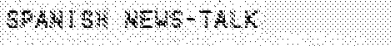 \\
\hline 2 & W० & 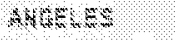 & $8 \times 1 \%$ & 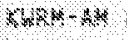 & e & 8 & 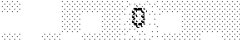 & 6 & 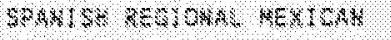 \\
\hline 2 & 48 & martos & 8. & $8+0 \times 44$ & 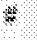 & 8 & tr & 9 & 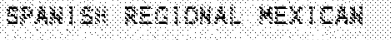 \\
\hline 2 & 43 & 4krks? & 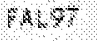 & $4 \% 4+4$ & 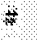 & 8 & 8 & 8 & 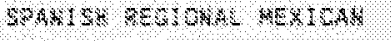 \\
\hline 2 & $4 \%$ & AN<C<\&? & 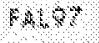 & $84, \times 8 \%$ & \% & 8 & 0 & 8 & $859418 \% 46$ \\
\hline 2 & 48 & 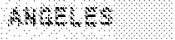 & 1408 & $8+34+8$ & t? & 8 & 0 & 6 & $4 \times 4,418<$ \\
\hline 2 & 148 & $2404=$ & Pl. & (P) & * & 8 & 0 & 8 & Horl \\
\hline 2 & 108 & $1868+8$ & $8 \mathrm{AP}$ & $80 \mathrm{EA}+4$ & ? & 8 & 8 & 6 & $48 \times 16$ \\
\hline 2 & $\mathrm{ss}$ & 480800 & 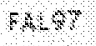 & $\mathrm{oc}-\mathrm{r}, \mathrm{r}$ & * & 4 & 0 & 6 & $6 \times 4 \times / 4$ \\
\hline 2 & 48 & SMUELE & $8+8$ & $4+4<4$ & 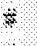 & 8 & 0 & ? & $4801 / 14$ \\
\hline 2 & 48 & 04648 & $\mathrm{k} / \mathrm{P}$ & $4+46-4$ & t. & 8 & 8 & 4 & $4+4,14 x$ \\
\hline 2 & 48 & $486-4=$ & 140 & 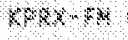 & 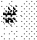 & 8 & 8 & 6 & 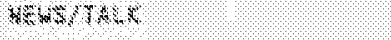 \\
\hline 2 & 808 & $448+48$ & $8+8 \%$ & $405 x+4$ & ध & 0 & 0 & 8 & $4+4 \times 148$ \\
\hline+ & 80 & $48+48$ & 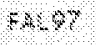 & $8 \times 7+4$ & 4 & 8 & 0 & 8 & HEA \\
\hline 2 & 808 & $\mathrm{n} / \mathrm{Q}<\mathrm{Cs}$ & $3 \times .07$ & $6 \mathrm{ser}+\mathrm{s}$ & e & 8 & 8 & 6 & 80601140 \\
\hline 2 & 808 & $48<8<-8$ & $84 \%$ & $848-\mathrm{A}$ & 4 & 8 & 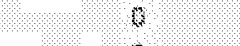 & f & $24 A B S 1 / 2$ \\
\hline 2 & 8 & $A, r s \leq s$ & 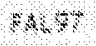 & $8 \mathrm{~s}+\mathrm{c}-\mathrm{s}$ & \% & 8 & $\mathrm{~s}$ & 0 & 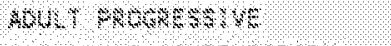 \\
\hline 8 & se & $488-4$ & 848 & 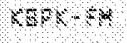 & 4 & 8 & 8 & 8 & HOH NDLS SOSEMTRARY \\
\hline 2 & 80 & $A \times s=\leq s$ & $1,4,4$ & $838->4$ & - & 48,306 & 18,900 & 30,200 & 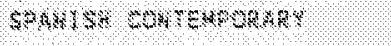 \\
\hline
\end{tabular}




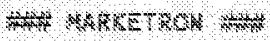

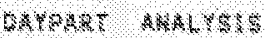

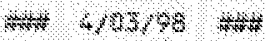

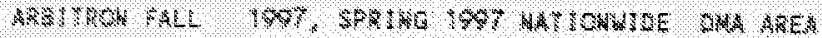

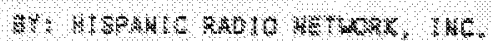

Pase 2

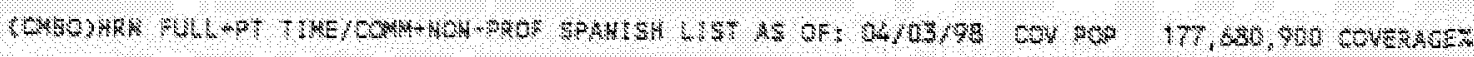
$42,80.222,30,900$, $7,2 \pi$

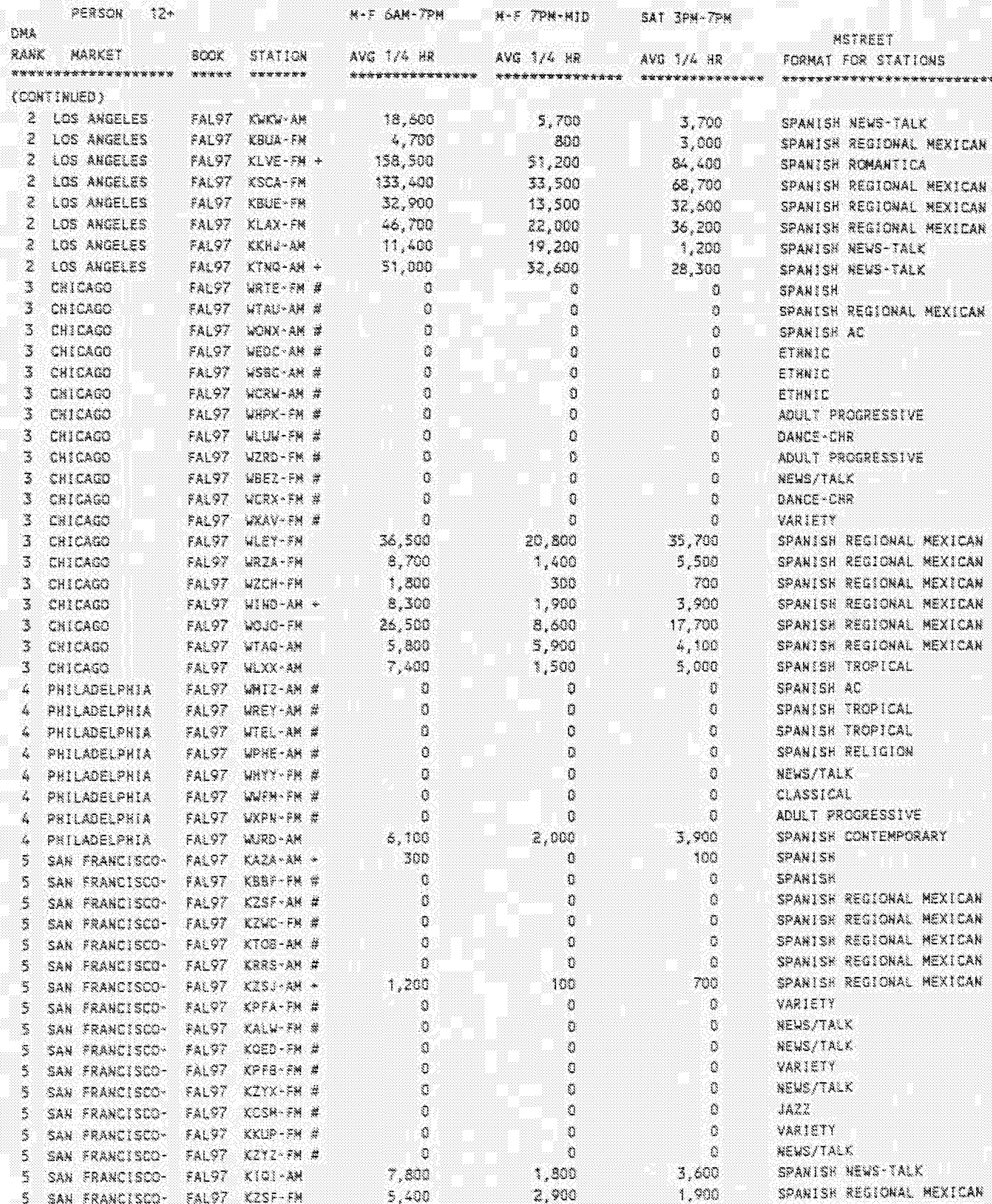




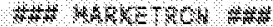

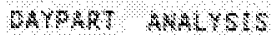

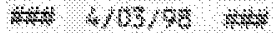

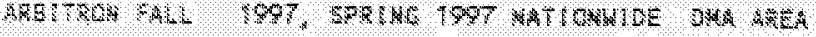

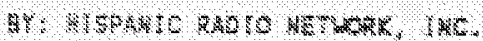

$\mathrm{nks}, 3$

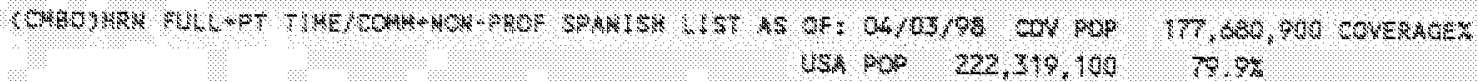

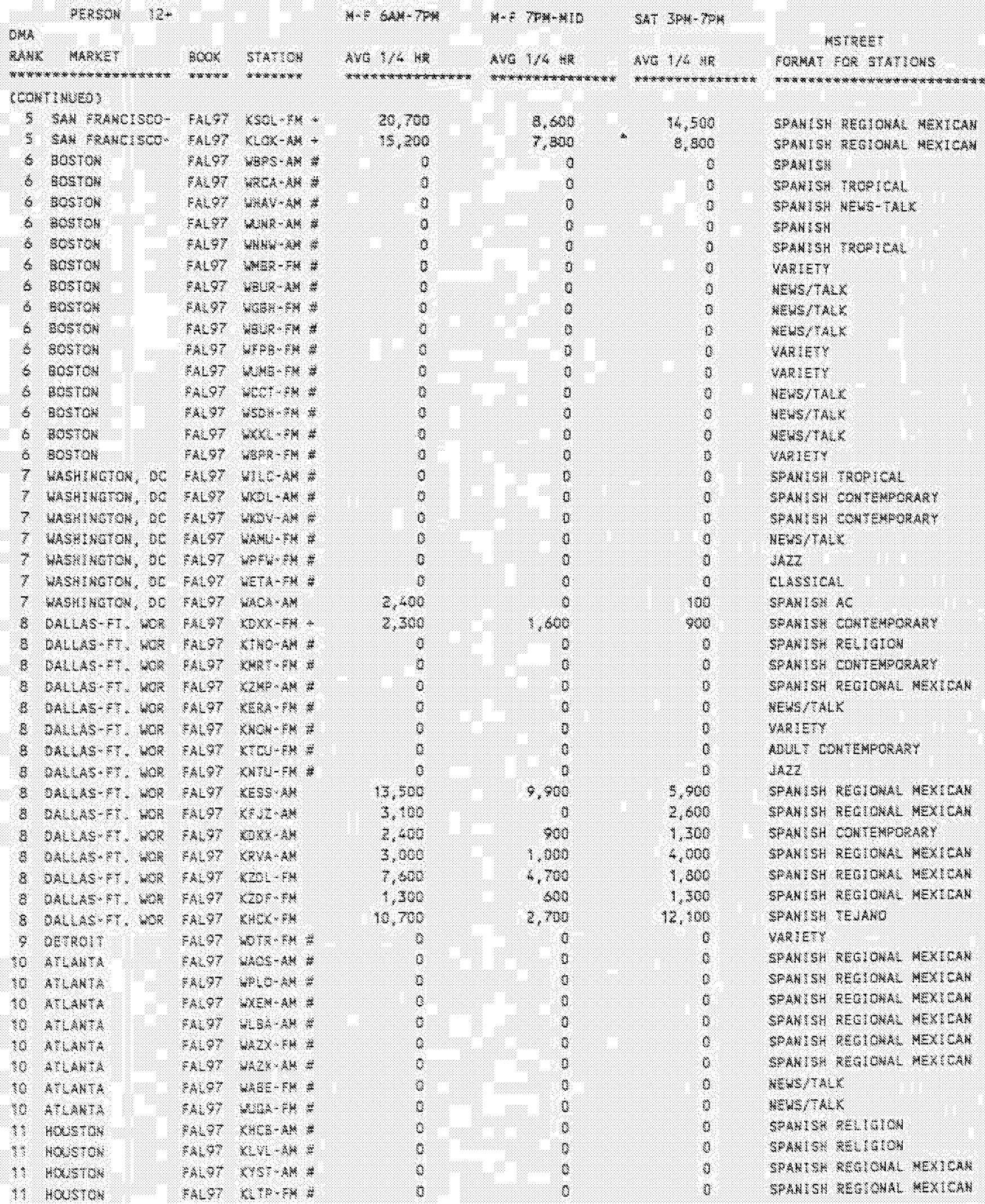




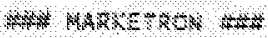

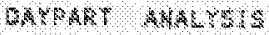

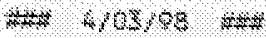

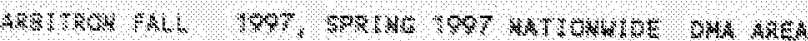

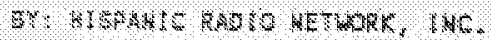

Bate 4

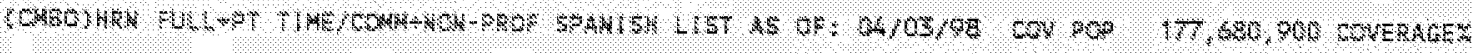

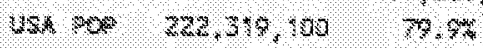

\begin{tabular}{|c|c|c|c|c|c|c|c|c|}
\hline & persen & & & & $4-844-784$ & $4-67+4-480$ & 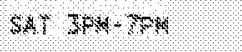 & \\
\hline 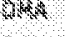 & & & & & & & & $48+888$ \\
\hline $244 \times$ & KKRES & $200 \%$ & 374:004 & & Aus $148 \%$ & 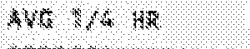 & A40 $1 / 4 \%$ & POOA T TOR STATSOKS \\
\hline$\infty$ & 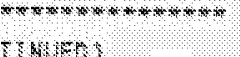 & soremention & $\infty+\infty \times+\infty$ & & 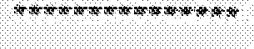 & 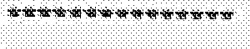 & 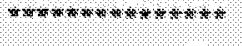 & 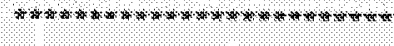 \\
\hline \multicolumn{9}{|c|}{ (CON SMEO } \\
\hline 13 & $14 x=98$ & 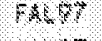 & $080 \times 4$ & 4: & \& & 0 & ๑ & 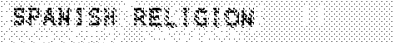 \\
\hline 11 & $104 \mathrm{~s}$ & 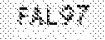 & $1847-8$ & ii & 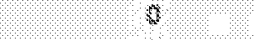 & 8 & 8 & 64848 \\
\hline 11. & $900 \mathrm{src}$ & 848 & $4 \times-8$ & 4 & 8 & 8 & 0 & wewer/ar \\
\hline 4 & 400800 & 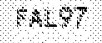 & $1848-78$ & 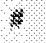 & 0 & 8 & 8 & 422 \\
\hline 19 & 403840 & $918 \%$ & moten & 4 & 9 & 6 & 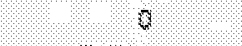 & $94 \times<4090:$ \\
\hline 11 & 10088 & s+cts & $x+8>-46$ & & 9,608 & 8,404 & 7,80 & 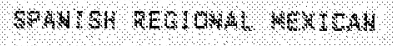 \\
\hline 41 & 4oustors & $94 . \%$ & $4814 \times 4$ & & 9 & a & 0 & 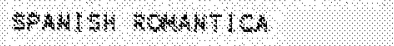 \\
\hline 4 & 800878 & 8007 & $x+0-\pi$ & & థ0) & T20 & 3no & 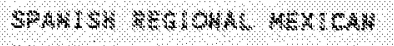 \\
\hline 81 & 100804 & $90 \%$ & $4 \times 1 \times 4$ & & 80,400 & 3,300 & 4,800 & 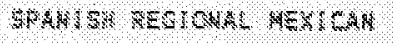 \\
\hline 18 & 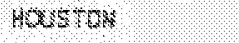 & F⿻十冖? & 80068 & & 17.200 & 2., 10 & 25,200 & $5+4 \times 85,88 \times 46$ \\
\hline 18 & 10050\% & 900 & $4 \times 4$ & & 0.901 & 3,300 & 8,600 & 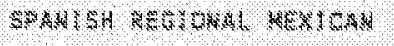 \\
\hline 11 & $30 \times+4$ & 84०० & $80 \mathrm{P}-\mathrm{Ar}$ & & 5,000 & 3, 00 & 1,700 & 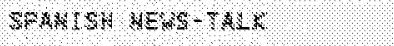 \\
\hline 11 & rausers & 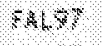 & $14 \times-4$ & $\because$ & 85,400 & 8,408 & 13,100 & 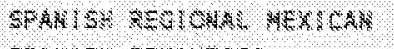 \\
\hline 1 & $1005 \%$ & $94 \%$ & 846 & & $7 \%$ & $4,90 \%$ & 9, $00 \%$ & 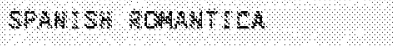 \\
\hline 82 & $\operatorname{sen}+4=6,4 k$ & $4+91$ & $12 \times 4+4$ & और & 0 & 0 & 0 & 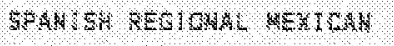 \\
\hline 8 & SEATC - PRCOMA & 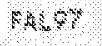 & 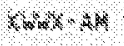 & ए & 8 & 8 & 6 & 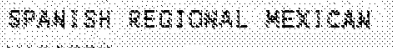 \\
\hline$\%$ & 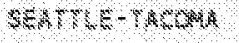 & +1, & $840-14$ & औ & 8 & 8 & 0 & $4 \times 184$ \\
\hline 12 & 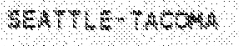 & $\times 4$, & 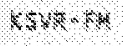 & 3 & 0 & 0 & 6 & $4 \times 1 \times 1 \%$ \\
\hline ४ & 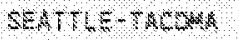 & $241 \%$ & $4 m+4$ & 4 & 8 & 8 & 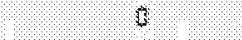 & 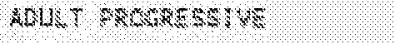 \\
\hline (2) & 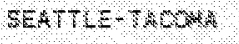 & 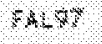 & \% & \% & 8 & 0 & 6 & 1,20 \\
\hline 8 & 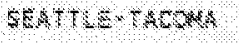 & 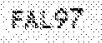 & $60-4$ & s & 6 & b & 8 & Whos Pat \\
\hline 8 & 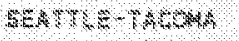 & $148 \%$ & s40, rex & « & 0 & 0 & 0 & WESCARLK \\
\hline 12 & $3 E A B=-R A O A A$ & 6487 & Bस+R & * & 4 & 8 & 0 & News, $\times 4 \times 2$ \\
\hline 16 & $\operatorname{seATCP} 3 \mathrm{CO} 4$ & 8407 & $80 \times 48$ & 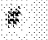 & 8 & 1 & to & 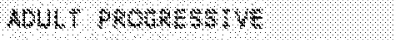 \\
\hline 12 & SEATH $\% \cdot A+4 \times t$ & 8407 & $\mathrm{H}^{4} \mathrm{~B}$ & 4 & 9 & 1) & c & 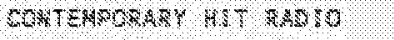 \\
\hline 13 & $06048-48$ & eAor & $4240 \%$ & * & 0 & 0 & 0 & s-4nss: \\
\hline 3 & ELESA & $8 \times, 07$ & 4044 & 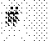 & 0 & s. & 1 & 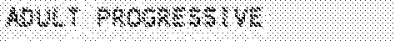 \\
\hline 3 & CLCYCLAKO & $8-87$ & $n_{3}, \mathrm{n}$, & 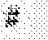 & 0 & 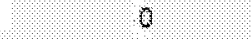 & 0 & GuLt FrovaEss rve \\
\hline 4 & HMKE MPO,$S-31$ & $8 \times 87$ & $4+1-14$ & 4 & 0 & 8 & 8 & 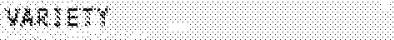 \\
\hline $4^{4}$ & 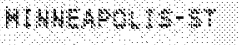 & S4P & dit-m & 4 & 8 & 6 & 8 & $4=851446$ \\
\hline 84 & 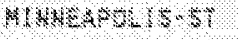 & $84 \%$ & $4 \times 48$ & e & 8 & 8 & 0 & Xexs/taL \\
\hline 1 & $44 \mathrm{KCAPO}, \mathrm{S}-\mathrm{st}$ & A $4 \%$ & Mror & $\%$ & 8 & 6 & 8 & 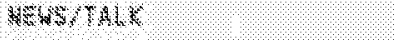 \\
\hline 4 & $4: 41-4>4<18-5$ & $>4<\%$ & $8<+<$ & 8 & 8 & 8 & 0 & 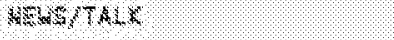 \\
\hline 84 & 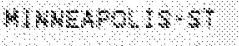 & 846) & $1884 \times 4$ & है & 8 & 0 & 8 & HEWS TIAK \\
\hline 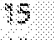 & $\mathrm{APCA} A \mathrm{P}, \mathrm{PET}$ & 8LP & $9 \times 4 \times 4$ & 8 & 8 & 0 & 8 & 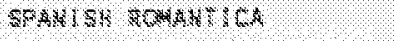 \\
\hline 83 & $848 \mathrm{~A}, \mathrm{~S}, \mathrm{AEAK}$ & $8 \times 4$ & $6 \times 1-4$ & 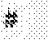 & 0 & 0 & 8 & 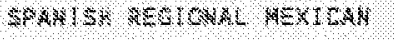 \\
\hline 8 & 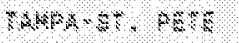 & $\mathrm{An}_{\mathrm{H}} \diamond$ & $4 \times 484$ & 8 & y. & 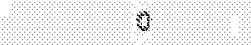 & 8 & 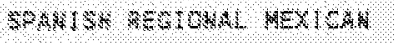 \\
\hline 88 & 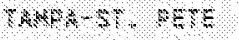 & $54>7$ & $\$ 008 \times 4$ & d. & 1) & 0 & 8 & $84118 \%$ \\
\hline 8 & $\mathrm{~B} \times \mathrm{PH} \cdot-4+\mathrm{PP}$ & BA, & $48+4$ & 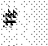 & 0 & 0 & 8 & SPRIS \\
\hline$\$$ & $14 \times 4,48 \%$ & $8,8 \%$ & 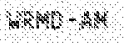 & 4 & 0 & 0 & 0 & 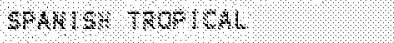 \\
\hline $8 \%$ & $1484+4$ & 84\%? & $1,40,4$ & te & 8 & b & 8 & stats $1 \times 0 \times 10 x$ \\
\hline 18 & $\mathrm{HAR}+\mathrm{r} / \mathrm{P}+\mathrm{P}^{\circ}$ & \$4\$ & $648 \times$ & \% & 0 & 8 & 4 & 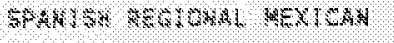 \\
\hline 8 & 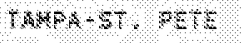 & 144\%? & $m+4$ & 4 & 0 & 0 & 6 & Hor $P$ r \\
\hline 15 & $18 \times 4 \cdot 51,0+4$ & 8887 & $x+8,4$ & 4 & 8 & 0 & ४ & New8 $1 \mathrm{x}$ \\
\hline 48 & 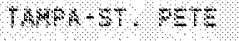 & 1487 & $4 \times 448$ & & 800 & 1,200 & 478 & 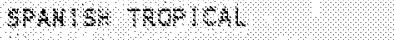 \\
\hline 86 & $\mathrm{ABA}+4,40 \%$ & 198\% & $4 \times 4+8$ & + & 28,060 & 12,800 & $26.82 \%$ & $\mathrm{srn} \times \mathrm{s} \times \mathrm{r}$ \\
\hline 18 & $m \times 4+1,40 \%$ & $84 \%$ & arks? & ? & 36,200 & 8, 000 & $8,5,00$ & 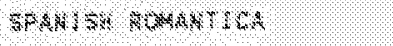 \\
\hline 18 & 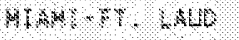 & 2.8\% & 60640 & 4 & 0 & 0 & 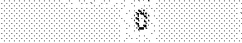 & 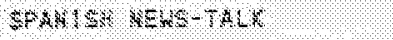 \\
\hline 18 & $9148-4.446$ & $8+8 \%$ & 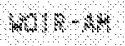 & 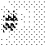 & e & 0 & 6 & 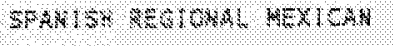 \\
\hline 18 & 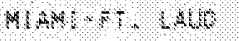 & \%०\% & $x+8-4$ & 8 & 8 & ๑ & to & $8 \times A+1 \%$ \\
\hline 16 & $M 44+4, \quad .40$ & $6 \times 47$ & $4=4<-4$ & ঝ & o & 8 & 8 & $4+4 \times 1340$ \\
\hline 18 & $M A \&-\theta, Q S O O$ & Bas? & $4 \times 46$ & 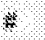 & 0 & 0 & 8 & $3 p \times 14 \mathrm{k}$ ? \\
\hline
\end{tabular}




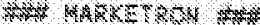

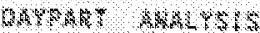
$4+401$,

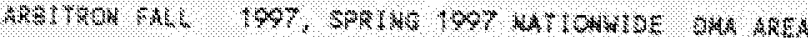

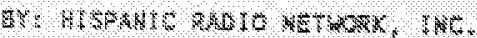

$\mathrm{ers}_{\mathrm{s}} \mathrm{s}$

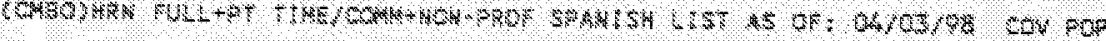
$1 \mathrm{NAO}, 22,31 \mathrm{O}, 13$

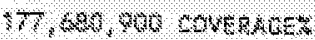
3. ,

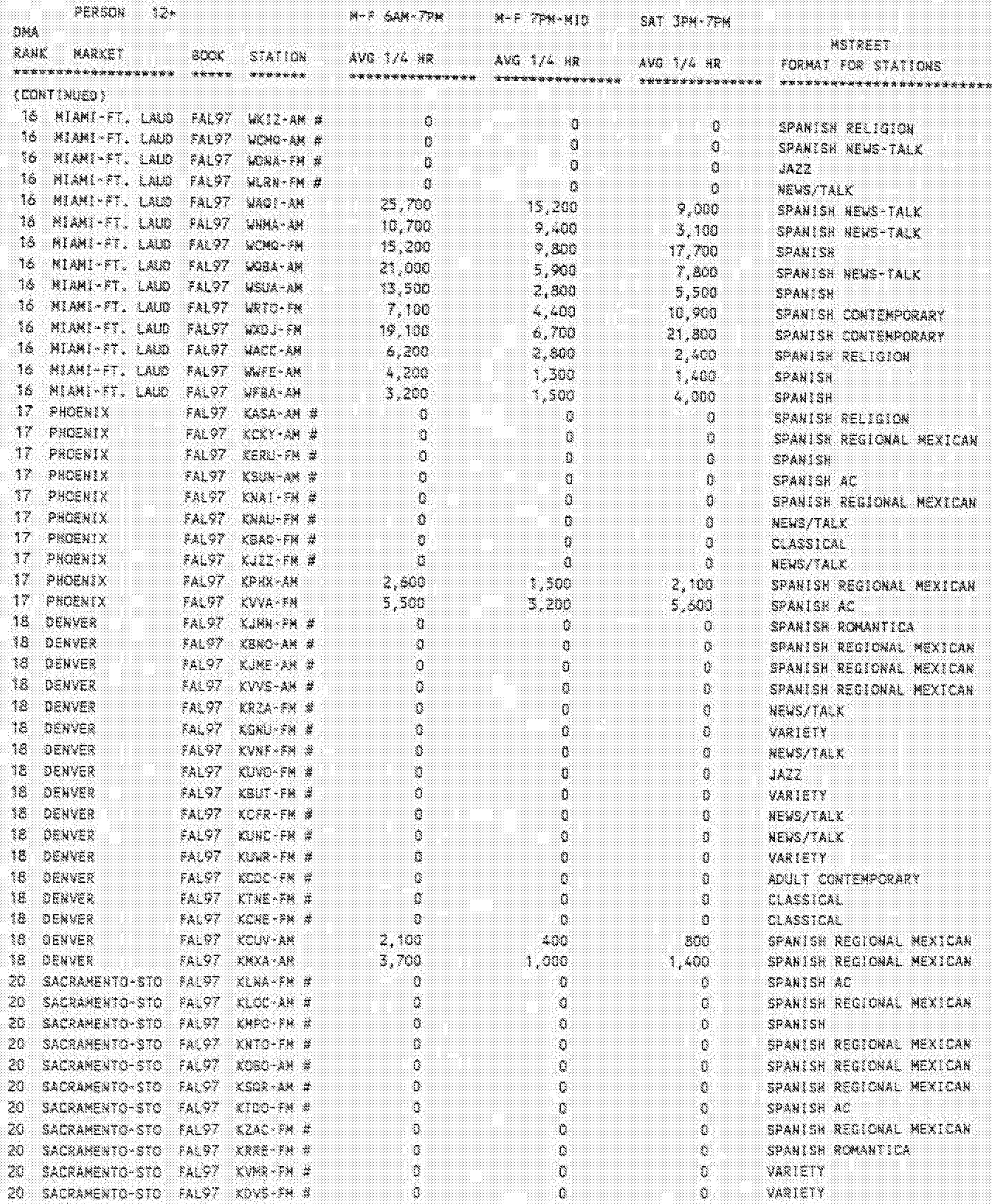




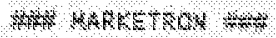

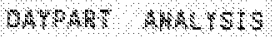

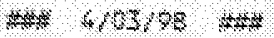

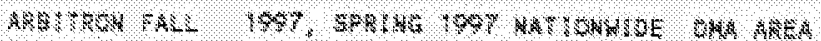

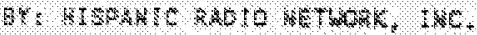

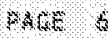

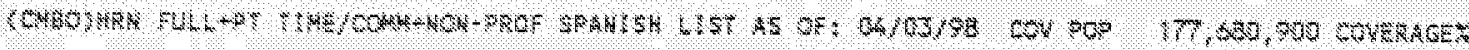

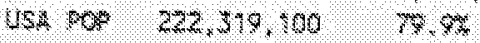

\begin{tabular}{|c|c|c|c|c|c|c|c|c|c|}
\hline He & sesora & 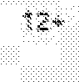 & & & & $\mathrm{H}-\mathrm{F} \Delta \mathrm{AB}-\mathrm{TQR}$ & $4-F+7 \times-410$ & SAI SPH- 76 & 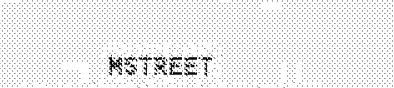 \\
\hline AN & $4 \times 8 \times=1$ & & 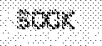 & $48+10 \%$ & & $4 \mathrm{HB} 184 \mathrm{k}$ & KU: $4<4 \%$ & ANG $1 / 4$ ME & Gorkat gro stotrous \\
\hline & 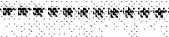 & $+\infty \operatorname{tat}^{+\infty}$ & $+\infty+\infty+\infty)$ & $+* * \infty \%$ & & 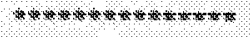 & 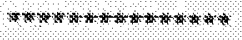 & 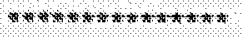 & 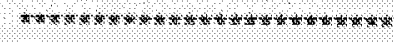 \\
\hline \multicolumn{10}{|c|}{ contrues) } \\
\hline 20 & Shrarexpo & - sto & 8467 & 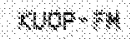 & * & 8 & 0 & s. & $y=x: 1 T A \times x$ \\
\hline 20 & $8 \mathrm{AC} \times \mathrm{M} \times \mathrm{Br}$ & 980 & $8 \mathrm{ntot}$ & $8,12-8$ & e & 8 & 0 & 6 & $\mathrm{AEN}=\mathrm{AA} \times$ \\
\hline 20 & $8 \times 6244018$ & $8-48$ & MAY & $\alpha<0-8$ & $*$ & 0 & 0 & 8 & aeus, $7 \times 4$ \\
\hline 20 & sherkmoks & 8.40 & FAOT & 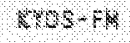 & 4 & 9 & 6 & $\mathrm{~b}$ & (40158? \\
\hline 26 & $86 \mathrm{~cm} 64 \mathrm{~km} / \mathrm{H}$ & $1 .+10$ & 8400? & $8,4 x-4$ & & 3,900 & 800 & 2,00 & 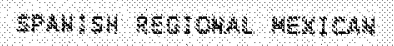 \\
\hline 20 & $84024 \times 4 \times 4$ & 130 & $246 \%$ & $84 \times 4 \times 8$ & & 2,500 & 8,408 & 900 & 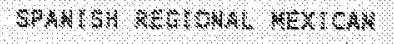 \\
\hline to & $\$ 4 \times 4 \times 15$ & $8+8$ & F⿻187 & $10+1-81$ & & 800 & \$or & 0 & 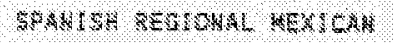 \\
\hline 28 & 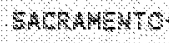 & 850 & $8 \times<\%$ & $x+x+4$ & $*$ & $3,90 \%$ & \%o & 1,400 & 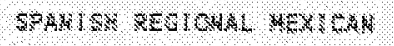 \\
\hline 20 & $8,46 \times 4 \times 18 \times 10$ & -85 & $\mathrm{RB}, \%$ & $84 \times-8$ & & 3,180 & 700 & s60 & 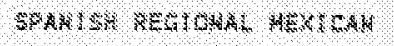 \\
\hline 20 & $8 \times 4 \times 4818$ & sto & Kas\%? & $+8,+\infty$ & & 9,080 & s.o & 1,80 & 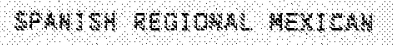 \\
\hline 20 & $48<\times 8 \times 8 \times 0$ & $\$ 10$ & $4 \times 87$ & $8410-4$ & & 6,400 & 3,000 & 3,600 & 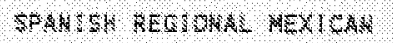 \\
\hline 20 & $2 \times 14 \times 1 \times 10 \%$ & sio & $4 \%, 7$ & 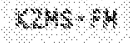 & & 4,600 & 4,800 & 1,808 & 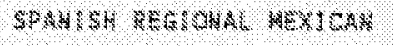 \\
\hline 2 & $00, \ldots<0+6$ & 184: & $4 \times 4$ & 48144 & ? & 0 & 0 & 8 & BRAN1 \\
\hline 22 & $01+1801-0 /$ & res: & $\times, \bullet 7$ & knk- & 4 & 0 & 4 & 8 & 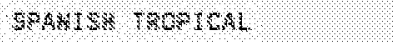 \\
\hline 2 & 100,40040 & 1804. & $8 \times 4 \%$ & $\mathrm{kRC}+\mathrm{a}$ & 4 & 6 & 8 & 8 & 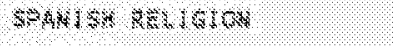 \\
\hline 3 & $00(10100+4)$ & 170\%: & 1840 & $00 \% 1-4$ & 4 & 4 & 4 & 9 & 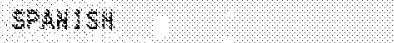 \\
\hline 27 & $4 \times 1 \times 1000$ & 100: & $8 \times 0 \%$ & $4 \times 4 \times 4$ & ४ & 0 & 8 & 9 & $3 \times \times 18 \times 14$ \\
\hline 22 & $040400+2$ & rots: & 4007 & $2+4+4$ & 4 & 8 & 8 & 4 & 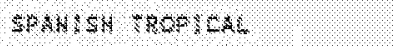 \\
\hline 22 & $0 \times 14 \times 0<-010$ & s.0\% & 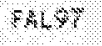 & Kar -4 & 4 & 0 & 8 & $a$ & 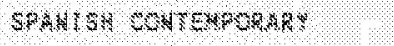 \\
\hline 22 & $9 \times 1+4 \times 20+4 \times$ & ron: & 90107 & 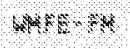 & 8. & 6 & 8 & 8 & $16 * 474 \%$ \\
\hline 28 & $00 \times 8 \% 00,2$ & rem & $84 \mathrm{~s}$ & 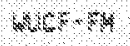 & * & 6 & 8 & 8 & $3 \times 2$ \\
\hline 23 & $94 \times 8 \times 404=$ & & $F 8 \%$ & $4014-94$ & 4 & 4 & 0 & 8 & 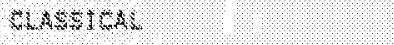 \\
\hline 25 & $848+8 \times 0 \times 5$ & & FALOt & अ $44-9 M$ & 4 & 6 & 8 & s & 14:4s:1 $48 x$ \\
\hline 3 & SAL P $400 \%$ & & $\mathrm{PA} ९ \mathrm{P}$ & $648+\pi$ & e & 8 & 0 & 6 & CLASTCAL \\
\hline 24 & $90 \% 14 \times 4,8$ & \&. & BASOT & $\mathrm{M}+\mathrm{2} \times \mathrm{M}$ & 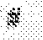 & 0 & 0 & 1 & 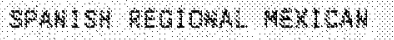 \\
\hline 24 & $90 \times r \times 18$, & 21 & Fast & $3 \times 3 \times 14$ & 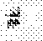 & 4 & $\mathrm{a}$ & 0 & 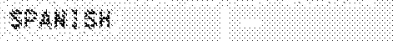 \\
\hline 26 & $90 \% 1 \times 1 \times 10$, & \% & M $40 \%$ & 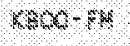 & 8? & 0 & 0 & 0 & KaBSES \\
\hline et & PGKT-ANS, & 8 & 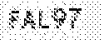 & $869, k$ & if & 1 & 9 & 0 & 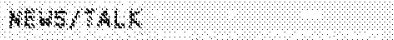 \\
\hline 24 & $80 \times \times \wedge \times 1$, & $\nabla$ & $84 \% 7$ & 4418-46: & & $2, \infty 0$ & 700 & 4,108 & 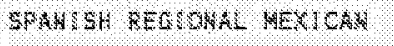 \\
\hline 28 & कAN 91080 & & 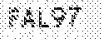 & $818 \times 4$ & $\bullet$ & top, $x$ so & 10,300 & 24,900 & \\
\hline 28 & $848<1800$ & & $8+407$ & 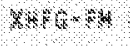 & F & 8 & 0 & 8 & \\
\hline 28 & 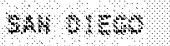 & & $=4,4$ & $x e x+4$ & 8 & 0 & 6 & 6 & \\
\hline 28 & sak 91800 & & $8 \times 20$ & $88804 A 4$ & औ. & 8 & a & 8 & \\
\hline 2 & \$AS PUCSO & & 940 & $x=30 \%$ & 4 & \% & @ & 0 & \\
\hline 26 & Eak P\&8\% & & $9 \times 6 \%$ & $40-84$ & ㅎ & \% & 3 & 8 & \\
\hline 28 & $5 \times 48480$ & & $8 \times 4$ & xes:un & s. & « & ४ & 4 & 4exs, 446 \\
\hline 28 & $\sin \mathrm{OLE}$ & & 8tosi & $4405-8$ & & 2,640 & 908 & 1,300 & 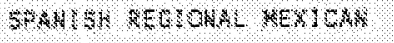 \\
\hline 2 & $3 \times 140466$ & & $8 \times 97$ & xprs 48 & & 2.804 & 4,800 & 4,78 & \\
\hline 2 & 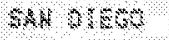 & & 8607 & 14848 & & 4,800 & 2,300 & 3,60 & \\
\hline 26 & $8 \times 16 \times 86$ & & (A. & 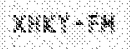 & & 3,800 & 2,300 & 3.100 & \\
\hline 24 & $94 \mathrm{Prs}$ & & 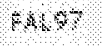 & $\times 40,4$ & & 8,200 & 2,70 & 9,180 & \\
\hline 26 & $8410<080$ & & $=4.8$ & 1448 & & 4800 & 3,000 & s, & 3 \\
\hline 86 & 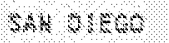 & & $8+48$ & $x+484$ & $\bullet$ & 4,80 & 3,80 & 1,600 & \\
\hline ४⿻ & $84 \mathrm{r} \quad \mathrm{r80} 3$ & & $8,8 \%$ & $440-4 \%$ & \% & $4, \$ 00$ & 8,100 & 800 & \\
\hline 8 & 1448488 & ४ 4 : & 8407 & $68 \times 8 \times 4$ & 4 & 0 & 0 & 8 & 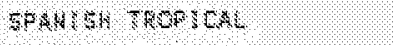 \\
\hline 4 & $14 \times+\infty<$ & $8 \%$ & $8 \times, ?$ & $4014-4$ & : & 0 & 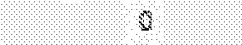 & 6 & 80448 \& $400 \%$ CA. \\
\hline 2 & $1 \times 1 \times 8+k<$ & $8 \%$ & $8+87$ & $6 \times 18648$ & + : & 6 & 0 & 8 & 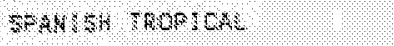 \\
\hline 2 & $14 \mathrm{~s}<\mathrm{s} / \mathrm{\alpha}$ & 486 & 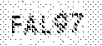 & 40,484 & *: & 0 & 0 & 0 & 844848 \\
\hline 3 & $4 \mathrm{k} \times \mathrm{man}$, & s:* & 8,4 & $4 \mathrm{NH}+\mathrm{Ar}$ & is & 8 & $\theta$ & 0 & 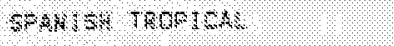 \\
\hline 2 & 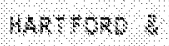 & (8) & $\mathrm{AR} \odot \mathrm{t}$ & $m+4$ & $*$ & 8 & 0 & 8 & 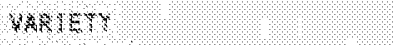 \\
\hline se & 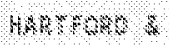 & Het & PA $\odot$ & $6460-74$ & 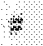 & 0 & 8 & 0 & $x=48+4,6$ \\
\hline $2 \%$ & hanstros & kites & $-\mathrm{AC} \bullet$ & $x+40$ & 8 & 0 & 8 & 8 & UEWBVTAP \\
\hline
\end{tabular}




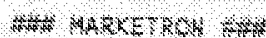

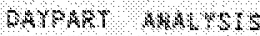
$4+4,4+4$

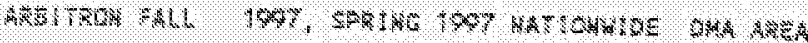

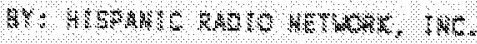

8,4,

1T, 400,900 conthate:

$\%, 8$

\begin{tabular}{|c|c|c|c|c|c|c|c|c|}
\hline 34 & 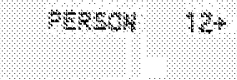 & & & & $4 \cdot+484 \times 7 \times$ & $4-578-816$ & $8 x=384-7+4$ & \\
\hline 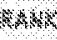 & 4ars: & $8 \times 4$ & $54 A+4$ & & 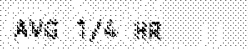 & 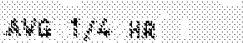 & AKG $/ 4+\%$ & 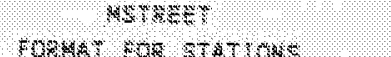 \\
\hline & 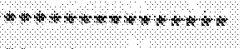 & thenowion & trterosos: & & 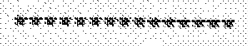 & 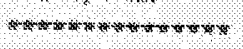 & trater & 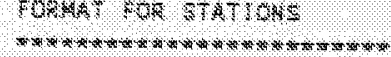 \\
\hline \multicolumn{9}{|c|}{ corr MLEO } \\
\hline 27 & $44 P F-C$ \& $M E M$ & 8469 & 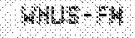 & 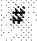 & 6 & 8 & 8 & WAR 5 H \\
\hline 27 & 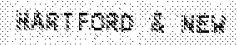 & CASOR & $6 \mathrm{~A}+\mathrm{AN}$ & & 1,600 & 700 & 600 & sonks:sh routsal \\
\hline 28 & CKAROSYE & PALOF & 460404 & e & 0 & 8 & 9 & 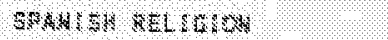 \\
\hline 26 & QnkR SOre & $5 \times 1,7$ & HA, -8 & 4 & 8) & 1 & 8 & 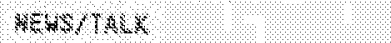 \\
\hline 28 & chorutre & 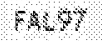 & $60 \times 6+4$ & 4 & 6 & 8 & 8 & 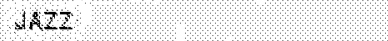 \\
\hline $2 *$ & 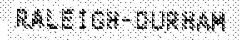 & BALOR & 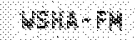 & 4 & 4 & 0 & 0 & $\sqrt{*} \geq 2$ \\
\hline 29 & 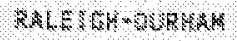 & 7157 & $3<s-4$ & & 1,502 & 1.800 & 3,580 & $48 \times 48$ \\
\hline s? & 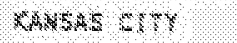 & 140\%? & $\mathrm{X}+\mathrm{l}-\mathrm{H}$ & 8 & 0 & 8 & 6 & $48 \mathrm{~s}=\mathrm{r}$ \\
\hline 32 & Gu whines & बASP & $48 \times 4 \times 4$ & « & 4 & 8 & 8 & $8>4184$ \\
\hline 3 & 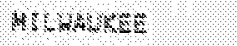 & $8 \times 47$ & $440-k$ & $\%$ & 8 & 8 & 8 & Ass $\sin <\mathrm{X}$ \\
\hline 32 & nowkrkes & 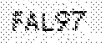 & $14 \times 8 \cdot 4$ & *. & 4 & 8 & 10 & 402 \\
\hline+2 & 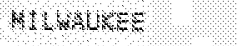 & $140 \%$ & 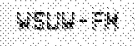 & *. & 8 & 9 & a & \&A2 \\
\hline 35 & $4 \times 8448+2=$ & M०० & $4=6, \mathrm{~m}$ & * & 8 & 1 & 0 & 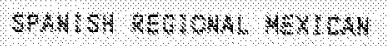 \\
\hline 36 & $3,4<4 \times 4 \times 4$ & 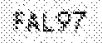 & 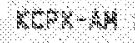 & 4 & 8 & 0 & 8 & 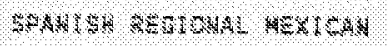 \\
\hline 36 & 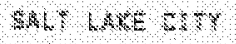 & 4,407 & 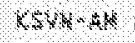 & si & 8 & 8 & 0 & 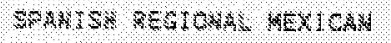 \\
\hline 80 & $4 \times, 4, k=4 r$ & 4067 & $80+4$ & 6) & 6 & 4 & 0 & 44848 \\
\hline 8 & $\mathrm{~s}, \mathrm{r}<\mathrm{k} \times \mathrm{H} / \mathrm{H}$ & (x $6 \%$ & $1084 \%$ & त) & 8 & 8 & 6 & 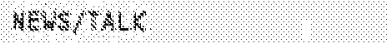 \\
\hline 36 & 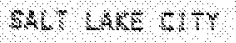 & (.407 & $484 \% 8 \%$ & 4 & 0 & 8 & 6 & 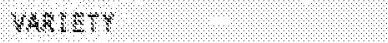 \\
\hline 36 & 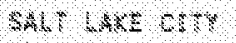 & Rls: & 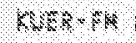 & ते & 0 & 0 & 6 & $4 \times 20,1 / 4 x$ \\
\hline 8 & 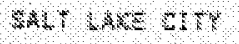 & nslot & $10 \times 1+8$ & w & 0 & 0 & 6 & 1exters \\
\hline 36 & 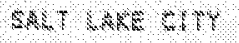 & SALT & $20 \pi+-4$ & \& & 8 & o & 8 & WhOES \\
\hline 38 & $3 \mathrm{AL} * \angle \mathrm{A}=\mathrm{CHT}$ & $8 \times 97$ & $x+40+4$ & \& & 6 & 0 & 0 & NAWS $/$ ALE \\
\hline 36 & 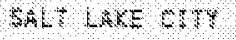 & $\mathrm{BA}, \mathrm{T}$ & $4 \times 18$ & क & 8 & 0 & 8 & Yom $\mathrm{SET}$ \\
\hline 37 & 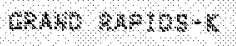 & 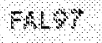 & $4 \mathrm{~T}, \mathrm{P} / \mathrm{N}$ & $m$ & 8 & 0 & 4 & $8=281 \%$ \\
\hline W\% & GRAN \&APIDS-X & (4⿻० & $4 m x<+2$ & F & 8 & 0 & 0 & HeWs/ ThLC \\
\hline 38 & $841641 \times+4,10$ & $+80 \%$ & $\mathrm{krk}-\mathrm{k}$ & - & $2, \infty$ & 9,700 & 25,800 & SPA4S sH TEAARO \\
\hline 38 & $4 A 4 \times 4+440$ & $\times 467$ & $\mathrm{sen}+\mathrm{AN}$ & 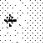 & 8,40 & 1,000 & 2,300 & 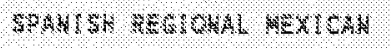 \\
\hline 38 & $34 \times 4 \times 1 / 10$ & PALF & $\mathrm{sec}-\mathrm{As}$ & 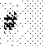 & 8 & 6 & 8 & 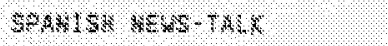 \\
\hline 38 & 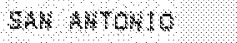 & $4 \mathrm{n}>7$ & $4.8 \times 1$ & 4 & 8 & 8 & c & 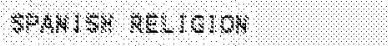 \\
\hline 38 & $64 \mathrm{ABN} \times \mathrm{AO}$ & Fast & KE & 4 & 0 & y & 8 & 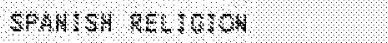 \\
\hline \%) & $3 \times 4 \times 48 \times 10$ & $8 \times \odot$ ? & $8+4+8$ & 8 & 8 & g) & 8 & 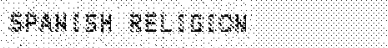 \\
\hline 38 & $2,4 \times 8 \times 10 \times 10$ & 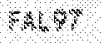 & $888 \times 4 \times$ & 4 & 8 & s) & 8 & 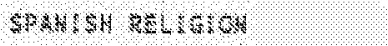 \\
\hline 38 & SAK ARTORPC & $640 \%$ & $x^{4}-1-4$ & 8 & 0 & 4 & 8 & 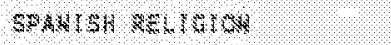 \\
\hline 38. & $248 \times 40410$ & m 48 & sstr-kn & a) & 8 & 6 & 0 & WE, STYMAS \\
\hline 38 & $\cos 1 \times 18 \times 140$ & 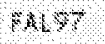 & 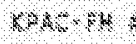 & $=$ & 3 & 8 & 8 & $4.45846 x$ \\
\hline 48 & skn kn+8k:0 & resol & $3 \times 4,4 \times$ & 8 & 8 & 8 & 8 & sonkrs? \\
\hline 30 & 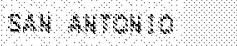 & 1007 & NHE-4: & $\Leftrightarrow$ & 8 & 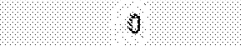 & 8 & 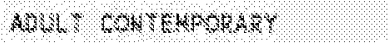 \\
\hline 18 & 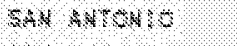 & 8487 & $854 \times 4 \times$ & 8 & 180 & 8 & 8 & 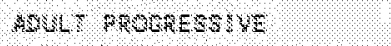 \\
\hline$a$ & $3484 \times 48,0$ & $8 \times 47$ & $\operatorname{sen}-\pi$ & & 4,800 & 2,100 & 3,860 & 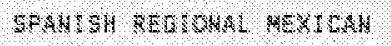 \\
\hline $8 \%$ & 848,46468 & BLP & सer +48 & & 2,000 & क्ष & 1,860 & $50 \times 4154,80 \times 40$ \\
\hline$t^{\prime}$ & $4 \times 4,48480$ & ents? & 405,4 & & 4,70 & 4,800 & 1, 80 & $4+4,54+3,4 \times 4$ \\
\hline & $444,100 / 8$ & $P A<>$ ? & 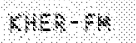 & & 2,000 & 4,80 & 1,200 & 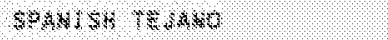 \\
\hline & 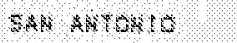 & $60 \%$ & 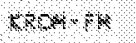 & & (c., 10 & T, 060 & 9,800 & $8 \times 8418 \times 48$ \\
\hline & $546 \times 6 \times 4+5$ & 14: 87 & k, 4 & & 3,40 & 4,060 & 1,600 & 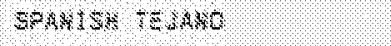 \\
\hline & $18 \times 1 \times 87860$ & 806 & $\mathrm{nnt}$ & & $4,2,0$ & 200 & 6 & 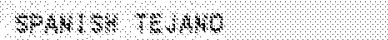 \\
\hline & $8+4<4>048$ & th, & $4+4,44$ & 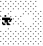 & 4,60 & 306 & 480 & \\
\hline & $46 \times P_{L}-84 \%$ & 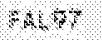 & $60104 \%$ & $*$ & 8 & 3 & 9 & 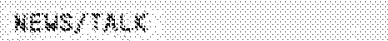 \\
\hline & $48 * 4<4 \times 6$ & (4) & $8 \% 4 \times 4$ & 8 & 4 & 0 & 9 & 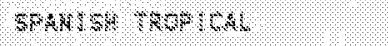 \\
\hline & $48,8 s, \mathrm{Ans}$ & Rest & $36,4,46$ & * & 8 & 0 & 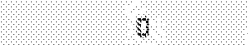 & shors sit 40 \\
\hline & $184 \times 1.848$ & \$A०० & 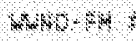 & 4 & 8 & 8 & 4 & AESS TRLA \\
\hline & $88404<4 k$ & PA० & $408-4$ & + & 3,500 & 3,900 & 9,890 & $70 \cdot s$ cussis pest \\
\hline 8 & Teratrs & $940 t$ & $3+3 \times 4+48$ & औ & 8 & 3 & 8 & seAns 8 \\
\hline
\end{tabular}
UEA $\infty 2027,3,0,10$

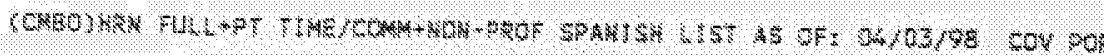

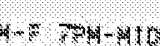

W6 $1 / 4$ Ha$$
\text { , }
$$

FOR4AT TOR STATIOKS

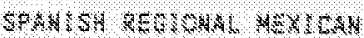

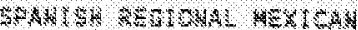
$4,1,060$ NCW $\$ 1 \mathrm{ALC}$ Wat 2 : wat $1 \times-x$

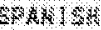

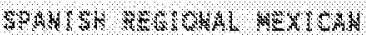
SAMIS \&EXS-TKK C) 0 A.

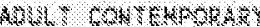

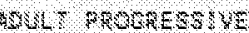
GNMUI SH TESAMO

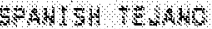

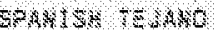

$1205 \times 1 \times 4$

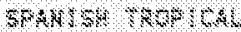
Sh: 1 ISW AC

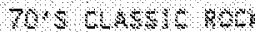
3NAn:s: 


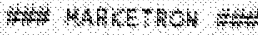

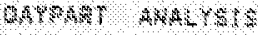
$4+40,030 \%$

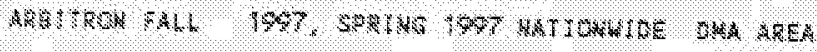

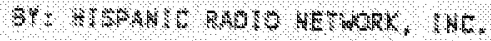

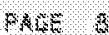

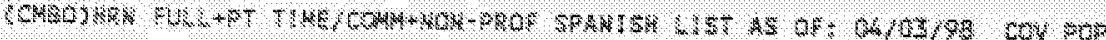

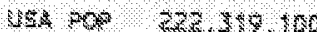

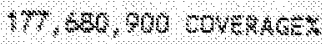
+,$\times$

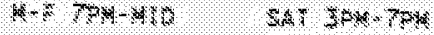

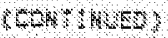

42 MEMP 10

EAB 47 WOST-AM $*$

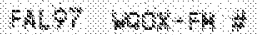

$4-6,84-704$

BALP $\quad 4,4 \%-4 \times$

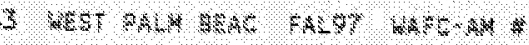

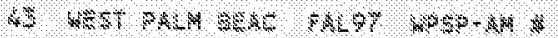

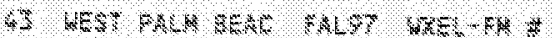

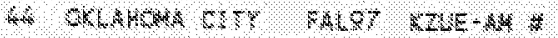

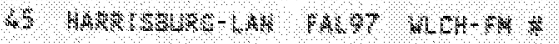

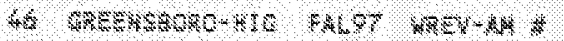

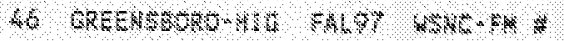

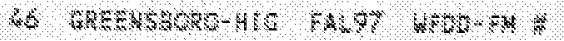

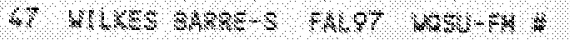

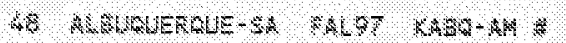

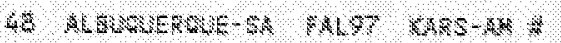

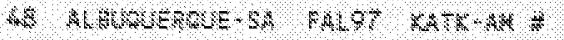

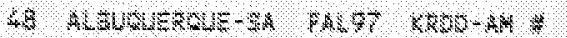

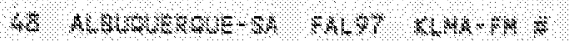

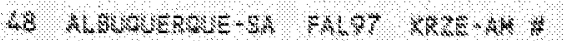

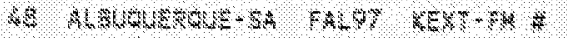

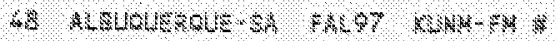

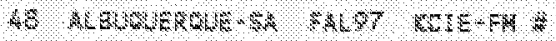

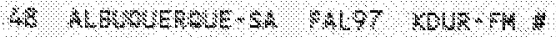

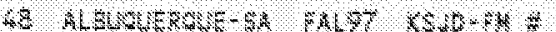

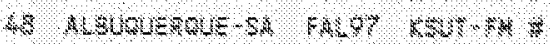

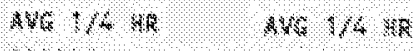

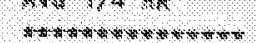

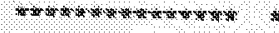

\begin{tabular}{|c|c|c|}
\hline 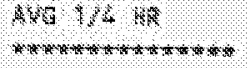 & 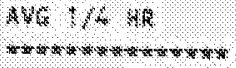 & Ris $14 \mathrm{kh}$ \\
\hline
\end{tabular}

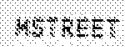

GOWh row STATIOUS

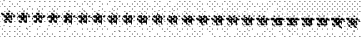

tapsinsts:

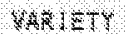

$8 \times 4854$ sest40

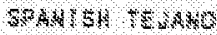

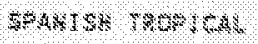

KEW $1 \mathrm{TAL}$

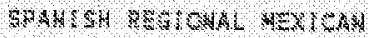

seanto

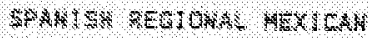

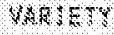

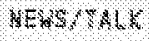

CuASTCA

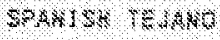

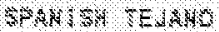

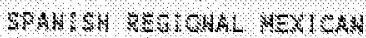

$58 \times 64: 5 \%$

semets

stans: :

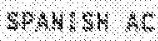

WENS/TALC

WAFIETT

Whater

NEWS TALK

NEWS R NLC

WRTETY

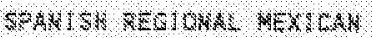

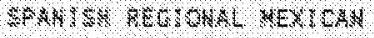

SPABS SH BECSOHRL AEXICHA SPAHISH RESIOUAL WEXICA

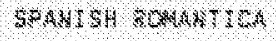

send is 5

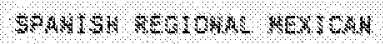

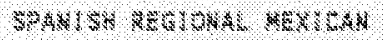

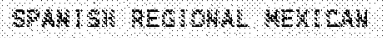

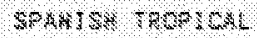

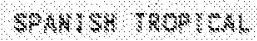

un: $5: 4$

wh:1818

NESS/ThLT

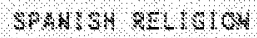

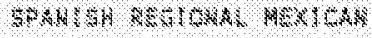

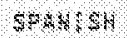

$40 \times 1484 \times$

seak \& s

s74hes:

$8, n+1.6$

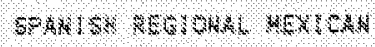

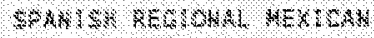

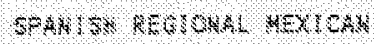

$\$ 4$ औis $\$$ s

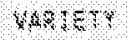

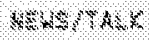




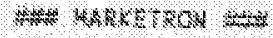

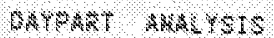

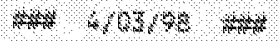

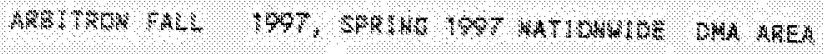

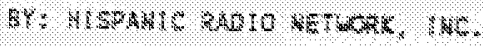

was 5

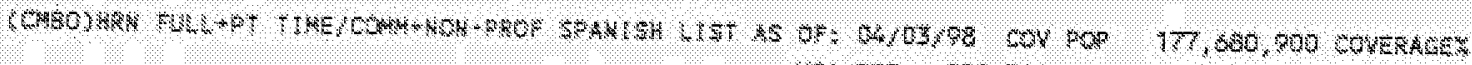

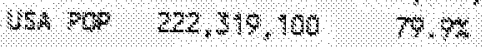

\begin{tabular}{|c|c|c|c|c|c|c|c|c|}
\hline $6 \times$ & reroos & & & & $x-8 \quad 84+2=4$ & $4-8$ Pex-3:30 & SAT $3 P R-R 4$ & Ner $x<1$ \\
\hline Am & Makret & 8006 & $8 \times 8404$ & & $1 \% 48$ & $\mathrm{NHS} 1 / 4 \mathrm{\& B}$ & NWo $1 / 4$ ख⿰氵 & 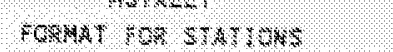 \\
\hline \multicolumn{9}{|c|}{ Eontrings } \\
\hline 88 & 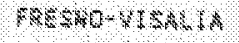 & \$67\% & 848,48 & \% & 3 & 0 & a & 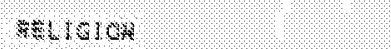 \\
\hline 83 & 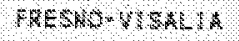 & 43.97 & ASP-AW & & $2, \infty 0$ & 300 & 1,000 & 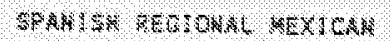 \\
\hline $5 \%$ & fecs $104 \mathrm{BALQH}$ & $448 \%$ & $1000 \times 14$ & $\bullet$ & 4,304 & 2,800 & 1,30 & 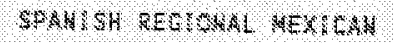 \\
\hline 98 & 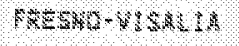 & $\times 17 \%$ & Kas $5+4 \mathrm{~K}$ & & $1, \times 00$ & 504 & 804 & 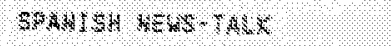 \\
\hline 5. & 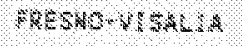 & Maror & $\times 00-8$ & & 3,100 & 3,400 & 1,809 & 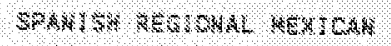 \\
\hline 85 . & $8 \mathrm{CSSO}+\mathrm{r} / \mathrm{sACA}$ & MAst & $x+x<+8$ & & 4,100 & 4.200 & 4,200 & 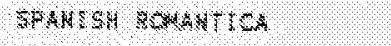 \\
\hline 88 & $4 x=8,0-4 \times<\times 16$ & $48 \%$ & $x+4+4$ & & 100 & 0 & 201 & 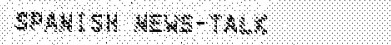 \\
\hline 9 & $4 R E \times 10-Y 8 R L A M$ & 4at? & $x \times x+4$ & & \%ol & reo & 200 & 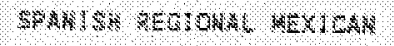 \\
\hline 3) & 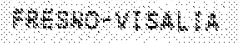 & \$1tor & 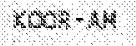 & & 4,008 & 480 & 1, 800 & 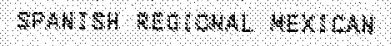 \\
\hline 3 & 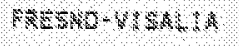 & $40+5 \%$ & $80,4-4$ & $*$ & 100 & 2,000 & \$o & 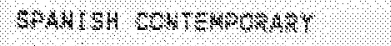 \\
\hline 55 & 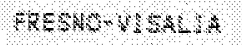 & $8 \times 877$ & 4648,8 & & 4,94 & 3,800 & ๑, 400 & 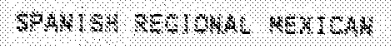 \\
\hline 88 & 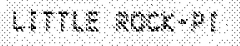 & IATY & $96 \%=8$ & ?: & 8 & 0 & o & 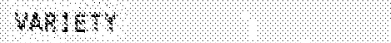 \\
\hline 87. & 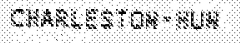 & $4 \times 8 \%$ & 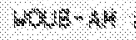 & 4 & 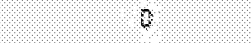 & 8 & 0 & . \\
\hline 98 & $4 \% 4$ & $4 \times 8 \%$ & 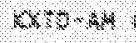 & 4 & 4 & 8 & 4 & 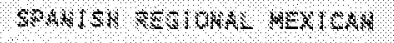 \\
\hline s\% & 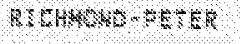 & M. & 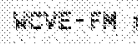 & \% & 4 & b & 0 & kestor $>A<x$ \\
\hline 6 & kusmo & sarot & $200+46$ & th & 8 & 4 & 6 & 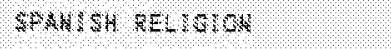 \\
\hline 60 & MSOTH & $4 \times 47$ & $6 \times+48$ & अै & $\theta$ & 8 & 8 & $98,4184 \mathrm{r}=4 \times 0$ \\
\hline 60 & $408 \times 1$ & Mrst & $1402 \times 4$ & $\$$ & 8 & 8 & 0 & 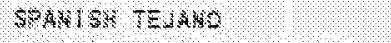 \\
\hline 6 & now 14 & 8977 & $4,+4$ & 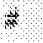 & $\bullet$ & 0 & 0 & 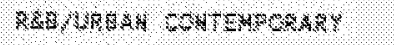 \\
\hline 6 & (608s? & \$48\% & $18 \%-4$ & \% & 0 & 0 & 0 & $10 \times 4, \times 14$ \\
\hline 8 & $453 \times 14$ & 4997 & $x=0-4 \times$ & & 2,700 & 300 & 4,500 & 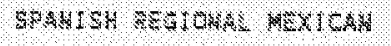 \\
\hline 80 & 4usti: & 8400 & $8 \times 1+4$ & & 800 & 200 & से। & 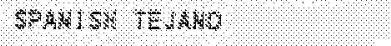 \\
\hline 80 & Austo & xistor & 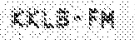 & & 2,000 & 9.400 & 4,008 & $48 \times 818488,460$ \\
\hline 61 & 4884848 & MATP? & $\mathrm{secs}-\mathrm{ks}$ & & 1,408 & 30 & 8,600 & 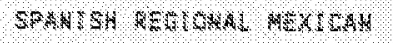 \\
\hline 81 & 448 ub4s & as? & $\mathrm{ks}-\mathrm{s}-\mathrm{m}$ & & 5,600 & 2,100 & 6.708 & 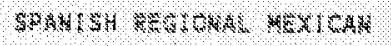 \\
\hline$\%$ & Herra-14reks & 1487 & $1 \% 8-4 \%$ & * & o & 0 & 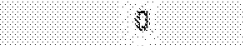 & 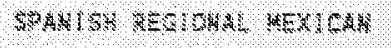 \\
\hline 65 & 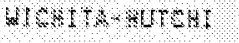 & $8 \times 98$ & 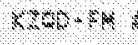 & $\forall$ & 8 & 6 & 0 & 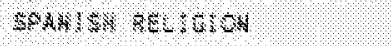 \\
\hline 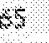 & $146454-48 \times 48$ & $4 \times 98$ & $443 \times 4$ & 4 & 8 & 8 & ○ & YAs $8 B 8$ \\
\hline 8 & 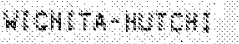 & 14,07 & 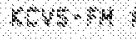 & 8 & 0 & 9 & o & 1416804 \\
\hline 36 & Wrott-4UTH & 446 & $304 \times 4$ & & 800 & 700 & 700 & 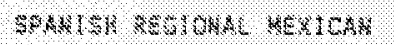 \\
\hline 78 & 80,04044 & $88 \times 07$ & 348,4 & 8 & 0 & 8 & 8 & $48 \times 4 r$ \\
\hline 7 & $80,04 \% 4$ & 1849? & $81>0,4$ & a & 0 & c & 8 & $68684 \pi \mathrm{k}, \mathrm{C}$ \\
\hline 7 & MomMLLS & 19487 & $x<+<-8$ & 4 & 8 & 8 & 8 & KMULF PROLEESSIVE \\
\hline 72 & Srascrs & $14 \% ?$ & $48 \mathrm{C} * \mathrm{k}$ & 8 & 0 & 8 & 2 & AOULT PEOARESSIVE \\
\hline 3 & somkink & $4 a+\%$ & $\mathrm{sech}-\mathrm{m}$ & $=$ & 6 & 9 & 3. & $80 \times 4184$ \\
\hline 8 & $800 \times 4 \times$ & 48432 & 20848 & 4 & 8 & 0 & 0 & GOLT PrOMRESS: VE \\
\hline 3 & $30 \times 48=$ & $1,48 \%$ & $90 \times-88$ & 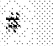 & 8 & 6 & 8 & 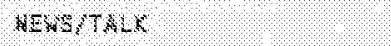 \\
\hline 78 & sen 4 & $4 \times>$ & x+4. 4 & e & 8 & 8 & 8 & 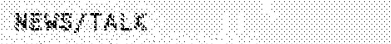 \\
\hline 2 & 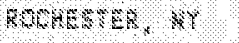 & $4 \times 467$ & $488-4$ & 4 & 4 & 0 & 9 & 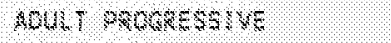 \\
\hline 78 & $60 \% 8=58 \pi, 61$ & $4 \times 4 \%$ & $4-\infty+4$ & 4 & 8 & 6 & 8 & 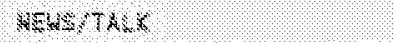 \\
\hline 8 & rireveresor & $89 \bullet \bullet ?$ & $0,6,4 \times 6$ & 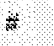 & 0 & 0 & 9 & 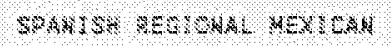 \\
\hline 78 & $4058 \times 1004=$ & $4 \times 4$ & $x+8 \times 4$ & (t) & 6 & c & 0 & sFrur : RE \\
\hline 78 & $140048018=$ & $8 \times 5 \%$ & $4 \times 4+8$ & 4 & 0 & i & 0 & 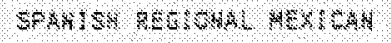 \\
\hline 18 & $14+40 \times 4 \% 48$ & $40 \% \%$ & 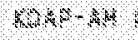 & e & 8 & 4 & b & 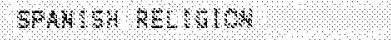 \\
\hline 8 & 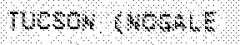 & 4.ro? & $4 \times 4 \times 4$ & 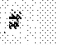 & 0 & ळ & 0 & 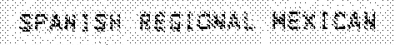 \\
\hline 8 & $440 \mathrm{~m} \times \mathrm{s} / \mathrm{m} / \mathrm{x}$ & 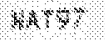 & $\times 4 \times, 4$ & 8 & 8. & 8 & 0 & $4+4,8 n$ \\
\hline 8 & 10401840048 & $18+4 \%$ & $40,+4$ & $*$ & 8 & s & 0 & 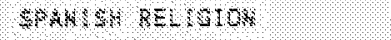 \\
\hline 4 & 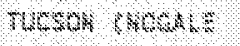 & 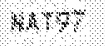 & $8 \times<+8$ & 6 & 8 & 8 & 0 & 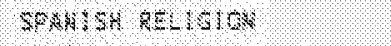 \\
\hline 8 & $100 \times 10400 \times 1=$ & xato & 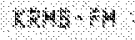 & * & 8 & 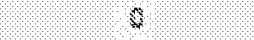 & 0 & SPRS S RESGTOR \\
\hline 8 & $100 \times 0 \times 400-4=$ & 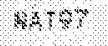 & 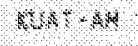 & \% & 8 & 8 & 0 & 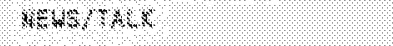 \\
\hline 4 & $146+64,6064=$ & $8 \times 87$ & $\times<8+\infty$ & * & 6 & 1 & 0 & HA, SY \\
\hline 8 & 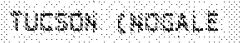 & $448 \%$ & 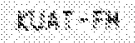 & 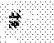 & 8 & 8 & 0 & 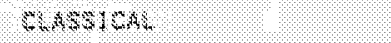 \\
\hline
\end{tabular}




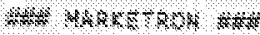

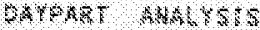

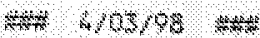

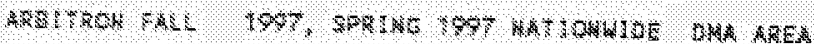

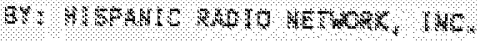

PRGE ID

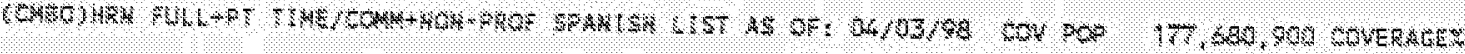

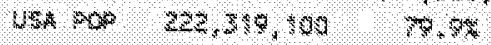

\begin{tabular}{|c|c|c|c|c|c|c|c|c|}
\hline 84 & $2 e \times 44$ & & & & A- $4-4$ त तथ & $x-8>9 x-412$ & $8473+x+74$ & $98 \mathrm{RP}=3$ \\
\hline 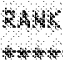 & 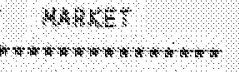 & $+\infty \times+\infty$ & strillat & & AVt $4 \%$ if & A4 140 HI & $186116+16$ & Ponut ror sursous \\
\hline \multicolumn{9}{|c|}{ COOAF BLP } \\
\hline 18 & 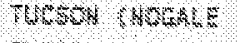 & MAOT & 14284 & 4 & 8 & 0 & a & 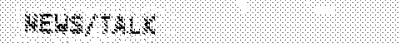 \\
\hline $7 \%$ & MUS6 $4084 \mathrm{C}$ & W.6.07 & $\sec x$ & & 2,00 & $1, \infty 00$ & 2,300 & 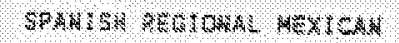 \\
\hline 8 & $18030 \times 40 \times x=$ & 4.497 & $20>-48$ & & 400 & 300 & 300 & 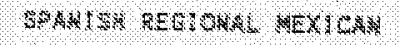 \\
\hline 73 & THown $600 \times 48$ & 19TOT & $x<-4$ & & 4,540 & \$48 & 800 & 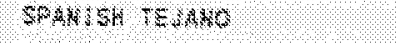 \\
\hline $7 \%$ & $140 \times 0 \times 10645$ & 14097 & $42+4$ & 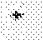 & 3,70 & 1,900 & $\infty 0$ & SPAU S4 BEG 108AL MEXTLAM \\
\hline 86 & 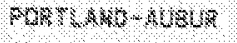 & 84787 & $4 \times \mathrm{PB}_{-4} 4$ & 4 & 8 & 8 & 8 & Y.1 14 \\
\hline 81 & $646848 \pi 8 \times 8$ & $10 \times 07$ & $\alpha+4$ & $*$ & 8 & 8 & 0 & AOISH \\
\hline 81 & $4+44 \times 1648 \%$ & Math & $4+10-40$ & 8 & 6 & 0 & 3 & YARE \\
\hline $8 \%$ & $4 \times 4 \times 4+4<>$ & 18407 & $60+-44$ & f. & 0 & 0 & v & WEst \\
\hline 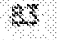 & $4,4 \times-3=-46$ & 14897 & $40+4-4$ & 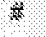 & 8 & 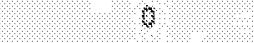 & 0 & 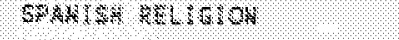 \\
\hline 0 & 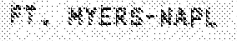 & No\%? & $40+4$ & 4 & 8 & 0 & 0 & $\mathrm{MENSTRL}$ \\
\hline 8 & $40,17=80-60$ & KMTOF & $40 .-46$ & & 1,400 & 8 & 40. & 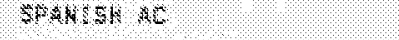 \\
\hline \% & 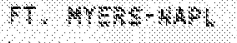 & 10.7.7 & $4+4$ & & 001 & T08 & 200 & $39418 \times+4,160$ \\
\hline 84 & $40180 \%$ & 10087 & $40 \mathrm{RP} \times$ & (4) & 0 & ४ & 8 & 1 $\times 1 \%$ \\
\hline 86 & 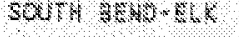 & MAS & 18884 & *. & 0 & 6 & ○ & $2 \times 4 \times 104$ \\
\hline 16 & $8404 \times 40 \mathrm{~m}$ & $100 \%$ & $4 \times 4,4$ & & 806 & 100 & 100 & 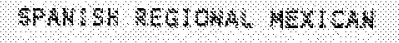 \\
\hline 87 & $8 \times 4 \times<+18+4$ & mor & 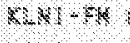 & म & 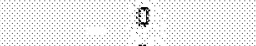 & 1) & 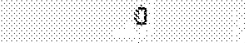 & Wewtras \\
\hline 80 & $140442 \times>0 \%$ & $4 \times 7$ & $4 \times 4-4$ & $\%$ & 0 & 0 & a & 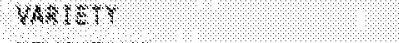 \\
\hline 4 & 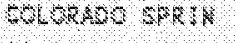 & 4907 & \%०+18 & 4 & 8 & o & to & $180,1 \% 4 \times$ \\
\hline 9. & $200 \times 10035 \%$ & $4 \times 8$ & $4 \times 4 \times+1 \%$ & & 800 & 100 & 300 & 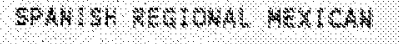 \\
\hline $9:$ & $60,0 \% 100 \$ 0 \% 1$ & $8 \times 197$ & $8 \times 4 \times-8 \times$ & & 8,000 & 400 & s00 & $\mathrm{SBN} \times 1 \mathrm{SO}, \mathrm{F}, \mathrm{NMO}$ \\
\hline 10 & $460-1468=8 \%$ & BNTP & $m-k$ & 4 & 0 & 9 & 8 & 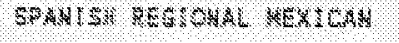 \\
\hline 96 & 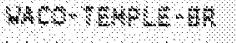 & आAs7 & $\mathrm{KBSPCH}$ & 4. & 0 & 0 & 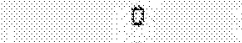 & $4 \times 1+15$ \\
\hline 86 & 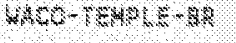 & 4077 & $4 \times 4-88$ & 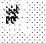 & 8 & 0 & 0 & dassche \\
\hline$\%$ & $4 A \infty-r+48,4,4$ & haro: & 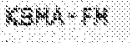 & & 1,100 & 800 & 2,000 & SPNASSU TEAKO \\
\hline$\%$ & $\mathrm{a}-\mathrm{NO}$ & $4 \times 97$ & $64 \cos$ & 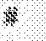 & 8 & 9 & $\theta$ & PPASY S $8 E 1,664$ \\
\hline$\infty$ & $=8,4=$ & $8 \times+\infty 7$ & $\mathrm{x} \times \mathrm{x}-\mathrm{A}$ & है & 8 & a & 0 & $4=65 / 4 K$ \\
\hline$\infty$ & $8<8<0$ & $810 \%$ & $x+1448$ & 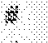 & \% & 6 & c & \\
\hline$\infty$ & \% 830 & $840 \%$ & $x \times</ 4$ & e. & 0 & 7 & h & \\
\hline 9 & $\triangle, P A B S$ & $8+187$ & $+4 * 44$ & 8 & 0 & 0 & 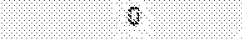 & \\
\hline 98 & $48 B$ & सe०? & $2 \times k+4$ & 8 & 9 & 8 & 8 & are? \\
\hline \% & $A(P A)$ & खA? & $\mathrm{x} \in \mathrm{P} \rightarrow \mathrm{B}$ & 4 & 8 & $\theta$ & 6 & WCWSTAK \\
\hline 9 & 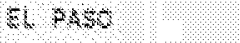 & $10 \times 8$ & $\mathrm{MNOH}$ & & 2,000 & 300 & 1,300 & 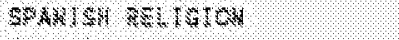 \\
\hline ๑ & 4494 & 198\% & $64-4$ & & 4,800 & 506 & 8,700 & $3 \times 134 \mathrm{kC}$ \\
\hline \% & 2485 & $18-97$ & $n=m-n$ & & 3,000 & 1,780 & 1,300 & 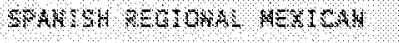 \\
\hline$\infty$ & 28484 & 4A & $\mathrm{rsma}_{4}=\mathrm{s}$ & & 18,606 & 5,680 & 90,700 & SFMUS SU REGTOKA MEXICAN \\
\hline ? & 84400 & $409 \%$ & 8454 & & 1,008 & 100 & 1,100 & 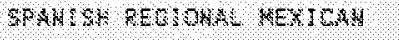 \\
\hline$\varnothing$ & W & 4,407 & $404 \%$ & & 6,4 & 2,10 & 3,80 & 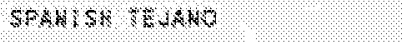 \\
\hline m. & 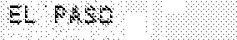 & 9.2\%०\% & $x+9 \cdot 48$ & & $3 \%$ & sot & 200 & \\
\hline ๑ & 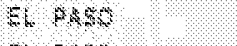 & $848 \%$ ? & $40+48$ & & 1, 00 & 400 & 800 & \\
\hline 8 & 4808 & $8 \times 8 \%$ & 6486 & & 80 & 400 & 200 & \\
\hline 9 & $4+48$ & Hor & $\mathrm{H}_{4}+\mathrm{m}$ & & 800 & 60 & 200 & \\
\hline 9. & $4 / 80$ & 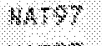 & K4: $91 \%$ & & 2980 & 200 & 2,100 & \\
\hline 90 & 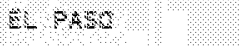 & 4407 & $448+4$ & & 8,200 & 30 & 96. & \\
\hline 9 & 6486 & $140 \%$ & $4+x<+94$ & & 606 & 800 & 800 & \\
\hline 9 & ब४िम & 1801 & $\times 4 \times n$ & \% & 4,10 & 780 & 70 & \\
\hline 8 & $44 \times$ & 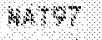 & \$ $4 \%$ & \% & 800 & 800 & 40 & 4.8 \\
\hline 18 & $4+4+46 \times<<4$ & $8 \times 8+$ & res +4 & 8 & 0 & 0 & 8 & $0 \times 6,4$ \\
\hline & $4 \times-4-4 B S+R$ & 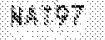 & $44 \times 44$ & t) & 8 & 6 & b. & $\mathrm{CAS} 8 \mathrm{t} \mathrm{R}$ \\
\hline 1 & 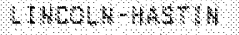 & 1.ค. & Kons 48 & त) & 9 & 6 & 8 & tusston \\
\hline 8 & $14606-4 x=84$ & $448 \%$ & $\mathrm{Ks} / 8 \mathrm{k}$ & 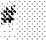 & 8 & 8 & 8 & C455184 \\
\hline 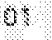 & 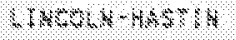 & $4.69 \%$ & $40 \% 104$ & 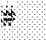 & t) & 6 & 8 & ¿LASGICA \\
\hline
\end{tabular}




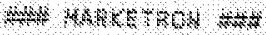
OHFART ABA $485 \mathrm{~s}$

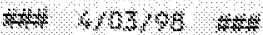

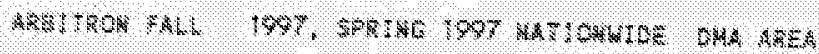

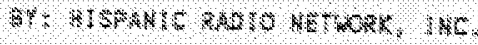

$x_{1}, 4,1$

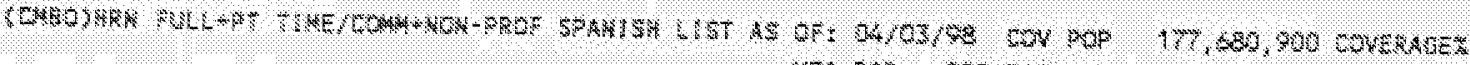

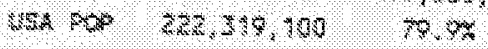

\begin{tabular}{|c|c|c|c|c|c|c|c|c|}
\hline D4: & $20 \times 804$ & & & & $4-8,8 \times-7+6$ & $M-F+3 *-819$ & $5 n+3 m-7+7$ & \\
\hline$\pi \times k$ & $44 \times 5$ & Aook & starou & & WE $14, H$ & & & 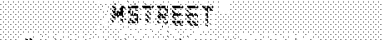 \\
\hline$+\infty \cdots$ & 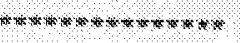 & traxime & $n+\infty \times m=$ & & 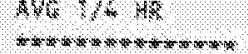 & $40 \% 48$ & $401 / 4 \%$ & 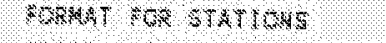 \\
\hline \multicolumn{9}{|c|}{$\begin{array}{l}\text { ConT THEO } \\
101\end{array}$} \\
\hline 101 & $40 \mathrm{cos} \times-4 \mathrm{sets}$ & 46507 & 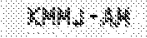 & & 201 & 0 & a & $50 \mathrm{AMl} 3 \mathrm{k}: \mathrm{k}=$ \\
\hline 102 & \%, wh Ne & WhYOT & $180<-4 \mathrm{~N}$ & ? & 0 & 0 & 6 & Whots \\
\hline 180 & $3+144+100-80$ & Mrtor & $4+8+\pi$ & 4 & 8 & 6 & 4 & 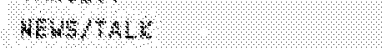 \\
\hline 483 & 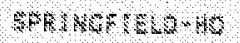 & $6 \times 3$ & $4,4-4$ & & 400 & 100 & 9 & 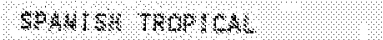 \\
\hline$\$$ & $3+2006010-80$ & $44 \% 7$ & $406-48$ & & 1,500 & 700 & 800 & 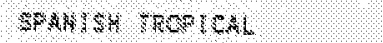 \\
\hline 104 & 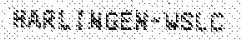 & 4atror: & $48 \mathrm{~m} /$ & 4. & 1 & 1 & 1 & 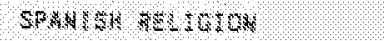 \\
\hline $10 \%$ & 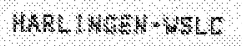 & $44+7$ & $8 \times 1 \times-8$ & ? & 8 & 0 & 8 & 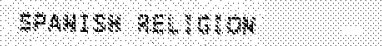 \\
\hline 104 & 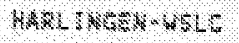 & Whot & $8018-84$ & $\approx$ & (c) & ๑ & 8 & 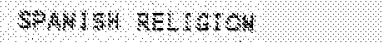 \\
\hline 16 & 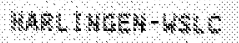 & NATOF & $180 \times 4$ & \% & 8 & 8 & 0 & 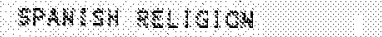 \\
\hline 10 & 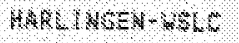 & $\mathrm{kAT}$ & $1 \times 18 \times 8 \times$ & 9 & 0 & 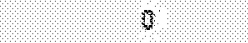 & 9 & 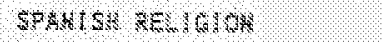 \\
\hline 804 & 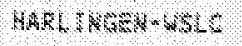 & $44+\%$ & $2084 \times 4$ & 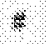 & 6 & 6 & 0 & $46,65 \times 1 \times 2 \times$ \\
\hline 10 & $4,4,40 \%+4,50$ & $146 \%$ & $40,+418$ & 4 & 4 & 0 & 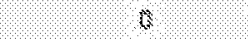 & 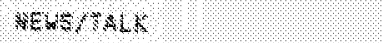 \\
\hline $18 \%$ & 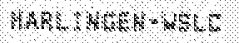 & \$8r & 100 $\times 14$ & 4 & 1 & 6 & 0 & $8 \times 8 \times 4$ \\
\hline 104 & 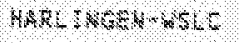 & astor & $\times>0 \times 4$ & & 800 & 840 & 70 & 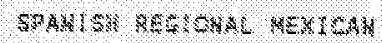 \\
\hline 48 & 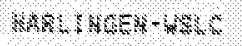 & 4,807 & KC+48\% & & 100 & 100 & 700 & 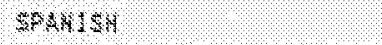 \\
\hline 148 & 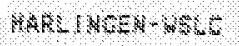 & $8 \times 40$ & $4+4$ & & 4,804 & 3,460 & 3.200 & 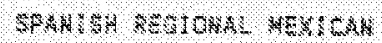 \\
\hline 104 & $4 \times 4 \mathrm{C} \times 4 \times 4 \times 6 \mathrm{~cm}$ & 40,87 & 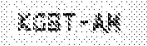 & & 8,00 & $2,2,1$ & $3, \% 10$ & 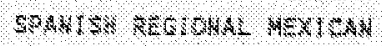 \\
\hline 80 & 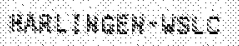 & $4, \mathrm{x} r \%$ & $x+8 \times 4$ & & 2,40 & 300 & 8, 100 & 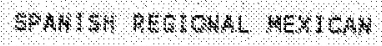 \\
\hline 104 & 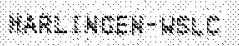 & $848 \%$ & $6+x \rightarrow+1$ & & 4,400 & $2,1,00$ & 1,60 & 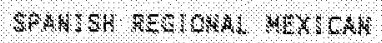 \\
\hline 104 & 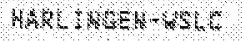 & 4467 & 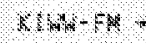 & ? & 4, 700 & 9,10 & 10,460 & SPAMIST LIE 140 \\
\hline 80 & 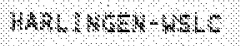 & xarar & $8+14+46$ & & 1,000 & 385 & 708 & 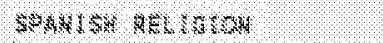 \\
\hline 104 & 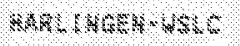 & N679\% & $60 \mathrm{sen} 8$ & & 11,900 & 3,900 & 6,360 & 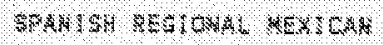 \\
\hline 10 & 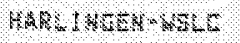 & Marot & $40 \mathrm{~s} \cdot \mathrm{An}$ & & 2,480 & 700 & 2,380 & 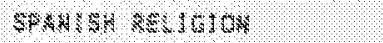 \\
\hline 104 & 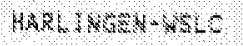 & $18 \times \% \%$ & $408+84 \%$ & $*$ & 91,000 & 0,80 & 7,100 & 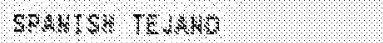 \\
\hline 10. & 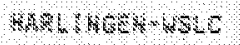 & nar? & 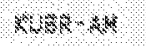 & & 80 & 306 & 400 & 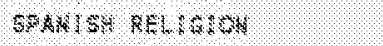 \\
\hline 104 & 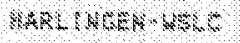 & 34.47 & $x \times 18 \times 8$ & & 2,80 & 800 & 1,200 & SFAB STH VEQS-TALK \\
\hline 104 & $M A R+1051-89.6$ & $48 \% 7$ & $x, 8+4,4$ & & $3 \times 1$ & 8 & twi & \\
\hline 84 & 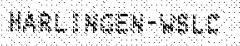 & $48 \% 7$ & $x=00 \times k=$ & 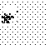 & 300 & 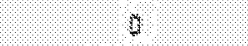 & 40 & \\
\hline 84 & $K A R<18 G Q R-4 B<C$ & $8 * 187$ & $\mathrm{xear}-\mathrm{Nh}=$ & $*$ & 600 & 300 & 380 & . \\
\hline 105 & 448546 & siso\% & Whan -40 & 政 & 8 & 6 & 0 & $6 \times 3,4 / 84 k$ \\
\hline 87 & 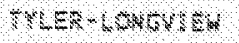 & MATOP & $12844-8 \times 4$ & 4 & 8 & 0 & 6 & 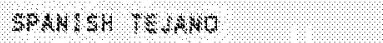 \\
\hline 807 & 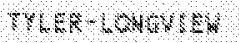 & $6 \times>+$ & $\mathrm{sPB}-\mathrm{-P}$ & & 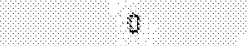 & 0 & 9 & $4 \times 58 \times 4 \times$ \\
\hline $10 \%$ & $11,60-4 \times 4 \times B=4$ & 148\% & $15 \times 4-64$ & & 1 & a & 0 & ink? \\
\hline 108 & $8100 \times 1448 \% 4$ & $145 \%$ & $130 \times-+48$ & 4 & 8 & o & 0 & VESSTTALK \\
\hline 166 & $8100 \times 4 k 860$ & $48 \% ?$ & $4248-9 \mathrm{~d}$ & * & 8 & 0 & 0 & WXSSIERL \\
\hline 111 & 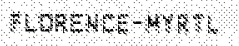 & 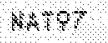 & $6>81-484$ & 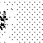 & 0 & c & 8 & a $-4 \mathrm{ck} \quad 0 . \mathrm{sin}=\mathrm{a}$ \\
\hline 18 & 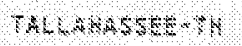 & $4>?$ & $4850-8 M$ & * & 8 & 18 & 6 & Wews $14 \mathrm{AK}$ \\
\hline 83 & $14 a c+4 \times 181<$ & $3 \times<>$ & $x+2-4 \times=$ & 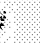 & 8 & 6 & 1 & Nexs,TAK \\
\hline 83 & 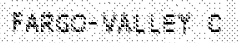 & $4 \times 6 \%$ & $847 \times 484 \%$ & & 8 & 0 & 0 & $4=x, 5,14<<$ \\
\hline 18 & 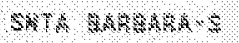 & 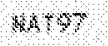 & $\mathrm{ker}-\mathrm{k})$ & & 0 & 6 & 8 & W 8 r $=\mathrm{Tr}$ \\
\hline 88\% & 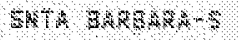 & k.P? & $8 \times 8 \times 84 \%$ & & 4 & 8 & 8 & $4 \times 1 \times 14<8$ \\
\hline 18 & 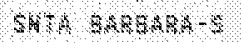 & 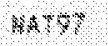 & $14 \times+48$ & & 9 & 6 & 6 & $26 \times 4804$ \\
\hline 185 & 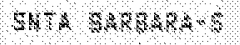 & $148 \%$ & $1001+18 \%$ & 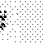 & 9 & 8 & 8 & $244580458581 \times 5$ \\
\hline 8s & 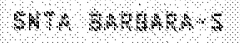 & 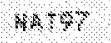 & $18 \times 6-4$ & & 960 & 360 & 800 & 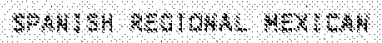 \\
\hline +8 & 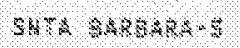 & nator & $\mathrm{rer}+\mathrm{tr}$ & & 4.800 & $\infty \varnothing 0$ & 4,000 & 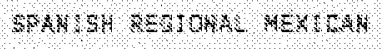 \\
\hline ४४ & $8 \times 8 \times 348 \% 4$ & 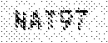 & $\mathrm{r}+\mathrm{H}=\mathrm{m}$ & & 30 & 8 & 8 & $3 r \times 4 s+40$ \\
\hline 48 & 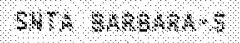 & 14\%? & $8+48$ & & 8,80 & 360 & 400 & 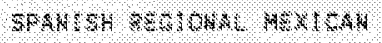 \\
\hline 48 & $4 \times, 4,8,4,48$ & 14.8\% & 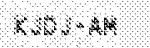 & & 80 & Set & 100 & 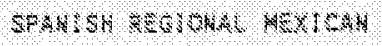 \\
\hline 38 & 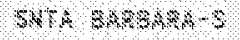 & $4 \times 8 \% 7$ & $48+8 k$ & & 2,800 & $+\infty$ & 4,200 & 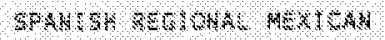 \\
\hline 18 & STIA Ahs & $4 \mathrm{Br}$ & $\times 01-7 \times$ & & 2,80 & 700 & 1, & 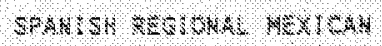 \\
\hline 18 & $r(, 4 \times 8+4)$ & $4887 \%$ & 128, 86 & 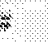 & 9 & 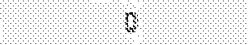 & 8 & 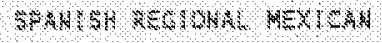 \\
\hline 38 & 8. & 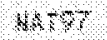 & $640,40 \%$ & 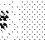 & o & 8 & 8 & $8+4 \times s+40$ \\
\hline
\end{tabular}




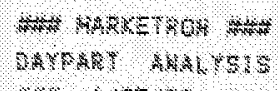

$+4+\infty,+\infty$

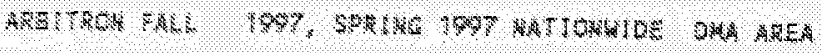

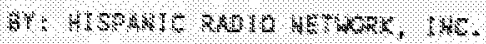

pas 12

17\%, 690,900 contranes

$\pi, \infty$

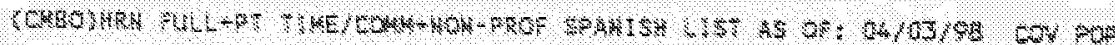
HA POF $222,31 \%, 110$

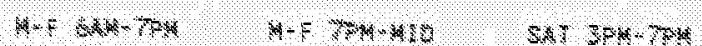

orses Pesso 12

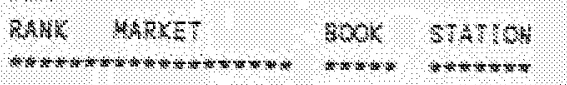

Cokr ranes:

$190 \mathrm{kENO}$

1489840

$\$ 48 . \quad 8 \% 3 \%$

14: $646 \mathrm{se}$

190 vlatere

$\$ 20$ PUENE

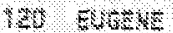

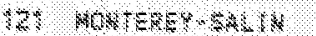

21 MGRTERE-SALSH

21 NOU TEEE-SALM

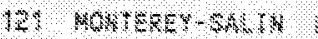

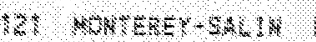

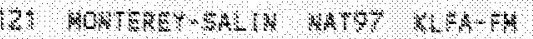

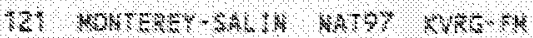

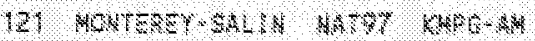

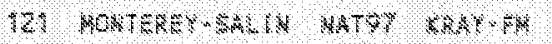

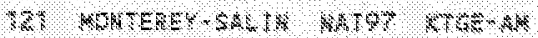

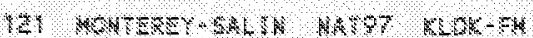

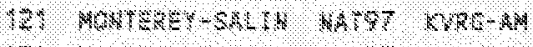

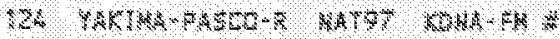

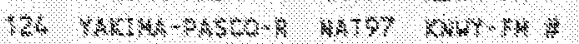

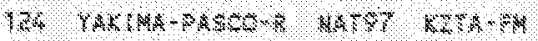

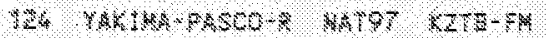

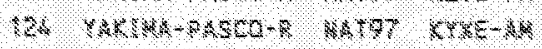

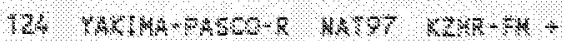

323015

3) $\mathrm{BOSE}$

$125,80,5$

126. $4 \times 4+1<10$

126 AMARISLO

\$26 4402: 560

\$26 $4 \times 68 \% 460$

$126 \quad 4 \times 12 \pi<18$

120 W6RTLO

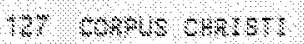

i2t cokus conkt

27 6OKMO CHRIST

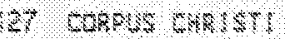

3

$\mathrm{sec}$

12?

43

12

12

12

\$)

12

कै:

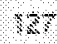

32

12

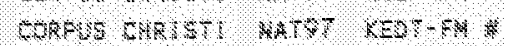

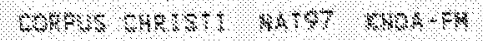

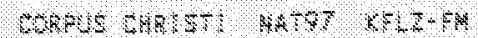

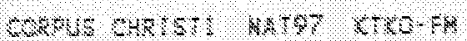

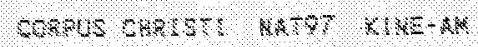

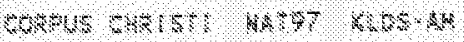

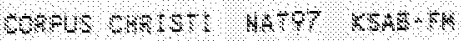

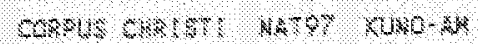

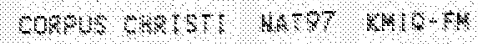

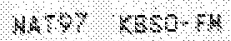

$4,687, \quad 34,4-80$

$4,+4,8+8,46$

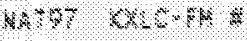

\begin{tabular}{|c|c|c|}
\hline A4G $9 / 4$ IR & AVR $14, \mathrm{HO}$. & 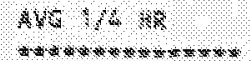 \\
\hline
\end{tabular}

4. $\div: 325$

ronht $=0$ e stet rows

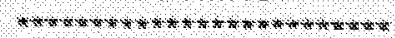

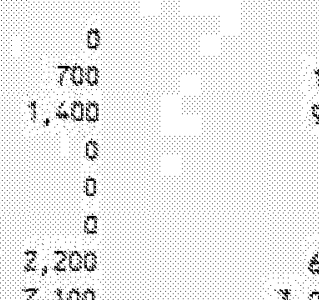

\begin{tabular}{|c|c|c|}
\hline 0 & c) & arws ratK \\
\hline $10 \%$ & 326 & 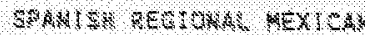 \\
\hline Q00 & 1,600 & sthin:St $=-\infty$ \\
\hline
\end{tabular}

XRS ET:

WE⿺S TH $1 \mathrm{~K}$

औR

wews, Tal

7,100

1,000

8

\&

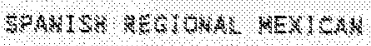

SPAn 15:

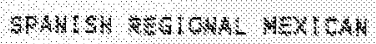

xistast

Whatery

706 200

$1,20018: 400$

$1,4,0$

400

3,30

3,300

$3,40 \%$

408

48

4.300

940

801

2,400

. 8

48

18

20

800

1,200

310

48

8

(1)

18

80

300

800

308

100

$7,4 \times$

3,10

800

T)

1,000

1,600

430

, 40

380

800

100

o

400

65

400

s00

40

40

4

( 1

0

8

500

201

40

18

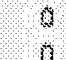

4

700

0

$4 \mathrm{CO}$

200

6,260

1,300

60

900

501

4012

8

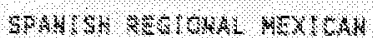
sganl

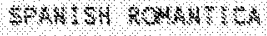

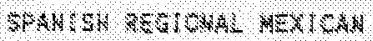

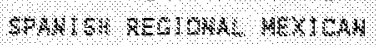

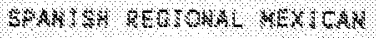

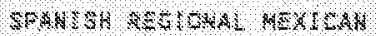

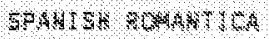
$34 \times 13$ WES

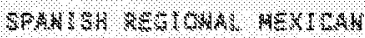
SFAMTSE CETHOH H MEICAN

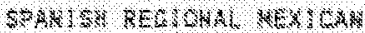
$34 \times 101$

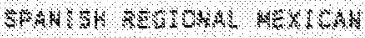
Sox41s:

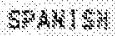

SPakn sh

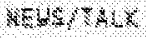

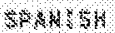

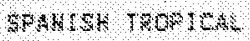

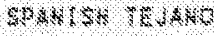

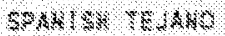

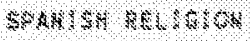

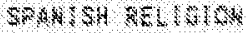

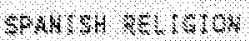

WE

SPAk:su TEAA

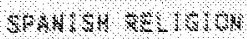

Pran BH TEIAMC

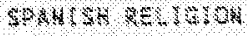

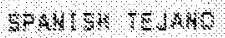

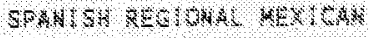

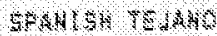

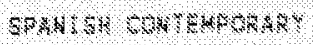

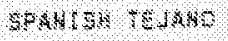

Sondis: TEMHO

NESW TMLE

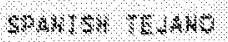

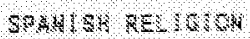




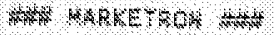

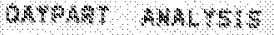

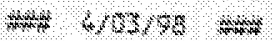

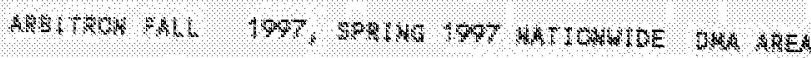

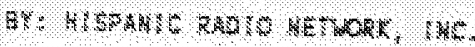

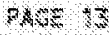

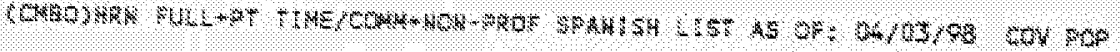
USA $70^{\circ} \quad 222,319,100$
$17,, 280,900$ wuER,AEX

$r, \%$

\begin{tabular}{|c|c|c|c|c|c|c|c|c|c|}
\hline 6m4 & $198 \times<\%$ & & & & $8-64-8 \times$ & 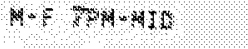 & 778 & \multirow{2}{*}{\multicolumn{2}{|c|}{ wertes }} \\
\hline 864 & HAKET & $800 \%$ & 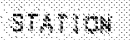 & & & & & & \\
\hline & 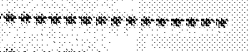 & *⿻心㇒: & $m+r+\pi=m$ & & 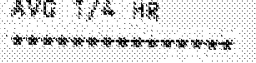 & 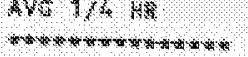 & T48 1/4 4R & 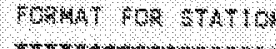 & \\
\hline \multicolumn{2}{|c|}{ cotortmuses } & & & & & & & & $*$ \\
\hline 130 & $4800458-84$ & Mas? & $604+4$ & \% & t) & 0 & & & \\
\hline 13 & $8 \% 1<0-8000 / 4 \mathrm{C}$ & axpr: & $x<x-4$ & 4 & 8 & 8 & 0 & 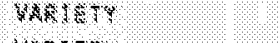 & \\
\hline 130 & arrot anosus & 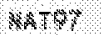 & $x+4 x-c k$ & 4 & (3) & 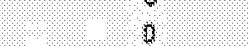 & 0 & $4808 \%$ & \\
\hline 30 & chro-8ensing & & 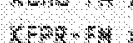 & $\infty$ & 0 & & 8 & arastrax & \\
\hline$\$ 30$ & $0 \times 160-40,4 \% \mathrm{~s}$ & 4087 & 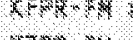 & $\%$ & $4, \infty 00$ & 4 & 0 & $148467 \mathrm{AL}$ & \\
\hline 131 & $8 \times 1 \mathrm{ersh}=\mathrm{s}$ & 4987 & $120+4$ & & & 408 & 800 & 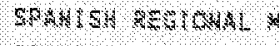 & 40806 \\
\hline & & $\mathrm{HAOH}$ & $94 \times 4$ & (3) & 9 & 8 & 0 & 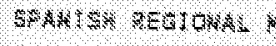 & Xextrans \\
\hline 131 & $46889>80$ & AN $\% 7$ & 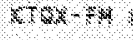 & 4 & 6 & 8 & 0 & SPAx 54 & \\
\hline $8 *$ & $44 x=5,8180$ & \$4०7 & $x, 3 \times m$ & 4 & 6 & 0 & 0 & 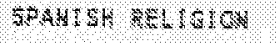 & \\
\hline 131 & 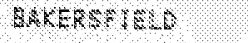 & $x \times 40$ & $84+4 \times$ & & $\$ \infty$ & 100 & 700 & 49441548 & \\
\hline 38 & 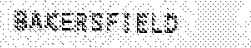 & $4 \times 5 \%$ & 44,44 & & $2,-90$ & 7wo & 2,300 & 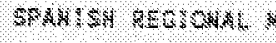 & 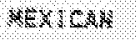 \\
\hline$* 3$ & 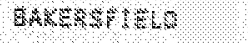 & Kल & 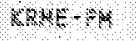 & & 460 & 800 & s00 & 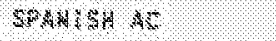 & \\
\hline 131 & $04 \times 8 \times 2480$ & 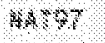 & $4 \times 4 \times 4$ & & $4,60 \%$ & 40 & 800 & 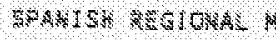 & 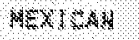 \\
\hline 13\% & 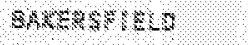 & $14+\odot$ & $04 \times 40$ & & 900 & 100 & rot & 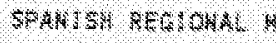 & $6 \mathrm{Ex}_{\mathrm{S}}$ \\
\hline 10\% & $44 \times 8 \times 5 \times 40$ & wart & $1 \times 14+4$ & & 2,100 & 1480 & 2., 0.00 & 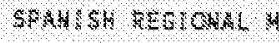 & 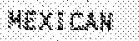 \\
\hline 4:? & 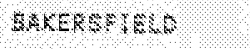 & आar & 40404 & & $1,8.08$ & 200 & 800 & 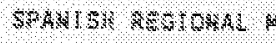 & ME $414 \times$ \\
\hline ts: & $8 \times 6 \times 1=1.40$ & $48 \%$ & $\mathrm{xc}+,+2$ & & 4,50 & 900 & 108 & a4tes & \\
\hline $18 \%$ & 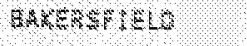 & 18707 & $6006+4)$ & & \%, & 4,000 & 7,800 & MAres-CMU & \\
\hline $14:$ & 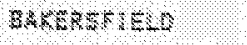 & $607 \%$ & $18 \times<+4$ & & 8,600 & 4,40 & 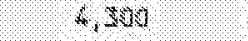 & - & \\
\hline 13 & 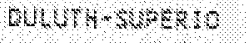 & $18 \times+$ & 40484 & 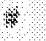 & 8 & फ & 0 & 10601425 & \\
\hline 34 & 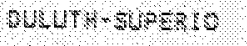 & $40 \% 7$ & 46464 & 4 & b & 0 & 0 & 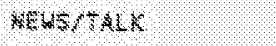 & \\
\hline 848 & $30004 \times 48$ & XATt? & $\mathrm{x}, \mathrm{s} \cdot \mathrm{Am}$ & 4 & 0 & 0 & s) & C.ASS 044 & \\
\hline 42 & 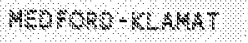 & 4967 & $48 \times 140$ & 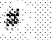 & 8 & 0 & 8 & 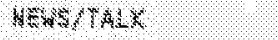 & \\
\hline 12 & ACOROFO- $4 \mathrm{AMA}$ & HAYT & $6 m+n=4$ & $\Leftrightarrow$ & 9 & \% & 8 & 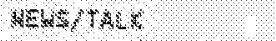 & \\
\hline 142 & $1 B O=O R O+B A M B T$ & XMTSt & $8+6 \cdot 44$ & e & 6 & 6 & 8 & Nexs, $\times 40$ & \\
\hline 142 & 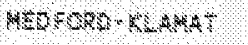 & 1XYOT & KHA,$A B$ & & 300 & 0 & 400 & 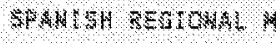 & $18 \times 86 \times N$ \\
\hline 84 & WETHA ALLS & 4,8 & $n+14 \times 4$ & 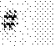 & 0 & 8 & 0 & 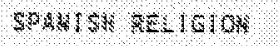 & \\
\hline 44 & 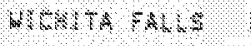 & 4ars? & $x=8,48$ & 4 & 0 & 6 & 0 & 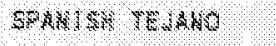 & \\
\hline 4 & 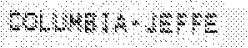 & $84 \times 4 \%$ & $x^{2} \mathrm{x} \cdot \mathrm{eH}$ & 3 & de & 8 & 4 & 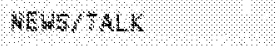 & \\
\hline 47 & $108808 x$ & QAPO & 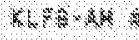 & 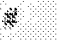 & 8 & 0 & 8 & SPAVISH RELISIOR & \\
\hline 86 & 4480606 & $499 \%$ & $\mathrm{MH}_{-}+\mathrm{H}$ & *ै & 4 & a & 0 & $8+4 \times 18$ & \\
\hline 140 & Lueser & 44897 & $\times 0 \%-4 \times$ & 4 & 8 & 3 & 0 & $8 \times 4,415 \%$ resson: & $x+x<54$ \\
\hline 147 & $48800 \mathrm{~s}$ & $18 \times-97$ & 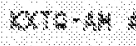 & F) & 8 & to & 0 & $8 r 4 m s+4$ & \\
\hline 140 & $46604 \mathrm{C}$ & $18 \times 8 \%$ & $894 \times 4$ & & 4,860 & 8 & 1,000 & 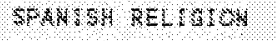 & \\
\hline 147 & $4.8804 \mathrm{C}$ & BAP? & $4 \times 4-4 \%$ & $\bullet$ & 3.860 & 2,20 & 3,700 & 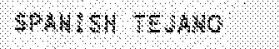 & \\
\hline$\$ 7$ & $4,8 \%<<$ & Mart? & 4828.84 & & 100 & 80 & 1,250 & 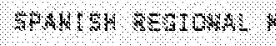 & 18 $\times 4 \times 4$ \\
\hline 48 & $48080 \times x$ & $4 \times-87$ & 184548 & & 800 & 800 & 1,900 & sonvres rewke & \\
\hline 50: & $6=504-4 \times 4,40$ & $4 \mathrm{st}$ & $x=28-4$ & 4 & 8 & 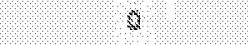 & 0 & 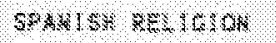 & \\
\hline 6 & $80 \times 844-480+4<0$ & 1479 & 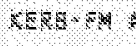 & 4 & 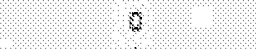 & 0 & 6 & 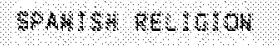 & \\
\hline 4 & 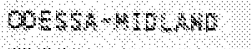 & Mrst & $\mathrm{An} \times 4 \cdot 4 \mathrm{H}$ & 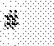 & 6 & $\diamond$ & 8 & 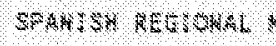 & $48 \times 40 \times$ \\
\hline 8 & $0080 \times-800,410$ & $4 \% \%$ & 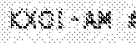 & \% & 4 & 8 & 0 & 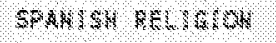 & \\
\hline 80 & $0-584-480468$ & $4 \times 8$ & 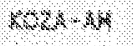 & & 908 & 200 & 1,100 & SPAMSSH REG:OMUL & $\mathrm{NBrC}$ \\
\hline$\$$ & $408 \times 8-18046$ & $4 \times 4$ & $4 \times k r k$ & & 3,000 & 1,80 & 2,40 & 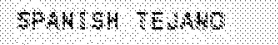 & \\
\hline & $04<-4-14<4 \times 4$ & M००\% & 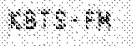 & & 40 & 900 & sir. & $844464 \%<4880$ & \\
\hline 80 & 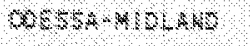 & \$ & $8 \times+4 \cdot-4$ & & 4,40 & 1,600 & 8,610 & 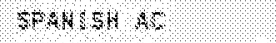 & \\
\hline 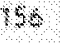 & $9400 \times 4=$ & $48 \%$ & 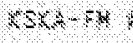 & 6 & 8 & 4 & 8 & xers 446 & \\
\hline 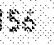 & $\mathrm{sucher+se}$ & 484 & $8+\bullet$ & *ै. & 8 & 8 & 0 & बes & \\
\hline 8 & 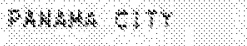 & k+1\% & $4 \times 4+8$ & क & 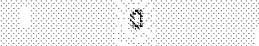 & 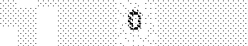 & 8 & $4 \times+s \times 48$ & \\
\hline 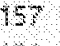 & $34 \times 484 \%$ & \$ $40 \%$ & $6 r=46$ & ? & 8 & 8 & 6 & $x=, s, A, x$ & \\
\hline 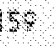 & $84448 \times 1 \times 48$ & $8,1,87$ & $40-8-4$ & है & 8 & $\theta$ & 0 & cass:cu & \\
\hline & $84 \times 58 R+65$ & $8+20$ & $48 \times<$ & 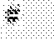 & 8 & 0 & 1 & WENSTALS & \\
\hline 9 & $9644 \times 848$ & 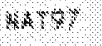 & $18.8-48$ & & 1,308 & 600 & 1,700 & 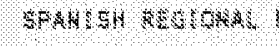 & $48 \times<0 \times$ \\
\hline 57 & PALA \$ 801406 & 8487 & wn. 4 & & 3,80 & 1,300 & 3,600 & 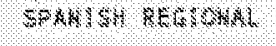 & 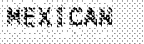 \\
\hline
\end{tabular}




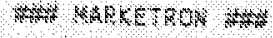

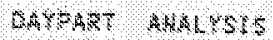

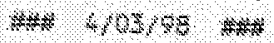

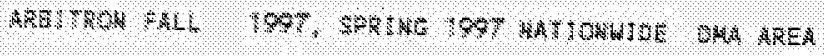

m:

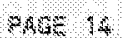

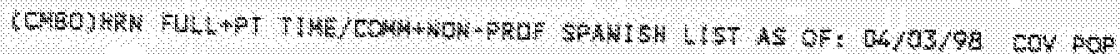
$4+24,310,04$

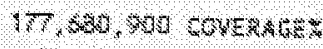

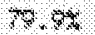

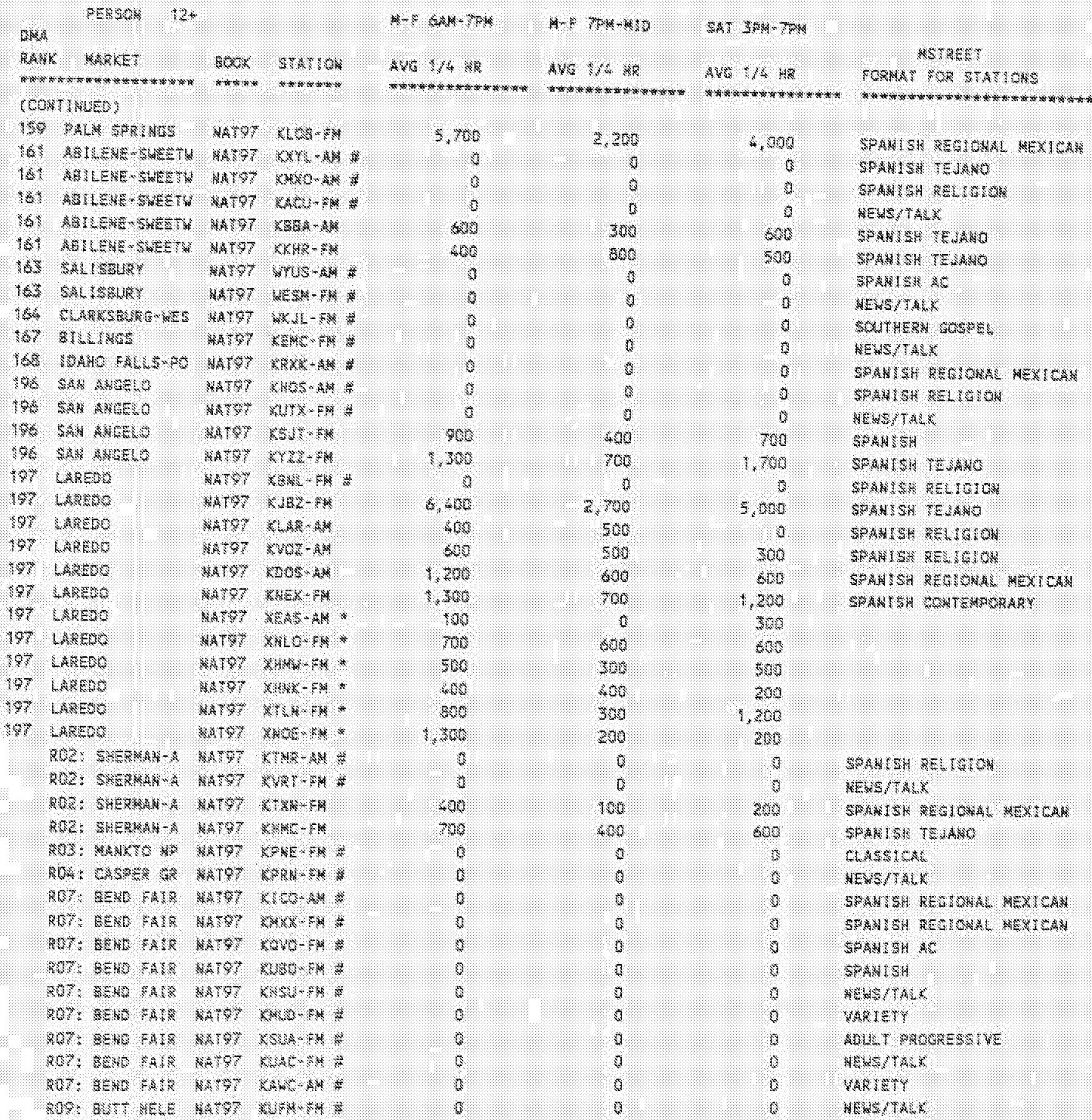

$823141 \% 0 \times 5$

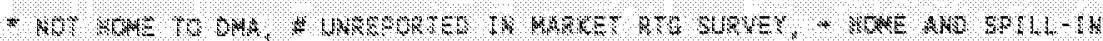




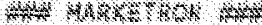

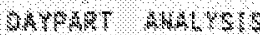

$x+403,46,4$

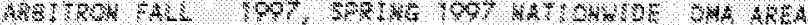

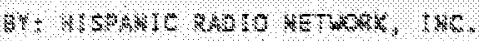

$4 \mathrm{sin}$

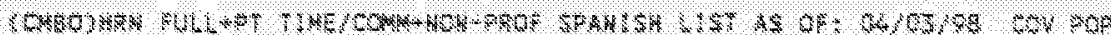

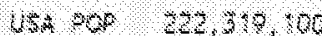

$17,69,507$ SWEW 7 . $\%$

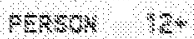

sem

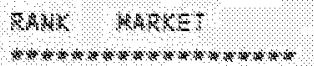

$3.45 \%$ ense

1.406 rak

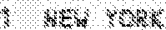

(1) ME. Y Y R

$\rightarrow$ her 408

New $90 \mathrm{~cm}$

ses rak:

i Wer rook

4 MES rom

4 BEW 908

BE W TOK

NEW YORK

WE Yomk

nEN rokK

WE Y York

rew rom

Bes $\$$ काk

$1046 \mathrm{mak}$

Hes ronk

\&ES ronk

406 MOLLE

LOS AREELE:

Los ARTELS:

ute anceles

LOS AMUELE:

400 M M

2 400 ANGLUE=

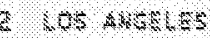

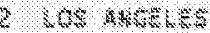

2 $106 \mathrm{Mre}=\mathrm{H}$

$7,6014,46$

2 $40 \%$ akt $\mathrm{k}: \mathrm{s}$

2 $160 \mathrm{~km} 6 \mathrm{k}=$

$2 \quad 1094668=$

2 LOS RALE E

2 LDS ABEELS.

2. $102 \times 146=5$

2. $\angle 0 \%$ AHES 3

- 40 S ARGE $S$ S

2 405 kats

2) 408 A HEZ S

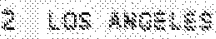

2100 AMBZLE:

$2 \quad \mathrm{NO} \mathrm{A} \times \mathrm{OL} 1 \mathrm{~S}$

$240 * 4 \times<* 158$

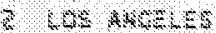

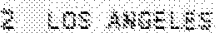

2. 405 axt

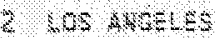

2408 ArEL LES

2 . 05 ALESLES

2 108 AREELES

2 प GS AMEES

$4-5<4 x+3$

Work stotron

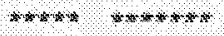

194.07 $\quad 40,2-44$ is

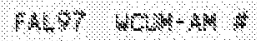

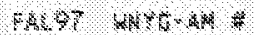

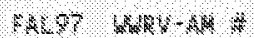

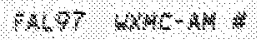

Ghor WEas ras :

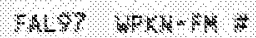

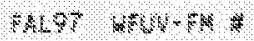

enwT whe and of

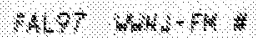

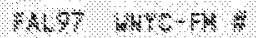

ALOT $4 \mathrm{kT}$ - $\mathrm{FH}$ ?

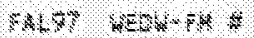

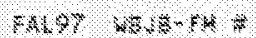

BALCT $: 460-7 \%$

PAT $\%$ :

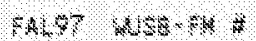

BaLT War a w

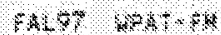

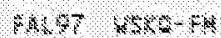

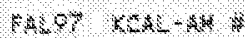

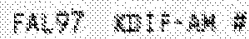

FKOO? $\quad \mathrm{ZZZ}+\mathrm{MH}$ \&

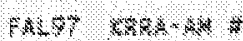

Mort xym-A* w

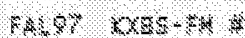

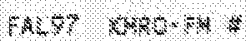

PACP KAEE.AH

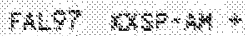

HAOT $\quad \mathrm{AOKR}-\mathrm{AM}=$

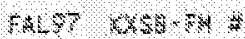

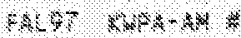

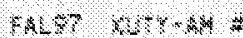

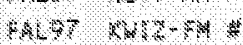

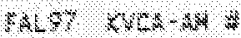

- 2067 1094-AM :

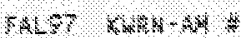

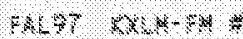

RALT $\quad a, A-F t$

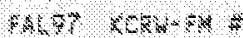

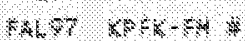

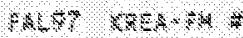

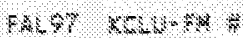

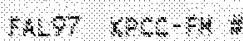

ench 2050 +4:

RA, $\times 3 \times-4 k$

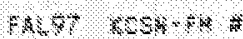

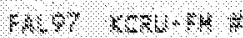

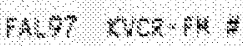

$8.497 \quad 6 \% 5 .-F K$ a

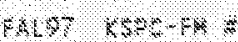

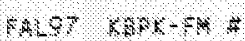

mas $\rightarrow$ kss-en a
4 stetest

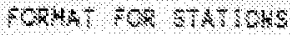

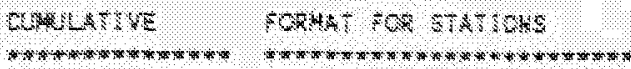

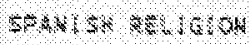

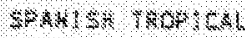

SPAts reorchi

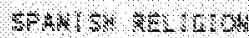

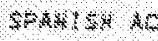

Whet?

VARIESY

Whas:

BESG ThL

Conso: int

NENS/ $34 \mathrm{X}$

UAR 15:

WEWTIRLC

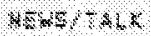

axis

WA: $: 5$

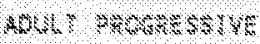

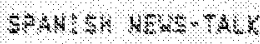

SHAH S ST NOWHTTCS

gphns an Gritekgromer

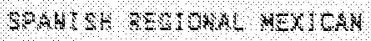

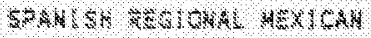

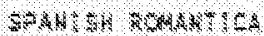

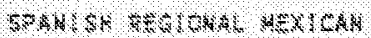

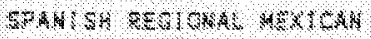

SPRM:

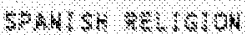

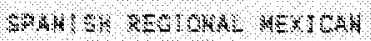

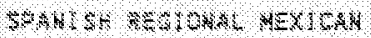

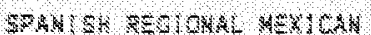

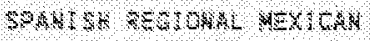

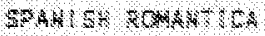

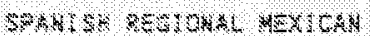

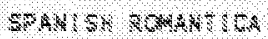

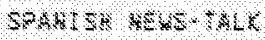

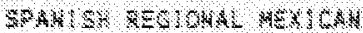

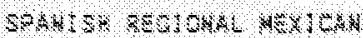

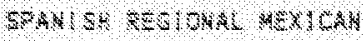

SRARSE AL

Nens TMLE

Hartor:

ETHALC

\&EW $S T$ TLL

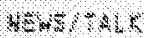

C...S5. +4

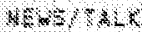

C.ass: 4 A

A $E, 5 \% \%<\mathrm{C}$

सहै⿴囗十 140

$0,40,24$

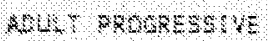

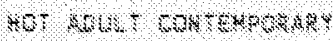

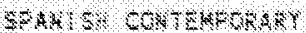




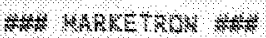

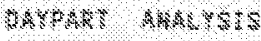
$4+40108$ sis

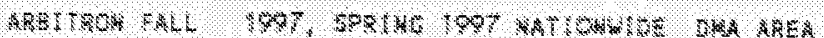

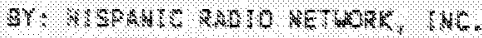

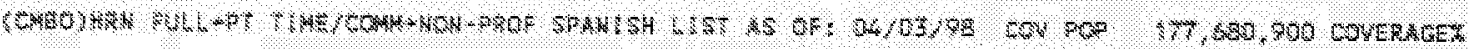
USA $90 \%, 22,39,900,7, \%$

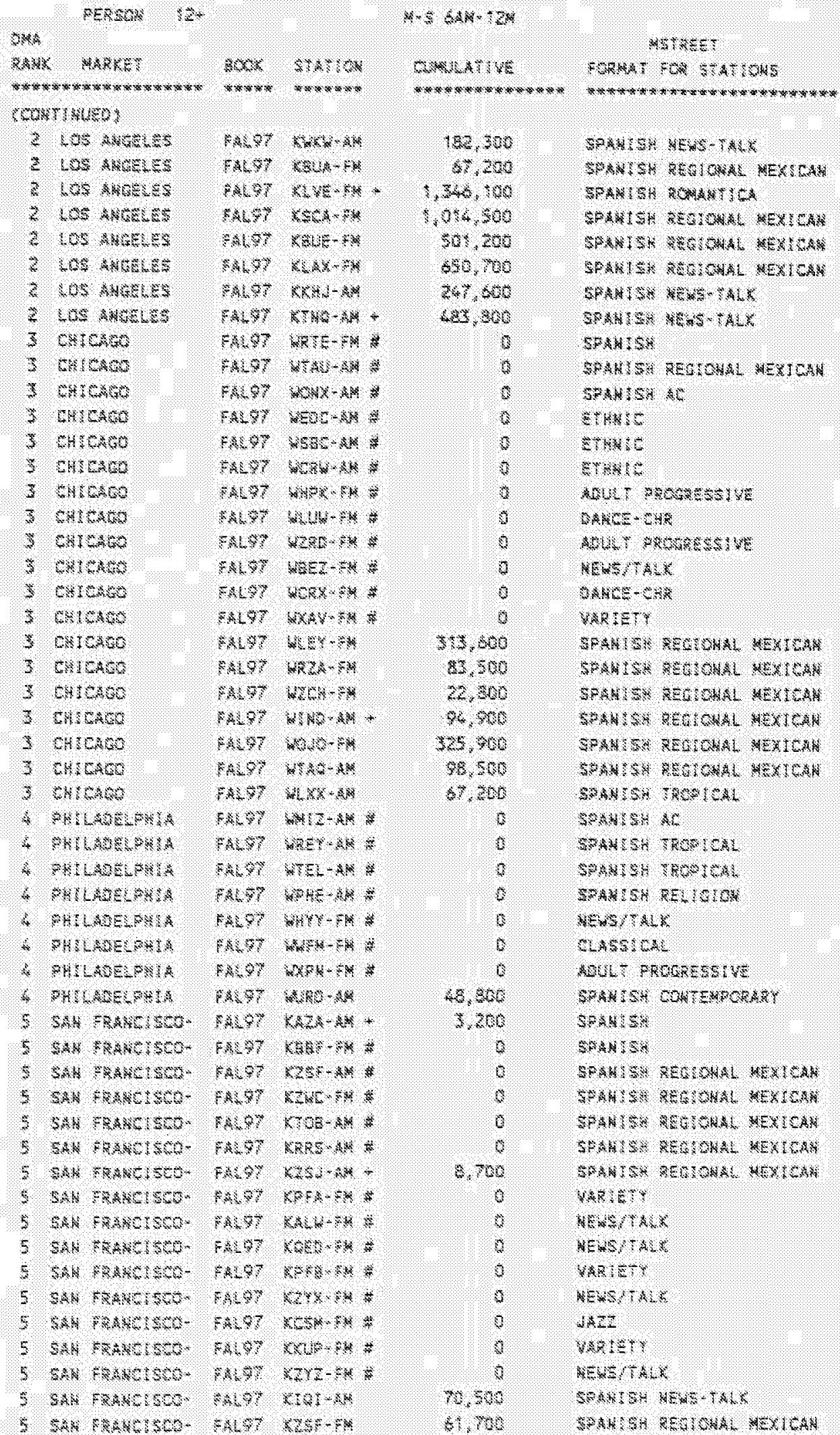




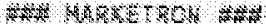
$348 \times 14,8$

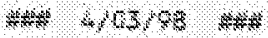

आR

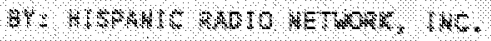

9.6E

(

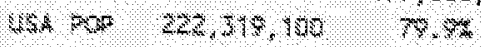

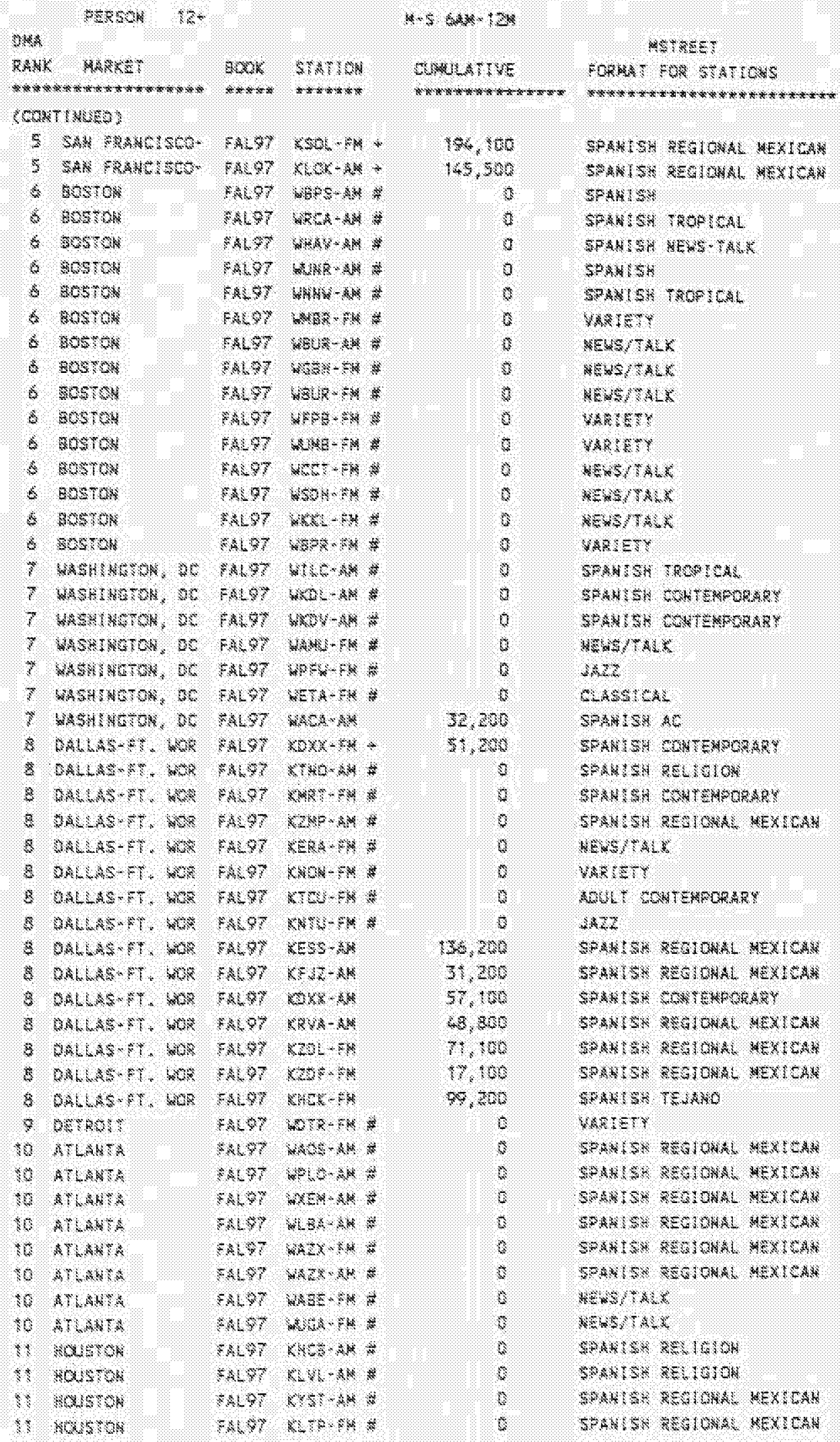




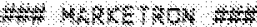
$\mathrm{AAPART}$ AMA 131

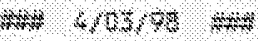

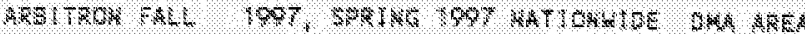

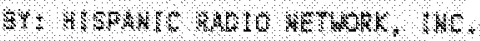

PAEE 6

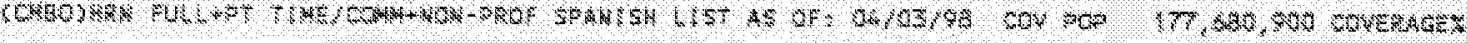

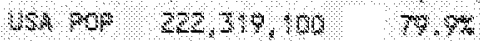

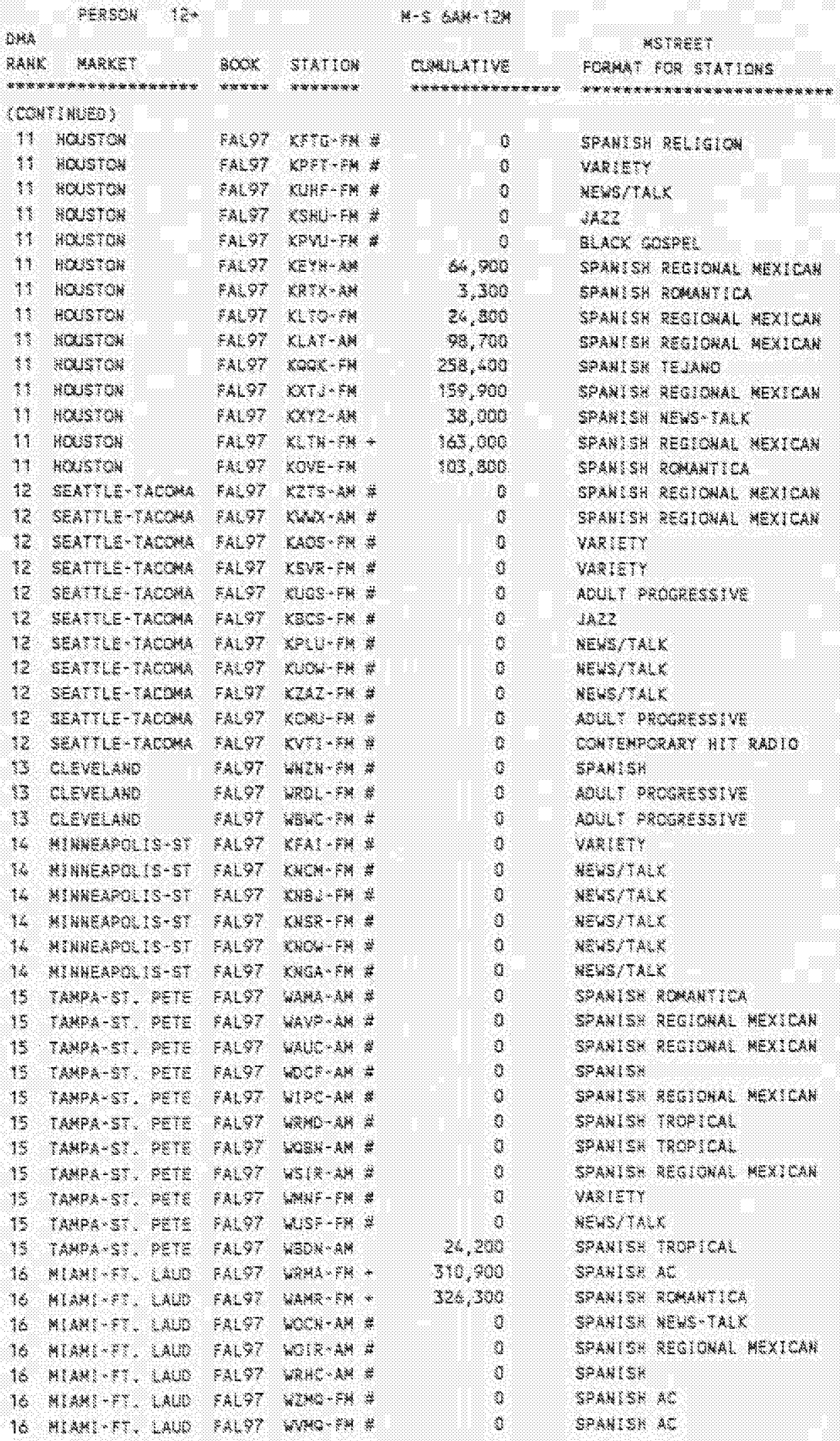




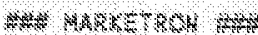
$010,1 \%$ mors $4 \%$, 100100 ,

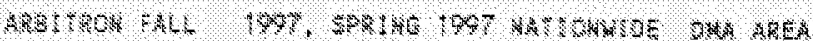

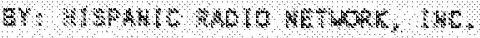

Fire 4

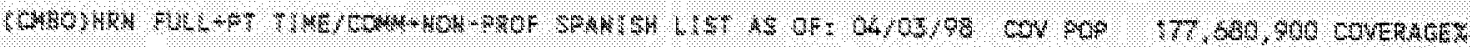

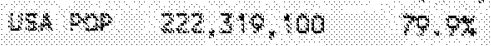

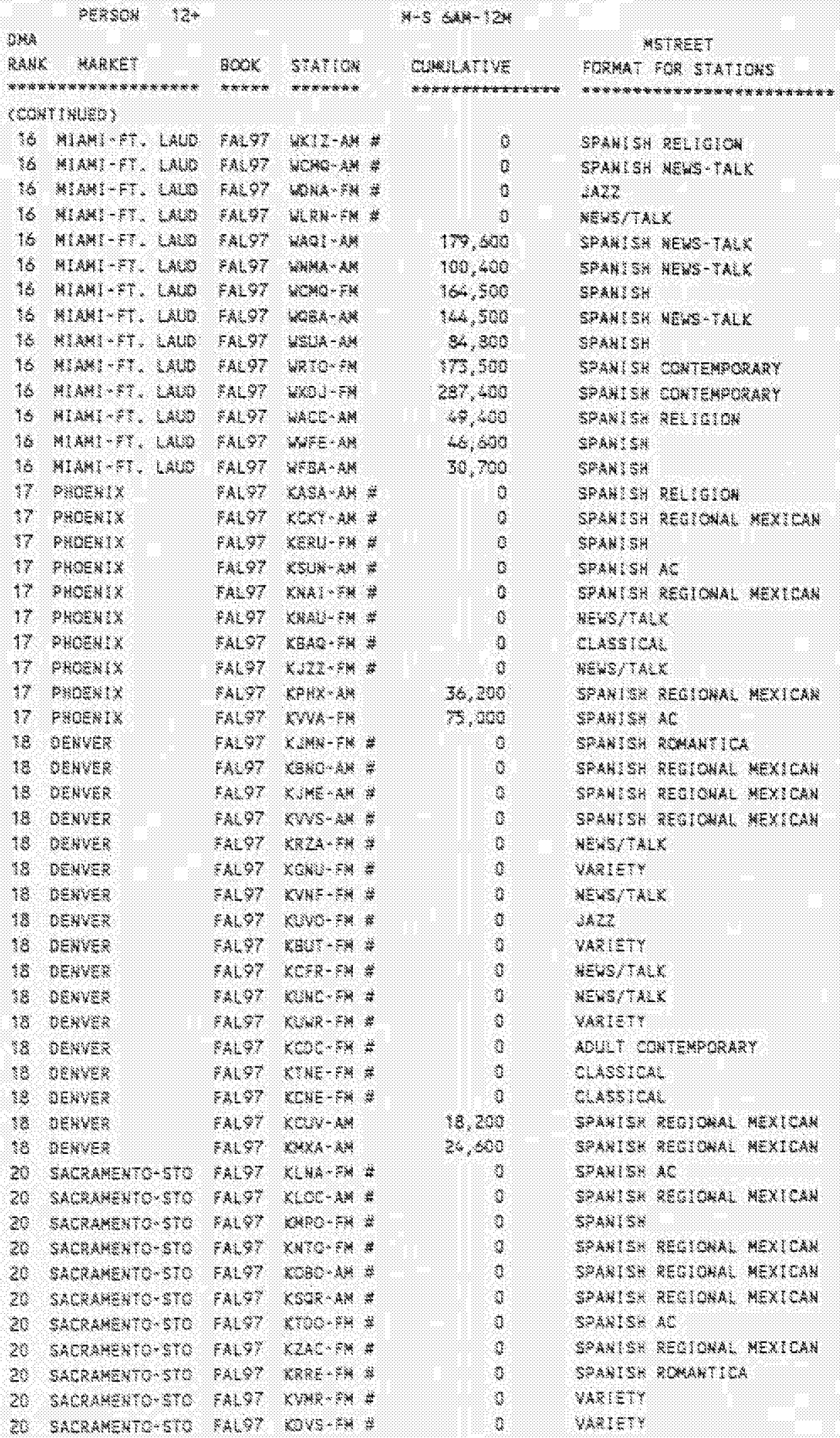




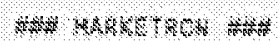

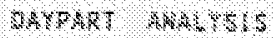
\% $18,1,8$

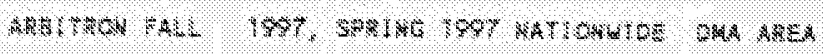

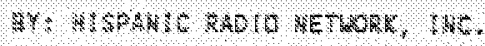

prat 6

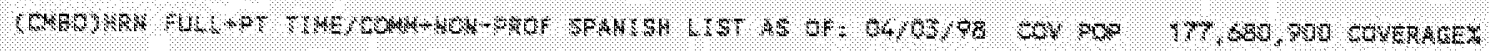

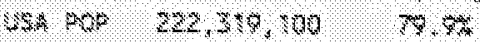

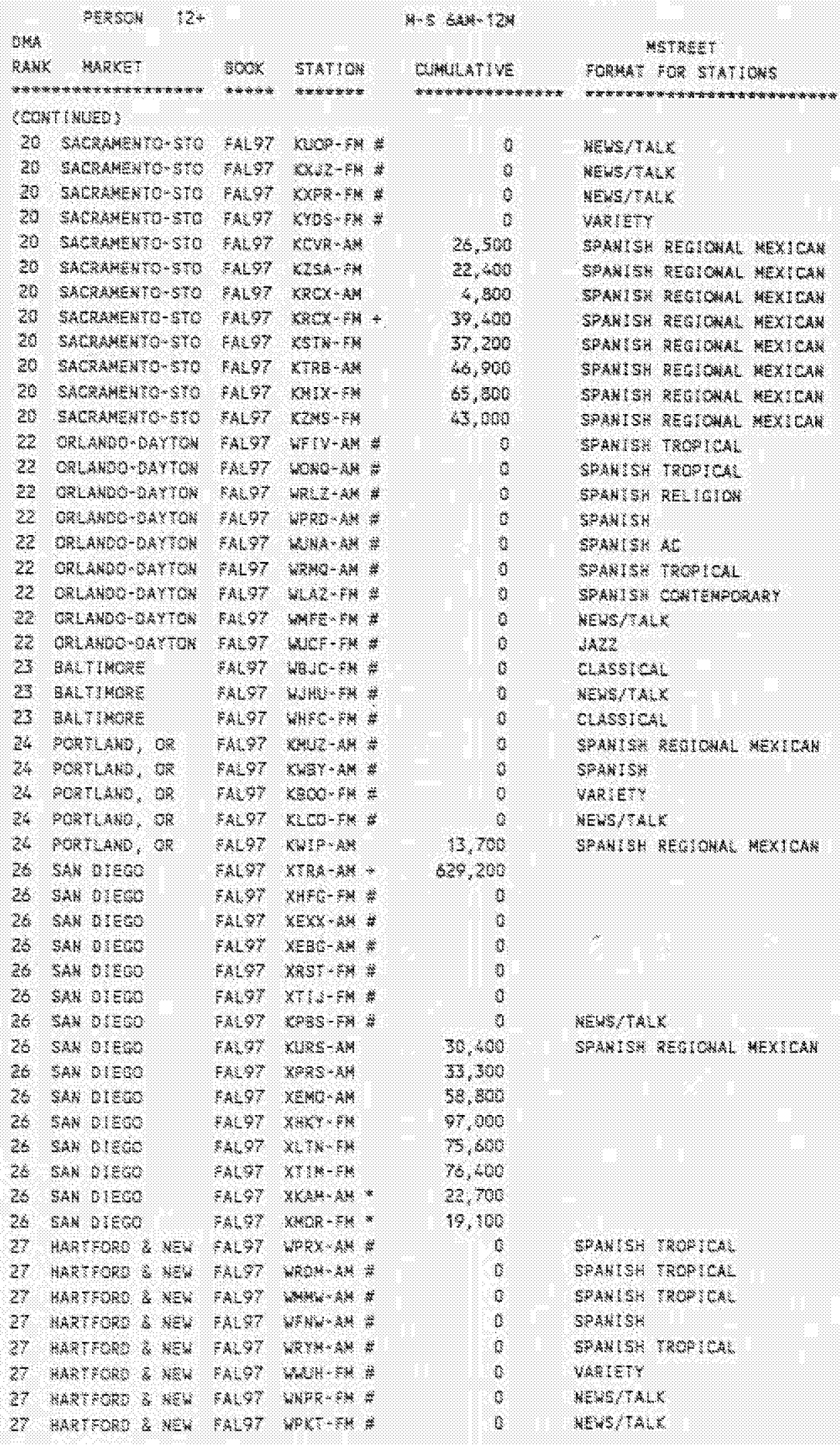




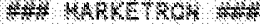

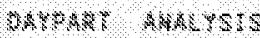

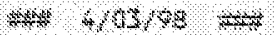

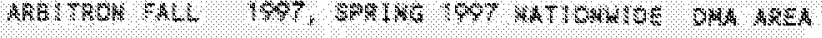

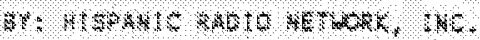

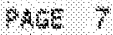

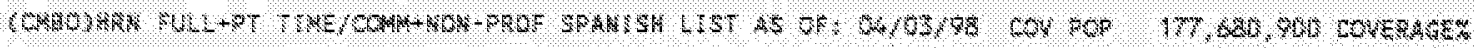

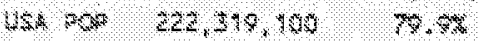

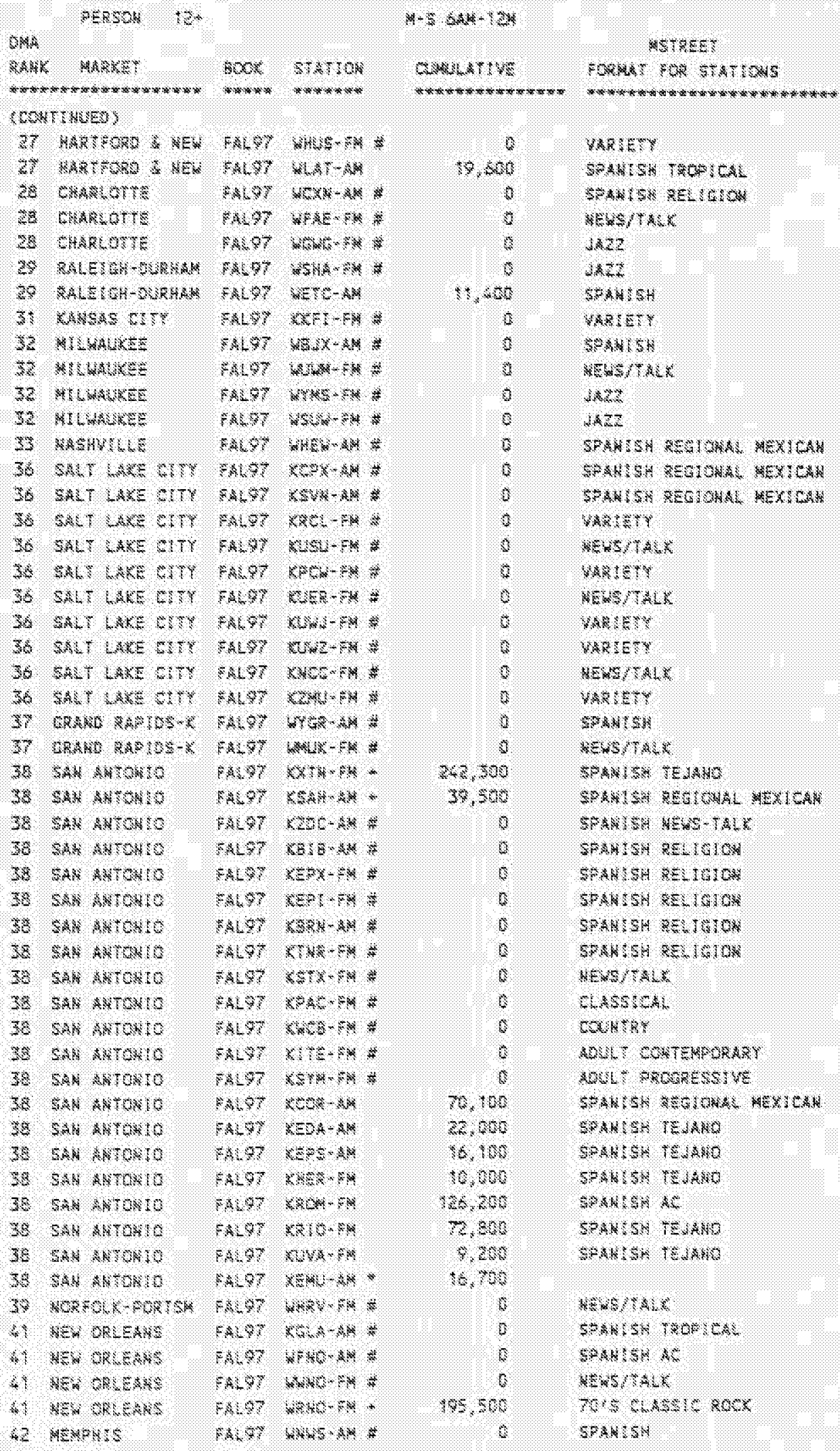




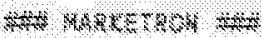

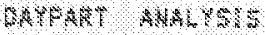
$4+40398,4$

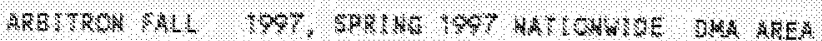

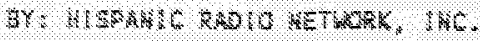

Pack :

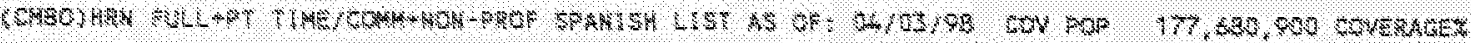

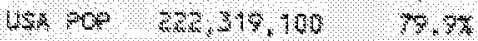

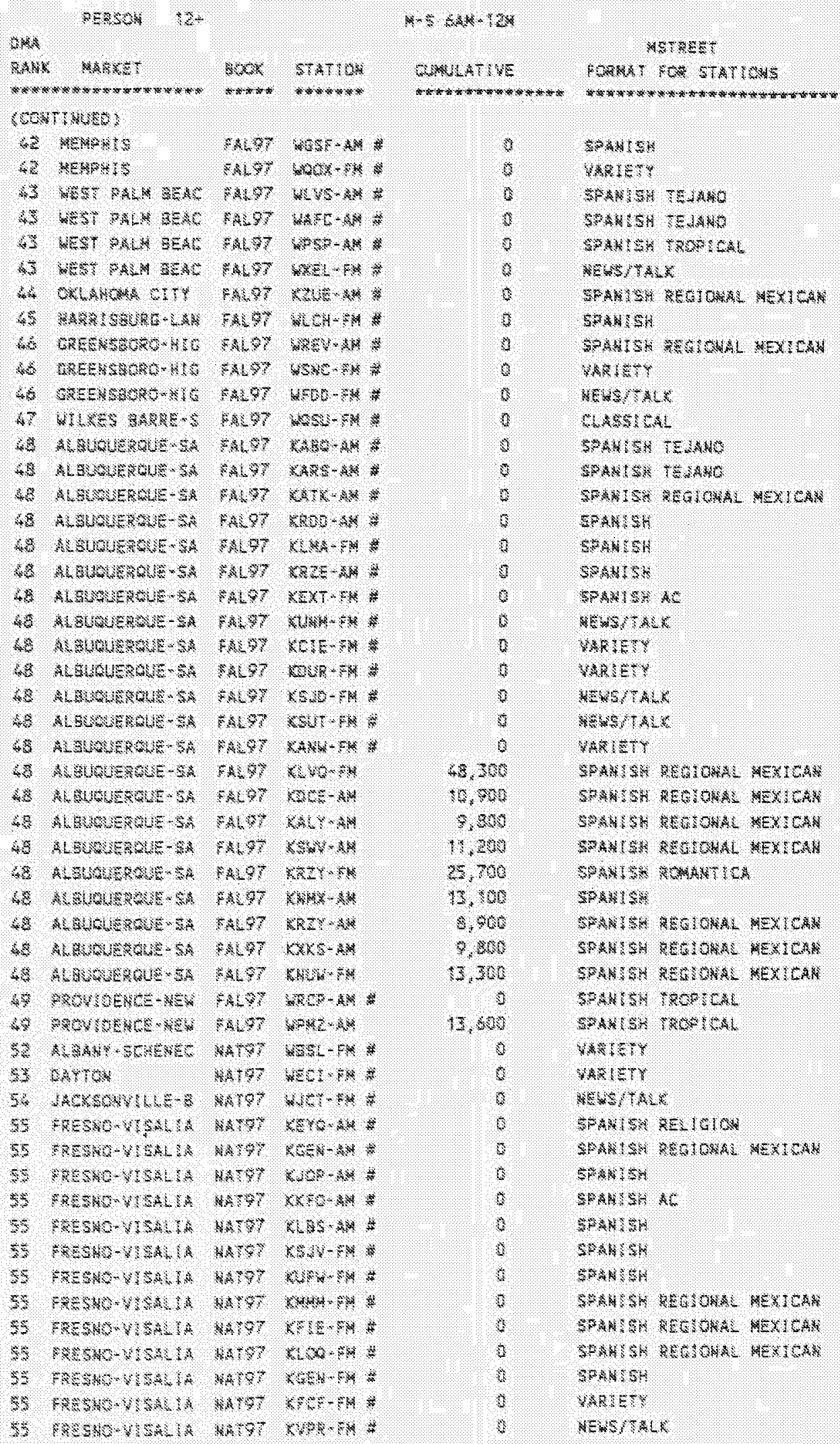


OHW MWESOH

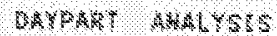
$+40,1039$

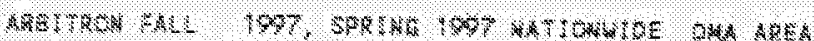

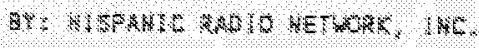

+ast 3

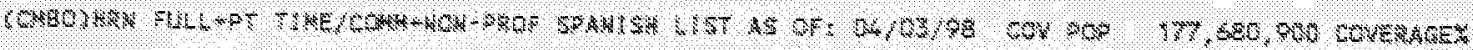

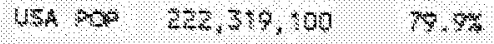

\begin{tabular}{|c|c|c|c|c|c|c|}
\hline $0 \times 4$ & 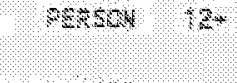 & & & & $\mathrm{m}+3+646 \cdot 124$ & 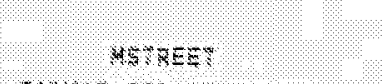 \\
\hline Renk & 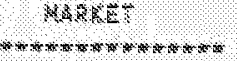 & 600 & $4,4+6$ & & CWOUAT:S & 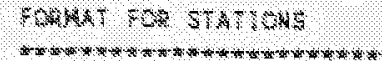 \\
\hline \multicolumn{7}{|c|}{ conts 1003} \\
\hline 95 & $72 \times 140-4 \operatorname{sen} 14$ & 40797 & 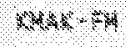 & 4 & 0 & $\mathrm{BE}, \mathrm{B}, \mathrm{O}$ \\
\hline 35 & 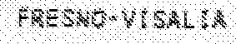 & Wx:T & CAMAB & & 27,300 & 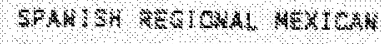 \\
\hline 35 & 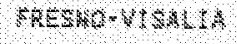 & 8608 & $1000-9 m$ & 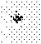 & 102,000 & 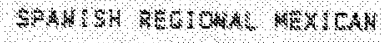 \\
\hline 35 & 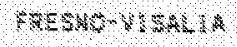 & $\times 4 \times \% 2$ & $608-44$ & & 19,800 & sPAKISK $4 \mathrm{CO}-7 \mathrm{ALC}$ \\
\hline 9 & 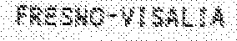 & NATQ & $2,20-98$ & & A, 70 & SPMWS S S REGTCAAL WEXICM \\
\hline 98 & MESHE-VISAL:B & MAST & 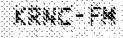 & & 68,60 & 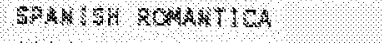 \\
\hline s) & 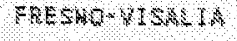 & $44 \%$ & $x+8 \times 4$ & & 1,200 & 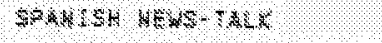 \\
\hline 55. & FBCSBO-USBRM & $401 \% 7$ & $8 x+4$ & & 10,100 & 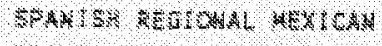 \\
\hline 35 & RRESHG UVSALKA & Mitor & $100 \times 4$ & & 17,500 & 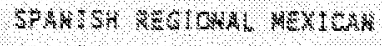 \\
\hline 3 & $8 \mathrm{BF} * 0+48 \times 4 \mathrm{~A}$ & $14+9$ & $x-4-44$ & 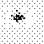 & 25.900 & SPARISH CATELFORMRY \\
\hline 85 & meso $18 \mathrm{Al} A$ & $10 \times 1 \%$ & $\mathrm{Han}+\mathrm{H}$ & & 23,300 & 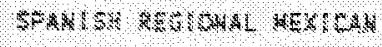 \\
\hline 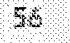 & $41 \mathrm{~L}=\mathrm{rcm} \times-\mathrm{r}$ & 4487 & $48-1+A$ & 8 & 0 & $4 a k+8$ \\
\hline 91 & $64 A \times \leq 870 \% \times 4 \%$ & $9467 \%$ & 100-48 & 8 & 1 & $48 \%$ \\
\hline 58 & $\mathrm{TULSA}$ & बatot & 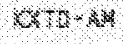 & \& & 8 & 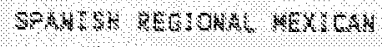 \\
\hline 59 & AISHOHOCESER & Neth & setert & \% & 2 & NeVtrak \\
\hline 60 & ALSAN & 8.9497 & $\mathrm{Mum}-\mathrm{ZM}$ & 4 & 0 & 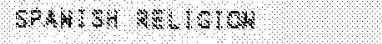 \\
\hline 6 & dusmen & $49 \% ?$ & $6 \times 2+4$ & है। & 8 & SPABst TE SABO \\
\hline 86 & nusts: & $x \times 79$ & $x k_{2}=4$ & 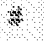 & 4 & 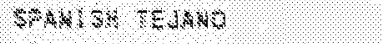 \\
\hline 80 & 4,584 & MATO & 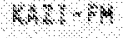 & * & 8 & 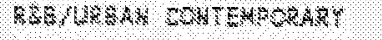 \\
\hline 6 & 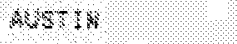 & $445 \%$ & $24+-14$ & r: & 8 & WESTrAtX \\
\hline 60 & $405+46$ & $4 \times+7$ & $x \leq 4+4$ & & 21,006 & 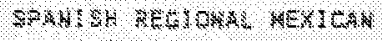 \\
\hline 60 & 105148 & xarot & $4 \times+4$ & & 90,00 & 48,4454 SEA4M0 \\
\hline 60 & N15 $1 \%$ & $\mathrm{MPT}$ & 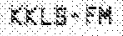 & & 34,000 & 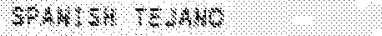 \\
\hline 4 & $4 \times 3 \times 444$ & ware? & $800-16$ & & 99,400 & 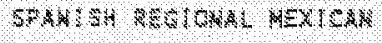 \\
\hline 81 & $4 \times s$ r 64 & 447? & 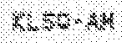 & & 30,300 & 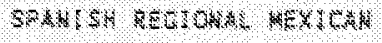 \\
\hline 65 & W1011M-4GT48 & nator & $160 \times-m$ & \& & 0 & SPABSOY XERSOHAL WEXSCAN \\
\hline 6 & 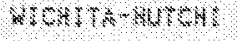 & whor & 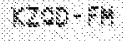 & * & s & 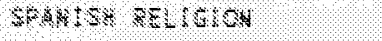 \\
\hline 8 & $45 \mathrm{CMC}$ - UMPAI & $8 \times 48$ & 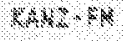 & * & i & 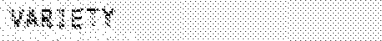 \\
\hline 65 & 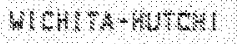 & Wasto? & 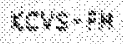 & 8 & 3 & $78-4606$ \\
\hline 68 & MLESA-HSTCH & $8 \times 107$ & 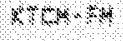 & & 2,900 & 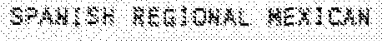 \\
\hline 71 & 1002010 & 1099 & $\mathrm{x}<4<\mathrm{N}$ & (4) & 0 & MAl $=7$ \\
\hline 71 & Honcks 4 & 3:407 & $4+8-x$ & 4 & 8 & Anos $/ 42$ \\
\hline 7 & BEWOLOS & 4087 & $90 \times-4$ & t & c & KULT 9ROLKESESVE \\
\hline 3 & STASCSBE & $4 \times 97$ & $418+4$ & 8 & 0 & AOULS PROKK \&SS YUE \\
\hline 73 & $\operatorname{sprk4}=$ & NATP & $2 \times 0-94$ & स & 8 & 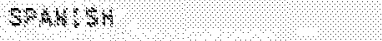 \\
\hline 3 & $89 \times 48$ & $101 \%$ & $x+8: 4$ & $\Leftrightarrow$ & 8 & ADUL PRothess W WE \\
\hline 3 & $3+1 \times k=$ & 18407 & 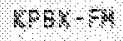 & e & 0 & WEWSMAL \\
\hline 74 & $2 \times 4+4$ & NAT & $48-8$ & 8 & 8 & BEAS $>A C X$ \\
\hline 3 & POHESFER, HT & wa9\% & $-854+1$ & t? & a & 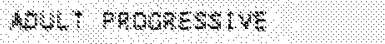 \\
\hline m: & $80,4: 5+2 \pi$, is & xart & 408,84 & 8 & 8 & MENSTOALS \\
\hline 76 & sesevport & BRTS & $10 \times 4-8$ & 4 & 8 & 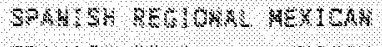 \\
\hline 8 & $3+4+24$ rorits & 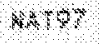 & $4,8=8,4$ & 8 & 8 & 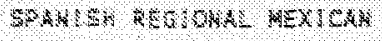 \\
\hline 78 & 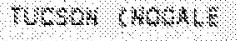 & $40+87$ & \$ 88,4 & 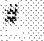 & 8 & 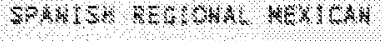 \\
\hline 8 & 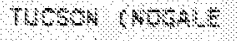 & $4+97$ & 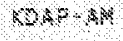 & 4 & 4 & 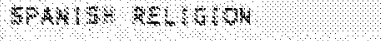 \\
\hline 18 & 145004 grows: & $40,9 \%$ & $0 \times 10.0 \%$ & is & 4 & 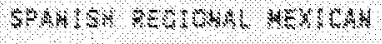 \\
\hline 78 & Mror Gomble & $4 \times 5 \%$ & CE4T,4 & 3. & 8 & $5 \times A, 4$ \\
\hline औs. & 7४80 $\angle$ HONLE & IAATH & $444-4$ & 4 & 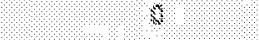 & 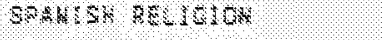 \\
\hline \%s & 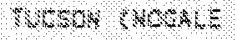 & 8.ro? & $x+4+44$ & 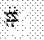 & 8 & 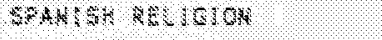 \\
\hline औ & 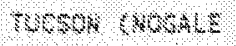 & $4 \times>7$ & $4048-48$ & 8 & 8 & 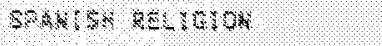 \\
\hline 78 & 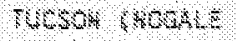 & $\mathrm{ABOB}$ & $4 \times+4$ & 4 & \% & $48 \times 5424$ \\
\hline 78 & THesh choses & $4 \times 587$ & $8 \times-4-4$ & \& & 8 & $4 \times 5 \%$ \\
\hline $\mathrm{be}$ & rusSO4 $\quad 80 \%$ \&e & $3 \times 5 \%$ & Rest-ses & a & 8 & 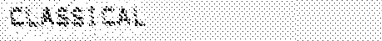 \\
\hline
\end{tabular}




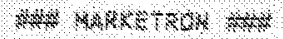

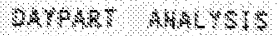
$4+40,4,4+x$

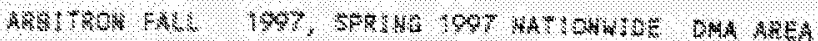

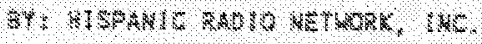

P\&\& 18

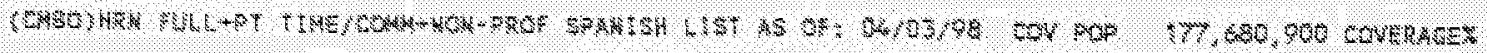
$1400,32,3,300$, 10 , st

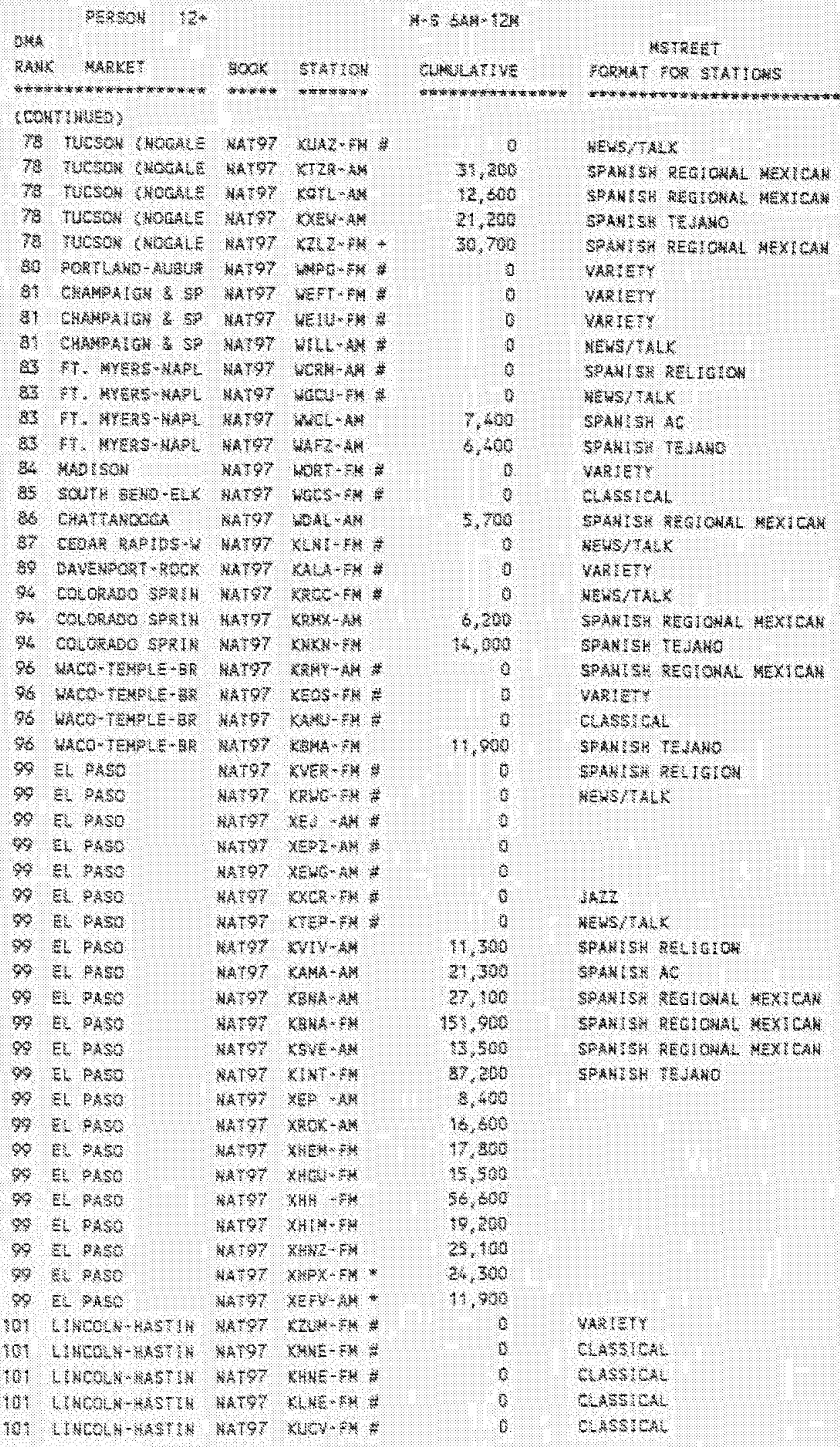




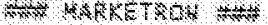

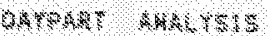

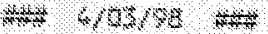

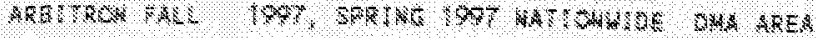

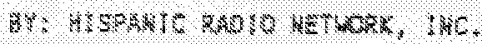

SAB 11

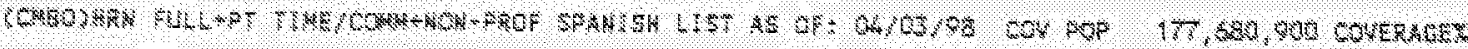

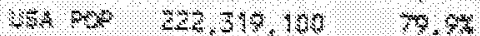

\begin{tabular}{|c|c|c|c|c|c|c|}
\hline 4 & 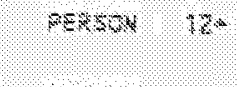 & & & & $5348+24$ & $4 s+<=1$ \\
\hline & Parser & $800 \%$ & 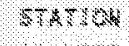 & & WuWh BTE & 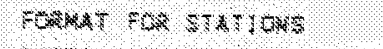 \\
\hline & 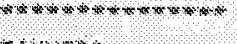 & $+\infty+\infty$ & ormonem & & 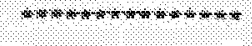 & werestion \\
\hline \multicolumn{7}{|c|}{ EEOT TUSE? } \\
\hline 001 & CINGOLN-HESTHN & $8 \times 997$ & $5046+164$ & & 12,20 & 5045046 \\
\hline 902 & $\mathrm{rr}, 46 \%$ & 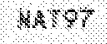 & 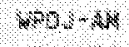 & 4 & 0 & 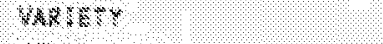 \\
\hline 90 & 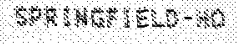 & 48507 & $4,8 s-4$ & 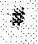 & 8 & NEWS/AIS \\
\hline+4 & SFERG & SAYPr & xPr-4M & & 4,700 & 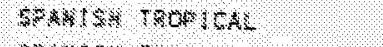 \\
\hline 103 & 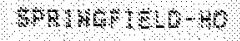 & 4,497 & $4+5<4$ & & 12,400 & 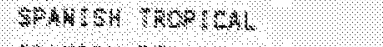 \\
\hline 104 & 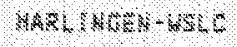 & 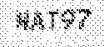 & $8 \times 4-4$ & $\%$ & 0 & $9+4015$ o $\quad 28-1610$ \\
\hline 806 & 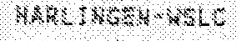 & 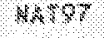 & 2an-m & s & o & sPanten ses 0,04 \\
\hline 186 & $14 R 454+4-6=$ & $4 \times 197$ & $1018 \cdot 94$ & s & 0 & 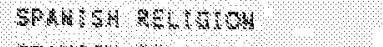 \\
\hline 3 & XNL WHENMOLO & $10 \times 107$ & $600-44$ & \& & 4 & 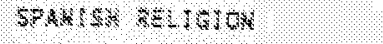 \\
\hline to & 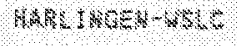 & $40 \% 9 \%$ & $\mathrm{xesc}, \mathrm{m}$ & 4 & 9 & 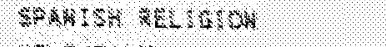 \\
\hline 106 & 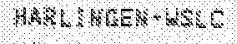 & 4h:57 & retsis-rus & 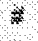 & 1 & Whate $/$ ALC \\
\hline$\$ 4$ & 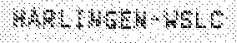 & mator & $642+4$ & * & 6 & $45, \infty / 4 \times x$ \\
\hline 14 & 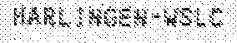 & 64597 & $x+8 x-84$ & * & 8 & 69024 \\
\hline 104 & 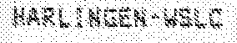 & 8487 & $680+48$ & & 13,500 & SFMNBH \&EG COAL MEXICAN \\
\hline 104 & 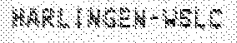 & 1440 & $24+4+4$ & & 7,700 & 58,458 \\
\hline 10 & 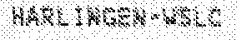 & Nast & $\mathrm{B}+28-6$ & & 6,300 & 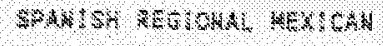 \\
\hline sos. & 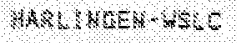 & 4atst & $\mathrm{RH} / \mathrm{TH}$ & & 8,80 & 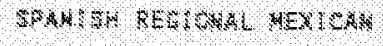 \\
\hline 106 & 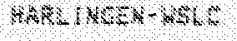 & Matry & $41 \mathrm{H} \cdot \mathrm{AM}$ & & 23,300 & 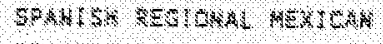 \\
\hline 104 & $148,1464-40,6$ & $4 A T 27$ & $84 x+84$ & & 8.64 & 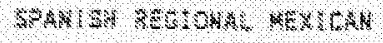 \\
\hline 106. & MACL SACEA- MSLC & 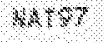 & \$4NAM & - & 206,400 & $5+46154 \quad 2 B+4 \times O$ \\
\hline 808 & $448<14<484540$ & MaTPR & $84 \mathrm{H} \cdot \mathrm{s}$ & & 8,868 & SPAKS EW $8=4,4,64$ \\
\hline 106 & HAR $M$ MSE - - CRLF & $4 \times 8 \%$ & qat +4 & & 13,384 & 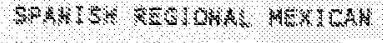 \\
\hline 184 & HAn $-466=4-48+$ & $4 a 6 \%$ & $8808 \times n$ & & 25,70 & 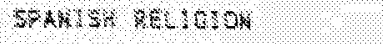 \\
\hline$\$ \%$ & NAKL BCES- BOSLE & $x+9$ & $2 \mathrm{se}-\mathrm{H}$ & + & 84,200 & 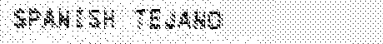 \\
\hline 804 & $14 R+14<-8-6+4<$ & BAT\% & $8 x-2=04$ & & 6,160 & SPMHTS 8416104 \\
\hline 108. & 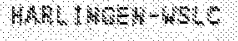 & $4 \times 57$ & $x+M-N$ & & 23,80 & 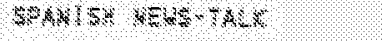 \\
\hline 104. & 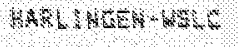 & 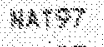 & xentar & & 9,300 & \\
\hline 104 & 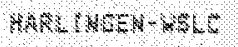 & $8 x+8 \%$ & $\times-81-48$ & * & 5,900 & \\
\hline 104 & $M B E L T R 6=4-46 L C$ & $4 \times 5 \%$ & $\mathrm{xech}-\mathrm{s} 4$ & $*$ & 6,500 & \\
\hline 185 & Mus as & 4957 & 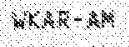 & 4 & 8 & kEN $>$ A $x$ \\
\hline$\$ 0 \%$ & $48=8-4 \times 448<$ & kint & $284-M$ & 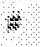 & 8 & 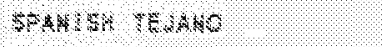 \\
\hline $10 \%$ & 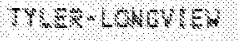 & 4487 & Btes- & $*$ & 6 & Cus5CK \\
\hline 167 & 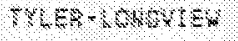 & $48 \times 7 \%$ & $x-4=4$ & * & 8 & $\sqrt{422}$ \\
\hline 108 & 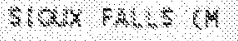 & Nator & 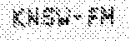 & 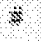 & 8 & AENBTASC \\
\hline 198 & $500 \times 846<8 \%$ & $8 x>7$ & Kans $\mathrm{FH}$ & 4 & 8 & $0-a s+4=$ \\
\hline 18 & 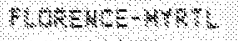 & $84+97$ & $\mathrm{NH}-\mathrm{kW}$ & 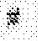 & 8 & $34 \times 6 \times 4+1$ \\
\hline 14 & 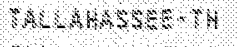 & $449 \%$ & HESO $M$ & 8 & 8 & ME WS/ 406 \\
\hline 113 & $4 \times m m-84<6<\div$ & 48007 & Ken-84 & 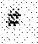 & 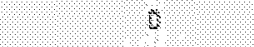 & WESPBAS \\
\hline 113 & $94 \times 0+4 \times 4 \times 46$ & $4 \times 47$ & xMrn & $\ll$ & 6 & WENSTASL \\
\hline 13 & 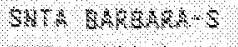 & $8.5 \% 7$ & $\mathrm{k}^{2} \mathrm{~s}+\mathrm{s}$ & * & 0 & 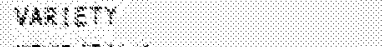 \\
\hline 85 & 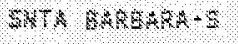 & BATP? & restans & e & s) & $48 \times 3 \tan x$ \\
\hline *8 & 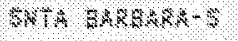 & \$.4.97 & $\mathrm{PAC}-\mathrm{B}$ & $\approx$ & 8) & 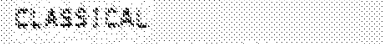 \\
\hline 189 & 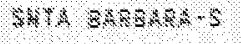 & WATPT & $\mathrm{BP}+\mathrm{AH}$ & * & 8 & 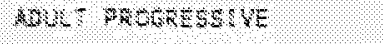 \\
\hline 115 & 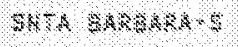 & Nator & $\mathrm{K}, \mathrm{X} \mathrm{O}-\mathrm{A}$ & & 4,90 & 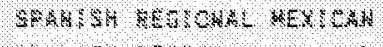 \\
\hline 119 & $34+8,8 R 8 \times 4 \times 8$ & $44 \div 37$ & $x<+8<1$ & & 3,500 & SEAUSA REGE SUAL WEXICAM \\
\hline 115 & SHR \& 8ABABA-s & nas? & 45046 & & ४००\% & $98 \times 4810$ \\
\hline 48 & $94964 \times 4 \times 43$ & $44 \times 6$ & $848-48$ & & Q.००3 & 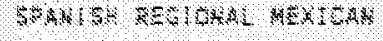 \\
\hline 48 & 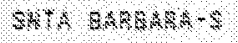 & $8 \times 4$ & $1.00 \% 4$ & & \% & 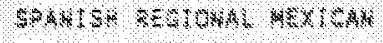 \\
\hline s & 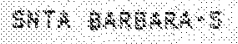 & nats & $80=-4$ & & 26.500 & 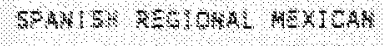 \\
\hline 18 & $567, \times \times 48 \times 4-6$ & 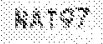 & 1 $550+48$ & & 20,96 & 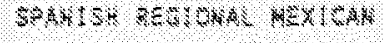 \\
\hline 13 & 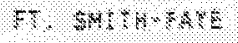 & 84A 8 & $2,20-14$ & 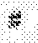 & 8 & 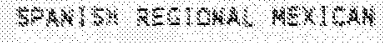 \\
\hline 4 & REA & $448 \%$ & wrom & \& & 9 & Soryisit a \\
\hline
\end{tabular}




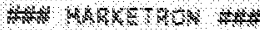

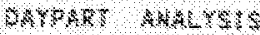

HW 400,96

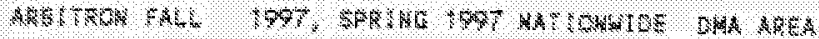

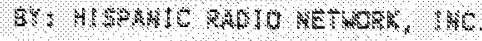

$8+12$

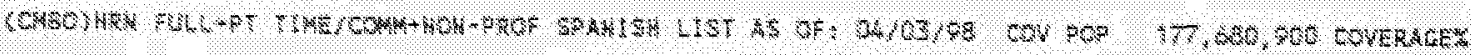

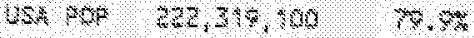

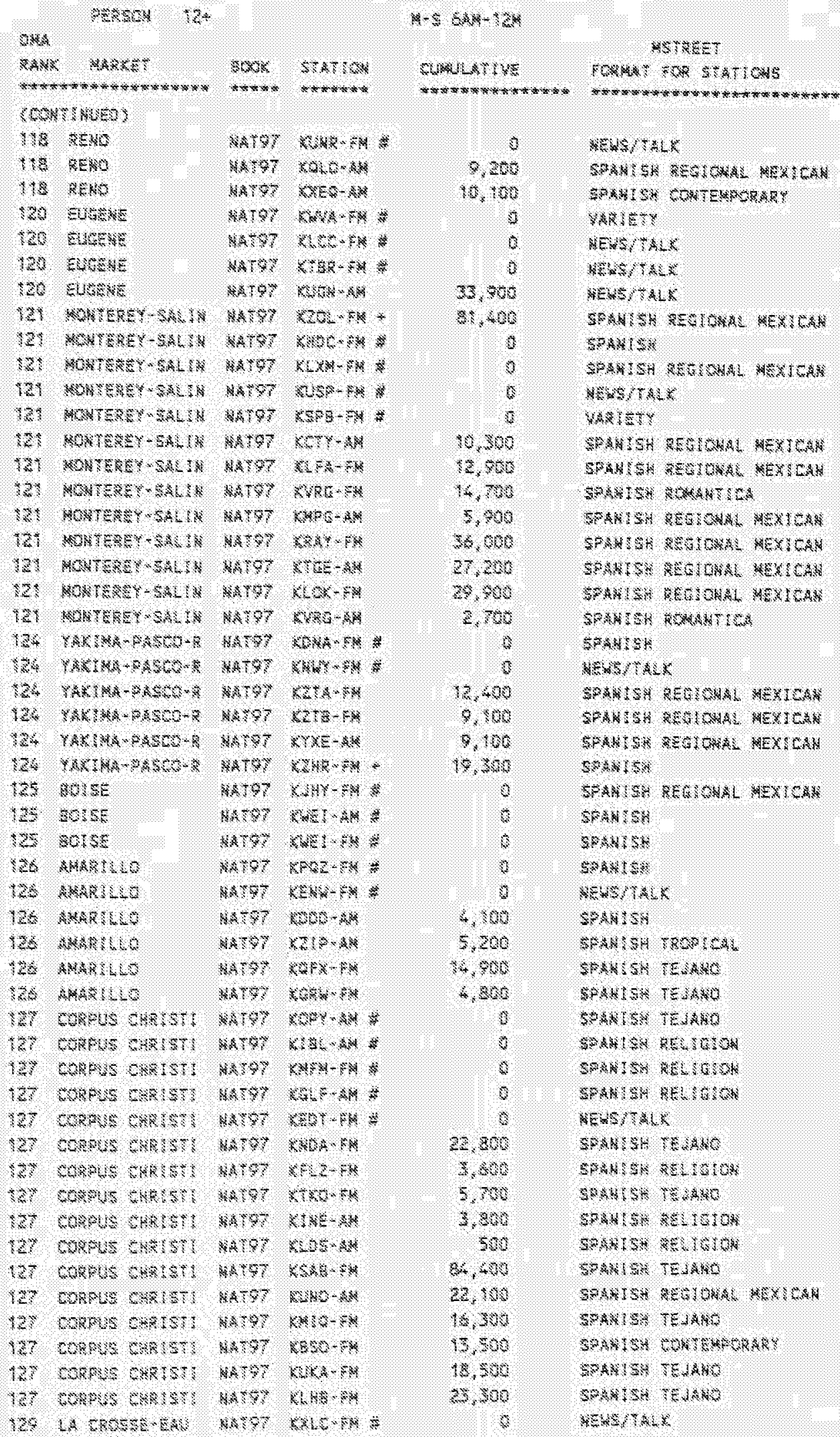




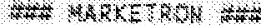

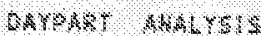

$+44034 \times$
Mas

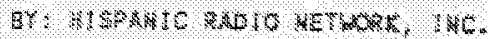

Pats

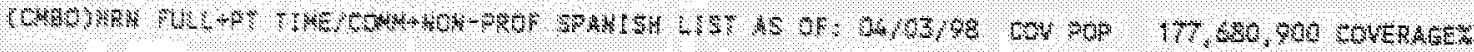

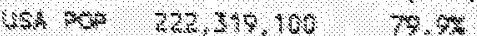

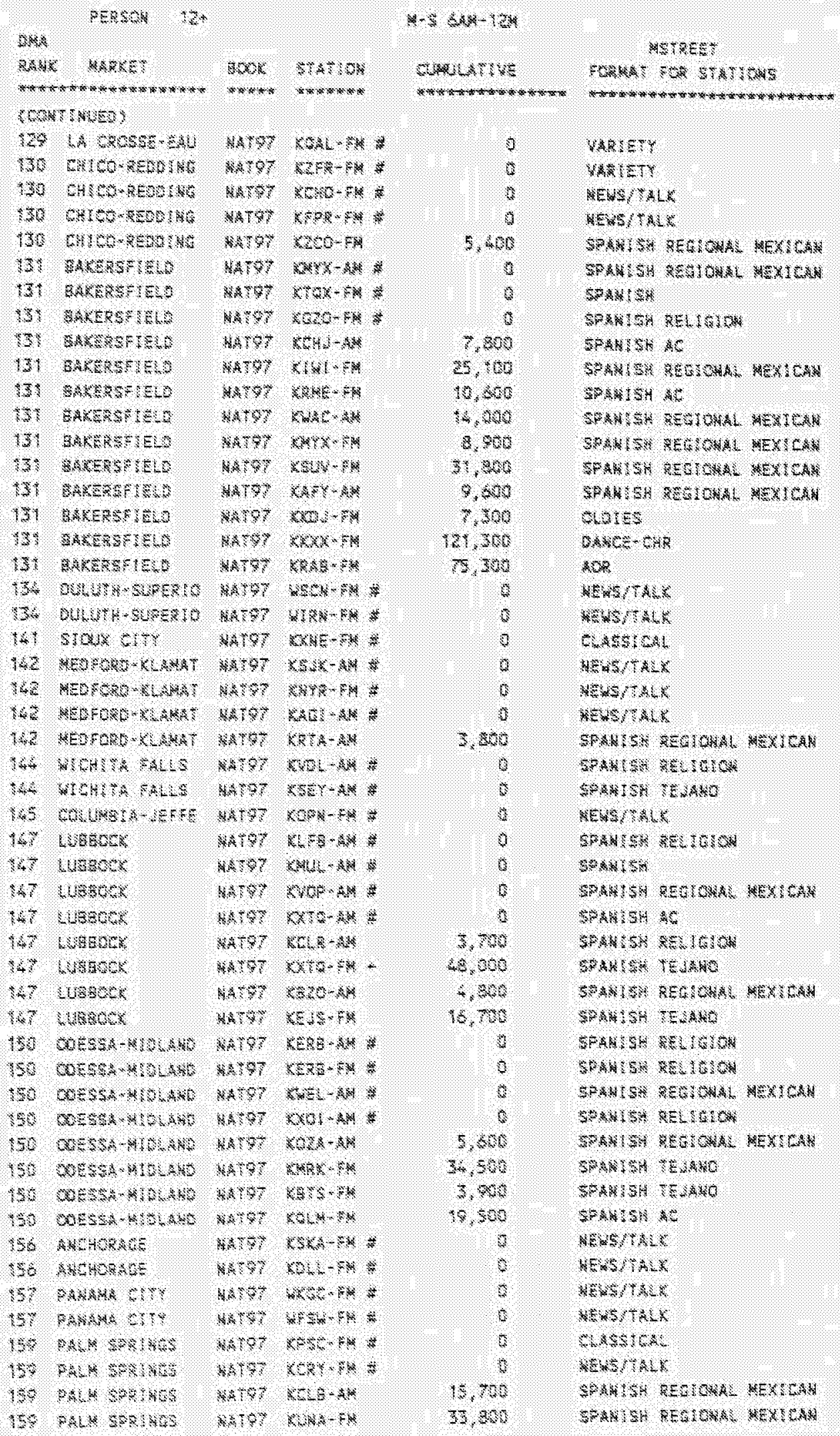




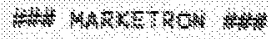
DAYPART ARMLTSIS

$4+10300$, twat

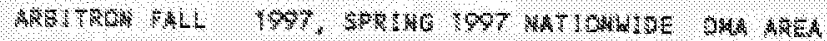

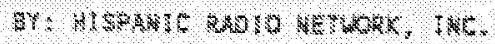

FHEE पै

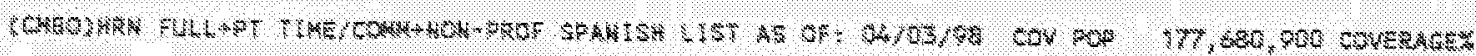

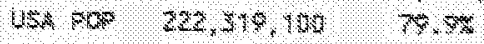

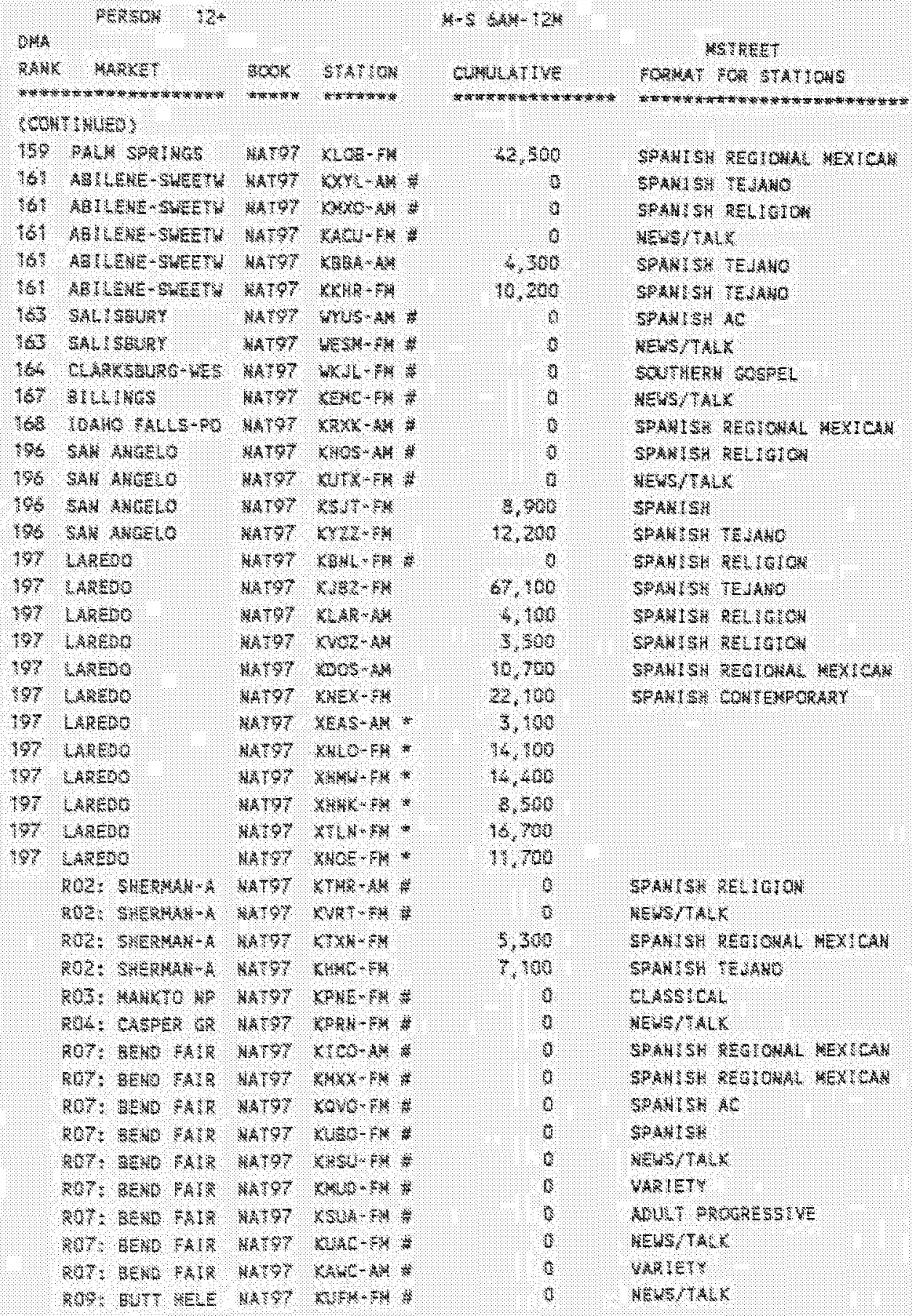

85. STATSOHS

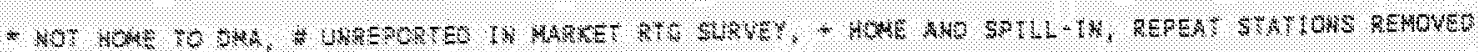

